\title{
Sabine Rivier
}

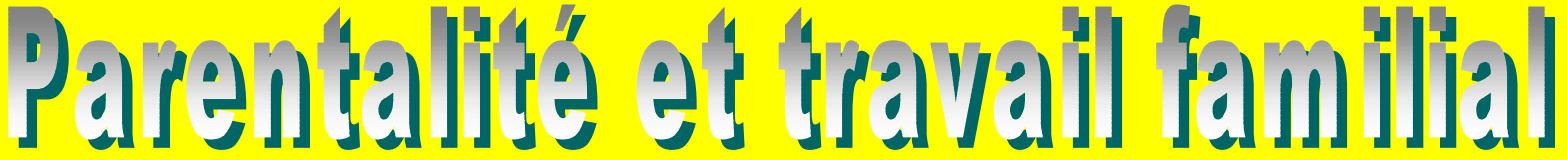 อง ไอ parentalisme,

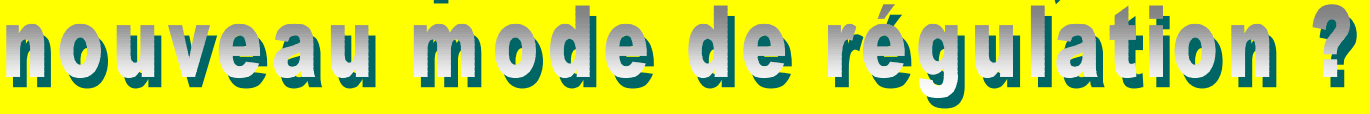

Dissertation zur Erlangung

des sozialwissenschaftlichen Doktorgrades

der sozialwissenschaftlichen Fakultät

der Universität Göttingen und der Universität Paris 1

Thèse pour obtenir le diplôme de

Doctorat en Sciences sociales

de l'Université de Paris 1 - Panthéon - Sorbonne

et de l'Université de Göttingen (Allemagne) 
Université de Paris 1 - Panthéon-Sorbonne

Sciences sociales

Discipline : Sociologie
Université de Göttingen (Allemagne) Georg-August-Universität Göttingen

Sozialwissenschaftliche Fakultät

\title{
Parentalité et travail familial en France et en Allemagne
}

\author{
- le parentalisme, \\ nouveau mode de régulation ? -
}

Thèse pour obtenir le diplôme de Doctorat en Sciences sociales de l'Université de Paris 1 Panthéon - Sorbonne (arrêté du 30mars 1992)
Dissertation zur Erlangung des sozialwissenschaftlichen Doktorgrades der sozialwissenschaftlichen Fakultät der Universität Göttingen

Présentée et soutenue publiquement le 14 juin 2002 à Göttingen par Sabine RIVIER (née à Bourges)

Tag der mündlichen Prüfung: 14. Juni 2002

\section{Jury}

Mme Marie-Agnès BARRERE-MAURISSON, Dr., Chercheur CNRS, MATISSE-Paris 1

M. Franz-Xaver KAUFMANN, Prof. em. Dr., Université de Bielefeld (Allemagne)

M. Claude MARTIN, Prof. Dr., Chercheur CNRS, LAPPS-Rennes, rapporteur (Zweitgutachter)

Mme Ilona OSTNER, Prof. Dr., Université de Göttingen (Allemagne), rapporteur (Erstgutachter)

\section{Directeurs de thèse}

Mme Marie-Agnès BARRERE-MAURISSON, Université de Paris 1

Mme Ilona OSTNER, Université de Göttingen 


\section{TABLE DES MATIERES}

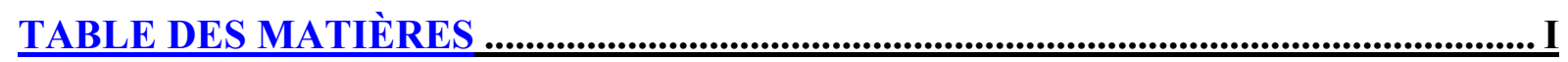

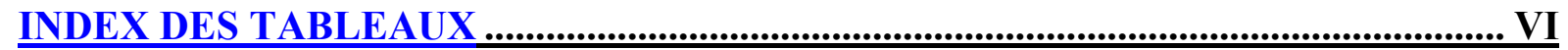

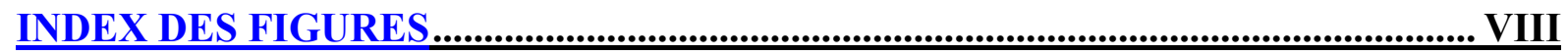

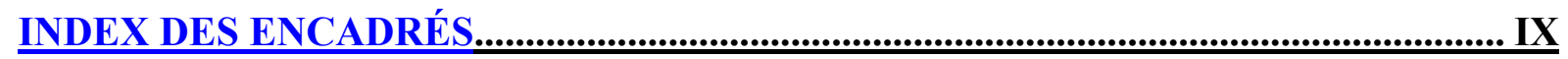

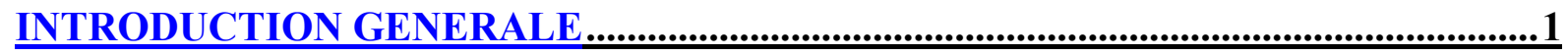

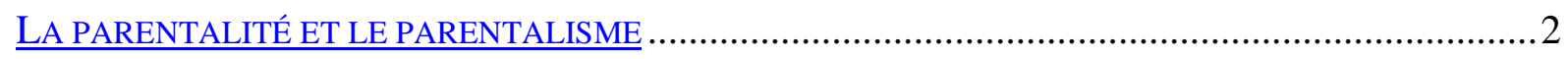

LA PARENTALITÉ AU CENTRE DU LIEN FAMILIAL................................................................

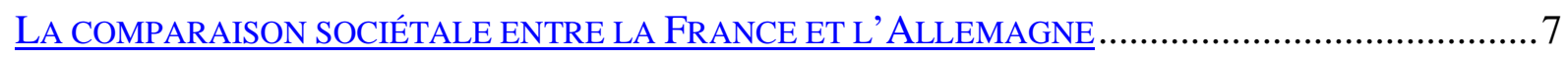

CHAPITRE I LE CADRE THÉORIQUE ET LES FORMES DE PARENTALITÉ : LA PARENTALITÉ EXCLUSIVE ET LA PARENTALITÉ CONCILIÉE ........................11

SECTION I.A LE CADRE THÉORIQUE ET CONCEPTUEL DE LA PARENTALITE................13

SOUS-SECTION I.A.1 LE CONJUGAL, L'ENFANT ET LE PARENTAL .....................................13

I.A.1.1 La famille : un groupe social universel ………….....................................................13

I.A.1.2 La famille nucléaire et la parenté .............................................................................. 14

I.A.1.3 Le couple conjugal au centre de l'analyse.................................................................15

I.A.1.4 L'enfant comme unité de référence......................................................................... 16

I.A.1.5 La notion de genre et la notion de parental ...............................................................17

SOUS-SECTION I.A.2 LA PARENTALITÉ COMME NOUVELLE UNITÉ THÉORIQUE.................20

I.A.2.1 La parentalité : la relation entre l'enfant et ses parents..............................................20

I.A.2.2 La redéfinition des types de familles.....................................................................24

SECTION I.B LA PARENTALITÉ DANS LES STATISTIQUES DÉMOGRAPHIQUES .............26

SOUS-SECTION I.B.1 LE MAINTIEN DE LA PARENTALITÉ HORS-MARIAGE DANS

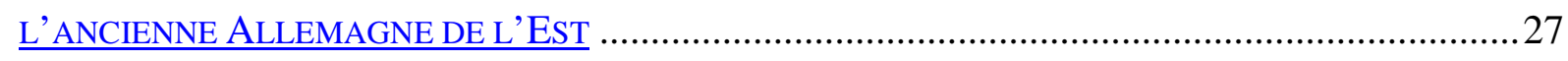

SOUS-SECTION I.B.2 LA PARENTALITÉ DANS LES FAMILLES LÉGITIMES ET NATURELLES 29 
I.B.2.1 La parentalité et le lien légitime .......................................................................... 30

I.B.2.2 La parentalité allemande au sein des familles naturelles........................................... 31

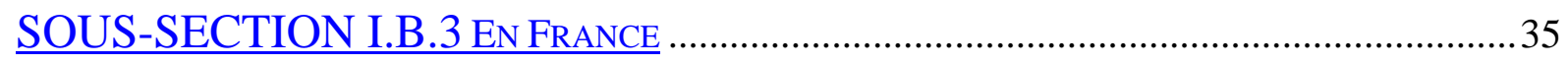

I.B.3.1 Les reconnaissances des pères et des mères et l'exercice de la parentalité ...................35

I.B.3.2 Les familles monoparentales et le contact avec le second parent.................................39

SECTION I.C LA PARENTALITÉ ET LA PARTICIPATION AU MARCHÉ DU TRAVAIL DES

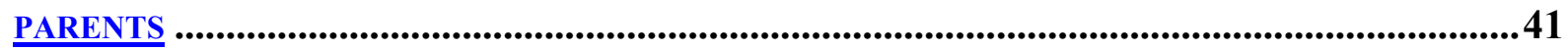

SOUS-SECTION I.C.1 L'ACTIVITÉ PROFESSIONNELLE DES COUPLES PARENTAUX EN

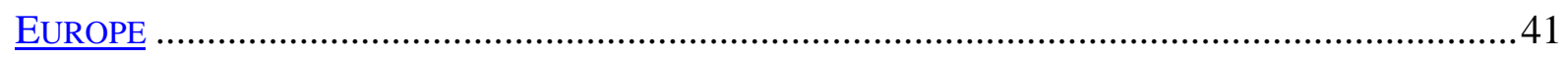

SOUS-SECTION I.C.2 EN AlLEMAGNE ..................................................................... 44

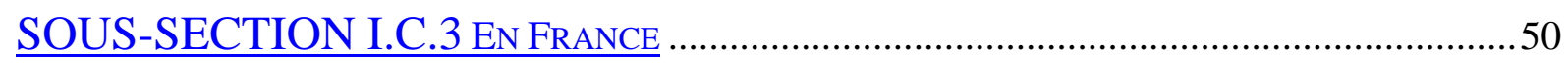

SOUS-SECTION I.C.4 LA PARENTALITÉ «EXCLUSIVE», LA PARENTALITÉ «CONCILIÉE»...52

SECTION I.D LE CADRE SOCIO-POLITIQUE ET SA SIGNIFICATION POUR LA

PARENTALITÉ …...........................................................................................................................55

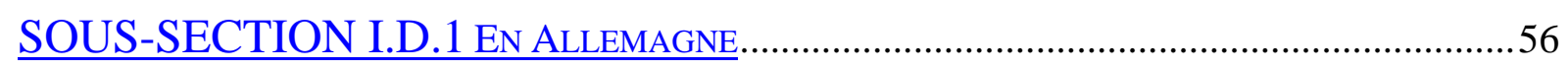

I.D.1.1 Le modèle très présent de la femme au foyer............................................................56

I.D.1.2 Les politiques familiales liées à la présence et à la charge de l'enfant ........................57

I.D.1.2.1 Le congé de maternité ...................................................................................57

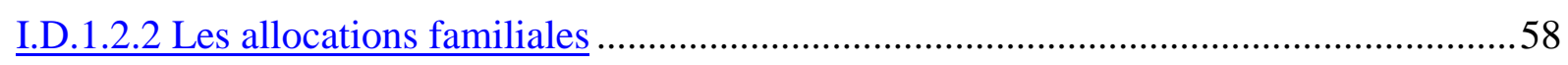

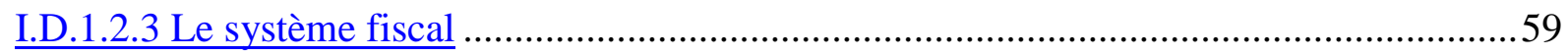

I.D.1.3 Les politiques familiales liées à une disponibilité des parents pour l'éducation des

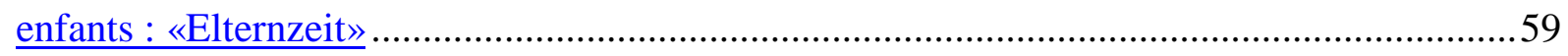

I.D.1.4 Les mesures de «conciliation» Travail-Famille ….....................................................61

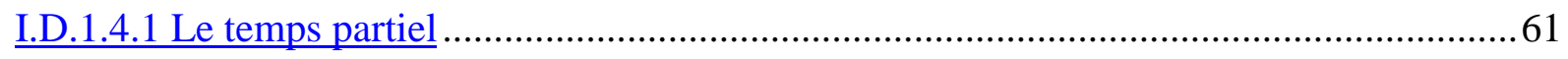

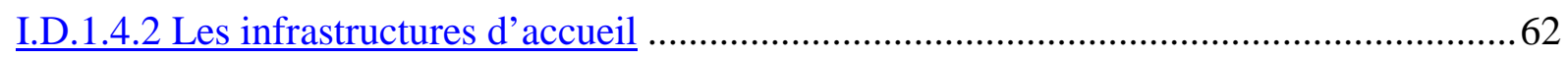

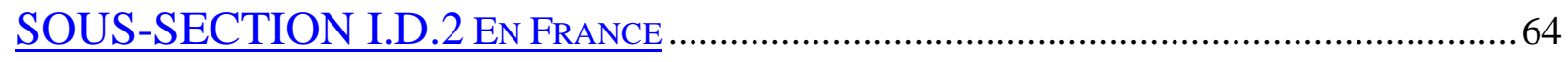

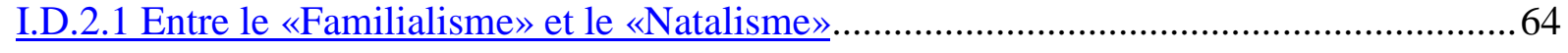

I.D.2.2 Les politiques familiales liées à la présence et à la charge de l'enfant ........................65

I.D.2.2.1 Le congé de maternité et de paternité......................................................................65

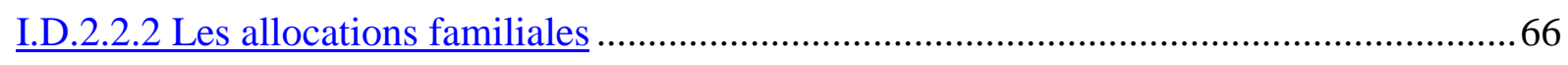

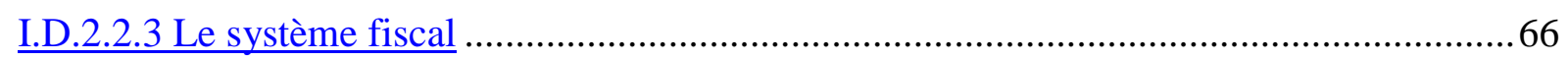

I.D.2.3 Le congé parental : pour une disponibilité des parents .............................................67

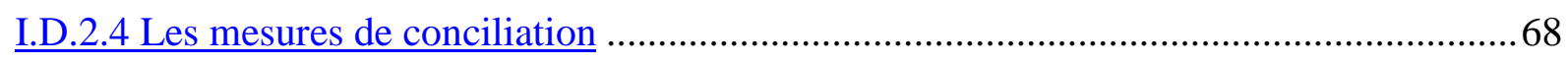

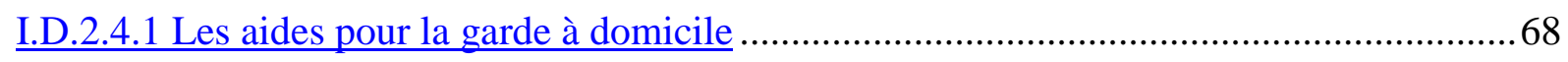

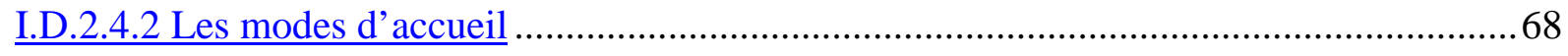

SOUS-SECTION I.D.3 LA PARENTALITÉ «EXCLUSIVE» ET «CONCILIÉE» EN FRANCE ET EN

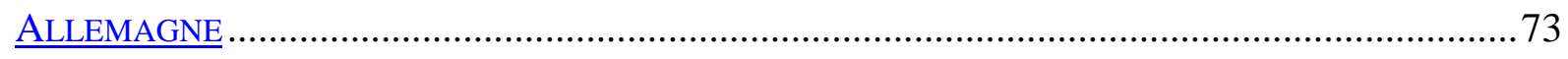

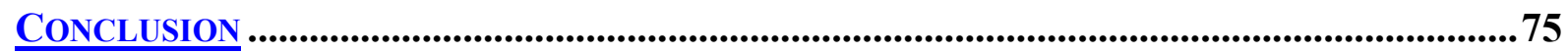




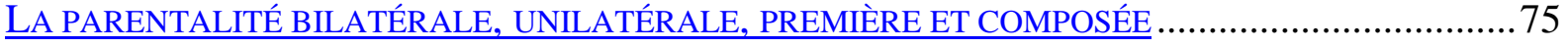

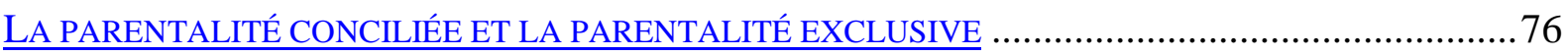

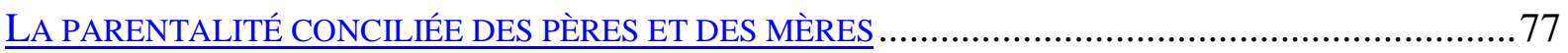

CHAPITRE II LES NORMES DE PARENTALITÉ : LA COPARENTALITÉ

FRANCAISE ET LA BIPARENTALITÉ ALLEMANDE .........................................79

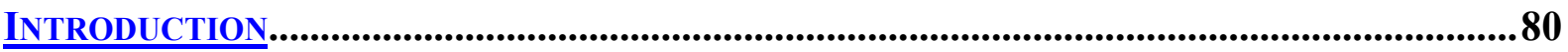

SECTION II.A HISTORIQUE DU RISQUE FAMILIAL ET DE SON TRAITEMENT SOCIAL ..83

SOUS-SECTION II.A.1 L'HISTORIQUE DU RISQUE FAMILIAL EN FRANCE ..........................84

II.A.1.1 La parentalité dans les familles légitimes et naturelles.............................................84

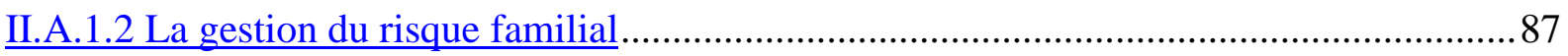

SOUS-SECTION II.A.2 L'HISTORIQUE DU RISQUE FAMILIAL EN ALLEMAGNE...................91

II.A.2.1 La parentalité en droit civil .................................................................................. 91

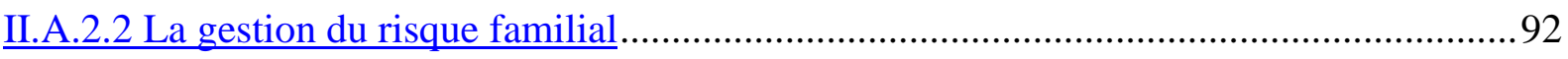

SOUS-SECTION II.A.3 LES NOUVELLES CARACTÉRISTIQUES DU «RISQUE FAMILIAL» ..... 95

SECTION II.B LA CO-PARENTALITÉ FRANCAISE ...............................................99

SOUS-SECTION II.B.1 LES PRINCIPES DE LA PARENTALITÉ DANS LES FAMILLES LÉGITIMES

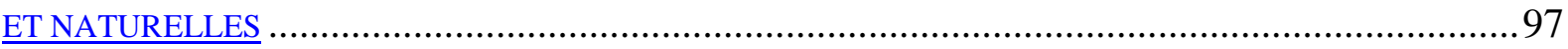

II.B.1.1 L'institutionnalisation de l'exercice bilatéral de l'autorité parentale...........................97

II.B.1.1.1 Les années 1970 et l'apparition de l'égalité parentale ...........................................97

II.B.1.1.2 Les années 1980 et la reconnaissance du principe de l'exercice conjoint de l'autorité

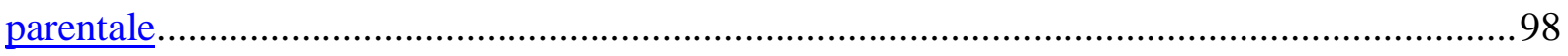

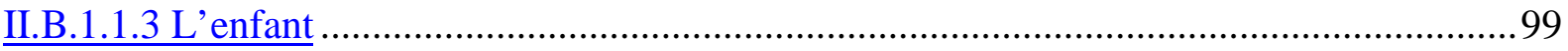

II.B.1.1.4 Les familles légitimes et naturelles ................................................................ 101

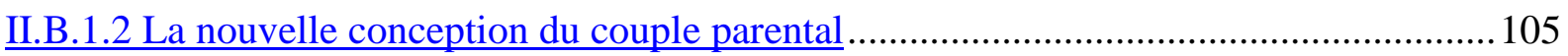

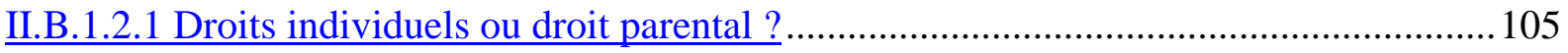

II.B.1.2.2 Les rôles parentaux et l'appréciation de leurs différences ......................................106

SOUS-SECTION II.B. 2 SÉPARATION CONJUGALE ET EXERCICE DE LA PARENTALITÉ.......107

II.B.2.1 Le partage de l'autorité parentale comme modèle de régulation de l'après divorce. 108

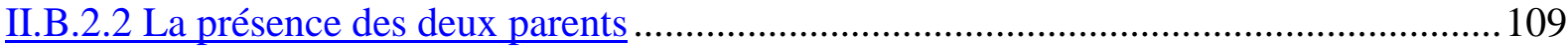

II.B.2.3 La coopération du couple parental dans l'intérêt de l'enfant ....................................111

II.B.2.4 La garde alternée et la médiation pour une plus grande coopération.........................112

II.B.2.5 Les quatre principes de la coparentalité ................................................................ 116

SOUS-SECTION II.B.3 LA GESTION SOCIALE DU RISQUE FAMILIAL .............................118

II.B.3.1 L'obligation d'entretien et l'obligation alimentaire ............................................. 118

II.B.3.2 La protection sociale du parent seul ................................................................... 118

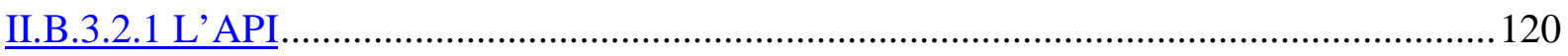


II.B.3.2.2 Les conditions attribution des prestations ....................................................... 123

II.B.3.2.3 Le système de recouvrement des pensions alimentaires ...................................... 125

II.B.3.3 La socialisation de la politique familiale : vers une gestion de la pauvreté...............127

II.B.3.4 L'activité professionnelle des mères et la coparentalité..........................................128

SECTION II.C LA BIPARENTALITÉ ALLEMANDE ........................................................131

SOUS-SECTION II.C.1 LES PRINCIPES DE LA PARENTALITÉ ..........................................131

II.C.1.1 L'autorité parentale conjointe dans le Code Civil....................................................131

II.C.1.2 L'intérêt de l'enfant comme vecteur de la présence des parents...............................133

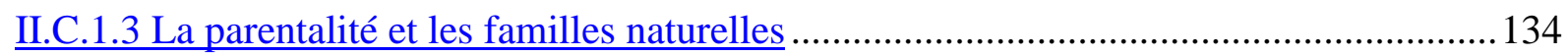

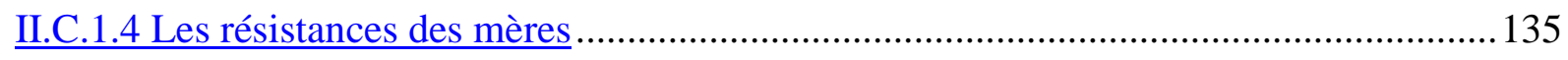

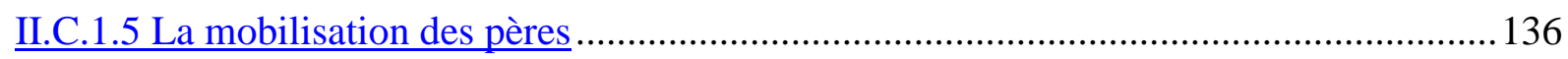

SOUS-SECTION II.C. 2 LA PROTECTION SOCIALE ET LE RISQUE FAMILIAL .....................137

II.C.2.1 Le risque familial et l'assistance sociale ................................................................137

II.C.2.2 L'obligation alimentaire des pères ................................................................... 138

II.C.2.3 Le système de recouvrement des pensions............................................................139

II.C.2.4 Les aides catégorielles destinées aux familles monoparentales ...............................142

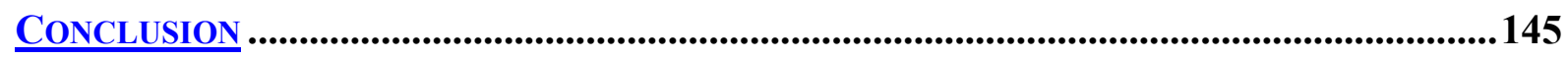

LES NORMES DE PARENTALITÉ : LA «COPARENTALITÉ» ET LA «BIPARENTALITÉ».....................145

Le couple parental et le principe de «non-conflictualité» (en Allemagne) et de partenariat (en

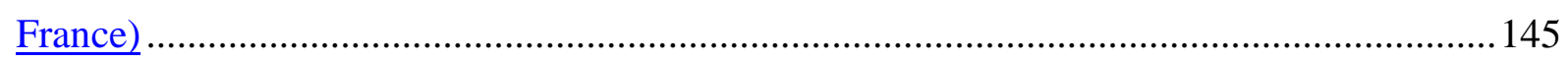

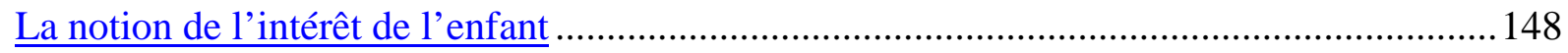

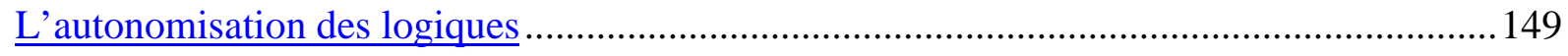

LE PARENTALISME COMME MODE DE RÉGULATION DU RISQUE FAMILIAL DEPUIS LES ANNÉES

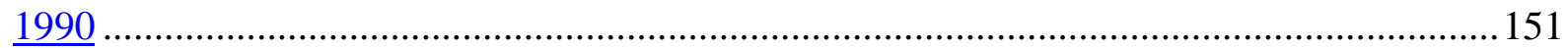

La «monoparentalité à la décharge du père» .................................................................... 151

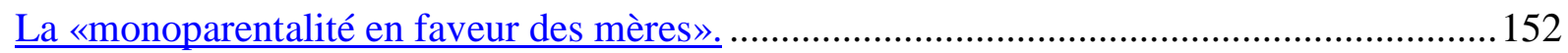

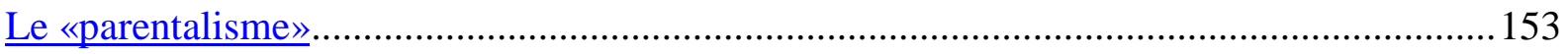

CHAPITRE III LES PRATIQUES DE PARENTALITÉ DANS LES FAMILLES ET LE

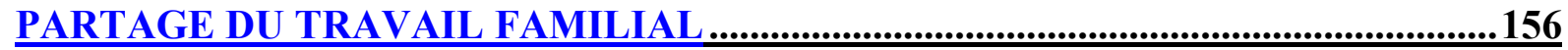

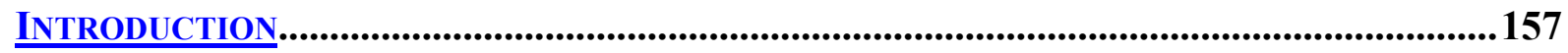

SECTION III.A LE PARTAGE DU TRAVAIL FAMILIAL EN FRANCE ...........................161

SOUS-SECTION III.A.1 LA PARENTALITÉ AU SEIN DU DOMESTIQUE DANS LES ANNÉES

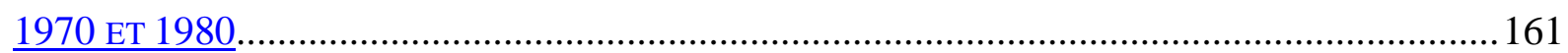

SOUS-SECTION III.A.2 LE TRAVAIL PARENTAL, LE TRAVAIL DOMESTIQUE ET LE TRAVAIL

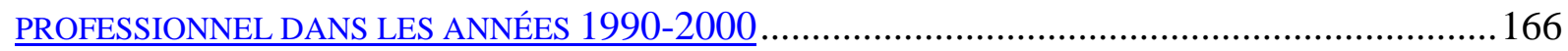

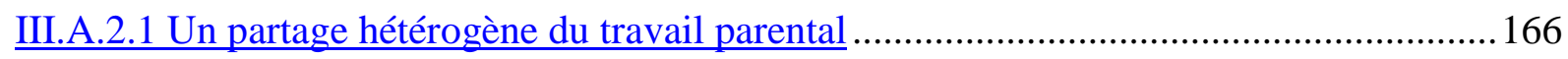

III.A.2.2 Le temps parental et le temps professionnel....................................................... 170 
III.A.2.3 Le travail domestique des pères (1980) et des mères (1990)...................................173

SOUS-SECTION III.A.3 LA DÉLÉGATION DU TRAVAIL FAMILIAL..................................179

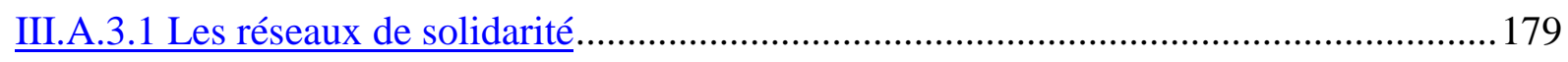

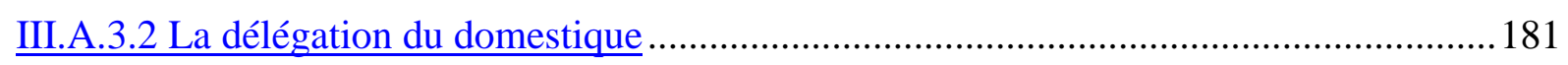

III.A.3.3 La délégation du parental............................................................................. 183

SECTION III.B LE PARTAGE DU TRAVAIL FAMILIAL EN ALLEMAGNE......................189

SOUS-SECTION III.B.1 LE TEMPS PARENTAL ET LE TEMPS DOMESTIQUE DANS LES ANNÉES 1960 ET 1970.

SOUS-SECTION III.B.2 LA PARENTALITÉ ENTRE DOMESTIQUE ET PROFESSIONNEL (1990

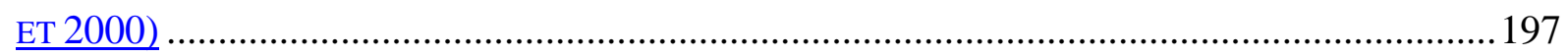

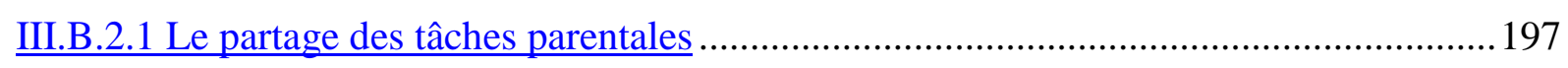

III.B.2.2 Une paternité individuelle et conjugale, une maternité consensuelle et sociale ......204

III.B.2.3 La «traditionnalisation» des rôles parentaux à la naissance de l'enfant ...................208

III.B.2.4 L'exercice de la parentalité et la «traditionnalisation» des rôles domestiques ........208

III.B.2.5 L'exercice de la parentalité et la situation professionnelle des parents ...................213

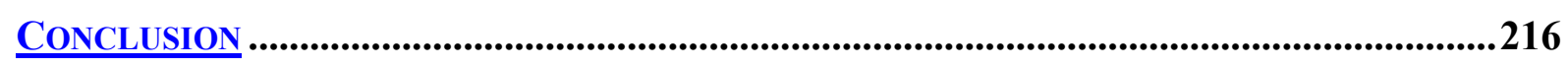

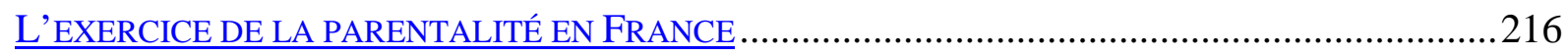

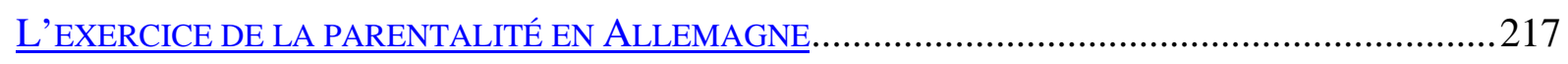

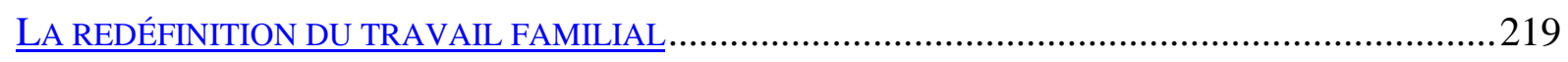

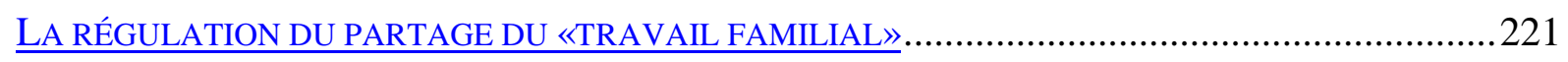

CONCLUSION : LE PARENTALISME COMME MODE DE RÉGULATION ?.......224

LES FORMES, LES NORMES ET LES PRATIQUES DE PARENTALITÉ .................................................. 227

LE FAMILIALISME, LE FÉMINISME ET LE PARENTALISME COMME MODES DE RÉGULATION EN

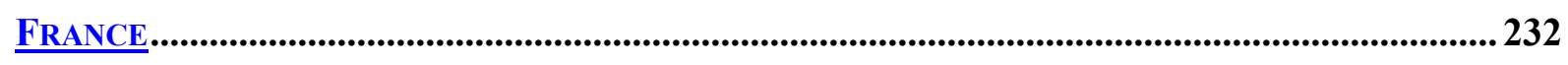

LE CONJUGALISME ET LE PARENTALISME COMME MODES DE RÉGULATION EN ALLEMAGNE ... 235

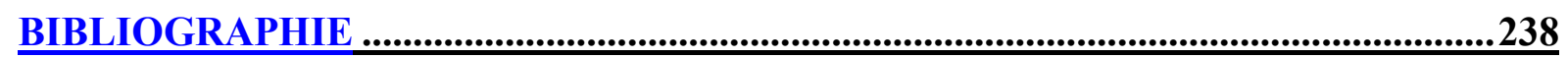

OUVRAGES GENERAUX ET SPECIALISES ................................................................238

ARTICLES ET CONTRIBUTIONS A DES OUVRAGES COLLECTIFS ..................241

NOTES, DOCUMENTS, COMMUNICATIONS, RAPPORTS ET THESES ...............247

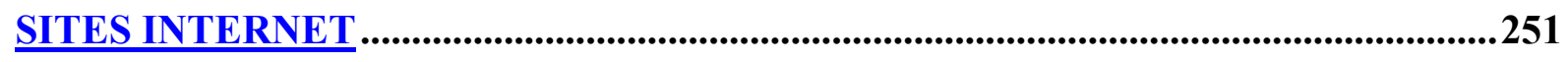

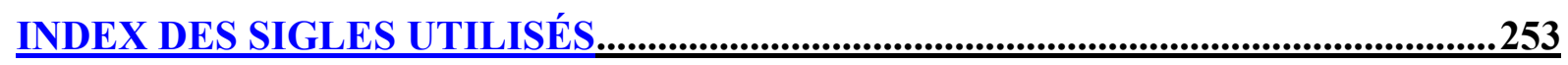

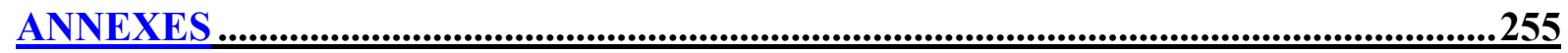




\section{INDEX DES TABLEAUX}

$\underline{\text { Tableau } 1}$ Répartition des types de famille en fonction de la situation conjugale et de la présence d'enfants, en Allemagne.....

$\underline{\text { Tableau } 2}$ Répartition des types de familles avec enfant en fonction de la nature de la relation conjugale, en Allemagne . 30

$\underline{\text { Tableau } 3}$ Rapport entre les familles naturelles avec enfant et sans enfant, en Allemagne .. 32

Tableau 4 Familles naturelles en fonction du statut matrimonial et de la présence d'enfant, comparaison entre les nouveaux et les anciens Bundesländer en 1991

Tableau 5 Proportion de couples avec et sans enfant en fonction du lien de conjugalité, en

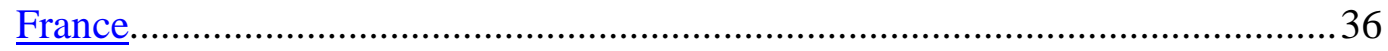

$\underline{\text { Tableau } 6}$ Répartition des mariages selon le nombre d'enfants légitimés..............................36

Tableau 7 Nombre de naissance hors mariage par rapport au nombre total de naissance depuis 1960, en France

Tableau 8 Modes pratiqués et souhaités de partage du travail professionnel dans les couples en Europe.

Tableau 9 Proportion de mères actives par rapport à l'ensemble des mères .46

Tableau 10 Taux d'activité des femmes en fonction du statut matrimonial. .47

Tableau 11 La situation professionnelle des mères, dans l'ancienne Allemagne de l'Ouest..49

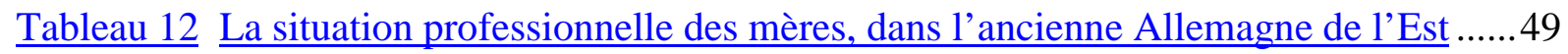

Tableau 13 Répartition des familles en fonction de leur participation professionnelle ..........51

Tableau 14 Nombre de places d'accueil en Allemagne..........................................................63

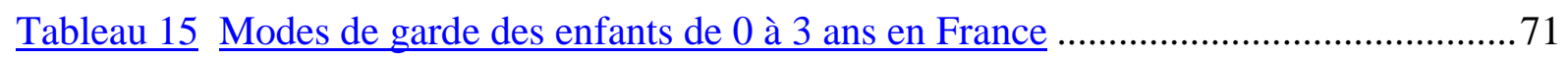

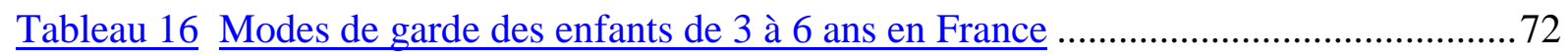

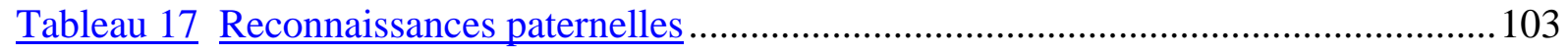

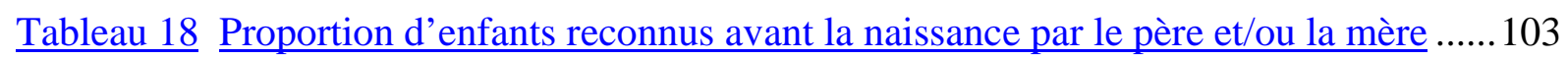

Tableau 19 Composition du revenu des familles monoparentales dans les deux pays .........120

Tableau 20 Temps parental hebdomadaire selon le type de famille.....................................167

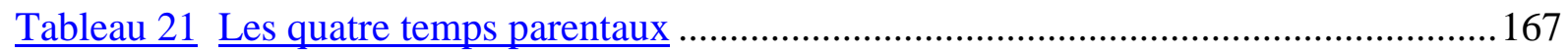

Tableau 22 Partage du temps parental dans les couples ......................................................168

Tableau 23 Temps parental individuel et temps parental total «normé»...............................169

Tableau 24 Répartition du temps professionnel et du temps parental ...................................172

Tableau 25 Temps parental selon l'activité professionnelle ................................................ 172

Tableau 26 Temps parental moyen pour les personnes vivant en couple..............................173

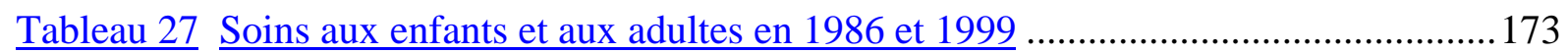

Tableau 28 La participation aux activités ménagères par sexe en 1998 ................................174

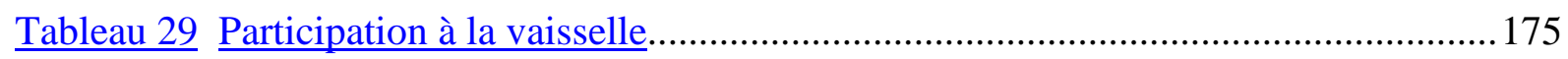

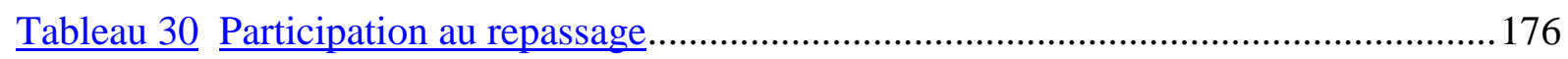


$\underline{\text { Tableau } 31}$ Volume horaire consacré au travail domestique en fonction du sexe et de la situation professionnelle

Tableau 32 Proportion de ménages qui ont reçu une aide informelle dans les quatre dernières semaines.

Tableau 33 Fréquence du recours à l'aide informelle 180

Tableau 34 Recours à l'aide informelle extérieure en fonction de la forme de famille .........180

Tableau 35 Composition du réseau qui apporte l'aide au foyer 181

Tableau 36 Recours aux services marchands par les ménages. 183

Tableau 37 Modes de gardes informels et institutionnalisés suivant l'âge de l'enfant..... 184

Tableau 38 Nombre d'heures de garde rémunérée en fonction de l'activité professionnelle 185

Tableau 39 Nombres d'heures de garde rémunérée en fonction de la forme de famille........186

Tableau 40 Modes de garde des enfants en fonction de la forme de famille. 187

Tableau 41 Temps parental des femmes actives, inactives et des hommes. 191

Tableau 42 Temps parental des hommes en pourcentage du temps parental des femmes....191

Tableau 43 Proportions relatives des activités domestiques et parentales, en fonction de la situation professionnelle 192

Tableau 44 Temps des femmes inactives et actives consacré aux taches domestiques.....

Tableau 45 Part du temps consacré à chaque tâche domestique par les femmes inactives et actives.

$\underline{\text { Tableau } 46}$ Temps absolus et relatifs consacrés par les hommes aux différentes activités domestiques en fonction de la situation professionnelle de la femme.................195

Tableau 47 Evolution du partage des tâches parentales 199

$\underline{\text { Tableau } 48} \underline{\text { Influence de la première naissance sur le partage des tâches domestiques }}$ 213 


\section{INDEX DES FIGURES}

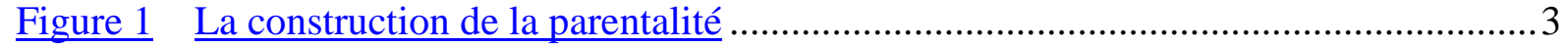

Figure 2 Les étapes de notre analyse : de la parentalité au parentalisme ................................4

$\underline{\text { Figure } 3}$ Opérationnalisation du lien Famille / Marché du travail / Etat ................................. 6

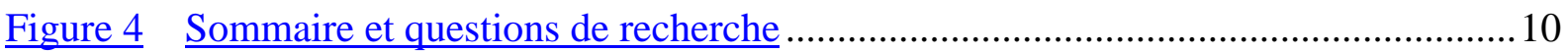

Figure 5 Trois relations qui déterminent les formes de parentalité ….................................... 12

Figure 6 La parentalité au centre des rapports de genre, de couple et de génération ............22

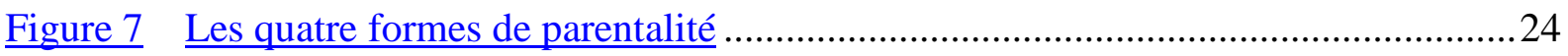

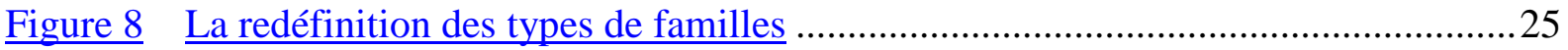

Figure 9 «Parentalité exclusive» et «parentalité conciliée» .................................................54

$\underline{\text { Figure } 10}$ Les politiques familiales et la prise en compte de la parentalité ...........................56

Figure 11 Formes de parentalité déterminées par le rapport institutionnel entre la famille et le marché du travail..................................................................................... 74

Figure 12 Les déterminants de la norme de parentalité.......................................................81

Figure 13 Chronologie des réformes du droit civil en France et en Allemagne...................146

Figure 14 Les normes de coparentalité et de biparentalité ................................................150

Figure 15 Les normes de parentalité et le nouveau mode de régulation du risque familial 154

Figure 16 Les déterminants du partage du travail parental ............................................... 157

Figure 17 Répartition des tâches domestiques selon les résistances dans le couple ............164

$\underline{\text { Figure } 18}$ Répartition des tâches parentales selon les résistances dans le couple ................170

Figure 19 Nombre d'heures d'aide rémunérée par semaine ...............................................182

Figure 20 Qui s'occupe du parental ?........................................................................198

Figure 21 Les modes de partage du travail parental, en Allemagne....................................201

Figure 22 Les souhaits des femmes relatifs aux modes de partage du travail parental.......201

Figure 23 Les logiques de partage du travail parental .......................................................203

Figure 24 Les modes de partage du travail domestique, en Allemagne .............................210

Figure 25 Les souhaits des femmes allemandes relatifs aux modes de partage du travail

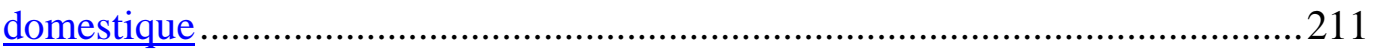

Figure $26 \quad$ L'articulation «Famille»-«Travail»..................................................................220

$\underline{\text { Figure } 27}$ Le partage du «travail familial» en fonction de la notion de travail ..................220

Figure 28 La régulation du partage du «travail familial» ...............................................223

Figure 29 Les modes de régulation des rapports sociaux en France et en Allemagne........236 


\section{INDEX DES ENCADRES}

Encadré $n^{\circ} 1$ Les évolutions du mariage dans 1'Allemagne réunifiée...................................30

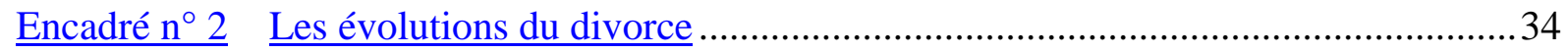

Encadré $n^{\circ} 3 \quad$ L'évolution du nombre de mariage depuis la seconde guerre mondiale ..........35

Encadré $\mathrm{n}^{\circ} 4$ L'activité professionnelle des hommes et des femmes en Allemagne en fonction de la distinction régionale Ouest/Est............................................. 44

Encadré $n^{\circ} 5$ Autres mesures catégorielles liées à la charge de l'enfant.................................66

Encadré $n^{\circ} 6 \quad$ La définition du travail parental par le groupe «Division Familiale du Travail».............................................................. 158

Encadré $\mathrm{n}^{\circ} 7$ Note méthodologique sur la mesure du travail parental et domestique, en

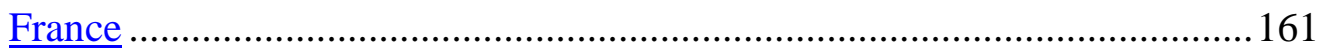

Encadré $n^{\circ} 8$ Note méthodologique sur les mesures du travail domestique et parental pour les années 1990 et 2000, en France ............................................................. 166

$\underline{\text { Encadré } n^{\circ} 9}$ Note méthodologique sur la mesure du travail domestique et parental dans les années 1960 et 1970, en Allemagne ............................................................ 189

Encadré $n^{\circ} 10$ Le budget temps en DDR dans les années 1970 ..........................................196

Encadré $n^{\circ} 11$ Note méthodologique sur la mesure du travail domestique et parental dans les années 1990 et 2000, en Allemagne ...........................................................200

Encadré $\mathrm{n}^{\circ} 12$ Les modifications de la répartition des tâches dans l'ancienne Allemagne de

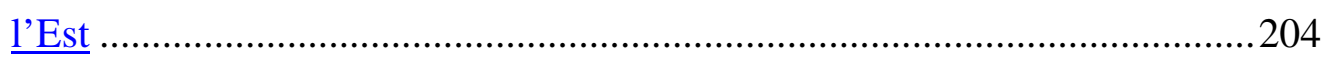

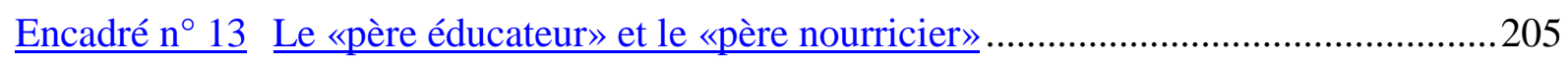




\section{Remerciements}

Mes remerciements vont tout d'abord à mes deux directrices de thèses qui m'ont soutenue et sans qui ce travail ne serait pas ce qu'il est devenu.

Je souhaite exprimer ma gratitude à Marie-Agnès Barrère-Maurisson, chercheur au CNRS-Paris I-MATISSE pour m'avoir associée au groupe Division Familiale du Travail qui m'a soutenue financièrement et matériellement, pour m'avoir fait découvrir un véritable travail d'équipe, les honneurs de la première page $\mathrm{du}$ Monde, les joies de la recherche et de la découverte. Je la remercie pour ses conseils qui ont fait mûrir ma réflexion, de sa grande disponibilité, de ses encouragements et de son accompagnement à chaque étape de mon travail.

Je suis reconnaissante à Ilona Ostner, professeur à l'Université de Göttingen, pour m'avoir associée à l'école doctorale de Göttingen, pour m'avoir offert les meilleures conditions de travail qu'il soit, pour sa confiance, son soutien inconditionnel, ses nombreuses suggestions, sa créativité et sa précieuse vision de l'essentiel.

Je remercie le DAAD, Office allemand d'échanges universitaires, pour son soutien financier et pour avoir reconnu, institutionnellement, la dimension francoallemande de mon travail.

Je remercie mon mari, pour sa présence et ses encouragements dans les moments de doute et d'abattements, pour avoir accepté un partage inégalitaire du travail familial en sa défaveur, pour avoir supporté la mise à l'épreuve du lien conjugal par un travail professionnel prenant et mes nombreux déplacements, pour ses conseils judicieux et son sens des mots.

Je pense aussi à mes parents pour leur généreuse solidarité intergénérationnelle, pour avoir toujours été à mon écoute à tout instant, pour leur soutien téléphonique et leurs e-mails, à l'aîné de mes frères et à ma grand-mère pour leurs relectures attentives, à mon frère cadet pour ses encouragements, à tous mes amis de Paris qui m'ont fait supporter la fatigue des voyages et l'éloignement, à ceux de Göttingen pour leur amitié.

Merci à vous, qui avait suivi chacun de mes pas vers l'aboutissement de ce travail, aventure scientifique et intellectuelle sur laquelle je m'étais engagée. 


\section{INTRODUCTION GENERALE}

«L'enfant, pour l'épanouissement harmonieux de sa personnalité, a besoin d'amour et de compréhension. Il doit, autant que possible, grandir sous la sauvegarde et sous la responsabilité de ses parents et, en tout état de cause, dans une atmosphère d'affection et de sécurité morale et matérielle ; l'enfant en bas âge ne doit pas, sauf circonstances exceptionnelles, être séparé de sa mère. (...)» Principe 6 de la déclaration des droits de l'enfant, proclamée par l'assemblée générale de l'Organisation des Nations unies le 20 novembre 1959.

«Les Etats parties respectent le droit de l'enfant séparé de ses deux parents ou de l'un d'eux d'entretenir régulièrement des relations personnelles et des contacts directs avec ses deux parents (...)»

Article, paragraphe 3 de la Convention internationale des droits de l'enfant ratification et adhésion par l'assemblée générale des Nations unies dans sa Résolution du 20 novembre 1989

Qu'advient-il de l'enfant après la séparation de ses parents ? Doit-on, avant tout, maintenir le contact avec sa mère comme la déclaration des droits de l'enfant le proclame en 1959 (Aidh 2002a) ou privilégier l'idée d'un couple parental à l'image de la Convention internationale des droits de l'enfant de 1989 (Aidh 2002b) ? La réponse dépend des époques, des pays, des conventions et des pratiques, mais en aucun cas elle n'est choisie par hasard. Les relations entre les membres de la famille ne s'improvisent pas, elles sont le résultat de la confrontation des normes et des pratiques : la «parentalité» (ou «Elternschaft» en allemand ${ }^{1}$ ), est une construction sociale que nous définissons comme l'ensemble des rapports sociaux entre l'enfant et ses parents. Quelles formes prend-t-elle, quels en sont les mécanismes constitutifs ? Ces questions sont d'autant plus importantes que la parentalité est au cœur du changement social et la manière dont elle est construite est un indicateur de la régulation du lien familial.

Les discours de crise des années 1990 sont de nouveau d'actualité en ce début du XXIe siècle : la famille est aux prises avec la montée des séparations et des divorces, la baisse des naissances et la montée de la cohabitation. La mise en avant, dans de nombreuses études récentes, du réseau d'entraide intergénérationnelle, n'a pu endiguer l'idée d'une déstabilisation du lien familial. Deux autres institutions, l'Etat-providence et le marché du travail, ne sont pas épargnées par les discours de crise. On parle d'un côté de la nécessité de rationalisation, des difficultés de gestion financière de l'Etat-providence, des problèmes de financement du système de sécurité sociale et des retraites, de l'autre, de la crise du pleinemploi, de la flexibilisation de l'emploi, de la précarité et du chômage. Cependant, cette interprétation, institution par institution, n'apporte qu'une compréhension parcellaire du changement social, insistant davantage sur les ruptures que sur les tendances intégratives. L'étude de la famille, plus encore, des changements du lien familial replacé dans son contexte, contribue à saisir le changement social dans son ensemble.

\footnotetext{
${ }^{1}$ Les termes en allemand concernant la famille, le marché du travail et les politiques familiales, ont été traduits et regroupés sous forme d'un glossaire bilingue à la fin du manuscrit (cf. Glossaire).
} 


\section{LA PARENTALITE ET LE PARENTALISME}

L'objet de notre travail est de contribuer à comprendre les évolutions du lien familial à partir de l'étude de la «parentalité» que nous avons défini comme concept analytique (cf. Chapitre I). Ce dernier intègre trois dimensions :

$\diamond$ la construction des relations intergénérationnelles entre chaque parent et l'enfant (le rôle de la mère et le rôle du père),

$\diamond$ la construction des rapports sexués à l'intérieur du couple parental

$\diamond$ et la construction des rapports entre le couple parental et l'enfant (la place de l'enfant).

La manière dont la «parentalité» est construite devient alors un indicateur des changements familiaux. A cet effet, nous proposons de mettre en évidence les mécanismes de la construction sociale de la «parentalité» et la figure 1 représente de quelle manière nous les avons opérationnalisés. A cette étape de notre travail, il s'agit de montrer en quoi la relation entre la famille et le marché du travail, entre la famille et l'Etat-providence, ainsi que les rapports familiaux, déterminent la «parentalité» dans ses formes, ses normes et ses modes d'exercice.

Premièrement, la «parentalité» est au cœur des rapports familiaux car elle désigne le lien social entre l'enfant et ses parents; elle dépend de la logique de genre, de la logique conjugale et de la logique générationnelle. Deuxièmement, elle est construite par la relation entre la famille et l'Etat-providence : le nombre croissant de divorces et de séparations augmente le risque de séparation parentale et menace le rapport entre l'enfant et ses parents. Devant l'ampleur de ce phénomène, ce que nous avons appelé le «risque familial» (cf. Chapitre II) est devenu un risque social à part entière et s'inscrit comme tel dans le système de protection sociale (Martin 1997a). Troisièmement, la parentalité est construite par la relation entre la famille et le marché du travail: le partage du travail parental ne pouvant se comprendre qu'en fonction du partage du travail professionnel, du travail domestique et réciproquement. Nous avons appelé ce phénomène «le partage du travail familial» (cf. Chapitre III).

La «parentalité» est ainsi à l'interface entre la famille et l'Etat-providence : «être parent» est aux frontières de ce qui relève de la sphère privée et de la sphère publique. L'éventail des prestations sociales dans le système de protection sociale français et allemand (liées à la présence de l'enfant et aux cas spécifiques des familles désunies) compose alors la parentalité. Elle est aussi à l'interface entre la famille et le marché du travail, entre la sphère de reproduction et celle de production. Les rapports sociaux entre l'enfant et ses parents se caractérisent alors en fonction des modalités de l'activité professionnelle: temps complet, temps partiel et inactivité (cf. Figure 1). 
Figure $1 \quad$ La construction de la parentalité

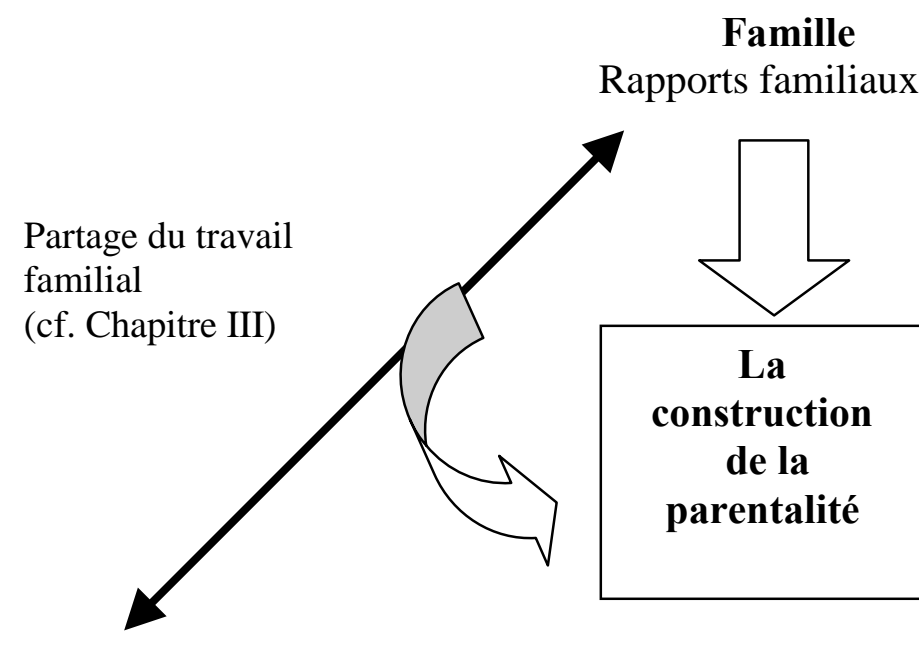

Marché du travail

Participation des parents au marché du travail

(Activité professionnelle à temps plein, à temps partiel, inactivité) (unies, désunies, légitimes et naturelles)

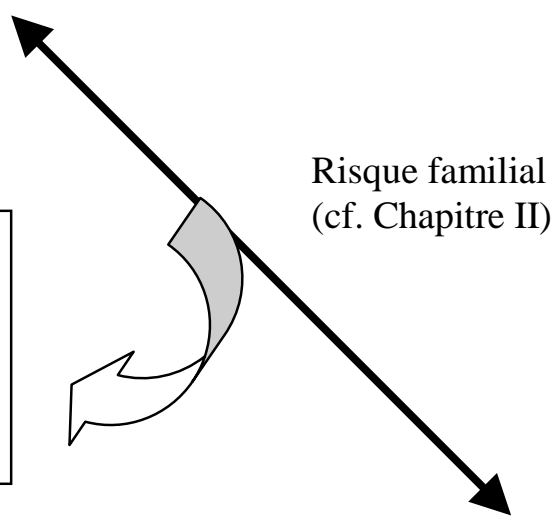

Etat-providence

Politiques familiales

La construction de la parentalité est un indicateur du changement social parce qu'elle tient compte de l'interdépendance entre le monde professionnel, la sphère publique et la sphère privée. D'où la question suivante : de quelle manière ces différents éléments déterminent-ils les formes, les normes et les modes d'exercice de parentalité ? Il s'ensuit différentes configurations :

$\diamond$ Chaque forme de participation, plus ou moins importante, des mères et des pères sur le marché du travail, agit directement sur la structure de la parentalité.

$\diamond$ De même, les aides à la conciliation de la vie familiale et professionnelle, à la prise en compte de la présence de l'enfant dans le ménage, construisent activement les modes de parentalité et influencent les arrangements familiaux.

$\diamond$ La place de l'enfant est un autre facteur de variation dans la construction de la parentalité. A ce titre, le droit civil et le droit social jouent un rôle normatif majeur par la production d'une norme de l'intérêt de l'enfant et de ses droits. Elle intervient dans la gestion des divorces et dans les critères d'attribution des prestations familiales. Pour identifier cela, on se réfère à la construction du risque familial, à la gestion du «Cash» et du «Care» relatifs aux enfants.

$\diamond$ Enfin, la redéfinition de l'identité masculine et paternelle depuis les années 1980, modifie de façon importante la définition des rôles sexués et des rôles parentaux. Ceci devient visible dans les pratiques familiales, le partage des tâches domestiques, professionnelles et parentales, ainsi que dans les discours qui les accompagnent.

Ainsi donc, il s'agit d'étudier les processus déterminant les formes, les normes et les modes d'exercice de la parentalité en fonction des situations familiales, des modalités du marché du travail, et du cadre socio-politique. La manière dont la parentalité est construite 
matérialise alors un mode de régulation du lien familial que nous avons identifié, grâce à la comparaison sociétale, comme étant le «parentalisme» ${ }^{2}$ (cf. Figure 2).

Figure 2 Les étapes de notre analyse : de la parentalité au parentalisme

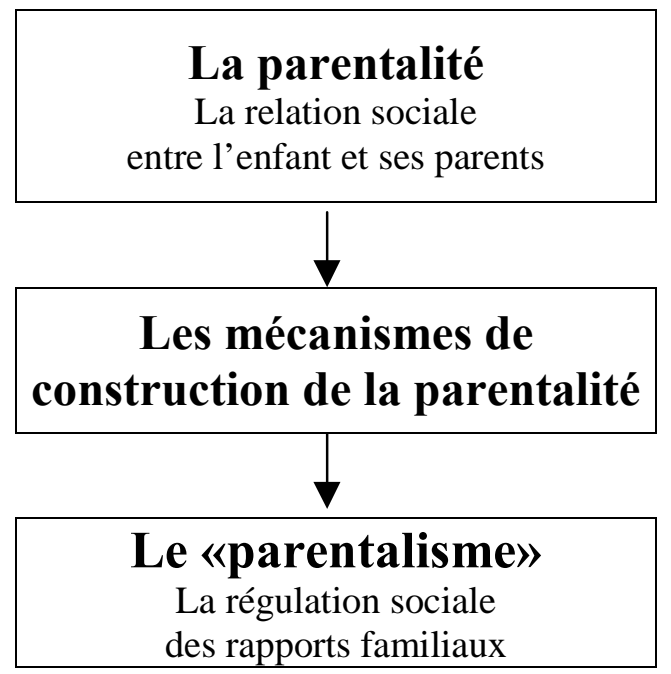

Le «parentalisme» ainsi identifié par nous, fait suite à un ensemble de modes de régulation des «affaires de famille» ${ }^{3}$ déjà identifiés auparavant :

$\diamond$ nous rappelons ici les définitions du «Familialisme», où la «famille» était au centre de la régulation, laquelle s'effectuait alors en direction d'un type particulier de famille : celle «fondée sur l'autorité du père et du mari» et de la «famille nombreuse» ;

$\diamond \mathrm{du}$ «Conjugalisme» ${ }^{4}$ qui décrit la régulation basée sur le «conjugal», en référence au «couple légitime»;

$\diamond$ du «Féminisme» fondé sur les «rapports de genre», qui faisait de la «femme» l'objet de l'action politique et sociale ;

$\diamond$ Et enfin donc, du «Parentalisme» qui place les «liens de parentalité» au centre de la régulation, qui s'oriente vers une norme fondée sur «les relations de l'enfant avec ses deux parents quelle que soit l'histoire familiale».

Ces modes de régulation autour de la «question familiale» (Martin 1997) sont des construits sociaux qui varient suivant les sociétés et les époques. De la même façon, le «parentalisme», qui est apparu en France et en Allemagne au cours des années 1990, y est de nature différente, laquelle ne se comprend que dans son contexte sociétal (cf. Conclusion Figure 29).

\footnotetext{
${ }^{2}$ Le groupe Division Familial du Travail a identifié et nommé pour la première fois ce nouveau mode de régulation (Barrère-Maurisson, Rivier 2002).

${ }^{3}$ D'après le titre de l'ouvrage écrit sous la direction de F. de Singly et F. Schultheis «Affaires de famille, Affaires d'Etat» (Singly de, Schultheis 1991).

${ }^{4}$ Ainsi nommé par nous pour décrire le cas de l'Allemagne depuis la période d'après-guerre (cf. Conclusion).
} 


\section{LA PARENTALITE AU CENTRE DU LIEN FAMILIAL}

Premièrement, nous avons placé la «construction de la parentalité» au centre même du lien familial et de ces évolutions. Cette démarche permet de comprendre l'ampleur des changements car nous identifierons tantôt la manière dont ils touchent les familles unies, qu'elles soient naturelles ou légitimes, tantôt les familles touchées par le phénomène de la séparation conjugale.

Notre objet est l'étude du lien social qui unit l'enfant à ses parents (cf. Figure 3 - ligne en gras -). Dans le but de déterminer ses modes d'exercice et son mode de régulation, nous retiendrons deux structures familiales où l'exercice de la parentalité varie par construction : nous étudions, en miroir, les situations des familles unies et désunies (cf. Figure 3). Les formes de parentalité s'interprètent au cœur de la mutation du lien familial : on privilégie ainsi «le centre et l'ordinaire » (Théry $1998: 19)$.

Deuxièmement, la famille n'est pas traitée comme un lieu autonome, au contraire, elle est replacée au cœur du contexte social. En ce sens, la parentalité a été discutée, non seulement comme un phénomène qui émerge des pratiques de la famille, mais également comme un phénomène social. Ainsi les mutations de la famille sont mises en relation avec les mutations du marché du travail et de l'Etat.

Le lien entre la famille et l'Etat (cf. Figure 3 - ligne en pointillés ) se compose :

$\diamond$ d'un côté des politiques familiales qui s'adressent respectivement à l'enfant, à la mère et au père. Au cours de cette analyse, nous recourons aux catégories classiques du statut matrimonial : en d'autres termes à la référence à la famille légitime, en opposant le statut des familles légitimes et à celui des familles naturelles. Enfin, le rôle des politiques familiales s'exprime par leur prise en compte de la parentalité et en termes de «conciliation» de la vie professionnelle et de la vie familiale.

$\diamond$ de l'autre côté, on étudiera le rapport au marché du travail à travers la participation professionnelle des pères et des mères (cf. Figure 3 - ligne en continu - 
Figure 3 Opérationnalisation du lien Famille / Marché du travail / Etat
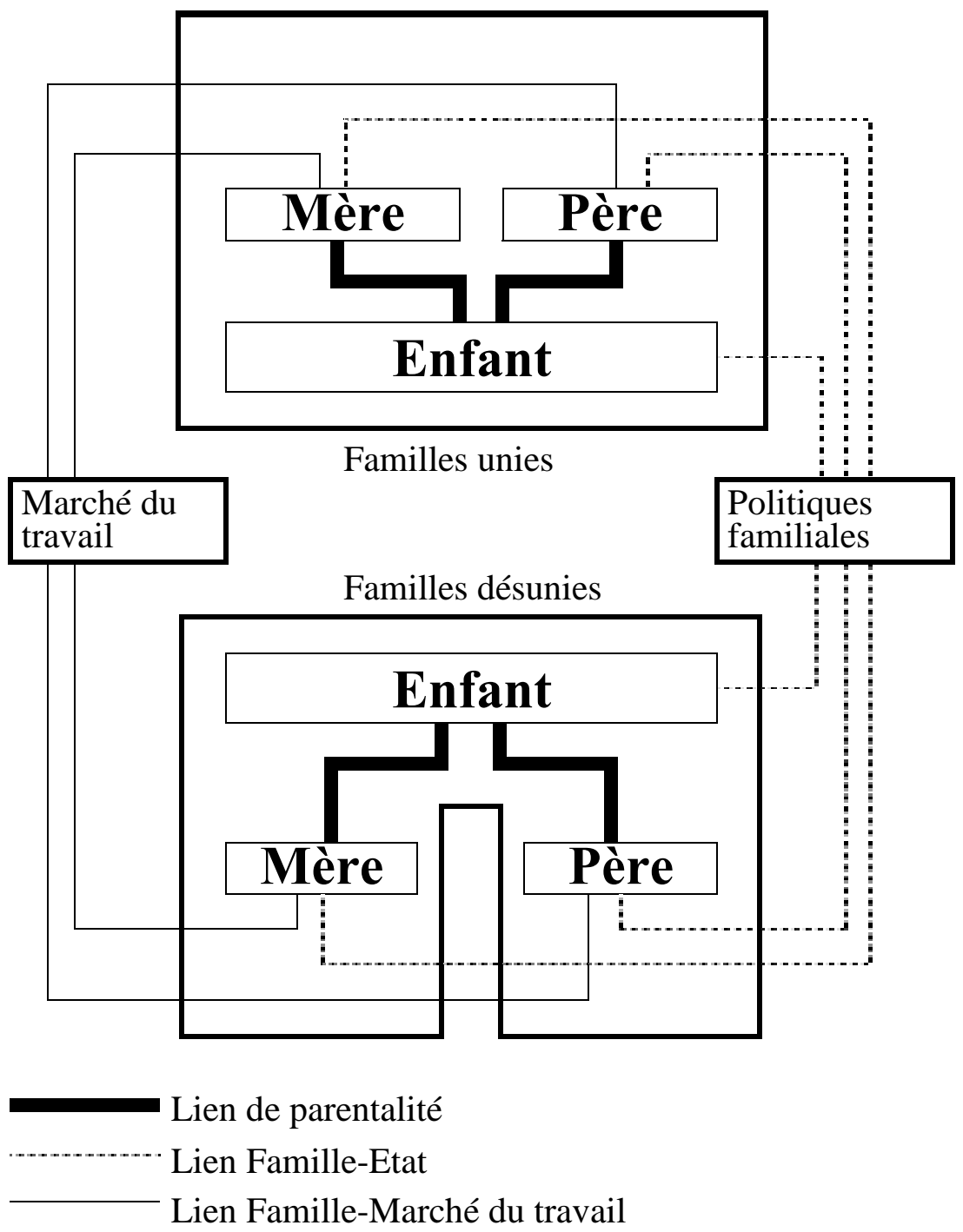

Troisièmement, seule une analyse historique, inscrite dans un temps long permet de repérer les grandes évolutions de la famille : elle évite d'accorder une trop grande importance à la rupture, bien que réelle, des années 1960 (F.X. Kaufmann 1996 ; Théry 1998 : 20). L'approche historique (cf. Chapitre II) a montré que la parentalité contemporaine n'est pas un phénomène nouveau en soi et que seule l'émergence de nouvelles caractéristiques lui donne sa forme actuelle.

Notre objectif est de montrer de quelle manière les représentations de la parentalité ont évolué, et comment, la famille, le marché du travail et l'Etat sont impliqués dans ces évolutions. Les réformes du droit relatif au divorce en 1975 en France et 1977 en Allemagne, déterminent le début de la période d'investigation, car elles symbolisent les évolutions décisives de la norme familiale, elles marquent l'érosion définitive du paradigme du «pater familias» (cf. Chapitre II). Parallèlement, les années 2000 illustrent le début d'une nouvelle phase. Les réformes relatives au droit de l'enfant, en 1993 en France et en 1998 en Allemagne, ont contribué à inscrire durablement une nouvelle définition de la famille centrée 
sur l'enfant, et ont donné une place dominante au rapport social entre l'enfant et ses deux parents dans la régulation du lien familial.

\section{LA COMPARAISON SOCIETALE ENTRE LA FRANCE ET L'ALLEMAGNE}

La comparaison sociétale permet d'aller plus loin dans la compréhension du sens des mutations actuelles. En faisant varier les caractéristiques qui interviennent dans la construction des rapports familiaux, elle apporte des indications précieuses sur les changements institutionnels et leur mode de régulation. La voie ouverte par «l'école d'Aixen-Provence» est une alternative aux approches culturalistes et universalistes (voir la position d'Iribane 1991). «Les objets comparés sont considérés comme autant de "construits sociaux" saisis au sein des processus qui les constituent dans leur spécificité sociétale. Une telle démarche ouvre sur de la généralité. En même temps, elle "donne à voir" les spécificités sans s'enfermer inexorablement dans des totalités culturelles closes pour elles-mêmes.» (Maurice, Sellier, Silvestre, 1992 : 77 ; Maurice 1989). Nous avons détourné la comparaison sociétale effectuée par ces trois auteurs sur la place de l'entreprise pour l'appliquer à la famille (souligné en italique par nous) ${ }^{5}$ : «Dès lors, il s'agit de mettre en évidence dans une société donnée les processus d'interdépendance (parentalitélsociété via le système de protection sociale et la participation au marché travail) qui contribuent à la construction des acteurs, à la forme d'existence de la famille dans la société».

L'ensemble des pays européens post-industriels sont confrontés aux mêmes mutations de la famille, du marché du travail et du régime d'Etat-providence depuis les années 1970. Or nous avons choisi le cas de la France et de l'Allemagne ${ }^{6}$ pour étudier la manière dont les rapports sociaux entre l'enfant et ses parents sont déterminés par la triade institutionnelle, Famille, Marché du travail, Etat.

En effet, les modèles familiaux y sont différents alors que les évolutions des pratiques au sein des familles sont semblables. En ce qui concerne le marché du travail, la participation des femmes varie [plus importante en France, elle est plus faible en Allemagne] mais les grandes évolutions macrosociologiques sont les mêmes [taux de chômage semblables, même développement du secteur tertiaire et du temps partiel]. Ces deux pays offrent également deux modalités de régime d'Etat-providence, l'un plus interventionniste, l'autre plus modéré, l'un plus nataliste, l'autre traditionnellement centré sur un modèle bourgeois de la famille. De

\footnotetext{
5 Citation originale: «Dès lors, il s'agit de mettre en évidence dans une société donnée les processus d'interdépendance (entreprise/société via le système d'éducation ou de régulation sociale) qui contribuent à la construction des acteurs et, pour ce qui nous concerne, à la forme d'existence de l'entreprise dans la société» (Maurice, Sellier, Sylvestre, 1992 : 85).

${ }^{6}$ L'Allemagne est un pays dont la configuration particulière oblige à différencier les termes de la comparaison. Pour la période antérieure à la réunification, on comparera plus spécifiquement la France à l'ancienne Allemagne de l'Ouest. Puis, à partir de la réunification, on comparera la France à l'Allemagne réunifiée, tout en distinguant les nouvelles des anciennes régions.

Au cours des dix dernières années qui ont suivi la réunification, le processus d'ajustement entre les deux régions ont provoqué, en particulier pour l'ancienne Allemagne de l'Est, des changements profonds, dans la structure du marché du travail et de la sphère familiale. Deux éléments sont à noter : les différences liées à la participation au marché du travail pour les femmes (similaires à la France pour l'ancienne Allemagne démocratique), et une conception différente du rôle du père (due en particulier à la place de l'Etat par rapport à la famille).
} 
plus, les deux pays ont un système de protection sociale comparable, même orientation Bismarkienne et prise en charge des même risques sociaux.

Derrière la similitude des mutations institutionnelles, chaque institution a une référence normative différente. Ceci nous permet de faire varier les liens institutionnels et d'en mesurer l'impact sur la construction des rapports entre l'enfant et ses parents. A ce niveau de l'observation, la France et l'Allemagne se définissent comme deux modèles dissemblables.

Les études comparatives du lien entre la famille et l'Etat viennent confirmer cette position. En effet, l'association des cas français et allemand dans la typologie de EspingAndersen (1990 : 26), qui regroupe à la fois le système français et allemand sous l'étiquette conservatiste-corporatiste ${ }^{7}$, est remise en cause par les travaux comparatifs (F.-X. Kaufmann 1995 : 710 ; Schultheis 1995 : 765). Elle est d'autant plus remise en question que ces comparaisons traitent des politiques familiales (Lessenich, Ostner 1995 : 781 ; Ostner 1995 : 9). D'un côté, ce sont les normes de la famille, la participation des femmes au marché du travail qui varient, de l'autre, le principe normatif d'ingérence plus ou moins légitime de l'Etat dans la sphère privée, dans une intervention nataliste dans le cas français ou «conjugaliste» dans le cas allemand.

$\mathrm{Au}$ cours des chapitres suivants, nous avons mis en évidence que les rapports parentaux suivent des motifs bien identifiés, venant contredire les positions théoriques qui soutiennent l'idée d'une anomie des comportements familiaux. La parentalité est un construit social dont l'étude donne «du sens» aux changements familiaux.

Les formes de la parentalité se regroupent en quatre catégories en fonction de l'existence ou non d'un couple parental pour l'enfant: «parentalité bilatérale» et «parentalité unilatérale», et de l'origine biologique ou sociale de la relation : «parentalité première» et «parentalité composée». Cette typologie permet de restituer les situations de fait dans les familles et se distancie des dénominations classiques fondées sur la forme du lien conjugal (en terme de légitimité et d'illégitimité) et du statut juridique des individus (marié, célibataire, divorcé ou veuf) (cf. Chapitre I).

Par ailleurs, nous avons vu que les modes de participation des parents au marché du travail et les mesures des politiques familiales construisent activement deux formes de parentalité. Elles sont fonction de l'exercice concomitant d'une activité professionnelle et des fonctions parentales : «parentalité conciliée», ou d'un exercice des fonctions parentales sans que le parent n'exerce d'activité professionnelle : «parentalité exclusive».

Dans les pratiques des familles, la «parentalité conciliée» est largement privilégiée par les deux parents : en référence au temps complet pour les pères (en France et en Allemagne) et pour les mères (dans l'ancienne Allemagne de l'Est et en France), et en référence au temps partiel pour les mères de l'ancienne Allemagne de l'Ouest. Les politiques familiales privilégient par leur orientation normative une forme particulière de parentalité. Les dernières mesures en France et les nouveaux objectifs politiques en Allemagne montrent que c'est la prise en compte du statut de parent pour les pères et les mères qui est au centre de la régulation politique des rapports familiaux.

\footnotetext{
${ }^{7}$ En effet, lorsque la recherche internationale en politique sociale se penche sur l'étude des régimes des Etatsprovidences, elle s'attarde davantage sur les systèmes anglo-saxons et scandinaves, faisant des régimes continentaux européens un seul et unique groupe homogène.
} 
La norme de parentalité dans les deux pays favorise le maintien du lien entre l'enfant et ses deux parents dans un exercice bilatéral de la parentalité (dans les familles unies mais également dans les familles désunies) malgré la montée des divorces et des familles recomposées. Si la place de l'enfant est bien la même, le rôle des pères et des mères, dans leurs relations personnelles à l'enfant, comme dans leur dimension conjugale, sont différents en France et en Allemagne. La différence de conception des rapports de genre mais aussi la nature des rapports entre l'Etat et la famille expliquent les différences dans le processus : on observe une norme de «coparentalité» en France (coopération parentale) et de «biparentalité» en Allemagne (juxtaposition du rapport de chaque parent à l'enfant) (cf. Chapitre II).

Les pratiques des familles déterminent la manière dont la parentalité est une réalité sociale, à ce titre nous avons choisi d'étudier le partage des tâches parentales. Il apparaît alors que la famille est le lieu de négociation du partage du «travail parental», du travail domestique et du travail professionnel, nous parlons alors de «travail familial». Le partage du «travail parental» dépend des mécanismes de partage du travail familial compris dans son ensemble. La participation grandissante des pères au parental et la participation de plus en plus généralisée des mères au professionnel définissent les nouvelles pratiques de partage. $\mathrm{Ce}$ dernier ne s'effectue pas uniquement suivant des différences de genre, mais surtout en fonction de l'exercice de la parentalité (laquelle s'oriente de plus en plus vers une norme égalitaire malgré des inégalités encore persistantes). Aujourd'hui, c'est le lien entre le professionnel et le parental qui est centre de la régulation du partage du travail familial pour les deux parents : non plus la «mère», la «travailleuse» ou «l'homme actif», mais la «mère active» et le «père actif» (cf. Chapitre III).

Ainsi, nous avons vu que les nouvelles orientations normatives dans la régulation du risque familial sont fondées sur la «relation entre l'enfant et ses deux parents» et que la régulation du partage du travail familial se base sur l'exercice de la parentalité. Les rapports entre l'enfant et ses parents sont au centre d'un nouveau mode de régulation des rapports familiaux, le «parentalisme» ou «Parentalismus» ${ }^{8}$ en allemand. Il s'affirme comme le mode de régulation familial vers lequel les deux pays convergent au-delà de leurs différences sociétales.

Notre question de recherche ${ }^{9}$ «Comment la parentalité met-elle en lumière les changements du lien familial ?» est déclinée en trois problématiques correspondantes aux chapitres de notre travail (cf. Figure 4).

Le premier chapitre traite tout d'abord de la parentalité du point de vue théorique, puis il montre l'influence du marché du travail et des politiques familiales dans la construction des formes de parentalité en France et en Allemagne.

Après avoir retracé les évolutions historiques dans la construction du lien entre l'enfant et les parents, le second chapitre définit la norme actuelle de parentalité. Il porte une attention particulière aux situations de séparation du couple parental. En effet, la forme du

\footnotetext{
${ }^{8}$ Nous nous référerons au terme de «Parentalismus» utilisé par S. Lessenich et I. Ostner (1995 : 796). Il est défini par ces deux auteurs comme la synthèse de la liberté de l'individu (ici la liberté de chaque parent de choisir une forme préférée de famille) et d'un ordre social qui protège l'individu le plus démuni (ici l'enfant). Pour notre part, les droits les parents et les enfants ne sont pas envisagés en opposition, mais en relation. De fait, nous définirons le «Parentalismus» comme un mode de régulation (politique) fondé sur le lien familial, lui-même centré sur la relation de l'enfant à ses deux parents, indépendamment de la forme de famille.

${ }^{9}$ La méthodologie est présentée en annexe (cf. Annexe 1).
} 
traitement social du «risque familial» révèle, par contraste, la conception normative de la parentalité.

Le troisième chapitre aborde les formes d'exercice de la parentalité dans les familles à partir du partage du travail parental. Ce dernier est étudié en fonction de la situation professionnelle (activité à temps complet, à temps partiel et inactivité) et de la participation des parents au travail domestique : à travers le partage du «travail familial».

Enfin, la conclusion synthétise les résultats de la comparaison. Les caractéristiques sociétales de la construction du rapport entre l'enfant et ses parents sont alors mises en parallèle faisant apparaît le «parentalisme» comme le nouveau mode de régulation du lien familial en France et en Allemagne.

Figure $4 \quad$ Sommaire et questions de recherche

\section{Introduction : Comment la parentalité met- elle en lumière les changements du lien familial ?}

Chapitre I : Le cadre théorique et les formes de parentalité : la parentalité exclusive et la parentalité conciliée

Chapitre II : Les normes de parentalité : la coparentalité française et la biparentalité allemande

Chapitre III : Les pratiques de parentalité dans les familles et le partage du travail familial

Conclusion : Le parentalisme comme mode de régulation?
Comment les évolutions socio-démographiques de la famille, ses modes de participation au marché du travail et le cadre socio-politique déterminentils les formes de parentalité ?

Comment se définit le mode privilégié de l'exercice de la parentalité en France et en Allemagne pour les familles unies et désunies: quel est le mode de régulation du risque familial ?

Quels déterminants influencent les modes l'exercice de la parentalité dans les familles ? Pourquoi la parentalité est-elle indissociable de la manière dont la famille partage le travail domestique et professionnel ?

Quel est le mode de régulation des rapports familiaux, des relations famille/marché du travail, et des liens famille/Etat-providence, dans les deux contextes sociétaux français et allemand? 


\section{CHAPITRE I LE CADRE THEORIQUE ET LES FORMES DE PARENTALITE : LA PARENTALITE EXCLUSIVE ET LA PARENTALITE CONCILIEE}

Introduction : Comment la parentalité metelle en lumière la régulation du lien familial ?

Chapitre I : Le cadre théorique et les formes de parentalité : la parentalité exclusive et la parentalité conciliée

Chapitre II : Les normes de parentalité : la coparentalité française et la biparentalité allemande

Chapitre III : Les pratiques de parentalité dans les familles et le partage du travail familial

Conclusion : Le parentalisme comme mode de régulation?
Comment les évolutions socio-démographiques de la famille, ses modes de participation au marché du travail et le cadre socio-politique déterminentils les formes de parentalité ?

Ce chapitre I présente, dans une première section, le cadre théorique et conceptuel. Il montre en quoi le concept de parentalité est l'aboutissement des réflexions sur les systèmes de parenté et de la famille élargie, des notions contemporaines de l'enfant et du parental. Nous déclinons ensuite quatre formes de parentalité, définies à partir de la relation entre l'enfant et ses parents : la «parentalité unilatérale», la «parentalité bilatérale», la «parentalité première» et la «parentalité composée».

Les sections, II, III, IV montrent successivement les transformations sociodémographiques de la famille, les évolutions du marché du travail, le cadre socio-politique, ainsi que leurs impacts respectifs sur la relation entre les parents et l'enfant (cf. Figure 5). Deux catégories typologisent, depuis le point de vue de la parentalité, les relations entre la famille et le marché du travail, et la famille et les politiques familiales : la «parentalité exclusive» et la «parentalité conciliée». On passe actuellement a un modèle de double «parentalité conciliée» dans les deux pays. Le statut de parent, des pères et des mères, est au centre de la régulation. Les différences sociétales s'expriment par la référence à un double temps complet pour les parents en France, et à un temps complet pour les pères et un temps partiel pour les mères en Allemagne. 
Figure 5 Trois relations qui déterminent les formes de parentalité

Famille

Les rapports familiaux

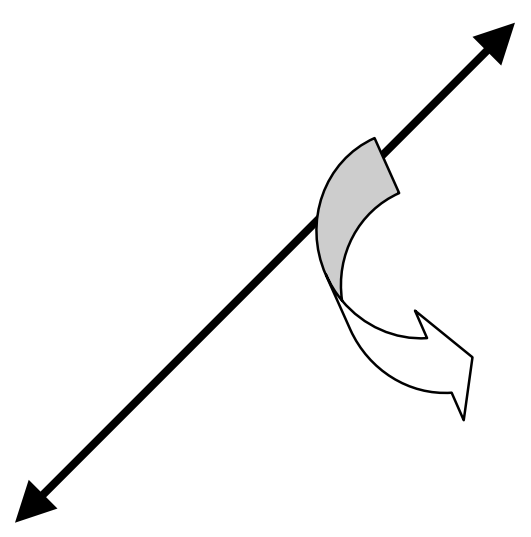

Marché du travail

La participation des parents au marché du travail

Les évolutions de la participation des familles au marché du travail et leurs impacts sur la parentalité
Les transformations démographiques de la famille et leurs influences sur la relation parents- enfant
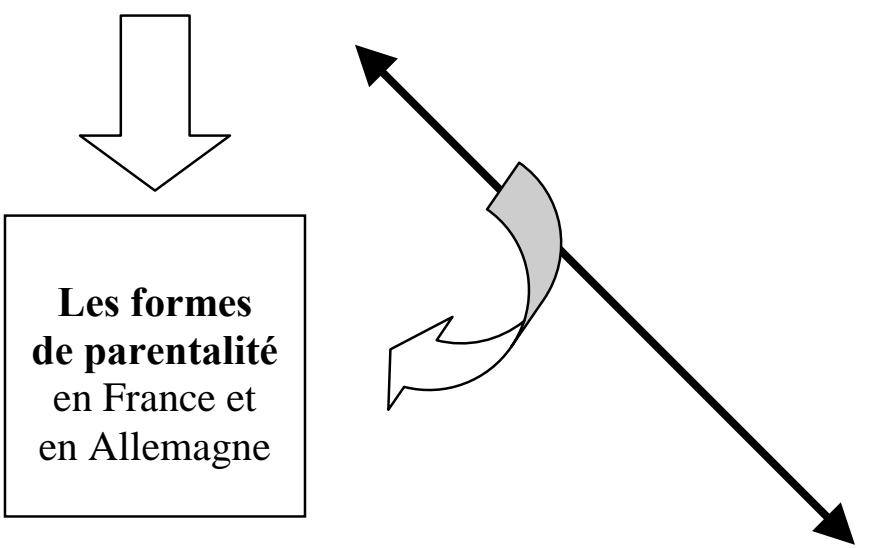

Etat-providence

Les politiques familiales

Le cadre socio-politique et sa signification pour la parentalité 


\section{SECTION I.A LE CADRE THEORIQUE ET CONCEPTUEL DE LA PARENTALITE}

L'objet de cette section est de montrer comment la notion actuelle de «parentalité» est le résultat des avancées théoriques en sociologie de la famille: d'abord, c'est la famille nucléaire qui a été l'objet de recherche, puis le couple conjugal. Plus récemment, c'est l'enfant qui est devenu l'unité de référence. Avec lui, les notions liées au parental se sont développées. Aujourd'hui, la parentalité est au croisement des études du lien conjugal et de l'enfant. Enfin, pour notre part, nous avons choisi de mettre la relation entre l'enfant et ses parents au cœur de l'analyse.

\section{SOUS-SECTION I.A.1 LE CONJUGAL, L'ENFANT ET LE PARENTAL}

\section{I.A.1.1 La famille : un groupe social universel}

Les travaux des ethnologues ont montré que la famille est un groupe social dont l'existence se retrouve dans l'ensemble des sociétés humaines. «(...), nous conclurons de ce qui précède, que la famille est bien un donné universel, en ce sens seulement qu'il n'existe aucune société qui soit dépourvue d'une institution remplissant partout une ou plusieurs des mêmes fonctions (unité économique de production et de consommation, lieu privilégié de l'exercice de la sexualité entre partenaires autorisés, lieu de la reproduction biologique, de l'élevage et de la socialisation des enfants) et obéissant partout aux mêmes lois : existence d'un statut matrimonial légal autorisant l'exercice de la sexualité entre deux des membres de la famille au moins (ou prévoyant des moyens d'y suppléer), prohibition de l'inceste (car les partenaires autorisés ne sont jamais les consanguins), division du travail selon les sexes. Cependant, même si le mode conjugal monogame, avec la résidence commune des conjoints est le plus répandu, l'extrême variété des règles qui concourent à l'établissement de la famille, à sa composition et à sa survie démontre qu'elle n'est pas, sous ses modalités particulières, un fait de nature mais au contraire un phénomène hautement artificiel, construit, un phénomène culturel.» (Héritier-Augé 1989 : 256).

Outre son caractère universel, les ethnologues ont montré que la famille a une nature sociale qui la rend dépendante des époques et des sociétés. L'organisation de la parenté, des rôles conjugaux et de la filiation, change d'une société à l'autre, obéissant à des règles différentes. Il n'y a pas un type de famille mais une multitude de types. «Aux besoins et aux désirs fondamentaux de l'individu et de l'espèce - le désir sexuel, le désir de reproduction, la nécessité d'élever, de protéger les enfants et de les conduire à l'autonomie - les diverses sociétés humaines ont apporté des solutions multiples, qui impliquent toujours l'existence d'une famille, si elles n'impliquent pas nécessairement l'existence de la cellule conjugale formée par un homme, une femme et leurs enfants. Non nécessairement biologique, la construction de cette cellule est donc en ce sens artificiel. On en prendra pour preuve quelques exemples où la notion de cellule conjugale est niée dans l'un ou l'autre de ces termes alors même que la réalisation pratiques en calque le modèle.» (Héritier-Augé 1989 : 255). Aussi, si 
la famille est un groupe social universel, la famille conjugale est, en revanche, un arrangement social spécifique ${ }^{10}$.

L'objet des sociologues est de décrire la structure et les rapports sociaux qui constituent la famille (rapport de parenté, de filiation, de conjugalité). Ils cherchent à en expliquer les évolutions structurelles et fonctionnelles. Aujourd'hui, l'étude de la famille se comprend définitivement au cœur des changements sociaux et on tiendra compte de ses relations à la sphère de production (en qualifiant sa relation avec le marché du travail) ${ }^{11}$.

\section{I.A.1.2 La famille nucléaire et la parenté}

En France, Le play (1875) a étudié la famille élargie d'origine paysanne (cohabitation des générations, autorité du père et du mari), voyant dans l'apparition de la famille conjugale le signe d'une déstabilisation des rapports sociaux.

En Allemagne, Riehl (1861) montre, au sein de la famille bourgeoise, le passage du modèle de la famille au sens de «grande maison», à celle de la «famille conjugale». La disparition de la famille élargie en tant que modèle dominant au profit de la famille conjugale est alors interprétée comme l'avènement des valeurs de l'individu. L'individualisation est perçue comme le passage de la référence au groupe, à celle de l'individu (Théry 1998) : la famille n'est plus un groupe prédéfini mais le réseau que dessine, à posteriori, des échanges interindividuels et électifs ; l'individu devient la cellule de base de la société.

$\mathrm{Au}$ cours de ces travaux, Durkheim définit la famille conjugale comme «le mari, la femme, et les enfants mineurs et célibataires» (1975): elle est l'aboutissement des changements de structure de la famille par un processus de «contraction de la famille» (et rappelle les thèses évolutionnistes de Le play).

Le passage de la famille élargie à la famille conjugale est interprété comme la conséquence (et le moteur) des changements sociaux provoqués par l'industrialisation (Parsons 1955).

La famille conjugale bourgeoise («bürgerliche Kleinfamilie») serait apparue avec le capitalisme dont les caractéristiques et sa structure trouvent leurs origines dans l'évolution industrielle de la société (Rosenbaum 1973 : 106-159).

On est passé alors de la famille comme unité de production, à la famille comme une unité de reproduction. La décohabitation des générations a fait disparaître la famille élargie et la famille conjugale est devenu le modèle dominant.

Ce n'est plus le changement de structure entre famille élargie et famille nucléaire qui est au centre de l'analyse mais les logiques de fonctionnement de cette dernière. Le couple conjugal devient alors l'unité de référence dans les travaux des sociologues de la famille.

\footnotetext{
${ }^{10}$ «Il va de soi, semble-t-il, que les partenaires de l'union conjugale sont de sexe différent, que cette union ne se noue qu'entre les vivants, que le géniteur des enfants est normalement le père dans le cadre de l'union conjugale, enfin que la famille conjugale (père, mère, enfants) est l'unité résidentielle économique élémentaire par laquelle passe l'éducation et l'héritage. Or l'expérience ethnologique montre qu'aucun de ces principes n'est universellement admis.» (Héritier-Augé 1989 : 255). Inversement, il a été montré que la famille élargie n'était pas l'unique mode d'organisation familiale dans le passé : la famille conjugale y était dèjà constitutée (Mitterauer 1979).

${ }^{11} \mathrm{La}$ relation de la famille avec l'Etat est également un objet d'étude en sociologie de la famille. Elle a été discutée en terme d'ingérence de l'Etat dans les rapports familiaux : de la perte de l'autorité du père et du mari, ou du déplacement des fonctions d'apprentissage de la famille vers l'école.
} 


\section{I.A.1.3 Le couple conjugal au centre de l'analyse}

C'est au cours des années 1970 que l'unité d'analyse des sociologues de la famille a changé. Deux orientations théoriques se distinguent alors en sociologie de la famille ${ }^{12}$ :

Les premières étudient le rapport du couple conjugal à la parenté. C'est à partir de leurs travaux qu'on sait désormais que la décohabitation des générations n'a pas supprimé les liens de solidarité familiale (Coenen-Huther, Kellerhals, Allmen 1994). La famille nucléaire se place au centre d'un réseau familial dont la potentialité d'entraide a été vérifiée. Sur l'ensemble du réseau potentiel, chaque ménage se constitue un réseau actif et solidaire de quatre à six personnes, qu'il recrute, pour les trois-quarts au sein des liens de filiation, c'est-àdire dans un lien intergénérationnel (les parents plus âgés ou les enfants adultes non cohabitants). Les aides occasionnelles sont largement répandues, les aides importantes sont moins fréquentes. Il semble que la décohabitation et la distance géographique entre les générations ne soient pas de nature à menacer l'existence de ce réseau de solidarités familiales.

Les secondes positions théorique traitent du lien conjugal et à sa nature : l'atomisation de la famille (élection des partenaires de manière relationnelle et non plus de façon économique ou normative) s'accompagne d'une autonomisation de la famille conjugale par rapport au lien de parenté (de Singly 1993 : 50). Ces travaux traitent de la cohabitation et du lien légitime, du mariage et du «démariage» (Théry 1993); le primat de l'affection dans la famille contemporaine implique alors les comportements de séparation et d'union (de Singly 1993 : 50). En d'autres termes, ces orientations théoriques envisagent l'ensemble des phénomènes liés au conjugal et aux rapports de genre et avec eux, à la différenciation sexuée des tâches.

Ce déplacement des valeurs au sein de la société, où domine le conjugal, est interprété comme une privatisation des rapports sociaux : la privatisation est une transformation des normes, l'individu prend alors le pas sur le groupe, et «toute intrusion de la règle, en particulier quand elle prend une forme prescriptive, apparaît comme une immixtion de la société dans l'espace privé de l'autonomie individuelle» (Théry 1998 : 16).

Actuellement, la famille nucléaire et la famille étendue vivent une mutation structurelle inachevée ${ }^{13}$. Cette mutation comporte de nouveaux risques pour le lien familial. Les nouveaux comportements d'unions et de désunions sont interprétés en fonction des catégories fondées sur le mariage. Il est l'unique lien légitime. On parle alors de «mariage», de «cohabitation (ou hors-mariage)», de «divorce», de «beau-parent» et de «démariage». Or «le démariage, plus que le refus ou la crise du mariage, désigne la situation historique nouvelle liée à la transformation du lien de conjugalité dans un sens plus égalitaire, plus privé et plus contractuel. Elle pose des problèmes radicalement inédits pour le lien familial.» (Théry 1998 : 33), car le «démariage», outre le fait de toucher à l'institution du couple, atteint également les principes de la filiation.

\footnotetext{
${ }^{12}$ Deux positions ont structuré les débats en sociologie de la famille pendant de nombreuses décennies (Déchaux 1995 : 525) : La sociologie de la famille doit-elle avoir pour objet le groupe conjugal ou la parenté ? Peut-on conclure à une desinstitutionnalisation de la famille ?

${ }^{13}$ Cette mutation trouve son origine dans trois grandes dynamiques (Théry 1998) : l'égalité des sexes, la progressive personnalisation du lien à l'enfant, et l'allongement continu de l'espérance de vie.
} 
Le pouvoir explicatif des analyses centrées sur le conjugal était réel lorsque les modes d'exercice de la parentalité au sein de la famille se déduisaient du lien conjugal : on pouvait alors décliner trois types de famille en fonction de la présence du conjoint :

les familles conjugales traditionnelles : père, mère et leur(s) enfant(s) biologique(s),

les familles monoparentales : un seul parent et son (ses) enfant(s),

les familles recomposées : plusieurs parents et beaux-parents en couple et des enfants d'une ou plusieurs unions.

Ainsi, les évolutions socio-démographiques de la famille depuis les années 1950 ont modifié deux choses: d'un côté la forme du lien conjugal [moins de mariage, plus de divorces, généralisation de la cohabitation, séparations des couples hors mariage, remises en couple]; de l'autre les comportements de reproduction [baisse globale du nombre des naissances, augmentation constante de celui des naissances hors mariage].

Le fait le plus marquant de ces dernières décennies est la dissociation progressive du lien conjugal et de la présence (ou de la charge) de l'enfant. Ainsi, l'exercice de la parentalité peut se dissocier de la conjugalité et de la filiation.

On ne parle plus d'un risque conjugal, mais d'un risque qui affecte le parental. Par conséquent, les catégories de la famille fondées sur le couple, rendent compte des modifications du lien conjugal, mais sans pouvoir expliquer les changements du point de vue de l'enfant. Partant de cette constatation, les sociologues ont alors tenté de reconstruire les catégories d'analyse à partir de l'enfant.

\section{I.A.1.4 L'enfant comme unité de référence}

Dans les années 1980 et plus particulièrement à partir des années 1990, l'enfant est devenu l'unité de référence théorique (Jesson 2000, Cadoret 2000). On s'est alors intéresser aux modifications du lien conjugal, mais depuis le point de vue de l'enfant. Ceci a eu pour effet de faire émerger la notion de «parental», puis celle de «couple parental».

En mettant l'enfant au centre de l'analyse, on discute autrement des effets du divorce et des séparations conjugales (Théry 1998, Dekeuwer-Défossez 1999). On s'intéresse à l'organisation des rapports parentaux après la séparation et aux pratiques de la résidence alternée (Neyrand 1994) et à la signification des familles éclatées et recomposées (MeuldersKlein, Théry 1993). On parle de pluralisation pour décrire la multiplication des situations pour l'enfant : la famille se diversifie, on a alors un pluralisme des modèles familiaux issues des phénomènes d'union et de désunion, un passage de la famille aux familles (Théry 1998 : 16). L'usage du terme de famille ne se justifie plus vraiment si ce n'est plus le couple qui est au centre, mais l'enfant (Villeneuve-Gokalp, 1999). En effet, l'enfant peut appartenir à différentes unités familiales en fonction de la décomposition et recomposition des «familles», ce qui rend la description des formes de familles particulièrement complexes (Leridon 1993).

Par ailleurs, «l'étude de l'évolution des savoirs sur la petite enfance montre ainsi que l'on est passé d'un modèle organiste dominant en matière familiale, selon lequel la nature détermine le rôle de chacun, à un modèle beaucoup plus ouvert à l'influence de la société sur les conduites.» (Neyrand 2000). Ceci remet en cause le pouvoir explicatif du déterminisme biologique pour comprendre les rôles parentaux. On choisit alors de les comprendre comme un construit social : ils ne sont plus uniquement le résultat des pratiques conjugales. 
De plus, l'évolution des savoirs sur la petite enfance montre l'influence déterminante des psychologues (en particulier de Lacan) car ils construisaient les rôles parentaux sur la fonction maternelle (maternage) et sur la fonction séparatrice du père. Le père reprend par-là de l'importance mais cette conception renforçait le clivage des rôles parentaux (Neyrand 2000).

Or, trois facteurs remettent en cause ce clivage: le mouvement contestataire des années 1968-70, les nouveaux pères depuis les années 1980 et les méthodes de procréation médicalement assistées (Neyrand 2000).

C'est donc au cours des années 1980 que les changements de normes concernant la place de l'enfant dans nos sociétés deviennent visibles. En droit par exemple, jusque dans les années 1980, le législateur définit la norme de «l'intérêt de l'enfant» comme la nécessité d'épargner à l'enfant le conflit parental, au prix même d'une perte de contact avec l'un des parents. Cette interprétation a été revisitée au cours des années 1990 : le bien être de l'enfant est aujourd'hui lié à l'existence d'une relation continue avec ses deux parents, quelle que soit l'histoire familiale et l'ampleur du conflit conjugal. C'est la «survie du couple parental» ${ }^{14}$ qui, du moins au niveau normatif, oriente les représentations (cf. Chapitre II). «L'expression de couple parental peut paraître ambiguë lorsque les parents ne vivent plus ensemble et ont parfois construit un autre couple, une autre famille, en mariage ou en hors mariage. Il ne reste pas moins que même si les pères et mères sont séparés, ils ne sont pas parents séparément : c'est pour bien signifier que l'enfant à deux parents, investis d'une mission éducative commune, que l'on utilisera la formule du couple parental.» (Dekeuwer-Défossez 1999 : 77). L'idée de «couple parental» souligne que la parentalité s'effectue à deux, car elle concerne le partage des responsabilités entre le père et la mère.

Les effets des séparations et recompositions conjugales ont montré que la dynamique familiale est de plus en plus complexe et que les situations pour l'enfant se multiplient. La progressive personnalisation du lien à l'enfant modifie les interprétations des changements de la famille, précédemment fondées sur les évolutions du couple conjugal. On est donc face à une nouvelle contradiction : désormais, il devient difficile d'affirmer d'un côté que l'enfant appartient à une seule et unique famille et de l'autre que le lien avec ses deux parents doit se maintenir quelle que soit l'histoire familiale. Désormais, la centralité de l'enfant a fait apparaître la notion de couple parental. Et c'est la relation entre l'enfant et ses parents qui émerge comme pivot de la famille.

\section{I.A.1.5 La notion de genre et la notion de parental}

Au cours des années 1970, les sociologues de la famille se sont intéressés au rapport entre les sexes, à la construction du «genre». Dans ces approches, on privilégie la femme comme objet d'étude, elle se discute principalement en opposition au statut de l'homme.

\footnotetext{
${ }^{14}$ Pour I.Théry (1993), l'idée de «couple parental» est l'expression normative de la volonté de faire survivre la famille au-delà du divorce. «La psychologisation du divorce a produit son idéal de la réussite, son modèle du bon divorce, son slogan «le couple parental survit au couple conjugal»» (Théry 1993 : 11). Les normes sociales «d'un nouveau modèle de mœurs, le bon divorce», reprennent l'idée que les parents restent parents même si les circonstances ne les définissent plus comme couple amoureux (Théry $1993: 115$ ). Or, à notre sens, cette notion ne signifie pas l'affirmation du lien familial fondé sur le conjugal, mais bien l'expression d'une nouvelle compréhension (automone) des rôles parentaux dans la famille (qui alors se dissocient des aléas du conjugal).
} 
L'étude des rapports de genre a montré que le contrat des sexes ${ }^{15}$ était fondé sur la répartition des rôles en fonction d'un rapport «patriarcal» (pour les années 1950 et le début des années 1960). Puis, la norme qui définissait la répartition des rôles au sein des familles, a évolué de façon très marquante dans les années 60. Dès 1955, Renata Mayntz (citée par Kettschau 1980 : 14) parle d'une abolition du modèle patriarcal et de l'émergence d'un modèle partenarial. Cette interprétation des changements dans les rapports de genre, se maintient dans les années 1970. Neithardt (1975) mentionne une tendance à l'émancipation au profit de la femme et d'une perte de légitimation du modèle patriarcal sous l'influence de trois facteurs : les changements économiques et en particulier l'activité professionnelle de la femme ; l'altération du modèle à un apporteur devant les difficultés économiques (un salaire, le plus souvent celui de l'homme, ne suffisant pas à garantir les revenus nécessaires à la famille); une nouvelle définition de l'identité masculine, moins centrée sur la sphère professionnelle et plus ouverte sur la famille et la sphère privée.

Les années 1970 contribuent à déconstruire le modèle traditionnel, fondé sur le partage sexué des rôles : «Les générations de Français actuellement adultes ont été évidement marqué par l'image classique des rôles masculins et féminins (...). En somme, l'homme empiète peu sur les travaux considérés comme le domaine réservé de la femme (lessive, repassage, couture, cuisine), sauf en ce qui concerne les soins à l'enfant pour les jeunes ménages (...) Peut-être chez les plus jeunes, quelque chose commence-t-il à bouger.» (Pitrou, 1972). Les opinions évoluent ; la nouvelle génération de parents n'est plus majoritairement favorable à l'exclusivité des soins au jeune enfant par la mère (Segalen, 1981); le nombre de partisans du modèle de la femme au foyer décroît, les jeunes se montrent favorables au travail professionnel de la femme.

Les années 1980 s'inscrivent dans le prolongement des années 1970 : le partage du professionnel et du parental sont désormais en redéfinition. Plus généralement, le lien entre la sphère familiale et la sphère professionnelle est définitivement acquis. L'articulation devient elle-même l'objet d'analyse (Barrère-Maurisson, 1987). La compréhension du partage, audelà du simple domestique, est alors possible. Le partage exclusif, entre les sexes, du professionnel, du domestique et du parental, cède la place à une redéfinition sociale du partage des fonctions et des rôles dans le couple et dans le couple parental.

Tout d'abord, l'analyse des rapports de genre a mis en évidence les mécanismes de domination et de hiérarchie en défaveur de la femme au sein de la sphère privée et au sein du marché du travail. Puis, après avoir eu exclusivement la femme comme objet de recherche dans les années 1980, les études ont intégré le point de vue de l'homme. Et plus récemment encore, le «gender mainstream» considère, de façon concomitante, une étude du masculin et $\mathrm{du}$ féminin: non plus dans une relation antinomique, mais dans leurs interactions respectives ${ }^{16}$.

\footnotetext{
15 Theobald (2000) rappelle le concept de contrat des sexes, développé par l'historienne Yvonne Hirtman, et qui définit les rapports de sexe comme un accord entre les hommes et les femmes, sur leurs places, leurs activités et leurs qualités. Il est hérité et transmis, mais également modifiable. Le contrat entre les sexes est, tout à la fois, une norme, la construction de cette norme dans la réalité quotidienne et le processus individuel d'examen de cette norme. Par cette approche, la ségrégation et la hiérarchie entre les sexes deviennent un mécanisme central, inhérent au marché du travail et à la famille. Dans ce contexte, le contrat des genres est au centre de l'analyse du partage sexué des tâches et du travail.

${ }^{16}$ C'est au cours des années 1970 que l'on parle de la femme, non plus comme «conjointe» et «mère» mais comme «sujet» (Castelain-Meunier 1998). On analyse alors la condition féminine dans l'ensemble de sa complexité. On montre un l'ensemble de tensions et de contradictions : à partir du moment où la femme était reconnue comme sujet, elle cumule alors trois statuts, celui de «conjointe», de «mère» et de «sujet».
} 
L'analyse conjointe des rôles et des fonctions parentales des pères et des mères est récente et encore restreinte. Par exemple, concernant les comportements de fertilité, les démographes parlent traditionnellement des comportements de la femme, très peu de ceux de l'homme et encore moins des deux sexes conjointement. Or la fertilité masculine ne coïncide pas avec celle des femmes (colloque INED). C'est donc avec un intérêt grandissant, que les démographes intègrent peu à peu l'homme dans les analyses concernant les comportements de reproduction : les facteurs de la fertilité masculine sont encore peu connus mais deviennent un objet de recherche (United Nations 2000 : 33). A l'échelle de la famille, il est tout aussi nécessaire de traiter à la fois les données concernant les mères et les pères afin de comprendre l'ensemble des mécanismes liés à la parentalité. Or, comme en réponse à ce nouveau défi, l'analyse se déplace lentement depuis une opposition homme/femme vers une vision conjointe des mères et des pères.

Actuellement, la notion de «parental» devient largement admise en France. Les études sur le «travail parental» et sa mesure «le temps parental» (Barrère-Maurisson, Marchand, Rivier 2000, Barrère-Maurisson, Minni, Rivier 2001, Algava 2002) contribuent à affirmer la place centrale de l'enfant et du couple parental. En Allemagne, le «parental» n'est pas constitué comme une catégorie sociologique, en revanche il devient l'objet de l'action publique (cf. Section IV).

Par ailleurs, «la femme pousse l'homme à s'affirmer en tant que sujet et partenaire »(Castelain-Meunier 1998). La dynamique à l'intérieur du couple provoque alors la redéfinition des rôles et des fonctions de homme dans la famille. Inversement, la mise en évidence des mécanismes de domination en défaveur des femmes, appelle aujourd'hui à une dénonciation des inégalités, et amorce le processus actuel de redéfinition de la place de l'homme. 


\section{SOUS-SECTION I.A.2 LA PARENTALITE COMME NOUVELLE UNITE THEORIQUE}

\section{I.A.2.1 La parentalité : la relation entre l'enfant et ses parents}

L'interprétation communément admise des mutations de la famille se rapporte à trois grandes transformations, l'individualisation, la privatisation et la pluralisation (Théry 1998 : 16-18). Or peut-on réellement parler d'individualisation et d'atomisation de la famille alors que l'on a montré à plusieurs reprises que les solidarités intergénérationnelles sont d'une grande intensité et que les jeunes n'ont pas perdu l'intérêt pour la famille ? Peut-on également parler de privatisation réelle des normes alors que les attentes sociales en matière familiale s'expriment sous la forme de l'appel au droit, en particulier la référence à l'intérêt de l'enfant qui appelle à un grand nombre de formes toujours plus actives du contrôle social ? Peut-on encore parler de pluralisation des modèles alors qu'il n'y a pas de réelles fractures sociologiques entre les familles légitimes et les familles naturelles, que de nouvelles formes de famille comme les familles monoparentales ou recomposées ne sont pas en fait des nouveaux modèles alternatifs, mais plutôt des séquences du cycle de vie familiale consécutive à une rupture de couple ? (Théry 1998 : 16-18).

On voit la nécessité de s'éloigner des approches centrées sur le couple conjugal car elles soulignent d'avantage les processus de décomposition et de complexification que ceux de transformation et d'invention. En revanche, les notions de «l'enfant» et du «couple parental» permettent désormais le nécessaire renversement de point de vue à adopter pour interpréter les changements du lien familial dans leur dynamique.

Les séparations parentales modifient le lien entre l'enfant et ses parents. Il prend alors deux formes : soit le couple parental existe après la séparation et la relation entre l'enfant à ses deux parents est maintenue ; soit le contact avec l'un des parents disparaît. Par ailleurs, la remise en couple de l'un [ou des deux] parents modifie aussi la relation car la parentalité sociale vient se mêler au biologique ${ }^{17}$. On voit ainsi se multiplier les formes de famille. Il n'est plus possible de les décrire du seul point de vue du couple conjugal et elles sont trop complexes pour être décrites du point de vue l'enfant.

Désormais, les séparations et les recompositions familiales s'accompagnent d'une nouvelle contradiction : en effet, on suppose toujours que l'enfant appartient à une seule et unique famille quelle que soit l'histoire conjugale, mais on affirme également que les liens entre l'enfant et ses deux parents sont indissociables.

On le voit, la complexification des relations familiales, par dissociation des processus de conjugalité et de filiation mais aussi par recompositions, conduit à un nécessaire renversement de perspective. On doit ainsi passer d'une catégorisation de famille fondée sur le couple (qui tentait ensuite de mettre en relation les parents et les enfants), puis d'une

\footnotetext{
${ }^{17}$ Certaines positions idéologiques insistent sur le phénomène d'éclatement des familles, en oubliant le phénomène de reconstitution. On parle alors de fragilisation de l'institution familiale, mettant en danger la cohésion sociale. Or, pour notre part, il nous semble important de souligner que les phénomènes d'éclatement, comme les phénomènes de recomposition, sont un phénomène moderne, qui souligne une complexification des interactions familiales, où se multiplient les négociations, les arrangements stratégiques, les interactions et les mobilisations de capitaux sociaux.
} 
catégorisation où l'enfant est au centre (confronté à la multiplication des situations), à une catégorisation où le rapport de l'enfant à ses parents est la nouvelle unité de référence (Barrère-Maurisson, Rivier 2002). «Il faut construire toute une terminologie de parenté absolument sans précédent, brouillant toutes les oppositions structurales que les analyses componentielles de la parenté ont l'habitude de dégager» (Bourdieu : 1993 : 32-36).

Ainsi, nous proposons de prendre la parentalité ${ }^{18}$ comme nouvelle référence théorique dans l'étude des rapports familiaux. Dans son acception commune, la parentalité est une notion centrée sur l'unité parentale: «la parentalité recouvre les attributions, droits, obligations et responsabilités des pères et des mères génétiques ou adoptifs pour ce qui touche la procréation, la naissance, l'établissement de la filiation génétique et/ou juridique, l'exercice de l'autorité parentale, l'éducation et l'ouverture de droits sociaux spécifiques, au regard de la dignité de la personne humaine.» Cette définition a été adoptée par la Commission Nationale Consultative des Droits de l'Homme à l'occasion de l'avis au gouvernement adoptée le 26 mars 1998 sur le projet «Parentalité et Droits de l'Homme en rapport avec les avancées scientifiques et technologiques» en France.

Pour notre part, il nous semble important de prendre en compte la relation entre l'enfant et le couple parental (la mère et le père). En effet, seule l'étude du lien (dans ses formes, ses normes et ses modes d'exercice) permet de discuter les changements familiaux dans leur dynamique et la place de la famille dans la société.

Nous définissons alors la parentalité comme l'ensemble des rapports sociaux entre l'enfant et ses parents. Elle est au croisement du conjugal et de la filiation ${ }^{19}$. En ce sens, elle intègre une compréhension du lien conjugal, du lien générationnel et des rapports de genre, tout en dépassant le cloisonnement théorique dans lequel ils étaient analysés jusqu'à présent (cf. Figure 6).

\footnotetext{
${ }^{18}$ En France, le terme de parentalité est un néologisme récent, dont l'usage s'est développé dans les années 1990 , pour devenir très fréquent depuis 1997. Ses significations sont multiples, et depuis 2000, il est surtout utilisé dans le cadre des réformes du droit de la famille (Damon 2001). La définition particulière developpée dans notre travail reprend puis prolonge cette signification, car elle intègre aussi le rôle déterminant du droit de la famille dans la construction sociale et normative des rapports entre l'enfant et ses parents.

${ }^{19}$ On placera, mais de manière plus marginale, les relations de parentalité dans l'ensemble du système de parenté, c'est-à-dire en relation avec la famille étendue. Le réseau de solidarité intergénérationnelle joue un rôle dans la gestion du parental et du domestique, c'est-à-dire lors du partage du travail familial dans le couple (cf. Chapitre III). Il s'agit de « situer la famille dans le système symbolique de la parenté (...) la parenté est l'institution qui articule la différence des sexes et la différence des générations, et les familles, si diverses soientelles concrètement, s'inscrivent dans cette dimension symbolique. » (Théry 1998 : 21).
} 
Figure 6 La parentalité au centre des rapports de genre, de couple et de génération

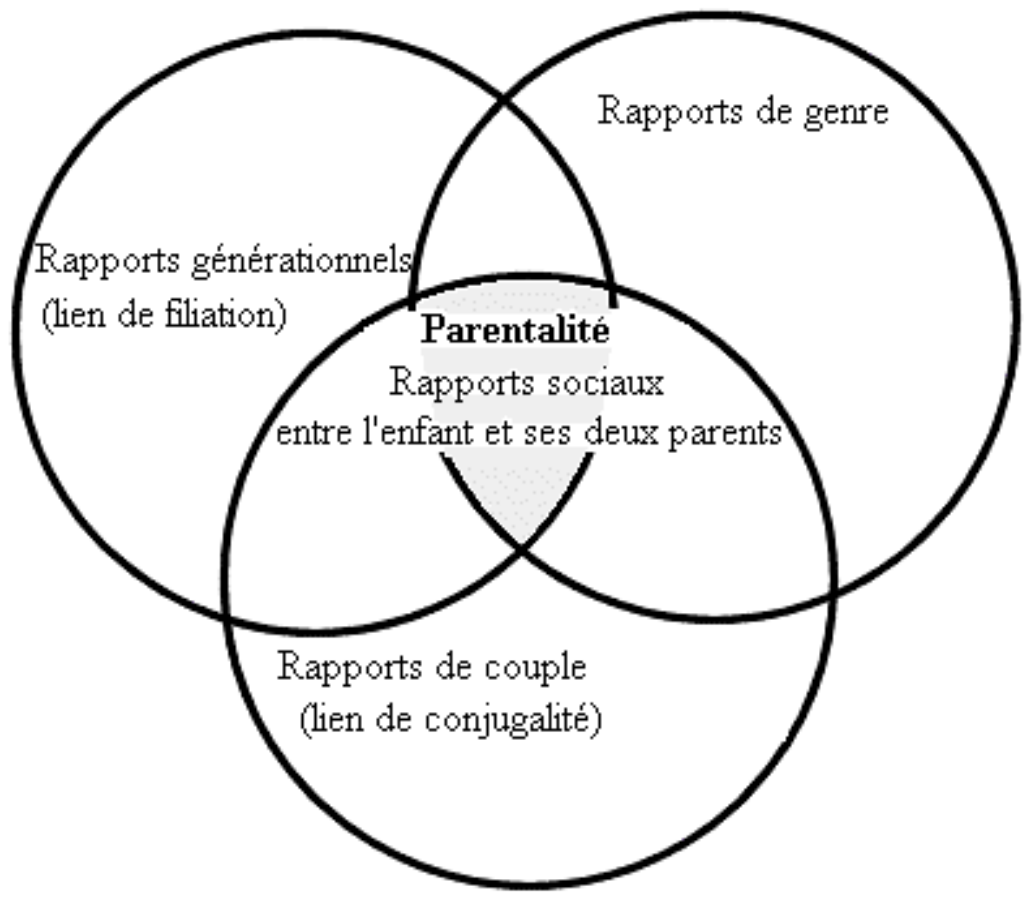

Si la relation de l'enfant à ses parents est le centre d'une unité conceptuelle revisitée, on a, à ce stade, quatre formes d'exercice de la parentalité :

- une «parentalité bilatérale» lorsque l'enfant entretient des relations avec les deux parents (que le couple soit uni ou désuni),

- une «parentalité unilatérale» lorsque la séparation conjugale se sanctionne par la perte de contact avec le deuxième parent,

- une «parentalité première» lorsque l'enfant vit toujours avec le couple parental d'origine,

- une «parentalité composée» où le biologique et le social sont mêlés pour l'enfant.

Ces catégories redéfinies, fondées sur les modes d'exercice de la parentalité, dépassent les dichotomies classiques «légitime/hors mariage», «unie/séparée», «cohabitation/noncohabitation», «social/biologique», «homme/femme», pour saisir principalement la forme du lien entre l'enfant et ses parents «bilatéral/unilatéral» et «premier/composé».

Les deux premières formes de parentalité se fondent sur la permanence relative du couple parental pour l'enfant: soit l'exercice est unilatéral et il exprime l'exclusivité des responsabilités éducatives de soins et de garde de l'enfant sur un parent ; soit il est bilatéral et il y a un exercice conjoint des responsabilités par les deux parents.

Les deux autres catégories décrivent les formes de parentalité à partir du couple parental : soit il est d'origine, dans le cas de la parentalité première ; soit il est le produit d'une séparation puis d'une recomposition familiale. Du point de vue de l'enfant, cela signifie dans le premier cas, une parentalité avec ces deux parents biologiques, dans le second cas, une parentalité composée alliant à la fois la relation biologique avec le parent d'origine, et une relation sociale avec le nouveau conjoint. 
En effet, il n'est plus possible d'affirmer, par souci de simplification, que l'enfant appartient à une seule famille, laquelle prendrait des formes différentes en fonction de l'histoire conjugale. Aujourd'hui, il faut tenir compte du fait que l'enfant puisse être impliqué simultanément dans différentes formes de parentalité. Par ailleurs, la description de la parentalité peut-être différente pour chaque membre de la famille. Ce que n'autorise pas les catégories classiques qui tenaient compte de la forme de lien conjugal, mais peu de leur implication pour l'enfant, et jamais de leur relation dialectique. En revanche, les quatre catégories proposées par nous résument à la fois, l'historique du lien conjugal, l'existence et la nature de la relation entre le parent et l'enfant, et la complexité de certaines constellations familiales.

En d'autres termes, ces quatre catégories proposent de repenser les changements socio-démographiques à l'intérieur des structures familiales à partir de la qualification du rapport de parentalité. Elles permettent de décrire le lien entre l'enfant et ses parents au cours des différentes étapes biographiques et historiques de la famille. Elles dépassent les catégories actuelles basées sur la description du lien conjugal: oppositions de genre, cohabitation des parents, union ou désunion, légitimation du lien matrimonial, pour décliner les formes de parentalité à partir de l'existence d'un couple parental premier ou composé et de l'exercice unilatéral ou bilatéral de la parentalité.

Dans le paragraphe suivant, les termes communément utilisés pour décrire la parentalité sont discutés en relation avec les nouvelles catégories proposées (cf. Figure 7).

Aujourd'hui, la «monoparentalité» a principalement pour origine les divorces et les séparations, elle est devenue une catégorie ambiguë : elle insiste sur la présence d'un seul parent (souvent la mère) qui a la responsabilité de la charge du soin de l'enfant tout en cachant l'existence du second parent. Aussi, pour notre part, nous proposons de parler de «parentalité bilatérale» lorsque le contact perdure avec le second parent et de «parentalité unilatérale» lorsque le contact est inexistant. Quant aux situations de monoparentalité où les parents se partagent l'autorité parentale, l'entretien et la résidence de l'enfant (on parle courrament de «coparentalité»), elle correspond, en fait, à une «parentalité bilatérale». L'apparition même du terme de «coparentalité» dans le langage courant, montre la tentative actuelle de résoudre cette ambiguïté. Cet exemple montre la nécessité de qualifier les formes de famille à partir de la relation entre l'enfant et ses parents.

L'«homoparentalité» définit les pratiques de parentalité au sein de couple homosexuel ; elle est actuellement discutée en terme de rapports de sexe. Cette discussion se rattache à la conception de la famille conjugale où la distinction de genre fonde la filiation. Or si on s'attache à comprendre cette forme familiale à partir des rapports entre l'enfant et ses parents, il apparaît une «parentalité composée» lorsque le biologique est mêlé au social et une «parentalité bilatérale» lorsque deux couples parentaux sont unis par le même enfant. Dans l'ensemble des cas, elle compose avec le social et le biologique. On dépasse alors les discussions centrées sur le genre pour apprécier, du point de vue de l'enfant, mais également du point de vue du couple parental, la nature de leurs relations réciproques.

Enfin, la «multiparentalité» exprime le phénomène, pour l'enfant, d'une multiplication des figures parentales. En réalité, il s'agit de la juxtaposition d'une figure «beau-parentale», à celle du couple parental «premier» qui, normativement, reste la référence pour l'enfant en cas de séparation des parents. La cohabitation et décohabitation, la filiation biologique et la filiation sociale y sont mêlées. 
Figure $7 \quad$ Les quatre formes de parentalité

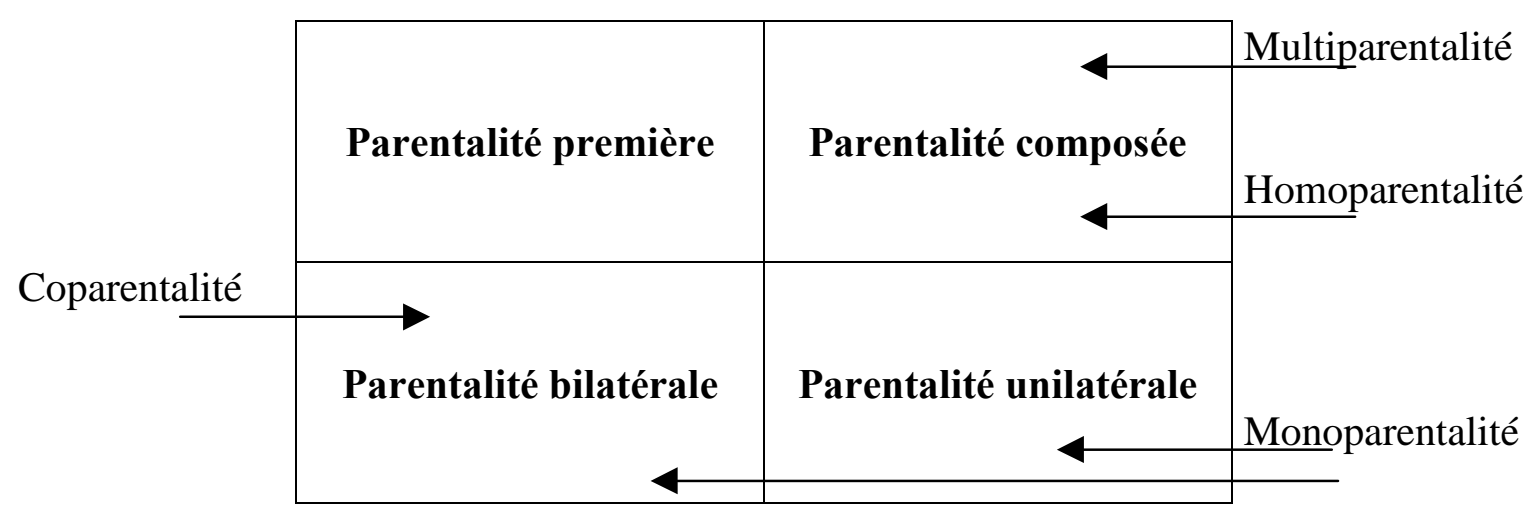

\section{I.A.2.2 La redéfinition des types de familles}

Dans ce contexte, nous définirons la famille comme un groupe social organisé autour des relations de parentalité, c'est-à-dire autour du lien social entre l'enfant et ses deux parents. La définition de la famille retenue ici est donc spécifique, puisqu'elle est centrée sur le lien avec l'enfant et non plus sur sa seule présence au sein du ménage (de sa résidence principale). Cela permet de rendre compte des dernières évolutions démographiques de la famille en France en Allemagne (cf. section suivante).

Il n'y a pas (ou plus) de superposition stricte entre les formes de parentalité et les formes de familles. En effet, on affirme dans les analyses actuelles que l'enfant appartient à une et une seule famille. Mais on lui reconnaît également de façon consensuelle aujourd'hui, un lien immuable avec ses deux parents. Or le phénomène généralisé de désunion et de recomposition familiale rend de plus en plus difficile cette coïncidence. Il s'avère alors qu'il n'est plus possible de dire que l'enfant appartient à une seule famille, au sens traditionnel du terme. La description systématique des rapports de l'enfant à ses parents montre que les critères de cohabitation, de filiation sociale et de filiation biologique ne suffisent plus à décrire la complexité des situations.

Nous proposons une définition de la famille fondée sur la parentalité. Ces catégories de familles sont définies à deux niveaux, à partir de la nature de la relation sociale ou biologique, et en fonction de l'existence d'un couple parental. Ce concept intègre l'idée d'une appartenance multiple à différents réseaux relationnels, accepte la proposition selon laquelle un enfant et un parent (père ou mère) appartiennent à différentes et plusieurs 'familles' (au sens de réseau) lorsque la biographie familiale devient complexe.

Nous montrons alors quatre types de famille fondés sur quatre types de parentalité : la «famille première», la «famille composée», la «famille bilatérale» et la «famille unilatérale» (cf. Figure 8). Ces nouvelles typologies des familles redistribuent de façon différente des catégories classiques : la «famille première» comprend la «famille légitime» (ou nucléaire) dans sa définition classique, mais également les «familles naturelles», qui n'ont pas connu de séparation conjugale. Les «familles composées» regroupent alors l'ensemble des «familles recomposées» qu'il y ait remariage ou non et les familles «homoparentales». Les «familles bilatérales» concernent l'ensemble des familles monoparentales lorsque le contact 
avec le père demeure. Enfin, les «familles unilatérales» comprennent une sous catégorie des familles monoparentales, puisqu'elles regroupent les familles monoparentales veuves (avant une recomposition familiale éventuelle) et les familles monoparentales lorsque que le contact avec le second parent a disparu.

Figure $8 \quad$ La redéfinition des types de familles

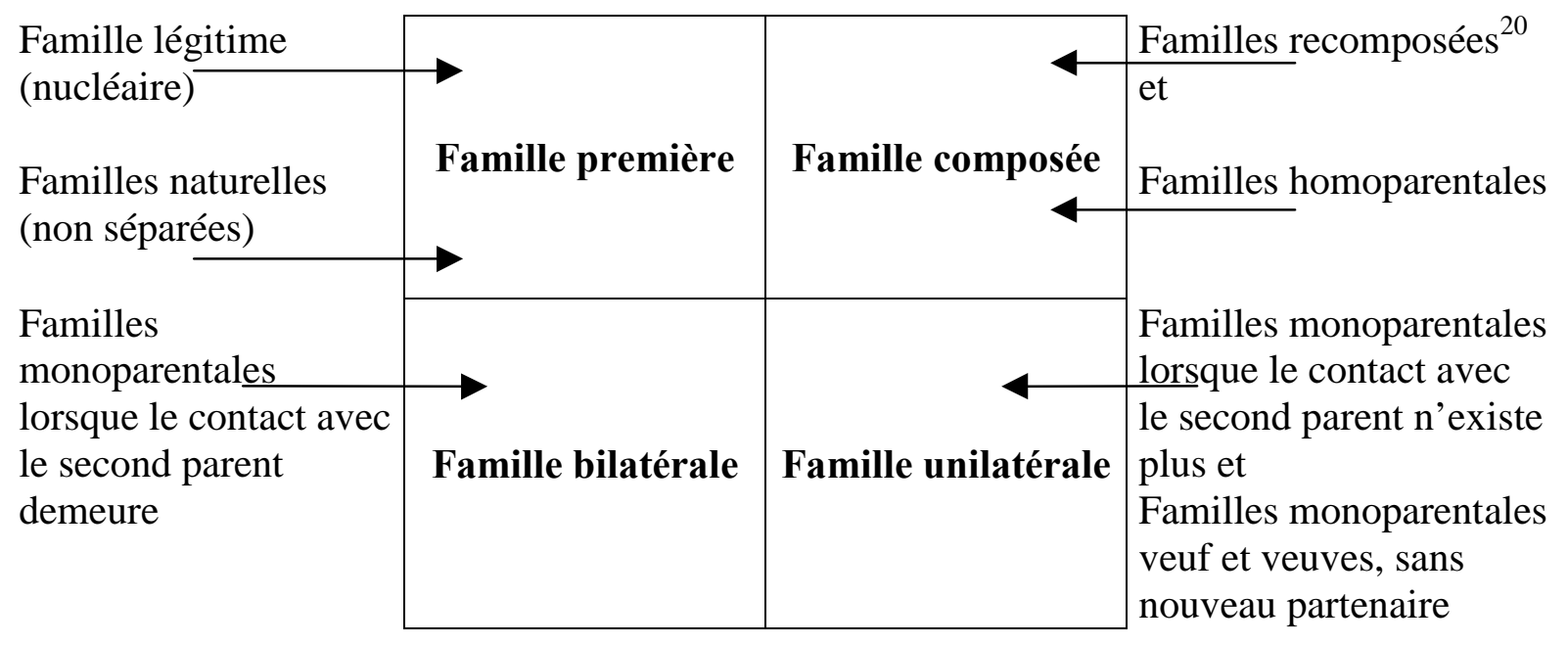

${ }^{20}$ Dans le cas des familles recomposées, plusieurs formes de parentalité se superposent : une parentalité composée (lorsqu'il y a une recomposition conjugale pour l'un des deux parents) qui s'ajoute à une parentalité bilatérale entre l'enfant et son couple parental d'origine (lorsque le contact avec l'ancien parent demeure). 


\section{SECTION I.B LA PARENTALITE DANS LES STATISTIQUES} DEMOGRAPHIQUES

Dans la partie théorique précédente, nous avons montré de quelle manière le concept de parentalité tient compte de la dissociation entre la filiation et la conjugalité, ainsi que de l'exercice réel du lien familial (en fonction des états de fait et non plus du statut juridique des individus). La grande difficulté actuelle est d'adapter les anciennes catégories (fondées sur le juridique) aux pratiques sociologiques. Or, il est difficile de mesurer les phénomènes de parentalité car les statistiques sont encore basées sur les critères traditionnels (marié, célibataire, veuf, divorcé).

En France, les catégories actuelles tiennent compte, certes, de la cohabitation (par les «enquêtes Emploi»), mais rendent difficilement compte des liens de paternité (en cas de séparation). Les problèmes de mesure de la décohabitation demeurent, puisqu'un individu est attaché à un ménage et un seul (et ne peut être pris en compte dans deux ménages), ignorant le cas de la garde alternée pour l'enfant, ou les phénomènes de «living apart together». En Allemagne, la mesure des phénomènes de parentalité est d'autant plus difficile que les statistiques officielles se basent uniquement sur les critères juridiques du statut matrimonial (elles tiennent peu compte des situations de cohabitation) (cf. Annexes 5 et 6).

Malgré ces difficultés méthodologiques, nous nous s'intéressons à une mesure qui tient compte de la présence de l'enfant (même temporaire). Les évolutions du mariage et du divorce sont présentées séparément (sous forme d'encadrés). En effet, au même titre que la dénatalité (qui la touche encore plus indirectement ${ }^{21}$ ), elles influencent directement l'émergence des nouvelles formes de famille, comme les couples de cohabitants (avec ou sans enfant), les familles monoparentales et les familles recomposées (Le Bras 1997 : 50). Cependant, elles n'en déterminent pas les pratiques.

\footnotetext{
${ }^{21}$ Ses évolutions sont présentées en annexe (cf. Annexe 4).
} 


\section{SOUS-SECTION I.B.1 LE MAINTIEN DE LA PARENTALITE HORS- MARIAGE DANS L'ANCIENNE ALLEMAGNE DE L'EST}

La réunification a modifié les comportements socio-démographiques des familles dans l'ancienne Allemagne de l'Est. Le recul démographique s'est maintenu après la réunification ; il est du au recul de l'immigration, mais surtout à la baisse radicale du nombre de naissances depuis 1992 : le recul des naissances atteint alors 40,5 \% entre 1989 et 1993 . Parallèlement, le nombre de mariage a baissé de 37,5\%.

Les résultats du rapport social sur les évolutions démographiques dans l'ancienne Allemagne de l'Est (Kurz-Scherf, Winkler 1994) montrent que se sont essentiellement les conditions et les structures sociales qui ont changé et non pas les représentations ${ }^{22}$. Le rapport nomme plusieurs raisons qui peuvent expliquer le recul du nombre de naissances : après la réunification, un nombre important de discriminations positives à l'égard des familles ont été supprimées (ensemble de crédits, habitat, structures d'accueil des enfants); il peut également s'agir d'une adaptation des comportements de fécondité à ceux de l'ancienne Allemagne de l'Ouest (comme par exemple l'ajustement de l'âge au premier enfant).

Les comportements socio-démographiques de l'ancienne Allemagne de l'Est se caractérisaient par un faible taux de couple sans enfant («Kinderlosigkeit»), un fort taux de mariage et de remariage et des comportements spécifiques selon l'âge : mariages et naissances précoces, fort taux de premier mariage, de divorce et de remariage (ce qui illustre un processus très dynamique de formation des familles). Par ailleurs, le mariage et la naissance du premier enfant étaient relativement indépendants, d'où un taux très important de familles monoparentales (et surtout de familles monoparentales célibataires). Le taux de naissances dans les familles naturelles est beaucoup plus important que dans l'ancienne Allemagne de l'Ouest, il s'accompagne également d'une forte proportion de légitimation. Contre toute attente, le nombre de familles naturelles et le nombre de naissances au sein de familles naturelles n'a pas diminué après la réunification, au contraire, entre 1989 et 1991, le taux de familles naturelles est passé de $33,6 \%$ à $41,7 \%$ et le taux de naissances dans les familles naturelles continue d' augmenter.

Le rapport évoque une tradition généralisée de mise en couple : le taux de personnes mariées est plus haut que dans l'ancienne Allemagne de l'Ouest, surtout pour les 25-35 ans mais également pour les 20-25 ans (depuis la réunification le nombre de jeunes couples mariés a largement diminué) ; la proportion de familles naturelles y est plus importante en particulier pour les jeunes de 20 à 25 ans ; enfin, la proportion de personnes seules (à l'exception des personnes âgées de plus de 55 ans) y est moins importante.

Mais, depuis la réunification, le nombre de mariage et le nombre de divorces ont diminués. Ce qui exprime l'incertitude face à la nouvelle situation législative et juridique. Par

\footnotetext{
${ }^{22}$ La réglementation de l'avortement a été modifiée au moment de la réunification : la réglementation beaucoup plus libérale de l'ancienne Allemagne de l'Est a été adaptée à la législation de l'ancienne Allemagne de l'Ouest par la modification du paragraphe 218 par décision du tribunal constitutionnel fédéral «Bundesverfassungsgericht». Depuis la réunification, le nombre d'avortements a diminué, ce qui prolonge la tendance depuis le milieu des années 1980. Il est à noter que le recul du nombre de naissances ne corrèle pas avec une utilisation de l'avortement comme instrument de contrôle des naissances, même s'il y a eu une légère reprise depuis 1990.
} 
exemple, dans le cas du divorce, les frais de procédure ont augmenté et la druée de séparation nécessaire au couple est plus longue que dans l'ancienne procédure.

En revanche, le nombre d'enfants de familles naturelles est plus important : en 1993, le taux est resté constant alors que le nombre de naissances en chiffre absolu s'est maintenu. Ainsi la part des naissances des familles naturelles continue de progresser : $40 \%$ des enfants naissent hors mariage, contre 10\% dans l'ancienne Allemagne de l'Ouest.

Le pourcentage de familles monoparentales célibataires parmi les familles monoparentales est beaucoup plus élevé que dans l'ancienne Allemagne de l'Ouest. Suivant les données de Mikrozensus $1991^{23}$, elles ont plus souvent un enfant et la proportion de familles monoparentales à deux enfants y est un peu plus importante.

L'impact de la réunification sur le comportement des familles de l'ancienne Allemagne de l'Est montre leur incertitude face à la redéfinition du lien légitime. Elle s'exprime surtout dans les comportements des jeunes qui choisissent de moins se marier. Le phénomène de mise en couple s'est restreint, et le recul brutal des naissances montre le scepticisme face aux changements sociaux. Cependant, le modèle des familles naturelles s'affirme comme une forme familiale qui permet le maintien des comportements de mise en couple et de parentalité face à l'incertitude et à la restructuration sociale (Kurz-Scherf ; Winkler 1994).

Avant la réunification, les comportements familiaux de l'ancienne Allemagne de l'Est se caractérisaient par une dynamique familiale forte: les séparations et remises en couples étaient relativement fréquentes. En d'autres termes, une «parentalité composée» suivait fréquemment la «parentalité première» (d'origine). Par ailleurs, la proportion de familles monoparentales célibataires y était plus importante, ce qui signifiait que la «parentalité première» était plus souvent une «parentalité unilatérale». La réunification a ralenti ces phénomènes, privilégiant les modèles de «parentalité première» et de «parentalité bilatérale».

\footnotetext{
${ }^{23}$ Les données issues de l'enquête Mikrozensus se basent sur la qualité juridique du lien matrimonial : marié, célibataire, divorcé ou veuf. De fait, les personnes seules avec enfant, les familles naturelles et les familles recomposées sont regropés dans la même catégorie. Pour cette raison, nous avons déduit le nombre de familles naturelles (estimé) de la catégorie des familles monoparentales afin d'en limiter le double décompte.

Par ailleurs, les catégories de familles naturelles sans enfant et des personnes vivant seules peuvent être redondantes, en particulier dans le cas de "living apart together". Le nombre de personnes vivant seules est alors probablement surestimé car il comprend les couples qui existent, mais qui ne cohabitent pas. Ces problèmes méthodologiques sont liés aux notions classiques de ménage (fondé sur le critère de cohabitation), de famille, et de conjugalité légitime (qui assimile dans la catégorie «hors-mariage» toutes les situations qui ne s'inscrivent pas dans le mariage ).
} 


\section{SOUS-SECTION I.B.2 LA PARENTALITE DANS LES FAMILLES LEGITIMES ET NATURELLES}

Dans l'ancienne Allemagne de l'Ouest, les enfants de moins de 18 ans vivent à $84 \%$ avec leurs deux parents mariés et à $13 \%$ avec une mère non-mariée ou séparée. Ces proportions atteignent respectivement $75 \%$ et $21 \%$ dans l'ancienne Allemagne de l'Est. Dans les deux cas, 3\% des enfants vivent avec leur pères (Engstler 1999: 43).

La proportion des familles monoparentales est restée la même entre 1991 et 2000, elle représente $6,2 \%$ de l'ensemble des familles ${ }^{24}$ (cf. Tableau 1). Les familles naturelles avec enfant représentent 3,6\% des familles en 2000 en Allemagne de l'Est, leur proportion est beaucoup plus faible et presque résiduelle pour l'Allemagne de l'Ouest avec 1,2\%.

«Etre en couple» est la forme de famille qui domine l'ensemble des modèles.

Tableau 1 Répartition des types de famille en fonction de la situation conjugale et de la présence d'enfants, en Allemagne, en \%

\begin{tabular}{|c|c|c|c|c|c|c|c|}
\hline & $\begin{array}{c}\text { Familles } \\
\text { naturelles } \\
\text { sans enfant }\end{array}$ & $\begin{array}{c}\text { Couples } \\
\text { mariés sans } \\
\text { enfant }\end{array}$ & $\begin{array}{c}\text { Personnes } \\
\text { vivant } \\
\text { seules }\end{array}$ & $\begin{array}{c}\text { Familles } \\
\text { naturelles } \\
\text { avec } \\
\text { enfant(s) }\end{array}$ & $\begin{array}{c}\text { Couples } \\
\text { mariés avec } \\
\text { enfant(s) }\end{array}$ & $\begin{array}{c}\text { Familles } \\
\text { monoparen } \\
\text {-tales }\end{array}$ & Total \\
\hline ANCIENNE ALLEMAGNE DE L'EST \\
\hline 1991 & 2,2 & 25,4 & 27,7 & 2,7 & 34,4 & 7,6 & 100,0 \\
\hline 2000 & 3,8 & 26,0 & 34,4 & $\mathbf{3 , 6}$ & 24,5 & 7,7 & 100,0 \\
\hline ANCIENNE ALLEMAGNE DE L'OUEST & \multicolumn{7}{|l|}{} \\
\hline 1991 & 3,1 & 23,7 & 35,5 & 0,7 & 31,2 & 5,9 & 100,0 \\
\hline 2000 & 4,0 & 25,4 & 37,0 & $\mathbf{1 , 2}$ & 26,6 & 5,9 & 100,0 \\
\hline ALLEMAGNE REUNIFIEE & & & & & \\
\hline 1991 & 2,9 & 24,0 & 34,0 & 1,1 & 31,8 & $\mathbf{6 , 2}$ & 100,0 \\
\hline 2000 & 4,0 & 25,5 & 36,5 & 1,7 & 26,2 & $\mathbf{6 , 2}$ & 100,0 \\
\hline
\end{tabular}

Source: nos propres calculs à partir des données de l'enquête Mikrozensus 2000 (Statistisches Bundesamt $2001: 61-68-71$ )

Le tableau 2 (ci-dessous) regroupe l'ensemble des familles avec enfants. L'exercice de la parentalité bilatérale (familles naturelles en couple + couples mariés) est majoritaire avec $84,2 \%$ en 1991 et $81,8 \%$ en 2000 .

Dans ce tableau, la catégorie des familles monoparentales est prise dans un sens stricte puisque les familles naturelles ont été isolées. Les chiffres donnent alors une estimation de l'exercice de la parentalité unilatérale : elle reprénte 15, 9\% en 1991 et 18,3\% en 2000. Les chiffres montrent que cette proportion est plus importante pour l'Allemagne de l'Est et la progression relative de ces familles y est également plus considérable.

\footnotetext{
${ }^{24}$ Les valeurs absolues de chaque catégorie de famille (cf. Tableaux 1 et 2 ) sont présentées en annexe (annexe 2 et 3).
} 
Tableau 2 Répartition des types de familles avec enfant en fonction de la nature de la relation conjugale, en Allemagne, en \%

\begin{tabular}{|c|c|c|c|c|}
\hline & $\begin{array}{c}\text { Familles } \\
\text { naturelles avec } \\
\text { enfant(s) }\end{array}$ & $\begin{array}{c}\text { Couples } \\
\text { mariés avec } \\
\text { enfant(s) }\end{array}$ & $\begin{array}{c}\text { Familles } \\
\text { monoparentales }\end{array}$ & Total \\
\hline ANCIENNE ALLEMAGNE DE L'EST & 6,1 & 77,0 & 16,9 & 100,0 \\
\hline 1991 & 10,0 & 68,4 & 21,6 & 100,0 \\
\hline 2000 & 1,9 & 82,6 & 15,6 & 100,0 \\
\hline ANCIENNE ALLEMAGNE DE L'OUEST & 78,9 & 17,5 & 100,0 \\
\hline 1991 & 3,6 & $\mathbf{8 1 , 4}$ & $\mathbf{1 5 , 9}$ & 100,0 \\
\hline 2000 & $\mathbf{2 , 8}$ & $\mathbf{8 1 , 9}$ & 100,0 \\
\hline
\end{tabular}

Source : Source : nos propres calculs à partir des données de l'enquête Mikrozensus 2000 (Statistisches Bundesamt $2001:$ 61-68-71)

\section{I.B.2.1 La parentalité et le lien légitime}

\section{Encadré $\mathrm{n}^{\circ} 1 \quad$ Les évolutions du mariage dans l'Allemagne réunifiée}

Sur l'ensemble de l'Allemagne, le nombre de mariages a baissé constamment entre 1990 et 1999 (Sozialpolitische Umschau 1998b, Statistisches Bundesamt 2000e). La baisse a été plus importante dans l'ancienne Allemagne de Ouest que dans l'ancienne Allemagne de l'Est ${ }^{25}$. Pour cette dernière, l'évolution n'a pas été linéaire. De légères augmentations du nombre d'unions légitimes entre 1993 et 1995, viennent marquer la tendance drastique à la baisse depuis 1989: le nombre de mariages a été divisé par deux entre 1990 et 1991, puis a encore baissé de 4,5 points en 1992 (Sozialpolitische Umschau 1997b).

On a vu une légère reprise des mariages en 1999 avec une augmentation de 2,1\% pour l'ancienne Allemagne de l'Ouest et de 10, $3 \%$ pour l'ancienne Allemagne de l'Est (Statistisches Bundesamt 2000e). Mais cette reprise de $1999^{26}$ est très ponctuelle puisqu'en 2000 le nombre de mariages a de nouveau baissé tout en restant légèrement supérieur à celui de 1998 (Statistisches Bundesamt 2001d) ${ }^{27}$. En 1999, en Allemagne de 1'Est, le nombre total de mariages atteint $59 \%$ de celui de 1990 (Statistisches Bundesamt 2002a).

\footnotetext{
${ }^{25}$ L'évolution du nombre de mariages ne se fait pas partout de la même manière, bien que la tendance générale en Europe, soit à la baisse. Le taux de nuptialité français a abaisse progressivement pour devenir un des taux les plus bas d'Europe, alors que l'Allemagne s'est maintenue avec un des taux les plus élevés (Hantrais 1994).

${ }^{26}$ La date du 9.09.1999 n'a pas été sans influence dans le choix de certains couples à célébrer leur union cette année là (Statistisches Bundesamt 2000e).

${ }^{27}$ En Allemagne, en 1999, l'âge au mariage était de 34,7 ans pour les hommes et de 31,7 ans pour les femmes (quel que soit leur statut matrimonial). Quant aux célibataires, l'âge au mariage est plus jeune (ce qui représente
} 
La proportion de couples mariés avec au moins un enfant de moins de 18 ans tend à diminuer par rapport à l'ensemble des familles. En l'intervalle de 10 ans, on a vu diminuer leur proportion de $7,1 \%$. Cela concerne surtout les familles avec un ou deux jeunes enfants. Le nombre de familles avec trois enfants ou plus s'est maintenu, ce qui s'explique par la structure familiale des familles d'origine étrangère (Statistisches Bundesamt 2000a). Aujourd'hui, on voit se développer une forme de famille qui s'inscrit dans la conjugalité, sans qu'il y ait d'enfant. La conjugalité n'est plus liée à l'exercice de la parentalité. On a précédemment rappelé le phénomène de dissociation de la filiation et du lien conjugal. Ici, c'est la conjugalité qui se dissocie de la filiation.

Entre 1991 et 1999, le nombre d'enfants de moins de six ans dans les familles monoparentales ou les familles naturelles a augmenté. Dans l'ancienne Allemagne de l'Est le nombre de familles monoparentales, recomposées et cohabitantes, qui recueillent un enfant de moins de six ans, a beaucoup moins diminué (-17,4\%), que le nombre de couples mariés (60,3\%) (Statistisches Bundesamt 2000b).

Entre 1991 et 2000, la proportion de familles monoparentales dont le chef de famille est un homme a augmenté de $63 \%$ mais leur part est très minoritaire par rapport à l'ensemble des familles monoparentales dont le chef de famille est une femme (leur nombre a augmenté de $31 \%$ entre 1991 et 2000). Pour l'année 2000, 436000 pères monoparentaux ont été recensés, ce qui représente $15 \%$ de l'ensemble des familles monoparentales (Statistisches Bundesamt 2001 : 68). Ces chiffres montrent que la parentalité unilatérale est majoritairement un phénomène féminin. En revanche, elle concerne désormais aussi les pères. Ce phénomène est nouveau car les chiffres des familles monoparentales «homme» était resté longtemps inchangé.

\section{I.B.2.2 La parentalité allemande au sein des familles naturelles}

Le nombre de familles naturelles a augmenté de façon constante depuis 1972. Leur nombre s'est multiplié par douze entre 1972 et 2000, et dans la dernière décennie, entre 1991 et 2000, leur nombre a augmenté de $52 \%$ (Statistisches Bundesamt 2000b).

Dans l'ancienne Allemagne de l'Ouest, les familles naturelles correspondent plus à un arrangement conjugal, qu'à une forme de famille. Encore actuellement, seules $20 \%$ des familles naturelles ont des enfant. Il en est autrement dans l'ancienne Allemagne de l'Est, puisque $40 \%$ des naissances s'effectuent dans des familles naturelles. Elles sont donc un arrangement familial autant qu'un arrangement conjugal.

Sur l'ensemble de l'Allemagne réunifiée, la proportion est donc de $30 \%$ de familles naturelles avec enfant pour $70 \%$ sans enfant ${ }^{28}$ (cf. Tableau 3 ).

la plus grande proportion) les hommes sont âges de 31 ans et les femmes de 28,3 ans (Statistisches Bundesamt 2002a: 69).

${ }^{28} \mathrm{~L}$ 'annexe 3 précise le nombre absolu pour chaque catégories de familles. 
Tableau 3 Rapport entre les familles naturelles avec enfant et sans enfant, en Allemagne, en $\%$

Dans L'AnCienne Allemagne de l'OUest

\begin{tabular}{|l|c|c|c|c|c|}
\hline Familles naturelles & 1982 & 1985 & 1988 & 1989 & 1990 \\
\hline Sans enfant & 86,2 & 89,8 & 88,2 & 88,5 & 88,9 \\
\hline Avec enfant & 13,8 & $10,2^{29}$ & 11,8 & 11,5 & 11,1 \\
\hline Ensemble & 100,0 & 100,0 & 100,0 & 100,0 & 100,0 \\
\hline
\end{tabular}

DANS L'AlLEMAGNe REUNIFIEE

\begin{tabular}{|l|c|c|c|c|c|c|c|c|c|}
\hline Familles naturelles & 1991 & 1992 & 1993 & 1994 & 1996 & 1997 & 1998 & 1999 & 2000 \\
\hline Sans enfant & 72,9 & 72,5 & 72,4 & 72,1 & 72,1 & 72,2 & 71,9 & 71,1 & $\mathbf{7 0 , 5}$ \\
\hline Avec enfant & 27,1 & 27,5 & 27,6 & 27,9 & 27,9 & 27,8 & 28,1 & 28,9 & $\mathbf{2 9 , 5}$ \\
\hline Ensemble & 100,0 & 100,0 & 100,0 & 100,0 & 100,0 & 100,0 & 100,0 & 100,0 & 100,0 \\
\hline
\end{tabular}

(Statistisches Bundesamt 2002a : 64)

Les familles naturelles sont plus souvent le fait de jeunes adultes : $46 \%$ de ces familles ont moins de 35 ans alors que la proportion n'est que de $12 \%$ pour les couples légitimes. $29 \%$ d'entre elles ont des enfants alors que c'est le cas de 51\% des couples légitimes.

$58 \%$ des familles naturelles sont formées de deux célibataires et $11 \%$ de deux divorcés (Statistisches Bundesamt 2000b). La part de «parentalité composée» dans les familles naturelles concerne la majorité des cas avec 53\% dans l'ancienne Allemagne de l'Est (statuts différents). A l'inverse, l'exercice de la parentalité dans les familles naturelles de l'ancienne Allemagne de l'Ouest correspond à une «parentalité première» dans 59,5\% des cas (cf. Tableau 4).

Tableau $4 \quad$ Familles naturelles en fonction du statut matrimonial et de la présence d'enfant, comparaison entre les nouveaux et les anciens Bundesländer en 1991 en \%

\begin{tabular}{|l|c|c|c||c|c|c|c||c|}
\hline \multirow{2}{*}{$\begin{array}{c}\text { Situation matrimoniale } \\
\text { du partenaire }\end{array}$} & \multicolumn{3}{|c|}{ Ancienne Allemagne de l'Ouest } & \multicolumn{3}{|c|}{ Ancienne Allemagne de l'Est } \\
\cline { 2 - 9 } & $\begin{array}{c}\text { Avec } \\
\text { enfant }\end{array}$ & $\begin{array}{c}\text { Sans } \\
\text { enfant }\end{array}$ & $\begin{array}{c}\text { Ensem- } \\
\text { ble }\end{array}$ & Total & $\begin{array}{c}\text { Avec } \\
\text { enfant }\end{array}$ & $\begin{array}{c}\text { Sans } \\
\text { enfant }\end{array}$ & $\begin{array}{c}\text { Ensem- } \\
\text { ble }\end{array}$ & Total \\
\hline $\begin{array}{l}\text { Les deux partenaires sont } \\
\text { célibataires }\end{array}$ & $10 \%$ & $90,0 \%$ & $100 \%$ & $\mathbf{5 9 , 5 \%}$ & $52,9 \%$ & $47,1 \%$ & $100 \%$ & $47,0 \%$ \\
\hline $\begin{array}{l}\text { Un partenaire est } \\
\text { célibataire -un partenaire } \\
\text { n'est pas célibataire }\end{array}$ & $32,2 \%$ & $67,8 \%$ & $100 \%$ & $18,7 \%$ & $68,5 \%$ & $31,5 \%$ & $100 \%$ & $\mathbf{2 1 , 3 \%}$ \\
\hline $\begin{array}{l}\text { Les deux partenaires ne } \\
\text { sont pas célibataires }\end{array}$ & $30,4 \%$ & $69,6 \%$ & $100 \%$ & $21,8 \%$ & $49,0 \%$ & $51,0 \%$ & $100 \%$ & $\mathbf{3 1 , 7 \%}$ \\
\hline Ensemble & $18,6 \%$ & $81,4 \%$ & $100 \%$ & $100 \%$ & $55,0 \%$ & $45,0 \%$ & $100 \%$ & $100 \%$ \\
\hline
\end{tabular}

Source : Bundesministerium für Familie und Senioren (1994: 51)

\footnotetext{
${ }^{29}$ Entre 1982 et 1985, la proportion absolue de familles naturelles avec enfants est restée la même, c'est le nombre de familles naturelles sans enfant qui a augmenté.
} 
A l'Est, le nombre de familles naturelles a augmenté en moindre proportion : plus 6 points entre 1991 et 2000. En 2000, environ $49 \%$ de l'ensemble des familles naturelles ont un ou plusieurs enfants (Statistisches Bundesamt 2001 : 73-75). L'exercice de la parentalité dans les familles naturelles est donc plus généralisé dans l'ancienne Allemagne de l'Est qu'à l'Ouest $^{30}$. La filiation est relativement plus dissociée de la conjugalité que dans l'ancienne Allemagne de l'Ouest où les deux phénomènes restent encore largement liés.

Le nombre de naissances dans les familles naturelles est en constante augmentation : leur proportion par rapport à l'ensemble des naissances représente $20 \%$ en 1998, $22 \%$ en 1999 et $23,4 \%$ en $2000^{31}$.

En Allemagne, la parentalité au sein de familles naturelles est limitée puisque la venue des enfants provoque généralement une union légitime des parents, au moins à partir du second enfant. Son exercice est donc essentiellement bilatéral. En ce sens, en Allemagne, l'exercice de la parentalité dans les familles naturelles est beaucoup moins l'affirmation d'un nouveau mode de parentalité qu'une phrase précédant l'inscription de la parentalité dans le conjugal.

Les enfants de familles naturelles vivent plus souvent une séparation des parents que les enfants de familles légitimes (Sozialpolitische Umschau 1998d). Ils subissent plus souvent que les autres la perte d'un père et la présence d'un beau-père. Pour l'ensemble de ces enfants qui vivaient dans une famille naturelle jusqu'à leurs trois ans, un peu moins de la moitié n'avait plus de contact personnel avec leur père. Et sur une période de dix ans, plus des deux tiers ont perdu tout contact avec lui (Sozialpolitische Umschau 1998d). La «parentalité première» dans les familles naturelles laisse plus souvent la place a une «parentalité unilatérale» que pour les familles mariées. Or si les «parentalités composées» sont plus répandues dans ce type de famille, elles semblent être le fait de la recomposition familiale d'un seul parent (le contact avec l'autre parent ayant disparu pour les deux tiers). Ainsi les «parentalités composées» se fondent sur une «parentalité unilatérale» et non sur le maintien du couple parental d'origine.

La proportion de couples sans enfant qui divorcent est supérieure à celle des couples avec enfant(s) (mineurs au moment du verdict), soit $52 \%$ contre $48 \%$ en $1999^{32}$. Et les divorces qui concernent des familles avec enfant, ne sont pas en constante augmentation. En d'autres termes, la «parentalité première» reste le modèle dominant de l'exercice de la parentalité.

\footnotetext{
${ }^{30}$ Le nombre moyen d'enfants dans les familles naturelles est de 1,4 contre 1,6 pour les couples mariés à l'Est (respectivement 1,4, contre 1,8 pour les couples mariés à l'Ouest) (Statistisches Bundesamt 2001 : 73-75).

${ }^{31}$ Nos propres calculs à partir des données de l'enquête Mikrozensus 2000 (Statistisches Bundesamt 2001d).

${ }^{32}$ Nos propres calculs à partir des données Mikrozensus (Statistisches Bundesamt 2002a : 77).
} 
Encadré $\mathrm{n}^{\circ} 2 \quad$ Les évolutions du divorce

La France et l'Allemagne appartiennent toutes les deux à la catégorie médiane des pays répartis selon leur taux de divortialité (Hantrais 1994). L'indice conjoncturel de divortialité, après une augmentation au cours des années 1970, est demeuré stable, autour de 0,3, entre 1985 et 1992, en France et en Allemagne (Bégeot, Fernandez - Cordon 1997 : 45). La tendance reste forte puisqu'environ un tiers des mariages se termine par un divorce avant d'atteindre 25 ans de vie commune (Sozialpolitische Umschau 1997a). Le taux de divorce en Allemagne de l'Ouest était inférieur à celui de l'Allemagne de l'Est. Après une constante baisse depuis 1991, il a de nouveau augmenté mais il reste encore inférieur au niveau relatif qu'il avait atteint dans les années 1980 (Engstler 1999 : 88).

Pour l'Allemagne réunifiée, on a vu une baisse du nombre de divorces en 1999 qui ne s'est pas maintenue en 2000 puisque leur nombre a de nouveau augmenté. Il reste supérieur à celui de 1998 (Statistisches Bundesamt 2001b, Statistisches Bundesamt 2001d, Statistisches Bundesamt 2002a : 76). Il prolonge ainsi la tendance à l'augmentation depuis les années 1970. 


\section{SOUS-SECTION I.B.3 EN FRANCE}

\section{I.B.3.1 Les reconnaissances des pères et des mères et l'exercice de la parentalité}

Encadré $\mathrm{n}^{\circ} 3 \quad$ L'évolution du nombre de mariage depuis la seconde guerre mondiale

En 1946 et 1947, le nombre de mariage est très élevé. Il est du en particulier au nombre d'unions qui n'avaient pas pu être légitimées pendant la guerre. Le nombre de mariage diminue jusqu'en 1956. Puis il augmente constamment jusqu'en 1972, soit une augmentation de 34 points en quinze ans. En 1972, l'âge au mariage est au plus bas et le nombre de mariages au plus haut. Depuis le nombre de mariage baisse continuellement, surtout pour les premiers mariages avec une baisse de $45 \%$ entre 1972 et 1994 (contre $39 \%$ pour l'ensemble des mariages). Sur cette même période le nombre de remariages a doublé (Daguet 1996).

Le poids des remariages est au plus bas en 1968 jusqu'en 1974, où ils ne représentaient que 8 $\%$ des mariages. En 1994, à peu près un quart des mariages est un remariage pour au moins un des deux époux.

Le nombre de divorces qui était de deux fois et demi plus élevé à la sortie de la guerre qu'en 1938 s'est stabilisé entre 1953 et 1963. Depuis il a continuellement augmenté avec une progression extrêmement rapide dans les années 1970. Puis il s'est relativement stabilisé au cours des années 1980. Depuis la hausse de la divortialité s'est ralentie (Daguet 1996).

Entre 1954 et 1968, la part des couples cohabitants était constante avec $3 \%$. Dans les années 1960, $86 \%$ de ces couples non-mariés comprenaient un veuf ou un divorcé. Part la suite, la cohabitation est devenue importante chez les jeunes célibataires et dans les années 1980, la cohabitation s'affirme comme le mode principal d'entrer en couple. Dans les années 1990, les couples hors mariage sont majoritairement des couples de célibataires et non plus, comme auparavant, des veufs et des divorcés.

Globalement, le nombre de mariage a continuellement baissé depuis 1972, à l'exception de la fin des années 1980. En 1996, il a brusquement augmenté de 10 \% (Beaumel, Kerjosse, Toulemon 1999). Cela concerne surtout les mariages de couples avec plusieurs enfants qui ont augmenté de $37 \%$, tandis que l'augmentation n'est que de $2,5 \%$ pour les couples sans enfant. Ces évolutions sont à rattacher à la réforme fiscale de 1996 qui a modifié le mode d'imposition des cohabitants, ceux-ci perdant le bénéfice de la demi-part supplémentaire accordée au premier enfant d'un parent célibataire. Cette réforme cible surtout les parents qui ont des revenus élevés, voire ceux dont la différence de salaire entre les conjoints est importante (ce qui explique son impact dans les couches sociales favorisées) .

La signification du mariage a changé de nature : il ne correspond plus au processus de mise en couple. En effet, au début des années 1990, un mariage sans cohabitation préalable concerne seulement une mise en couple sur neuf. Cela représentait un quart des mariages annuels (Beaumel, Kerjosse, Toulemon 1999). La cohabitation est devenue le mode exclusif de mise en couple et concerne les personnes les plus jeunes. Les cohabitants sont les plus nombreux dans la tranche d'âge des 26-28 ans (respectivement pour les femmes et les hommes). La cohabitation devient alors un mode de vie en couple durable. 
De plus, la parentalité ne s'inscrit plus uniquement dans le lien du mariage et l'entrée dans la parentalité s'inscrit maintenant indépendamment du mariage.

Tableau 5 Proportion de couples avec et sans enfant en fonction du lien de conjugalité, en $\underline{\% \text {, en France }}$

\begin{tabular}{|l|c|c|c|c|c|c|c|c|c|}
\hline $\begin{array}{l}\text { FAMILLES } \\
\text { NATURELLES }\end{array}$ & $\mathbf{1 9 9 0}$ & $\mathbf{1 9 9 1}$ & $\mathbf{1 9 9 2}$ & $\mathbf{1 9 9 3}$ & $\mathbf{1 9 9 4}$ & $\mathbf{1 9 9 5}$ & $\mathbf{1 9 9 6}$ & $\mathbf{1 9 9 7}$ & $\mathbf{1 9 9 8}$ \\
\hline Sans enfant & 64,2 & 62,3 & 60,3 & 60,4 & 59,4 & 59,4 & 57,7 & 56,4 & 55,7 \\
\hline Avec enfant & 35,8 & 37,7 & 39,7 & 39,6 & 40,6 & 40,6 & 42,3 & 43,6 & $\mathbf{4 4 , 3}$ \\
\hline Ensemble & 100,0 & 100,0 & 100,0 & 100,0 & 100,0 & 100,0 & 100,0 & 100,0 & 100,0 \\
\hline \multicolumn{10}{|c|}{} \\
\hline COUPLES MARIES & $\mathbf{1 9 9 0}$ & $\mathbf{1 9 9 1}$ & $\mathbf{1 9 9 2}$ & $\mathbf{1 9 9 3}$ & $\mathbf{1 9 9 4}$ & $\mathbf{1 9 9 5}$ & $\mathbf{1 9 9 6}$ & $\mathbf{1 9 9 7}$ & $\mathbf{1 9 9 8}$ \\
\hline Sans enfant & 53,9 & 54,7 & 55,4 & 56,0 & 56,4 & 56,6 & 57,2 & 57,4 & 58,2 \\
\hline Avec enfant & 46,1 & 45,3 & 44,6 & 44,0 & 43,6 & 43,4 & 42,8 & 42,6 & $\mathbf{4 1 , 8}$ \\
\hline Ensemble & 100,0 & 100,0 & 100,0 & 100,0 & 100,0 & 100,0 & 100,0 & 100,0 & 100,0 \\
\hline
\end{tabular}

Champ : personnes majeures, vivant en couple dans le même logement ; enfants mineurs résidant avec le couple (avec l'un des deux parents ou des deux)

Source : nos propres calculs d'après INSEE, Enquêtes Emploi, 1990 à 1998 (Beaumel, Kerjosse, Toulemon 1999)

La légitimation des enfants et du couple intervient également beaucoup plus tard, indépendamment des naissances. La venue d'un enfant n'entraîne plus nécessairement le mariage, ce que confirme la proportion de conceptions prénuptiales qui est passée de 11,1\% en 1975 à 3,8 \% en 1996 (Beaumel, Kerjosse, Toulemon 1999). En 1996, 40 \% des naissances s'inscrivent dans des familles naturelles, mais $52 \%$ des premières naissances $(29 \%$ des naissances de rang deux et $20 \%$ des naissances de rang trois et plus).

La majeure partie des mariages ne légitiment pas d'enfant. Leur proportion a diminué de 10 points au cours de la première moitié des années 1990 (cf. Tableau 6). En d'autres termes, le mariage précède de moins en moins le passage à la parentalité.

Tableau 6 Répartition des mariages selon le nombre d'enfants légitimés (en \%)

\begin{tabular}{|c|c|c|c|c|c|}
\hline \hline Années & $\begin{array}{c}\text { Mariages ne } \\
\text { légitimant } \\
\text { pas d'enfant }\end{array}$ & $\begin{array}{c}\text { Mariages } \\
\text { légitimant un } \\
\text { enfant }\end{array}$ & $\begin{array}{c}\text { Mariages } \\
\text { légitimant } \\
\text { deux enfants }\end{array}$ & $\begin{array}{c}\text { Mariages } \\
\text { légitimant } \\
\text { trois enfants } \\
\text { ou plus }\end{array}$ & Total \\
\hline 1990 & 82,4 & 13,2 & 3,5 & 1,0 & $100 \%$ \\
\hline 1991 & 81,5 & 13,6 & 3,8 & 1,1 & $100 \%$ \\
\hline 1992 & 80,5 & 14,3 & 4,1 & 1,1 & $100 \%$ \\
\hline 1993 & 79,3 & 14,9 & 4,5 & 1,3 & $100 \%$ \\
\hline 1994 & 78,1 & 15,5 & 5,0 & 1,4 & $100 \%$ \\
\hline 1995 & 77,4 & 15,6 & 5,4 & 1,6 & $100 \%$ \\
\hline 1996 & 71,9 & 18,5 & 7,7 & 1,9 & $100 \%$ \\
\hline
\end{tabular}

Source : INSEE, statistiques de l'état civil (Beaumel, Kerjosse, Toulemon 1999) 
Entre 1989 et 1993, 81 sur 100 mariages ne légitimaient pas d'enfant et 19 légitimaient au moins un enfant. Sur ces dix-neuf couples, 7 d'entre eux légitimaient des enfants en présence de demi-frère et de demi-sœur. Sur les 81 mariages restants, 11 concernaient des couples dont au moins un des conjoints avait un enfant. Sur l'ensemble des unions formées entre 1989 et 1993, avec ou sans mariage, $11 \%$ des hommes et des femmes avaient déjà un enfant (Beaumel, Kerjosse, Toulemon 1999). Les maris vivent avec les enfants de leur conjointe dans neuf cas sur dix, ce qui n'est le cas que d'une belle-mère sur deux. Globalement, un des conjoints devient un beau-parent dans $18 \%$ des mariages (Beaumel, Kerjosse, Toulemon 1999). On a la même proportion de beaux-parents quelque soit le sexe : un tiers de ces mariages concerne les hommes qui étaient seul à avoir des enfants auparavant, un tiers concerne les femmes et un tiers les deux conjoints.

La part des naissances hors mariage est en progression constante ${ }^{33}$ depuis les années 1960. En 1960 et 1970, les naissances hors mariage, encore confinées dans les milieux populaires ouvriers et employés de petits commerces, se diffusent dans les autres catégories sociales, elles restent cependant sous représentées dans les catégories supérieures (Ministère de l'emploi et de la solidarité 2000 : 50). Elles représentaient 6,1\% de l'ensemble des naissances en 1960 pour atteindre à peu près un quart au cours des années 1980. Dans les années 1990, elles représentaient plus du tiers des naissances et dépassent $40 \%$ en 1998. Ce taux est similaire à la proportion de naissances hors mariage dans l'ancienne Allemagne de l'Est.

L'exercice actuel de la parentalité dans les familles naturelles est différent de celui des années 1970, comme le montre l'attribution du nom de l'enfant à la naissance. En effet, au début des années 1970, les trois quarts des enfants nés hors mariage se voyaient attribuer le nom de la mère. Aujourd'hui, sous l'influence de l'augmentation des reconnaissances paternelles et surtout des reconnaissances prénatales, les proportions se sont inversées : désormais, 7 enfants sur 10 portent le nom du père. Et sur ceux enregistrés sous le nom de la mère, les deux tiers d'entre eux sont susceptibles de changer rapidement de nom (Ministère de l'emploi et de la solidarité 2000). En 1994, un mois après la naissance, 8 enfants sur 10 portent le nom du père en 1994 (respectivement un enfant sur 3 en 1965-1970).

En ce qui concerne les comportements de reconnaissances paternelles et de légitimation, ce ne sont plus les femmes les plus jeunes qui obtiennent le plus rapidement et le plus fréquemment une reconnaissance paternelle. Au contraire, à l'heure actuelle, le nombre de reconnaissances paternelles est le plus important pour les femmes âgées de 25 à 30 ans. Un fort écart d'âge avec le père continue de retarder les reconnaissances et les légitimations. L'influence des catégories socioprofessionnelles joue un rôle déterminant dans la fin des années 1990, leur impact s'est inversé depuis les années 1960 et 1970 : en effet, l'inactivité de la mère ne favorise plus la reconnaissance paternelle, au contraire se sont les enfants de mères avec une activité intermédiaire et supérieure qui sont le plus proprement reconnu par les pères, et ce d'autant plus que le père appartient à la même catégorie sociale (Ministère de l'emploi et de la solidarité 2000 : 52).

\footnotetext{
${ }^{33}$ Le nombre de familles naturelles est en constante augmentation. La France se situe parmi les pays européens dont les taux sont les plus hauts, rejoignant les pays scandinaves. L'Allemagne appartient au groupe de pays ayant les taux les plus bas (tendance plutôt suivie par les pays européens du Sud) (Hantrais 1994).
} 
Aujourd'hui presque tous les enfants sont reconnus et l'impact de la catégorie sociale est beaucoup plus atténué. Seules les reconnaissances prénatales restent socialement marquées. Les mères exerçant une profession intermédiaire ou supérieure obtiennent une reconnaissance paternelle avant la naissance dans une proportion deux fois et demi plus forte que les mères sans profession (Ministère de l'emploi et de la solidarité 2000). Les catégories populaires, qui reconnaissaient autrefois le plus largement et le plus vite les enfants sont aujourd'hui devancées par les catégories supérieures et moyennes (Munoz-Pérez, Prioux 1999b).

Ainsi, les catégories supérieures ont moins souvent d'enfants hors mariage, mais les reconnaissances prénatales des pères et les reconnaissances conjointes prénatales sont plus nombreuses. Ils dotent ainsi les enfants, dès la naissance, d'une filiation à l'égard des deux parents, comme les enfants nés dans le mariage (Munoz-Pérez, Prioux 1999b : 860).

En résumé, les catégories sociales se différencient moins qu'autrefois dans leurs usages de la reconnaissance et de la légitimation ${ }^{34}$. Les naissances hors mariage se répartissant sur l'ensemble des milieux, mais elles restent quand même plus fréquentes dans les milieux populaires. Toutefois les reconnaissances prénatales conjointes sont une pratique socialement marquée dans les classes supérieures (Ministère de l'emploi et de la solidarité 2000 : 50). La biparentalité dans les familles naturelles est essentiellement une parentalité bilatérale et première. La distinction entre familles légitimes et familles naturelles perd de sa signification quant à l'exercice de la parentalité. En effet, les reconnaissances par les pères avant la naissance de l'enfant (et les reconnaissantes conjointes (même si elles se font en moindre proportion)) montrent la volonté des parents d'institutionnaliser l'exercice bilatéral de la parentalité. On voit ici très précisément que la «parentalité bilatérale» relève des pratiques sociales et dépasse les catégories juridiques de légitimité et d'illégitimité.

Pendant, les deux premiers tiers du XXe siècle, la proportion des naissances hors mariage est restée stable et marginale (Daguet 1996). Depuis 1965, la proportion de naissance hors mariage a augmenté sans interruption, elle s'est accélérée dans les années 1980 et sa progression s'est ralentie depuis. La part de reconnaissance paternelle montre que de nombreuses naissances s'inscrivent dans un couple (Daguet 1996). La présence enfant demeure plus fréquente lorsque le couple est marié et en 1990, on a parmi les couples où l'homme à moins de 35 ans, $80 \%$ de couple marié et $40 \%$ des couples non mariés qui ont des enfants. (Daguet 1996).

Le tableau 7 montre la progression de la part des naissances hors mariage par rapport à l'ensemble des naissances entre 1960 et 1998 en France. La hausse des naissances hors mariage est essentiellement le fait de couples, puisque la proportion de naissances survenant chez des femmes seules est restée relativement inchangée depuis la fin des années 1960 et approche environ $4 \%$ (Couet 1996).

\footnotetext{
${ }^{34}$ La catégorie socioprofessionnelle des parents ne semble plus jouer un rôle significatif pour la légitimation des enfants par le mariage. Elle est devenue moins fréquente et plus tardive : moins de la moitié des enfants reconnus sont légitimés de cette façon alors que plus des deux tiers l'étaient il y a moins de trente ans (Ministère de l'emploi et de la solidarité 2000 : 50).
} 
Tableau $7 \quad$ Nombre de naissance hors mariage par rapport au nombre total de naissance depuis 1960, en France

\begin{tabular}{|c|c|c|c|c|c|c|c|c|}
\hline Année & 1960 & 1965 & 1970 & 1975 & 1980 & 1985 & 1990 & 1991 \\
\hline$\%$ & $6,1 \%$ & $5,9 \%$ & $6,8 \%$ & $8,5 \%$ & $11,4 \%$ & $19,6 \%$ & $30,1 \%$ & $31,8 \%$ \\
\hline
\end{tabular}

\begin{tabular}{|c|c|c|c|c|c|c|}
\hline 1992 & 1993 & 1994 & 1995 & 1996 & 1997 & 1998 \\
\hline $33,2 \%$ & $34,9 \%$ & $36,1 \%$ & $37,6 \%$ & $38,9 \%$ & $40,0 \%$ & $40,7 \%$ \\
\hline
\end{tabular}

Source : Ministère de l'emploi et de la solidarité, 29e rapport sur la situation démographique en France (2000: 37).

Au cours des quinze années qui précèdent le recensement, $26 \%$ des enfants nés hors mariage ont été légitimés, $30 \%$ vivent avec un couple de célibataires, $16 \%$ dans une famille monoparentale célibataire, $27 \%$ avec un couple dont l'un des deux parents n'est pas célibataire, et moins de $1 \%$ sont décédés (Couet 1996).

Ainsi, $83 \%$ des enfants vivent avec un couple parental et $16 \%$ sont des enfants qui ont connu une séparation parentale sans recomposition familiale. Pour les enfants qui vivent avec un couple parental, $26 \%$ d'entre eux vivent au sein d'un couple marié, $57 \%$ dans une famille naturelle (Couet 1996). Ces familles naturelles se composent de couples célibataires (30 \%) qui exercent vraisemblablement une «parentalité première», et de familles recomposées $(27 \%)$ fondées sur une «parentalité composée».

La nouvelle signification du mariage, ainsi que la nouvelle signification du hors mariage ${ }^{35}$ montre que la parentalité se discute en termes d'inscription double de la filiation de l'enfant. L'institutionnalisation d'une reconnaissance de la «parentalité première», quelle que soit la forme de lien conjugal, inscrit la bilatéralité du lien entre l'enfant et ses parents.

\section{I.B.3.2 Les familles monoparentales et le contact avec le second parent}

En France, les familles monoparentales représentent 9,3\% du nombre de familles avec enfants en 1968 et 13,2\% en 1990 (16,2\%, sans limite d'âge des enfants) (INSEE 1994 : 17). Le nombre de familles monoparentales a continué à augmenter dans l'absolu, prolongeant des décennies antérieures (Herpin, Olier 1997). En 2000, sur l'ensemble des familles, dont un enfant est âgé de moins de six ans, 90, 3\% sont des couples et 9,7\% sont des familles monoparentales, alors qu'elles représentaient 8,8\% des familles en 1990 (Fenet, Leprince, Périer $2001:$ 12).

\footnotetext{
35 Nous rappelons que l'inscription de la parentalité hors mariage s'accompagne d'une progression des reconnaissances paternelles. En 1996, les trois-quarts des enfants nés hors mariage sont reconnus par le père dès la naissance (la moitié dans les années 1980 et 1/5 au début des années 1970 (Beaumel, Kerjosse, Toulemon 1999)). La reconnaissance paternelle est non seulement plus répandue, elle est également plus rapide, et souvent conjointe.
} 
Le maintien d'un lien parental bilatéral est fonction de la durée d'exercice de la paternité avant la séparation parentale. Plus la séparation intervient après une longue période de cohabitation avec l'enfant, plus le lien sera maintenu dans les familles naturelles (par rapport aux familles légitimes) (Villeneuve-Gokalp 1993). Par contre, si le temps de parentalité est court avant la séparation, les familles monoparentales, issues de familles naturelles, maintiennent moins bien le lien paternel que les couples mariés.

Ainsi, l'exercice d'une parentalité bilatérale après la séparation dépend plus de la durée de cohabitation avec l'enfant que de la forme du lien conjugal (légitime ou naturel). Cependant, le maintien d'une «parentalité première» est plus fréquent dans les couples mariés que dans les familles naturelles. Ces dernières vivent plus souvent une parentalité composée (qui n'exclue pas l'exercice bilatéral de la parentalité avec le couple d'origine).

La rupture conjugale est plus souvent suivie d'une seconde union pour les couples sans enfant, que pour les couples avec enfant. La présence enfant ralentit la remise en couple (Blöss 2001 : 59). En 1990, $71 \%$ des femmes et $64 \%$ des hommes sans enfant âgés de moins de 40 ans, se sont remariés 5 ans après la rupture. Avec la présence d'enfants au sein du couple, seulement $51 \%$ des mères et $56 \%$ des pères vivent à nouveau en couple (Thélot et Villac 1998): la présence enfant a une influence très forte dans les trajectoires post divorce des mères ; l'impact est moins prononcé pour les pères (mais les écarts entre les sexes est plus forte pour les couples qui n'ont pas enfant)

Il faudra à l'avenir observer les phénomènes de remise en couple, pour voir de quelle manière le maintien d'une parentalité bilatérale (après la séparation) limite les remises en couple (et donc l'exercice de la parentalité composée) au profit d'un phénomène de «living apart together» : en d'autres termes, de quelle manière le maintien du lien à l'enfant pour les deux parents signifie une décohabitation pour la nouvelle relation de couple. 


\section{SECTION I.C LA PARENTALITE ET LA PARTICIPATION AU MARCHE DU TRAVAIL DES PARENTS}

Dans cette partie nous nous attachons à montrer de quelle manière les parents participent au marché du travail en France et en Allemagne et de quelle façon cette participation influence la structure familiale, c'est-à-dire la présence ou non d'un conjoint. Inversement, nous discuterons, dans le chapitre III, de l'influence de la participation au marché du travail sur l'exercice de la parentalité, à travers le partage du travail familial.

Afin, d'appréhender l'impact de la participation du marché du travail sur l'exercice du lien familial, nous ne considérons ici que l'activité professionnelle des pères et des mères. Cependant, et afin de replacer ce phénomène dans son contexte, nous rappelerons les tendances de la participation au marché du travail en fonction des genres sous forme d'encadrés.

\section{SOUS-SECTION I.C.1 L'ACTIVITE PROFESSIONNELLE DES COUPLES PARENTAUX EN EUROPE}

Actuellement, en Europe, il existe un large consensus (les 3/4 des individus) selon lequel les deux membres du couple souhaitent être actifs. L'enquête montre une moindre généralisation de ce modèle dans les pratiques, même s'il reste majoritaire. Il ne s'agit pas pour autant d'un partage équivalent du travail professionnel puisque seuls $30 \%$ le partagent de façon équivalente (à temps plein). Le modèle de la femme au foyer fait de moins en moins l'objet d'un consensus ( $15 \%$ des cas) mais il reste cependant pratiqué par $1 / 3$ des couples ${ }^{36}$ (Bielenski, Kauppinen $1999^{37}$ ).

Sur l'ensemble de l'Europe, on observe une préférence très nette pour un modèle où l'homme travaille à temps plein et la femme à temps partiel. Ce mode est celui qui apporte la plus grande satisfaction pour ceux qui l'ont choisi. Le modèle de l'homme à temps complet et de la femme au foyer n'est plus majoritaire ni dans les pratiques ni dans les représentations. On note un intérêt marqué pour un travail à temps partiel principalement chez les femmes, mais également chez les hommes. Par ailleurs, lorsque les deux partenaires travaillent à temps partiel, il semble que la majorité des individus ayant choisi ce mode de partage soient très

\footnotetext{
${ }^{36}$ Dans les pays scandinaves, $52 \%$ de l'ensemble des couples favorisent des pratiques où l'homme et la femme sont à temps complet. Dans les pays méditerranéens (Grèce, Irlande, Espagne, Portugal ) la majorité des couples (52\%) favorisent un modèle où l'homme travaille à temps complet et la femme est au foyer. Il est remarquable que, que ce soit dans les pays scandinaves ou dans les pays méditerranéens, $37 \%$ des coupes souhaitent un modèle à double temps complet. Cette préférence dépasse largement le nombre de réponse aux autres modalités. Pour les autres pays (dont la France et l'Allemagne) on a à part égale (28\%) une préférence pour un modèle où l'homme et la femme sont actifs à temps complet tous les deux et où l'homme est à temps complet et la femme à temps partiel. Les pays scandinaves, par contre, préférent en second modèle, celui où les deux sont à temps partiel.

${ }^{37}$ Il s'agit des résultats d'une enquête représentative effectuée sur 30.000 personnes dans quinze pays de l'union européenne et la Norvège effectuée en 1998 et commanditée par la fondation européenne pour l'amélioration des conditions de vie et de travail, ainsi que par le ministère du travail norvégien.
} 
satisfaits de cette formule, c'est d'ailleurs le mode pour lequel il y a un taux de satisfaction le plus important par rapport à la forme exercée et souhaitée ${ }^{38}$ (Bielenski, Kauppinen $\left.2000: 4\right)$.

«Il est surprenant de noter que la présence d'enfants dans le ménage a très peu d'influence sur le temps de travail cumulé du couple, qu'il s'agisse du temps de travail actuel ou des préférences» (Bielenski, Kauppinen 2000 : 6). Par contre, la façon dont le travail est réparti au sein du couple semble être influencé par la présence d'enfants et notamment par l'âge du dernier-né ${ }^{39}$ (cf. Tableau 8).

Lorsque le dernier-né est âgé de moins de 6 ans, un peu moins de la moitié des couples ont un mode de partage dans lequel l'homme travaille à temps complet et la femme est inactive. Pour un $1 / 4$ des couples, l'homme et la femme sont à temps plein ; pour le dernier $1 / 4$, l'homme est à temps plein et la femme à temps partiel. Ce dernier modèle a la préférence de $45 \%$ des couples.

Dans le cas où le dernier-né à plus de 6 ans, plus d'un tiers des couples suivent le modèle de la femme au foyer, un tiers où les deux sont à plein temps et un peu moins d'un quart pour un partage temps plein - temps partiel. Lorsqu'il n'y a pas (ou plus) d'enfant dans le ménage, $40 \%$ des couples sont à temps plein tous les deux dans $27 \%$ des couples l'homme travaille et la femme est au foyer. Plus les enfants sont âgés et moins le modèle de la femme au foyer est pratiqué et plus le modèle à deux temps plein devient majoritaire (cf. Tableau 9).

\footnotetext{
${ }^{38}$ Les deux auteurs Bielenski et Kauppinen (1999) ont également évalué les préférences concernant les modes de partage du travail professionnel dans les couples en fonction du sexe de la personne interrogée et en fonction du modèle de partage pratiqué. De façon générale on remarque que les différences entre les préférences des femmes et des hommes ne varient pas de façon très importante. C'est surtout le mode d'arrangement professionnel du couple qui explique la variation dans l'appréciation :

premièrement, lorsque les deux travaillent à temps plein, plus de 55\% des individus vivant dans ces couples sont satisfaits du mode de partage choisi. L'alternative est un mode d'organisation où l'homme serait à temps plein et la femme à temps partiel. Le modèle de la femme au foyer est peu préféré, moins de 5\% (voire $1 \%$ pour les femmes). Le modèle où les deux sont à temps partiel est préféré à $13 \%$ par les hommes et $16 \%$ par les femmes. Deuxièmement, dans le cas d'un couple où l'homme est à temps plein et la femme à temps partiel, plus de la majorité des couples qui ont choisi ce mode de partage en sont satisfaits, plus particulièrement les femmes que les hommes. Un double temps partiel serait une alternative pour environ $17 \%$ à $18 \%$. Le double temps complet ne représente une alternative que dans environ dans $11 \%$ à $12 \%$ des cas. Un partage très inégal du travail professionnel entre un homme à temps plein et une femmes au foyer, serait une alternative pour un peu plus d'hommes que de femmes, ces dernières préfèrent ce modèle dans moins de $2 \%$ des cas.

Troisièmement, dans le cas où l'homme travaille à temps complet et la femme est au foyer, seulement $1 / 3$ des hommes sont satisfaits de cet arrangement contre $40 \%$ des femmes, cette proportion est inférieure au nombre de couples qui sont dans cette situation. Pour eux, la première alternative serait un temps plein pour l'homme et un temps partiel pour la femme pour $1 / 3$ des femmes et pour $20 \%$ des hommes, ou un double temps complet pour $20 \%$ des hommes et de $17 \%$ des femmes. Ces couples adhèrent le moins au modèle du double temps partiel qui serait une alternative pour $12 \%$ des hommes et $7 \%$ des femmes.

${ }^{39}$ Le seul modèle où la répartition ne semble pas être influencée par la présence et l'âge des enfants est le modèle où les deux partenaires sont à temps partiel ; il est pratiqué par seulement $1 \%$ à $2 \%$ des couples.
} 
Tableau $8 \quad$ Modes pratiqués et souhaités de partage du travail professionnel dans les couples en Europe ${ }^{(1)}$, en fonction de l'âge des enfants dans le ménage ${ }^{(2)}(\mathrm{en} \%)$

\begin{tabular}{|l|c|c|c|c|c|c|}
\hline & \multicolumn{2}{|c|}{$\begin{array}{c}\text { Le dernier-né a } \\
\text { moins de } 6 \text { ans }\end{array}$} & \multicolumn{2}{|c|}{$\begin{array}{c}\text { Le dernier-né a } \\
6 \text { ans ou plus }\end{array}$} & \multicolumn{2}{|c|}{$\begin{array}{c}\text { Pas (ou plus) d'enfant } \\
\text { dans le ménage }\end{array}$} \\
\cline { 2 - 7 } & $\begin{array}{l}\text { Modèle } \\
\text { pratiqué }\end{array}$ & $\begin{array}{c}\text { Modèle } \\
\text { préféré }\end{array}$ & $\begin{array}{c}\text { Modèle } \\
\text { pratiqué }\end{array}$ & $\begin{array}{c}\text { Modèle } \\
\text { préféré }\end{array}$ & $\begin{array}{c}\text { Modèle } \\
\text { pratiqué }\end{array}$ & $\begin{array}{c}\text { Modèle } \\
\text { préféré }\end{array}$ \\
\hline $\begin{array}{l}\text { Les deux partenaires travaillent à plein } \\
\text { temps }\end{array}$ & 24 & 26 & 32 & 33 & $\mathbf{4 0}$ & $\mathbf{3 8}$ \\
\hline $\begin{array}{l}\text { L'homme travaille à temps plein et la } \\
\text { femme à temps partiel }{ }^{(3)}\end{array}$ & 24 & $\mathbf{3 9}$ & 23 & $\mathbf{3 4}$ & 15 & 21 \\
\hline $\begin{array}{l}\text { L'homme travaille et la femme ne } \\
\text { travaille pas }\end{array}$ & $\mathbf{4 7}$ & 15 & $\mathbf{3 5}$ & 14 & 27 & 14 \\
\hline $\begin{array}{l}\text { Les deux partenaires travaillent à temps } \\
\text { partiel }\end{array}$ & 1 & 18 & 2 & 14 & 2 & 17 \\
\hline Autre modèle (4) & 4 & 2 & 8 & 4 & 16 & 9 \\
\hline Total & 100 & 100 & 100 & 100 & 100 & 100 \\
\hline
\end{tabular}

Source : Bielenski, Kauppinen 1999, European Foundation for the Improvement of Living and Working Conditions

${ }^{(1)}$ Europe des «15» et la Norvège

${ }^{(2)}$ Individus âgés de 16 à 64 ans

${ }^{(3)}$ Dans cette étude, le temps plein est supérieur à 35 heures de travail par semaine.

(4) Cette catégorie comprend le cas où la femme travaille et l'homme est inactif et le cas où les deux ne travaillent pas mais dont au moins l'un des deux souhaite travailler.

Les pratiques et les représentations relatives au modèle à deux pleins temps sont en adéquation : il y a très peu d'écart entre le nombre de couples qui le pratique et le nombre de couples qui le préfère. C'est le modèle à temps plein/temps partiel pour lequel on a un grand nombre de couples qui préféreraient ce partage. La présence d'enfants et en particulier de jeunes d'enfants, favorise la pratique d'un modèle où l'homme travaille et la femme est inactive $^{40}$, tout en remarquant qu'il n'est pas majoritairement souhaité.

\footnotetext{
${ }^{40}$ L'enquête faite par le journal BRIGITTE effectuée en 1998 a montré que dans la catégorie des $14-49$ ans, $48 \%$ déclarait qu'il était naturel que la femme s'occupe de la famille et du foyer. Il faut cependant remarquer que pour cette même classe d'âge, $85 \%$ ont répondu que l'activité professionnelle était aussi importante pour une femme que pour un homme.
} 


\section{SOUS-SECTION I.C.2 EN ALLEMAGNE}

Encadré $\mathrm{n}^{\circ} 4 \quad$ L'activité professionnelle des hommes et des femmes en Allemagne en fonction de la distinction régionale Ouest/Est

En Allemagne de l'Ouest, les femmes sont de plus en plus présentes sur le marché du travail ; la moitié d'entre elles travaillaient en 1980 à 50,2 \% (soit un taux d'activité de $58,5 \%$ ), ce pourcentage atteignait 61,7\% en 1999.

Dans l'ancienne Allemagne de l'Est, leur taux d'activité à chuté depuis la réunification, il était de 77,2\% en 1991, pour atteindre 73\% en 1999 (Institut der deutschen Wirtschaft Köln 2001 : 12). Dans l'ancienne Allemagne de l'Est, la norme du temps plein concerne l'ensemble des familles quelles que soient leur forme, à l'exception des personnes vivant seules (majoritairement des retraités). Le souhait de travailler à temps partiel est relativement peu répendu, sauf éventuellement chez les couples mariées avec des enfants. Mais leur proportion reste faible avec 17,3\% en 1993 (le taux de pratique du temps partiel pour les femmes en DDR était de 27 \%) (Kurz-Scherf, Ingrid ; Winkler, Gunnar, 1994).

L'exercice d'une activité professionnelle chez des hommes est général. En 1999, ils travaillent tous à plus de $80 \%$ : cependant, les hommes de l'ancienne Allemagne de l'Est sont moins actifs qu'en 1991, où ils avaient un taux d'activité de $86 \%$. Pour les hommes de l'ancienne Allemagne de l'Ouest, leur taux d'activité a baissé de 84,4\% en 1980 à 82,7\% en 1990 pour atteindre 80,3\% en 1999 (Institut der deutschen Wirtschaft Köln 2001 : 12). Ils exercent très rarement une activité professionnelle à temps partiel et ils sont moins touchés par le chômage que les femmes.

La proportion globale d'hommes actifs occupés, a diminué de 5 points entre 1991 et 2000 sur l'ensemble de l'Allemagne. Inversement, l'activité des femmes s'est maintenue et leur taux d'activité a légèrement augmenté, montrant très clairement la généralisation de l'activité professionnelle des femmes en Allemagne (Statistisches Bundesamt 2001 : 37) (cf. Annexe $10)$.

En mai 2000, 19,8 \% de l'ensemble des salariés allemands travaillent à temps partiel, 38,3\% pour les femmes et 4,8\% pour les hommes (Statistisches Bundesamt 2001 : 29) (cf. Annexe 7). La part des activités professionnelles à temps partiel pour les femmes a augmenté de 8 points entre 1991 et 2000 (seulement 5 points dans l'ancienne Allemagne de l'Est) (Statistisches Bundesamt 2001 : 29). Pour les hommes, on a une légère augmentation de leur participation puisqu'elle n'était que de $2 \%$ en 1991. Le taux d'activité à temps partiel est de $\mathbf{2 2 , 8} \%$ en 2000 pour les femmes dans l'ancienne Allemagne de l'Est, et plus du double avec 41,9\% pour l'ancienne Allemagne de l'Ouest.

Les activités marginales ou occasionnelles «geringfügige Beschäftigung» concernent tout particulièrement les femmes (cf. note 1 de l'encadré). En Allemagne, en mai 2000, $44 \%$ des femmes sont actives dans ce type d'activités. Par ailleurs, sur l'ensemble de ces activités marginales et occasionnelles, $77 \%$ sont exercées par des femmes. Ces dernières sont surtout des femmes mariées (75\% d'entre elles). La majorité (49\%) sont âgées de moins de 40 ans. Ce type d'activité concerne donc essentiellement une stratégie conjugale du partage du travail professionnel, qui concerne avant tout les mères. Les hommes sont très peu concernés par ce 
type d'activité et le statut matrimonial a moins d'impact sur leur activité (45,4\% sont mariés) (Statistisches Bundesamt 2001 : 31) (cf. Annexe 9).

La forte proportion de femmes travaillant occasionnellement ou à temps partiel se reflète dans le volume horaire global travaillé par semaine (Statistisches Bundesamt 2002a : 103). Sur l'ensemble de l'Allemagne, en mai 2000, 26,3\% des femmes travaillent moins de 21 heures par semaine et $44 \%$ entre 21 h et 39 heures. En revanche, $56 \%$ des hommes travaillent plus de 40 heures. Ils sont 4,2\% à travailler moins de 21 heures et $39,9 \%$ à travailler entre $21 \mathrm{~h}$ et 39 heures (cf. Annexe 8).

Entre 1991 et 2000, le nombre de demandeurs d'emploi a augmenté de $24 \%$ pour les femmes (32 \% à l'Est et $19 \%$ à l'Ouest) et de $60 \%$ pour les hommes (Statistisches Bundesamt 2001 : 36). Le nombre de chômeurs masculins a augmenté (Statistisches Bundesamt 2001 : 36) et le chômage touche proportionnellement plus ceux de l'ancienne Allemagne de l'Est. En comparant 2000 et 1991, le nombre de chômeurs hommes a augmenté de $76 \%$ pour l'ancienne Allemagne de l'Est, contre $52 \%$ pour l'Allemagne de l'Ouest.

La progression absolue du chômage touche plus les hommes que les femmes, mais le rapport entre le taux d'activité professionnelle et le taux de chômage, montre que les femmes sont relativement plus touchées que les hommes. Ceci est plus vrai pour 1991, tandis quand 2000, l'impact est proportionnellement équivalent pour les deux sexes (source : nos propres calculs à partir des chiffres de l'enquête Mikrozensus (Statistisches Bundesamt 2001)).

Note 1 : Les emplois «flexibles» concernent plus de la moitié des actifs en Allemagne. C'est surtout dans l'ancienne Allemagne de l'Est que la flexibilité des activités professionnelles a augmenté (Statistisches Bundesamt 2001 : 12-13). Il est cependant important de remarquer que ce ne sont pas les activités régulières qui ont augmenté au cours de cette décennie, mais plutôt le travail occasionnel le week-end et les jours fériés. Il est plus juste de qualifier la montée du travail flexible comme la généralisation d'une flexibilité occasionnelle des horaires de travail. Le déplacement de l'activité professionnelle sur le week-end, vient directement s'inscrire en concurrence d'un temps familial et d'un temps parental.

Sur l'ensemble des mères d'au moins un enfant mineur, $63 \%$ sont actives sur l'ensemble de l'Allemagne en 2000 (et $7 \%$ cherchent du travail). La proportion est inférieure dans l'ancienne Allemagne de l'Ouest avec $61 \%$ (et $5 \%$ demandeuses d'emploi), contrairement à celle de l'ancienne Allemagne de l'Est avec $72 \%$ (et $18 \%$ qui cherchent un travail) (Statistisches Bundesamt 2001a).

Le tableau 9 indique la proportion de mères actives, par rapport à l'ensemble des mères en âge d'être actives et ayant au moins un enfant mineur. Sur l'ensemble de l'Allemagne réunifiée, au cours des années 1990, on voit que la proportion de mères actives a augmenté de 3,2 points. En revanche, les évolutions sont inverses entre l'Ouest et l'Est. Dans les régions de l'Ouest, la proportion des mères actives a augmenté de 8,1 points dans la dernière décennie, alors qu'elle a chuté d'environ 10 points pour les régions de l'Est sur la même période. 
Tableau 9 Proportion de mères actives par rapport à l'ensemble des mères ${ }^{(*)}$ en $\%$

\begin{tabular}{|c|c|}
\hline & $\begin{array}{c}\text { PourCentage de } \\
\text { MERES ACTIVES }\end{array}$ \\
\hline \multicolumn{2}{|l|}{ AllemaGne } \\
\hline 1991 & $59,1 \%$ \\
\hline 1999 & $62,3 \%$ \\
\hline \multicolumn{2}{|c|}{ AnCienne Allemagne de l'OUest } \\
\hline 1991 & $51,5 \%$ \\
\hline 1999 & $59,6 \%$ \\
\hline \multicolumn{2}{|c|}{ AnCIENNe AlLEMAGNe de L'Est } \\
\hline 1991 & $83,3 \%$ \\
\hline 1999 & $73,3 \%$ \\
\hline
\end{tabular}

Source : Statistisches Bundesamt 2000c

${ }^{(*)}$ mères âgées de 15 à 65 ans et ayant au moins un enfant mineurs

Globalement, sur l'Allemagne réunifiée, la différence entre les femmes mariées et célibataires pour l'année 1999 est faible. Les femmes mariées travaillent à 62,8 \%, les célibataires à $65 \%$ (Institut der deutschen Wirtschaft Köln 2001 : 12). Mais les femmes mariées travaillent plus à l'Est qu'à l'Ouest avec une différence de près de 20 points $(59,4 \%$ contre 78,6\%). Par contre, les différences du taux d'activité des femmes célibataires entre l'Allemagne de l'Ouest et l'Allemagne de l'Est sont très faibles (pour les années 1980 et 1990).

Le rapport entre les taux d'activité des femmes montre une grande différence régionale. Pour Allemagne de l'Est, le rapport entre l'activité des femmes célibataires et des femmes mariées est à peu près resté constant entre 1980 et la fin des années 1990. Pour l'ancienne Allemagne de l'Ouest, le rapport était constant dans les années 1980, en revanche, à la fin des années 1990, la différence qui était de l'ordre environ 14,5 points, n'est plus que de 6 points. Ce phénomène est le fait de l'augmentation de la participation des femmes mariées qui atteint $60 \%$ à la fin des années 1990.

Enfin, la plus grande différence régionale concerne les comportements des femmes mariées : au début des années 1990, 81,5 \% des femmes mariées étaient actives dans l'Ancienne Allemagne de l'Est, soit une différence de 27,6 points avec l'Ouest. L'écart reste encore important à la fin des années 1990 même s'il s'est réduit de 10 points (cf. Tableau 10). 
Tableau 10 Taux d'activité ${ }^{(*)}$ des femmes en fonction du statut matrimonial

\begin{tabular}{|l|c|c|c|c|}
\hline \multirow{2}{*}{} & \multicolumn{2}{|c|}{$\begin{array}{c}\text { Femmes célibataires } \\
\text { (statut juridique) }\end{array}$} & \multicolumn{2}{c|}{ Femmes mariées } \\
\cline { 2 - 5 } & $\begin{array}{c}\text { Allemagne de } \\
\text { l'Ouest }\end{array}$ & $\begin{array}{c}\text { Allemagne de } \\
\text { l'Est }\end{array}$ & $\begin{array}{c}\text { Allemagne de } \\
\text { l'Ouest }\end{array}$ & $\begin{array}{c}\text { Allemagne de } \\
\text { l'Est }\end{array}$ \\
\hline 1980 & 60,7 & - & $\mathbf{4 6 , 1}$ & - \\
\hline 1990 & 68,1 & - & 53,9 & - \\
\hline 1991 & - & 67,8 & - & 81,5 \\
\hline 1999 & 65,4 & 63,3 & $\mathbf{5 9 , 4}$ & $\mathbf{7 8 , 6}$ \\
\hline
\end{tabular}

Source : Institut der deutschen Wirtschaft, Köln (2001 : 12)

${ }^{(*)}$ Le taux d'activité se calcule à partir des femmes qui exercent une activité professionnelle et des demandeuses d'emploi, par rapport à la population totale.

Le tableau 11 représente la situation professionnelle des femmes dans l'ancienne Allemagne de l'Ouest en fonction de l'âge du plus jeune enfant dans le ménage. Dans les cas où le plus jeune enfant à moins de trois ans, les mères sont sur-représentées dans la catégorie des inactifs (qu'elles soient mariées ou non). Leur taux de participation au marché du travail est sous-représenté. Par ailleurs, elles sont très nombreuses à prendre un congé parental (par effet de structure car le congé parental est attribué pour la présence de jeunes enfants).

Dans les cas où l'enfant est âgé de trois à six ans, les mères sont encore légèrement sur-représentées dans la catégorie des inactives. Elles sont sur-représentées également dans la catégorie du chômage (le taux est le plus élevé quel que soit le statut conjugal et quel que soit l'âge de l'enfant ce qui montre une volonté d'exercer une activité professionnelle dès que l'enfant est âgé de 3 ans). On a une légère sur-représentation de l'activité à temps partiel. Par contre l'activité professionnelle à temps complet est sous-représenté pour ces mères.

Lorsque le dernier enfant est âgé de 6 à 10 ans, c'est l'activité à temps partiel qui est largement sur-représentée, et surtout dans le cas des familles monoparentales (au sens large). Lorsque l'enfant a plus de 10 ans, les mères sont sous-représentées dans la catégorie des inactives, sur-représentées pour le temps complet. Leur participation à temps partiel est proche de la moyenne générale (sauf pour les femmes mariées qui sont sur-représentées).

Ainsi, quel que soit l'âge de l'enfant, on voit l'effet du lien conjugal : les mères mariées sont plus souvent inactives et moins souvent à temps complet, et légèrement plus à temps partiel. Ces chiffres ne permettent pas de mettre en évidence l'impact du couple (le relevé des données est fonction des catégories juridiques et non de la présence ou non d'un conjoint).

Les familles dites «monoparentales» ${ }^{41}$ sont plus souvent actives à temps complet que les femmes mariées, elles travaillent moins à temps partiel que ces dernières. Elles sont plus souvent au chômage et moins souvent inactives, dans un rapport du simple au double.

\footnotetext{
${ }^{41}$ On rappellera que selon la définition de Mikrozensus, la catégorie des familles monoparentales comprend l'ensemble des familles veuves et divorcées ainsi que les familles mariées et séparées, qu'elles soient ou non avec un partenaire. Elle comprend, de fait, l'ensemble des familles naturelles et recomposées.
} 
Lorsque l'enfant est plus âgé, les mères travaillent relativement plus, mais l'inactivité concerne toujours un tiers des femmes mariées. En revanche pour les familles «monoparentales», l'activité à temps complet atteint $47 \%$.

Le profil de l'Allemagne de l'Est est différent de celui de l'ancienne Allemagne de l'Ouest (cf. Tableau 12). Les écarts entre les familles monoparentales et les familles mariées sont faibles. $49 \%$ des familles monoparentales et $53 \%$ des femmes mariées travaillent à temps complet. Ces catégories se distinguent des autres car le temps partiel y est très faible et le taux de chômage important. Lorsque les enfants ont moins de trois ans, un tiers des femmes sont inactives (quel que soit leur statut matrimonial). $20 \%$ ont recours à un congé parental. Elles sont aussi moins nombreuses à temps partiel et légèrement plus à temps complet que les femmes de l'ancienne Allemagne de l'Ouest. Lorsque les enfants ont entre 3 à 6 ans, l'inactivité des femmes ne représente plus que 5 à $7 \%$ de l'ensemble (contrairement à un tiers auparavant). Elles sont plus souvent au chômage qu'actives. Mais lorsqu'elles sont actives, c'est le plus souvent à temps complet avec 38\%. Quant aux femmes mariées, elles travaillent dans la même proportion à temps complet.

Lorsque les enfants ont de 6 à 10 ans, les femmes travaillent majoritairement à temps complet entre $52 \%$ et $54 \%$, quel que soit leur statut matrimonial. Leur taux de chômage est important ; elles sont seulement $4 \%$ à être inactives. Le temps partiel est légèrement plus représenté chez les femmes mariées.

Lorsque les enfants sont âgés de 10 à 15 ou de 15 à 18 ans, la situation est sensiblement la même (alors qu'en Allemagne de l'Ouest, on avait encore une forte progression du temps complet). Ici, le taux d'activité à temps complet atteint environ 52\% quel que soit l'âge de l'enfant et la situation matrimoniale. Les taux de chômage représentant un peu moins du $1 / 5$ des mères. Le temps partiel est uniquement exercé par $10 \%$ des mères, et 16 à $18 \%$ des mères mariées.

En 1997, en Allemagne de l'Ouest, les familles monoparentales sont moins actives que les femmes mariées vivant avec leur conjoint : Elles ont un taux d'activité de $71 \%$ alors que les familles monoparentales sont actives à $53 \%$. Par contre, lorsqu'elles sont actives, elles sont plus souvent à temps complet que les femmes mariées vivant avec leur conjoint. Le taux de mères monoparentales au chômage dans l'ancienne Allemagne de l'Ouest atteint $12 \%$ contre $5 \%$ chez les femmes mariées ; pour l'ancienne Allemagne de l'Est on a respectivement $28 \%$ contre $18 \%$ (Engelbrech, Jungkunst 2001). 
Tableau 11 La situation professionnelle des mères ${ }^{(*)}$, en fonction de l'âge du plus jeune enfant, pour l'ancienne Allemagne de l'Ouest, en \%

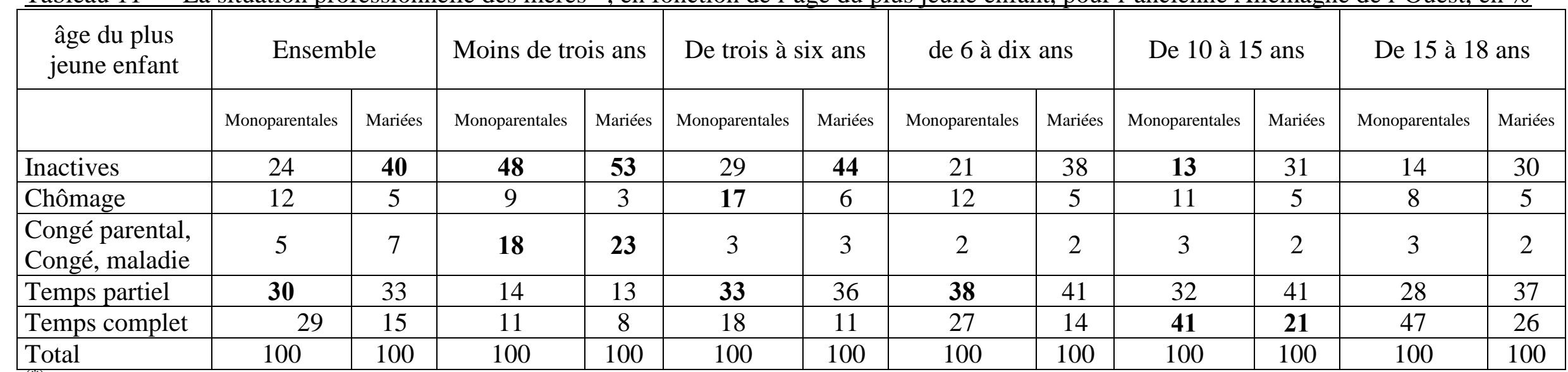

${ }^{(*)}$ Les enfants du ménage sont âgés de moins de 18 ans.

Source : enquête de Mikrozensus 1997 (Engelbrech, Jungkunst 2001)

Tableau 12 La situation professionnelle des mères ${ }^{(*)}$, en fonction de l'âge du plus jeune enfant, pour l'ancienne Allemagne de l'Est, en \%

\begin{tabular}{|l|c|c|c|c|c|c|c|c|c|c|c|c|}
\hline $\begin{array}{c}\text { âge du plus } \\
\text { jeune enfant }\end{array}$ & \multicolumn{2}{|c|}{ Ensemble } & \multicolumn{2}{c|}{ Moins de trois ans } & \multicolumn{2}{c|}{ De trois à six ans } & \multicolumn{2}{c|}{ de 6 à dix ans } & \multicolumn{2}{c|}{ De 10 à 15 ans } & \multicolumn{2}{c|}{ De 15 à 18 ans } \\
\hline & Monoparentales & Mariées & Monoparentales & Mariées & Monoparentales & Mariées & Monoparentales & Mariées & Monoparentales & Mariées & Monoparentales & Mariées \\
\hline Inactives & 8 & 7 & 32 & 35 & 5 & 7 & 4 & 4 & 2 & 3 & 5 & 5 \\
\hline Chômage & $\mathbf{2 8}$ & $\mathbf{1 8}$ & 23 & 12 & $\mathbf{4 2}$ & $\mathbf{3 2}$ & 31 & 20 & 23 & 17 & 22 & 16 \\
\hline $\begin{array}{l}\text { Congé parental } \\
\text { Congé, maladie }\end{array}$ & 5 & 4 & $\mathbf{2 2}$ & $\mathbf{2 4}$ & 2 & 1 & 1 & 1 & 2 & 1 & 2 & 1 \\
\hline Temps partiel & 10 & 18 & 6 & 11 & 13 & 22 & 12 & 21 & 10 & 18 & 9 & 16 \\
\hline Temps complet & $\mathbf{4 9}$ & $\mathbf{5 3}$ & 17 & 18 & 38 & 38 & 52 & 54 & 63 & 61 & 62 & 62 \\
\hline Total & 100 & 100 & 100 & 100 & 100 & 100 & 100 & 100 & 100 & 100 & 100 & 100 \\
\hline
\end{tabular}

${ }^{(*)}$ Les enfants du ménage sont âgés de moins de 18 ans.

Source : enquête de Mikrozensus 1997 (Engelbrech, Jungkunst 2001) 


\section{SOUS-SECTION I.C.3 EN FRANCE}

Les caractéristiques de l'activité féminine sont largement connues, elles sont communes aux deux pays : généralisation de l'activité féminine depuis les années 1960, développement du secteur tertiaire, montée du temps partiel. Les femmes sont également moins nombreuses que les hommes dans les activités professionnelles hautement qualifiées et d'encadrement. Elles sont également, de façon générale, beaucoup plus touchées par le chômage. Leur activité professionnelle à temps partiel s'inscrit dans la problématique du temps partiel contraint ou choisi.

Le taux d'activité des femmes n'a cessé de croître jusqu'à aujourd'hui. Le pourcentage de femmes actives âgées de 25 à 49 ans est passé de $49 \%$ en 1970 à 78,7\% en 1998. La part des femmes âgées de 50 à 59 ans a augmenté de 53,7\% en 1990 à 63,2\% en 1998. Les mères sont aussi majoritairement actives, $72,3 \%$ des mères de deux enfants et $51 \%$ des mères de trois enfants et plus ; ces dernières n'étaient que 16\% en 1982 (Données Sociales, 1999).

«La féminisation de l'emploi féminin associée à l'essor rapide du salariat dans les années 1960, s'est accompagnée d'une transformation dans l'organisation interne de la vie familiale, si on en juge par la généralisation d'un double modèle, celui des couples «biactifs», mais aussi celui des mères de famille qui cumule l'activité professionnelle et l'activité parentale (Maruani, 1998), la norme étant actuellement pour une femme de «garder son emploi» et de faire garder ces enfants. Mariage et maternité ne semblent donc plus un obstacle à l'autonomie économique des femmes. Les évolutions de l'emploi attestent à la fois que le taux d'activité professionnelle des mères s'est accru plus fortement que celui des femmes célibataires sans enfant, mais aussi que l'activité professionnelle féminine est plus continue et moins tributaire du calendrier des naissances. De manière encore plus significative, on remarque que le taux d'activité des mères de deux enfants est maintenant à peine inférieure à celui des mères d'un seul enfant ( $74 \%$ contre $78 \%$ ), et que celui des mères de trois enfants ou plus en 1990 était identique à celui des mères d'un seul enfant en 1960 (46 \%)» (Blöss $2001: 53)$.

En France, les données du recensement de 1982 montrent que désormais les familles à deux apporteurs sont plus nombreuses que les familles à un apporteur principal. Et si le partage du professionnel est un fait, il reste néanmoins à préciser que ce partage n'est pas égalitaire. Les deux apporteurs ne sont pas équivalents, le temps partiel étant principalement exercé par les femmes.

En 2000, la norme du couple à deux actifs s'est maintenue (cf. Tableau 14) et le temps complet pour les femmes s'est généralisé. Le double temps complet devient le modèle le plus répendu. 
Tableau 13 Répartition des familles en fonction de leur participation professionnelle

\begin{tabular}{|l|c|}
\hline FORMES DE FAMILLES & $\mathbf{\%}$ \\
\hline Couple de deux actifs & 56,3 \\
\hline Couple homme actif/femme inactive $^{*}$ & 32,3 \\
\hline Couple dont l'homme est inactif $^{(*)}$ & 2,5 \\
\hline Familles monoparentales femmes & 8,4 \\
\hline Familles monoparentales hommes & 0,5 \\
\hline Total & $100 \%$ \\
\hline
\end{tabular}

Source : enquête emploi, INSEE, mars 2000

${ }^{(*)} 1,2 \%$ où l'homme est inactif et la femme active et $1,3 \%$ où l'homme est inactif et la femme inactive

45, $4 \%$ des enfants de moins de 3 ans vivent avec deux parents actifs occupés et 4,2\% avec une mère monoparentale active occupée. La proportion de mères de jeunes enfants travaillant à temps partiel a augmenté de 10 points au cours des dernières années (Enquête emploi INSEE 2000). «Entre 1995 et 2000, le taux d'activité des parents de deux enfants dont le plus jeune avait moins de trois ans, a connu une baisse sensible (de 60 à $56 \%$ ) en raison notamment de l'extension de l'allocation parentale d'éducation au deuxième enfant. En revanche, les taux d'activité des mères qui se situaient dans toutes les autres configurations familiales ont continué de progresser» (Fenet, Leprince, Périer 2001 : 14). Au total, 58,6 \% de l'ensemble des enfants de moins de 3 ans ont des prents actifs occupés ou à la recherche d'un emploi. 


\section{SOUS-SECTION I.C.4 LA PARENTALITE «EXCLUSIVE» ET LA PARENTALITE «CONCILIEE»}

La participation au marché du travail des hommes montre une grande stabilité. La quasi-totalité des hommes sont actifs à temps complet. Ce n'est qu'au cours de la dernière décennie que leur activité professionnelle à temps partiel a légèrement progressée.

Par ailleurs, on remarque que les statistiques différencient très rarement les chiffres en fonction de la situation familiale de l'homme. Cependant, on peut penser que le taux d'activité professionnelle des pères est équivalent à celui des hommes. En effet, très peu de pères interrompent leur activité professionnelle pour prendre un congé parental $(1,5 \%$ en Allemagne). Ce chiffre est resté quasiment inchangé, malgré les nombreuses incitations politiques (cf. section suivante). Ainsi, la majorité des pères cumulent à la fois un rôle parental et un rôle professionnel. Nous voyons ainsi apparaître que la conciliation «travailfamille» concerne l'ensemble des pères. Leur contrainte est liée à une activité professionnelle à temps complet et continue.

L'augmentation de la participation des femmes au marché du travail depuis ces trente dernières années est constante ; elle est générale sur l'ensemble des femmes, quel que soit le statut matrimonial, la constellation familiale ou l'âge de l'enfant.

La différenciation du taux d'activité des femmes en fonction de leur situation familiale, a permis de mettre en évidence que la majorité des mères exerce une activité professionnelle. En France, les mères sont majoritairement actives à temps complet depuis les années 1980. A la fin des années 2000, les couples parentaux sont composés de deux actifs à temps complet.

Dans l'ancienne Allemagne de l'Est, à la fin des années 1990, la norme du temps complet concerne les femmes et les hommes. Les chiffres montrent que l'activité professionnelle des mères est également continue, puisque la prise d'un congé parental n'est une alternative que pour $1 / 5$ des femmes et uniquement lorsque l'enfant est âgé de moins de trois ans. L'activité professionnelle des mères varie en fonction de l'âge du plus jeune enfant, mais dès qu'il a trois ans, elles sont $38 \%$ à être active à temps complet, quel que soit leur statut conjugal. Dès les 6 ans de l'enfant, elles sont de nouveaux très majoritairement actives à temps complet.

En 1997, dans l'ancienne Allemagne de l'Ouest, l'inactivité est une alternative pour un grand nombre de mères, surtout lorsqu'elles sont mariées. Globalement, lorsque les mères ne sont pas mariées, elles exercent une activité professionnelle à $60 \%$ : la moitié à temps partiel et la moitié à temps complet (ce chiffre exclut le taux de chômage).

À la fin des années 1990, le modèle de participation des mères pour l'ancienne Allemagne de l'Est se fait en référence au temps complet. En Allemagne de l'Est, comme en France, la majorité des mères doivent concilier un rôle parental et un rôle professionnel en fonction de la norme du temps professionnel à temps complet.

Dans le cas des mères de l'ancienne Allemagne de l'Ouest, l'inactivité est encore largement répandue, elle est majoritaire dans le cas des enfants très jeunes. Les mères non mariées sont majoritaires lorsque l'enfant à plus de trois ans, et les femmes mariées lorsque l'enfant à plus de 6 ans. Pour ces dernières, c'est le modèle du temps partiel qui domine 
encore largement. Dans le cas des mères non mariées, le temps partiel domine jusqu'aux dix ans de l'enfant.

Dans l'ancienne Allemagne de l'Ouest, l'exercice d'un rôle parental, s'il concerne dorénavant l'ensemble des mères, nécessite surtout une conciliation avec une activité professionnelle à temps partiel.

A l'échelle du couple parental, cela signifie que pour l'ancienne Allemagne de l'Est et pour la France, il s'agit majoritairement de couples de parents, actifs, équivalents, à temps complet. Pour l'ancienne Allemagne de l'Ouest, la norme suit le modèle de deux parents actifs inéquivalents. Cependant, le modèle où le père est actif à temps complet et la mère inactive («Ernährermodell») (Ostner 1995) reste encore largement représenté et proportionnellement plus lorsque les parents sont mariés.

Par ailleurs, le pouvoir déterminant des enfants sur la trajectoire professionnelle des mères perd de sa force ${ }^{42}$ : la naissance du premier enfant signifie de moins en moins une cessation d'activité. En France, jusque dans les années 1990, les mères de trois enfants arrêtaient de travailler. Or des études récentes ont montré que l'arrêt professionnel de la mère n'était pas lié à la venue du troisième enfant, mais avait déjà été arrêté avant les projets de maternité. Dans l'ancienne Allemagne de l'Est, avant la réunification, le taux d'activité des femmes était proche de celui des hommes, les mères maintenaient leur activité professionnelle lors des naissances excessives. Depuis la réunification, leur participation au marché du travail a légèrement diminué, tout en restant très élevé. Dans l'ancienne Allemagne de l'Ouest, le taux d'activité des mères qui maintiennent leur activité professionnelle, augmente de façon constante.

Enfin, l'âge de l'enfant est sans doute le facteur qui influence le plus la participation des mères sur le marché du travail. Cette dernière est directement liée aux possibilités structurelles de délégation et de prise en charge du parental (cf. section suivante).

Les modèles classiques analysent la position respective des membres du couple dans leur relation individuelle au marché du travail. Cette dernière est alors discutée en terme de genre «homme / femme» et en terme de conjugalité «marié / non marié».

Pour notre part, nous avons choisi de décrire la participation professionnelle des hommes et des femmes en fonction de leur statut de parent et non plus en fonction de leur lien conjugal (marié ou non). Nous proposons de tenir compte, pour chaque individu, de la relation entre son rôle parental et son rôle professionnel. Les différentes relations entre la famille et le marché du travail déterminent donc deux formes de parentalité : soit le parent n'est pas actif et il exerce exclusivement un rôle parental dans une "parentalité exclusive», soit le parent est actif et il concilie un rôle parental et un rôle professionnel dans une «parentalité conciliée».

Les modes de participation au marché du travail à temps complet ou à temps partiel sont des catégories développés depuis le point de vue du marché du travail. Pour notre part, nous proposons les catégories de «parentalité exclusive» et de «parentalité conciliée» car elles rendent mieux compte des implications dans la famille, par une prise en compte de l'exercice de la parentalité et des pratiques de partage du travail (cf. Chapitre III).

\footnotetext{
${ }^{42}$ La forme conjugale a également de moins en moins d'influence sur l'activité professionnelle des mères. Cependant, en cas de séparation conjugale, les familles monoparentales sont plus souvent présentes sur le marché du travail.
} 
Depuis ce nouveau point de vue, il apparaît alors clairement que la parentalité des hommes s'exerce exclusivement sous la forme d'une «parentalité conciliée». Celle des femmes prend la forme d'une «parentalité exclusive» lorsque la mère est inactive, et la forme d'une «parentalité conciliée» lorsqu'elle est active. Les mères sont majoritairement actives en France depuis les années 1980, en Allemagne de l'Ouest depuis le début des années 1990, et en Allemagne de l'Est depuis les années 1970. La «parentalité exclusive» est un modèle très marginal en France, il est devenu un modèle minoritaire en Allemagne. Aujourd'hui, la «parentalité conciliée» concerne la majorité des parents et l'ensemble des couples. Ainsi, il n'est plus possible de parler de conciliation d'un rôle parental et d'un rôle professionnel, mais au contraire d'une cumulation des deux fonctions pour les deux parents.

Au niveau conjugal, nous parlerons alors d'un exercice double de «parentalité conciliée» dans le cas où les deux parents sont actifs (équivalents ou non-équivalents). Dans le cas où la femme est au foyer, la parentalité est «conciliée» pour le père et «exclusive» pour la mère (cf. Figure 9).

Figure 9 «Parentalité exclusive» et «parentalité conciliée»

\begin{tabular}{l|l|l|l}
\cline { 2 - 3 } $\begin{array}{l}\text { Familles à un } \\
\text { apporteur } \\
\text { principa }\end{array}$ & $\begin{array}{c}\text { Famille à une } \\
\text { parentalité exclusive } \\
\text { et une parentalité } \\
\text { conciliée }\end{array}$ & $\begin{array}{c}\text { Famille à deux } \\
\text { parentalités } \\
\text { conciliées }\end{array}$ & $\begin{array}{l}\text { Familles à deux } \\
\text { apporteurs }\end{array}$ \\
-inéquivalents \\
ou \\
-équivalents
\end{tabular}




\section{SECTION I.D LE CADRE SOCIO-POLITIQUE ET SA SIGNIFICATION POUR LA PARENTALITE}

Chaque pays a une représentation particulière de la 'question sociale' ; elle influe sur la tradition nationale de la politique sociale : «la question du travailleur» pour l'Allemagne, «la question de la famille» pour la France (F.X Kaufmann 1987 : 15). En Allemagne, la protection de la famille a été très tôt inscrite au niveau constitutionnel, sans pour autant s'exprimer concrètement dans une réelle volonté politique (F.X. Kaufmann 1997b). S. Lessenich et I. Ostner (1995 : 788) parle d'une «rhétorique sur la famille à la place d'une politique familiale». Quant à la politique française, elle a une très longue tradition, elle est explicite et elle symbolise une reconnaissance naturelle et publique de la famille, «une rhétorique et une politique de la famille».

Ces éléments déterminent la manière dont le «membre de la famille», le «travailleur» ou «l'individu» est construit par les politiques sociales et familiales. C'est alors, alternativement, la forme de famille, le sexe ou le lien conjugal qui est visé. Pour notre part, nous étudions la manière dont l'individu, en tant que parent, est appréhendé dans les politiques familiales.

La figure 10 représente les différentes politiques sociales en fonction de leur degré de proximité par rapport à la parentalité. Le premier niveau se compose des mesures sociales qui sanctionnent directement la présence de l'enfant à travers les congés de maternité et de paternité, les prestations familiales et le système fiscal. Le second niveau concerne l'ensemble des mesures qui dégagent une disponibilité en temps des parents pour se consacrer, sinon à temps plein, du moins principalement, à l'éducation de l'enfant. Le troisième niveau regroupe l'ensemble des mesures de conciliation entre les activités professionnelles et les activités familiales ; il rassemble tantôt les mesures liées au professionnel comme le temps partiel, tantôt la délégation du travail domestique et du travail parental à travers les structures d'accueil des enfants et du système scolaire. 
Figure $10 \quad$ Les politiques familiales et la prise en compte de la parentalité

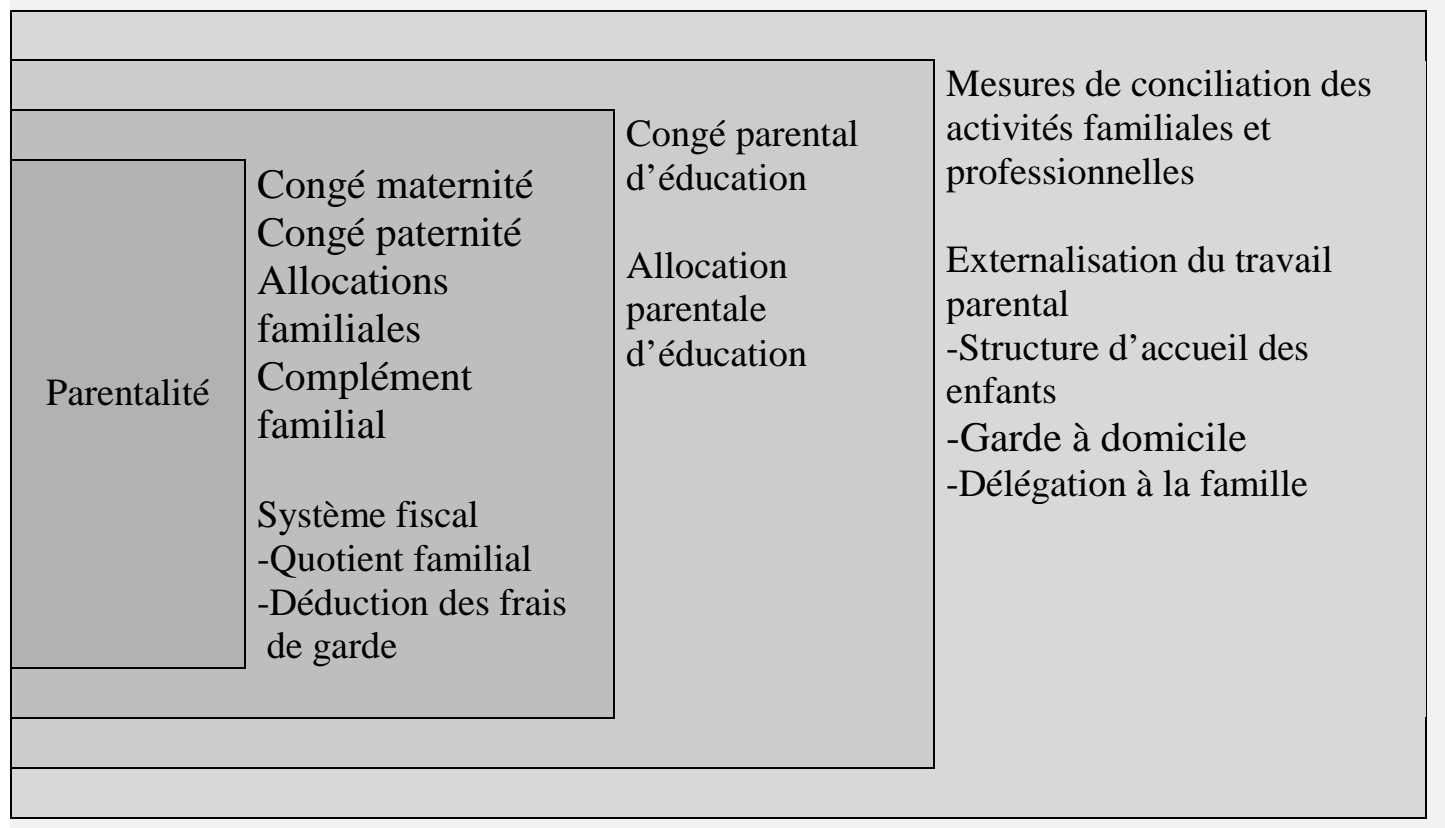

\section{SOUS-SECTION I.D.1 EN ALLEMAGNE}

\section{I.D.1.1 Le modèle très présent de la femme au foyer}

La représentation idéale de la famille correspond à la famille bourgeoise, basée sur le mariage (Lampert 1991 : 344 ; Ostner 1993 ; Voegeli 1991 : 110) et le principe de «l'homme soutien de famille» «Ernährermodell»(Ostner 1995), bien que la représentation familiale s'émancipe de plus en plus de cet idéal-type (Walter 1995). A cette norme correspond une forme politique de la famille que Schultheis définit de „conjugaliste“ et qui place son point fort sur le couple marié (Schultheis : 150).

La politique familiale, née dans les années 1950 sous la période Adenauer, cherchait à se distancier de l'idéologie nationaliste - socialiste et de l'idéologie communiste de la DDR (Bast, Ostner 1992 : 250). Sous le régime nazi, l'institution de la famille et du couple ont été dissociés et l'homme relevé de ses fonctions par le «Führer». Dans l'ancienne Allemagne de l'Est, le père remplissait un rôle sans réelles attentes sociales, de nombreuses thèses sur «une société sans pères» ont été développées à propos de l'ex - DDR (Bast, Ostner 1992 : 250). Une politique familiale en réaction à ces deux courants, où la place du père avait été affaiblie, s'est alors développée. La famille et le couple ont été institutionnalisés comme une sphère privée dont l'Etat se teindrait à l'écart. L'arrangement intrafamilial devait suivre sa propre logique, le père et l'époux étaient la première instance régulatrice des affaires familiales. La décision d'une maternité et le nombre d'enfants relèvent du domaine privé (Bast, Ostner 1992 : 250). La famille s'intègre dans une conception privatiste et non-interventionniste des affaires de famille. Elle n'est pas reconnue comme une branche pertinente du droit social (Schultheis : 150) et cet héritage limite la légitimité d'une ingérence publique dans les affaires de familles. 
En 1950, le travail des femmes n'était pas souhaité, il était un danger pour «l'équilibre du sexe féminin». En 1960, il n'est plus une menace pour la femme, mais pour le bien de l'enfant. Le modèle familial se construit alors autour de l'individualisation des droits de l'enfant : il est dans l'intérêt de l'enfant que quelqu'un se dévoue à son éducation.

La représentation selon laquelle la femme peut à la fois exercer une activité professionnelle et avoir une famille s'est généralisée dans les années 1960, de façon concomitante avec les évolutions socio-démographiques et l'entrée massive des femmes sur le marché du travail. Parallèlement, les tentatives de conciliation entre la vie familiale et professionnelle avaient été mises en place dans les années 1970 par le gouvernement socio libéral, contrecarrées par le retour des chrétiens-démocrates (Schultheis : 150). La réforme de la loi sur la famille de 1977 contribue à marquer l'érosion du modèle dominant de la femme au foyer. Cette nouvelle représentation vient rompre avec un modèle classique d'une biographie où la femme arrête son activité professionnelle au moment du mariage et plus encore à la naissance des enfants. Elle a été remplacée progressivement par un modèle à «3 phases» qui tient compte de la reprise de l'activité professionnelle lorsque les enfants quittent le foyer. Ce n'est qu'à partir des années 1970, et plus encore des années 1980, qu'apparaît une biographie des femmes alliant activité professionnelle et vie familiale. Ce modèle «à double orientation» «Doppelorientierung» ou «doppelter weiblicher Lebensentwurf» pour les femmes se généralise au niveau normatif, peu encore dans les pratiques : les deux tiers des femmes interrogées en 1995 ont une biographie qui correspond au modèle classique de biographie féminine en trois phases, où les séquences professionnelles et familiales se suivent (Hullen 1995 : 150).

\section{I.D.1.2 Les politiques familiales liées à la présence et à la charge de l'enfant}

\section{I.D.1.2.1 Le congé de maternité}

Le congé de maternité «Mutterschaftsurlaub»a été l'objet d'une réforme en décembre 2001. La durée du congé de 14 semaines a été étendue à l'ensemble des naissances. Les jours de congé peuvent être reportés après la naissance de l'enfant, si celle-ci survient avant la date prévue (cette mesure a pris effet en été 2002) (Bundesministerium für Familie, Senioren, Frauen und Jugend, 2001a). Si la mère le souhaite, elle peut continuer son activité professionnelle pendant les 6 semaines avant la naissance, la durée de 8 semaines après l'accouchement ne pouvant être réduite «Mutterschutzfrist». Sa durée est prolongée dans le cas de naissances multiples (12 semaines en cas de naissance avant terme ou naissances multiples) et non pas uniquement pour le troisième enfant comme en France.

Le congé de paternité n'existe pas sous la forme qu'on lui connaît dans les pays scandinaves ${ }^{43}$ ou depuis peu en France : cependant, les pères peuvent demander une mise en disponibilité «Sonderurlaub», à leur employeur. Suivant le $§ 616$ Abs 1 BGB toute personne, empêchée de travailler pour des raisons personnelles, peut prétendre à un congé payé. Les modalités sont le plus souvent réglées par le contrat de travail «Arbeitsvertrag», par les accords de branche «Tarifvertrag» ou la convention collective dans l'entreprise

\footnotetext{
${ }^{43}$ En Finlande les pères ont droit à un congé de paternité de 18 jours, d'un mois en Suède, et de 2 semaines au Danemark.
} 
«Betriebsvereinbarung» : la jurisprudence actuelle reconnaît la naissance d'un enfant comme un empêchement personnel et accorde au père un à trois jours de disponibilité suivant les accords spécifiques (AOK 2001). Mais le congé n'est pas un droit au sens juridique du terme. Par ailleurs, ce congé ne revient pas de droit au père d'un enfant naturel «Bundesverfassungsgericht» (BVerfG 1998a, 1998b), lequel peut demander un congé sans solde ou prendre des jours de congé payé.

\section{I.D.1.2.2 Les allocations familiales}

Dans le cadre de la «politique de redistribution horizontale entre les ménages suivant le critère de présence d'enfants» («Familienleistungsausgleich»), l'allocation familiale correspond à une exonération d'impôt sur le revenu, dans la proportion du minimum vital de l'enfant («Existenzminimum»). Elle prend deux formes, un abattement à la base suivant le $\S$ 32 Abs. 6 EstG ou le versement d'une allocation familiale («Kindergeld»). Dans un premier temps, l'allocation est versée sous forme d'une indemnité. Dans un second temps, le fisc «Finanzamt» calcul l'impôt sur le revenu, vérifie si l'allocation familiale correspond au montant de l'exonération souhaitée et décide s'il est plus avantageux pour le ménage de procéder à un abattement à la base. L'allocation familiale tient également compte des besoins pour la garde, l'éducation et la formation de l'enfant. Le paiement est effectué par les caisses familiales du service public ${ }^{44}$. Le montant de l'allocation familiale depuis le 1er janvier 2002 se monte à 154 Euros pour le premier, second et troisième enfant, et à 179 Euros pour chaque enfant supplémentaire. Cette allocation familiale est attribuée à chaque enfant qui réside en Allemagne, jusqu'à sa majorité ${ }^{45}$, indépendamment de sa nationalité.

Le calcul de l'allocation tient compte des enfants dont le lien de parenté est direct avec le bénéficiaire, mais également des enfants du conjoint (suivant leur domiciliation). Mais le critère de résidence habituelle ne s'applique pas aux situations où l'enfant est présent quelques jours par semaine, comme par exemple dans le cas d'une résidence alternée (Bundesanstalt für Arbeit 2002). Un seul bénéficiaire perçoit l'allocation familiale pour un seul et même enfant ${ }^{46}$ et elle est attribuée à la personne qui en a la charge financière.

Lorsque les parents vivent ensembles, ils peuvent s'accorder entre eux pour savoir qui sera le bénéficiaire de l'allocation. Cette latitude permet un calcul plus avantageux du montant de la prestation en fonction de la situation familiale, par exemple dans le cas où il $\mathrm{y} \mathrm{a}$ des enfants d'une première union. Deux facteurs interviennent alors dans le calcul de l'allocation familiale : le nombre d'enfants à charge pour le ménage (en fonction du critère de résidence habituelle), mais également le nombre d'enfants qui ont un lien de parenté avec le bénéficiaire. Ces derniers influencent sur le rang des naissances et donc indirectement sur le montant de l'allocation, ils sont alors comptabilisés mêmes s'ils ne résident pas avec le bénéficiaire.

\footnotetext{
44 L'allocation familiale est délivrée depuis le 1er janvier 2001 par la caisse nationale familiale («Bundesfamilienkasse»), il s'agit d'une nouvelle institution au sein du ministère des finances («Bundesamt für Finanzen») dont le premier objectif est de gérer le calcul et le paiement de l'allocation familiale. En ce qui concerne le paiement d'autres suppléments familiaux («Familienzuschlag»), il relève du service public auquel le bénéficiaire est rattaché.

${ }^{45}$ Dans le cas des enfants de plus de 18 ans, les parents peuvent bénéficier de l'allocation familiale jusqu'à ses 27 ans si ce dernier se trouve en formation ou fait des études supérieures, jusqu'à ses 21 ans, s'il est inscrit au chômage comme demandeur d'emploi.

${ }^{46}$ Dans le mois qui suit le mariage de l'enfant, les parents ne sont plus bénéficiaires de l'allocation familiale, car c'est son conjoint qui a alors le devoir de soutien.
} 
Lorsque les parents sont divorcés, séparés ou non mariés, l'allocation est allouée au parent qui a la résidence habituelle. Si elle n'est pas fixée, elle revient à celui qui participe le plus à l'entretien de l'enfant. (Bundesanstalt für Arbeit, 2002).

\section{$\underline{\text { I.D.1.2.3 }} \underline{\text { Le }} \underline{\text { système fiscal }}$}

Le système d'impôt («Ehegattensplitting») favorise les couples mariés qui ont de grands écarts de revenus : le revenu du ménage est d'abord divisé par deux avant d'être multipliée par deux après le calcul de l'imposition (Information für Familien, Reihe Ratschläge und Hinweise 1995 :148 ). Il n'y a pas de quotient familial au sens français (cf. partie suivante). Les frais engagés dans le soutient matériel de l'enfant (pour sa formation et son éducation) sont pris en compte dans le calcul des allocations familiales («Kinderfreibetrag» et «Kindergeld»). En revanche, les grilles d'imposition tiennent compte du statut familial du conjoint (divorcé, veuve ou marié), de son activité professionnelle ${ }^{47}$ et de la présence d'enfant (Unsere Steuern von A bis Z 1996 : 113).

\section{I.D.1.3 Les politiques familiales liées à une disponibilité des parents pour l’éducation des enfants : «Elternzeit»}

Le congé parental a été institué en 1986 en Allemagne de l'Ouest et en 1991 dans les nouveaux Bundesländer («Elternzeit»). Depuis la réforme de 1992, sa durée est de trois ans. Si la personne cotise ( «Pflichtmitglied»), son assurance maladie est prolongée le temps du congé parental. Elle peut exercer une activité professionnelle jusqu'à 19 heures par semaine. Depuis son entrée en vigueur en 1986, 96 \% des parents ayant droit ont choisi de prendre un congé parental (pratiquement exclusivement les mères ${ }^{48}$ ), près de $100 \%$ si la mère ne travaillait pas auparavant, et à 98\% si elle travaillait auparavant (Presse-und Informationsamt der Bundesregierung, 1995 : 59). Les parents peuvent le prendre trois fois à tour de rôles pendant la durée du congé, ce qui est très rarement le cas dans les faits. Très peu d'hommes, moins de $2 \%$, choisissent de prendre un congé parental ${ }^{49}$. Les deux premières années sont

\footnotetext{
${ }^{47}$ Il existe un supplément familial «Familienzuschlag» pour les fonctionnaires : il est fonction de la situation matrimoniale des bénéficiaires «Verheiratetenzuschlag» et du nombre d'enfants à charge «Kinderzuschlag». Lle supplément familial niveau 1 est un supplément de traitement pour tout fonctionnaire marié, veuf ou divorcé ; le supplément familial niveau 2 correspond à un supplément de traitement versé au bénéficiaire pour la présence d'enfant. Le supplément familial dépend également de la classe de rémunération : à titre d'indication et pour les groupes de rémunération des fonctionnaires A1 jusqu'à A8, le niveau 1 est de 95,96 Euros et le niveau 2 de 182,17 Euros. Le supplément familial augmente de 86,21 Euros pour le second enfant, de 220,74 Euros pour le troisième et pour chaque enfant supplémentaire (Niedersächsisches Landesamt für Bezüge und Versorgung 2001).

${ }^{48}$ La durée du congé parental, que les femmes envisage de prendre, se varie très nettement en fonction de la région : à l'Ouest, les femmes projettent un congé parental beaucoup plus long qu'à l'Est : en effet, à l'Ouest, $12 \%$ des mères ont prévu de prendre un congé parental inférieur à un an, $15 \%$ d'une durée de un à deux ans, $63 \%$ d'une durée deux à trois ans et 10\% de plus de trois ans. A l'Est, $25 \%$ souhaitent le prendre pour un an, $37 \%$ pour un à deux ans, $38 \%$ pour deux à trois ans. Aucune femme n'a prévu de le prendre pour une durée supérieure à trois ans (Beckmann 2001).

${ }^{49}$ Le ministre fédéral de la famille, Christine Bergmann (SPD), s'est engagé dans une campagne d'opinion en mai 2001 «Mehr Spielraum für Väter» («Plus de place pour les pères»). L'intention de la campagne est de réduire l'écart entre un investissement potentiel des pères évalué à $20 \%$, et les 1,5\% d'hommes qui prennent un
} 
financées au niveau fédéré et le montant de l'allocation est de 307 Euros par mois («Erziehungsgeld») ${ }^{50}$. Par la suite, le montant du congé parental dépend des régions qui prolongent son financement par des aides supplémentaires pour une durée comprise entre 6 mois et un an («Landeserziehungsgeld») (Wingen 1997 : 336-337). Leur politique en la matière relève non seulement de leurs fonds financiers mais aussi de leur orientation politique : les régions à majorité chrétienne-démocrate favorisent les aides individuelles par le biais d'allocations, celles à majorité sociale-démocrate développent d'avantage les aides concernant les infrastructures collectives et les services (Wingen 1997 : 335).

Le gouvernement vient de mettre en place une nouvelle loi sur le congé parental «Elternzeit» en janvier 2001 (BerzGG). Elle a pour ambition de permettre un partage plus égalitaire du travail parental pendant les trois premières années après la naissance de l'enfant. $\mathrm{Au}$ cœur de cette loi, se situe le principe selon lequel le père et la mère pourront prendre le congé parental en commun. Durant cette période, les deux parents ont le droit d'exercer un temps partiel, jusqu'à 30 heures par semaine dans une entreprise de plus de 15 salariés. La troisième année du congé parental peut être prolongée avec l'accord de l'employeur jusqu'à ce que l'enfant ait atteint l'âge de huit ans. Enfin, le plafond de revenus a été relevé.

Les résultats du projet IAB (Beckmann 2001) viennent confirmer les données relevées en 1996 dans le cadre d'un projet mis en place par le ministère de la famille en Allemagne et effectué sous la direction de Vaskovics (Vaskovics; Rost 1999): seuls 1,5\% des pères prennent un congé parental. Pour quelles raisons les hommes ne font pas appel à cette mesure de congé parental?

La première raison serait la perte de revenu non suffisamment compensée par l'activité professionnelle de la femme et par l'aide financière du congé parental ${ }^{51}$ : cette réponse est nommée dans plus de 70\% des cas, par les hommes (Vaskovics; Rost 1999) et par les femmes (à l'Est et à l'Ouest) (Beckmann 2001).

La seconde réponse avancée par les hommes se différencie selon les deux régions : à l'Ouest, les raisons sont liées à la carrière et à la situation professionnelle, contrairement à l'Est où les inquiétudes sont liées à l'emploi et au poste de travail (à la peur de ne pas retrouver une situation d'emploi) (Vaskovics ; Rost 1999). Les réponses des femmes montrent qu'elles ne font pas de distinction nette entre les items de réponses liées à la situation professionnelle et ceux liés à l'emploi. Mais on retrouve sur certaines réponses une préoccupation en écho de celle des hommes : à l'Est, les femmes craignent que l'homme ne retrouve pas une situation d'emploi après le congé parental (Beckmann 2001).

congé parental. Il s'agit donc d'encourager un nouveau du partage du parental, soutenant une participation des deux parents au moment du congé parental. Cette campagne souhaite participer à un changement de l'image du père et de l'homme en particulier en agissant en coopération avec le monde de l'entreprise qui, selon le ministre, montre encore une grande réserve quant à l'implication des hommes dans le monde de la famille (bmfsfj 2001).

${ }^{50}$ Le montant de 307 Euros concerne les couples mariés qui ne dépassent pas la limite de 51130 Euros par an et de 38350 Euros pour les familles monoparentales. Après les 7 mois de l'enfant, le plafond est abaissé : soit par exemple 16470 Euros pour un enfant (en dessous de cette somme le ménage reçoit la somme de 307 Euros dans sa totalité). Pour les familles cohabitantes, les revenus du partenaire sont comptabilisés. De plus, cette allocation est réservée aux personnes inactives ou à temps partiel (Bundesministerium für Familie, Senioren, Frauen und Jugend, 2001b).

${ }^{51}$ Ce que confirme le fait que le nombre de père, qui ont pris le congé parental, augmente avec la qualification et le revenu de leur femme (Beckmann 2001). 
La troisième réponse donnée concerne les attitudes et les opinions des hommes. Les 2/3 des femmes considèrent que l'attitude de l'homme concernant les tâches familiales a été un frein à la prise en charge du congé parental par les pères (Beckmann 2001). Mais seulement $1 / 3$ des hommes sont du même avis. On observe donc un décalage très important entre les réponses des pères et des mères concernant les représentations des pères. Ce décalage peut être interprété comme une moindre résistance des pères quant à leur participation et une surévaluation par les mères des tendances traditionnelles des pères ${ }^{52}$.

\section{I.D.1.4 Les mesures de «conciliation» Travail-Famille}

\section{I.D.1.4.1 Le temps partiel}

Le temps partiel est beaucoup plus développé dans l'ancienne Allemagne de l'Ouest que dans l'ancienne Allemagne de l'Est. En mai 2000, près de $22 \%$ des salariés travaillaient à temps partiel à l'Ouest ${ }^{53}$, contre $13 \%$ à l'Est (Statistisches Bundesamt $2001: 28$ ).

Derrière ces chiffres se cachent des raisons structurelles différentes : dans l'ancienne Allemagne de l'Ouest, les $2 / 3$ des femmes travaillant à temps partiel déclarent le faire pour des raisons personnelles ou familiales : pour l'éducation des enfants ou les soins d'un membre de la famille (Statistisches Bundesamt 2001). Elles ne sont que $21 \%$ dans l'ancienne Allemagne de l'Est. La proportion d'hommes travaillant à temps partiel est de $12 \%$ à l'Ouest et de $5 \%$ à l'Est. La raison la plus fréquemment donnée pour justifier leur pratique du temps partiel est une formation ou une reconversion professionnelle. Ce qui n'est le cas que de $4 \%$ des femmes.

Dans les régions de l'Est, les femmes travaillant à temps partiel déclarent à $52 \%$ ne pas avoir trouvé de travail à temps complet (contre $6 \%$ à l'Ouest). Les écarts chez les hommes sont aussi marqués, bien que moins importants : $33 \%$ à l'Est et $14 \%$ à l'Ouest (Statistisches Bundesamt 2001).

La logique est donc différente : à l'Ouest, les raisons pour l'exercice d'un temps partiel pour les femmes sont très majoritairement familiales. A l'Est, les raisons sont liées aux évolutions du marché du travail, surtout pour les femmes, mais également pour les hommes.

\footnotetext{
${ }^{52}$ Les femmes qui n'ont jamais envisagé de partager le congé parental, déclarent à plus de $50 \%$ que leur partenaire n'avait jamais envisagé de prendre un congé parental, à l'Est comme à l'Ouest. Ce pourcentage est beaucoup plus faible lorsque la femme envisageait de partager le congé parental (s'il n'y avait pas de baisse de revenu). Dans ce cas, elles estiment à 1/3 pour l'Ouest et à $25 \%$ pour l'Est que l'homme n'avait pas envisagé de congé parental (Beckmann 2001). On voit ainsi que la projection sur le partenaire de certaines attitudes est liée aux propres conceptions des femmes et joue sur la prise en charge du congé parental.

${ }^{53}$ On rappellera que le travail à temps partiel reste un domaine féminin : à l'Ouest, en 2000, $42 \%$ des femmes actives travaillent à temps partiel, cette proportion est de $5 \%$ pour les hommes. Dans les nouveaux Länder, $23 \%$ des femmes salariées travaillent à temps partiel contre $4 \%$ des hommes. Selon les données de l'office fédéral de la statistique (Statistisches Bundesamt 2001), $90 \%$ des temps partiels était effectué par des femmes.

Par ailleurs, c'est essentiellement dans les nouveaux Länder que l'irrégularité des temps de travail a augmentée dans l'intervalle des dix dernières années (Statistisches Bundesamt 2001).
} 
La dernière loi sur le temps partiel («Teilzeitarbeitsgesetz») est rentrée en application en janvier 2001 : elle a pour but de développer le temps partiel en particulier pour les employés qui souhaitent une réduction de leur temps de travail. Si ce dernier n'arrive pas à s'entendre avec la direction, il peut entreprendre une action juridique. Les effets de cette loi, qui vise un partage plus égalitaire du travail familial, devront être nuancés, notamment si le temps partiel reste essentiellement féminin, et n'offre pas suffisamment de garanties pour les hommes.

\section{I.D.1.4.2 Les infrastructures d'accueil}

Devant l'incapacité actuelle de faire face à la demande croissante de la prise en charge des plus jeunes enfants, l'Etat, après avoir privilégié les solutions privées comme la famille et les jardins d'enfant privés, réoriente son action vers le développement des structures. La capacité de l'aide publique actuelle ne couvre pas plus de $5 \%$ des demandes. «On observe en Allemagne une double subsidiarité découlant d'une compétence forte des différents pouvoirs territoriaux et d'une place importante des acteurs non étatiques par rapport aux institutions publiques (ce qui s'illustre par l'existence d'équipements comme les jardins d'enfants ou les crèches qui sont largement privatisées).» (Commaille 1996 : 224).

Les infrastructures d'accueil pour les enfants âgés de zéro à trois ans couvrent $3 \%$ des besoins $^{54}$, ils sont ouverts pendant quelques heures le matin. Pour les enfants de moins de trois ans, il n'y a pratiquement pas de système public de prise en charge. Les structures sont essentiellement privées $^{55}$ : parents, associations de parents ou autres organismes caritatifs. La région et surtout la commune, prend en charge le financement des places de jardin d'enfants (après trois ans) et de crèches (avant 3 ans) (Wingen 1997 : 336-337). Depuis le $1^{\text {er }}$ janvier $1996^{56}$, un droit à une place d'une demi-journée en garderie est garanti pour les enfants de plus de 3 ans. Mais l'infrastructure ne peut permettre la couverture des besoins (Dienel 1996 : 49). $75 \%$ des enfants âgés de 3 à 6 ans sont accueillis dans des centres, dont seuls $12 \%$ sont ouverts toute la journée. Enfin, l'école accueille les enfants en âge d'être scolarisés pour 4 à 5 heures le matin (Oelschläger, Schunter-Kleemann, 1992 ; Lohkamp-Himmighofen, 1993). Les repas sont pris au domicile, après l'école (Dienel 1996 : 45). Le début et la fin de la demijournée varient d'un jour sur l'autre, ce qui limite l'activité professionnelle éventuelle à un temps partiel (Lohkamp-Himmighofen 1993 : 349 ; Dienel 1996 : 46).

\footnotetext{
${ }^{54}$ L'activité professionnelle des femmes est une variable déterminante, dans la mesure où la garde des enfants les plus jeunes est essentiellement assurée par les mères : ce sont donc les contraintes ou les choix quant à leur activité professionnelle qui déterminent le besoin d'accueil des jeunes enfants (occasionnel ou régulier). Plus globalement, la diversification des temps de travail des parents induit une diversification des besoins d'accueil et une complexité croissante des réponses à apporter (Fenet, Leprince, Périer 2001 : 14). Par ailleurs, les modes d'accueil des enfants sont des indicateurs intéressants pour évaluer l'action de l'Etat sur la problématique de la conciliation (Lohkamp-Himmighofen, $1993: 321$ ).

${ }^{55}$ Les objectifs de la politique familiale jalonnent le parcours de la femme active et peuvent tout aussi bien la contraindre, lui faciliter ou lui interdire l'accès au marché de l'emploi. Ils facilitent (ou non) l'articulation de la vie familiale et professionnelle. Or, les familles monoparentales sont très concernées par ces mesures, plus que les couples, car elles doivent à la fois assurer les ressources économiques du ménage et l'éducation des enfants (Lohkamp-Himmighofen, 1993 : 317-318).

${ }^{56}$ Cette réforme est liée à la réunification allemande. Elle vient compenser, pour les nouveaux Länder, les restrictions du droit à l'avortement, conséquents à l'adaptation du droit de la famille de l'ex-DDR à celui de la BRD (Dienel 1996 : 49).
} 
Le tableau 14 (ci-dessus) indique le nombre de places d'accueil pour 1000 enfants entre 3 et 6 ans et demi. En 1995, dans l'ensemble des régions de l'ancienne Allemagne de l'Est, le nombre absolu de place offerte approche le nombre d'enfants. En revanche dans l'ancienne Allemagne de l'Ouest, les différences entre les régions sont encore très importantes, la couverture des besoins allant du simple au double.

L'association allemande pour les familles monoparentales (VAMV) souligne les déficits actuels, et revendique, une amélioration de la compatibilité enfant/travail pour tous les parents, une offre suffisante en matière de gardiennage, et une mise en place d'un système scolaire sur toute la journée pour les parents qui le désirent (Publication de l'association VAMV 1996).

$\underline{\text { Tableau } 14 \quad \text { Nombre de places d'accueil pour } 1000 \text { enfants âgés de } 3 \text { à } 6 \text { ans et demi }}$

\begin{tabular}{|l|c|}
\hline \multicolumn{2}{|c|}{ AnCienne Allemagne de L'OUeST } \\
\hline Baden-Württenberg & 924 \\
\hline Rheinland-Pfalz & 904 \\
\hline Saarland & 836 \\
\hline Hessen & 782 \\
\hline Bayern & 755 \\
\hline Bremen & 656 \\
\hline Schleswig-Holstein & 649 \\
\hline Niedersachsen & 641 \\
\hline Nordrhein-Westfalen & 630 \\
\hline Berlin-Ost & 514 \\
\hline Hamburg & 509 \\
\hline
\end{tabular}

\begin{tabular}{|l|c|}
\hline \multicolumn{2}{|c|}{ AnCIENNE AlLEMAGNE DE L'EST } \\
\hline Thüringen & 1019 \\
\hline Sachsen & 979 \\
\hline Berlin-Ost & 976 \\
\hline Bandenburg & 973 \\
\hline Sachsen-Anhalt & 920 \\
\hline Mecklenburg-Vorpommern & 891 \\
\hline
\end{tabular}

Source : Statistisches Bundesamt 1995 (Sozialpolitische Umschau 1997c) 


\section{SOUS-SECTION I.D.2 EN FRANCE}

\section{I.D.2.1 Entre le «Familialisme» et le «Natalisme»}

Depuis le XIXème siècle, les politiques familiales reprennent alternativement deux motifs politiques, le familialisme et le natalisme. Le familialisme est une morale de la vie sociale dont les bases sont, le rayonnement de l'Eglise, l'entreprise familiale comme mode de production, et l'exclusion des femmes de la scolarisation et du travail non familial. Ce courant prône la famille nombreuse, l'ordre moral fondé sur le respect des hiérarchies, la tradition chrétienne, la morale catholique, la propriété, la femme comme épouse et mère, le foyer (Lenoir 1985a, 1985b). En revanche, le natalisme est guidé par une perception du monde social d'inspiration scientifique et rationaliste, qui encourage les objectifs démographiques afin de renforcer la puissance économique et militaire de la patrie. La famille nombreuse est encouragée de fait, l'illégitimité des naissances n'est plus stigmatisée. Cette typologie permet de saisir, ce que R. Lenoir appelle la «production des représentations de la famille». La dialectique entre les deux courants, nataliste et familialiste, structure le discours sur la famille en France.

La seconde guerre mondiale et la fin des années 1940 sont l'âge d'or du familialisme. A titre d'exemple, la Fête des mères institutionnalisée en 1950 illustre «Le stéréotype de la cause familialiste : la famille nombreuse dont la mère est au foyer» (Lenoir 1985 : 37, Prost 1984 : 20). Puis, dans les années 1950, la position des «familiaux» est ébranlée par les positions des responsables économiques du pays et la perte de l'influence du Mouvement Républicain Populaire (Lenoir 1985 : 35). La mouvance familialiste ne s'y était pas renouvelée dans les années soixante, l'arrivée au pouvoir du gaullisme n'y étant pas propice. R. Lenoir définit trois transformations des bases sociales du familialisme: le déclin de l'Eglise, la disparition des entreprises familiales, et l'accès des femmes à la scolarisation et à l'activité professionnelle. Entre 1950 à 1970, le familisme cède devant la modernisation morale (Lenoir 1985).

Les catégories «enfants naturels», «mariage», «concubinage», relèvent d'une régulation de la famille qui repose sur la famille légitime et sur une économie domestique et patrimoniale. Les transformations morales de l'époque éloignent le nouveau mode de régulation de la famille de ces référents. Ceci est facilité par une gestion plus technocratique de la famille. Dans les années 1960 et 1970, les politiques familiales s'orientent vers une action sociale en faveur de certaines catégories de ménages (Prost 1985 : 20). Il s'agit de «répartir équitablement la charge économique que représente l'éducation et l'entretien des enfants entre les différentes catégories de ménage» (Lenoir 1991 : 798). L'action sociale se réfère à la famille multiple et non plus à la famille unique et indivisible. Dès le début des années 1960, les modes de redistribution des prestations familiales (allocations pour les plus modestes) se transforment et depuis début des années 1970, les populations cibles sont redéfinies (Lenoir 1985 : 37, Prost $1984: 20$ ).

On ne s'intéresse plus à la forme familiale mais aux situations sociales à l'origine de ces choix : les nouvelles prestations sociales sont soumises à des conditions de revenus, les aides destinées aux familles monoparentales sont créées. «Ainsi une condition sociale comme celle de fille mère, de femme divorcée, (...) est complètement modifiée selon que les mères sont perçues et pensées comme le produit d'une déchéance morale ou comme le résultat d'une 
conduite rationnelle et volontaire. A ce travail de conversion du regard sur le monde social, contribuent de plus en plus les spécialistes des sciences sociales et les nouvelles générations de travailleurs sociaux. Ce travail va du changement de vocabulaire à la transformation du droit lui - même. On passe ainsi de la condition de fille mère au statut de parent isolé» (Lenoir $1985: 804)$.

Dans les années 1980, l'Etat s'est exprimé très fermement sur sa décision de ne pas privilégier un modèle familial particulier (Lenoir 1991 : 179).

Enfin, depuis les années 1990, les politiques familiales s'appliquent de plus en plus à favoriser l'exercice de la fonction parentale, pour les deux parents (cf. paragraphes suivants). La multiplication des modes de garde collectifs et individuels est un instrument de promotion de la parité professionnelle, laquelle est de plus en plus dépassée par un souci de promouvoir la parité parentale, l'une étant irrémédiablement liée à l'autre. En ce sens, la parentalité et le souci d'une plus grande égalité parentale deviennent centrales dans les politiques familiales en France. La réforme récente sur le congé de paternité et le livret de paternité en est l'inscription la plus forte.

\section{I.D.2.2 Les politiques familiales liées à la présence et à la charge de l'enfant}

\section{I.D.2.2.1 Le congé de maternité et de paternité}

Le congé de maternite ${ }^{57}$ a une durée totale de seize semaines dont 6 et 10 semaines avant et après l'accouchement ${ }^{58}$. A partir du troisième enfant, le congé est de vingt-six semaines $^{59}$ (CAF 2001). Le calcul des indemnités journalières résulte de la situation professionnelle et du régime d'affiliation à la sécurité sociale. Elles sont attribuées uniquement en cas d'interruption d'activité salariée : de façon générale, l'indemnité est égale au salaire journalier de base, calculé sur la moyenne des salaires des trois mois qui précédent le repos prénatal. La caisse primaire d'assurance maladie verse les indemnités, son montant maximum est de 62 Euros. Au-delà de ce montant, certaines conventions collectives permettent de maintenir le niveau du salaire antérieur ${ }^{60}$ (Cnamts 2002).

Le congé de paternité est une mesure qui a été introduite récemment en France, il vient s'ajouter à l'ancien "congé de solidarité familiale" dévolu aux pères par le Code du travail (Article L.226-1). Depuis le premier janvier 2002, les pères ont donc la possibilité de prendre un congé de paternité de 11 jours consécutifs (y compris les samedis et les dimanches) qui vient s'ajouter aux trois jours qui leur étaient accordé antérieurement. La durée totale de leur congé peut donc atteindre 14 jours. Le congé de paternité ne suit pas obligatoirement les trois jours, mais il doit débuter dans les 4 mois qui suivent la naissance. Il n'est pas fractionnable,

\footnotetext{
${ }^{57}$ En cas d'adoption, le repos postnatal peut être réparti entre le père et la mère, l'un et l'autre peuvent y prétendre (Cnamts 2002).

${ }^{58} \mathrm{Si}$ la mère décède du fait de son accouchement, le père de l'enfant peut percevoir des indemnités journalières de repos postnatal à condition de cesser son travail pendant cette période (Cnamts 2002).

59 La durée du congé peut être prolongée de 2 semaines supplémentaires avant l'accouchement en cas de grossesse pathologique. Dans le cas de naissances multiples, la durée est de 34 semaines pour des jumeaux, et de 46 semaines pour des naissances de plus de 2 enfants (Cnamts 2002).

${ }^{60} \mathrm{Si}$ la personne est demandeur d'emploi, le calcul sera fait sur la moyenne des trois derniers bulletins de salaires précédant le chômage (Cnamts 2002).
} 
mais si le père le souhaite, le congé peut ne pas être pris dans toute sa durée ${ }^{61}$. Le congé de paternité peut être allongé en cas de naissances multiples à 18 jours calendaires. L'indemnité journalière versée au père par la caisse d'assurance maladie répond aux même conditions d'ouverture des droits et aux même règles de calculs que l'indemnité journalière du congé de maternité ${ }^{62}$ (il est rémunéré à 100\% du salaire sous le plafond de 2279 Euros mensuels fixé par la Sécurité sociale) (Cnamts 2002).

Encadré $\mathrm{n}^{\circ} 5 \quad$ Autres mesures catégorielles liées à la charge de l'enfant

L'allocation pour jeune enfant (APJE) est attribuée, sous condition de ressources, à partir du 5ème mois de la grossesse et après la naissance, jusqu'aux 3 ans de l'enfant. Elle est de 156 Euros, par mois si les ressources du ménage ne dépassent pas 17045 Euros pour un couple à un actif (22 526 Euros pour une personne seule ou un couple de deux actifs) (CAF 2001).

Le complément familial est une prestation liée à la charge de l'enfant. Elle est attribuée sous condition de ressources. Elle s'adresse aux ménages qui ont au moins trois enfants à charge, tous plus âgés de 3 ans. Le montant de l'aide est de 141 Euros par mois, si le revenu du ménage ne dépasse pas les limites calculées en fonction du nombre d'enfant et de la situation conjugale. Dans le cas de trois enfants, le plafond est fixé à 24545 Euros pour un couple a un seul revenu et à 30025 Euros pour un parent isolé ou un couple à deux actifs (CAF 2001).

L'allocation de rentrée scolaire est une autre prestation sous condition de ressources d'un montant de 243 Euros par enfant, si les ressources du ménage ne dépassent pas 15885 Euros (CAF 2001).

\section{I.D.2.2.2 Les allocations familiales}

Les allocations familiales sont attribuées sur le seul critère de la charge d'enfant, quelle que soit la situation familiale et le montant du revenu du ménage. Elles sont attribuées à partir du second enfant avec un montant de 108 Euros par mois et de 248 Euros pour trois enfants (plus 139 Euros pour chaque enfant supplémentaire), ces allocations sont majorées suivant l'âge des enfants (CAF 2001).

\section{I.D.2.2.3 Le système fiscal}

En France, le calcul de l'impôt sur le revenu tient compte de la situation familiale du contribuable et du nombre de personne à charge ${ }^{63}$ : le nombre d'enfants célibataires de moins

\footnotetext{
${ }^{61}$ En cas de décès de la mère, le père a droit au congé maternité qu'il peut ajouter à son congé paternité (Cnamts 2002).

${ }^{62}$ Les indemnités journalières du congé de maternité et de paternité ne sont cumulables ni avec les indemnités journalières versées pour un arrêt de travail en cas de maladie ou d'accident du travail, ni avec l'allocation parentale d'éducation à taux plein, ni avec l'allocation de présence parentale, ni avec l'indemnisation par l'assurance chômage ou le régime de solidarité (Cnamts 2002).

${ }^{63}$ Le supplément familial de traitement (équivalent du «Familienzuschlag» allemand) est un droit du fonctionnaire, il est indissociable de sa rémunération. Ce supplément de traitement à caractère familial est constitué d'une partie fixe et d'une partie proportionnelle dont le montant varie en fonction de l'indice de rémunération et du nombre d'enfants à charge. Lorsque les deux parents sont fonctionnaires, le supplément
} 
de 21 ans (25 ans, s'ils poursuivent leurs études), d'enfants infirmes ou qui accomplissent leur service militaire, ainsi que des personnes invalides vivant dans le ménage (Patrimoine.com 2002). Chaque foyer est alors imposé en fonction de son revenu divisé par le nombre total de parts auxquelles il a droit. Le barème progressif de l'impôt sur le revenu s'applique à chaque part et, donc, à revenu égal, l'impôt diminue avec le nombre d'enfants «système du quotient familial» (Ministère de l'emploi et de solidarité, 1998). Puis on multiplie ce résultat par le nombre de parts (Patrimoine.com 2002) (cf. Annexe 12).

Un enfant compte pour une demi-part (ou une part s'il s'agit du troisième). Dans le cas des célibataires ou des divorcés ne vivant pas en concubinage, le premier enfant est compté pour une part. Les veufs ou les veuves élevant des enfants issus du mariage avec le conjoint décédé, conservent le nombre de parts dont ils bénéficiaient du vivant de leur conjoint. Lorsqu'ils n'ont plus d'enfant à charge, ils conservent une demi-part supplémentaire (Ministère de l'emploi et de solidarité, 1998). Dans les autres cas, les célibataires, divorcés ou séparés (en concubinage ou non) sont assimilés à un célibataire ou à divorcé avec personnes à charge (Patrimoine.com 2002).

Les frais de garde d'un enfant âgé de moins de 7 ans peuvent se déduire de l'impôt sur le revenu ${ }^{64}$. Elle concerne d'ensemble des dépenses nécessitées par la garde des enfants à l'extérieur du domicile par une assistante maternelle agréée (salaires nets et cotisations sociales) ou dans un établissement de garde agréé : crèche, halte-garderie, garderie périscolaire et post-scolaires, centres de loisirs sans hébergement (Ministère de l'Économie, des Finances et de l'Industrie, 1998). La base maximale de déduction est de $25 \%$ des dépenses retenues dans la limite annuelle de 2300 Euros par enfant et dans la limite du montant des revenus professionnels nets de frais (Patrimoine.com 2002). Pour bénéficier de la réduction d'impôt, les deux parents doivent travailler au moins à mi-temps. Les couples mariés dont l'un des conjoints est sans emploi ou occupe un emploi partiel (inférieur à un mi-temps) sont exclus de la mesure. Si l'enfant est gardé à domicile par une personne salariée, les dépenses correspondantes relèvent de la réduction d'impôt pour l'emploi d'un salarié à domicile.

\section{I.D.2.3 Le congé parental : pour une disponibilité des parents}

En France, le congé parental d'éducation est une suspension du contrat de travail (il n'est pas rompu) pendant une période d'un an, renouvelable deux fois. Pendant cette période, le salarié n'est pas rémunéré. Ses droits en matière d'assurance maladie maternité, d'invalidité et de décès sont maintenus. Tout salarié peut bénéficier du congé parental d'éducation à partir de la fin du congé maternité, jusqu'au troisième anniversaire de l'enfant, s'il est salarié au sein de l'entreprise depuis au moins un an au jour de la naissance de l'enfant. Il ne peut être

\footnotetext{
familial de traitement ne peut être versé qu'à l'un des deux agents. Dans l'ensemble des cas, c'est le père le qui perçoit. La mère perçoit éventuellement une allocation complémentaire dont le montant est égal à la différence existante entre le supplément familial de traitement perçu par son conjoint et celui auquel elle pourrait prétendre de son propre chef (Administration française 2000, 2002).

${ }^{64}$ Une réduction de l'impôt sur le revenu est accordée aux contribuables ayant des enfants à charge qui poursuivent des études secondaires ou supérieures. Le montant de la réduction d'impôt due aux frais de scolarisation se monte à 61 Euros par enfant au collège, 153 Euros par enfant au lycée et de 183 Euros par enfant poursuivant des études supérieures (Patrimoine.com 2002).
} 
refusé par l'employeur. Le salarié a la possibilité de demander un congé parental d'éducation ou de travailler à temps partiel (Administration française 2001).

Le droit à l'allocation parentale d'éducation dépend de l'activité professionnelle actuelle et passée ainsi que de l'âge et du nombre d'enfant à charge. Elle n'est pas attribuée pour un enfant. Dans le cas d'un ménage à deux enfants dont le dernier est âgé de moins de trois ans, le parent doit avoir arrêté de travailler ou travailler à temps partiel (en d'autres termes ne pas percevoir d'allocation chômage). Il doit avoir également exercé au moins 2 ans dans les 5 ans qui précédent la naissance du second enfant. Le parent qui ne travaille plus, perçoit l'allocation à taux plein soit 484 Euros (320 Euros s'il est salarié à mi-temps et 242 Euros pour un temps partiel compris entre 50 et 80\%) (CAF 2001).

\section{I.D.2.4 Les mesures de conciliation}

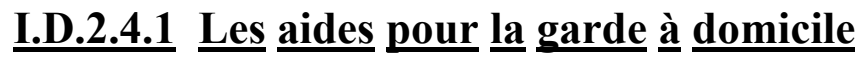
formes :

Les aides pour la garde à domicile d'un enfant de moins de 6 ans prennent deux

premièrement, si l'enfant est gardé par assistante maternelle agréée, les parents ont la possibilité de bénéficier de l'aide pour l'emploi d'une assistante maternelle agréée (AFEAMA) sauf si l'un d'eux a arrêté de travailler et à recours à l'APE. L'allocation est trimestrielle, elle ne peut dépasser $85 \%$ du salaire de l'assistante maternelle, elle peut être éventuellement majorée en fonction des revenus du ménage, et elle est plus importante pour un enfant de moins de trois ans ${ }^{65}$.

Deuxièmement, l'allocation de garde d'enfant à domicile (AGED) concerne la garde d'un enfant de moins de 6 ans par une personne au domicile de la famille. Son montant varie en fonction de l'âge de l'enfant et des ressources. Les deux parents doivent travailler, le montant de l'allocation est directement versé à l'URSSAF. Elle couvre 50 à $75 \%$ des cotisations sociales. (CAF 2001).

\section{I.D.2.4.2 Les modes d'accueil}

En France, depuis le début des années 1970 et plus encore depuis les années 1975, on constate l'émergence d'une politique de l'enfant «lente élaboration d'une politique sociale en faveur de la petite enfance.» (Fenet, Leprince, Périer 2001 : 6). Cette nouvelle orientation politique trouve son origine dans l'activité professionnelle des femmes qui s'est généralisé au cours des années 1970, mais plus encore, dans le maintien de l'activité professionnelle pour ces femmes au moment des maternités. Par ailleurs, la diminution de la mortalité infantile à partir des années 1970, qui n'est plus que de $14 \%$ en 1973, alors qu'elle était de 67\%o en 1945, réorientent les objectifs politiques sanitaires et de conservation de l'enfant vers une politique centrée sur sa socialisation précoce et son éducation. La politique de l'enfant, trouve d'abord son origine dans les politiques de socialisation et de valorisation de la petite enfance. Depuis peu, depuis la fin des années 1990, on voit également apparaître les prémices d'une politique parentale «d'accompagnement de la fonction parentale» dont les orientations ont été

\footnotetext{
${ }^{65}$ Par exemple, pour un enfant de moins de trois ans, dans un ménage où les revenus sont inférieurs 12708 Euros, l'allocation se monte à 200 Euros (respectivement 100 Euros lorsque l'enfant est âgé de 3 à 6 ans) (CAF 2001).
} 
réaffirmées, au cours des conférences de la famille en juin 2000 et juin 2001 (Ministère de l'emploi et de solidarité, 2001). Par ailleurs, depuis le début des années 1990, l'accompagnement de la fonction parentale est un nouvel objectif des politiques d'accueil des jeunes enfants, au même titre que le «développement des emplois de proximité», et de la «prévention précoce des exclusions» (Fenet, Leprince, Périer 2001 : 9).

Les besoins des familles pour la garde des enfants non scolarisés sont directement liés à l'activité professionnelle des femmes : or le taux d'activité féminin a constamment augmenté ; la fléxibilisation récente des temps de travail complexifie les situations; le développement du temps partiel, l'individualisation des temps de travail et la précarisation des emplois, sont des évolutions qui touchent particulièrement les mères de jeunes enfants. $\mathrm{Au}$ cours des dernières années, on observe une individualisation de la demande et une diversification de l'offre (Silvéra 1998). «Par ailleurs, l'aménagement et la réduction du temps de travail ainsi que l'autorisation du travail de nuit des femmes devraient conduire à la poursuite voire à l'intensification de ces évolutions» (Fenet, Leprince, Périer 2001 : 14). «Les structures d'accueil sont appelées à jouer un rôle nouveau de soutien aux parents. C'est au quotidien que la crèche peut aider les jeunes parents qui ont moins que leur aînés une expérience auprès des tout-petits. L'accueil de l'enfant, ponctuel ou en urgence, peut aussi, dans des situations matérielles ou familiales problématiques, atténuer la charge pesant sur la famille et aider l'enfant à vivre cette période difficile pour le parent. La participation de la vie de la structure permet aux parents d'en rencontrer d'autres, de confronter, de valoriser leurs compétences et de dédramatiser les situations.» (Fenet, Leprince, Périer 2001 : 33).

L'évolution de l'accueil des jeunes enfants est étroitement liée aux connaissances psycho-sociologiques et au statut du jeune enfant dans la société. «L'importance croissante conférée à l'enfant et l'attention grandissante portée aux relations enfants-parents sont des facteurs à prendre en compte» (Fenet, Leprince, Périer 2001 : 15). En effet, les lieux d'accueil et de garde se définissent aussi comme des espaces d'accompagnement de la fonction parentale. «Le besoin accru des parents d'être accompagnés dans leurs relations avec leur enfant, d'être rassurés, d'échanger sur les difficultés qu'ils peuvent rencontrer en tant que parents. C'est une nouvelle qui s'exprime, d'une façon ou d'une autre, à l'écart des modes d'accueil des jeunes enfants. Ce sont tout particulièrement les crèches qui ont à y répondre, dans la mesure où se sont majoritairement les familles avec un premier enfant, qui les fréquente (58\% des usagers des crèches).» (Fenet, Leprince, Périer 2001 : 15).

Les préoccupations natalistes et le souci de conservation des enfants ont justifié très tôt l'intervention de l'Etat français au sein de la sphère privée. La baisse de la mortalité infantile, l'augmentation de la participation professionnelle des femmes et le maintien de leur activité lors des maternités, ont changé quelque peu l'orientation de l'intervention de l'Etat, qui dès le début des années 1970, porte un intérêt accru sur la socialisation et l'éducation du jeune enfant. Un décret de 1977 définit le statut d'assistante maternelle qui remplace celui des nourrices et affirme leur fonction éducative et d'accompagnement. À la fin des années 1970, on constate une forte pénurie de l'offre par rapport à la demande des familles, malgré l'effort de réglementation et de développement de l'infrastructure. Puis la diversification des réponses publiques au problème de la garde des enfants devient centrale dans les années 1980 : il s'agit d'un côté de développer l'accès aux structures accueil collectif et individuel pour les parents qui continuent à travailler et de l'autre côté de permettre aux familles d'assumer elle-même la garde de leur enfant par la création d'allocations spécifiques. Le souci est de mettre en adéquation, par une aide publique, la multiplication des réponses à la diversité des besoins de 
garde de jeunes enfants, sans pour autant privilégier un modèle de famille particulier : par exemple en dissociant l'accueil de l'enfant dans une structure collective et la situation professionnelle de ses parents (le chômage, temps partiel).

En France, l'infrastructure d'accueil des enfants de moins de trois ans a considérablement progressé, «on est passé de 36000 places en structures collectives en 1969 à 267000 places en 1999 avec une diversification croissante de l'offre de services» (Fenet, Leprince, Périer 2001). Les évolutions qui ont eu lieu dans les années 1990 sont marquées à la fois par la multiplication de l'offre, la multiplication des services, et un recours cumulé aux différents de mode de garde. Un décret du 1er août 2001 relatif aux établissements et services d'accueil des enfants de moins de 6 ans reconnaît officiellement les structures parentales et les accueils multiples (Fenet, Leprince, Périer 2001 : 10).

$43,5 \%$ des enfants de moins de trois ans sont gardés par un de leurs parents (cf. Tableau 15) et $11 \%$ sont scolarisés régulièrement (ce taux de scolarisation est plus élevé encore pour les enfants de deux à trois ans puisque plus d'un tiers de ces enfants sont inscrits à l'école maternelle). Plus de la moitié sont pris en charge par un mode de garde. Pour ces derniers, $19 \%$ sont accueillis par une assistante maternelle agréée, $16 \%$ sont en dehors du système d'accueil organisé, $9 \%$ sont accueillis en crèches et $1,5 \%$ sont gardés à leur domicile par une employée de maison. 
Tableau 15 Modes de garde des enfants de 0 à 3 ans en France

\begin{tabular}{|c|c|}
\hline \multicolumn{2}{|c|}{ OU SONT LES ENFANTS DE MOINS DE TROIS ANS? } \\
\hline $\begin{array}{l}43,5 \% \text { sont gardés par un de } \\
\text { leurs parents }\end{array}$ & $\begin{array}{l}24,8 \% \text { (sur l'ensemble des enfants de moins de trois } \\
\text { ans) sont gardés par un parent bénéficiant de l'APE }\end{array}$ \\
\hline $11 \%$ sont scolarisés ${ }^{66}$ & $\begin{array}{l}\text { un certain nombre d'entre eux sont gardés, en } \\
\text { complément de l'école, par une assistante maternelle, } \\
\text { une employée de maison (et font donc partis des } \\
\text { effectifs comptabilisés ci-après) }\end{array}$ \\
\hline \multicolumn{2}{|c|}{ Au moins $45,5 \%$ des enfants de moins de trois ans fréquentent un mode d'accueil : } \\
\hline $\begin{array}{l}19 \% \text { sont accueillis par } \\
\text { une assistante maternelle } \\
\text { agrée }\end{array}$ & $\begin{array}{l}\text { dont un certain nombre fréquente une école } \\
\text { maternelle }\end{array}$ \\
\hline $\begin{array}{l}16 \% \text { se situent hors du } \\
\text { système d'accueil } \\
\text { organisé. } \\
\text { Ce chiffre est difficile à } \\
\text { établir compte tenu du } \\
\text { cumul possible entre les } \\
\text { différents modes d'accueil. }\end{array}$ & $\begin{array}{l}\text { des estimations indiquent que : } \\
* \text { les trois-quarts seraient gardés par un membre de la } \\
\text { famille (grands-parents notamment) } \\
* \text { un quart par une autre personne (garde non } \\
\text { déclarée). } \\
\text { Cependant les déclarations de garde par un membre } \\
\text { de la famille sont surestiméees au regard de la ,garde } \\
\text { au noir“. }\end{array}$ \\
\hline $\begin{array}{l}9 \% \text { sont accueillis en } \\
\text { crèches }\end{array}$ & $\begin{array}{l}\text { dont } 66 \% \text { en crèches collectives, } 30 \% \text { en crèches } \\
\text { familiales et } 4 \% \text { en crèches parentales }\end{array}$ \\
\hline $\begin{array}{l}1,5 \% \text { sont gardés à leur } \\
\text { domicile par une employée } \\
\text { de maison }\end{array}$ & un certain nombre fréquente une école maternelle \\
\hline
\end{tabular}

Source : bilan mensuel de statistiques, janvier 2001 et statistique de la DRESS et de CNAF, juin 2001 (Fenet, Leprince, Périer 2001 : 21)

L'accueil des enfants de 0 à 6 ans se caractérise en France par la coexistence d'un accueil public ou associatif (les crèches collectives, les crèches familiales, les crèches parentales, les haltes garderies, les relais assistantes maternelles) et d'un accueil individuel. La garde est alors assurée par une assistante maternelle, par une employée de maison ou par un réseau informel, comme les parents eux-mêmes, les grands-parents, à la famille élargie ou le voisinage. Pratiquement l'ensemble des enfants âgés de 3 à 6 ans sont scolarisés. La moitié des enfants sont gardés à domicile par un des parents, un peu moins d'un quart sont gardés par le réseau informel de garde. $14 \%$ sont gardés dans un centre de loisirs sans hébergement, $11 \%$ à domicile par une assistante maternelle et $2 \%$ par une employée de maison (cf. Tableau $16)$.

\footnotetext{
${ }^{66}$ Plus du tiers des enfants entre 2 et 3 ans sont inscrit à l'école maternelle. En 1960, seuls $36 \%$ des enfants de trois ans étaient scolarisés.
} 
Tableau 16 Modes de garde des enfants de 3 à 6 ans en France

\section{OU SONT LES ENFANTS DE 3 A 6 ANS ?}

Quasiment tous les enfants de 3 à 6 ans fréquentent une école maternelle ou plus marginalement un jardin d'enfants : $88 \%$ d'entre eux sont à l'école publique. Un recours aux modes d'accueil est souvent nécessaire en complément de l'école :

\begin{tabular}{|ll|}
\hline $51 \%$ sont gardés à leur domicile par un de leurs parents (le plus souvent la mère) \\
\hline $22 \%$ sont gardés autrement & $\begin{array}{l}\text { une partie d'entre eux sont gardés par leurs } \\
\text { parents lorsqu'ils travaillaient à temps partiel (ou } \\
\text { s'ils peuvent aménager leur temps de travail). } \\
\text { Enfin, on ignore les modalités d'accueil de la } \\
\text { population restante. }\end{array}$ \\
\hline $14 \%$ fréquente un centre de loisirs sans hébergement \\
\hline $\begin{array}{l}11 \% \text { sont accueillis par une } \\
\text { assistante maternelle }\end{array}$ & leurs parents bénéficiant de l'AFEAMA \\
\hline $\begin{array}{l}2 \% \text { sont gardés à domicile } \\
\text { par une employée de maison }\end{array}$ & leurs parents bénéficiant de l'AGED \\
\hline
\end{tabular}

Source : bilan mensuel de statistiques, janvier 2001 et statistique de la DRESS et de CNAF, juin 2001 (Fenet, Leprince, Périer 2001 : 21)

«Le rythme de développement des crèches et haltes-garderies a été beaucoup plus long que celui de l'accueil individuel déclaré. L'évolution marquante de ces dernières années a été la substitution de l'accueil individuel déclaré à la garde gratuite ou „au noir“» (Fenet, Leprince, Périer 2001 : 23). Les grandes lignes d'évolution des modes de garde collectifs montrent une multiplication des structures. Cette évolution est relativement récente et montre un souci d'adéquation entre la souplesse de fonctionnement et les besoins individuels. Quant à évolution de l'accueil individuel, le nombre d'assistantes maternelles agréées a augmenté, en particulier depuis la création de l'AFEAMA en 1991: en moins de dix ans, le nombre de familles bénéficiaires s'est multiplié par 5. Quant aux familles qui bénéficient de l'AGED, leur nombre s'est multiplié par huit entre 1988 et 1999 . La progression a été très forte à partir de 1995 en raison de l'augmentation du montant de l'allocation, de son extension aux enfants de 3 à 6 ans, et d'une réduction supplémentaire de l'imposition. 


\section{SOUS-SECTION I.D.3 LA PARENTALITE «EXCLUSIVE» ET «CONCILIEE» EN FRANCE ET EN ALLEMAGNE}

Les politiques familiales contribuent activement à l'institutionnalisation de la parentalité, leurs interventions se situent à trois niveaux : premièrement elles sont fonction de la charge et de la présence de l'enfant dans les familles, deuxièmement du temps dégagé pour s'occuper de l'éducation de l'enfant par un parent et troisièmement de la conciliation des activités familiales et professionnelles.

Une classification de la France et de l'Allemagne suivant ces trois types de mesures montre que le premier niveau d'allocations et de prestations familiales directement liées à la charge de l'enfant, est relativement similaire en France et en Allemagne. En revanche, la France se distingue de Allemagne par l'introduction récente d'un congé de paternité.

Le système allemand d'allocations familiales construit plus activement la parentalité car l'allocation ne dépend pas du rang de naissance l'enfant, bien que son attribution soit unilatérale comme en France. De plus, elle tient compte de l'ensemble des relations de parentalité: l'ensemble des enfants sont pris en compte dans le calcul, indépendamment du lieu de résidence, ce qui n'est pas le cas en France. En revanche, en France, c'est le congé de paternité qui vient compléter la prise en compte de la parentalité.

Le système fiscal français inclut le principe du quotient familial et reconnaît une demipart par enfant, le système allemand retient celui du «quotient conjugal». Mais plusieurs études ont montré que les effets du quotient familial en France incitent les familles moyennes au mariage et s'apparentent alors aux effets du quotient conjugal en Allemagne.

Au second niveau d'intervention publique, nous avons vu que le congé parental existe en France et en Allemagne où le financement est plus généreux et où l'ouverture des droits se fait dès le premier enfant.

Enfin, la troisième catégorie de mesures qui concerne la conciliation du travail professionnel et du travail parental, montre que les modes de garde des enfants sont plus développés en France qu'en Allemagne, tant par l'importance que par la diversité de l'offre. L'ensemble des enfants âgés de 3 ans y est pris en charge et préscolarisé. En revanche, les besoins de prise en charge des plus petits sont encore importants. Dans l'ancienne Allemagne de l'Est, l'ancienne infrastructure permet d'accueillir un plus grand nombre d'enfant que dans l'ancienne Allemagne de l'Ouest où l'offre est très restreinte.

En résumé, derrière un dessin semblable des politiques familiales à l'égard de la parentalité, les plus grandes différences entre la France et l'Allemagne sont l'innovation récente du congé de paternité en France et le développement des différents structures d'accueil du jeune enfant. On favorise ainsi l'exercice du parental et du professionnel. La parentalité prend alors une forme «conciliée» pour les parents. Les politiques familiales en France reconnaissent la «parentalité conciliée» des mères, elle commence à envisager la «parentalité conciliée» des pères (cf. Figure 11).

En Allemagne c'est la généralisation du congé parental pour l'ensemble des enfants et de son financement plus généreux qui détermine une forme «exclusive» de parentalité, c'està-dire d'un retrait d'un parent du marché du travail pour se consacrer aux fonctions 
parentales. Alexandra Wagner (2000) énonce un ensemble de facteurs qui viennent construire et renforcer la norme du modèle «à un apporteur et demi» («Zuverdienst»). Le système social ainsi que le système d'imposition mais également l'infrastructure concernant l'accueil expliquent le choix de partage traditionnellement sexué entre l'homme et la femme. Ceci explique la répartition traditionnelle des rôles mais également la participation professionnelle des femmes à temps partiel: le système d'imposition («Ehegattensplitting») favorise l'inactivité de la mère voire son activité partielle, au même titre que la prise en charge dans le système de couverture du système maladie par droit dérivé de la femme mariée, du nombre insuffisant de structures d'accueil, des heures d'ouverture très contraignantes des structures collectives. Par conséquent, les couples à deux actifs équivalents, c'est-à-dire où l'homme et la femme effectuent tous les deux un temps complet, doivent compter avec un taux d'imposition beaucoup plus fort et avec une organisation beaucoup plus lourde pour la garde des enfants.

Ces facteurs montrent que la politique familiale en Allemagne encourage une «parentalité exclusive» pour les mères. Cependant, les nouvelles orientations politiques pour le développement des modes d'accueil des enfants vont dans le sens d'une prise en compte d'une «parentalité conciliée». Quant aux pères, leur «parentalité conciliée» est devenue récemment un objet de l'attention politique (cf. Figure 11).

Figure 11 Formes de parentalité déterminées par le rapport institutionnel entre la famille et le marché du travail

\begin{tabular}{|c|c|c|c|}
\hline & $\begin{array}{c}\text { Mesures relatives à } \\
\text { la présence et à la } \\
\text { charge de l'enfant }\end{array}$ & $\begin{array}{c}\text { Mesures dont l'objectif est } \\
\text { de dégager du temps pour } \\
\text { s'occuper de l'enfant }\end{array}$ & $\begin{array}{c}\text { Mesures de } \\
\text { conciliation }\end{array}$ \\
\hline France & $=^{+ \text {le congé de paternité }}$ & + & ++ \\
\hline Allemagne & + & ++ & parentalité conciliée \\
\hline
\end{tabular}




\section{CONCLUSION}

\section{LA PARENTALITE BILATERALE, UNILATERALE, PREMIERE ET COMPOSEE}

Dans la partie théorique de ce chapitre, nous avons souligné que les nouvelles mutations de la famille mettent à l'épreuve la force heuristique des notions sociologiques actuelles. Les changements du lien familial nécessitent un renversement de point de vue, depuis le conjugal, vers l'enfant, puis vers le parental. Et plus encore, nous avons montré l'importance d'étudier leurs relations respectives.

Les évolutions socio-démographiques de la famille (naissances hors mariage, reconnaissances paternelles, séparations et remises en couple) ont modifié la parentalité : la conjugalité se dissocie de la filiation et l'enfant n'appartient plus obligatoirement à une seule et unique famille lorsque la situation familiale devient complexe.

Aussi, une description du lien conjugal, en terme de légitimité, ne suffit plus pour rendre compte des relations au sein de la famille. Désormais, les séparations conjugales influencent les formes des rapports sociaux que l'enfant entretient avec ses parents : soit le couple parental existe après leur séparation (par un exercice conjoint de la parentalité par les deux parents), soit le contact avec le second parent disparaît et l'exercice de la parentalité devient unilatéral. De plus, la remise en couple, de l'un ou des deux parents, après la séparation conjugale, modifie également la forme des relations entre l'enfant et ses parents : dans le cas d'une recomposition familiale, la parentalité biologique est mêlée au social, elle s'ajoute aux liens qui existent entre l'enfant et son couple parental d'origine. Il devient très difficile de décrire la multiplicité des formes de famille depuis le point de vue de l'enfant ou du point de vue du couple conjugal.

Pour ses raisons, nous avons choisi la «parentalité» comme nouvelle unité de référence pour d'étudier les changements au sein de la famille. La parentalité traite des rapports sociaux entre l'enfant et ses parents. Ce concept rend compte des évolutions dynamiques de la structure familiale, à partir de la relation dialectique entre l'enfant et le parental. Elle se décline selon quatre formes : une "parentalité bilatérale» lorsque les rapports parentaux existent avec les deux parents, une «parentalité unilatérale» lorsque l'enfant perd le contact avec un parent, une «parentalité première» lorsque l'enfant vit avec le couple parental d'origine et une «parentalité composée» lorsque le biologique et le social sont mêlés.

Ces catégories, fondées sur le lien de l'enfant avec ses parents, permettent de repenser les catégories familiales courantes. Or, ces dernières sont confrontées actuellement à deux difficultés. Premièrement, elles se basent sur le statut juridique des individus et plus particulièrement sur la relation conjugale et sa légitimité, elles sont alors discutées suivant la distinction «marié»/《non-marié», et non pas en suivant l'existence réelle d'un couple. Deuxièmement, elles tentent d'intégrer les nouvelles situations de fait comme les séparations des parents. On parle alors de «familles monoparentales» et de «coparentalité» : par-là, et de façon contradictoire, on tente de rendre compte de l'existence du second parent tout en le 
niant dans le terme de monoparentalité. De plus, ces catégories fondées sur le conjugal, ne saisissent pas les phénomènes d'homoparentalité qui remettent en cause le fondement même de la conjugalité légitime basée sur les différences de sexe («seul espace légitime pour la reproduction»).

Pour notre part, nous proposons les termes de «familles unilatérales», de «familles bilatérales», de «familles premières» et de «familles composées» (cf. Figure 7 et 8 ) de la section I). Chaque forme de famille est fondée sur une parentalité spécifique. Ces catégories conçoivent une appartenance simultanée à différentes unités familiales. Les relations de parentalité se construisent alors en réseau. Ainsi, nous avons adapté les catégorie pour saisir, dans les faits, la norme actuelle de parentalité qui se définit à partir du lien indissociable entre l'enfant et ses deux parents.

Dans la section démographique de ce chapitre, nous avons proposé de recomposer les catégories statistiques afin de mesurer les phénomènes de parentalité. La difficulté a été alors d'adapter les catégories statistiques fondées sur les distinctions juridiques (marié, célibataire, veuf, divorcé) pour saisir l'exercice de la parentalité dans les faits. Il s'avère que la «parentalité première» reste le modèle dominant de parentalité, la très grande majorité des enfants vivant avec leurs deux parents.

Les pratiques réelles de parentalité ne s'expliquent plus par la forme du lien conjugal, en terme de légitimité et d'illégitimité. En effet, la «parentalité première» est aujourd'hui majoritairement un phénomène hors mariage : elle se construit d'abord dans les familles naturelles, avant d'être légitimée par la suite, mais moins fréquemment et plus tardivement. Ce phénomène est visible en France et dans l'ancienne Allemagne de l'Est. Pour l'ancienne Allemagne de l'Ouest, la «parentalité première» coïncide encore avec une union conjugale légitime. Les couples développent alors des nouvelles stratégies d'institutionnalisation de la «parentalité bilatérale»: les reconnaissances paternelles et les reconnaissances conjointes prénatales viennent s'ajouter aux comportements de légitimation de la filiation par le mariage.

Par ailleurs, une «parentalité composée» succède le plus souvent à une «parentalité première» lorsque les parents se séparent. La période de transition peut être plus ou moins longue, à l'issue de laquelle une «parentalité composée»s'ajoutera éventuellement à une «parentalité bilatérale», si le lien avec le deuxième parent se maintient.

Enfin, la «parentalité bilatérale» domine dans l'ensemble des familles car les enfants vivant le plus souvent avec leurs deux parents. Cependant, lorsque les parents se séparent, son exercice au quotidien prend le plus souvent la forme d'une «parentalité unilatérale» (le contact personnel à l'enfant étant fréquemment rompu pour le père). En revanche, le maintien de l'obligation alimentaire des pères, en cas de séparation, s'interprète comme une marque institutionnelle qui fait de la «parentalité bilatérale» la norme d'exercice reconnue (cf. Chapitre II).

\section{LA PARENTALITE CONCILIEE ET LA PARENTALITE EXCLUSIVE}

La section III traite de la manière dont la participation des parents au marché du travail construit la parentalité. Nous avons fait émerger deux modes d'exercice de la parentalité : la «parentalité conciliée» et la "parentalité exclusive». Dans le premier cas, un rôle parental vient s'ajouter à un rôle professionnel, dans le deuxième cas le parent n'exerce pas d'activité 
professionnelle. Ces deux modèles sont répartis de façon différente en France et en Allemagne

Le modèle de la «parentalité exclusive» est plus pratiqué en Allemagne de l'Ouest. Il s'exerce comme un mode stable d'organisation des rapports familiaux, ou comme un mode temporaire lorsque le parent interrompt son activité professionnelle pour une durée plus ou moins longue. La «parentalité exclusive» est essentiellement exercée par les mères : elle existe comme mode stable surtout pour les femmes mariées, et elle est très répandue comme mode d'exercice temporaire. Dans l'ancienne Allemagne de l'Est, l'exercice de la «parentalité exclusive» est limité aux mères de jeunes enfants de moins de trois ans.

Dans l'Allemagne de l'Ouest, les pères exercent tous une «parentalité conciliée», les mères la pratique en référence à un travail professionnel à temps partiel (de même pour les mères d'enfants en bas-âge (Ostner 2001)). Dans l'ancienne Allemagne de l'Est, la «parentalité conciliée» est comprise en référence au travail professionnel à temps complet, pour les pères, comme pour les mères. En France, la situation est similaire à celle de l'ancienne Allemagne de l'Est, la «parentalité conciliée» est exercée par les deux parents.

Les différences sociétales s'expriment dans la référence au double temps complet des parents français, et au temps complet des pères et au temps partiel des mères dans l'Allemagne réunifiée.

\section{LA PARENTALITE CONCILIEE DES PERES ET DES MERES}

La section IV du chapitre montre le rôle de l'Etat-providence dans la construction de la parentalité. La place de la parentalité dans les différentes mesures politique reflète les orientations normatives de l'action publique envers la famille. Inversement, les politiques familiales régulent les rapports entre l'enfant et ses parents, et donc, la forme du lien familial.

En ce sens, les politiques familiales construisent une forme spécifique d'exercice de parentalité dans la manière dont elles tiennent compte du statut de parent au sein de la société. Elles régulent, par les différentes mesures ou l'absence de mesures, les relations sociales suivant le principe social en vigueur. Les catégories «de parentalité conciliée» et de «parentalité exclusive» ont permis de montrer qu'il y avait, au sein de la société française, une prise en compte de la «parentalité conciliée» pour les hommes. En Allemagne, à l'heure actuelle, on commence à prendre en compte la «parentalité conciliée» des mères (par le biais de leur activité professionnelle) et des pères (par le biais de leur rôle parental). Le modèle d'une famille à deux «parentalités conciliées» s'affirme en France, il émerge en Allemagne. Ce dernier est concomitant avec la disparition progressive de la «parentalité exclusive» comme un mode stable d'exercice de la parentalité dans les familles.

En Allemagne, la «parentalité exclusive» devient de plus en plus une forme temporaire de parentalité. De plus, la reconnaissance concomitante de la «parentalité conciliée» pour les pères et les mères participe à diminuer, normativement, le caractère sexué du partage des tâches. Ceci explique que le «politique» fait de la «parentalité exclusive» un objet d'action, qu'il conçoit comme un instrument de «conciliation»entre la «famille» et le «marché du travail», dont il cherche à modifier le caractère sexué. Il tente, actuellement, à inciter les pères à recourir à cette mesure.

Chaque régime socio-politique se réfère à une compréhension particulière de la famille. Dans le cas de la France, la forme était centrale : les interprétations familialistes et 
natalistes sont l'émanation d'une référence tantôt à la famille légitime, tantôt à la famille nombreuse. Dans le cas de l'Allemagne, son rapport au système social est discuté en terme de conjugalité : le couple, dans sa forme légitime est au centre de la définition de la famille. Aujourd'hui, c'est la prise en compte du statut de parent, pour les pères et les mères, qui est au centre des dernières mesures en politiques familiales : le congé de paternité en France, le développement des centres d'accueil et les campagnes d'opinion récentes pour la reconnaissance de la «parentalité conciliée» des pères en Allemagne. 


\section{CHAPITRE II LES NORMES DE PARENTALITE : LA COPARENTALITE FRANÇAISE ET LA BIPARENTALITE ALLEMANDE}

Le chapitre précédent a traité du cadre théorique et des formes de parentalité qui sont déterminées par les rapports familiaux, par les relations entre la famille et le marché du travail, et par les relations entre la famille et l'Etat-providence. L'objet de ce chapitre II est d'étudier les normes de parentalité et leur construction sociale en France et en Allemagne.

Introduction : Comment la parentalité metelle en lumière les changements du lien familial ?

Chapitre I : Le cadre théorique et les formes de parentalité : la parentalité exclusive et la parentalité conciliée

Chapitre II : Les normes de parentalité : la coparentalité française et la biparentalité allemande

Chapitre III : Les pratiques de parentalité dans les familles et le partage du travail familial

Conclusion : Le parentalisme comme mode de régulation?
Comment se définit le mode d'exercice privilégié de la parentalité en France et en Allemagne pour les familles unies et désunies : quel est le mode de régulation du risque familial ? 


\section{INTRODUCTION}

Face aux dernières évolutions de la famille dans nos sociétés postindustrielles, un discours strictement moraliste a été abandonné au profit d'une destigmatisation des unions illégitimes. Mais le «discours de crise» n'a pas pourtant disparu, l'institution du mariage serait, plus que jamais, en danger. De la même manière, les phénomènes récents qui touchent la parentalité peuvent s'interpréter comme une nouvelle crise de la famille : la parentalité hors mariage est devenue courante, la généralisation des divorces et des ruptures en multiplie les formes.

Or, pour notre part, nous pensons que ces changements ne sont pas le résultat d'une perte des repères normatifs et d'une fragilité du lien familial. En effet, une étude sociétale et historique, depuis le droit canon en France et le droit coutumier en Allemagne jusqu'à nos jours, montre la variabilité de la norme de parentalité. Depuis les années 1990, et plus encore aujourd'hui, la norme de parentalité a pris un nouveau sens et une forme bien particulière. Nous avons mis en évidence que la relation entre l'enfant et ses parents suit des motifs bien identifiés. L'objet de ce chapitre II est de mettre en évidence les normes actuelles de la parentalité.

Dans un premier temps, nous nous intéressons aux règles qui modèlent la relation entre l'enfant et ses parents dans le cas des familles «unies» (légitimes et naturelles). Le couple conjugal et le couple parental forment alors le plus souvent une seule et unique entité : la «parentalité première».

Dans un second temps, notre but est d'identifier la norme qui détermine les comportements parentaux après une séparation conjugale. Quelle forme d'exercice des rapports entre l'enfant et ses parents est alors socialement privilégiée ? A l'image d'I.Théry qui, pour mettre en place son concept de démariage, s'appuie sur le mariage : «Aujourd'hui encore et plus que jamais, les débats sur le divorce sont fondamentalement des débats sur le mariage» (1993 : 12) ; à la manière de P. Bourdieu pour qui «il faut traiter les nouvelles formes sociales comme des analyseurs de la famille dite normale» (1996:3); à l'exemple de J. Lewis pour qui la position des familles monoparentales est particulièrement pertinente pour l'analyse car elles sont révélatrices par leur nature de «bordeline case» (1993:10); on étudie alors la construction sociale de la norme de parentalité à partir de la régulation de la séparation parentale.

En effet, dans ce cas d'une séparation parentale (divorce ou séparation), le couple parental se dissocie du couple conjugal, mettant à vif les modes d'exercice de la parentalité. Cette situation oblige alors à redéfinir les rôles parentaux des deux parents. Quatre grands facteurs influencent la définition du parental : les parents eux-mêmes, leur arrangement familial antérieur, leur relation au marché du travail, mais aussi la relation entre la famille et l'Etat-providence. Dans ce cas précis, il s'agit de l'action publique face aux conséquences de la séparation, pour l'enfant. Devant statuer sur les contentieux familiaux, le législateur gère «l'après-divorce» et il se réfère alors explicitement à un ensemble de principes qui inscrivent les rôles, les droits et les devoirs de chaque parent face à l'enfant.

La divortialité n'est plus le lot d'une minorité de familles, leur nombre est en constante augmentation depuis ces trente dernières années. Le risque de séparation du couple parental se généralise sur l'ensemble des familles et devient un nouveau risque social : le «risque familial». Ce terme exprime le risque d'éclatement de l'unité parentale et les 
conséquences de la séparation conjugale pour la parentalité, en d'autres termes pour les rapports entre l'enfant et ses parents. Il côtoie aujourd'hui le risque de pauvreté dû à la présence des enfants, et le risque relationnel, consécutif à l'isolement après la séparation. On parlera de «risque» au sens où ce phénomène mobilise l'intervention de nombreuses institutions et d'un ensemble d'acteurs (Martin 1997b). Leurs interventions forment un mode spécifique de régulation du risque. Inversement, cette régulation détermine, en retour, l'action sociale au sein du familial, de la relation avec le marché du travail et de l'orientation de l'intervention publique.

Notre objectif est de mettre en évidence le mode de régulation du «risque familial» et de ses évolutions. L'analyse se fonde sur trois éléments : premièrement, les composantes individuelles du risque pour les pères et les mères; deuxièmement, la relation des parents au marché du travail ; et troisièmement, l'influence du système de protection sociale. En effet, il existe tout un arsenal de mesures politiques et sociales qui déterminent les nouveaux arrangements parentaux à respecter après la séparation. Ils institutionnalisent les principes constitutifs de la parentalité et l'ensemble des composantes dessine ainsi une logique particulière de la régulation du «risque familial».

Le premier axe de l'analyse traite de l'émergence de l'autorité parentale conjointe, à travers les réformes du droit civil, les pratiques des magistrats relatives à l'attribution de la garde des enfants, mais également aux principes de régulation des conflits parentaux (le nonconflict, l'autorité parentale conjointe et l'intérêt de l'enfant).

Le second axe identifie les mécanismes d'attribution des responsabilités parentales des pères et des mères autour de l'obligation alimentaire et de l'obligation de soutien de l'enfant. Comment s'articulent ces différentes composantes dans la couverture sociale du risque ? (cf. Figure 12). Quelle place ont alors le père, la mère, leur participation au marché du travail, la protection publique des familles monoparentales (les allocations catégorielles et le recouvrement des pensions alimentaires) et les réformes du législateur concernant la famille et l'enfant? (Martin 1997a ; Choquet, Sayn 2000).

\section{Figure 12 Les déterminants de la norme de parentalité}

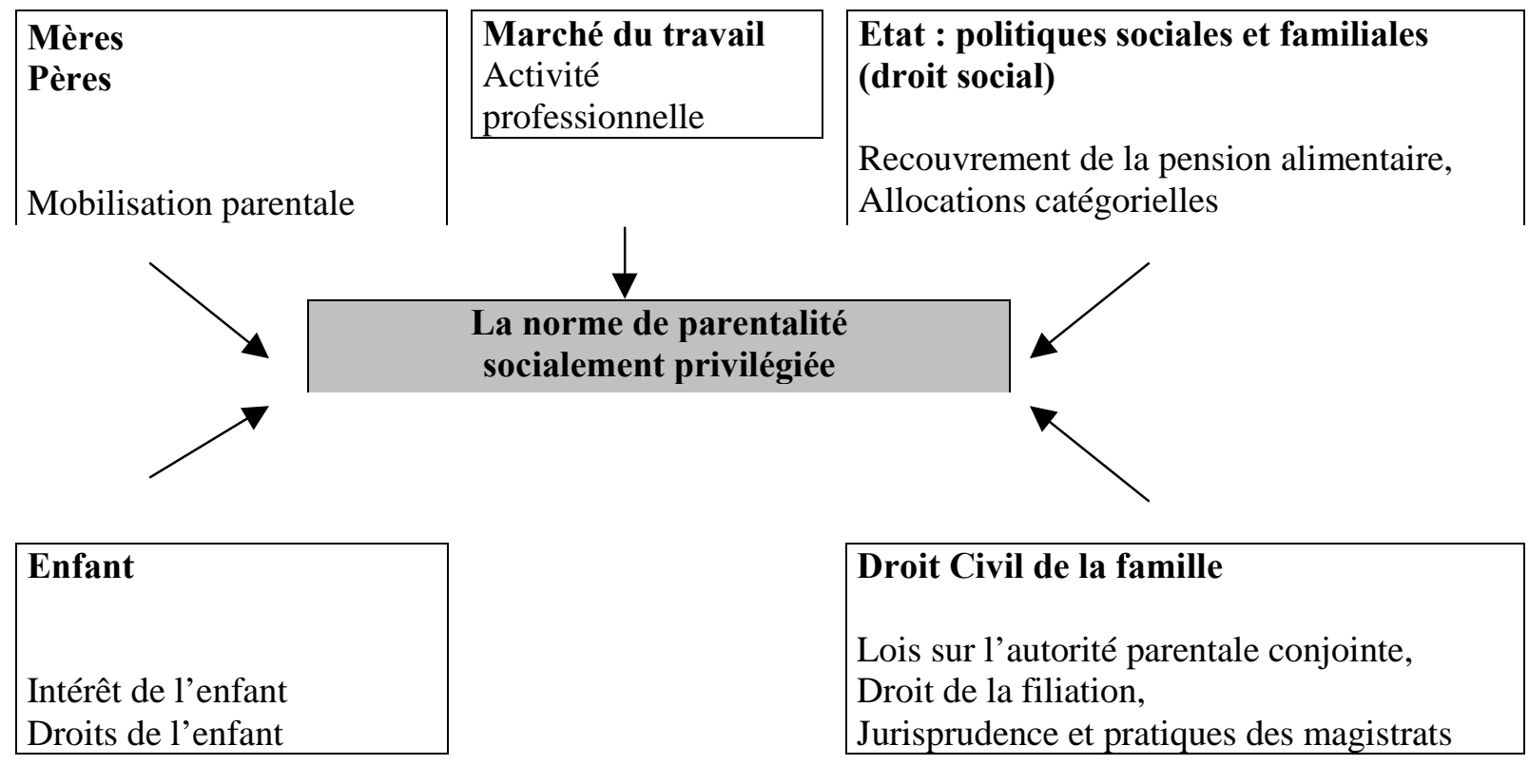


Nous observons un changement dans la nature du risque familial et dans ses modes de régulation (cf. sections suivantes) : on passe ainsi d'un «modèle monoparental» de régulation du «risque familial», vers une «norme d'exercice bilatéral de la parentalité». Cette dernière régule aujourd'hui les relations familiales, dans le sens d'un maintient de la relation de l'enfant avec ses deux parents, après la séparation conjugale. Plus généralement, la régulation de l'exercice de la parentalité montre la volonté normative de maintenir ce double lien quelle que soit la forme de famille. Aussi, la nouvelle norme de parentalité définit la forme privilégiée du maintien d'une relation bilatérale de l'enfant avec ses deux parents, quelle que soit la forme de familles. Elle fonde un nouveau mode de régulation du «risque familial» : le «parentalisme».

Quatre dimensions construisent le mode de gestion de la séparation parentale: premièrement, le «couple parental» formé par les deux parents dans les familles unies, désunies, légitimes et naturelles ; deuxièmement, la place centrale de l'enfant dans la famille et la référence à «l'intérêt de l'enfant» ; troisièmement, la «norme de non-conflictualité», voire de médiation et de coopération; et enfin, quatrièmement, l'autonomisation des logiques, conjugale, parentale, de genre et générationnelle les unes par rapport aux autres.

Ces quatre éléments se composent différemment sous l'influence de facteurs sociétaux spécifiques en France en Allemagne. L'action publique, en France, intervient sur le maintient du lien bilatéral entre l'enfant et ses deux parents mais également dans le couple parental. Par ailleurs, le législateur dissocie le conjugal du parental en distinguant explicitement les termes du contentieux. De fait, il privilégie les principes normatifs d'une coopération parentale et la justifie par rapport au principe de l'intérêt de l'enfant. Ces éléments constituent la norme de «coparentalité».

En Allemagne, la logique du parental est étroitement liée à celle du conjugal, qui domine les termes de la séparation parentale. Par ailleurs, le principe de non-ingérence dans la sphère familiale et la spécification des sexes et des rôles, maintiennent une vision privée de la séparation parentale. Cependant, le parental est traité en référence à la norme de nonconflictualité. Une situation paradoxale apparaît alors entre la règle de non-ingérence dans le conjugal et celle de non-conflictualité du parental. Les fortes résistances face à une éventuelle institutionnalisation de la coopération parentale, par le biais d'une médiation, illustre cette contradiction. De fait, la régulation contemporaine du risque familial s'effectue en fonction d'une double responsabilité parentale des pères et des mères, d'une juxtaposition et une complémentarité des rôles (non dans une équivalence et une coopération comme dans le cas français). La norme parentalité prend alors la forme de la «biparentalité».

La première section de ce chapitre traite de l'apparition de la norme contemporaine de parentalité : quand la parentalité est-elle apparue sous la forme qu'on lui connaît aujourd'hui ; en quoi ce phénomène est-il nouveau; et comment les conditions sont apparues, depuis la norme familiale du «pater familias», pour faire émerger un nouveau mode d'exercice de la parentalité et de gestion du risque familial?

La seconde et troisième section présente la construction de la norme de parentalité et le mode de régulation du risque familial dans le cas français et allemand. Dans chacune de ces deux sections, l'analyse est construite autour de trois axes : premièrement, une présentation des évolutions du cadre social et juridique dans le cas des familles «unies» légitimes et naturelles ; deuxièmement, l'étude des familles désunies, et troisièmement, les politiques familiales et le droit social. 


\section{SECTION II.A L'HISTORIQUE DU RISQUE FAMILIAL ET DE SON TRAITEMENT SOCIAL}

Cette section II.A montre de quelle manière le risque familial a changé de forme au cours de l'histoire et comment ses modes de régulation ont évolué.

Dans les années 1970, le risque familial a changé radicalement de nature sous l'impact des bouleversements démographiques et sociologiques de la famille, de la redéfinition du droit social et des modifications du droit civil. L'ancien modèle dominant a alors définitivement cessé d'être lié à la norme du Pater Familias et bien que certaines caractéristiques marquent encore le modèle contemporain, l'émergence d'un nouveau contexte social détermine une nouvelle logique de gestion du risque familial. L'ensemble de ces nouvelles données conditionne de nouveaux droits et devoirs des parents.

Historiquement, le veuvage est la forme première du risque familial. Ce risque s'inscrit en référence au mariage et de la famille légitime. Les débats autour des naissances «illégitimes» rendent visible le mode de régulation du risque familial car ils discutent ouvertement des rôles parentaux, des institutions et de la place du père. Les naissances «scandaleuses» sont traitées en marge de la norme dominante : «(...) les uns comme les autres [historiens, administrateurs, réformateurs] se sont peu intéressés aux orphelins ou aux enfants des familles légitimes «désertées» par le mari/père, pourtant beaucoup plus nombreux que les bâtards ou les enfants abandonnés. Nous disposons de peu de sources pour repérer les réponses apportées avant l'émergence des Etats sociaux à la question «qui doit nourrir les enfants que le mari de leur mère ne peut pas ou ne veut pas entretenir ? ». C'est sans doute parce que l'existence de ces enfants ne posait que des problèmes d'ajustement par rapport aux normes (...) Alors que l'existence d'enfant dont la mère n'était pas mariée, défiant ouvertement ces normes, était identifiée et représentée comme hautement problématique, obligeant les contemporains à répondre pratiquement et/ou théoriquement à la question de l'attribution de la responsabilité de leur entretien.» (Lefaucheur 1997 : 7). 


\section{SOUS-SECTION II.A.1 L'HISTORIQUE DU RISQUE FAMILIAL EN FRANCE}

\section{II.A.1.1 La parentalité dans les familles légitimes et naturelles}

A la fin de l'Ancien régime, le droit canon légifère le mariage et la filiation : l'union est de nature sacramentelle et les naissances ne sont légitimées que dans le cadre du mariage. Juridiquement, seul l'époux devient père «Pater is est quem nuptiae demonstrant» ${ }^{67}$.

Lorsque la naissance est légitime, le père exerce une paternité biologique en sa qualité de géniteur, et une paternité sociale parce qu'elle s'exprime dans le cadre du mariage et qu'elle est reconnue et légitimée par la société. Les devoirs qui lui incombent en tant que père, sont des devoirs d'éducateur et de nourricier. Lorsque la naissance est illégitime, l'enfant a un géniteur mais pas de père au sens social du terme. Juridiquement, l'enfant n'est pas soumis à la puissance paternelle puisque, légalement, il n'y a pas de lien de famille entre lui et son géniteur. L'enfant n'a alors aucune prétention juridique à la succession de son père biologique.

Néanmoins, si le droit monarchique admet «la recherche en paternité», elle se limite à la recherche du géniteur. Il «n'a, à l'égard de l'enfant, qu'un devoir moral qu'il remplit, éventuellement, en lui fournissant les aliments nécessaires» (Mulliez 1990 : 281). Si le père refuse de donner une aide alimentaire, alors la jurisprudence admet deux actions en recours pour la mère ${ }^{68}$ : la première basée sur la maxime «Creditur virgini parturienti jurantique se aliquo cognitam» est destinée à assurer les frais de gésine ; l'autre tend, en recherchant la matérialité du lien, à pourvoir définitivement à l'entretien matériel de l'enfant (Mulliez 1990 : 281). Devant la difficulté des preuves à fournir et les conditions à remplir pour que la demande soit recevable, peu de cas ont pu aboutir en gain de cause pour la mère et l'enfant ${ }^{69}$.

A la fin du XVIIIème siècle, la mortalité infantile est considérée aussi gravement que l'abandon et l'infanticide. On se mobilise pour garantir la conservation des enfants dans le but d'assurer la puissance militaire, coloniale et économique de la France. «Or cet objectif paraît compromis par les pratiques de transfert du soin de l'enfant vers la collectivité ou vers d'autres familles : l'allaitement mercenaire ou artificiel, consécutif à l'abandon ou au placement nourricier fréquent des enfants illégitimes, décime leurs rangs. Apparaît donc comme le moindre mal, tout ce qui peut favoriser l'allaitement de ces enfants par leur mère, y compris la destigmatisation de l'illégitimité (...). La prévention de l'abandon par l'assistance

\footnotetext{
${ }^{67}$ La mère quant à elle, devient automatiquement mère par l'acte naturel de l'accouchement et le cordon ombilical: "Mater certa est" (Sullerot 1992 : 71). Cet état de fait justifie, au-delà des époques, le lien dit «naturel» entre la mère et l'enfant.

${ }^{68}$ Seules 3\% des filles-mères épousent l'homme qu'elles ont désigné comme étant le père, lors de la déclaration (Chiffre pour la ville de Nantes au XVIIIème siècle, Cabantous (1990 : 341)).

${ }^{69}$ Les solutions de recours pour une participation financière du père sont limitées. Seulement, même si la recherche en paternité a un faible impact coercitif, elle permet un recours en justice. Or ceci est bien particulier à la période de l'Ancien régime car, à la Révolution française, la recherche en paternité sera interdite pour ne réapparaître qu'en 1912.
} 
aux filles-mères et non plus autour du recueil des enfants trouvés. Sa première inscription dans la législation fut l'œuvre de la Convention nationale, qui, en 1793, décida la création d'asiles de maternité et l'octroi de secours aux filles-mères» (Lefaucheur 1991 : 119). La prise en charge des enfants n'est plus collective et l'intérêt national justifie une ingérence dans les familles à risques. Les mères célibataires deviennent un moindre mal devant les préoccupations liées à la survie de l'enfant. Le paradigme sociopolitique devient alors une «variante démographique de l'angélisme chrétien de l'Ancien régime» (Lefaucheur 1991: 119).

Ce modèle a progressivement évolué vers une variante socio-patriotique ou «patriotique-familialiste» (Lefaucheur 1997 : 9) : le mal suprême est alors la socialisation défectueuse des enfants. Le rôle de l'Etat était tel que «le principe selon lequel la charge de l'élevage des enfants incombe à leurs auteurs peut être remis en cause si l'éducation des enfants ne semble pas correctement ou suffisamment assurée par la famille» (Lefaucheur 1991 : 120). Cette ingérence dans les prérogatives éducatives de la famille se justifie au nom d'une bonne socialisation de l'enfant. L'Etat collectivise une partie de l'entretien des enfants et sollicite des familles d'accueil.

Une nouvelle conception du mariage émerge du droit révolutionnaire : le mariage peut être soit solennel, soit privé. Un contrat privé de mariage est une union dont «l'état de fait» suffit pour lui donner sa légitimité. Le droit intermédiaire reconnaît une valeur juridique au contrat privé de mariage. Le concubinage est ainsi reconnu au même titre qu'un mariage solennel. L'illégitimité des enfants issus de «concubins»n'est plus pertinente car un couple non marié s'unit, de fait, par le projet de maternité. Et si le père ne reconnaît pas l'enfant par la suite, alors l'enfant reste légitime par sa mère. Il en résulte que tout enfant voulu est légitime (Mulliez 1990).

Le droit révolutionnaire a porté une attention particulière au bonheur et à la sécurité de l'enfant. Les rôles parentaux se définissent comme un ensemble de droits et de devoirs : «surveillance et protection, voilà le droit des parents ; nourrir, élever, établir leurs enfants, voilà leurs devoirs» (Fenet cité par Mulliez 1990).

Le mariage-contrat ne devait plus être subi mais librement contracté par les deux époux et son caractère contractuel devait permettre de le résilier plus facilement. Ainsi, dans l'idéalisme révolutionnaire, l'institutionnalisation du divorce par consentement mutuel devait permettre la disparition de l'adultère. Les mariages solennels, privés ou subséquents, devaient permettre de régulariser facilement la situation, de limiter les abandons d'enfants et de donner un père aux enfants. Ainsi, toute naissance serait l'expression de la volonté des parents. «Le mariage est une convention par laquelle l'homme et la femme s'engagent, sous l'autorité de la loi, à nourrir et élever les enfants qui peuvent naître de leur union» (article premier du premier projet de Code civil de la Convention) (Mulliez 1990). Les soins de l'enfant seraient assurés par l'amour maternel et paternel qui pourraient être pleinement vécus (Mulliez 1990). La définition du père qui était liée à celle de l'époux dans l'Ancien régime change de sens. De plus, le droit révolutionnaire rétablit l'adoption ${ }^{70}$. Elle devait pallier l'absence de lien familial

\footnotetext{
${ }^{70}$ En revanche, la recherche en paternité étant interdite dans le droit intermédiaire, la paternité est plus que jamais la conséquence de la volonté de l'homme. Le droit révolutionnaire, par l'article premier de la Déclaration des droits de l'Homme, abolit la différence entre les enfants légitimes et illégitimes. Dans cet esprit, la recherche en paternité aurait été un critère discriminant puisqu'il aurait révélé la nature du lien et donc aurait rétabli une hiérarchie entre les enfants légitimes, illégitimes, incestueux et adultérins.
} 
ou la quasi-absence de lien familial dans les cas des familles monoparentales. L'ensemble des enfants aurait été ainsi inscrit dans une filiation.

La base contractuelle du lien conjugal émancipe le lien parental des contraintes matrimoniales dans le but de favoriser un exercice volontaire de la parentalité.

Le Code civil rétablit la primauté de la légitimité au début du XIX siècle. Il marque un retour à la tradition en rétablissant le mariage solennel comme unique union légitime ${ }^{71}$. Les enfants illégitimes sont à nouveau discriminés par rapport aux enfants légitimes. On distingue à nouveau trois catégories : les enfants naturels, adultérins et incestueux.

Dans le Code Napoléon, les règles d'exercice de la paternité obéissent au principe suivant : le mari est le père de tous les enfants nés de sa femme, il n'est jamais le père de ses enfants adultérins ; non marié, il n'est le père que des enfants qu'il reconnaît ${ }^{72}$ (Sullerot 1992 : 140).

La prohibition de la recherche en paternité est maintenue alors que le mariage reprend sa qualité de contrat indissoluble ${ }^{73}$ : les naissances naturelles perdent alors leur caractère légitime qu'elles avaient sous le droit intermédiaire et les enfants sans père se multiplient (Mulliez 1990). Les naissances adultérines ne peuvent plus être suivies d'un «divorceremariage» du père avec la mère naturelle car l'adultère ne peut plus être «régularisé». «Dans le cas de divorce admis en justice pour cause d'adultère, l'époux coupable ne pourra jamais se marier avec son complice» (loi du 30 Ventôse, an XII, «loi sur le divorce», chapitre IV, Article CCXCII). Le divorce redevient exceptionnel et grave de conséquence (Roussin 1992 : 15 ) et l'idée du divorce-sanction (surtout pour la femme) redevient actuel.

La puissance paternelle est établie le 24 mars 1803, par l'article 373 du Code civil, titre IX du projet du Code civil intitulé «De la puissance paternelle» : «Les articles du présent titre sont communs aux pères et mères des enfants naturels reconnus». Si l'enfant est reconnu par la mère seule, il n'est pas soumis à l'autorité paternelle et le père géniteur n'a pas d'obligation alimentaire. Si l'enfant est reconnu par son géniteur, (ou par ses deux parents naturels), alors il relève de la même réglementation que celle des enfants légitimes.

Mais la parentalité illégitime n'est pas pour autant assimilée à la parentalité légitime : l'enfant né hors mariage n'a pas de droit de succession.

Premièrement, il s'agit de réaffirmer l'obligation coutumière des parents naturels d'élever leurs enfants, de les nourrir et de les établir. Tronchet ${ }^{74}$ demande, lors de la discussion sur la reconnaissance de l'enfant naturel, le 26 brumaire an $\mathrm{X}$, «l'enfant reconnu n'aura droit qu'à une créance sur les biens de celui qui l'a reconnu». Cette proposition aura été retenue par le Conseil ; Cambacérès ${ }^{75}$ précisa alors que cette part, appelée créance, devrait

\footnotetext{
${ }^{71}$ Mais la laïcisation du mariage demeure, en héritage de la Révolution.

${ }^{72}$ Dès que l'homme se déclare père, il se voit dans l'obligation de remplir ses devoirs de soutien alimentaire.

${ }^{73}$ La loi du 8 mai 1816 rend le mariage indissoluble. Il faudra attendre 1884 pour que le divorce soit de nouveau possible. Entre temps, la France aura connu plusieurs changements de pouvoir, et à chaque époque la question du divorce sera systématiquement rediscutée (Roussin 1992 : 16).

${ }^{74}$ Tronchet était avec Portalis, Bigot de Préameneu et Maleville, l'un des quatre auteurs de l'Avant-projet du Code civil présenté au conseil d'Etat présidé par Bonaparte, et voté dans sa forme définitive le 21 mars 1804.

${ }^{75} \mathrm{Il}$ aura été un des pères du Code civil. Son premier projet de Code, lu le 9 août 1793, se prolonge par un second, le 9 septembre 1794, qui préserve les avancées égalitaires. A la mort de Robespierre, la nouvelle Constitution nomme de nouveau Cambacérès pour un troisième projet (qui sera plus retenu quant aux objectifs égalitaires). Puis un quatrième projet, commandé à Jacqueminot, fondera la trame sur laquelle les quatre auteurs du Code Napoléon vont se baser (Sullerot 1992 : 65).
} 
plutôt être qualifiée «d'aliments» (Mulliez 1990 304). On retrouve là, la solution du droit coutumier, pour laquelle «qui fait l'enfant doit le nourrir».

En contrepartie, les parents naturels se voient attribuer des droits supplémentaires qui n'existaient pas sous l'Ancien régime : leurs enfants sont soumis à leur droit de correction et dépendent de leur consentement pour se marier.

En résumé, à la Révolution, l'exercice de la paternité est socialement marqué par les références à l'amour et au sentiment paternel ; la paternité ne devait pas être forcée.

Puis le tournant conservateur rétablit les pouvoirs parentaux et une stigmatisation des naissances illégitimes. L'obligation morale du géniteur à être pourvoyeur d'aliments est toujours présente, mais elle est subordonnée à la volonté du père, lui permettant de se désengager au nom de la morale familialiste et chrétienne.

\section{II.A.1.2 La gestion du risque familial}

Nous nous intéressons maintenant au lien qui existe entre les représentations collectives qui motivent les actions de l'Etat et la législation qui met en place les obligations alimentaires du père envers ses enfants. Aussi, quatre paradigmes historiques de l'aide étatique retracent les mesures législatives relatives à l'obligation alimentaire : «l'angélisme chrétien», «l'angélisme, mais sous une forme malthusienne», «l'angélique libéral» et le «patriotique nataliste».

L'illégitimité et l'abandon sont deux formes du risque familial qui mobilisent l'action de l'Etat sous l'Ancien régime. «L'angélisme chrétien» (Lefaucheur 1991) a été une réponse au problème de l'infanticide et de l'abandon sous l'Ancien régime. «Il fallait leur laisser la possibilité de l'abandonner : le recueil des bâtards dans les hôpitaux et hospices d'enfants trouvés se généralisera à partir du XVIème siècle, tandis que les hôpitaux offraient aux filles grosses et aux femmes adultères la possibilité d'accoucher secrètement» (Lefaucheur 1991 : 118).

La collectivisation d'une partie de la charge de l'entretien de l'enfant était un moindre mal, mais les questions de la responsabilité financière et de l'organisation de l'élevage restaient très discutées. Le problème était en partie résolu par la forte mortalité infantile qui était peu combattue. Plus que le problème de la conservation des enfants, c'est le maintien de la morale chrétienne, de la morale conjugale et du combat de l'infanticide, qui justifient l'action de l'Etat.

La loi du 4 juin 1793 permet de reconnaître les naissances illégitimes et de légitimer les enfants adultérins («bâtards»). Elle permet aux enfants naturels d'être reconnus par leur mère, leur ouvrant l'accès à l'héritage maternel, mais aussi par leur père, s'il en exprime la volonté. La reconnaissance est effective par la décision personnelle du père, même si elle doit être ensuite corroborée par la mère (Sullerot 1992 : 75).

Le père n'assume la charge que des enfants dont il se déclare volontairement le père. La femme engrossée est dépendante de la volonté de l'homme puisque la recherche en paternité lui est interdite (Article 340). Le géniteur n'a pas à pourvoir aux besoins alimentaires s'il ne reconnaît pas l'enfant.

La loi du 12 brumaire an II accorde aux enfants adultérins ou incestueux, à titre d'aliment, un tiers en propriété de la portion des biens du géniteur à laquelle ils auraient eu droit s'ils étaient nés d'un mariage solennel ou privé (Mulliez 1990 : 295). Ce droit ne prend effet qu'à la mort du père biologique. Mais cette disposition est très limitée dans ses effets car 
la recherche de paternité est interdite. Et, les droits de ces enfants ne sont pris en compte que lorsque la paternité apparaît au cours d'un procès ou si les déclarations du défunt étaient couchées sur testament (ce qui était assurément la minorité des cas).

Si les révolutionnaires considéraient que la paternité ne devait pas être forcée par une recherche en paternité, en revanche, ils estimaient que les pères assumeraient spontanément et volontairement leur paternité tant naturelle que légitime.

Et dans les cas où le géniteur ne peut (ou ne veut) pas se constituer père ${ }^{76}$, l'adoption, réhabilitée par la Révolution ${ }^{77}$, devait donner un père aimant ${ }^{78}$ à ces enfants qui aurait eu les devoirs d'un père légitime.

Les réformes de la Révolution française au sujet du mariage avaient pour objectif de réduire le nombre d'enfants légitimes non désirés, et de favoriser l'adoption des autres. Il s'agissait de prôner l'amour paternel et ses obligations alimentaires subséquentes.

Le risque familial prend une nouvelle forme avec la loi sur le divorce en France en 1792. A partir de cette date, le risque familial ne concerne plus uniquement les familles illégitimes et les naissances scandaleuses mais également les séparations des unions légitimes. L'article V concerne la pension et les soins post-divorce des enfants : «Soit que les enfants, garçons ou filles, soient confiés au père seul ou à la mère seule, soit à l'un \& à l'autre, soit à des tierces personnes, le père \& la mère ne seront pas moins obligés de contribuer aux frais de leur éducation \& entretien ; ils y contribueront en proportion des facultés \& revenus réels \& industriels de chacun d'eux». L'entretien de l'enfant est autant du ressort du père que de la mère. De plus, les droits de succession des enfants divorcés sont maintenus à part égales avec les éventuels enfants de second lit (Article VII) ${ }^{79}$.

En résumé, les dispositions sont telles que les différences entre les enfants illégitimes et légitimes relatives aux subsides du père, s'amenuisent. Cependant une grande différence demeure entre les enfants illégitimes non reconnus et les autres : les enfants non reconnus, en

\footnotetext{
${ }^{76}$ C'est à dire les cas où le mariage solennel ou privé ne peut pas prendre effet : séduction de la mère, exercice de la prostitution ou enfant incestueux ou adultérin.

${ }^{77}$ Cette mesure a été prise devant l'urgence sociale que posaient les abandons d'enfants. A titre d'illustration, nous citerons les chiffres suivants : de 1700 abandons parisiens entre 1700 et 1720, on passe à une moyenne annuelle de 5000 à 6000 entre 1760 et 1789 (Cabantous 1990 : 341). Suivant l'extrait de l'arrêt du Conseil du roi du 10 janvier 1779 : «S.M. a remarqué avec peine que le nombre d'enfants exposés augmentoit tous les jours et que la pluspart provenoient aujourd'hui de noeuds légitimes». Cependant, les deux tiers des abandons restent ceux d'enfants illégitimes. La situation de pauvreté de l'époque reste sûrement l'explication principale de ce phénomène.

${ }_{78}^{78}$ Pour s'assurer des intentions paternelles du père adoptif, les droits de succession des enfants adoptés étaient limités. Cela pour contrecarrer certaines pratiques, héritées de l'Ancien Régime, de vieux célibataires qui, en choisissant un héritier, évitaient la dispersion de leur patrimoine en de multiples parts.

${ }^{79}$ Les dispositions et les prérogatives testamentaires que la communauté institutionnalise pour un enfant, révèlent la qualité du lien qu'on reconnaît entre l'enfant et son père. Si l'enfant a droit à une part de l'héritage de son père, alors il est reconnu comme étant socialement de sa descendance.

Or la loi du 2 novembre 1793, précédée du décret du 8 avril 1791, établit l'égalité stricte entre tous les héritiers en ligne directe. Les enfants illégitimes sont assimilés aux enfants légitimes dans les droits de succession. Mais elle s'applique à une catégorie privilégiée d'enfant illégitime, ceux que le père a reconnus (Sullerot 1992:64).

De plus, la loi du 4 juin 1793 établit un nouveau droit pour les enfants naturels de concubin en leur permettant d'hériter de leur parent. Ainsi les distinctions entre enfants légitimes, illégitimes reconnus, et naturels reconnus sont abolies en ce qui concerne l'héritage.
} 
particulier les enfants adultérins, incestueux ou «scandaleux», n'ont droit ni à une pension alimentaire, ni à l'héritage.

La paternité reste un acte subordonné à la volonté du père. Cela est surtout vrai pour la période révolutionnaire où la recherche en paternité, pour faire valoir les droits alimentaires de l'enfant, n'existe plus. L'obligation morale qui pèse sur le père est le seul contrôle social.

Le paradigme dominant au lendemain de la Révolution est marqué par un retour à «l'angélisme» mais sous une forme «malthusienne». Les naissances illégitimes et le coût d'entretien des enfants abandonnés étaient ressentis comme le pire des maux, la charge était jugée trop importante pour la collectivité : «à partir de 1811, la pratique préconisée par la législation de l'assistance fut ainsi celle des «tours»» (Lefaucheur 1991 : 121). Les fillesmères ne pouvant être prises en charge par la société, l'abandon des enfants, par le biais des tours, était considéré comme un moindre mal. Mais cette solution était largement controversée : elle encourageait les femmes à se décharger du "fruit de leur pêché" et favorisait l'illégitimité. Et le Code civil marque le retour moralisateur du XIXème siècle : au nom de la morale chrétienne conjugale, le père n'est pas sollicité dans ses devoirs alimentaires envers l'enfant illégitime, la recherche en paternité reste interdite.

Cette empreinte morale s'exprime aussi dans la réglementation du divorce et de la garde des enfants : cette dernière n'est plus fixée en fonction de l'intérêt de l'enfant mais en fonction de la moralité du parent. L'article CCXCVI «Les enfants seront confiés à l'époux qui a obtenu le divorce, à moins que le tribunal, sur la demande de la famille, ou du commissaire du Gouvernement, n'ordonne, pour le plus grand avantage des enfants, que tous ou quelquesuns d'eux soient confiés aux soins, soit de l'autre époux, soit d'une tierce personne» contraste avec l'article I, § I V de la loi précédente du 20 septembre 1792: la garde des enfants était alors partagée suivant l'accord des parents, attribuée à la mère pour les enfants de moins de 7 ans, ou encore au père pour les garçons plus âgés.

L'article CCXCVII du Code civil napoléonien exprime différemment les droits et les devoirs des parents que l'article V, § IV de la loi du 20 septembre 1792 : «Quelle que soit la personne à laquelle les enfants sont confiés, les père et mère conserveront respectivement le droit de surveiller l'entretien et l'éducation de leurs enfants, et seront tenus d'y contribuer à proportion de leurs facultés». Les droits des parents sont énoncés alors qu'ils ne l'étaient pas précédemment ; leurs devoirs d'entretien sont d'ordre moral ; l'expression «sont tenus de...» remplace la mention «sont obligés de...».

Avec le retour de la question de la dépopulation au cours du XIXème siècle, «le modèle démo-patriotique ${ }^{80}$ de la régulation du risque d'abandon des enfants illégitimes l'emporte sur le modèle «angélique libéral» (Lefaucheur 1991 : 122). Dans ce contexte, l'objectif est de contrecarrer l'abandon et la mortalité infantile et les filles-mères deviennent l'objet de l'attention bienveillante de la collectivité.

L'Etat multiplie les aides pour les mères célibataires ; des «maisons maternelles» sont crées pour permettre aux filles-mères de se reposer en fin de grossesse et d'allaiter l'enfant. Ces structures deviennent obligatoire par les lois de 1939 et 1943. L'ordonnance de 1945 créa la protection maternelle et infantile. Les préoccupations natalistes s'accompagnent ainsi d'une «déstigmatisation» de la maternité hors mariage. «Si le devoir de maternité est assimilable, devant la Patrie, au devoir de sang des hommes, la femme mariée qui devient mère est pareille au conscrit qui suit le sort de sa classe mais la fille-mère, c'est le volontaire qui devance

\footnotetext{
${ }^{80}$ ou «patriotique nataliste»
} 
l'appel. Il faut lui rendre les honneurs. Sans la fille mère, il n'y a plus de France, dès lors, il faut réhabiliter ce rôle» (Charles de Rouvre ('Les filles-mères et la Patrie 'in La Rumeur 1927) cité par Lefaucheur (1991: 123)).

La seconde partie du XXème siècle compose avec deux motifs : le patriotisme nataliste et familialiste. Le premier considère la position des familles monoparentales comme un moindre mal par rapport aux objectifs de repopulation ; le second met l'accent sur les carences familiales et la socialisation à risque des enfants de famille monoparentale.

Quant aux dispositions du Code civil à l'encontre du père, elles restent les mêmes jusqu'au début du XXème siècle où ses droits, comme «Pater Familias», vont être progressivement restreints. A partir des années 1980, le père retrouve une nouvelle place à travers ses nouveaux droits civils et son statut d'éducateur. 


\section{SOUS-SECTION II.A.2 L'HISTORIQUE DU RISQUE FAMILIAL EN ALLEMAGNE $^{81}$}

\section{II.A.2.1 La parentalité en droit civil}

Traditionnellement, le droit coutumier, hérité en partie du droit romain, faisait loi sur le territoire allemand malgré les forts particularismes régionaux. Au cours du 18ème et du 19 ème siècle, plusieurs tentatives de codification du droit ont été entreprises par les Etats allemands. Trois codes sont nés de ces réformes : le «Codex Maximilianeus Bavaricus» de 1756, le «Allgemeine Landrecht für die preussischen Staaten» en 1794 et le «Badische Landrecht» de 1810. Ces codes ont levé les pratiques du droit commun (Blasius 1987 : 99).

\section{Le code prussien et son libéralisme}

L'«allgemeine Landrecht für die preußischen Staaten» (ALR dans le reste du texte), œuvre de Carl Gottlied Svarez, est rentré en application le 1 juin 1794. Nous l'avons retenu car il inspira fortement le législateur du Code civil allemand actuel «Bürgerliches Gesetzbuch» qui entra en application en 1900. La question du soutien alimentaire des enfants légitimes est réglée comme suit : «le devoir primordial du couple marié est la reproduction et l'élevage des enfants» (ALR II, 1. T., 1, §§ 1 et 2). L'accent est mis sur les devoirs conjugaux lesquels organisent l'exercice de la fonction parentale. La parentalité est avant tout l'expression du conjugal ; les devoirs parentaux n'existent pas comme tels.

Le divorce est permis mais il est soumis à une législation beaucoup plus conservatrice que le droit révolutionnaire français de l'époque. En effet, l'ALR maintient fermement le principe de faute au centre du divorce. Seule la partie innocente peut faire la demande de divorce (ALR II, 1. T., 8, § 670) et une fois le divorce prononcé, le remariage avec la personne de l'adultère est interdit (ALR II, 1. T., 1, § 25).

Cependant, deux éléments nuancent cette procédure. Premièrement, il existe une clause de divorce par accord mutuel ( $\$ 716$ et suivants, ALR II, 1. T., 8), mais à condition que le couple soit sans enfant. Deuxièmement, la clause d'accord mutuel a été inspirée des pratiques jurisprudentielles de l'époque, même si son inscription dans la loi restreint son application. En effet, Carl Gottlied Svarez, initiateur de la forme définitive du ALR, négocia, avant la rédaction finale de la loi, l'inflexion du projet en faveur de ces clauses plus permicives. Il s'appuya sur un rapport du cabinet de Frédéric le Grand de 1783, et évita une dérive vers une décision plus conservatrice. Le rapport citait les cas de divorce qui auraient été prononcés sur la demande conjointe des partis. La justification populaire de cette pratique, soulignait le rapport, était que la situation de conflit entre les parents pouvait mettre la morale de l'enfant en danger. L'intervention de Svarez a eu pour suite la rédaction des articles $\S \S$ 716, 717, 718a, respectivement «demande mutuelle», «aversion» et «aversion insurmontable», dans les clauses de divorce de l'ALR.

\footnotetext{
${ }^{81}$ Les citations et des expressions allemandes, mentionnées entre guillemets dans cette partie, ont été traduites par nous-mêmes (sauf mention contraire).
} 
La fin de la première moitié du XIXème siècle a été marquée par le retour de la rigueur morale. Vers 1840 , le statut des mères célibataires redevient un sujet de critique pour la morale conservatrice et religieuse de l'époque. Friedrich Carl von Savigny, ministre de la Justice, considère le nombre de grossesses illégitimes comme le résultat d'une législation privilégiant l'adultère et l'atteinte à la morale publique. Il souhaite rétablir la reconnaissance du lien sacré du mariage et la condamnation de l'adultère. Il s'oppose cependant à la transposition du Code Napoléon, en vigueur dans la Rhénanie qu'il juge trop libéral car le code limite les recours des femmes «engrossées», mais ne les condamne pas moralement. Finalement, son projet de réforme en 1845 n'aura pas le soutien nécessaire pour être adopté.

L'année 1854 marque l'apogée des tendances réactionnaires-conservatrices en Prusse (Weber 1907 : 340) : le concubinage et l'illégitimité sont sévèrement critiqués.

Par la suite, l'orientation conservatiste du «Bürgerliches Gesetzbuch» (BGB), créé à la fin du 19ème siècle, domine le droit, et l'autorité parentale est remise entre les mains du père.

\section{II.A.2.2 La gestion du risque familial}

Traditionnellement, l'illégitimité était gérée de façon très discriminante par la morale publique. L'ALR va marquer la rupture avec le droit coutumier car le code prussien donne un nouveau statut juridique aux mères célibataires et à leurs enfants illégitimes, il leur donne accès à une nouvelle position sociale. Le statut juridique des enfants illégitimes est fixé dans la seconde partie de l'ALR : «Qui engrosse une femme, en dehors du couple légitime, doit dédommager la victime et s'occuper de l'enfant» (article 1027) $)^{82}$.

En France et en Allemagne, le géniteur n'est pas de lien de parenté avec son enfant illégitime non reconnu. En revanche, la situation de la mère célibataire et de son enfant est plus avantageuse en Allemagne qu'en France où elle reçoit plus rarement une pension alimentaire (Weber $1907: 340$ ).

En Allemagne, la mère célibataire peut revendiquer une pension alimentaire, à condition de désigner l'homme sous serment et de ne pas s'être prostituée. Le contre argument selon lequel la mère aurait eu des rapports sexuels avec d'autres hommes pendant la période de conception est alors irrecevable. Dans ces cas précis, le tribunal décide, soit d'attribuer la charge alimentaire à l'homme ${ }^{83}$ désigné par la mère, soit d'impliquer les autres partenaires.

Le dédommagement de la mère se fait proportionnellement aux circonstances (Blasius $1987: 100)$ :

$\diamond$ S'il y a eu promesse de mariage et si la femme a connaissance du nom, de l'état et du rang du séducteur, alors elle a les mêmes droits qu'une femme divorcée jugée innocente ( $\S$ 1049-1050). Elle peut prétendre à une amende que le mari doit payer pour avoir était condamné en procès.

\footnotetext{
${ }^{82}$ Le premier titre «du couple légitime» ( $\$ 11$, articles 1027 à 1137) traite des femmes «engrossées», et le paragraphe 9 du deuxième titre s'intitule «des droits et devoirs respectifs des parents et des enfants» (\$§ 592665).

${ }^{83}$ Les parents du père sont juridiquement sollicités si le père n'est pas en mesure de s'acquitter de la pension alimentaire (Weber $1907: 340$ ).
} 
$\diamond$ S'il n'y a pas eu de promesse de mariage, il n'en reste pas moins une pension alimentaire pour la mère.

Le statut juridique de l'enfant illégitime est lui-aussi amélioré par rapport au droit commun, mais il reste lié au sort de la mère. Si elle s'est vu reconnaître les droits d'une femme divorcée, alors son enfant a les mêmes droits qu'un enfant élevé dans un couple légitime (ALR II, 2.T., 9, § 592). Si le père a déjà une descendance légitime, alors l'enfant n'a pas de droit de succession. Sinon on lui reconnaît un 1/6 de l'héritage (ALR II, 2. T., 9, §§ 651 et 652) (Blasius $1987: 100$ ).

Or cette législation a peu de sens s'il est facile au père de contester sa paternité. Le législateur allemand, pour assurer en quelque sorte un droit de protection des mères célibataires, va mettre en place le système jurisprudentiel suivant (Blasius 1987 : 101) : si les rapports sexuels sont niés par le père, alors le tribunal dispose d'une procédure particulière (ALR II, 1.T., 11, §§ 1144-1119) : soit le juge accepte la validité du «serment de réalisation ${ }^{84}$ de la mère ; soit le «serment de purification» ${ }^{85}$ du père. Pour avoir gain de cause, chacun des parents devait prouver son intégrité et la falsification de l'autre. Dans la majorité des cas, où il n'était pas possible d'incomber les torts à un des deux protagonistes, l'ALR règle le conflit de la sorte : «S'il est difficile d'attribuer la faute à l'un des deux partis et que le doute persiste, alors entre le serment de réalisation et celui de purification, il sera reconnu le premier plutôt que le second».

Cependant, le père a la possibilité, au lieu de payer la pension alimentaire, de prendre à ses côtés l'enfant âgé d'au moins 4 ans. Le renoncement de la pension alimentaire pouvait permettre à la mère de garder l'enfant (Weber 1907 : 340).

1854 marque le retour de la rigueur morale. Les changements effectués sur les paragraphes 11 (Titre 1 partie II) et 9 (Titre 2 partie II) de ALR sont publiés le 24 avril 1854 dans le journal officiel de la Prusse. Le statut juridique de la mère célibataire se dégrade ; ses statuts sociaux et économiques ne sont plus garantis juridiquement. Seules les femmes violées ou abusées par une promesse de mariage, pourront avoir droit aux nouvelles dispositions de l'ALR (Blasius 1987 : 108). Toutes les autres femmes ne peuvent revendiquer, auprès du père de l'enfant, que les frais d'accouchement et de baptême, une pension de 6 semaines et les dépenses nécessaires à leur état (Weber 1907 : 560, Blasius 1987 : 109).

De plus, la procédure de réclamation des mères devant le tribunal a changé, elle limite leur recours juridique. Le rôle du juge n'est plus d'étudier les circonstances de la conception, mais c'est à la femme de prouver son intégrité (Blasius 1987 : 109). Le géniteur peut, à partir de cette date, invoquer les relations multiples de la mère pendant la période de conception. Cet argument est recevable et le dégage de ses devoirs alimentaires. Tout enfant, dont la mère avait déjà un enfant illégitime, ne touchait plus la pension du père. Et enfin, les parents du géniteur ne sont plus pris à parti dans le paiement de la pension alimentaire (Weber 1907 : $560)$.

La gestion du divorce dans le code civil allemand (BGB) montre l'orientation conservative des réformes du droit à la fin du XIXème siècle et la réinscription du rôle patriarcal de l'homme.

\footnotetext{
${ }^{84}$ «Erfüllungseid», déclaration assermentée déclarant que les relations sexuelles ont bien eu lieu.

${ }^{85}$ «Reinigungseid», déclaration assermentée par laquelle il nie avoir eu les relations sexuelles.
} 
En cas de divorce, la mère qui assume l'entretien quotidien de l'enfant par son patrimoine et son travail, doit verser sa part à son mari, même s'il a eu les torts du divorce (§ 1585). Elle dépend du bon vouloir du mari pour qu'il lui reverse l'argent en y ajoutant sa propre participation (Weber $1907: 456$ ).

Un enfant illégitime est juridiquement membre de la famille de sa mère, il a donc tous les droits et devoirs d'un enfant légitime (en matière d'héritage par exemple). La mère possède l'autorité parentale. Le père ne possède ni devoir ni droit : il ne peut ni réclamer une pension vieillesse à son enfant, ni partager l'autorité parentale (Weber 1907 : 570).

Juridiquement, l'affiliation de l'enfant à la parenté du père est techniquement réalisable. Le père illégitime peut, sur l'approbation de l'autorité administrative, légitimer l'enfant ( $\$ 1723$ ) (pour un homme déjà marié sa femme doit donner son aval ( $\$ 1726)$ ). Et cette reconnaissance peut se faire contre l'avis de la mère de l'enfant, il suffit que la tutelle voit dans le fait de ne pas légitimer l'enfant un désintérêt pour l'enfant ( $\$ 1717$ ). La mère perdra alors l'autorité maternelle ( $\$ 1738$ ) puisque l'enfant prend la place d'un enfant légitime auprès du père. Il sera donc soumis à son autorité. En d'autres termes, il est facile à un père fortuné de retirer l'enfant illégitime à sa mère. 


\section{SOUS-SECTION II.A.3 LES NOUVELLES CARACTERISTIQUES DU «RISQUE FAMILIAL»}

Notre analyse a porté essentiellement sur la condition de la mère célibataire. Cette forme de famille révélait le mieux le risque familial et son traitement «passé». Aujourd'hui, les mères célibataires n'illustrent plus le traitement social de la monoparentalité en France et en Allemagne car elles représentent moins d'un quart de l'ensemble des familles monoparentales.

Jusque dans les années 1970, la majorité des situations de monoparentalité était consécutive au veuvage : 54,2\% au recensement de 1968 en France. A l'heure actuelle, la monoparentalité par suite d'un décès d'un parent est très restreinte (20\% en 1990) (Mendras 1997 : 217).

En revanche, le nombre de familles monoparentales, conséquentes à une désunion ${ }^{86}$, est en constante augmentation depuis les trente dernières années (INSEE 1994 : 17). Aujourd'hui, les femmes divorcées et séparées forment la très large majorité des familles monoparentales.

Actuellement, en Europe ${ }^{87}$, environ un tiers des couples divorcent ou se séparent. Leur nombre a constamment augmenté depuis le début des années 1970 et le divorce est devenu un risque social qui touche une partie importante de la population. La divortialité n'est plus le lot d'une minorité, la société est en face d'une situation de «démariage» (Théry 1993). Il y a une généralisation du risque de séparation sur l'ensemble des familles. Nous parlerons de «risque familial». Le père devient une nouvelle composante dans la gestion du risque familial ${ }^{88}$.

Les années 1970 marquent le début d'un changement de pratique et de norme dans l'exercice de la parentalité pour les familles légitimes, naturelles, unies et désunies, en France et en Allemagne.

Premièrement, les réformes sur le divorce amorcent le renversement de paradigme et l'institutionnalisation de la parentalité devient possible. L'introduction de la procédure de divorce par consentement mutuel contribue à déstigmatiser le divorce. En 1975 en France et 1977 en Allemagne, la possibilité de divorcer «à l'amiable» permet de dissocier les différents conjugaux de la garde des enfants. Ainsi, l'un des deux parents n'est plus systématiquement exclu pour la garde des enfants. Le divorce par consentement mutuel prépare une conjoncture favorable à l'exercice en commun de l'autorité parentale conjointe.

Jusque là, le divorce était une crise grave et un déchirement de la famille : les torts étaient incombés à un parti, l'un des deux était fautif. L'enfant était alors confié au parent «innocenté» par la procédure de divorce. «Il y a vingt ans, quand la loi sur le divorce a été votée en 1975, on avait encore l'idée d'un divorce très contentieux, c'était forcement une

\footnotetext{
${ }^{86}$ Par désunion, nous entendons le divorce ou la séparation d'un couple. Mais La difficulté du comptage des familles monoparentales amène le statisticien à inclure des couples de concubins dans la catégorie des maternités célibataires.

${ }^{87}$ La France et l'Allemagne se regroupent toutes les deux dans la catégorie médiane des pays répartis selon le taux de divortialité (Hantrais 1994 ; Begeot, Fernandez-Cordon 1997 ).

${ }^{88}$ Cette nouvelle caractéristique du groupe fait s'interroger Sullerot (1992 : 103) sur la pertinence de la dénomination «familles monoparentales»; or il n'existe pas de «familles monoparentales» mais des «foyers monoparentaux», les seules familles monoparentales sont celles des veufs et des veuves.
} 
bagarre, forcement une situation difficile à dénouer, et donc la question de la garde conjointe des enfants était inimaginable. Depuis, il y a eu une évolution sociale très importante.» (Entretien avec Madame le juge aux affaires familiales 1997).

Deuxièmement, la généralisation du divorce déstigmatise les séparations parentales et conjugales. «Les comportements pouvaient être considérés comme déviants tant qu'ils demeuraient minoritaires» (Commaille1996 : 51). Aujourd'hui, le divorce n'est plus le fait d'une minorité en marge de la norme sociale.

Or, la situation qui pouvait être le lot d'une minorité n'est plus obligatoirement acceptable pour un grand nombre. I. Théry ${ }^{89}$ soulignait que «les séparations de couple sont aujourd'hui banalisées. Devenant le cas d'un grand nombre, il est devenu insupportable pour la société de se satisfaire d'une situation qui était le cas d'une minorité : l'éloignement du père» (Théry 1997).

Historiquement, la mère a toujours été le parent de référence pour les enfants en bas âge. La certitude biologique du lien a été le plus sûr garant de la force de la relation mèreenfant. Par contre, si les soins incombaient à la mère, l'autorité parentale ne lui fût accordée qu'au XXème siècle. L'ancienne conception du «Pater Familias» définissait le père comme l'éducateur au sein de la famille légitime. Sa fonction de nourricier pouvait s'étendre, mais de façon très restrictive, au cas des naissances illégitimes. La gestion du risque familial, dans le cas des naissances illégitimes et des divorces, s'est effectuée à la décharge du père, la mère devant souvent assumer seule les charges de l'enfant. Le lien de parenté entre le père et l'enfant existait très rarement dans le cas des naissances illégitimes et en aucun cas contre sa volonté. La régulation du risque familial s'effectuait selon le mode de la «monoparentalité à la décharge du père».

Depuis, les années 1970 ont définitivement remis en question la dominance exclusive de l'autorité paternelle et maritale de l'homme. Les pratiques jurisprudentielles concernant les familles naturelles, octroyaient à la mère l'exclusivité des droits et des devoirs envers les enfants. Le père est absent de «l'après-divorce». La garde de l'enfant est systématiquement donnée à la mère. Ces pratiques sont accréditées par les discours scientifiques de l'époque. La relation mère-enfant est très fortement institutionnalisée, tant en France qu'en Allemagne. Ces pratiques furent très favorables à un exercice monoparental de l'autorité parentale en faveur de la mère.

La disparition de la procédure contentieuse du divorce et l'augmentation du nombre de personnes concernées, sont deux facteurs qui ont facilité l'apparition d'un paradigme fondé sur l'exercice partagé de la parentalité. Cette coresponsabilité, institutionnalisée dans le droit civil, est semblable aux évolutions des politiques familiales, du droit social, et des pratiques (en jurisprudence, des magistrats et des parents). L'exercice conjoint de la responsabilité parentale prend deux formes : la «coparentalité» en France et la «biparentalité» en Allemagne (sections suivantes).

\footnotetext{
${ }^{89}$ Intervention d'I.Théry lors du colloque sur la «coparentalité» qui a eu lieu à la Maison du Barreau de Paris le 12 juin 1997. Il a été organisé par la commission Typhaon avec le concours de l'ordre des avocats de Paris, le syndicat de la magistrature et le syndicat des avocats de France (une partie des actes du colloque a été publiée dans la revue Dialogue (1998).
} 


\section{SECTION II.B LA CO-PARENTALITE FRANCAISE}

\section{SOUS-SECTION II.B.1 LES PRINCIPES DE LA PARENTALITE DANS LES FAMILLES LEGITIMES ET NATURELLES}

\section{$\underline{\text { II.B.1.1 L'institutionnalisation de l'exercice bilatéral de l'autorité }}$ parentale}

\section{$\underline{\text { II.B.1.1.1 Les années }} \underline{1970} \underline{\text { et l'apparition de l'égalité parentale }}$}

Les premiers principes contemporains de l'autorité parentale sont apparus dans le code civil en 1970, la puissance paternelle est alors abandonnée au profit de l'autorité parentale (loi du 4 juin 1970, article 371-2 C. civ. ) : «L'autorité parentale appartient aux pères et mères pour protéger l'enfant dans sa sécurité, sa santé et sa moralité».

La suppression de la puissance paternelle dans les textes législatifs montre de quelle manière s'effectue la révolution normative des rapports familiaux (cf. la chronologie juridique en Annexe 13).

«Quand en 1970, la réforme aboutit, on attache la plus grande importance à la suppression du mot «puissance» paternelle. Le changement de mot se veut changement d'esprit. «L'autorité parentale» n'est plus une prérogative mais un complexe de droit et de devoir qui correspond à ce qu'on nomme aujourd'hui une fonction ${ }^{90}$. Sa finalité n'est plus la domination mais la protection. Enfin, elle est égalitaire, elle est exercée par les deux parents, chacun des deux époux étant supposé agir avec l'accord de l'autre quand il fait seul un acte usuel relativement à la personne de l'enfant. Voilà bien appliqués les principes d'égalité, entre père et mère et l'individualisme des fonctions.» (Sullerot 1992 : 127).

Cette réforme illustre deux nouvelles caractéristiques de ce que nous avons appelé le «lien générationnel», c'est-à-dire l'ensemble des rapports sociaux entre les parents et les enfants.

Premièrement, il s'agit d'un ensemble complexe de droits et de devoirs qui inscrit la relation générationnelle dans une dimension fonctionnelle. Le lien générationnel se construit autour de responsabilités parentales, qui ont pour finalité la protection de l'enfant, et de droits, qui déterminent les modes d'exercice de l'autorité parentale.

Deuxièmement, le lien générationnel contemporain suit le nouveau principe d'égalité de droits et de devoirs entre les pères et les mères.

On rappellera deux réformes du droit civil qui ont marqué la deuxième partie du XXème siècle, et qui ont permis, par la suite, l'émergence du critère d'égalité parentale et la montée de l'exercice bilatéral de l'autorité parentale.

\footnotetext{
${ }^{90}$ Le fait de définir l'autorité parentale comme une fonction, dont l'exercice est un devoir autant qu'un droit pour les parents, signifie qu'elle ne relève pas de la volonté individuelle des parents. Elle prend alors une dimension sociale, laquelle autorise en retour une régulation normative des rapports générationnels par la société.
} 
$\diamond$ Premièrement, le principe d'égalité de droits et de devoirs entre les deux parents, est l'aboutissement des réformes pour l'égalité individuelle entre les hommes et les femmes. Un premier changement concerne l'inscription dans le préambule de la constitution en 1946, du principe d'égalité des droits entre les hommes et les femmes ; le second modifie, le 13 juillet 1965, les régimes patrimoniaux. Ce dernier rééquilibre l'égalité dans les rapports conjugaux : les pouvoirs de la femme mariée sur les biens communs sont garantis et le mari ne peut plus s'opposer à ce que la femme exerce une activité professionnelle. Cette inscription progressive des rapports égalitaires hommes-femmes, à l'intérieur des rapports conjugaux, participe à la redéfinition des rapports parentaux et facilite l'inscription des nouveaux principes en 1970.

$\diamond$ Deuxièmement, la loi de 1972 institutionnalise l'égalité des filiations entre les enfants naturels et les enfants légitimes, leur déclarant les mêmes droits et les mêmes devoirs (article 334 C. civ.). En effet, avant 1972, l'enfant naturel, même reconnu par ces deux parents, était discriminé face à l'enfant légitime. On ne lui reconnaissait pas de filiation paternelle (sans accès au patrimoine paternel, et sans devoir d'assistance à son encontre). Ce principe d'égalité horizontale entre les enfants s'ajoute au principe d'égalité entre le père et la mère.

A partir de cette date, et tout au long des années 1980 et 1990, l'idée selon laquelle il n'est plus possible d'accepter une discrimination de statut entre les enfants naturels et légitimes s'affirme à nouveau. Aujourd'hui encore, cette idée anime les propositions de réforme du droit civil de la famille.

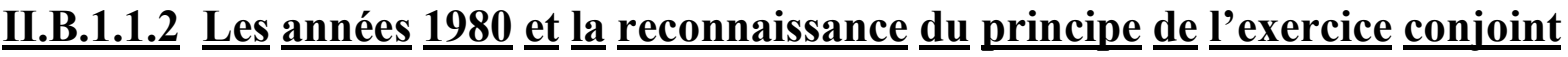 de l'autorité parentale}

Des projets de réforme de l'autorité parentale furent déposés de façon récurrente à l'Assemblée nationale et au Sénat dans les années 1980. Ils proposent principalement une réforme des articles 256, 287, 288 et 372, 373, 374 du Code civil, pour l'extension de la notion d'autorité parentale, pour les parents légitimes et naturels.

Le 22 juillet 1987, la loi Malhuret affirme la possibilité, pour les parents naturels, d'obtenir une autorité parentale conjointe par simple déclaration devant le juge de tutelle. L'autorité parentale (article 372 du Code civil) est exercée en commun par les deux parents s'ils sont mariés. Elle est également exercée en commun si l'enfant naturel est reconnu par ses deux parents avant qu'il ait atteint l'âge d'un an, et s'ils vivent en commun au moment de la reconnaissance concomitante ou de la seconde reconnaissance.

En 1993, une réforme a modifié l'exercice de l'autorité parentale en cas de rupture familiale mais peu celui des familles «unies», quelles soient légitimes ou naturelles (section suivante).

Depuis, une réforme du droit civil de la famille est en préparation : le nouveau Code regroupera les dispositions touchant la famille, aujourd'hui éparses dans différents codes (Code civil, Code de la santé publique, de la famille et de la sécurité sociale). L'objectif de la 
commission préparatoire ${ }^{91}$ n'est pas un bouleversement du droit de l'autorité parentale, «mais plutôt l'achèvement d'un mouvement commencé en 1970, afin de tirer toutes les conséquences des principes posés à l'époque et de donner plus de cohérence à des règles retouchées depuis à différentes reprises.» (Dekeuwer-Défossez 1999 : 71).

En effet, les transformations des modes de vie familiaux et en particulier la constitution de plus en plus fréquente de famille hors mariage, ainsi que l'augmentation des ruptures familiales (qu'elles soient hors mariage ou en mariage), poussent à revisiter les principes de l'autorité parentale. La commission Dekeuwer-Défossez estime qu'il est de son devoir de tenir compte des nouveaux besoins, mais également d'affirmer le principe d'égalité, entre les enfants, et entre les parents.

«(...) Les transformations qu'ont connu les modes de vie familiaux, rendent nécessaires une réforme, non seulement pour répondre à de nouveaux besoins, mais encore pour affirmer (ou réaffirmer) un certain nombre de principes. En particulier, la constitution de plus en plus fréquente de familles hors mariage, l'augmentation des séparations, en mariage ou hors mariage, ne peuvent que retentir sur le droit de l'autorité parentale. Le premier phénomène se conjugue d'ailleurs avec une exigence de plus en plus forte d'égalité entre enfants, quelles que soient les circonstances de leur naissance ; or, abolir les différences entre enfants naturels et légitimes conduit à repenser un certain nombre de règles en matière d'autorité parentale. Les deux autres phénomènes posent de façon nouvelle le problème de la coparentalité et, au-delà, de la place du père. On sait en effet que pour des raisons diverses, de plus en plus d'enfants sont assurés au quotidien par leur mères et ne voient leur père que de façon épisodique. Comment redonner aux pères toute la place qui lui revient auprès de l'enfant ? Il s'agit moins là de reconnaître de prétendus «droits des pères» que de respecter le droit de l'enfant à être élevé par ces deux parents : les circonstances la vie peuvent éloigner le père de l'enfant, s'il ne peut l'en empêcher, le droit ne doit pas, dans ces dispositions, comme par ces silences favoriser la rupture.» (Dekeuwer-Défossez 1999 : 71-72).

\section{$\underline{\text { II.B.1.1.3 L'enfant }}$}

La référence à l'enfant a été un véritable moteur de réforme de l'autorité parentale. Il s'agit beaucoup moins de prendre en compte les droits individuels des pères et des mères que d'accorder la priorité à l'exercice commun de la parentalité et de maintenir le couple parental pour l'enfant.

Outre les législations nationales, de nombreux textes internationaux et européens posent des principes qui constituent une sorte de droit commun de l'enfance. (DekeuwerDéfossez : 72). «Quatre grandes idées s'en dégagent avec une force particulière : droit de l'enfant à une famille, primauté de la famille par rapport aux autorités publiques, sociale ou judiciaire, droit de l'enfant à être élevé par ces deux parents et en toute hypothèse de

\footnotetext{
${ }^{91}$ Le projet de réforme du droit de la famille a été amorcé en 1997. Le commanditaire politique avait chargé les commissions successives d'adapter le droit civil aux évolutions contemporaines afin d'éviter « que ne se creuse un fossé entre les aspirations de nos citoyens et le droit » (lettre de mission d'Isabelle Guigou, ministre de la justice, (Dekeuwer-Défossez 1999 : 71)). Deux rapports ont marqué les projets de réforme : le premier rapport «couple, filiation et parenté aujourd'hui» a été rédigé par Irène Théry (1997) à la demande conjointe de la ministre de l'emploi de la solidarité et du garde des sceaux. Puis, sur la base de ces travaux, Françoise DekeuwerDéfossez (1999) a rédigé, dans un second rapport, les conclusions de la nouvelle commission pour le garde des sceaux et le ministre de la justice.
} 
conserver des relations avec ses deux parents, prise en compte de la vie, et plus généralement des besoins d'expressions et d'information, de l'enfant.» (Dekeuwer-Défossez 72-73).

La Convention Internationale des Droits de l'Enfant ratifiée par la France en 1990, demande aux états de prendre «les mesures appropriées pour que l'enfant soit effectivement protégé contre toutes les formes de discrimination ou de sanctions motivées par la situation juridique, les activités, les opinions déclarées ou les convictions de ses parents» (Art. 2).

La notion de couple parental, présente dès les années 1990, a été réitérée lors de la ratification de la convention européenne des droits de l'enfant en 1996. «Les Etats parties s'emploient de leur mieux à assurer la reconnaissance du principe selon lequel les deux parents ont une responsabilité commune pour tout ce qui est d'élever l'enfant et d'assurer son développement» (Art. 18-1). L'article 7-1 recommande que l'enfant ait, «dans la mesure du possible, le droit de connaître ses parents et d'être élevé par eux».

Dans le cas de la France, outre la convention européenne des droits des enfants, la discrimination encore présente dans le droit civil, entre les enfants naturels et légitimes, pousse actuellement à recomposer l'autorité parentale.

En amont de l'autorité parentale se situe la filiation. Effectivement, ce n'est que lorsqu'elle est juridiquement établie, que les pères et les mères sont titulaires de l'autorité parentale. Or, encore actuellement dans la législation française, l'établissement juridique de la filiation est différent pour les enfants naturels et pour les enfants légitimes. Cette distinction a deux conséquences dans les pratiques :

Dans les familles légitimes, le partage de l'autorité parentale se fonde sur le principe de l'autorité parentale conjointe, il est immédiat et trouve son origine dans le mariage. Dans les familles naturelles, la reconnaissance de l'enfant est un acte volontaire qui doit être effectuée, séparément ou conjointement, par les deux parents afin d'instituer le lien de filiation. Une reconnaissance conjointe de l'enfant lui donne une double filiation sans impliquer l'exercice conjoint de l'autorité parentale.

Les propositions de réforme de la commission de Dekeuwer-Défossez souhaite abolir la distinction entre la filiation naturelle et la filiation légitime ${ }^{92}:$ «Il a semblé à la commission qu'il était aujourd'hui bien plus important que le droit souligne davantage la communauté de fondement du rapport de filiation, quel que soit le lien civil unissant les parents. (...) Pour la réalisation de cet objectif, et dans la mesure où la société admet ouvertement la coexistence de plusieurs formes de conjugalité, il devient peu significatif d'articuler l'ensemble du droit de la filiation à partir ou non d'un mariage.» (Dekeuwer-Défossez 1999 : 31). Il s'agit pour la commission de mettre en avant les droits et les devoirs induits par la filiation, et ce, quelle que soit la nature du lien de conjugalité entre les parents.

Ces propositions argumentent pour une uniformisation des statuts suivant le principe de non-discrimination des enfants. Les demandes de réformes vont donc dans le sens d'une dissociation des logiques conjugale-matrimoniale et parentale ${ }^{93}$.

\footnotetext{
${ }^{92}$ Cela a été le cas en Allemagne le 16 décembre 1997.

${ }^{93}$ La Déclaration Universelle des Droits de L'homme de 1948 qui a consacré un important article à la famille (Art. 16), avait déjà précisé que le droit des enfants à la protection sociale ne doit pas dépendre du statut matrimonial de leurs parents (Art.25-2).
} 


\section{II.B.1.1.4 Les familles légitimes et naturelles}

À l'heure actuelle, les parents sont titulaires de l'autorité parentale dès que la filiation est établie, que ce soit dans les familles légitimes ou naturelles. La filiation est automatiquement établie dans les familles légitimes, et chaque parent est titulaire de l'autorité parentale. Et suite à la réforme de 1993, ils l'exercent en commun.

Contrairement aux familles légitimes, la loi de 1993 inscrit deux conditions à l'exercice en commun de l'autorité parentale dans les familles naturelles : premièrement, la filiation doit être établie à l'égard des deux parents dans l'année de la naissance de l'enfant, deuxièmement, les parents doivent vivre ensemble lors de la reconnaissance concomitante (c'est-à-dire du père et de la mère) ou de la seconde reconnaissance (par le second parent) (article 372 al. 2 C. civ.).

Lorsque ces conditions ne sont pas remplies, il existe une règle subsidiaire qui attribue, à défaut, l'exercice de l'autorité parentale à la mère. La dominance maternelle à titre subsidiaire maintient une inégalité de droit entre les deux parents. Dans ce contexte, la commission préparatoire de la réforme du droit civil de la famille propose de substituer à la règle qui affirme la prééminence de la mère, une règle plus simple et plus égalitaire : le parent dont la filiation est déjà établie continue à exercer seul l'autorité parentale (DekeuwerDéfossez 1999 : 79).

Cependant l'exercice en commun de l'autorité parentale peut être officialisé par les parents naturels de l'enfant, lors d'une déclaration conjointe devant le greffier en chef du tribunal de grande instance ou par une demande aux juges aux affaires familiales (article 374 C. civ.).

La mise en place de l'autorité parentale conjointe, comme principe de régulation du risque familial pour les familles naturelles, rencontre des résistances dans son institutionnalisation.

La discrimination récurrente des familles naturelles par rapport aux familles légitimes exprime la référence traditionnelle à l'ultime modèle de la famille unie et légitime. C'est elle qui est au centre du code civil. Les règles d'application de l'autorité parentale (Art. 372 et s.) dans les familles naturelles étaient traitées en fin de section.

«Dans le code civil, les règles de l'autorité parentale ont été construites autour du modèle de la famille légitime unie. Certes, le législateur est intervenu depuis 1970 pour étendre aux enfants nés hors mariage la plupart des règles relatives aux enfants nés en mariage. Mais la structure traditionnelle est encore visible dans les textes. (...) De plus, sont traités au titre du divorce, un certain nombre de problèmes qui intéressent en réalité tous les cas de séparations : séparations de fait des époux, séparation des concubins. Cette présentation est non seulement complexe, mais encore peu cohérente au regard des principes d'égalité de coparentalité qui anime aujourd'hui le droit de l'autorité parentale. Il semblerait donc opportun d'une part, de reconstruire les règles de l'autorité parentale autour de ces deux principes, d'autre part, de créer une sorte de droit commun de la séparation.» (DekeuwerDéfossez 1999 : 75).

Le législateur se distance peu à peu du référent unique à la famille légitime. La loi Malhuret de 1987 apporte, pour les couples naturels, la possibilité d'exercer, par déclaration conjointe devant le juge des tutelles, l'autorité parentale conjointe (Article 5). Sans cette 
démarche, le père n'a pas d'autorité parentale même s'il a reconnu l'enfant, et même si elle est concomitante avec celle de la mère. Avec la loi de 1993, la reconnaissance concomitante avant l'âge de six mois de l'enfant, enclenche la procédure d'autorité parentale conjointe, ou bien, après ce délai, s'ils vivent en commun lors de la reconnaissance du second parent (Article 23 sexie de la loi). Mais elle n'est pas encore une règle de principe comme pour les situations de «l'après-divorce».

La commission Dekeuwer-Défossez propose que les règles d'exercice en commun de l'autorité parentale s'appliquent à tous les enfants (dés lors que sa filiation est établie), puis de spécifier dans un second temps des situations particulières qui feraient exceptions à cette règle, lorsque l'exercice de l'autorité est unilatéral ou lorsque l'enfant est confié à un tiers (Dekeuwer-Défossez $1999: 76)^{94}$.

L'institutionnalisation de la parentalité conjointe pour les familles naturelles rencontre beaucoup de résistance et si elle se met en place, c'est surtout à travers les droits de l'enfant. Historiquement, les réformes pour une harmonisation des situations des familles naturelles et légitimes, ont toutes été motivées par la volonté de ne pas discriminer les enfants entre eux. Aujourd'hui, les droits des enfants et la norme de l'intérêt de l'enfant s'imposent comme référents dans les législations nationales et internationales.

En 1997, les naissances hors mariage représentent $40 \%$ de l'ensemble des naissances, contre 6\% en 1965 (Munoz-Pérez ; Prioux 1999a : 481). Parallèlement, la proportion d'enfants non reconnus par le père diminue fortement : sur l'ensemble des enfants naît hors mariage en 1965 et en 1970, un peu plus des trois quarts ont été reconnus par le père, les enfants nés en 1975 sont reconnus à $81 \%$, et ceux nés en 1985 à $88 \%$ (estimation) et on peut penser qu'en 1994 l'estimation atteindra 92 \% (Munoz-Pérez ; Prioux 1999a : 483). Non seulement les reconnaissances paternelles augmentent proportionnellement, mais elles sont également de plus en plus précoces. Un mois après la naissance, plus de $80 \%$ des enfants naturels sont déjà reconnus, contre 33,4\% en 1965 (cf. Tableau 17).

\footnotetext{
${ }^{94}$ Premièrement, la commission propose de regrouper l'ensemble des règles relatives à l'autorité parentale sous un titre commun «De l'autorité parentale» et d'y intégrer les dispositions qui figuraient au titre de divorce et qui pourraient être appliquées à l'ensemble des séparations, comme c'est le cas dans les faits (Dekeuwer-Défossez 1999 : 77-78). Deuxièmement, il s'agit de dépasser les distinctions faites entre les enfants selon les articles 372 et 374 C. civ. pour tenter de ne faire aucune différence entre les enfants, quelle que soit leur naissance. Troisièmement, «l'instauration de règles posant le principe d'un exercice en commun de l'autorité parentale quels que soient les aléas de la vie du couple : juridiquement, la séparation ne doit plus apparaître comme une coupure dans l'exercice de l'autorité parentale, mais comme une simple donnée rendant nécessaire un aménagement des modalités déversées de celle-ci.» (Dekeuwer-Défossez 1999: 78).
} 
Tableau 17 Reconnaissances paternelles ${ }^{(1)}$ (pour 100 enfants nés hors mariage) selon l'âge de l'enfant

\begin{tabular}{|l|c|c|c|c|c|c|c|}
\hline & \multicolumn{7}{|c|}{ Année de naissance } \\
\cline { 2 - 8 } & $\mathbf{1 9 6 5}$ & $\mathbf{1 9 7 0}$ & $\mathbf{1 9 7 5}$ & $\mathbf{1 9 8 1}$ & $\mathbf{1 9 8 5}$ & $\mathbf{1 9 9 0}$ & $\mathbf{1 9 9 4}$ \\
\hline Reconnaissance avant un mois $^{*} 33,4$ & 33,8 & 1,1 & 63,2 & 68,5 & 67,5 & 80,6 \\
\hline proportion finale $^{(2)}$ & 76,2 & 76,7 & 80,6 & 84,5 & 88 & 90,5 & 92 \\
\hline
\end{tabular}

(1) Il s'agit de l'ensemble des reconnaissances par le père, y compris celles effectuées devant notaire ; les filiations paternelles établies lors d'un jugement sont comprises.

${ }^{(2)}$ Il s'agit de la proportion totale de reconnaissances paternelles (quelle qu'en soit la date).

Source : Munoz-Pérez ; Prioux (1999a : 484)

Premièrement, la forte progression du nombre des reconnaissances entre 1970 et 1975, plus de 17 points, est liée à la réforme du 3 janvier 1972 sur la filiation, à la possibilité de reconnaître les enfants adultérins, et au meilleur statut de l'enfant naturel reconnu (MunozPérez ; Prioux 1999a). Les années 1980 marquent une autre césure et le pourcentage de reconnaissances paternelles avoisine $70 \%{ }^{95}$. Dès la fin des années 1990, on dépasse les $80 \%$.

Le phénomène des reconnaissances prénatales est relativement récent et il s'est très développé depuis la deuxième moitié des années 1980 (Munoz-Pérez ; Prioux 1999 : 486). A l'heure actuelle, pour les générations nées en 1994, 37 \% des enfants nés hors mariage ont été reconnus avant la naissance, par le père et la mère. Les reconnaissances prénatales unilatérales sont minoritaires.

Ainsi, le phénomène des reconnaissances prénatales est essentiellement dû aux reconnaissances conjointes des deux parents (cf. Tableau 18); il s'inscrit dans l'exercice commun de la parentalité.

Tableau 18 Proportion d'enfants reconnus avant la naissance par le père et/ou la mère (pour 100 enfants nés hors mariage)

\begin{tabular}{|c|c|c|c|c|c|c|c|}
\hline \multirow[b]{2}{*}{ PROPORTION D'ENFANTS RECONNUS PAR } & \multicolumn{7}{|c|}{ Année de naissance } \\
\hline & 1965 & 1970 & 1975 & 1980 & 1985 & 1990 & 1994 \\
\hline - le père et la mère & 0,9 & 1,3 & 4,6 & 79 & 13,4 & 23,3 & 35,8 \\
\hline - le père et la mère conjointement & $\mathbf{0 , 8}$ & 1,1 & 4,5 & 7,6 & 13,1 & 22,7 & 35,0 \\
\hline - la mère seule & 3,4 & 4,7 & 4,8 & 5,9 & 7,4 & 6,8 & 5,8 \\
\hline - le père seul & 0,4 & 0,3 & 0,5 & 0,4 & 0,3 & 0,6 & 1,1 \\
\hline
\end{tabular}

Source : Munoz-Pérez ; Prioux 1999a : 486

\footnotetext{
${ }^{95}$ Depuis les années 1990, plus des trois-quarts des enfants hors mariages sont reconnus dans le premier mois après la naissance. Ceci s'explique par la nature des unions dans les familles naturelles : il s'agit d'unions de fait qui accèdent ensuite à la parentalité. Le nombre des reconnaissances paternelles, qui concernent plus de $90 \%$ des enfants nés hors mariage, montre à quel point la paternité au sein de cette forme familiale est assumée et revendiquée.
} 
Une reconnaissance prénatale conjointe signifie pour l'enfant, à la manière des enfants nés dans le mariage, qu'ils ont une double affiliation -paternelle et maternelle- dès la naissance, premier pas vers l'exercice conjoint de l'autorité parentale. Cette démarche concerne un très large tiers des familles naturelles et signe une inscription forte de la norme de coparentalité.

La situation professionnelle a un impact sur la reconnaissance parentale : «Les catégories sociales se différencient moins qu'autrefois dans leurs usages de la reconnaissance et de la légitimation. Toutefois, les reconnaissances prénatales conjointes, initiées par les classes supérieures, reste une pratique socialement marquée (...) les mères exerçant une profession intermédiaire ou supérieure obtiennent une reconnaissance paternelle avant la naissance dans une proportion de deux fois et demi plus forte que les mères sans profession.» (Ministère de l'emploi et de la solidarité 2000). «Ainsi, c'est dans les catégories supérieures nous avons vu qu'elles ont encore aujourd'hui moins souvent des enfants nés hors mariageque les pères reconnaissent leurs enfants avant la naissance, le plus souvent et ceci conjointement avec la mère : ils les dotent ainsi dès la naissance d'une filiation à l'égard des deux parents, comme les enfants nés dans le mariage.» (Munoz-Pérez ; Prioux 1999b : 860). Les catégories supérieures, qu'elles choisissent le mariage ou la cohabitation, ont le souhait d'inscrire l'enfant dans un double lien parental, qu'il soit conséquent à une légitimation ou à une reconnaissance conjointe prénatale. L'enfant, dès sa venue au monde, est alors inscrit dans un rapport de parentalité où la double filiation est établie. Inversement, les pratiques unilatérales de la reconnaissance sont beaucoup plus répandues dans les couches de milieux populaires $^{96}$.

L'inactivité a une influence sur les reconnaissances prénatales : lorsque la mère est inactive, la probabilité pour que l'enfant soit reconnu avant la naissance est faible, elle est d'autant plus faible que l'homme est lui-même inactif ${ }^{97}$ (Munoz-Pérez et Prioux 1999b : 865). «Ainsi par exemple en 1994, lorsque la mère est inactive, la probabilité d'une reconnaissance prénatale est de $38 \%$ si le père appartient à l'un des deux groupes professionnels [cadres et profession intermédiaire, employé], de $31 \%$ s'il est ouvrier et de $26 \%$ s'il est inactif.» (Munoz-Pérez et Prioux 1999b : 865). La probabilité d'une reconnaissance prénatale tombe de $67 \%$ chez un couple de cadres ou de profession intermédiaire à $45 \%$ chez un couple d'ouvriers et à $26 \%$ chez un couple d'inactifs (Munoz-Pérez et Prioux 1999b : 866).

Ces chiffres montrent l'importance de la catégorie socioprofessionnelle et de la situation professionnelle des parents, en premier lieu de la mère ${ }^{98}$, en second lieu du père, sur

\footnotetext{
${ }^{96}$ Mais la reconnaissance conjointe prénatale n'est pas l'unique privilège des couches intermédiaires et supérieures. Elle touche, quoiqu'en moindre proportion, l'ensemble des couches sociales C'est dans le milieu ouvrier que les parents légitiment le moins souvent leurs enfants (Munoz-Pérez et Prioux 1999b : 865).

${ }^{97}$ Le 29ème rapport sur la situation démographique de la France, du ministère de l'emploi de la solidarité, paru en 2000, basé sur les travaux de Munoz-Pérez et Prioux, nous renseigne de façon très précise, sur les circonstances et les caractéristiques de la reconnaissance paternelle et parentale des enfants nés hors mariage. Le rapport développe le cas des couples de parents inactifs, groupe marginal mais en augmentation : les parents tardent davantage à reconnaître leurs enfants et ils se marient plus rarement pour les légitimer (Ministère de l'emploi et de la solidarité $2000: 52$ - 53).

Cette remarque a d'autant plus d'importance, qu'actuellement l'ensemble des enfants nés hors mariage font l'objet d'une reconnaissance paternelle, les différences entre les catégories sociales s'amenuisent. Par contre, l'inactivité semble garder une signification particulière.

98 «L'appartenance socioprofessionnelle de la mère est prépondérante dans le comportement des pères vis à vis de la reconnaissance prénatale. » (Munoz-Pérez et Prioux 1999b).
} 
la reconnaissance prénatale. Cela influence donc la conception de la parentalité et sa nature «conjointe».

De façon générale, l'inactivité de la mère ne favorise plus la reconnaissance de l'enfant par le père comme cela a été le cas avant les années 1980. Aujourd'hui, l'inactivité tend à faire reconnaître l'enfant beaucoup plus tardivement par les parents. Il faut ajouter à cela que se sont les mères qui exercent une profession intermédiaire et supérieure pour qui l'enfant est le plus promptement reconnu, et surtout, lorsque le père appartient aux mêmes catégories sociales (Ministère de l'emploi et de la solidarité 2000 : 52).

«Les années 1990 nous offrent ainsi un panorama exactement inverse de celui des années 60. Les catégories populaires qui, autrefois, reconnaissaient le plus fréquemment et le plus vite leurs enfants, sont aujourd'hui en retrait, largement devancées par les catégories supérieures et moyennes. D'autre part, les enfants des mères inactives, plus souvent reconnus dans le passé, le sont plutôt moins aujourd'hui, en particulier avant la naissance.» (MunozPérez et Prioux 1999b : 866).

\section{II.B.1.2 La nouvelle conception du couple parental}

\section{II.B.1.2.1 Droits individuels ou droit parental $\underline{\text { ? }}$}

L'année 1970 marque le passage définitif vers une nouvelle définition de la norme de parentalité. La puissance paternelle a fait place à l'idée d'autorité parentale et pour la première fois, le parental est à la fois «paternel» et «maternel». On coupe alors définitivement avec l'idée de chef de famille, notion qui a été alors supprimée des textes.

De la même manière, certaines prérogatives attribuées à la mère, en particulier son exercice subsidiaire de l'autorité parentale dans les familles naturelles, sont aujourd'hui fortement critiquées. Une des propositions de la commission est d'inscrire, de façon symbolique, en tête des dispositions relatives à l'autorité parentale, un article 371-1 alinéa 2 selon lequel «sauf disposition légale ou décision judiciaire, nul ne peut faire obstacle à l'exercice par les pères et mères de leurs droits et devoirs de parents».

Une des volontés actuelle du législateur est de dépasser les droits individuels des pères et des mères : «il s'agit moins là de reconnaître de prétendus : «droits des pères» que de respecter le droit de l'enfant à être élevé par ses deux parents» (Dekeuwer-Défossez 1999 : 72).

Néanmoins, deux situations dans le droit actuel montrent de quelle manière les droits individuels peuvent venir contredire les droits de l'enfant et l'exercice paritaire de la parentalité : l'accouchement sous $\mathrm{X}$ et la recherche en filiation.

L'accouchement sous X est au croisement des droits de la mère et de ceux de l'enfant. Cette procédure d'abandon de l'enfant permet de respecter le souhait de la mère de maintenir son identité secrète (Art 341-1 du Code civil). Le droit de l'enfant à connaître ses deux parents est alors subordonné au souhait d'anonymat de la mère.

L'établissement judiciaire des filiations paternelles par la loi du 8 janvier 1993, exige que l'enfant (ou la mère qui le représente) fournisse au tribunal des présomptions ou indices graves pour ouvrir la procédure. Ce n'est qu'après avoir reconnu le bien-fondé de ces présomptions que le juge ordonne un examen comparé des sangs et/ou une expertise 
génétique. Ceci afin d'écarter des demandes malveillantes. Le père peut alors refuser en invoquant la loi du 29 juillet 1994 qui l'y autorise.

\section{$\underline{\text { II.B.1.2.2 }} \underline{\text { Les }} \underline{\text { rôles parentaux }} \underline{\text { et l'appréciation de leurs différences }}$}

En France, le discours d'interchangeabilité des rôles paternel et maternel dans les années 1980 a facilité et encouragé la présence du père dans les familles (Sullerot, 1992 : 170). «La paternité est associée à la parentalité, autour du partage des responsabilités. Si la notion de différence existe, c'est de manière diffuse» (Castelain-Meunier, 1992 : 94).

«Dans bien des cas, maternité et paternité peuvent désormais être traitées par le droit de manière identique : l'accès à la certitude sur la paternité et la promotion dans le droit de la filiation d'une éthique de la responsabilité, par nature égalitaire, permet de favoriser la recomposition du droit de la filiation autour de règles communes à la paternité et à la maternité : ainsi, les actions judiciaires consacrées à l'établissement forcé de la filiation ou à la contestation de la filiation, n'ont plus besoin de distinguer en fonction de la différent de sexe.» (Dekeuwer-Défossez 1999 : 54). Il existe un consensus social et moderne pour l'égalité des valeurs entre les rôles parentaux.

En revanche, certaines dispositions montrent les difficultés à appliquer juridiquement la norme égalitaire entre les parents. Les débats autours d'une réforme possible des règles de transmission du nom illustrent ce conflit entre les principes égalitaires et les prérogatives attachées aux rôles paternel et maternel.

Le droit français relatif à la transmission du nom, est resté l'un des plus rigides, malgré une timide réforme dans les années 1980. En effet depuis 1985, la loi autorise l'usage du double nom maternelle et paternel (dans l'ordre voulu). Mais seul le patronyme paternel peut être transmis. Cette suprématie patriarcale dans la dévolution du nom, a été débattue à l'Assemblée Nationale le 8 février 2001, lors de la présentation de la proposition de loi par Gérard Gouzes (PS, Lot-et-Garonne).

Cette proposition relance les débats sur le sens de la paternité et de la maternité. La transmission du nom, était jusqu'à présent un privilège du père. Cette réforme permet de promouvoir un principe d'égalité entre les sexes et, pour cette raison, elle est soutenue par la délégation aux droits des femmes. «Le plus important, finalement, c'est que l'on ouvre ici aussi une brèche dans la représentation traditionnelle de la parentalité, qui apparaît moins unique, exclusive, et qui s'oriente vers la pluriparentalité, la composition. Cette libération du nom va dans le sens d'une libération de nos conceptions de la parentalité.» (Entretien Michel Tort 2001).

Cependant, cette proposition ne fait pas l'unanimité et la discussion se polarise : les uns soutiennent cette réforme, au nom d'une plus grande égalité entre les hommes et femmes, et veulent se distancer d'une position réactionnaire selon laquelle la mère donne la vie et le père donne le nom. Les autres recommandent de conserver le nom comme «marqueur» de paternité.

«Quelques dispositions juridiques propres à la paternité ou à la maternité se justifient encore : elles se situent en quelque sorte en miroir et naissent du fait que la maternité suppose la gestation alors que la paternité, au contraire invisible, commence toujours par une désignation. (...) Dans cette optique, il paraît souhaitable de maintenir certaines spécificités liées à la gestation, ainsi que le principe d'une articulation de la nomination sur la paternité. (...) La prééminence paternelle dans la transmission du nom est héritée de l'histoire. Il est 
actuellement très fortement contesté au nom de l'égalité des sexes. Elle offre pourtant l'avantage d'équilibrer socialement les liens de filiation paternelle et maternelle : la maternité est toujours visible en raison de la gestation et la paternité est toujours a priori incertaine et découle d'une désignation : le port par l'enfant du nom du père est la manifestation sociale et publique de cette désignation de la filiation paternelle. (...) La commission pense qu'il n'est pas opportun de «fragilisé la transmission du nom du père», alors que, par ailleurs, les lignes directrices des réformes proposées s'attachent à ne pas dévaloriser la place de celui-ci dans le droit de la filiation ou de l'autorité parentale. Dans notre société, le lien social de maternité est toujours clairement repéré ; en revanche, la place du père a subi depuis quelques décennies de profondes mutations qui sont allées de paire avec le partage de l'autorité parentale (loi du 4 juin 1970) et l'accroissement du nombre des séparations entre le père et les enfants du fait de la désunion du couple : ouvrir la transmissibilité au nom de la mère créent de nouveaux déséquilibres et de nouveaux enjeux qui contiennent indirectement le risque de porter une atteinte supplémentaire à l'image de la paternité.» (Dekeuwer-Défossez 1999 : 54-68).

Devant le nombre de séparations, le nom serait la seule marque de filiation et de paternité devant l'affaiblissement du lien enfant-père après les séparations conjugales. Cet argument est également repris par différentes associations paternelles en particulier «l'enfant et son droit» : le nom de famille est tout ce que les enfants gardent de la filiation paternelle ${ }^{99}$.

Pareillement, certaines positions féministes n'adhérent pas totalement à cette nouvelle réforme : effectivement, le collectif droit des femmes estime que le nom de famille ne doit pas faire l'objet d'un choix entre la lignée du père et celle de la mère puisque les deux lignages sont à l'origine de l'enfant. Ce choix serait à l'origine de nouvelles tensions à l'intérieur de la famille, source de conflit mais également enjeu de pouvoir.

Cette réforme génère les incertitudes profondes mais elle reste un indicateur du mouvement réformateur concernant les nouvelles conceptions égalitaires de la parentalité. L'attribution du patronyme comme nom de famille ne serait plus la marque incontestable de paternité. La construction de la parentalité est ébranlée par la disparition de ce référent jusquelà immuable et l'oblige à se reconstruire autrement.

\section{SOUS-SECTION II.B.2 LA SEPARATION CONJUGALE ET L'EXERCICE DE LA PARENTALITE}

La partie qui suit traite de l'exercice de la parentalité dans les familles désunies. Les situations de désunions sont révélatrices des principes et des pratiques de parentalité pour deux raisons : la première est d'ordre démographique, en effet, la séparation conjugale touche de nombreuses familles et elle est devenue une modalité dont il faut tenir compte ; la seconde est d'ordre sociologique, la régulation sociale qui s'effectue dans le cas des séparations conjugales ne définit pas seulement le partage des responsabilités parentales, mais elle régule aussi la relation entre les deux parents. Par-là, elle définit explicitement, à un niveau normatif, les rôles parentaux et leurs modes d'exercice. Cette ingérence sociale est beaucoup plus visible au moment de la séparation parentale que dans les familles unies, car, pour ces dernières, on estime que l'application des règles de parentalité relèvent strictement de la sphère privée.

\footnotetext{
${ }^{99}$ Aujourd'hui, l'écrasante majorité des enfants naturels porte le nom de leur père (INED, 1999).
} 


\section{II.B.2.1 Le partage de l'autorité parentale comme modèle de régulation de l'après divorce}

Comme on l'a rappelé précédemment dans la partie historique, les réformes du divorce du 11 juillet 1975 marquent définitivement une rupture avec le principe unique de faute. La nouvelle procédure permet l'application d'une norme non-conflictuelle de la séparation conjugale, laquelle modifie alors la gestion du parental. On abandonne ainsi la référence de faute dans la procédure de divorce pour l'attribution de la garde des enfants (Art. 287 du Code civil) : Il n'y a plus de bon et de mauvais parent. La loi découple la gestion du lien générationnel de la faute conjugale : le parental s'émancipe du conjugal.

$\mathrm{Au}$ cours des années 1970 et 1980 de nouvelles pratiques jurisprudentielles se développent marquant le début d'une pratique de garde conjointe de l'enfant (cf. section suivante).

Sur le plan politique, le principe de non-conflictualité de la séparation parentale ainsi que celui de la présence bilatérale des parents pour le bien de l'enfant domine de plus en plus les discours : «Il s'agit d'instituer une véritable responsabilité parentale égale, doublée d'une répartition équitable de l'hébergement. Seul le défaut d'accord des parents entraînerait le tribunal à statuer, ce qui aurait pour double avantage de désengorger les juridictions, (en 1980, près de $60 \%$ de la totalité des affaires civiles concernait les interventions en justice en matière de droit de la famille), et de dédramatiser les procédures du divorce et de séparation» (exposé des motifs de l'avant projet de loi, mis à l'étude au sein du PS, 1980).

Cette incitation au «non-conflit» est explicitement exprimée au nom de l'enfant. On évoque que le conflit met l'enfant dans une situation de déchirement l'obligeant à choisir entre ses deux parents : «L'intérêt de l'enfant réside essentiellement dans sa possibilité de pouvoir pareillement aimer et être aimé de ses deux parents, à en faire un enfant qui soit, dans la mesure du possible, au-dessus du conflit parental et qui ne soit pas amené, comme c'est trop souvent le cas, dans une garde distincte, à prendre parti. Cette hypothèse est exclue en cas de conflit persistant entre ceux-ci. Il convient donc de recourir chaque fois qu'il est possible aux décisions judiciaires qui, renonçant à l'attribution unilatérale de l'enfant à un seul parent, empêcheront soit l'éviction, soit la démission de l'autre et avec la garde conjointe ou le nouveau principe de garde associée, offriront aux deux la possibilité de dépasser leurs griefs pour remplir ensemble leur fonction parentale» (projet de loi de 1981 N$^{\circ} 36$ enregistré à la présidence de l'Assemblée nationale le 2 juillet 1981, annexe au procès-verbal de la séance du 8 juillet 1981).

La fin des années 1980 propose un nouveau principe d'exercice de la parentalité après la séparation des parents. Il vient s'ajouter au modèle, jusque là unique, de l'exercice unilatéral. Par la loi «Malhuret» de 1987 l'expression «garde des enfants» est supprimée en faveur de celle «d'autorité parentale». Elle offre la possibilité aux parents de choisir entre un exercice conjoint ou unilatéral. Le libellé de la loi marque la préférence du législateur pour la nature «conjointe» de l'autorité, la famille devant surmonter la rupture conjugale : la loi «doit permettre de maintenir la famille au-delà même de la séparation» (propos de C. Malhuret, J.O, Assemblée nationale, 1987).

L'exercice en commun de l'autorité parentale par les parents divorcés est la règle de principe depuis 1993. Elle n'est plus une possibilité, mais elle devient un principe de gestion du risque familial. Nous considérons cette loi comme l'aboutissement institutionnel de la reconnaissance du couple parental. «En cas de séparation, on ne part pas de l'idée qu'un seul 
des parents exercera l'autorité parentale, on part de l'idée inverse, ce qui renverse quand même très profondément la problématique générale. C'est le point de départ. Puis on voit si c'est réalisable ou non, s'il y a des difficultés, s'il n'y en a pas. Cela situe déjà différemment les parents au moment de la séparation» (Entretien avec Madame le Juge aux Affaires Familiales 1997). Cette conception présuppose une entente entre les parents.

L'article 287 al. 1 et 2 du Code civil dispose qu'en cas de divorce «l'autorité parentale est exercée en commun par les deux parents. Le juge définit à défaut d'accord amiable ${ }^{100}$ ou si cet accord lui apparaît contraire à l'intérêt de l'enfant, le parent chez lequel les enfants ont leur résidence habituelle. Si l'intérêt d'un enfant le demande, le juge peut confier l'exercice de l'autorité parentale à l'un des parents». En pratique, le partage en commun de l'autorité parentale est retenu dans $90 \%$ des cas (Fulchiron 1997) ${ }^{101}$.

Un projet de réforme du droit civil de la famille a été initié en 1997. Ses grandes lignes ont été réaffirmées le 15 juin 2000 durant la conférence de la famille: harmoniser les filiations légitimes, naturelles et adultérines; améliorer le droit successoral du conjoint survivant; simplifier le divorce et développer la médiation; faciliter les pratiques de résidence alternée en supprimant du Code civil l'application faite aux tribunaux de fixer la résidence habituelle de l'enfant. Enfin, la place de la médiation sera accrue : en cas de conflit familial, le juge pourra l'ordonner, sans avoir à obtenir l'accord des parties.

La volonté du gouvernement est de faciliter les instruments de conciliation et de partage des responsabilités parentales entre les deux parents pour une meilleure parité parentale.

\section{II.B.2.2 La présence des deux parents}

Jusque dans les années 1970, les pratiques jurisprudentielles donnaient systématiquement la garde à la mère.

«La réponse jurisprudentielle est : quand les enfants sont petits, on les laisse plutôt avec leur mère, avec l'idée qu'un enfant tout petit a plutôt besoin de sa présence (...) en cas de séparation on voit apparaître certaines règles, par exemple, on ne donnera pas la résidence $\mathrm{d}$ 'enfant en bas âge au père, sauf si la mère est incapable. C'est une règle implicite qui dessine la jurisprudence. Ce n'est que lorsque la mère est totalement défaillante, folle, toxicomane, incapable, qu'on accorde la résidence au père, s'il y a un père. Voilà comment la jurisprudence dessine la jurisprudence de l'enfant. Lorsque l'enfant est un peu plus âgé, la jurisprudence appréciera quel parent, il n'y a pas de règle générale, on est dans des considérations beaucoup plus au cas par cas, on apprécie soit l'avis des enfants qui souhaitent précisément vivre avec tel ou tel parent, soit l'environnement familial de tel ou tel parent qui serait plus ouvert ou pas ; au moins quand les enfants sont petits. Dans la majorité des procédures de divorce avec des enfants en bas âge, oui, c'est la mère qui a la garde des enfants.» (Entretien avec Madame le juge aux affaires familiales 1997).

\footnotetext{
${ }^{100} \mathrm{Au}$ moment de la séparation, les accords préalables des parents sur la garde de l'enfant, sont uniquement obligatoires dans le cas des divorces sur requête conjointe, et pas dans les autres procédures de divorce. Et dans le cas des séparations de familles naturelles, le législateur n'a pas de droit de regard sur les conventions liées à l'enfant.

${ }^{101}$ Actuellement l'article 286 du Code Civil stipule «le divorce laisse se substituer les droits et devoir des pères et mères à l'égard de leurs enfants, sous réserve des règles qui suivent ». Les projets de réforme de la commission vont jusqu'à souhaiter que les aléas de la vie du couple ne remettent pas en cause la fonction parentale et propose de reformuler l'article 286 comme suit : «le divorce n'emporte par lui-même aucun effet sur le droit et devoirs de parents à l'égard de leur enfant» (Dekeuwer-Défossez 1999 : 78).
} 
En France, ces pratiques concernent l'attribution de la résidence principale de l'enfant. Elle est resté longtemps fermée aux pères, qui d'ailleurs se risquent très peu à la demander. Cela est du à une jurisprudence en défaveur des pères et une difficulté à penser le divorce autrement que dans un paradigme de conflit.

$\mathrm{Au}$ cours des années 1970 et 1980, de nouvelles pratiques jurisprudentielles se développent marquant le début d'un exercice conjoint de la garde, la garde alternée étant de plus en plus souhaitée. Le 21 mars 1983, la cour de cassation rend un arrêt qui validite la garde conjointe mais refuse celle de la garde alternée. Elle réaffirme sa position avec un nouvel arrêt le 2 mai 1984 en faveur de la garde conjointe.

Le colloque sur la coparentalité, organisé par l'association Typhaon ${ }^{102}$ nous a permis d'être confrontée aux discours et aux pratiques des professionnels du Barreau sur la question des pratiques jurisprudentielles relatives à l'autorité parentale conjointe. Même s'il n'y a pas l'unanimité entre les magistrats, comme l'a rappelé Madame le Juge aux Affaires Familiales en entretien (1997), deux grandes appartenances se dégagent :

Les uns, défenseurs de la norme de l'institution familiale, légitiment des pratiques jurisprudentielles qui accordent la résidence principale à la mère.

Les autres, appartenant au mouvement «éducatif», favorisent la présence éducative du père, la circulation de l'enfant entre les deux parents et le partage du temps de présence. Ils s'assurent que la place de chaque parent sera respectée. L'idée n'est pas de sanctionner un parent mais plutôt d'assurer l'accès de l'autre parent à l'enfant.

La majorité des magistrats partage l'idée selon laquelle la présence du père doit être favorisée après le divorce. «Un principe jurisprudentiel est en train de se dessiner aujourd'hui : quel est le parent qui favorise le mieux les relations avec l'autre parent ? C'est à dire le parent qui fait obstruction à la présence de l'autre parent se verra sanctionné, c'est un critère qui est relativement récent. C'est un critère d'ailleurs inspiré d'une jurisprudence américaine (californienne), mais que l'on voit apparaître de plus en plus en France.» (Entretien avec Madame le juge aux affaires familiales 1997). Il s'agit donc de permettre aux deux parents d'avoir accès à l'enfant.

Ce critère rentre en concurrence avec les pratiques jurisprudentielles liées au sexe du parent, surtout pour les jeunes enfants. Les règles de visite d'un week-end sur deux et l'attribution d'une résidence principale pour l'enfant, sont vivement discutées, néanmoins encore largement pratiquées.

Une autre pratique des magistrats illustre bien la tendance actuelle à favoriser le couple parental. Les magistrats aimeraient pouvoir fixer, plus souvent, une pension alimentaire symbolique pour le père (Colloque 1997), car, dans les nombreux cas où le père n'est pas solvable, la possibilité de fixer une pension, même très faible, lui permettrait de s'acquitter de son devoir de soutien alimentaire. Le lien, même symbolique, serait alors maintenu. Or, le droit social limite cette pratique. En effet, cette «pension alimentaire symbolique» désavantageraient les mères puisque cela supprimeraient leur droit à l'ASF. Car si la pension, par exemple de 100 Fr. [15 Euros], est réellement payée, alors la mère ne remplit plus les critères d'attribution, tandis que l'ASF pourrait être plus avantageuse pour elle.

\footnotetext{
${ }^{102}$ Le colloque sur la «coparentalité» a eu lieu à la Maison du Barreau de Paris le 12 juin 1997.
} 
Les couples eux-mêmes choisissent un exercice partagé de la parentalité. «On voit se dessiner la préoccupation de parvenir à un accord pour les enfants, même si la séparation est difficile ou douloureuse. Le souci de parvenir à un accord pour les enfants, c'est surtout, que les deux parents demeurent présents dans l'éducation des enfants d'une manière ou d'une autre. Et en cela, moi je considère que le principe d'autorité parentale correspond réellement à une réalité, et pas simplement à une fiction juridique, purement imaginaire, ou qu'un législateur a voulu imposer ses vues, comme cela a souvent été dit, ou l'idée d'un couple qui se maintiendrait de manière mythique, moi je constate, au jour le jour, que les parents font en sorte qu'ils existent l'un et l'autre, après une séparation».

«Il y a des accords très élaborés pour que les pères soient très présents, $\mathrm{y}$ compris une fois par semaine. Non seulement ils sont plus présents, mais il y a un accord pour que les deux parents participent à l'éducation des enfants (...) on voit de nombreux accords, où ce n'est pas un week-end sur deux, mais au moins une fois par semaine. C'est une chose très usuelle à Paris, quand il y a un accord entre les parents» (Entretien avec Madame le juge aux affaires familiales 1997).

Malgré cette tendance, le père est encore très souvent absent de l'après-divorce. Il voit peu ses enfants. Plus d'un tiers des enfants perdent tout contact avec le parent non-gardien ${ }^{103}$ (Mendras 1997 : 220).

Globalement, plus d'un enfant français sur deux n'a pas de contact suivi avec son père (Villeneuve-Gokalp 1993). La relation s'atténue avec le temps. Plus la séparation est ancienne, moins le lien est intensif ${ }^{104}$. Quand la séparation est inférieure à 6 ans, $40 \%$ des enfants continuent à voir leur père ( $25 \%$ quand elle est plus ancienne).

Le mariage est un autre facteur déterminant. La différence est très forte pour les enfants qui ont vécu moins de 2 ans avec leur père : $61 \%$ des enfants de cohabitants ont perdu tout contact avec lui, contre $32 \%$ des enfants de divorcés. Mais dès que la cohabitation a duré plus de deux ans, l'écart disparaît. Au contraire, «les couples choisissant l'union libre sont plus nombreux que les couples légitimes à préserver les liens père-enfant, en adoptant un mode de garde partagée» (Villeneuve-Gokalp 1993 : 77).

Enfin, plus le milieu socio-économique du père est élevé, plus les rapports avec l'enfant sont réguliers. Cela s'explique par les moyens qu'il peut mettre en œuvre pour préserver la relation.

\section{II.B.2.3 La coopération du couple parental dans l'intérêt de l'enfant}

Comme on l'a vu précédemment, le principe de l'autorité parentale conjointe a pour but d'inscrire normativement l'entente entre les deux parents, dans les familles «unies» ou «désunies». Elle s'exprime sous la forme de la norme de «non-conflictualité» ou de «partenariat».

\footnotetext{
${ }^{103}$ Un autre souhait de la commission est de combattre les désaffectations parentales, et en particulier le détachement de l'un des parents. Elle propose d'inscrire dans les textes «quelles que soient les modalités d'exercice de l'autorité parentale, chacun des pères et mères doit maintenir des relations personnelles avec enfant.» (Dekeuwer-Défossez 1999 : 87).

${ }^{104}$ Néanmoins, plus le père a connu son enfant avant la séparation, plus le lien sera entretenu. Si la vie commune avec l'enfant a durée au moins 5 ans, les relations restent plus étroites malgré le temps. 30\% le voit même longtemps après la séparation.
} 
I. Théry analysa les dossiers de 253 affaires de divorce. Trois valeurs fondamentales forment le modèle du «bon divorce» : la stabilité (une bonne moralité parentale), le dépassement de la crise conjugale (le divorce doit être effacé) et la stigmatisation du conflit (l'interdit suprême est le conflit parental car cela nuirait à l'enfant). L'enfant doit avoir accès à ses deux parents et le couple parental a l'obligation normative de coopérer, chaque parent se doit de respecter la fonction de l'autre. La préoccupation de l'intérêt de l'enfant et de ses droits légitime cette nouvelle conception de la parentalité. «(...) L'indéfinition de l'intérêt de l'enfant ne renvoie par à la pure objectivité, mais au contraire, des nouvelles normes très puissantes ont progressivement émergé (...)» (Théry 1993 : 143).

La définition de l'intérêt de l'enfant influence le critère d'attribution de la garde (ou de sa résidence). En fait, la jurisprudence s'appuie sur la définition des dangers qui menace l'intégrité et le bien-être de l'enfant. Elle fonde les règles de gestion de la séparation familiale. Paradoxalement, l'intérêt de l'enfant est une notion floue sur laquelle tout le monde est unanime. «Tout ce qui peut être bon pour lui» est donc défini par contraste, à partir de ce qui n'est pas dans son intérêt ${ }^{105}$.

Il serait faux d'assurer que l'exercice de la «coparentalité» serait seulement conflictuel en cas de séparation des parents. Le législateur a également statué en cas de conflit dans les familles légitimes, comme le montrent les dispositions suivantes : «si les pères et mères ne parviennent pas à s'accorder sur ce qu'exige l'intérêt de l'enfant, la pratique qu'ils avaient précédemment pu suivre dans des occasions semblables, leur tiendrait lieu de règles. À défaut d'une telle pratique, ou en cas de contestation sur son existence ou son bien-fondé, «le parent le plus diligent pourra saisir le juge aux affaires familiales» qui statuera après avoir tenté de concilier les parties.» (Art. 372-1-1 du Code civil, Loi n93-vingt-deux du 8 janvier 1993).

«Au quotidien, c'est l'autorité parentale qui permet aux parents d'exercer leur responsabilité d'élever leur enfant. La stabilité et la force de ce lien sont nécessaires à la fois pour donner aux parents les moyens de leur mission, et pour assurer à l'enfant l'équilibre et les repères dont il a besoin. Cette autorité mérite d'être revalorisée, et plus clairement confiée aux deux parents ensemble, quelles que soient les vicissitudes de leur vie de couple. Droits de l'enfant et responsabilité des parents ne sont pas antinomiques mais s'appuient l'un sur l'autre dans une dynamique de croissance (...).» (Dekeuwer-Défossez 1999 : 22).

\section{II.B.2.4 La garde alternée et la médiation pour une plus grande coopération}

\section{La garde alternée}

La «résidence paritaire» ou «résidence alternée» est un mode d'organisation de l'après-divorce, qui permet à l'enfant d'avoir deux domiciles où il réside alternativement. Elle est un instrument privilégié de la «coparentalité», car elle s'appuie sur la présence des deux parents.

La «résidence alternée» trouve ces origines dans «la garde alternée» du divorce par consentement mutuel de 1975. Elle devait remplacer la traditionnelle répartition entre l'hébergement principal (accordé à la mère dans $80 \%$ des cas) et le droit de visite pour le

\footnotetext{
${ }^{105}$ Pour cette raison, I. Théry parle d'une «illusion absolue d'une définition scientifique de l'intérêt de l'enfant, susceptible de se décliner ensuite en propositions législatives» (Théry 1993 : 127).
} 
second parent. Cette pratique des «1-3-5» cantonnait les droits de visite, généralement du père, à un week-end sur deux et à la moitié des vacances scolaires.

Les pratiques de la garde alternée n'ont pas de véritables fondements juridiques. Néanmoins, le projet de réforme du droit la famille, discuté le 31 janvier 2001 en conseil des ministres, devrait lui donner une assise juridique et favoriser son exercice.

Les deux derniers rapports commandés par le gouvernement sur la famille (Théry 1998 ; Deuwerker-Defossez 1999) ont appelé à une reconnaissance juridique de la règle de l'hébergement partagé. Le gouvernement envisage donc de faire sauter le verrou législatif de l'article 287 du code civil qui oblige, à l'heure actuelle, le juge à statuer sur la résidence habituelle de l'enfant. Il favorise ce qu'il considère être un instrument d'apaisement des conflits et promeut ainsi la parité parentale.

La résidence habituelle, inscrite dans les textes actuels, véhicule une conception unilatérale de l'autorité parentale. En effet, «on constate que trop souvent les débats se focalisent sur la définition de cette résidence habituelle qui donne à son titulaire l'impression d'être un parent principal, et à l'autre parent celle de ne jouer qu'un rôle secondaire. Quant au tiers, il semble n'avoir que trop tendance à ne traiter qu'avec le parent auprès de qui l'enfant à sa résidence habituelle. De ce point de vue, la résidence habituelle tend à se substituer à l'ancienne garde, au risque de nier ce qui fait l'essence même de l'exercice en commun de l'autorité parentale, c'est-à-dire, le partage par les pères et mères de leur responsabilité de parents.». La résidence habituelle donnait effectivement l'impression de récompenser le parent «non fautif» (en particulier la mère). Cela favorise dans certaines institutions, particulièrement à l'école, une représentation unilatérale de l'autorité parentale. «Il paraitrait donc opportun de recourir à une formule très générale : seraient déterminées «les modalités d'exercice de l'autorité parentale», le juge veillant, en toute hypothèse, à ce que soit protégé l'intérêt de l'enfant. Une telle formule n'empêcherait nullement les parents ou le juge de fixer une résidence habituelle s'il l'estime nécessaire, du moins ne serait-ce plus une obligation.» (Dekeuwer-Défossez 1999: 80 2).

En 1994, si la pratique des résidences alternées ne concerne que $6 \%$ des parents après la séparation (INED 1994), elle prend progressivement de plus en plus d'importance. En effet, «les jeunes pères sont conscients que la paternité, auparavant garantie par institution, passe désormais par la construction, et l'entretien, du lien père-enfant» (Castelain-Meunier 1998). Cette reconstruction de la paternité a lieu alors que la maternité est en redéfinition sous l'influence de l'activité professionnelle des femmes; l'institution familiale ne garantissant plus, par les anciens repères, l'identité parentale.

Un nouveau modèle, «la coparentalité», trouve écho dans la parité des sexes. Il s'agit d'une redéfinition des rapports parentaux plus que d'une disparition complète des repères, la norme de «coparentalité» étant le cadre moderne de la définition de la paternité et de la maternité.

Malgré la loi de 1993 instituant le principe le partage l'autorité parentale conjointe, d'anciennes pratiques relatives à l'attribution de la résidence se maintiennent. Elles étaient conformes aux arrêtés jurisprudentiels antérieurs, en particulier au verdict de la cour de cassation de 1984. Elle se prononce contre l'alternance. A l'époque, le parent qui avait la garde de l'enfant disposait aussi de l'autorité parentale, et la cour a jugée l'exercice alterné impossible. 
En effet cette pratique ne fait pas l'unanimité chez les juges, les avocats et les psychologues $^{106}$. La définition de l'intérêt de l'enfant par les psychologues et les psychiatres influence les positions des magistrats ${ }^{107}$ : à l'heure actuelle, de nombreuses études tentent de rétablir un avis favorable par rapport à la garde alternée. Un arrêt du 10 février 1999 de la cour d'appel de Paris a jugé que «rien ne permet d'affirmer que l'hébergement partagé soit néfaste à l'enfant». Pour une partie de ces praticiens, la situation conflictuelle serait envenimée par la garde alternée qui ne peut être imposée aux parents.

«Lors de ma recherche, en 1994, seuls deux juges aux affaires familiales sur les 20 interrogés n'étaient pas opposés à la résidence alternée, depuis, les choses ont évoluées, mais on est encore loin d'atteindre une majorité de juges favorables. Certains JAF refusent toujours quand même cette solution est proposée par les deux parents.» (Entretien avec Gérard Neyrand 2000).

Outre les associations des pères, comme «SOS papa» ou la «fédération des mouvements de la condition paternelle» qui revendiquent un partage équitable de la résidence de l'enfant, de nombreux témoignages d'avocat mais également de juges, montrent un intérêt grandissant de la part des parents pour un partage de la résidence.

Les JAF accusés publiquement de plus en plus souvent de sexisme envers les hommes, et de donner systématiquement la garde à la mère, se montrent de plus en plus ouverts aux pratiques de la parité parentale. Mais l'évolution reste lente et rencontre de nombreuses résistances.

Les positions entre le ministère de la famille, qui se fait l'avocat des pratiques des individus, et le ministère de la justice qui représente le système judiciaire et en particulier les pratiques des juges, sont visibles dans les débats autour de la réforme actuelle sur le droit la famille. Le ministère de la famille, initiateur de la réforme, estime que la résidence alternée doit devenir un mode d'organisation aussi banale que les autres, quitte à revoir l'attribution des prestations sociales relatives à l'enfant (notamment lallocation logement). La résidence alternée est considérée comme un moyen d'apaiser les conflits en poussant à la parité parentale. Au contraire, pour le ministère de la justice et l'association des avocats de la famille, la garde alternée accentuerait le conflit des parents au détriment de l'enfant.

\section{La médiation}

Aujourd'hui, la médiation familiale a pour objectif d'aider les parents à mieux gérer leurs désaccords parentaux sans décision autoritaire extérieure ; elle est également en dehors du système judiciaire. La médiation familiale ne prend pas partie, contrairement à l'enquête sociale.

\footnotetext{
${ }^{106}$ Dans le cas des psychanalystes, Françoise Dolto plaidait dans les années 1980 contre la garde alternée la déclarant très néfaste pour les enfants. En particulier parce qu'elle imposerait la fréquentation de deux écoles pour l'enfant. Elle renforce l'idée selon laquelle la garde alternée déstabilise l'enfant.

${ }^{107}$ Gérard Poussin préconise une répartition plus équitable par la résidence alternée. «Ce n'est pas forcément du 50/50. C'est aux parents de trouver une solution adaptée aux besoins de l'enfant, avec l'aide des travailleurs sociaux. Mais, pour cela, il faudrait un changement de mentalité. La résidence alternée n'a pas la côte chez les juges (...) en culpabilisant les gens qui sont pour la garde alternée. On dit que c'est l'horreur pour l'enfant, alors que le peu d'étude réalisée montre que ce n'est pas vrai.» (Entretien avec Gérard Poussin, 2000).
} 
La commission insiste sur le fait qu'en situation de conflit aigu, l'accent doit être mis sur la recherche du dialogue, afin de favoriser l'émergence d'accord au sujet des enfants ${ }^{108}$. Il s'agit d'éviter une réponse judiciaire, la procédure pénale venant souvent aggraver les choses. Le médiateur souligne la place centrale de l'enfant, pour dénouer le conflit ${ }^{109}$ : l'enfant se retrouve donc au centre de la problématique parentale et non plus conjugale. La médiation familiale tente de répondre aux enjeux normatifs de la coparentalité.

«La restauration du dialogue entre les parents, une plus grande autonomie dans l'établissement de leurs accords, sont de nature à garantir, pour l'avenir, mieux que par une décision judiciaire ponctuelle, le respect des règles de la coparentalité. (...) C'est pourquoi le groupe propose d'accroître le pouvoir d'intervention du juge dans la vie privée, lorsque les parents sont enlisés dans un conflit aigu qui fait obstacle à tout contact entre l'enfant et l'un de ses parents : «en cas de violation manifeste et réitérée des droits et devoirs de l'autre parent, le juge peut imposer la rencontre des parents en présence d'un médiateur» (DekeuwerDéfossez 1999 : 180). La commission estime qu'il peut être préférable, dans un premier temps, de contraindre les parents à se rencontrer en présence d'un médiateur afin d'examiner les moyens à mettre en œuvre pour respecter les règles de la «coparentalité».

Autant la médiation familiale est reconnue comme un instrument moderne de l'exercice non conflictuel de la parentalité, autant l'enquête sociale est dénoncée comme un instrument pervers qui maintiendrait des pratiques conflictuelles et traditionnelles dans les procédures de divorce et de séparation. Elles servent actuellement de recours au juge des affaires familiales pour statuer sur l'exercice de la parentalité lorsque le désaccord parental persiste.

L'enquête sociale introduite par ordonnance le 12 avril 1945, modifiée par la loi de 1975 (Art. 287-1), puis en 1987 (Art. 287 -2), reste l'instrument d'une vision encore conflictuelle du divorce et de la séparation parentale. Elle incite à la déresponsabilisation d'un des parents, le marginalisant, l'affaiblissant dans les rapports de pouvoir. Et effet, elle est souvent un rapport déséquilibré de la situation, un ensemble d'arguments pour avoir raison ou tout au moins pour ne pas avoir tort. Elle concourt à l'aspect conflictuel en prenant partie. Elle est souvent ordonnée comme réponse à un désaccord du couple, or, elle-même, de par nature envenime les conflits car elle recherche la faute de l'un des parents.

Les écueils de l'enquête sociale sont nombreux, on évoquera la dérive psychologique induite par le jugement moral et l'avis motivé de l'enquêteur, à l'intention du juge des affaires familiales, mais aussi les orientations traditionnelles concernant la définition de l'intérêt de

\footnotetext{
${ }^{108}$ La commission Dekeuwer-Défossez (Dekeuwer-Défossez 1999 : 81) propose d'aller beaucoup plus loin et de faire «des conventions passées entre parents, le mode de règlement de principe des conséquences de la séparation à l'égard des enfants». La convention propose alors de valoriser, au plus haut point, la convention établie entre les parents au moment de la séparation (quel que soit le mode de séparation des parents), et de faire de cet accord, une convention qui organiserait les modes de l'exercice de l'autorité parentale, mais également les règles de la contribution à l'entretien et à l'éducation de l'enfant. Il y aurait alors possibilité, quel que soit le couple parental (d'origine légitime ou non ), de faire homologuer cette convention par le juge aux affaires familiales. Le couple parental serait donc alors valorisé par le fait que le juge parte d'abord de la convention, établie volontairement entre les parents, tout en lui laissant sa mission de gardien de l'intérêt de l'enfant. Ils seraient les premiers responsables de l'organisation du couple parental et de l'exercice des fonctions maternelles et paternelles.

${ }^{109}$ Une psychologue d'un cabinet de médiation, lors du colloque sur la coparentalité (Colloque 1997) évoque que ce qui permet de débloquer de nombreuses situations, c'est d'insister sur la souffrance de l'enfant lors des entretiens avec les parents «et je vous assure que cela marche». La norme «anti-conflictuelle» de la logique parentale est lisible dans les pratiques sociales.
} 
l'enfant, et l'attribution de la garde à un seul parent (la mère) suivant la conception traditionnelle du parent «fautif».

Le mouvement de la conciliation paternelle (fmcp) dénonce de nombreuses résistances dans le système judiciaire, en particulier dans les enquêtes sociales ${ }^{110}$. Le mouvement de la conciliation paternelle définit l'intérêt primordial de l'enfant comme celui de garder ses deux parents. «L'enfant a besoin de stabilité affective, du maintien de la continuité de ses repères où les deux parents doivent être dans une relation égalitaire, par principe, sans discrimination, sans déséquilibre des pouvoirs entre eux, sans que l'enfant ne devienne ni un enfant chantage, pas plus d'un enfant-otage, ni encore un enfant-marchandage.».

\section{II.B.2.5 Les quatre principes de la coparentalité}

Tout d'abord, le premier principe de l'autorité parentale est sa nature de fonction «c'est-à-dire un ensemble de droits et de devoirs corrélatifs : chaque droit reconnu aux pères et mères n'est que l'autre face d'un devoir qui leur ait imposé ; cette fonction est finalisée : elle appartient aux pères et mères pour protéger l'enfant et assurer son éducation.» (DekeuwerDéfossez 1999 : 71).

Le second principe est celui de d'égalité «la loi a posé le principe égalité des pères et des mères : égalité de droit, mais aussi égalité de devoir.» (Dekeuwer-Défossez 1999 : 71).

Le troisième principe constructif de l'autorité parentale est celui de couple parental : «c'est-à-dire, l'idée selon laquelle il est de l'intérêt de l'enfant d'être élevé par ces deux parents, dans la famille fondée sur le mariage comme dans la famille créée hors mariage, que le couple parental soit uni ou qu'il soit désuni.» (Dekeuwer-Défossez 1999 : 71).

S'ils ont été réitérés au moment des différentes réformes, chaque principe s'est affirmé avec une force différente à chaque époque. Historiquement, c'est d'abord le principe de l'autorité parentale comme fonction qui s'est affirmé. La fin des années 1980 et le début des années 1990, ont inscrit définitivement le concept d'égalité entre les pères et les mères. Aujourd'hui, c'est l'idée d'un couple parental qui s'affirme de plus en plus et domine les projets de réforme en France.

Le principe de parité parentale s'ajoute aux trois principes précédents : en effet, l'obligation des parents de s'inscrire dans leurs fonctions, à l'intérieur du binôme parental, est l'un des nouveaux devoirs qui leur incombe, au nom de l'enfant. L'idée est celle d'une coopération parentale après la rupture familiale.

«A l'égard des tiers de bonne foi, chacun des parents est réputé agir avec l'accord de l'autre, quand il fait seul un acte visuel de l'autorité parentale relativement à la personne de l'enfant.» (Article 372-2). Cet article inscrit la présomption de coopération entre les parents dans l'exercice de l'autorité parentale (que le couple soit uni ou séparé ${ }^{111}$ ). Les situations conflictuelles sont interprétées comme une exception à cette règle de principe.

\footnotetext{
110 Le «fmcp» critique la procédure de l'enquête sociale en quatre points : elles sont le reflet d'idées traditionnelles ; les techniques des enquêtes sociales desservent les enfants ; les enquêtes sociales sont contraires à la loi et elles servent le conflit, non le consensus.

${ }^{111}$ Lors des recompositions familiales, la «beau-parentalité» se distingue de la «coparentalité». «Il y aurait quelque incohérence à affirmer d'un côté le principe de coparentalité, à insister sur la place et sur le rôle du
} 
De plus, le principe d'égalité entre les enfants est un élément constitutif de l'exercice de l'autorité parentale: l'exercice de l'autorité parentale, dans ses effets, ne doit pas différencier les enfants légitimes des enfants naturels.

Par «coparentalité» on entend donc la forme actuelle de l'exercice de l'autorité parentale basée sur les principes d'égalité, de partenariat, s'exerçant conjointement pour les pères et mères, concernant l'ensemble des enfants, quelle que soit la situation matrimoniale des parents. Mais les résistances des institutions à l'exercice de la «coparentalité» sont nombreuses.

parent qui ne vit pas avec l'enfant et, de l'autre, à institutionnaliser une fonction de beaux-parents qui risque fort d'entrer en concurrence avec la fonction parentale.» (Dekeuwer-Défossez 1999 : 89). La commission a choisi de ne pas assimiler le beau-parent à une figure parentale mais de l'assimiler à un tiers pour lequel la commission propose d'étendre les moyens d'action. Le couple «recomposé» est en premier lieu un nouveau couple conjugal. Sans nier la charge et les relations quotidiennes avec les enfants, elle la distingue de la fonction parentale, liée à l'autorité parentale et à la filiation. On aboutit donc ici à une distinction de plus en plus grande du couple conjugal et de la fonction parentale. 


\section{SOUS-SECTION II.B.3 LA GESTION SOCIALE DU RISQUE FAMILIAL}

\section{II.B.3.1 L'obligation d'entretien et l'obligation alimentaire}

Nous nous intéressons maintenant à l'expression, dans le code civil, des droits et des devoirs qui unissent les membres d'une même famille. L'obligation parentale d'entretien et les obligations alimentaires, sont inscrites dans le code civil sous le chapitre «des obligations qui naissent du mariage» (Art. 203 et s.). Elle s'inscrit différemment dans les familles légitimes et les familles naturelles.

Pour les premières, l'obligation est liée au lien matrimonial du couple parental, pour les secondes, c'est le lien de parenté avec l'enfant qui la détermine. Le souhait d'harmoniser les statuts des familles légitimes et les familles naturelles, pousse le législateur à se distancier de plus en plus d'une conception restrictive du mariage. Indirectement, c'est donc la référence au couple conjugal légitime qui disparaît, et de façon plus profonde, au couple conjugal ${ }^{112}$. Les rapports générationnels entre les parents et les enfants, s'autonomisent de plus en plus des rapports conjugaux.

La commission Dekeuwer-Défossez a constaté que de nombreuses situations ne respectent pas les accords sur les pensions alimentaires, les droits de visite et l'hébergement : or, selon l'avis de la commission, plus on supprimera les procédures conflictuelles, plus on favorisera les accords entre parents et plus ceux-ci seront respectés. «Des évolutions se dessinent : les enfants, s'ils résident majoritairement chez leur mère, voient plus régulièrement leurs pères, les pensions impayées traduisent probablement davantage l'insolvabilité des débiteurs que leur mauvaise foi.» (Dekeuwer-Défossez 1999 : 177).

\section{II.B.3.2 La protection sociale du parent seul}

Les familles monoparentales sont au centre de ce que Claude Martin appelle «l'apparition de la nouvelle pauvreté dans le courant des années quatre-vingts, c'est à dire une pauvreté conçue comme une menace pesant sur des personnes aptes au travail, qui ont connu un «accident de trajectoire» (...) Parmi les accidents de trajectoire, on trouve bien sûr au premier rang la perte de l'emploi, mais les ruptures familiales apparaissent également comme une source de désinsertion et d'exclusion. Le CERC (1993) a ainsi mis en évidence récemment, à partir des résultats de l'enquête «situation défavorisée» de 1986-1987, les rapports qui existent entre chômage et rupture conjugale, l'un entraînant l'autre et réciproquement, jusqu'à provoquer des situations de précarité ou d'exclusion sociale» (Martin 1996 : 55).

Une étude du CERC de 1993 (Etude citée par Commaille (1996 : 70)) montre, par la composition des revenus, que le poids des transferts est plus important dans les familles

\footnotetext{
${ }^{112}$ Dans le cadre des réformes sur le droit civil, ce sont les liens de parenté qui sont privilégiés. La commission insiste sur la distinction entre, d'un côté le lien de parenté d'où découle la fonction d'autorité parentale et l'obligation parentale d'entretien, et de l'autre côté, la prise en charge d'un enfant comme dans le cas de la beauparentalité à la manière d'un tiers (Dekeuwer-Défossez 1999 : 170). C'est pour ces raisons que la commission s'exprime contre le fait de fonder une obligation alimentaire pour le beau-parent.
} 
monoparentales qui ont connu une rupture conjugale. $24,5 \%$ des hommes contre $43 \%$ des femmes séparées tirent plus de $20 \%$ de leur revenu disponible des transferts sociaux ; ce taux atteignant la moitié des revenus pour $23,4 \%$ des femmes. Cette dépendance s'atténue, sans cependant disparaître totalement, lorsqu'il y a reconstitution d'un nouveau couple.

Ainsi, les événements déclencheurs de la demande de soutien public, en France, sont le chômage, puis la rupture conjugale en seconde place.

De plus, $80 \%$ des mères en situation de monoparentalité perçoivent des prestations familiales et des aides au logement pour un montant moyen de 2570 Fr. [390 Euros] par mois, $20 \%$ ne sont pas ou peu couvertes par les prestations familiales (celles qui n'ont pas d'enfant de moins de trois ans ou celles qui ont un seul enfant) (INSEE 1994 : 82). Cette somme prend une part plus ou moins importante dans le budget du ménage, en fonction du niveau de vie et des autres ressources financières. Les écarts sont très importants.

Dans ces $80 \%$, sont comptées les femmes en situation de monoparentalité qui dépendent d'un revenu minimum ${ }^{113}$, soit $26 \%$ d'entre elles (pour elles la part de l'aide publique est supérieure à $18 \%$ ). Pour les $74 \%$ qui ne sont pas dépendantes d'un revenu minimum, plus de la moitié d'entre elles ne reçoivent pas plus de 1000 Fr. [150 Euros] (pour elles la part de l'aide sociale dans le budget est inférieure à $18 \%$ ).

Les femmes qui n'ont pas d'une activité professionnelle, qui n'ont pas droit aux aides familiales ou qui sont en fin de droit aux allocations chômage et familiales, sont à $26 \%$ dépendantes d'un revenu minimum. En 1993, $35 \%$ des parents célibataires reçoivent un minimum social (API ou RMI (Revenu Minimum d'Insertion )) (Martin 1996: 56). Une minorité des familles monoparentales font l'objet de mesure de revenu minimal (environ un quart d'entre elles).

Ces chiffres ont été avancés afin de nuancer l'interprétation de la part de l'aide sociale dans les budgets des ménages monoparentaux en rappelant qu'il s'agit d'un groupe très hétérogène.

Les aides publiques représentent respectivement $18 \%$ et $12 \%$ (cf. Tableau 20) dans la composition financière du budget des mères seules en France ${ }^{114}$ et en Allemagne ${ }^{115}$.

\footnotetext{
${ }^{113}$ Les parents isolés se sont révélé particulièrement vulnérables face à la pauvreté et au dénuement (Whiteford, Bradshaw 1994 : 79). Le pourcentage de familles monoparentales situées en dessous du seuil des bas revenus est passé de 18 à 27\% entre 1984 et 1990, et de 11 à 16\% pour les très bas revenus (Martin 1996 : 56). En 1993, $35 \%$ des familles monoparentales reçoivent un «minimum social». Leur situation de précarité se distingue de ce qu'on appelle traditionnellement la grande pauvreté. «La précarité sociale liée à la monoparentalité représente une autre forme de pauvreté, résultat d'une vulnérabilité produite par la brusque chute des ressources non compensée par un statut socioprofessionnel avantageux, par la charge exclusive des enfants» (Commaille 1996 : 70).

${ }^{114}$ La part de l'Etat est donc importante dans le budget des familles monoparentales. Pour les couches sociales les moins défavorisées, il s'agit d'un apport d'appoint (allocations familiales ou aide au logement). Pour celles en situation de précarité, le revenu minimum de l'aide sociale est très important, jusqu'à ce que l'entrée sur le marché du travail, ne les en fasse sortir.

${ }^{115}$ Le débat relatif à la dépendance par rapport à l'assistance publique ne les épargne pas. Ces discussions sont récurrentes en France et en Allemagne, surtout relayées par les partis les plus conservateurs et les libéraux, même si il n'y a pas de «panique sociale» comme en Grande-Bretagne. «Le débat politique sera en permanence traversé par un débat sur les limites du devoir d'assistance en relation avec le degré de responsabilité de l'individu confronté à une situation de pauvreté(...) Dans tous les débats sur les instruments d'une politique de solidarité étatique, apparaît à la fois la crainte de leur effet désincitateur au travail, de leur effet de «déresponsabilisation», de leur détournement par des stratégies astucieuses de la part de bénéficiaires abusifs» (Commaille 1996 : 47-49). Nous avons vu que ces arguments sont apparus lors de la mise en place des allocations spécifiques aux familles monoparentales.
} 
Tableau 19 Composition du revenu des familles monoparentales en France et en Allemagne

\begin{tabular}{|l|c|c|}
\hline & FrAnCE $\left.^{(* *}\right)$ & AlLEMAGNE $^{(*)}$ \\
\hline Activité rémunérée & $54,9 \%$ & $54 \%$ \\
\hline Retraite & $7,1 \%$ & $12 \%$ \\
\hline Pension alimentaire & $6,6 \%$ & $15 \%$ \\
\hline Aide sociale & $18,3 \%$ (prestations familiales) & $12 \%$ \\
\hline Indemnité chômage & $3,2 \%$ & $6 \%$ \\
\hline Autres & $9,9 \%$ & $1 \%$ \\
\hline
\end{tabular}

${ }^{(*)}$ Sources : Mikrozensus1985, Sonderauswertung des Stat. Bundesamtes

${ }^{(* *)}$ Sources : INSEE, enquête Budget Famille 1989

Cette différence s'explique par le fait que la France fait partie des quelques rares pays d'Europe qui ont des aides sociales spéciales aux familles monoparentales, comme l'API. La population d'allocataires ayant droit à cette allocation de soutien recouvre donc toutes les situations de monoparentalité en dessous du seuil de cette allocation différentielle ${ }^{116}$.

\section{$\underline{\text { II.B.3.2.1 L'API }}$}

Historiquement, les premières aides sociales dont ont bénéficié les familles monoparentales étaient liées à leur statut de veuve. Puis, en 1938, outre le décès du conjoint, c'est le risque familial lié à l'absence ou à la disparition de l'un des deux parents qui est pris en compte. Ces familles bénéficient d'une majoration des prestations familiales. L'aide est principalement indirecte, par le biais de remises fiscales. Cette solution est d'ailleurs encore privilégiée en Allemagne. «A la Libération, la première dérogation à la norme liant le droit aux allocations familiales à l'exercice d'une activité salariée, s'effectuera au profit des veuves de guerre, tandis que le bénéfice de l'allocation de salaire unique était étendu aux mères célibataires» (Lefaucheur $1991:$ 125).

Il faudra attendre les années 1970 pour voir de nouveaux changements dans la couverture du risque de monoparentalité. L'allocation pour orphelin créée en 1970 a étendu ses critères en 1975. Puis finalement, c'est en 1976 qu'est apparue l'Allocation pour Parent Isolé (API). Cette allocation marque le pas vers une reconnaissance et une prise en charge collective de la

Effectivement, dans un contexte où les débouchés sur le marché du travail sont de plus en plus difficiles, «la question de l'Assistance est à nouveau posée pour celles qui n'ont pas les atouts nécessaires pour intégrer le marché du travail (en termes de qualification, d'expérience, mais aussi et peut-être surtout de l'âge) (Martin 1996 : 91).

${ }^{116}$ En fait deux groupes se distinguent dans les familles monoparentales : Pour les premières, celles à très faible revenu (inférieur à 65448 Fr. [9978 Euros]), l'aide étatique est primordiale dans leur budget, elle se partage entre les prestations familiales $(40,2 \%)$ et les indemnités chômage $(11,3 \%)$. Le salaire représente moins d'un tiers des ressources $(29,1 \%)$, l'apport des pensions alimentaires est extrêmement bas $(3,7 \%)$. Pour les secondes, dés que le revenu du ménage est plus élevé, on voit une autre tendance se profiler : la première source de revenu est le salaire (supérieur à 79704 Fr. [12151 Euros] ) (pour un peu moins des deux tiers du budget), les pensions pour environ 6\% (deux fois plus que pour les ménages les plus pauvres). En France, les salaires des couples représentent 70,7\% et les prestations familiales 15,6\% de leur budget (INSEE 1989). 
situation de pauvreté dans laquelle se trouvent les familles monoparentales. Il ne s'agit plus uniquement de traiter des situations de veuvage, mais de tenir compte des séparations.

L'API est une allocation différentielle, donnée sous condition de ressource. Son montant est dépendant du nombre d'enfants. Sa durée est déterminée par l'âge du dernier-né (jusqu'à ses trois ans) ou bien limitée à un an, à partir de la séparation.

Lors de sa création, l'API fut discutée, dans un relatif consensus, à l'Assemblée nationale et au Sénat (Martin 1996 : 70). Des principaux arguments avancés, nous avons retenu les suivants : les dispositions de l'API permettaient d'éviter le recours à l'aide sociale à l'enfance (pour les enfants), d'éviter le recours à l'interruption de grossesse, et de permettre la sortie du dispositif d'aide sociale ou d'assistance (Martin 1996 : 69). L'API évite d'associer la stigmatisation de l'assistance sociale au risque familial. Les familles monoparentales sont conceptualisées comme une catégorie du droit social.

Créée en 1976, en pleine montée de l'incertitude économique, l'Allocation pour Parent Isolé est le résultat de l'intérêt grandissant à l'égard des familles. La politique familiale du gouvernement Raymond Barre (1976-1981), pendant le mandat du président Giscard d'Estaing, renoue avec les orientations «familialistes». Dans ce contexte, l'API fut la tentative de reconduction de la femme dans un rôle de mère au foyer. Ce salaire maternel n'a pu compenser la perte du deuxième salaire et la détermination des femmes à rester présentes sur le marché du travail. Les mesures ont été justifiées par l'idée que les familles d'au moins trois enfants devaient être soutenues et que la baisse du taux de fertilité devait être combattue. Les mesures prises à l'époque, étaient plus liées aux objectifs natalistes qu'à la défense de l'institution familiale : la multiplication des facilités a aidé l'insertion des femmes dans la vie professionnelle, du moins à mi-temps ; le renoncement à l'activité professionnelle n'est plus un critère d'attribution des aides familiales; et le gouvernement a créé une aide spéciale aux familles monoparentales. Aussi, nous retiendrons plutôt le terme de «néo-natalisme» de N. Martin-Papineau (1994) que celui de «familialisme» employé par Lenoir (1991:176) pour décrire cette période des années 1970.

Dans les paragraphes suivants, nous discuterons de la logique de l'API : du critère d'enfant à charge, du critère d'isolement, et des discussions relatives au rapport «aides publiques/du travail rémunéré» [1'API joue-t-elle un rôle de désincitation au travail professionnel ?].

L'API est définie par l'article L. 524-1 du Code de la sécurité sociale de la manière suivante : c'est une allocation destinée à toute personne isolée résidant en France, ayant seule la charge d'un ou plusieurs enfants. Les conditions d'application de l'API et du critère $\mathrm{d}^{\text {'isolement }}{ }^{117}$ varient selon les Caisses d'allocations familiales (CAF) (Commaille 1996 : 126). Le critère d'isolement dépend des pratiques locales et plus encore de la seule évaluation du contrôleur des CAF. Les règles internes et arbitraires ont pour effet de provoquer une

\footnotetext{
${ }^{117}$ Deux critères relativisent l'application stricte de ce critère dans la pratique : premièrement, toutes les caisses n'appliquent pas de la même manière le critère d'isolement ; deuxièmement, ce critère concerne peu de situation réelle. En effet, les résultats de l'étude de Claude Martin cité par H. Mendras (1997 : 218) montre que 65\% des parents gardiens n'ont pas de nouveau partenaire, et parmi les $35 \%$ qui entretiennent une nouvelle liaison cohabitent avec le nouveau partenaire (seulement $9 \%$ de l'échantillon). Ces données sont à relativiser par rapport à la durée écoulée depuis la séparation et l'âge de la femme : 45\% des jeunes femmes de moins de 30 ans se remettent en ménage, seulement $5 \%$ après 45 ans.
} 
gestion des situations selon des critères «de fait» et non pas selon des critères «relatifs à la situation personnelle» de l'allocataire (Commaille 1996 : 129). «L'isolement reste une question appréciée au cas par cas et se fonde plus sur la «mise en couple» que sur une éventuelle collaboration économique» (Sayn 1996 : 14). Les Caisses d'allocations familiales contrôlent «l'isolement» de l'allocataire car l'aide de l'Etat se limite à la situation de célibat. En effet, on présuppose que le nouveau couple installera une solidarité économique et matérielle. L'Etat joue un rôle de subsidiarité par rapport à la solidarité du couple. Par-là, elle relève plus de la logique conjugale que de la logique parentale.

Néanmoins, le montant de l'allocation augmente en fonction du nombre d'enfants. Cette majoration est une reconnaissance explicite de la fonction reproductive, puisque ce supplément s'adresse directement au parent dans une logique nataliste.

Une activité professionnelle n'est pas explicitement interdite, mais elle est limitée par le fait que l'allocation soit différentielle. On rappellera que l'API a été créée dans l'idée d'un salaire maternel et son pouvoir «décommodificateur» ${ }^{118}$ avait été explicitement recherché. Mais, l'interdiction d'exercer un travail a été levé pour rendre possible un travail à temps partiel.

L'API est une alternative au marché du travail pendant au moins 12 mois. Mais elle a les effets pervers des allocations «décomodificatrices»: elle offre au bénéficiaire une alternative au marché du travail mais lui demande, après la durée de perception, de s'y réintégrer (Lewis 1993 : 20). Or les difficultés de réinsertion sont beaucoup plus grandes pour les femmes que pour les hommes, faisant des familles monoparentales un groupe vulnérable sur le marché du travail.

Même si l'API est conçue comme un revenu de remplacement, elle n'est qu'une phase hors du marché du travail (une transition). C'est un ««revenu de remplacement» mais ce n'est que pour permettre la transition et la recherche d'un emploi, pour éviter le pire [«le placement de l'enfant»] si les femmes ne pouvaient concilier les deux (Martin 1996 : 69).

Et c'est bien là où les opposants trouvent les arguments contre l'API, l'incitation au travail serait insuffisante. Ce sont les effets pervers dénoncés dans les années $1980^{119}$ (Le Gall ; Martin 1987 : 79-82) : des situations d'assistance se développeraient, encourageant ainsi les situations de fraude ${ }^{120}$.

«Une équipe d'économistes, dirigée par Jean-Claude Ray, a spécifiquement étudié cette question des effets de l'API (effets souhaités, affichés, mais aussi jugés indésirables ou pervers) en distinguant les objectifs explicites ou affichés et les objectifs implicites. Ainsi, par exemple, l'accroissement des revenus de certaines familles monoparentales ou la limitation des cas d'avortement sont des objectifs explicites ${ }^{[121]}$. En revanche, la volonté de limiter la progression du chômage en diminuant le nombre de demandes d'emploi non satisfaites est un objectif inavoué (et non pervers). Pour certaines des mères isolées, la désincitation au travail n'est pas redoutée mais souhaitée. Parmi les effets jugés indésirables, on trouve l'installation

\footnotetext{
${ }^{118}$ Ce terme, traduit de l'allemand et de l'anglais, illustre l'idée selon laquelle l'incitation des politiques sociales se fait en direction de la sortie du marché de travail.

${ }^{119}$ Ces réflexions soulevées en France dans les années 1980 à propos de l'API, rappellent les critiques faites au programme allemand d'aide aux familles monoparentales.

${ }^{120}$ Le contrôle de l'Etat est moindre pour les situations de monoparentalité qu'il ne l'est pour d'autres situations qui relèvent de l'assistance sociale. Ce contrôle est d'autant moins ingérant que l'allocation est nationale et qu'elle n'a pas les obligations propres à un programme comme c'est le cas en Allemagne.

${ }^{121}$ En Allemagne, la volonté explicite de l'aide aux familles monoparentales est aussi d'offrir une garantie contre l'avortement (Schultheis $1987: 44)$.
} 
dans la dépendance de certaines allocataires, la fraude ou le pseudo-isolement, les séparations favorisées par l'existence de la prestation, la programmation de naissance pour prolonger l'accès au droit. Mais il existe des effets imprévus souhaitables, comme le travail de certaines allocataires à temps partiel, près d'une femme sur cinq travaille à temps partiel pendant l'API.» (Martin 1996 : 72).

\section{II.B.3.2.2 Les conditions attribution des prestations}

Les conditions d'attribution des prestations destinées aux familles monoparentales polarisent l'exercice de la parentalité : «il serait souhaitable de ne pas exiger certains critères d'attribution (isolement, non-reconnaissance d'un enfant par le père, etc...), non seulement parce que la preuve de ces situations est difficile à établir, mais surtout parce que ces exigences poussent les mères à rester seules, à couper l'enfant de l'autre parent, pour pouvoir toucher ce secours.» (Commission nationale consultative des droits de l'homme «portant sur parentalité et droits de l'homme en rapport avec les dispositions juridiques et les pratiques sociales», Adopté le 14 janvier 1999).

«L'aide au parent isolé (API) et l'allocation de soutien familial (ASF) sont assorties de conditions qui permettent aux caisses de poursuivre le parent absent pour exiger de lui une pension alimentaire. Lorsqu'il s'agit de couples en grandes difficultés financières, il arrive que le parent isolé (souvent la mère) renonce à ces allocations pour éviter à son ancien compagnon des poursuites judiciaires. En corollaire, celui-ci n'ose pas reprendre contact avec les enfants ou renouer avec sa femme puisqu'il est dans l'incapacité de contribuer financièrement. Or, souvent l'homme a quitté le foyer parce qu'il avait honte et se culpabilisait de ne pouvoir subvenir au besoin de sa famille. En conséquence, il parait souhaitable de réviser cette pratique administrative qui renforce l'éclatement des couples.» (Commission nationale consultative des droits de l'homme «portant sur parentalité et droits de l'homme en rapport avec les dispositions juridiques et les pratiques sociales», Adopté le 14 janvier 1999).

L'article L. 513-1 et R. 513-3 du Code de la Sécurité sociale stipule que les prestations familiales sont attribuées à une seule personne -l'allocataire-, celle qui a la charge effective et permanente de l'enfant. Mais lorsque l'enfant est à la charge des deux parents, c'est celui qui en fait la demande le premier qui percevra l'allocation. Or, ce principe est en contradiction avec le principe de l'autorité parentale conjointe du droit civil. Actuellement, de nombreux magistrats cherchent à favoriser une meilleure circulation de l'enfant, ce qui implique donc une répartition de la charge de l'enfant. Mais il y a de nombreuses résistances de la part des CAF (institution garante du droit social en matière de famille) à appliquer ces principes de partage de la charge.

La position normative de la CAF comme agent de l'action sociale est particulièrement révélatrice de la place qui peut être donnée à l'enfant dans le traitement de la situation de monoparentalité. En fait, il s'avère que ce critère obéit à une règle de droit plus qu'à une règle de fait : avoir à charge l'enfant ne suffit donc pas pour devenir allocataire.

«Tout en affirmant ne pas vouloir prendre en considération les arrangements de famille, les caisses appliquent le refus aux seuls arrangements qui confient l'enfant à un tiers (...) C'est un moyen de laisser le fait (la prise en charge effective et permanente) en dehors de la matière strictement juridique, en préférant s'attacher à la qualité de parent au sens du droit civil pour apprécier les conditions requises de l'allocataire (...) En tout cas, le but recherché de 
la réduction du domaine d'application des prestations familiales n'est pas remis en cause. Parmi ces justifications, il apparaît à l'évidence que la question de l'obligation d'entretien des parents à l'égard de leurs enfants a pris une importance considérable. L'inexécution par le ou les parents de leurs obligations pécuniaires est présentée comme justifiant, en droit, le refus de verser des prestations aux tiers demandeurs» (Sayn 1996 : 11).

Par cette interprétation de la charge de l'enfant, c'est le lien mère-enfant que l'on cherche à maintenir : la mère se voit reconnaître le droit à une aide financière pour l'entretien de l'enfant car c'est elle qui a, dans la majeure partie des cas, la résidence de l'enfant (exclusivement pour les enfants en bas âge). Ce lien est maintenu au delà des recompositions familiales puisque les prestations ne sont pas supprimées (Sayn 1996). Ce qui est implicitement défendu, ce n'est donc pas l'enfant lui-même et son coût, mais une position normative de la CAF.

Mais cette notion d'enfant à charge se heurte aux nouveaux comportements familiaux, comme celle de la garde alternée. Lors du colloque sur la «coparentalité», les pratiques de la CNAF ont été ainsi énoncées par son représentant : si la garde est partagée entre les parents, alors c'est celui qui en fait la demande le premier qui recevra les prestations familiales. Or, cette pratique n'est pas adaptée au couple parental, car elle institutionnalise plutôt le lien mère-enfant. Le père est conceptualisé comme un payeur de pension alimentaire, ni comme un ayant-droit, ni comme un parent qui partage, en fait, la charge de l'enfant (Colloque 1997). En d'autres termes, il s'agit d'une pression des pouvoirs publics sur les obligations alimentaires du père de l'enfant ou des grands-parents.

La participation financière des pères est un élément important dans la protection $\mathrm{du}$ risque familial. Le montant mensuel des pensions ainsi que la régularité de leur paiement, dépend de plusieurs facteurs. Si le niveau conflictuel de la séparation agit sur le paiement des pensions (Festy, Valetas 1993 : 97-120), le premier facteur est la solvabilité du payeur. Début 1986, le montant moyen des pensions par enfant et par mois était de 650 Fr. [100 Euros] en France. Mais une sur cinq était inférieure à 300 Fr. [46 Euros].

En France, «la pension alimentaire est non négligeable. C'est très important financièrement. Il y a toujours des contentieux très importants autour des pensions alimentaires. Et nous voyons revenir des personnes pour des modifications modestes, de 200, 300, 400 Fr.» [30, 45, 60 Euros] (Entretien avec Madame le Juge aux affaires familiales, 1997). Les pères français paient beaucoup moins, et moins régulièrement que les pères allemands. A peu près un tiers des pensions alimentaires est payé irrégulièrement ou partiellement et un quart n'est jamais payé.

Le niveau conflictuel de la séparation est un facteur qui joue dans le paiement des pensions. Ce sont les conditions de la séparation, elles-mêmes reflet du passé conjugal des époux, qui pèsent sur le déroulement financier de l'après-divorce... Si la situation conflictuelle entre les deux ex-partenaires permet un accord à l'amiable, alors les défaillances du père seront moins grandes. Corrélativement, les couples qui s'entendent le mieux sont issus des couches les plus aisées. La situation économique du père y est généralement assurée, le paiement des pensions est facilité. La pension est d'autant plus élevée que l'accord est large et que celui-ci a été obtenu directement par les conjoints. En cas d'accord direct entre les partenaires sur tous les points, la pension dépasse de moitié celle fixée dans les divorces où le désaccord a été total. 
Si le montant a été déterminé par le système judiciaire pendant la procédure de divorce, il est possible de mettre en évidence certains mécanismes qui influencent le montant de la pension (Festy ;Valetas 1993) : l'augmentation du nombre d'enfants s'accompagne d'un recul du montant unitaire des pensions; les femmes plus âgées à la séparation reçoivent plus que les femmes plus jeunes; le montant des pensions est plus bas lorsque l'ex-mari est sans emploi au moment de la séparation.

Paradoxalement, la défaillance des débiteurs devient plus rare à mesure que la somme en jeu s'accroît, car les pensions les plus faibles sont fixées pour des catégories sociales en difficulté (donc peu solvables). Si le juge a décidé d'attribuer une pension alimentaire élevée, c'est que la solvabilité du père a été éprouvée. A titre d'exemple, les pensions sont perçues à $73 \%$ pour les professions libérales et les cadres, à $45 \%$ pour les inactifs et à $22 \%$ pour les commençants et les artisans (Mendras 1997 : 221). En bref, le niveau socioprofessionnel et économique du débiteur est déterminant, car plus la pension est élevée, plus elle est régulièrement payée, plus le débiteur est solvable. Cette corrélation est soulignée par différents auteurs (Martin 1996 : 54 ; Festy, Vatelas 1993).

Le montant moyen des pensions françaises qui est environ égal à la moitié du montant légal allemand montre que la première explication de la défaillance des pères français est de nature économique. En France comme en Allemagne, le facteur économique qui pèse sur les pères est incompressible. Le non-paiement des pensions alimentaires a obligé les Etats à institutionnaliser une couverture publique des pères défaillants.

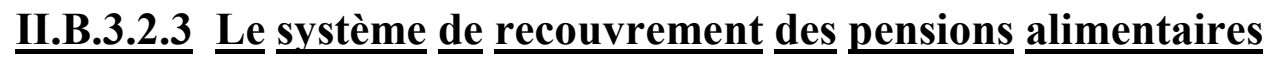

La première allocation de recouvrement des pensions alimentaires est l'Allocation orphelin (AO dans le reste du texte) crée en $1970^{122}$. «Cette prestation ne s'adressait alors qu'aux catégories traditionnelles de la veuve et de l'orphelin, de la fille-mère et du bâtard» (Lefaucheur 1991 : 125). Les femmes victimes d'impayés avaient alors deux moyens d'action, le recours au droit pénal pour abandon de famille, et le recours au droit privé pour faire exécuter la créance du débiteur (saisi de ses biens et débit de son salaire). En 1973, les moyens de recours des femmes sont simplifiés : la procédure de paiement direct peut s'effectuer par un huissier. La demande préalable au juge n'est plus nécessaire.

L'AO est ouverte en 1975 aux enfants reconnus par leurs deux parents mais manifestement abandonnés par l'un des deux. Puis, avec la réforme du divorce en 1975, les enfants dont le parent non-gardien ne s'est pas soumis à l'obligation alimentaire depuis plus de deux mois, deviennent également allocataires (l'Allocation de Parent Isolé est crée l'année suivante).

Quant à l'AO, elle est transformée en une Allocation de Soutien Familial qui, depuis 1984, joue le rôle d'avance sur les pensions alimentaires impayées. Son champ d'attribution a été élargi pour être recevable pour tous les enfants «manifestement ou réellement abandonnés». La distinction entre les enfants légitimes, illégitimes, ainsi que la distinction entre les familles monoparentales, divorcées, séparées, veuves, célibataires, et mariées, n'existe plus.

\footnotetext{
${ }^{122}$ En France, dans les années 1970, la gauche s'est faite l'initiatrice de deux combats culturels, le divorce et le projet de recouvrement des pensions alimentaires par la création d'un fond public (Théry $1993: 78$ ). Ce courant politique définit les responsabilités parentales devant les choix familiaux comme faisant parties de la responsabilité collective. L'idée était d'aider les personnes les plus démunies devant les situations de divorce. Les mêmes combats sont portés par la gauche allemande dans la même période.
} 
L'ASF a été explicitement créée pour protéger les droits alimentaires de l'enfant par l'intervention des CAF en cas de non-recouvrement des pensions. Il y a deux types d'ASF : une recouvrable et une non-recouvrable (les CAF engagent les fonds sans que les débiteurs soient solvables). La prise en compte du risque familial concerne alors l'enfant et non plus la femme comme dans le cas de l'API.

L'Etat a du tenir compte des nouveaux besoins et des difficultés économique des pères et des mères. C'est sous ce double mouvement que c'est développé une lutte contre la pauvreté et la précarité. «Avant 1973, la position du législateur était claire. Il considérait que le non-paiement d'une créance alimentaire ne justifiait pas que la collectivité se substitue financièrement au débiteur» (Martin : 1996 : 73).

Mais devant l'irrégularité des paiements des pensions alimentaires pour l'enfant, l'Etat a mis en place une procédure de recouvrement des pensions alimentaires non payées. En France et en Allemagne, le système permet à toutes les familles monoparentales, même si elles ne reçoivent pas d'aide publique, de recourir à des moyens juridiques pour faire aboutir le paiement des pensions. Cette prestation française et allemande a été instituée à la même époque, suivant deux principes identiques : le recouvrement «à perte» des pensions pour les payeurs non solvables et un avancement de la pension dans l'attente du versement régulier des «mauvais payeurs». Ce système se couple à une procédure de poursuite judiciaire à l'encontre du père. Il s'agit d'un dispositif de l'Etat pour réaffirmer les rôles du père comme nourricier.

La norme d'application du recouvrement des pensions est semblable en France et en Allemagne : premièrement, l'enfant doit rester à la charge d'au moins un de ses parents, deuxièmement, l'Etat n'intervient qu'en cas de défaillance de la solidarité du couple parental, et troisièmement, l'Etat offre son soutien matériel contre l'assurance que le parent non gardien sera réellement sollicité dans ses devoirs alimentaires. L'Etat veut s'assurer de n'intervenir que de manière subsidiaire.

De plus en plus de dossiers sont classés comme non recouvrables, $30 \%$ en décembre 1986, contre 42\% en décembre 1990 (Villac, Renaudat 1991 : 95). Or, si l'ASF est maintenue malgré son inefficience relative et malgré les difficultés économiques qui rendent la solvabilité des pères de plus en plus aléatoire, c'est qu'elle remplit un autre rôle : ce service a une fonction de police normative : le principe d'obligation alimentaire des parents doit être assuré au-delà de la séparation familiale. Le non-respect de cette norme est alors considéré comme une déviance qui renforce, de fait, la force normative de la prestation (Commaille, 1994). On remarque que les deux pays ont la même orientation normative face au risque familial : le rapport Etat-Famille est tel que l'Etat, à travers ses services sociaux, applique une logique stricte de subsidiarité par rapport à la famille.

En France, les nouveaux pouvoirs juridiques des CAF garantissent à l'Etat que les relais de la sphère privée ont bien été épuisés avant d'engager sa protection. Ainsi, le caractère subsidiaire de l'ASF est équivalent à celui qui a été mis en évidence, dans l'attribution du RMI, par Villac et Renaudat (1991 : 95). 


\section{II.B.3.3 La socialisation de la politique familiale : vers une gestion de la pauvreté}

En Allemagne, les familles monoparentales «tombent» sous la politique de la pauvreté. En France, elles sont une catégorie du droit social, mais l'aide publique s'oriente vers une socialisation du risque familial. Les aides spécifiques aux familles monoparentales se révèlent globalement plus généreuses qu'en Allemagne où l'aide publique est vécue comme une alternative au marché du travail.

Dans les années 1970, le gouvernement français marque son intention de redéployer les transferts sociaux vers les plus démunis. La création de l'Allocation Orphelin, première étape d'une reconnaissance du risque familial s'inscrit dans ce processus. La politique familiale s'oriente vers une couverture des risques sociaux.

L'orientation de la politique familiale des années 1970 exprime une coupure avec la politique des natalistes et des familiaux. «Ainsi l'Etat cessait de se proposer pour objectif d'encourager les familles à se conformer à un modèle dont l'évolution sociale s'éloignait, avec le développement du travail féminin et l'acceptation encore timide des familles monoparentales, tandis que la politique des transferts sociaux prenait le pas sur celle de péréquation des charges familiales» (Prost $1984: 21$ ).

De nombreuses logiques politiques se sont superposées pour former aujourd'hui une politique familiale à qui on reproche un manque de cohérence. Les logiques de redistribution horizontales ${ }^{123}$ et verticales ${ }^{124}$ se sont ajoutés aux objectifs natalistes et familialistes.

A notre avis, le principe de redistribution verticale s'affirme de plus en plus comme la logique qui domine les choix politiques et les discours, laissant apparaître une forte tendance à la lutte contre les inégalités, et plus précisément contre la pauvreté. L'orientation des politiques familiales dans le sens d'une politique de pauvreté et de la gestion de la précarité a été notée par de nombreux auteurs J. Commaille (1992), J. Fagnani (1996), M. Villac (1993).

La part des allocations soumises à condition de ressources est en constante progression depuis les années 1970, et la récente réforme des allocations familiales s'inscrit dans cette tendance générale.

«La mise sous condition de ressources est, en effet, une des réponses possibles aux préoccupations de lutte contre la pauvreté en accentuant le rôle de redistribution verticale des systèmes de protection sociale, et plus globalement de l'ensemble des systèmes de transfert, (...) en France, la part du montant total des prestations familiales versées sans condition de ressources est passée de près de 60\% en 1971 à environ 47\% en 1995» (Fagnani 1996 : 31).

Parallèlement, l'intérêt public se préoccupe vivement de la situation économique des familles monoparentales, les risques de socialisation des enfants n'est plus au premier plan de

\footnotetext{
${ }^{123}$ L'objectif est de soutenir l'ensemble des ménages, en tenant compte du nombre et de l'âge des enfant et non plus uniquement les familles nombreuses (Martin 1996 : 66). Cette logique s'impose surtout jusqu'au début des années soixante-dix.

124 «La redistribution verticale, c'est à dire une redistribution des ressources des familles à moyens et hauts revenus vers les familles les plus défavorisées, à l'aide de deux types de prestations : celles qui sont soumises à condition de ressources et des prestations spécialisées» (Martin 1996 : 66).
} 
l'action sociale, tant en France qu'en Allemagne. «Au thème des effets psychologiques de la dissociation de la famille a succédé vers le milieu des années soixante-dix, celui des risques économiques et sociaux.(...) On parle moins dès lors [les années 1980] d'inadaptation ou de déviance des enfants élevés dans les familles dissociées et plus de précarité voire de pauvreté de ces ménages» (Martin 1996 : 85). La protection sociale des familles monoparentales modifie son orientation pour rejoindre les objectifs d'une politique sociale de la pauvreté et de la précarité. Commaille (1996 : 110-111) appelle ce processus «la socialisation de la politique familiale» ${ }^{125}$. L'aide sociale pour les familles monoparentales se justifie alors dans leurs difficultés à s'intégrer sur le marché et non plus dans «leur statut de monoparentalité».

Ce changement d'intérêt participe au processus de centration du politique sur la situation de précarité économique conséquente au risque familial. L'orientation sociale des politiques familiales tend à constituter le risque familial comme un risque social.

De fait, les familles monoparentales, de par leur activité professionnelle, sont confrontées de plus en plus aux risques sociaux liés au marché de travail. En effet, la situation de précarité de ces femmes, n'est pas toujours déclenchée par la rupture conjugale. La perte d'un emploi est parfois le facteur qui les contraint à l'aide sociale. En outre, la sortie de l'aide sociale est conditionnée de plus en plus, en France comme en Allemagne, par une réintégration sur le marché du travail.

\section{II.B.3.4 L'activité professionnelle des mères et la coparentalité}

L'étude des ressources des mères seules montre que le revenu de l'activité professionnelle est le premier apport financier ${ }^{126}$. Mais les familles monoparentales françaises et allemandes ont des rapports de nature différente avec le marché du travail car leur activité professionnelle dépend de la conceptualisation du travail des femmes en général.

En 1992, 82\% des mères seules avec au moins un enfant de moins de 25 ans étaient actives, alors que les mères en couple ne l'étaient qu'à 68,3\% (INSEE 1992). Le taux d'activité des mères seules âgées de 25 à 54 ans, continu de progresser. De 84,3\% en 1990, il a atteint $86,1 \%$ en 1994 (72,7\% pour les mères en couples et contre 56\% en Allemagne (1986)) (INSEE 1995). Seulement 18,1\% des salariées travaillent à temps partiel contre $29 \%$ des femmes vivant en couple (Martin 1996 : 50). Les mères seules travaillent à 93,2\% avec un enfant âgé de 6 à 17 ans. Avec deux enfants, ce taux varie entre 70 et $92 \%$ selon l'âge des enfants. Avec trois enfants, les chiffres atteignent $43 \%$ si le plus jeune à moins de 3 ans (INSEE 1992).

Paradoxalement, la différence du taux d'activité des femmes allemandes et françaises ne se reflète pas dans la part du salaire dans leur revenu. Le salaire ne représente que $55 \%$ du

\footnotetext{
125 Néanmois, «la socialisation de ce risque familial ne semble pourtant pas très avancée en France (...), la protection contre le risque de pauvreté que la France offre à ses citoyens en situation monoparentalité reste limitée : en 1979, la proportion de familles «pauvres» parmi les ménages monoparentaux était portée de $38 \%$ à $18 \%$ par l'incidence des transferts sociaux, l'incidence étant à peu près du même ordre en Allemagne fédérale (de $26 \%$ à $12 \%) »$ (Lefaucheur $1991: 130)$.

126 L'activité professionnelle permet également de bénéficier de la protection sociale, de la retraite, de l'assurance maternité, et de l'assurance maladie. Cette protection est aussi accessible par certaines prestations familiales (donc par l'intermédiaire de l'Etat), ou par le régime d'affiliation en fonction du statut matrimonial (donc en fonction du mari).
} 
revenu des femmes françaises, alors qu'elles travaillent à plus de $82 \%$. Une des raisons est le niveau plus bas des salaires. Un quart des femmes monoparentales gagnent moins de $5300 \mathrm{Fr}$. [808 Euros] par mois en France en 1992. Elles ont plus fréquemment des emplois moins qualifiés que les femmes mariées.

En 1993, le salaire moyen d'une mère seule à un temps complet, était de $8174 \mathrm{Fr}$. $\left[1246\right.$ Euros ${ }^{127}$. Lorsque les mères travaillent, elles sont $16 \%$ à avoir des revenus très faibles (ce qui est le cas de plus de la moitié des mères inactives).

Les femmes sont plus touchées que les hommes par les difficultés du marché du travail : moins bonnes qualifications, emplois précaires, chômage, activité professionnelle marquée par le cycle familial (c'est à dire dépendante du nombre et de l'âge des enfants), salaires moins élevés ${ }^{128}$ (Voges, Ostner 1995 : 124). Dans la catégorie des familles monoparentales, le pourcentage des mères seules touchées par la pauvreté, est en moyenne deux fois plus important que celui des pères seuls (Roll 1992 : 25).

En 1992, 17,4\% des femmes en situation de monoparentalité étaient au chômage (INSEE 1992). Or ce chiffre augmente ; leur taux de chômage en France est de 12,4\% en 1984, de $14 \%$ en 1989 et de 17,4\% en 1992. Les difficultés économiques, la norme sociale, et le combat de l'isolement social, incitent ces mères à vouloir intégrer le marché du travail.

L'activité professionnelle des mères, qu'elles soient seules ou en couple, détermine les modes d'exercice de la parentalité. Lorsque les deux parents travaillent à temps plein, ils ont plus tendance à partager les tâches domestiques que les familles où seulement l'un des deux conjoints travaille :

«Ce qui me frappe, c'est que, même dans les milieux moins favorisés matériellement, et parce que les deux parents travaillent, il y a des accords très élaborés pour que les pères soient très présents, y compris une fois par semaine. (...) Et cela a sûrement joué dans l'évolution du couple. On le voit aussi quand on compare Paris avec la Province. Dès que les femmes ne travaillent pas, la situation est déjà différente» (Entretien avec Madame le Juge aux affaires familiales 1997). Ainsi, plus l'activité économique des femmes est importante, plus les divorces sont négociés, et plus les couples présentent au juge un accord préétabli sur les conditions de l'après-divorce, et en particulier sur les modalités de garde de l'enfant.

L'exercice bilatéral de la parentalité est favorisé par l'exercice d'une activité professionnelle de la mère parce qu'il implique un partage des tâches éducatives entre les parents. En effet, M.-A. Barrère-Maurisson a mis en évidence le lien qui existe entre les sphères du travail professionnel et du travail domestique. Ce lien oblige les couples à un partage du travail qu'il soit égalitaire ou non (Barrère-Maurisson 1992).

Si l'un des conjoints ne travaille pas, alors le domestique (qui comprend ici le parental) est à sa charge. Dans les couples à deux apporteurs équivalents, et si les carrières des deux partenaires sont à fortes valeurs promotionnelles, le domestique est délégué à l'extérieur. Si par contre, les deux conjoints qui travaillent, n'ont pas de carrière promotionnelle, alors il y aura un partage relativement équivalent du domestique et du professionnel (BarrèreMaurisson 1992). La même observation a été faite en Allemagne où, lorsque les deux parents travaillent à temps plein, ils ont plus tendance à partager les taches du domestique, que les

\footnotetext{
${ }^{127}$ De 6230 Fr. [950 Euros] pour une ouvrière, de 13636 Fr. [2079 Euros] pour les cadres supérieurs.

${ }^{128}$ Le salaire moyen d'une mère monoparentale est inférieur, dans la proportion de 2400 Fr. [366 Euros], par rapport au salaire moyen des pères seuls (INSEE 1994).
} 
familles où seul l'un des deux conjoints travaille (Schütze 1988 : 106). Cette constellation familiale du «couple équivalent», semble le plus propice à l'exercice de la parentalité, dans les familles unis ou désunies.

L. Roussel détermine plusieurs modèles de fonctionnement de couple. Nous retiendrons la «famille-club» et la «famille-histoire» au sein desquelles le partenaire est vécu comme un partenaire réel et non fantasmé, autonome: les rapports familiaux ne sont pas basés sur des relations fusionnelles. L. Roussel met alors en concordance ces modèles conjugaux et les attitudes parentales (Roussel 1989 : 198).

C'est uniquement dans ces deux idéaux-types que l'enfant est placé au centre de la famille comme sujet de droit. Pour l'auteur, la parentalité place l'enfant comme sujet de droit dans la famille et va de pair avec une conception non-fusionnelle de la famille, l'activité féminine étant une marque d'autonomie des époux l'un par rapport à l'autre.

Ces conclusions qui concerne les familles «unies» seront étendues aux situations de «l'après-divorce». Effectivement, l'arrangement entre conjoints caractérise l'historique de la relation du couple et leur autonomie respective conditionnée par l'exercice d'une activité professionnelle. Cela détermine les conditions du «divorce» et, comme l'a souligné le juge aux affaires familiales, de «l'après-divorce». Ainsi, la parentalité dépend de la conception plus ou moins fusionnelle du couple, de l'activité féminine, et de l'importance de la conception de l'enfant comme sujet de droit. Selon ces deux auteurs, on peut s'attendre à une croissance du nombre de familles fonctionnant sur ces modes, formant ainsi un terrain favorable à l'exercice bilatéral de la parentalité. 


\section{SECTION II.C LA BIPARENTALITE ALLEMANDE ${ }^{129}$}

\section{SOUS-SECTION II.C.1 LES PRINCIPES DE LA PARENTALITE}

A la différence de la France, pour qui la nouvelle norme de parentalité s'est institutionnalisée dans les années 1970, les principes d'exercice de l'autorité parentale dans les familles «unies» et «désunies» ont changé dans les années 1980 en Allemagne.

\section{II.C.1.1 L'autorité parentale conjointe dans le Code Civil}

On rappellera que, pour la première fois dans l'histoire du droit allemand, la réforme du divorce de 1977 permet de concevoir le contentieux conjugal d'une manière moins conflictuelle. Avec la possibilité d'un divorce à l'amiable, la gestion du parental change : en effet, l'attribution de la garde parental de l'enfant n'est plus exclusivement affectée en fonction du critère du bon et du mauvais parent. Jusque dans les années 1980, l'autorité parentale était obligatoirement attribuée à l'un des deux parents. La garde de l'enfant était pratiquement exclusivement attribuée à la mère, sauf critères très restrictifs. L'attribution de la garde obéira très longtemps encore à cette distinction de genre.

Or, l'attribution unilatérale de l'autorité parentale donne les pleins pouvoirs à l'un des parents, c'est à dire l'ensemble des droits et des devoirs parentaux, les droits de visite en faisant partie. Aussi, le parent décide qui peut avoir accès à l'enfant, car il est le seul juge de ce qui relève de l'intérêt de l'enfant (Entretien avec Monsieur le juge aux affaires familiales 1997). Puis la réforme du 1er janvier 1980 change la qualité des relations générationnelles (parents-enfant) : la notion de «Gewalt» est supprimée des textes en faveur du terme de «Sorge», faisant glisser la notion de «puissance parentale» vers celle de «soins parentaux».

En 1982, la Cour constitutionnelle allemande rend un verdict très attendu sur l'autorité parentale. Elle déclare que la procédure d'attribution jusque là en usage, est anticonstitutionnelle : le fait d'attribuer l'autorité parentale à un seul parent s'oppose au droit constitutionnel de l'autre parent. Elle condamne l'autorité parentale unilatérale comme unique principe de garde des enfants parce que celle-ci légifère contre les droits du second parent. Elle fait référence à l'article 6 de la Constitution allemande sur la protection de la famille et du couple, lequel s'exprime indirectement en faveur d'un partage bilatéral de l'autorité parentale. A partir de cette date, les parents divorcés peuvent, sur leur demande, exercer conjointement l'autorité parentale.

Malgré cet arrêté de la cour constitutionnelle, les magistrats continuent d'appliquer très largement le principe unilatéral de l'attribution de l'autorité parentale lors de la séparation des parents.

Lorsque les juges français partaient du principe, dès les années 80, que les parents étaient susceptibles de s'entendre, les juges allemands argumentaient qu'il n'était pas possible de forcer les parents à coopérer et que l'autorité parentale conjointe devait rester une exception. L'enfant devait se voir offrir un nouveau milieu familial où les conflits du couple

\footnotetext{
${ }^{129}$ Les citations et des expressions allemandes mentionnées entre guillemets dans cette partie ont été traduites par nous-mêmes (sauf mention contraire).
} 
parental lui étaient épargnés. La rupture du contact, souvent définitive, avec l'autre parent avait alors une moindre importance (Bode 1999: 1401). Ainsi, il suffisait que l'un des parents, la mère dans la majorité des cas, refuse la coopération avec l'autre parent, pour que le droit de garde soit unilatéral ${ }^{130}$, en principe à son avantage. Le droit de visite de l'autre parent pouvait être supprimé et le contact interrompu, indépendamment de la qualité de la relation avec les enfants, si les visites devenaient une source de conflit entre les parents ${ }^{131}$.

Il faudra attendre la fin des années 1990 pour que ce modèle unilatéral perde de sa force normative. Aujourd'hui, le fait de refuser l'autorité parentale conjointe ne justifie plus l'attribution unilatérale de l'autorité parentale. La présence des deux parents après le divorce devient le nouveau caractère de la gestion de la garde des enfants en France et en Allemagne (Breithaupt, 1993). Le principe de partage de l'autorité parentale s'inscrit en 1998 dans le code civil ( $\$ 1626$, BGB KindRG).

Un an de pratique depuis la réforme de 1998 sur les droits des enfants montre que l'influence de la jurisprudence basée sur la tolérance du lien avec l'autre parent est récente mais devient la norme de référence ${ }^{132}$ (Motzer, 1999 : 1103).

L'évolution des pratiques jurisprudentielles ont apporté des changements normatifs concernant la présence et le rôle du père dans les années 1980 et 1990 . Aujourd'hui, une majorité de magistrats favorise la présence éducative du père, la circulation de l'enfant entre les deux parents et le partage du temps de présence ${ }^{133}$.

Pour la sociologue du droit, J. Limbach (1988 : 21), la jurisprudence, en matière de divorce, cherche plus à assurer l'éducation de l'enfant, que l'égalité stricte entre les exconjoints.

L'évolution a été plus lente en Allemagne qu'en France et la représentation du couple parental après le divorce y est récente ${ }^{134}$. Il faut noter que ces positions ne font pas l'unanimité chez les magistrats (Oelkers; Kasten, 1994). Leurs résistances relatives à l'attribution de l'autorité parentale conjointe ont été beaucoup plus importantes en Allemagne qu'en France ${ }^{135}$. Ces résistances sont dues à différents facteurs, tels que la représentation de la famille et les enjeux identitaires des mères ${ }^{136}$.

\footnotetext{
${ }^{130}$ Décision du tribunal OLG (Oberlandesgericht) Bamberg du 14.3.1995, (FamRZ 1995 : 1509)

${ }^{131}$ Décision du tribunal OLG Hamm du 31.10.1994 (FamRZ 1995 : 314)

${ }^{132}$ Les magistrats partisans de l'autorité parentale conjointe ont trouvé dans les pratiques jurisprudentielles de l'Etat de Californie aux Etats-Unis un modèle de gestion de l'après-divorce, tant pour les magistrats français, que pour les magistrats allemands (Luthin, 1996). En effet, l'Etat fédéral californien a institutionnalisé le partage de l'autorité parentale dès 1979 .

${ }^{133}$ Cela concerne aussi les cas où le contact entre le père et les enfants a été interrompu. Décision du tribunal OLG de Karlsruhe du 23. 09. 1998 et du tribunal OLG de Braunschweig du 14. 10. 1998 (FamRZ, 1999 : 184).

134 «Sous les auspices du divorce, il est clair que le conflit est plus important, mais ce n'est pourtant pas une raison suffisante pour exclure le fait qu'il puisse aussi y avoir de la compréhension mutuelle» (M. von Renesse 1996).

${ }^{135}$ Bien que des résistances aient pu être mises en évidence en France (Rubellin-Devichi 1996), le processus a été plus rapide.

136 «En général, c'est toujours le père qui est actif toute la journée. Il n’a donc pas le temps de s'occuper des enfants dans la journée, du moins quand les enfants sont petits. Dans la plupart des divorces que nous avons à juger avec des petits enfants, oui, c'est la mère qui est auprès des enfants et c'est le père qui gagne l'argent, pourrais-je dire. Et dans des circonstances normales, c'est la mère qui est plus dans la situation de pouvoir s'occuper des enfants que le père. C'est la seule raison. Ils sont peut être aussi capables l'un que l'autre mais je dois prendre en compte des conditions extérieures.» (Monsieur le juge aux affaires familiales du tribunal de Göttingen, 1997).
} 
Actuellement, elle juxtapose les deux parents mais elle juge encore de manière sceptique une possible coopération (Maccoby ; Mnookin, 1995 ; Salgo, 1996).

Dans ce contexte, le changement de référence pour un partage de l'autorité parentale a pris forme indirectement grâce à l'institutionnalisation des droits de l'enfant. Ce processus qui place l'enfant au centre a permis de justifier la réforme des droits de l'enfance (qui contient l'exercice de l'autorité parentale).

Cette discussion montre surtout l'attache à l'institution familiale et les résistances au changement de paradigme «Pourquoi est-il question d'une plus grande prise en compte de l'intérêt de l'enfant dans le cadre des droits de l'enfant et non pas d'un changement de perspective qui placerait son intérêt comme principe premier de la nouvelle réforme sur les droits de l'enfant ?» (B. Höll député PDS).

«Nous devons dépasser l'idée que l'enfant est simplement un objet à éduquer et une sous-partie de la famille. Il a sa personnalité propre. Cela doit être autrement plus affirmé que cela n'apparaît actuellement (...). La réforme des droits de l'enfant serait vraiment d'importance. Je serais redevable au gouvernement si cela pouvait être préparé plus rapidement que cela ne l'a été jusqu'à aujourd'hui.» (S. Rahard-Vahldieck, députée CDU).

Or, comme le montre les paragraphes suivants, la prise en compte de l'intérêt de l'enfant est un pas supplémentaire vers l'institutionnalisation de l'exercice bilatéral de la parentalité.

\section{II.C.1.2 L'intérêt de l'enfant comme vecteur de la présence des parents}

Aujourd'hui, le refus de coopération entre les parents n'est plus un argument valable pour demander l'exercice unilatéral de l'autorité parentale. Il est subordonné à la nouvelle définition de l'intérêt de l'enfant ${ }^{137}$ : en effet, celui-ci doit pouvoir garder le contact avec ses deux parents dans le but de lui éviter des conflits de fidélité. Le conflit, qu'il soit potentiel ou réel, est subordonné à la double socialisation de l'enfant, et don lieu de résidence est attribuée à celui qui favorise le plus le contact avec l'autre parent. Les décisions sont laissées au couple parental, supposé capable de prendre des décisions communes au-delà de leurs différents (Wagenitz ; Barth, 1996) pour le bien de leur enfant.

La notion de l'intérêt de l'enfant «Kindeswohl» a joué un rôle important dans la mise en place des réformes de l'autorité parentale. La France et l'Allemagne ont ratifié la «Convention internationale des droits de l'enfant» (New York, 1990) qui définit l'intérêt supérieur de l'enfant comme «le droit de connaître, de disposer de ses deux parents» (Article 9). Cette convention associe l'intérêt de l'enfant et la présence des deux parents. Cette association a été un moteur réel pour les réformes de l'autorité parentale conjointe.

Le fait même d'avoir ratifié cette convention a relancé les débats (Stöcker, Ullmann, 1992) sur le rôle dominant de la mère (Herdegen, 1993), dans l'attribution de la garde et de l'autorité parentale, après la séparation conjugale. A l'occasion d'un rapport du gouvernement sur les mesures mises en place depuis la signature de la Convention mondiale des droits des enfants du 20 novembre 1989, l'enfant et ses droits ont été discuté au parlement à la session du 25 mars 1993 (Das Parlament : 1993). Au-delà du consensus entre les partis qui s'accordent sur l'importance de l'enfant, deux conceptions s'opposent : les droits des enfants

\footnotetext{
${ }^{137}$ Décision du tribunal OLG Groß-Gerau du 22.10.1997 (FamRZ 1998)
} 
trop spécialisés pourraient mettre la famille en danger ${ }^{138}$; les opposants estimant le contraire $^{139}$. Plus précisément, il s'agit d'une opposition de conception entre le droit des parents et le droit des enfants.

Enfin, la réforme de 1998 sur les droits de l'enfant, autorise la présence d'un «avocat des enfants» («Verfahrenpfleger»), en d'autres termes d'un curateur ad hoc, nommé pour veiller aux intérêts de l'enfant lors de la procédure de divorce. Ceci montre le souhait normatif de l'Etat de placer l'enfant au centre de la procédure de la séparation parentale. Mais les résistances sont nombreuses, tant au niveau des parents que des magistrats, et cette procédure est très rarement utilisée.

Ainsi, malgré la nouvelle définition de l'intérêt de l'enfant, la conception d'un couple parental après la séparation ne fait pas l'objet d'un consensus social. On parlera dans le cas de l'Allemagne d'une juxtaposition des deux parents et de leur présence bilatérale dans une dynamique d'opposition des droits des pères et des mères.

\section{II.C.1.3 La parentalité et les familles naturelles}

Jusqu'en 1998, le père naturel n'a aucune autorité parentale. Celle-ci est entièrement déléguée à la mère depuis 1969. Si le père reconnaît l'enfant, il n'obtient pas de droit, mais une obligation alimentaire. C'est la mère qui, par l'autorité parentale, détermine, au nom de l'enfant, ce qu'elle considère comme relever de son bien-être. Elle a donc tout pouvoir pour permettre ou non les contacts entre l'enfant et son père. Dans le cas des familles naturelles, les visite entre le père et son enfant ne sont pas un droit.

Si la mère souhaite interdire l'accès à son enfant, le père n'a aucun contre-pouvoir efficace. Néanmoins, depuis 1979, il peut avoir recours au juge des tutelles, mais il lui faudra prouver que ce contact correspond au bien-être de l'enfant, preuve rendant la démarche concrètement impossible.

La réforme sur les droits de l'enfant en 1998 permet d'accéder à l'autorité parentale conjointe lorsque les parents naturels effectuent une déclaration d'exercice commun «Sorgeerklärung». Dans cette procédure, les couples n'ont pas à faire preuve de leur cohabitation. Dans les autres cas, seule la mère a l'exercice de l'autorité parentale ; la règle de subsidiarité en sa faveur a été maintenue.

\footnotetext{
${ }^{138}$ K. Riegert (député CDU-CSU) rappelle que la politique du gouvernement est une politique de la famille, comme cela est spécifié dans la Constitution. Il souhaite donc dénoncer la stratégie des socio-démocrates qui selon lui mettent les droits des enfants en «guerre» contre ceux des parents. Nous rappellerons l'idéologie du parti conservateur CDU par l'intervention de la député S. Geiger pendant ce même débat parlementaire «les enfants vivent sous la protection de la famille (...) et ils ont besoin de parents, qui sont conscients de leur responsabilité, et à qui on ne doit pas non plus les retirer».

139 Pour le député N. Eimer (FDP centre gauche) la réforme est à saluer comme «le fait que ce ne soit plus l'intérêt des parents ou bien un principe d'ordre abstrait qui soit le principe de départ de la réflexion, mais les droits des enfants».
} 


\section{II.C.1.4 Les résistances des mères}

En Allemagne, la représentation de la famille correspond à celle de la famille bourgeoise basée sur le mariage et son principe de «l'homme soutien de famille» (Ostner, 1993). L'idée que la mère se dévoue à l'éducation de(s) l'enfant(s) est centrale dans la représentation allemande de la famille «unie» et «désunie» (Limbach, 1981).

Dans ce contexte, l'exclusivité des droits de garde est un enjeu identitaire très important pour les mères allemandes. Ainsi, devant la montée des droits du père, les associations de soutien des familles monoparentales se sont opposées à l'exercice de l'autorité parentale conjointe ${ }^{140}$. En effet, les nouveaux droits des pères (droit de garde et de présence auprès de l'enfant) ont été vécus par les associations de défenses des mères comme une restriction de leurs propres droits, et non comme un nouveau principe égalitaire de partage des tâches (Heiliger, 1992 ; M. Breithaupt, 1993).

La fédération des associations de familles monoparentales (VAMV) se déclare défavorable à la réforme proposée par le SPD sur l'autorité parentale conjointe, argumentant que le père s'éloignant de ses enfants (les liens se réduisent considérablement après 6 mois), il serait inconcevable de lui accorder des droits alors qu'il ne remplit aucun devoir ${ }^{141}$. La désaffectation paternelle, ainsi comprise, justifie la mise en balance des devoirs et des droits parentaux, les opposant dans une situation de concurrence. Les droits respectifs de chaque parent sont discutés en opposition.

L'enjeu est d'autant plus important que l'identité sociale des femmes allemandes se construit essentiellement dans la sphère familiale. En effet, leur présence sur le marché du travail, est très différente de celle de leurs homologues françaises. Le travail des femmes en France a une valeur sociale qui n'a pas son équivalent en Allemagne (Martin, 1996). En Allemagne, la norme de «l'homme soutien de famille» détermine la faible acceptabilité sociale de la participation au marché du travail des femmes et des épouses, et a fortiori des mères.

Cette norme se reflète dans les situations de l'après-divorce. En effet, conformément aux articles 1361 I, II et 1570 du Code Civil allemand, après une séparation ou un divorce, il n'est pas attendu de la personne, chez qui l'enfant réside, qu'elle exerce une activité professionnelle; elle a droit à une pension alimentaire tant que les soins et l'éducation de l'enfant l'empêchent d'être active.

Pour M. von Renessse ${ }^{142}$, deux formes de facteurs s'opposent à une réforme du droit de la famille ${ }^{143}$ : la première est qu'un divorce conflictuel provoque chez les mères, dont la plus part vivent en «intensive symbiose» avec l'enfant, une volonté de couper définitivement avec l'ancien partenaire, la forte attache passionnelle à l'enfant provoquant l'idée que faciliter

\footnotetext{
${ }^{140}$ A titre d'exemple, la fédération des associations de familles monoparentales (VAMV) a fait paraître un livret, distribué à grande échelle par le ministère allemand de la famille, intitulé «Comment m'en sortir toute seule ?» lequel ne traite ni du père, ni de ses droits, ni d'une éventuelle coopération parentale.

${ }^{141}$ Cette discussion est d'autant plus intéressante, que la désaffectation paternelle (au niveau financier) dans les faits est beaucoup moins importante qu'en France, où cet argument n'est pas avancé.

${ }^{142}$ M. von Renesse, juge des affaires familiales, est ancienne députée SPD au siège à la commission du SPD de la politique intérieure et du droit.

${ }^{143}$ Nous rappellerons que le SPD a été porteur des réformes sur les droits de l'enfant en Allemagne.
} 
l'accès à l'autre parent équivaut à une perte ; la seconde vient des groupes de défense des mères qui voient d'abord l'intérêt de la mère dans le divorce, au détriment de celui de l'enfant.

«En réalité, le plus grand paradoxe est né du fait que l'intérêt de la femme sera séparé de celui de son enfant. Au fond le législateur souhaite que le changement de paradigme se fasse comme suit : que l'on tienne compte de l'intérêt de l'enfant afin de proposer une réponse aux séparations problématiques des parents» (Entretien avec M. von Renesse : 1996).

\section{II.C.1.5 La mobilisation des pères}

Dans son article sur les changements des rapports enfant-parents depuis la seconde guerre mondiale, Y. Schütze retrace l'évolution des préoccupations relatives à l'éducation des enfants à travers l'orientation des travaux scientifiques de l'époque. Les intérêts se focalisent «de plus en plus sur la qualité psychologique de la relation mère-enfant dans les premières années de la vie de l'enfant (...) la relation mère-enfant n'est plus donnée comme naturelle, mais au contraire elle est problématisée comme $l a^{144}$ variable décisive pour l'équilibre physique et psychique de l'enfant» (Schütze 1988 : 100). Ce témoignage sur les années 1970 est précieux car il montre l'origine d'un trait spécifique allemand qui justifie le rôle dominant de la mère dans l'éducation des petits enfants.

Puis, dans les années 1980, la présence du père comme éducateur remet en question la dominance du lien mère-enfant. «La tendance à l'émotionnalisation des rapports parentenfant, qui se prolonge dans les années 80, accuse une modification (...) le rapport parentenfant n'est plus défini de façon dominante comme un rapport mère-enfant, mais au contraire, le père gagne en importance en tant que personne de référence» (Schütze, 1988 : 100).

Ce discours se prolonge dans les années 1990. Le 17 novembre 1997, l'hebdomadaire allemand «Der Spiegel» titrait en première page : «Die vaterlose Gesellschaft» («La société sans père»). Les débats, hautement polémiques, ont été relancés sur la place des pères après le divorce, sur le pouvoir des mères et du système judiciaire.

Depuis, les associations de défense des droits des pères se sont multipliées et ont contribué à inverser l'image du père désintéressé du sort de ses enfants, en un père responsable et discriminé.

En d'autres termes, l'Allemagne construit la parentalité sur une argumentation basée sur la guerre des sexes et sur une vision très polarisée des rôles. Ces caractéristiques ont donné une coloration particulière au changement de norme de la parentalité : la juxtaposition des parents dans une relative concurrence au sein du couple parental.

\footnotetext{
${ }^{144}$ En italique dans le texte
} 


\section{SOUS-SECTION II.C.2 LA PROTECTION SOCIALE ET LE RISQUE FAMILIAL}

Au niveau national, les familles monoparentales ne sont pas conceptualisées comme une catégorie de l'intervention sociale: il n'existe pas d'allocation spécifiques pour les familles monoparentales dans le régime général de la protection sociale. En revanche, elles relèvent de la «Sozialhilfe» («assistance sociale») ${ }^{145}$. A niveau des Etats fédérés, l'aide sociale prend la forme d'une protection catégorielle (correspondante à l'API en France). Les différents Länder ont développé des programmes spécifiques destinés aux familles monoparentales, néanmoins, ils ne donnent pas lieu à un droit social en soi.

\section{II.C.2.1 Le risque familial et l'assistance sociale}

L'aide sociale est régie par le principe de subsidiarité qui hiérarchise les ressources dans l'ordre suivant : premièrement, les ressources privées (la force de travail, le salaire et la propriété ) ; deuxièmement, la pension (à travers les personnes impliquées pour un soutien alimentaire); troisièmement, les aides sociales de prévention de compensation et de dédommagement; et quatrièmement, en dernier recours, l' «assistance sociale» (Bauer 1992). $\mathrm{Au}$ sein de l'aide sociale, ces ressources vont être activées (pour des familles monoparentales) dans une logique particulière qui vient contredire le principe général de subsidiarité. En effet, leur statut de mère modifie la hiérarchie présentée ci-dessus.

Le premier pendant de la couverture sociale des familles monoparentales est celui de l'assistance sociale, par l'attribution d'un minimum social. Les principes de l'assistance sociale allemande divise le groupe des allocataires potentiels suivant deux critères de précarité : «l'aide en cas de revenu défaillant» («Hilfe zum Lebensunterhalt» et «l'aide pour situation difficile» (loi du 1.6.1962 Bundessozialhilfegesetz (BSHG)). Les familles monoparentales appartiennent à la dernière catégorie.

Une étude comparative entre l'Allemagne et les Etats-Unis permet d'appréhender la qualité de cette dépendance (Voges/Ostner 1995). Il semble que pendant les douze premiers mois, les mères seules aient moins de difficultés à sortir de l'aide sociale qu'aux Etats-Unis. Au-delà de ces douze mois, les chances s'inversent. Après 24 mois, 38\% des femmes allemandes, en situation de monoparentalité, relèvent du régime de l'assistance sociale contre $20 \%$ aux Etats-Unis. Mais globalement, en comparaison avec le Canada, la Grande-Bretagne, et les Etats-Unis, ce sont les familles monoparentales allemandes qui s'émancipent le plus rapidement de l'aide publique, en moyenne 9,4 mois (contre 10,3 aux Etats-Unis).

\footnotetext{
${ }^{145}$ La protection publique des familles monoparentales s'étend dans de nombreux domaines. Une étude approfondie de la mosaïque des avantages sociaux dépasserait le cadre de notre analyse. Néanmoins, nous nommerons les mesures les plus importantes (à noter que l'allocation logement joue un rôle indirect important). En Allemagne, les familles monoparentales reçoivent les allocations familiales comme les autres familles, soit en 1997, 720 Fr. [110 Euros] pour le premier et le second enfant, 990 Fr. [151 Euros] pour le troisième et $1150 \mathrm{Fr}$. [175 Euros] pour les rangs suivants. Les familles monoparentales peuvent, comme les couples, choisir entre les allocations ou une déduction d'impôt.

Elles peuvent déclarer $18530 \mathrm{Fr}$. [2825 Euros] de «frais de fonctionnement du ménage», qui pourront être déduits des impôts, sorte de compensation du quotient conjugal qu'elles ne peuvent appliquer.
} 
En Allemagne, la raison pour laquelle les mères seules demandent l'aide sociale, est majoritairement liée aux événements familiaux, mais leur situation s'améliore quand elles accèdent à une activité professionnelle. Ce phénomène est beaucoup plus marqué que dans les autres pays de l'étude. En Allemagne, ce sont le divorce et la séparation qui sont les premières raisons pour recourir au secours du système social (Voges/Ostner 1995). Ce risque de pauvreté lui est très spécifique car pour le Canada et les Etats-Unis, c'est le chômage qui a la plus grosse influence.

Sans être le facteur le plus déterminant, la remise en couple joue un rôle très important dans l'amélioration de la condition des familles monoparentales. En revanche, c'est la (re)prise d'une activité professionnelle qui a la plus grande influence pour sortir de l'assistance sociale, pour la France, et plus encore pour l'Allemagne.

Les familles monoparentales, en tant que bénéficiaires de l'assistance sociale, sont contraintes au principe de «Selbsthilfe», d' «autonomie par rapport à l'aide publique». C'est donc à ce titre qu'elles sont relativement plus présentes sur le marché du travail que les mères en couple.

Cependant, en tant que mères, elles remplissent un rôle éducatif auprès du jeune enfant qui est considéré comme primordial. Par-là, elles accèdent à un statut particulier au sein de l'assistance sociale. A ce titre, on toléra qu'elles puissent profiter de l'aide sociale pour se consacrer à plein temps à l'éducation de leurs enfants. Le rôle d'éducatrice leur donne un statut d'axception, qui précède leur intégration sur le marché du travail. De fait, pour les famille monoparentales, l'assistance sociale bascule en première place de la logique de subsidiarité de l'aide sociale, devançant le recours aux ressources privées.

Le second niveau de l'aide sociale pour les familles monoparentales, se constitute autour de la pension alimentaire, elle prend alors deux formes :

Premièrement, c'est la famille d'origine de la mère qui peut être sollicitée. Ce qui est, dans les faits, peu pratiqué. Effectivement, si la femme est enceinte, l'aide sociale lui sera attribuée sans faire appel à la famille d'origine (afin d'éviter un éventuel avortement). Et si la mère est divorcée, séparée, ou veuve, elle est statistiquement plus âgée, et dans ce cas, ses propres parents sont généralement retraités; il leur est alors difficile d'être les garants économiquement pour la mère et son enfant. Aussi, ce principe de soutien financier par la parenté directe est peu pratiqué, comme le confirme le témoignage du directeur du service social de recouvrement des pensions alimentaires de Göttingen (1997).

Deuxièmement, l'Etat sollicite un autre relais de la sphère privée qui ne soit ni la mère, ni sa parenté : c'est l'aide du père des enfants qui, par le principe de subsidiarité de l'assistance sociale, va être requise.

\section{II.C.2.2 L'obligation alimentaire des pères}

Les pères allemands s'acquittent à plus de $76 \%$ de la pension alimentaire des enfants (Neubauer, 1989 : 414), laquelle constitue une part importante du budget des familles monoparentales. Par exemple, pour les femmes qui ont suivi le programme «Mütter-Kind» (cf. § II.C.2.4. les aides catégorielles destinées aux familles monoparentales), la pension alimentaire versée par le père des enfants peut atteindre $58 \%$ de leur budget.

«Les pères sont obligés d'être actifs à la recherche d'emploi. (...) Ils doivent donc prouver leur bonne foi, lorsqu'ils sont chômeurs par exemple (...) si la période de chômage 
est plus longue qu'un ou deux ans, alors, oui, nous lui réclamons des documents» (Entretien avec le Directeur du service social de recouvrement des pensions alimentaires de Göttingen, 1997). Par ailleurs, un père chômeur qui ne pourrait pas exercer un travail à temps plein peut être obligé de prendre un travail à temps partiel ${ }^{146}$.

Le non-paiement des pensions alimentaires a obligé les Etats allemand et français à institutionnaliser une couverture publique des pères défaillants. Les femmes qui font appel au service de recouvrement des pensions sont en majorité des femmes qui sont confrontées à la non-solvabilité des pères. En effet, les cas traités par le service de recouvrement des pensions alimentaires concernent souvent les couches sociales les plus défavorisées.

«Sur l'ensemble de toutes les demandes de recouvrement, la moitié provient de femme de l'assistance publique. Théoriquement, toutes les classes sociales pourraient être représentées mais en majorité, ce sont les couches sociales les plus basses ou moyennes qui viennent. Je pense que les mères qui ont de bons revenus n'ont pas besoin de l'aide, ou n'estiment pas nécessaire de venir, ou bien encore peuvent, avec l'aide d'un avocat, faire valoir leurs droits, ou bien leur partenaire est solvable et paye.» (Entretien avec Monsieur le directeur du service social de recouvrement des pensions alimentaires 1997).

La relation entre les pères et le système de protection sociale est différente de celle entre les mères et l'aide sociale. Le père a le devoir de s'intégrer au marché du travail, son obligation d'entretien l'oblige à rester solvable. Elle domine totalement la conception de la position du père dans l'après-divorce.

\section{II.C.2.3 Le système de recouvrement des pensions}

La mise en place d'un système de recouvrement des pensions a été portée par les partis de gauche. En France, dans les années 1970, la gauche s'est faite l'initiatrice de deux combats culturels, le divorce et le projet de recouvrement des pensions alimentaires par la création d'un fonds public (Théry 1993 : 78). Ce courant politique définit les responsabilités parentales devant les choix familiaux comme faisant partie de la responsabilité collective. L'idée était d'aider les personnes les plus démunies devant les situations de divorce. Les mêmes combats sont portés par la gauche allemande dans la même période ${ }^{147}$.

En Allemagne, lors du débat sur le projet de l'UVG (allocation de recouvrement des pensions alimentaires) en 1978, la député I. Karwatzki (CDU) fait remarquer aux partis FDP et SPD qu'il semblerait normal que ce soit le parti du CDU qui introduisent cette mesure, car, étant à l'origine des réformes sur le divorce, il serait logique qu'ils assument les risques familiaux issus de cette libéralisation du divorce.

Ce discours marque le passage vers une responsabilité collective des choix personnels. La vocation du droit social prévu dans les années 1970 perd de la force pour devenir une protection contre la précarité.

\footnotetext{
${ }^{146}$ Décision du tribunal OLG Oldenburg du 24.10.1995 (FamRZ 1996 : 672)

${ }^{147}$ Le projet de l'UVG a été présenté par les partis du SPD et du FDP et discuté au Bundestag pour la première fois le 5 octobre 1978. La protection de l'enfant est au centre des préoccupations : «le but que ma fraction politique souhaite atteindre, est de garantir la pension alimentaire de l'enfant, pour qu'il n'ait pas à souffrir des conséquences lorsque son droit alimentaire n'est pas honoré» (Propos de la députée SPD E. Eilers, Das Parlament : 1978).
} 
En Allemagne, la situation de pauvreté des familles monoparentales est présente dans les discours politiques. Elle fut énoncée lors des débats parlementaires sur la mise en place de $\mathrm{l}^{\prime} \mathrm{UVG}^{148}$. Le problème du versement irrégulier des pensions alimentaires est vu, par les politiciens, comme le premier respons de la précarité des familles monoparentales. Ainsi, la protection sociale, autour des familles monoparentales, ne s'oriente pas vers des mesures catégorielles comme en France, mais relève du système de couverture de la pauvreté.

Le député N. Eimer (FDP) lors de la présentation du projet de UVG au Bundestag ${ }^{149}$, souligne les deux objectifs de la réforme :

D'un côté, la mesure a pour objectif d'éviter l'avortement pour les mères en situation précaire, provoquée par le non-payement de la pension alimentaire. Pour les mêmes raisons, l'obligation de payement des parents de l'allocataire est annulée si la femme est enceinte. Et, à titre exceptionnel, les mères seules peuvent recevoir l'allocation de congé parental sans la déduire de la «Sozialhilfe» («l'assistance sociale»).

D'un autre côté, si l'activité professionnelle est possible (le salaire n'est pas déduit de l'allocation), elle ne fait pas l'objet de mesure d'incitation. Ces arguments ont surtout pour but de rassurer le parti du CDU/CSU, afin de diminuer leur résistance à la réforme. Mais le travail des familles monoparentales n'est pas une réelle préoccupation, contrairement à leur rôle éducatif. La référence au droit à l'éducation, à la présence à plein temps d'un parent pour l'enfant est récurrente.

L’UVG est versée sous forme de revenu minimum, et dans sa version discutée en 1978, elle peut être versée pendant 3 ans et dans les limites des 6 premières années de l'enfant «(...) afin de soulager de nombreux pères et mères en situation de monoparentalité, et surtout de leur permettre dans les années les plus décisives de la vie de l'enfant d'accomplir, tout particulièrement, leurs devoirs d'éducation» (Propos de I. Karwatzki (CDU), Das Parlament $\mathrm{N}^{\circ} 43 / 38$ octobre 1978$)^{150}$.

Le montant de la pension de recouvrement se calcule à partir du montant de la pension de référence «Düsseldorfer Tabelle», moins la moitié de l'allocation familiale pour le premier enfant. Or, l'allocation familiale par enfant a augmenté, faisant diminuer la pension alimentaire de recouvrement.

Comme l'UVG se calcule en fonction d'une moyenne, il n'y a pas de relation entre la pension alimentaire fixée par le tribunal et le montant de l'aide. La prestation est donc découplée de la situation familiale et de la décision du juge. La justice et l'administration

\footnotetext{
${ }^{148}$ Les résultats d'une recherche commanditée par le ministère de la Justice auprès de l'institut «Max-Plank» permettent d'étayer par des arguments scientifiques le projet de UVG proposé par la fraction SPD, soutenue au Bundestag par la députée E. Eilers (Das Parlament 1978).

${ }^{149}$ La discussion sur l'UVG se fait dans une approbation générale. Le parti du CDU/CSU approuve cette mesure en faisant remarquer que l'amélioration des conditions de vie des familles «incomplètes» a toujours été inscrite à son agenda politique.

En France, la nécessité d'une couverture publique fait l'unanimité dans les formations politiques. «lors de la préparation du projet de loi par Mme Roudy, alors ministre des droits des femmes, l'accord des différentes formations politiques est quasi-unanime pour une prise en charge, par les pouvoirs publics, du phénomène du non paiement des pensions alimentaires» (Martin $1996: 74$ ).

${ }^{150}$ Cette orientation de l'allocation est mise très clairement en valeur, nous semble-t-il, par la contre-remarque de la député I. Karwatzki : le fait de limiter la période possible de l'attribution de l'allocation aux six premières années de la vie de l'enfant n'est pas soutenable. En effet, c'est la situation de précarité qui doit déterminer l'attribution de l'allocation et non pas l'âge de l'enfant. Cette remarque souligne bien l'orientation que le SPD et FDP donnaient à l'allocation : une sorte d'allocation d'éducation pour les 6 premières années de la vie de l'enfant.
} 
chargée du recouvrement des pensions fonctionnent en parallèle et de façon plus indépendante qu'en France ${ }^{151}$.

Suivant le principe de subsidiarité de l'aide publique par rapport à la sphère privée, l'allocataire de l'UVG se doit d'épuiser tous les soutiens privés avant de faire appel à l'aide de l'Etat. La logique d'attribution de cette aide sociale a trois niveaux d'intervention :

Le premier est celui de la mère, laquelle n'est pas tenue de prendre un emploi si elle a la charge du jeune enfant : «en fait la mère nous intéresse peu. Bien sûr il lui sera demandé ses activités financières, mais ça n'a rien à voir avec l'attribution de l'allocation UVG.» (Entretien avec Monsieur le directeur du service de recouvrement des pensions alimentaires 1997)

Le second niveau d'intervention concerne la parenté. «Théoriquement oui, il existe, en Allemagne, le principe selon lequel les parents de ligne directe doivent, par la loi payer la pension alimentaire. Mais, la pratique ne tient pas compte de ce principe. Cela est dû au fait que les grands-parents d'un enfant doivent déjà pouvoir conserver une somme, de «Selbstbehalt» «somme considérée nécessaire pour leurs propres besoins financiers», par exemple 6000 Fr. [92 Euros] par mois, en dessous de cette somme il n'est pas possible de leur réclamer de l'argent. C'est à dire, qu'il est très irréaliste de croire que l'on peut exiger d'eux une pension. C'est pourquoi, le niveau de vie des parents ne sera pas mis en examen par nos services.» (Entretien avec Monsieur le directeur du service de recouvrement des pensions alimentaires 1997).

Troisièmement, d'un côté, les mères sont peu sollicitées parce qu'elles remplissent les devoirs éducatifs auprès de l'enfant, de l'autre, le père est institué comme le premier responsable de l'entretien de l'enfant. Et s'il n'est pas solvable, alors, il a l'obligation de s'intégrer sur le marché du travail.

«Les pères sont obligés d'être actifs à la recherche d'emploi. La réglementation est telle que le débiteur doit prouver et documenter jusqu'à 30 demandes d'emploi par mois. Nous pouvons l'obliger légalement à nous les donner. Ils doivent donc prouver leur bonne foi, (lorsqu'ils sont chômeurs par exemple). Cela ne suffit pas de dire qu'on est chômeur; néanmoins, dans un premier temps, cela nous suffit. Par contre, si la période de chômage est plus longue qu'un ou deux ans, alors, oui, nous lui réclamons des documents. La jurisprudence a décidé que le service de recouvrement pouvait réclamer jusqu'à 30 demandes d'emploi par mois. Mais ce nombre théorique de demandes d'emploi n'est jamais demandé.» (Entretien avec Monsieur le directeur du service de recouvrement des pensions alimentaires 1997).

Avant que soit mis en place l'UVG, le «Land» de Hambourg avait mis en place un système pilote de recouvrement de pensions alimentaires dans les années 1970. En 1978, lors du débat sur le projet de UVG, la député I. Karwatzki rappela les chiffres d'efficience de ce

\footnotetext{
${ }^{151}$ Par ailleurs, les critères de non-attribution de l'allocation pension de recouvrement sont importants car ils définissent l'orientation normative de l'allocation. La demande n'aboutira pas, si l'enfant est confié à un tiers, si l'enfant vit en présence d'un beau-parent, si le parent refuse de donner l'identité et les coordonnées de l'autre parent et si l'autre parent a remplit ses devoirs alimentaires.

En Allemagne (comme en France où les critères d'attribution sont les mêmes), la norme d'application du recouvrement des pensions est semblable. L'enfant doit rester à la charge d'au moins un de ses parents. L'Etat n'intervient qu'en cas de défaillance de la solidarité du couple parental et avec l'assurance que le parent non gardien sera réellement sollicité dans ses devoirs alimentaires.
} 
service (Das Parlament 1993). L'objectif était de récupérer 25\% des pensions avancées, 10\% ont été finalement recouvertes. L’UVG a pris sa version moderne en 1984.

En 1997, l'efficience du service est pratiquement restée la même, soit $17 \%$ de recouvrement, pour un objectif de $30 \%$. «Je pense que les objectifs de recouvrement sont de plus en plus poussés vers le haut. On va essayer de récupérer le plus grand nombre de pensions, par rapport à la situation difficile en Allemagne, à l'heure actuelle, bien que la situation économique soit aussi difficile pour les débiteurs. Les objectifs sont montés mais à cause de la situation de chômage, ils sont de plus en plus difficiles à appliquer» (Entretien avec Monsieur le directeur du service de recouvrement des pensions alimentaires 1997).

La pension alimentaire de l'enfant est d'abord une affaire qui relève de la sphère privée. C'est uniquement en cas de défaillance des débiteurs privés, que l'aide publique prend le relais. Cette procédure montre le glissement normatif du rôle protecteur de l'Etat vers le principe suivant : «les parents doivent être les premiers responsables pour l'entretien financier et alimentaire de leur enfant».

Dans les deux pays, le recouvrement des pensions alimentaires se fait surtout à perte pour l'Etat car les pensions sont peu payées par les pères. Malgré les contraintes économiques, l'opinion politique est unanime : ce service est essentiel pour la protection sociale des familles monoparentales. A notre avis, le consensus entre les partis montre la fonction normative du service de recouvrement des pensions alimentaires (au delà de sa fonction économique). La norme sociale est que la responsabilité et les devoirs alimentaires incombent en premier lieu à la sphère privée.

\section{II.C.2.4 Les aides catégorielles destinées aux familles monoparentales}

Dans l'ensemble des formes d'aides catégorielles réservées aux familles monoparentales, nous avons retenu le programme «Mère et Enfant» de l'Etat de BadenWürttemberg. Ce dernier a deux particularités intéressantes pour notre analyse. D'une part, il fut projet-pilote en 1975 dans la République Fédérale Allemande ; d'autre part, il a été soumis à une évaluation en 1993 par le ministère de la Famille, de la Femme, de la Formation et de la Culture.

Ce programme offre un soutien financier et pédagogique aux mères seules d'enfants en bas âge. Les participantes perçoivent ce soutien pédagogique ${ }^{152}$, non comme un contrôle, mais comme l'opportunité de rompre l'isolement social : pour $86 \%$ des femmes, l'échange d'expériences étant jugé comme un point très bénéfique du programme.

En fait, la recherche d'un emploi est une préoccupation moindre par rapport à l'importance que revêt l'éducation des enfants dans les trois premières années de leur vie : la présence éducative de la mère est primordiales pour l'enfant et l'aide sociale est vécue comme une alternative au marché du travail ${ }^{153}$. La relation entre l'emploi et la protection sociale des familles monoparentales s'organise suivant le principe de l'Etat-père. «L'aide financière du

\footnotetext{
${ }^{152}$ Le soutien pédagogique est organisé sous forme de groupes de réunion et de discussion. Les thèmes des groupes de soutien s'articulent autour trois axes: les problèmes rencontrés dans les administrations, les problèmes financiers, et enfin, l'aide et les conseils pour la recherche d'un emploi. Ce dernier thème gagne en signification surtout en fin de programme.

${ }_{153} 82 \%$ des femmes en situation de monoparentalité travaillent en France (1992), contre 56\% en Allemagne (1986)
} 
programme «Mère et Enfant» doit permettre aux familles monoparentales de se libérer de la nécessité de l'activité professionnelle pour des raisons économiques afin d'être dans une situation qui leur permette de se consacrer à l'éducation et aux soins de leurs enfants dans les trois premières années de leur vie». (Landtagsdrucksache, 1993).

Néanmoins, en 1994, 31\% des participantes souhaitent un soutien concret pour la recherche d'un emploi dès la première année, et plus de $50 \%$ le souhaite au moins en fin de programme. 36\% des anciennes participantes jugent qu'une aide pour l'emploi aurait été souhaitable. En conclusion du rapport, les enquêteurs proposent d'améliorer le programme en renforçant le dispositif de recherche d'emploi.

Par ailleurs, bien que le programme permette une activité professionnelle à temps partiel, elles sont inactives à plus de $80 \%$, actives à $13 \%$, et $7 \%$ en formation. La composition des ressources des mères monoparentales qui suivent le programme, nous apprend que la pension alimentaire est la seconde source de revenu pour la majorité d'entre elles (58\%), le recouvrement de la pension alimentaire par le service social pour $39 \%$. 8\% reçoivent le salaire d'une activité professionnelle irrégulière et $6 \%$ d'une activité régulière.

Bien que $70 \%$ des femmes monoparentales à l'Ouest et $54 \%$ à l'Est soient divorcées, mariés, séparées ou veuves, la pension alimentaire n'est le revenu principal que pour $11 \%$ des familles monoparentales à l'Ouest et $1 \%$ à l'Est.

C'est l'activité professionnelle des familles monoparentales qui représente la première ressource pour $57 \%$ dans l'ancienne Allemagne Ouest et $61 \%$ à l'Est. Les prestations sociales, en particulier l'assistance sociale, est la seconde source de revenus pour $27 \%$ d'entre elles (équivalent au RMI français). Enfin, l'allocation chômage est la première source de revenus pour $22 \%$ des mères seules à l'Est (elles sont plus nombreuses qu'à l'Ouest) (Engelbrech, Jungkunst 2001).

L'accueil sur le marché du travail des femmes en fin de droit ne leur est pas favorable, bien qu'à la sortie, elles doivent compter, pour plus de la moitié d'entre elles, sur une activité professionnelle comme principale source de revenu: une majorité trouvera une activité professionnelle, $24 \%$ à temps plein et $34 \%$ à temps partiel.

Par ailleurs, ce programme a un effet «décommodificateur» certain (Schultheis 1987) : sur $50 \%$ des participantes qui avaient un travail avant, une sur deux exercent une activité professionnelle après le programme, et $26 \%$ sont dépendantes de l'aide sociale (complètement ou en partie $)^{154}$.

Finalement, 58\% des anciennes participantes assurent leurs ressources au moyen d'une activité professionnelle régulière, $26 \%$ reçoivent l'aide sociale en complément ou comme ressource principale, $6 \%$ ont des allocations chômage comme première source de revenu, et $9 \%$ vivent de la pension alimentaire. Globalement, le même nombre de mères monoparentales travaille à la sortie du programme, mais un quart des femmes n'a pas pu se réinsérer dans le marché du travail.

En résumé, l'Allemagne n'a pas d'allocation catégorielle liée au statut des familles monoparentales, dans le régime social national. Mais il existe des programmes à chaque Land (à l'image du programme d'aide aux familles monoparentales spécifique du BadenWürttemberg).

\footnotetext{
${ }^{154}$ Quant au petit tiers des participantes qui recevaient l'aide sociale ou le chômage avant le programme, 40\% auront une activité salariale à la sortie du programme.
} 
L'Etat allemand a d'autres dispositifs, comme une grande diversité d'exonération d'impôt ${ }^{155}$ (reconnaissance des frais de garderie des enfants pour les familles monoparentales...).

Traditionnellement, la politique de la famille, centrée sur la femme au foyer, ne permet pas d'avoir une couverture directe des familles monoparentales. Cette reconnaissance explicite serait en contradiction avec l'idéal du couple conjugal légitime «Ehe». Commaille (1996 : 114) reprend l'étude de Schultheis pour spécifier le système de protection en Allemagne : il s'agit d'une construction globale de la situation individuelle du demandeur. Le système est plus orienté sur le «besoin»; la France aurait plus une logique «d'ayant droit». Cela explique la différence de protection du risque familial entre la France et l'Allemagne. La protection sociale allemande se baserait plutôt sur une reconnaissance des besoins des familles monoparentales, ces dernières relevant alors de l'assistance sociale.

Enfin, la gestion du risque familial maintient le père et la mère à des niveaux différents, dans le système de protection sociale, en juxtaposant les mesures et les aides ${ }^{156}$. Leurs relations à l'aide sociale et au marché du travail sont très marquées par le cloisonnement des rôles masculins et féminins. L'exercice de la parentalité reconnaît l'existence de la fonction parentale comme un ensemble de droits et de devoirs, ainsi que le principe d'égalité entre les parents. Mais la notion de couple parental n'existe pas comme telle, on parlera de «biparentalité»: en d'autres termes d'une présence bilatérale de deux parents où la conflictualité des rapports domine les représentations.

\footnotetext{
${ }^{155}$ La fiscalité joue un rôle indirect mais non moins important dans le façonnement de la protection sociale. Elle conditionne les passages d'un relai à l'autre de l'économie mixte. «les systèmes de transfert, et surtout la fiscalité, peuvent plus ou moins pénaliser ou encourager le travail salarié des mères (...) la France, pour ne pas pénaliser les familles à double revenu, les plafonds de ressources liés à l'octroi de certaines prestations familiales, ou aux aides au logement, sont relevés lorsque la conjointe exerce une activité professionnelle» (Fagnani 1996 : 32). En France, le travail professionnel est d'autant plus encouragé que les déductions fiscales sont couplées à des prestations pour diminuer les frais de garde des enfants. Deux prestations des années 1980 s'inscrivent dans cet ordre : l'aide à la famille pour l'emploi d'une assistante maternelle agrée (AFEAMA) et l'allocation de garde d'enfant à domicile (AGED).

L'Allemagne donne le choix et l'imposition des revenus des conjoints peut se faire séparément. La pratique possible du «Ehegattensplitting» est avantageuse pour les couples à un revenu, et désincite les femmes à prendre une activité professionnelle.

${ }^{156}$ La réforme des droits de l'enfant de 1998 a modifié le statut des enfants naturels et avec lui le statut des familles monoparentales. La procédure obligatoire de mise sous tutelle juridique du chef de famille monoparentale est suprimée, elle est remplacée par un possible suivi par le service d'aide sociale à l'enfance (Sozialpolitische Umschau 1998). Ce changement de statut des familles monoparentales modifie la nature de la relation entre la famille et l'Etat. C'est le rôle de «l'Etat père» qui disparait au profit d'une forme de protection sociale face au risque familial beaucoup moins interventionniste. La suppression de la mise sous tutelle obligatoire des familles monoparentales naturelles, est une reconnaissance de l'existence, dans de nombreux cas, d'un second parent, le père. Il s'agit également une reconnaissance de l'indépendance des femmes et des mères, que la mise sous tutelle avait rangées dans la catégorie des individus assistés et non responsables.
} 


\section{CONCLUSION}

Dans ce chapitre, notre objectif a été d'identifier les caractéristiques et les formes de la norme actuelle de parentalité. Pour cela, nous avons étudié le «risque familial» et sa régulation, à travers la protection de l'enfant face aux conséquences de la séparation parentale. Cette dernière met en évidence la manière dont la norme de parentalité est définie socialement: quelle est la forme d'exercice de la parentalité normativement privilégiée?

Même si l'adage «le couple parental survit au couple conjugal» est le même en France qu'en Allemagne, sa construction après le divorce est différente. La régulation du risque familial s'effectue dans le sens d'un maintient de la relation de l'enfant avec ses deux parents, c'est-à-dire dans un exercice bilatéral de la parentalité. En revanche, la composition des caractéristiques constitutives de la norme de parentalité se fait différemment dans les deux pays : deux variations sociétales apparaissent: la «coparentalité» française et la «biparentalité» allemande (cf. Figure 14). Elles posent ainsi les bases du «parentalisme» (cf. Figure 15).

\section{LES NORMES DE PARENTALITE : LA «COPARENTALITE» ET LA «BIPARENTALITE»}

La norme de parentalité est une construction sociale. Le principe contemporain de la parentalité, en France et en Allemagne, s'articule autour de quatre dimensions constituantes : une notion de couple parental, des règles égalitaires, des règles partenariales ou non conflictuelles, et une autonomisation de la logique parentale par d'autres logiques familiales. Elle privilégie actuellement un exercice bilatéral de l'autorité parentale au nom de l'enfant. Cette conception bilatérale des droits et des devoirs des parents, est relativement récente. Elle a remplacé, dans le droit civil, et beaucoup plus lentement dans le droit social, une conception unilatérale de l'exercice de la parentalité.

\section{Le couple parental et le principe de «non-conflictualité» (en Allemagne) et de partenariat (en France)}

La parentalité actuelle se définit à partir de l'idée de «couple parental», qui se fonde sur l'égalité de droits et de devoirs entre les pères et les mères. Elle coïncide avec une évolution des mœurs, redéfinissant la place des pères au sein de la famille. Elle résulte de la prise de conscience devant l'étendue du phénomène de la «fausse monoparentalité» et en particulier de «l'éloignement du père comme d'une situation insupportable» (Théry 1993). L'exercice de la parentalité des familles «séparées», et surtout «divorcées», oblige le législateur à émettre des référents pour gérer la séparation parentale. Ces référents sont alors explicitement discutés ; ils deviennent plus visibles. Il est alors plus aisé de reconstruire la logique sous-jacente aux principes d'exercice de l'autorité parentale, et de façon plus générale, celle de la parentalité. 
Progressivement, les lois et les décisions jurisprudentielles vont se succéder pour permettre un partage de plus en plus important des devoirs et des droits entre les parents. La figure 13 représente les réformes en droit civil de la famille, en France et en Allemagne, concernant l'autorité parentale et le droit des enfants. Elles marquent l'émergence du caractère bilatéral de la parentalité, à travers les principes de l'autorité parentale conjointe qui en sont l'expression la plus directe.

Dans l'intervalle des trente dernières années, de nouvelles pratiques des rôles parentaux se sont mis en place, lesquels sont en rupture avec les principes antérieurs. La forme d'exercice de l'autorité parentale s'est déplacée depuis un exercice unilatéral des droits et devoirs parentaux vers un exercice bilatéral. Cette évolution trouve ses racines dans trois phénomènes nouveaux : premièrement, la destigmatisation des familles naturelles par rapport aux familles légitimes, deuxièmement, une unification des principes liés à l'exercice de l'autorité parentale entre les familles "unies" et "désunies" en droit civil, troisièmement, la prise en considération au niveau du droit social, en particulier sous l'impulsion de réformes internationales, des nouveaux concepts de l'intérêt de l'enfant (son intérêt se définit comme son droit à avoir un couple parental, que ce soit au sein des familles légitimes, naturelles, unies ou désunies ou, depuis peu, que les parents soient du même sexe ou non).

Figure 13 Chronologie des réformes du droit civil en France et en Allemagne

\begin{tabular}{l}
19751980 \\
\hline 1975 réforme du divorce \\
|1977 réforme du divorce \\
|1982 première réforme sur l'autorité parentale \\
|1987 Loi Malhuret première réforme sur l'autorité parentale \\
|1990 Ratification de la convention internationale des droits de l'enfant \\
|1992 Ratification de la convention internationale des droits de \\
l'enfant par l'Allemagne réunifiée \\
|1993 L'autorité parentale conjointe comme principe \\
|1997-... Projet de réforme du droit civil de la famille \\
France \\
Allemagne
\end{tabular}

L'abandon du principe unique du divorce pour faute en 1975 en France et en 1977 en Allemagne, marque l'apparition d'une nouvelle conception du divorce. Grâce à l'introduction du divorce par consentement mutuel, les époux peuvent se séparer sans que l'une des parties soit accusée et condamnée. La logique de conflit jusque-là dominante, se modifie pour autoriser une logique de coopération entre les parents.

L'introduction de la pratique conjointe de l'autorité parentale dans les années 1980 inscrit plus avant la notion de coopération parentale dans le droit civil. En France, la loi Malhuret de 1987 offre la possibilité aux parents divorcés de choisir entre une autorité parentale conjointe et une autorité parentale unilatérale. En Allemagne, les années 1980 sont marquées par le verdict très attendu de la Cour constitutionnelle sur l'autorité parentale en 1982 : le fait d'attribuer obligatoirement l'autorité parentale à un seul parent s'oppose au droit constitutionnel de l'autre parent. A partir de cette date, les parents divorcés peuvent exercer conjointement l'autorité parentale. Le principe de l'autorité parentale conjointe est reconnu.

Les années 1990 apportent avec elles la consécration institutionnelle du partage de l'autorité parentale. En France, l'autorité parentale conjointe devient un principe en 1993. En 
Allemagne, l'autorité parentale conjointe, comme principe, est entrée en application le ler juillet 1998 avec l'adoption de la nouvelle loi relative aux droits des enfants.

Ainsi, le législateur a légiféré depuis ces 25 dernières années dans le sens d'un divorce de moins en moins contentieux. En diminuant le caractère conflictuel du divorce, le législateur facilite l'existence du binôme parental. Cette nouvelle gestion des différents conjugaux influence directement l'organisation de la garde des enfants. L'un des deux parents, le père dans la majorité des cas, n'est plus systématiquement exclu de l'aprèsdivorce. Ainsi, la présence des deux parents est le nouveau caractère de la gestion de la garde des enfants en France et en Allemagne. L'entente éventuelle des parents est centrale, parce que souhaitée. Deux éléments caractérisent les dernières réformes de l'autorité parentale conjointe : l'entente présupposée des parents et l'implication restreinte des tribunaux en cas de conflit. L'existence du couple parental, au-delà de la rupture conjugale, est le résultat de la poussée normative de l'Etat qui institutionnalise le principe d'une entente des parents par la mise en avant de la «non-conflictualité». Le couple parental s'appuie sur la règle de partenariat pour la France ou encore de «non-conflictualité» pour l'Allemagne. Le couple parental se définit à partir de l'idée d'égalité et de partenariat (ou dans sa forme moins aboutie de non-conflictualité).

Les pratiques des magistrats relatives à l'attribution de l'autorité parentale, inscrivent une conception particulière du divorce et de l'après-divorce dans les normes sociales. Les pratiques jurisprudentielles des années 1970 donnaient systématiquement la garde de l'enfant à sa mère, tant en France qu'en Allemagne. Le rapport mère-enfant avait été problématisé comme la variable décisive pour l'équilibre physique et psychique de l'enfant et le père était traditionnellement absent de l'après-divorce. La présence dominante de la mère dans les premières années de la vie de l'enfant se retrouve dans la jurisprudence et les pratiques des années 1980, lorsqu'il s'agit de fixer la résidence principale et la garde du petit enfant.

Puis, les changements normatifs des années 1980 et 1990, sur la présence et le rôle du père, ont fait évoluer les pratiques jurisprudentielles. Aujourd'hui, les magistrats favorisent majoritairement la présence éducative du père, le partage du temps de présence et la circulation de l'enfant entre les deux parents, bien que le jeune enfant soit presque toujours à la charge principale de la mère.

Les résistances des magistrats, telles que la représentation de la famille et les enjeux identitaires des mères, quant à la pratique de l'autorité parentale conjointe, ont été beaucoup plus importantes en Allemagne qu'en France,.

Les pratiques des magistrats allemands expriment leur scepticisme face à l'entente des parents et favorisent encore davantage la présence des mères. La conflictualité de l'aprèsdivorce est resté très ancrée, dans les représentations et les pratiques des magistrats, ce qui a pour conséquence d'entretenir une rivalité entre les parents et rend difficile une adéquation avec la nouvelle norme de l'intérêt de l'enfant et le principe d'autorité parentale conjointe concrétisé tardivement dans les années 1990.

De plus, l'enjeu identitaire pour les mères allemandes augmente leurs résistances face au partage de l'autorité parentale. En Allemagne, la représentation de la famille correspond à la famille bourgeoise basée sur le mariage et son principe de «l'homme soutien de famille». Leur faible participation sur le marché du travail renforce encore ces résistances. Les modes de conciliation entre l'activité professionnelle et la vie familiale, comme les structures d'accueil des jeunes enfants, sont peu développés et favorisent peu leur intégration sur le 
marché du travail. Dans ce contexte, l'exclusivité des droits de garde est un enjeu identitaire très important pour les mères allemandes. En conséquence, chaque parent revendique ses droits en opposition à l'autre parent. L'Allemagne construit la parentalité sur une vision très polarisée des rôles et des logiques de genre, à la différence de la France, où le discours d'interchangeabilité des rôles paternel et maternel a facilité et encouragé la présence du père dans la famille. En d'autres termes, la représentation du couple parental en Allemagne après le divorce s'exprime sous la forme d'une «biparentalité», un couple parental à deux parents juxtaposés.

En France, la coopération des parents est plus importante qu'en Allemagne ; elle est tout d'abord plus encouragée par les pratiques des magistrats et elle découle d'une plus forte présence des mères sur le marché du travail. Leur activité économique a une incidence sur le maintien du couple parental après le divorce. Lorsque les deux parents travaillent à temps plein, ils ont plus tendance à partager les tâches domestiques que les familles où seulement l'un des deux conjoints travaille. La représentation du couple parental en France, après le divorce, s'exprime sous la forme d'une «coparentalité», un couple parental coopérant.

Les modes de régulation des relations parentales sont différents dans les deux pays. La parentalité prend deux formes différentes, la «coparentalité» en France et la «biparentalité» en Allemagne. Les rapports de genre sont plus traditionnels en Allemagne ; ils entretiennent une guerre des sexes, qui trouve par ailleurs écho dans un moindre investissement professionnel des femmes et dans les mesures «patriarcales» de l'Etat-providence. Par contre, la parentalité française est basée sur une coopération plus forte des parents, bien que, au contraire de l'Allemagne, l'ingérence de l'Etat dans les affaires de familles soit plus importante, mais paradoxalement, cette ingérence a pour objectif de pousser à la coopération des parents, plus que d'avoir un rôle subsidiaire dans les affaires de familles.

\section{$\underline{\text { La notion de l'intérêt de l'enfant }}$}

Une autre caractéristique de la parentalité moderne est la redéfinition de l'intérêt de l'enfant : au nom de l'enfant et de ses droits, les parents se doivent de maintenir le «couple parental» après la désunion familiale, dans une logique de «non-conflictualité». Son intérêt est d'être en relation avec son père et sa mère, qu'ils soient séparés ou non. Les logiques égalitaires et partenariales du couple parental y trouvent leur légitimation.

C'est encore grâce à cette notion d'intérêt de l'enfant, et du principe de nondiscrimination des statuts de l'enfant en fonction de la situation matrimoniale des parents, que se généralise l'application du principe d'autorité parentale conjointe (expression pratique du principe de parentalité).

La notion de l'intérêt de l'enfant («Kindeswohl») a joué un rôle important dans la mise en place des réformes de l'autorité parentale. La France et l'Allemagne ont ratifié la Convention internationale des droits de l'enfant (New York, 1990). Par-là, les deux pays reconnaissent son intérêt supérieur : «le droit de connaître et de disposer de ses deux parents» (Article 9). Cette convention associe l'intérêt de l'enfant et la présence des deux parents. Cette association est un moteur réel pour les réformes. En Allemagne, l'institutionnalisation définitive du principe de partage de la garde des enfants en 1998 a pu être réalisée par ce biais. Mais avant d'aboutir à cette réforme, le fait même d'avoir ratifié cette convention avait relancé les débats sur le partage de l'autorité parentale et du droit de véto de la mère. 
La notion de l'intérêt de l'enfant est un repère normatif nécessaire à la gestion des situations conflictuelles, elle permet aux magistrats d'y faire référence afin de désamorcer certains conflits parentaux. Mais elle reste pour cela une notion vague, dont le sens change en fonction du contexte social. A la fin des années 1970, jusqu'au milieu des années 1980, on définissait l'idée de l'intérêt de l'enfant par le principe suivant : l'enfant devait être tenuu à l'écart du conflit parental, quitte à rompre le contact avec l'autre parent (le père), afin de lui offrir une situation fixe et non conflictuelle (en particulier auprès la mère). Il s'agissait d'une gestion du risque familial par le principe de monoparentalité, au profit des mères et en défaveur des pères.

C'est progressivement au cours des années 1990, que la notion de l'intérêt de l'enfant a été redéfinie : à l'heure actuelle, l'enfant doit avoir accès à ces deux parents, et le conflit parental, sans être nié, doit être dépassé au nom de l'intérêt de l'enfant. Et il est du devoir des parents de chercher l'entente, pour constituer ce couple parental nécessaire à l'enfant. Cette gestion bilatérale du risque familial dissocie la séparation conjugale de la séparation parentale. C'est en cherchant à isoler le parental du conjugal, que le législateur espère favoriser le principe de «non conflictualité», voire de partenariat.

\section{$\underline{\text { L'autonomisation des logiques }}$}

La norme de parentalité contient le principe d'autonomisation de la logique conjugale, de la logique de genre et de la logique intergénérationnelle. En effet, la logique parentale, tout en étant au croisement du conjugal, des relations de genre et des relations intergénérationnelles, n'en est pas moins de plus en plus indépendante : l'idée de couple parental dépasse la rupture conjugale et la logique conjugale peut se soustraire à la logique de genre, comme dans le cas de l'homoparentalité. On a vu également que la logique conjugale (basée sur le lien matrimonial), devient de plus en plus autonome et indépendante des logiques de genre, mais également des logiques générationnelles. Or cette autonomisation des logiques les unes par rapport aux autres a permis l'émergence de la nouvelle logique du parental, qui n'est plus la résultante de leur interaction. La parentalité se définit comme une logique autonome au sein des familles. Elle a ses propres caractéristiques : partenariale (ou non-conflictuelle), égalitaire, centrée sur l'enfant (cf. Figure 14).

L'axe vertical de la figure 14 (ci-dessous) représente la dimension parentale et coujugale. Il hiérarchise les principes de la norme de parentalité contemporaine selon leur apparition historique : le premier principe "droits et devoirs parentaux" signifie que la parentalité est une fonction, finalisée par les droits et les devoirs parentaux; le second souligne la règle d'égalité des droits et des devoirs entre les pères et les mères (un tel principe est apparu au cours des années 1970 et des années 1980); le troisième principe est celui du couple parental, c'est-à-dire de l'exercice bilatéral des droits et devoirs basés sur le principe égalité ; enfin, le quatrième et dernier principe exprime l'exercice bilatéral du parental dans le sens d'une non-conflictualité.

L'axe horizontal représente la dimension de l'enfant par les principes d'exercice de la parentalité. On voit là un principe unique d'égalité entre les enfants, quel que soit le lien de conjugalité entre les parents : légitime, naturel, uni ou désuni. 
Figure 14 Les normes de coparentalité et de biparentalité

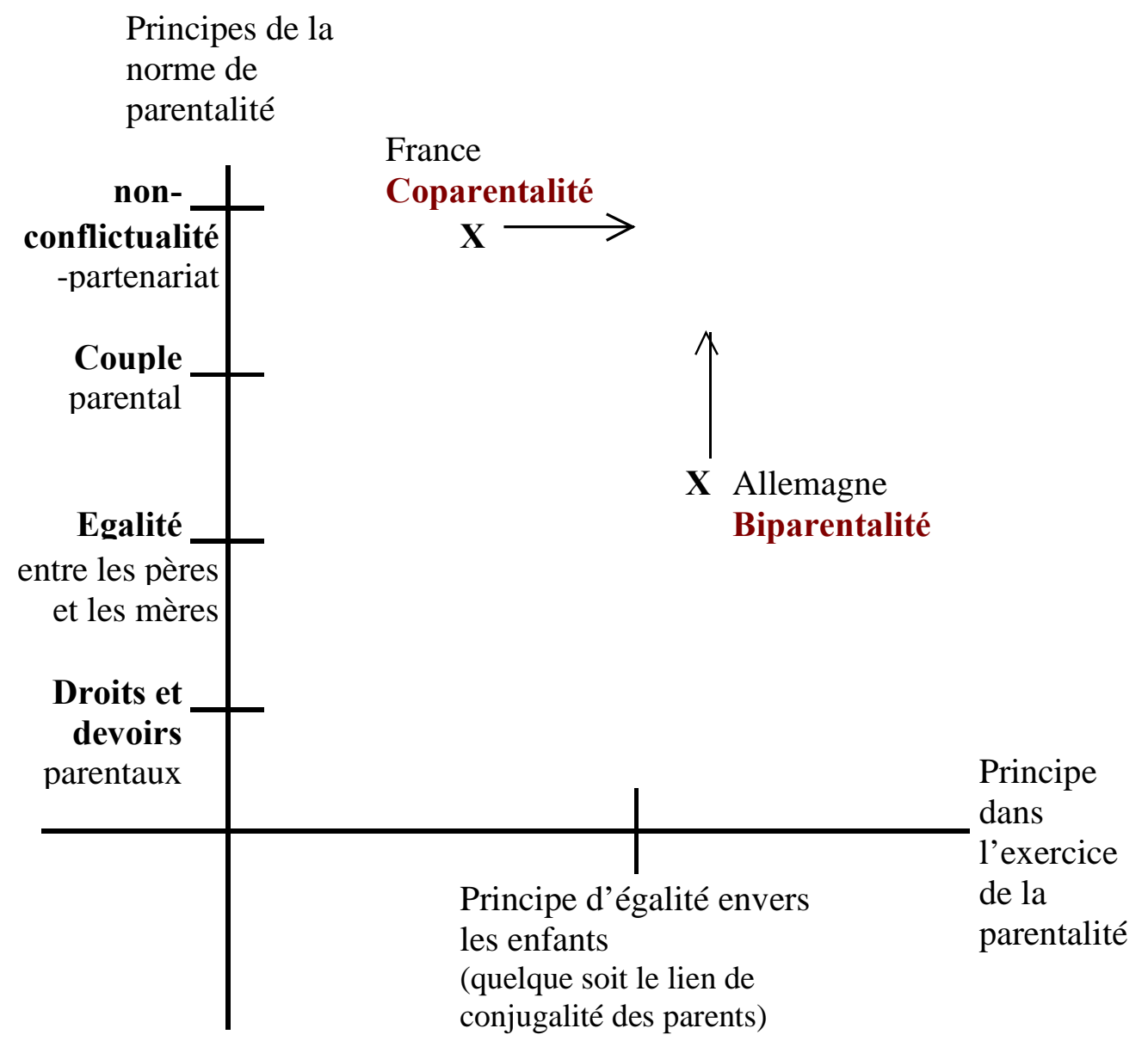

Pour la France, la norme de parentalité cumule l'ensemble des quatre principes et les deux derniers, ceux de partenariat et de parité parentale, sont fortement institutionnalisés. Par contre, elle s'inscrit moins dans le principe d'égalité envers les enfants car il existe toujours, dans le droit civil français, une distinction entre filiation légitime et filiation naturelle. Cependant, cette distinction est remise en cause dans les projets actuels de réforme du Code civil de la famille (Dekeuwer-Défossez 1999) : la volonté du législateur est d'inscrire l'autorité parentale conjointe, quelle que soit la situation de conjugalité des parents, d'où le sens de la flèche vers la droite. La parentalité s'inscrit dans un cadre de partenariat, de parité et de coopération, on parlera de «coparentalité».

Dans le cas de l'Allemagne, l'exercice de la parentalité s'articule autour des normes d'égalité entre les pères et des mères, et bien que le principe du couple parental soit inscrit, c'est-à-dire de l'exercice conjoint de la parentalité, il est encore largement dépassé par les inégalités de genre. Les résistances au couple parental et en particulier à sa coopération sont nombreuses, ce qui explique sa position centrale sur l'axe vertical. Cependant, le mouvement actuel des évolutions montre une inscription plus avant du couple parental, tant dans les pratiques que dans les dernières réformes des lois sur la famille. La règle de non-conflictualité est de plus en plus largement acceptée, d'où la flèche vers le haut. Quant à l'exercice de l'autorité parentale, l'égalité envers les enfants est largement plus réalisée qu'en France, et a été inscrite dès 1997. La parentalité se base sur la structure égalitaire de la parentalité, binôme 
parental, mais plus dans une juxtaposition, que dans une coopération entre les parents : on parle alors de «biparentalité».

Cette représentation graphique des normes de parentalité (cf. Figure 14) montre que la France et l'Allemagne convergent vers une norme commune de parentalité qui allie à la fois l'égalité des droits et devoirs des pères et des mères, l'exercice conjoint de la parentalité d'une manière sinon partenarial (en France) du moins «non-conflictuelle» (en Allemagne), tout en respectant les principes d'égalité envers les enfants. On voit alors que la logique du parental s'autonomise définitivement du lien conjugal en dépassant la référence au lien matrimonial.

Le maintien du lien entre l'enfant et chacun de ses deux parents est au centre de la régulation des relations sociales dans les familles unies et désunies. La parentalité qui est au centre de la régulation du lien familial, montre une prise en compte du statut de parent, pour les mères et plus récemment pour les pères. Le lien à l'enfant devient l'enjeu de la régulation juridique, politique et sociale face au risque familial contemporain, définissant le "parentalisme» comme un nouveau mode de régulation familiale (cf. paragraphe suivant et Figure 15).

\section{LE PARENTALISME COMME MODE DE REGULATION DU RISQUE FAMILIAL DEPUIS LES ANNEES 1990}

\section{$\underline{\text { La «monoparentalité à la décharge du père» }}$}

Jusqu'au milieu du XXe siècle, le risque familial était conséquent à la rupture du couple parental par veuvage (par la disparition de l'un des deux parents); il était également lié aux naissances dites «honteuses» (les naissances hors mariage, voulues ou non). Il s'agissait donc d'un ensemble de situations où les droits et les devoirs parentaux étaient exercés unilatéralement, par le parent survivant en cas de veuvage, ou par la mère, en cas d'abandon famille ou de non légitimation du couple. Le risque familial était alors essentiellement un risque féminin et la logique de l'action publique, en France et en Allemagne, suivait le principe d'une régulation unilatérale de la parentalité : le père, dans le cas des naissances naturelles, était considéré d'avantage comme un géniteur qu'un père. En France, la gestion publique du risque familial visait l'équilibre entre le degré de collectivisation de l'enfance et les obligations alimentaires des pères. En Allemagne, le débat portait sur le niveau d'implication du père payeur, l'action de l'Etat étant alors plus coercitive qu'en France. Le traitement social du risque familial découlait directement de la référence à la famille légitime, au paradigme du «pater familias», sous le régime du «Familialisme». La régulation du risque familial s'effectuait sur le mode de la «monoparentalité à la décharge du père» (cf. Figure 15). 


\section{$\underline{\text { La «monoparentalité en faveur des mères». }}$}

Au cours du XXe siècle, le risque familial a changé. Il n'est plus lié au veuvage, ou aux naissances naturelles et scandaleuses, mais à la généralisation des séparations conjugales. Le père devient alors une nouvelle composante qui modifie le mode de régulation du risque familial fondé jusqu'ici sur une norme privilégiant un exercice unilatéral de la parentalité (suite au veuvage ou à la décharge -plus ou moins forte- du père dans le cas des naissances hors mariage). Dans ce nouveau contexte, le modèle de régulation du risque familial se modifie, et il se prolonge jusqu'au début des années 1990 pour la France et jusqu'au milieu des années 1990 pour l'Allemagne.

L'intervention de l'Etat existe, en France comme en Allemagne, dans un rapport de subsidiarité par rapport à la sphère privée, favorisant alors, par sa non-ingérence, les accords de couples. Cette caractéristique existe aussi dans le droit civil. En effet le législateur a opté pour une autorité parentale conjointe comme règle de principe, limitant de fait, l'ingérence des tribunaux aux cas de conflits. De même, le système de recouvrement des pensions alimentaires réaffirme le rôle du père comme nourricier.

Par ailleurs, l'aide publique donne aux familles monoparentales allemandes un statut particulier : on tolérera qu'elles puissent profiter de l'aide sociale pour se consacrer à plein temps à l'éducation de leurs enfants, leur rôle d'éducatrice passe avant leur intégration sur le marché du travail.

En France, notre analyse permet de corroborer les résultats de C. Martin en ce qui concerne les ressources des familles monoparentales. Le modèle français permet une combinaison des ressources. «Même si la contribution des ex-conjoints à l'entretien des ménages monoparentaux reste faible, le support fournit par l'Etat en terme de services à la petite enfance et d'allocations diverses, permet aux femmes de trouver dans l'emploi à temps plein la solution pour échapper à la pauvreté» (Martin : 1996 : 86). C'est surtout à travers la participation au marché du travail des mères que la sphère privée est impliquée dans la régulation du risque familial.

Le père est le premier protagoniste à qui revient la charge des enfants sous la forme d'une pension alimentaire. La pension alimentaire est non négligeable dans le budget des familles monoparentales, plus encore en Allemagne qu'en France. Le père est le premier payeur et l'Etat ne participe que de manière subsidiaire. Le rôle de l'Etat est de faire pression sur les pères en engageant éventuellement des poursuites ou en contrôlant leur solvabilité. Ce principe est autant valable en France qu'en Allemagne. C'est uniquement en cas de défaillance que l'Etat intervient. Les systèmes publics de protection sociale français et allemand ont mis en place en 1984 une prestation sociale pour couvrir le non-paiement des pensions alimentaires. Les systèmes de recouvrement de la pension alimentaire obéissent à deux principes identiques : le recouvrement à perte des pensions pour les payeurs non solvables et un avancement de la pension dans l'attente du versement du débiteur. Ce système se couple à une procédure de poursuite judiciaire à l'encontre du père. Parallèlement, les services de recouvrement ont de grandes difficultés à récupérer les pensions avancées.

Jusque dans les années 1980, les décisions jurisprudentielles accordent le plus souvent la garde de l'enfant à la mère, tant en France qu'en Allemagne. Par ailleurs, les pères allemands payent des pensions alimentaires plus élevées, les payent plus régulièrement et sont 
plus solvables que les pères français. Ils restent le premier responsable de la charge alimentaire de l'enfant après la séparation en Allemagne ; en France, la situation est différente car la solvabilité des pères est moindre et les mères exercent plus fréquemment une activité professionnelle. Le père est exclu de l'exercice de l'autorité parentale comme éducateur. C'est la mère qui remplit le rôle éducatif auprès de l'enfant et pratiquement exclusivement chez le petit enfant. La gestion du risque familial se fait alors selon le mode de la «monoparentalité en faveur des mères».

\section{$\underline{\text { Le «parentalisme» }}$}

Puis, progressivement, en France comme en Allemagne, et depuis les années 1980, la catégorie des familles monoparentales se compose désormais majoritairement de femmes divorcées et séparées. Le risque familial concerne la protection des enfants face aux conséquences de la séparation des parents. La construction de la parentalité est marquée par la prise en compte récente du lien entre l'enfant et le père car les représentations du rôle du père changent et se généralisent: sa place de co-éducateur au sein du couple parental doit se maintenir après la séparation pour le bien de l'enfant. A l'heure actuelle, la définition des droits et des devoirs (juridiques et sociaux) du père envers l'enfant renforce progressivement les liens institutionnels avec le père, face à ceux de la mère qui avaient été très fortement institutionnalisés au cours des vingt dernières années.

Outre la présence du père, la généralisation du travail professionnel des mères et l'érosion du rôle de l'Etat-providence comme celui d'un «père protecteur», modifient fondamentalement les données de la régulation. L'organisation de la protection de l'Etat subit des modifications très importantes dans les années 1980. Le droit social s'organise plutôt autour d'une protection du risque de pauvreté que d'une protection du risque familial. L'action protectrice de l'Etat se cantonne de plus en plus aux risques sociaux issus des défaillances du marché du travail plutôt que des bouleversements du marché matrimonial. Par ailleurs, il laisse la place aux parents pour une gestion individualisée du risque familial : la mention «à défaut d'accord entre conjoints» illustre ce renversement de logique.

Jusque dans les années 1980, l'exercice de la parentalité bilatérale des familles unies (traitées en référence à la famille légitime) s'opposait fortement à une norme de parentalité unilatérale dans le cas des familles désunies. Depuis, les principes d'exercice de la parentalité s'harmonisent de plus en plus, quelle que soit la forme de la famille.

Sous l'influence de la montée des valeurs égalitaires dans les deux sociétés, de la volonté du législateur d'harmoniser les pratiques des institutions sociales aux valeurs actuelles, des évolutions démographiques et sociales de la famille, des changements internes au système de protection sociale, de la participation croissante des femmes sur le marché du travail et des droits des enfants, le traitement du risque familial évolue vers une norme d'exercice bilatéral de la parentalité.

La nouvelle régulation des séparations familiales (de gestion du risque familial) est fondée sur un exercice bilatéral de la parentalité, suivant deux versions sociétales, la «coparentalité» française et la «biparentalité» allemande (cf. Figure 15). Elle supplante progressivement l'ancien mode de gestion de la séparation parentale basé sur l'exercice unilatéral de la parentalité par un parent, dans sa version historique «la monoparentalité à la 
décharge du père», puis dans sa version moderne «la monoparentalité en faveur de la mère».

Aujourd'hui, depuis le milieu des années 1990, la norme de parentalité définie la forme privilégiée d'une relation bilatérale entre l'enfant et ses deux parents quelle que soit la forme de familles (unie ou désunie, et a fortiori légitime ou naturelle). Le mode de régulation du risque familial fondé sur cette norme prend alors les caractéristiques du «parentalisme» (cf. Figure 15).

Figure 15 Les normes de parentalité et le nouveau mode de régulation du risque familial

les normes privilégiant un exercice unilatéral des relations entre l'enfant et ses parents les normes privilégiant un exercice bilatéral de la parentalité

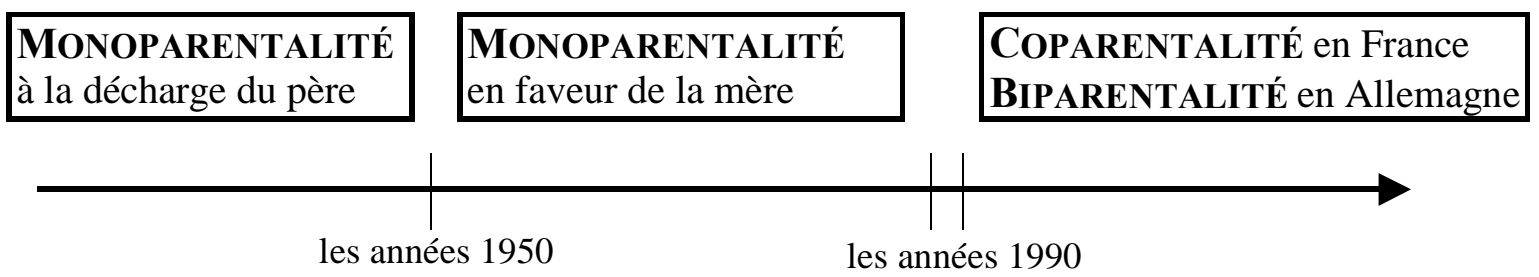

Parentalisme

Cette évolution s'accompagne d'un ensemble de résistances et de contradictions. Des situations de conflit apparaissent entre les pratiques sociales et les normes. En France, on voit par exemple s'opposer le principe d'autorité parentale conjointe à celui de l'attribution de la résidence habituelle en droit civil, ou encore, les réticences du droit social qui continue d'attribuer unilatéralement les allocations familiales même si la charge de l'enfant est partagée entre les deux parents après leur séparation. En Allemagne, la norme de nonconflictualité fait difficilement l'unanimité chez les magistrats qui privilégient encore une attribution unilatérale de la garde de l'enfant.

«La question du divorce et des modes de vie familiale qui en résultent montrent, par exemple, que «le modèle contractualiste» (Martin, 1997) mis en oeuvre dans les lois récentes sur la famille, basé sur le consentement mutuel et l'exercice conjoint de l'autorité parentale, ne s'applique en définitif qu'à certaines franges de la population concernée. Il masque l'essentiel des situations de rupture conjugale où le conflit est si présent qu'il conduit généralement l'Etat (le juge), à utiliser des mesures de sauvegarde de l'intérêt de l'enfant ou à défaut d'accord entre conjoints- la mère est désignée comme dépositaire du lien de filiation.» (Blöss 2001 : 63-64).

L'adéquation entre le modèle contractualiste et les pratiques réelles des couples ne concernent qu'une minorité. Les pratiques de garde alternée (Neyrand 1994), comme expression première du partage conjoint de l'autorité parentale est surtout le fait des catégories supérieures. «Ce pari de la «coparentalité» post-divorce n'a pas pour autant obéré l'inégal statut de parents que la loi maintient selon leur appartenance de sexe, ni le traitement social différencié qu'elle leur réserve. » (Blöss 2001 : 64). Il demeure aujourd'hui un décalage entre ce nouveau principe normatif et les pratiques qui sont fondées sur l'exercice unilatéral de la parentalité en faveur de la mère. En effet, des mécanismes de reproduction maintiennent 
des inégalités parentales (Blöss 2001) qui rendent difficile le maintien effectif de la relation entre l'enfant et ses deux parents.

Dans ce contexte, il devient nécessaire d'approfondir les pratiques réelles des parents dans le partage du travail parental. Or, comme elles ne peuvent se comprendre que dans leur contexte familial et social, l'objet du prochain chapitre est d'étudier le partage du travail parental au sein du «travail familial» (cf. Chapitre III). 


\section{CHAPITRE III LES PRATIQUES DE PARENTALITE DANS LES FAMILLES ET LE PARTAGE DU TRAVAIL FAMILIAL}

A la suite du chapitre II qui traite de la norme de «parentalité», de sa construction et de sa régulation, le chapitre III étudie les modes d'exercice de la parentalité dans les familles et leurs déterminants.

Introduction : Comment la parentalité metelle en lumière les changements

du lien familial ?

Chapitre I : Le cadre théorique et les formes de parentalité : la parentalité exclusive et la parentalité conciliée

Chapitre II : Les normes de parentalité : la coparentalité française et la biparentalité allemande

Chapitre III : Les pratiques de parentalité dans les familles et le partage du travail familial

Conclusion : Le parentalisme comme mode de régulation?
Quels déterminants influencent les modes l'exercice de la parentalité dans les familles ? Pourquoi la parentalité est-elle indissociable de la manière dont la famille partage le travail domestique et professionnel ? 


\section{INTRODUCTION}

L'objet de ce chapitre est d'identifier les pratiques de la parentalité dans les familles. Dans ce but, nous avons étudié la répartition les tâches parentales entre les pères et les mères.

Notre analyse concerne quatre facteurs qui déterminent le partage du travail parental : les possibilités de délégation, institutionnelle et informelle, du travail parental ${ }^{157}$; la participation des parents au marché du travail ; et enfin la participation des pères et des mères au travail domestique (cf. Figure 16).

$\underline{\text { Figure } 16 \quad \text { Les déterminants du partage du travail parental }}$

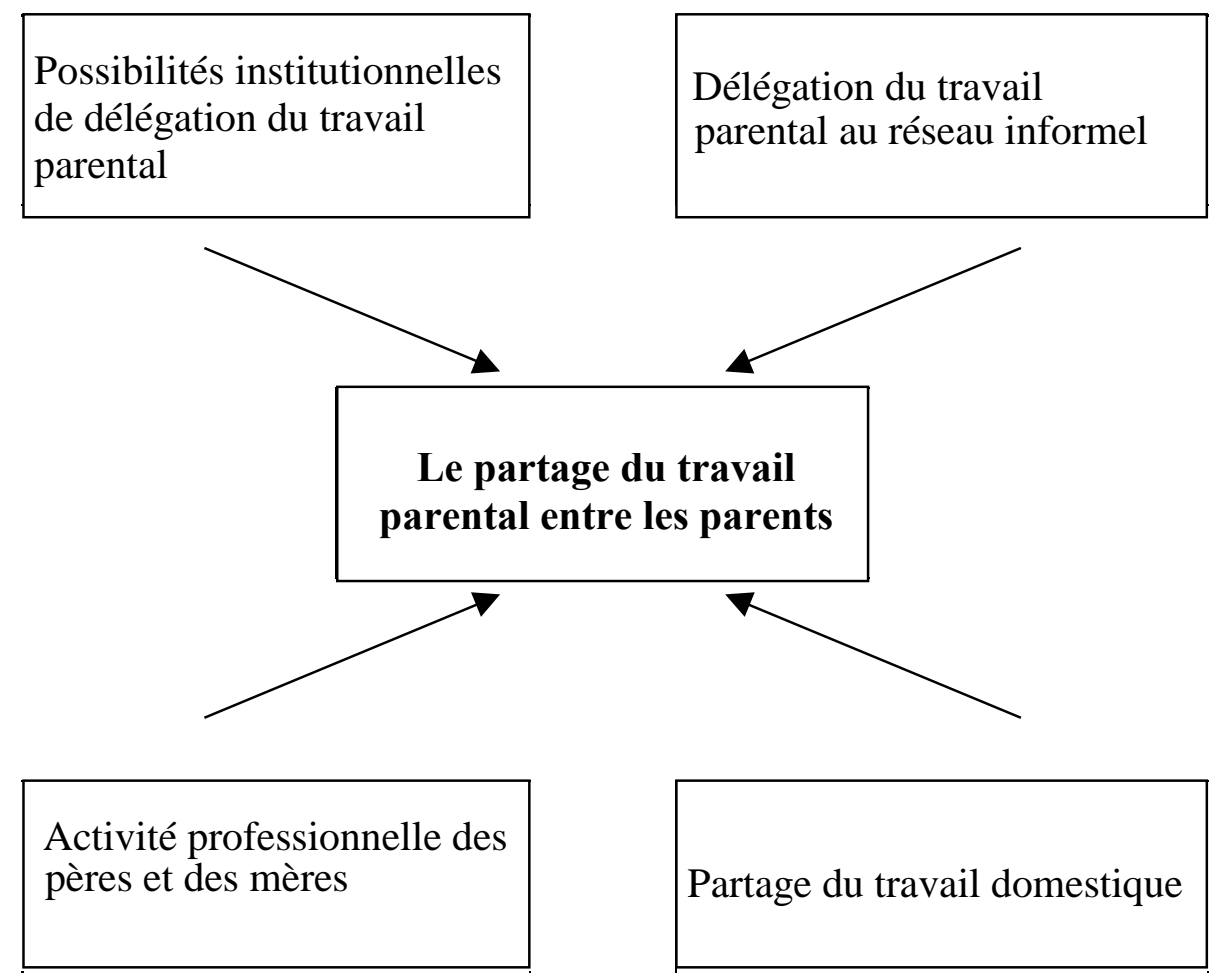

La notion de travail que nous avons retenue ici, est prise au sens d'activité : le but de cette dernière est de produire de «l'utile» pour la communauté ; le résultat a directement des effets identifiables pour la famille; et l'exercice de l'activité peut faire l'objet d'une rémunération, mais non nécessairement. Nous parlerons de travail professionnel, au sens classique d'activité économique rémunérée.

Quant au statut des tâches domestiques, il prend la notion de travail depuis les discussions des années 1970, sous l'impulsion des mouvements féministes. Le travail domestique, devient une catégorie constituée au début des années 1980 . On entend par-là l'ensemble des tâches effectuées (sous-entendu par les femmes) au sein de la famille ; elles

\footnotetext{
${ }^{157}$ Nous parlerons de délégation du travail parental au même titre que de délégation du travail domestique, dans le sens d'un mode d'organisation du travailet non pas comme jugement moral (qui fait appel à un tout autre débat sur la responsabilité des parents envers leur enfant).
} 
comprennent alors tout ce qui relève du foyer, c'est-à-dire l'ensemble des tâches ménagères mais également des soins aux enfants.

Enfin, les tâches liées aux enfants ont d'abord été comprises à partir des soins matériels, puis peu à peu, à partir des tâches de nature relationnelle et de socialisation (qui définissent la sociabilité parentale). Et c'est très récemment que les tâches parentales ont été envisagées sous l'angle du travail (Barrère-Maurisson ; Marchand ; Rivier 2000) : l'exécution des différentes tâches parentales formant ainsi le «travail parental», au même titre que le travail professionnel et domestique.

Encadré $n^{\circ} 6 \quad$ La définition du travail parental par le groupe Division Familiale du $\underline{\text { Travail }}$

En isolant le travail parental du travail domestique, ce dernier prend un sens strict, il concerne alors l'ensemble des tâches ménagères et exclut les tâches effectuées directement «avec» et «pour» l'enfant (lesquelles relèvent alors du «parental»).

Le travail parental (Barrère-Maurisson; Marchand; Rivier 2000) regroupe toutes les activités effectuées par les parents avec et pour les enfants.

Il s'agit, d'une part, des tâches relatives aux jeunes enfants : les habiller, leur faire la toilette, leur faire prendre les repas, jouer avec eux, passer avec eux du temps à l'extérieur, les conduire à l'école, les accompagner à des activités extra-scolaires, les aider à faire leurs devoirs ; d'autre part, des activités effectuées auprès des adolescents : parler, regarder ensemble une émission de télévision, etc..

La notion de travail parental ainsi construite permet de qualifier quatre types de tâches classés ci-dessous par ordre d'importance dans l'emploi du temps des pères et des mères, en France, en 1999 :

La sociabilité parentale recouvre les activités de présence auprès des enfants, c'est-à-dire les jeux effectués avec eux, à la maison ou à l'extérieur, et les échanges avec les adolescents.

Les activités parentales domestiques concernent essentiellement les petits enfants puisqu'il s'agit de leur faire prendre les repas, de les habiller et de faire leur toilette.

Le travail parental «taxi» est calculé à partir des tâches qui consistent à accompagner les enfants à leurs activités extra-scolaires et de les conduire à l'école.

Le travail parental scolaire est consacré à l'aide aux devoirs scolaire.

Le premier niveau de notre analyse est la construction sur le plan individuel des règles d'exercice de la paternité et de maternité.

Le second niveau est centré sur l'interaction conjugale et plus particulièrement sur l'interaction parentale en tant que résultat de la confrontation des compréhensions individuelles des rôles et des fonctions du père et de la mère. En focalisant sur l'interaction conjugale, le modèle théorique prend une dimension interactionniste qui accorde à l'acteur un pouvoir déterminant sur le partage du travail parental. Par-là, la négociation globale de l'ensemble de ces tâches et la norme des rapports de genre sont mises en rapport direct (Koppetsch ; Burgkart 1999 ; Huinink 2001 : 15). En effet, la norme des rapports de genre et à la signification qu'elle prend dans les différentes configurations sociales détermine les stratégies individuelles des acteurs ${ }^{158}$. Cette norme latente des rapports de genre détermine la

\footnotetext{
${ }^{158}$ Les représentations des individus concernant les rôles sexués influencent le partage des tâches au sein du conjugal. A une représentation selon laquelle les hommes et les femmes sont fondamentalement différents, correspond un partage des tâches basé sur une différenciation des rôles (fonctions et devoirs) : d'un côté les tâches familiales, de l'autre le monde professionnel. On peut définir cette conception comme l'extrémité d'un
} 
fonction symbolique ${ }^{159}$ du travail familial et détermine par-là, son organisation entre les partenaires.

Le partage du travail parental se réalise aux différents niveaux de l'action sociale, à la manière du travail domestique. «Le piège se construit dans le monde des gestes, des injonctions, dans le silence des habitudes. Il est donc logique qu'ils ne soient guère perçus par les acteurs qui donnent d'autres explications à la répartition inégalitaire des tâches ménagères : les perspectives professionnelles différentes entre hommes et femmes et les rôles sexuels. En fait, ces trois éléments interagissent étroitement, les obstacles rendant plus difficile la réussite professionnelle des femmes, poussent à la reproduction des rôles anciens mais ces obstacles viennent autant de la famille que du marché du travail ; et dans la famille, les rôles sont à la fois producteurs d'un cadre de pratiques et le produit des pratiques. Il serait donc erroné de surestimer le poids des gestes, en oubliant l'impact persistant des rôles et le marché du travail. Mais il est pareillement erroné de ne prendre en compte que ces deux facteurs visibles : bien que n'expliquant pas tout, la différence des manières est centrale (et cela de plus en plus) dans les choix de répartition et la définition d'une organisation domestique.» (J. C. Kaufmann 1992 : 169).

Nous avons alors montré, dans ce chapitre, que le partage du travail parental dépend de la négociation de l'ensemble du «travail familial». Ce concept décrit l'ensemble du travail négocié au sein de la famille. Il comprend alors le travail parental, le travail domestique et le travail professionnel. Il rompt avec les analyses classiques fondées sur l'opposition entre «Famille» et «Travail» (cf. Conclusion de ce chapitre Figure 26 et Figure 27).

Le mode de partage du travail familial, de part sa nature sociale, varie suivant les époques et le contexte sociétal. La segmentation des rôles et des fonctions entre les sexes qui fondait l'ancien modèle de partage des tâches parentales est remise en question. Aujourd'hui, la répartition des tâches parentales change, et si elle est loin d'être égalitaire, elle est, pour la première fois, en pleine évolution dans le sens d'une plus grande parité parentale. La participation au marché du travail des pères comme des mères est une donnée importante dans la répartition du travail familial. Le modèle de partage en France s'effectue sur la base d'un double temps complet, en Allemagne, sur la base d'un temps complet pour le père et d'un temps partiel pour la mère. Enfin, le partage du travail domestique évolue peu et très lentement, seules certaines tâches commencent à être partagées entre les sexes.

La régulation du travail familial montre que la relation qui domine les rapports familiaux aujourd'hui est la relation entre le travail parental et le travail professionnel pour les mères et pour les pères (ce qui n'était pas le cas précédemment $\mathrm{cf}$. Figure 28). Elle est déterminée par la participation croissante des pères au parental et par l'exercice généralisé de l'activité professionnelle des mères. Le «père actif» et la «mère active» sont au cour de la régulation du partage du travail familial.

\footnotetext{
continuum des représentations et des attitudes des rôles sexués des individus. A l'autre extrémité, se situe la représentation égalitaire selon laquelle les individus sont égaux en droits et en devoirs, se devant de partager chaque tâche (Fthenakis ; Minsel 2001 : 19).

${ }^{159} \mathrm{La}$ notion de fonction symbolique du travail familial est empruntée à la théorie du «Doing Gender». L'arrangement conjugal choisi au cours de l'interaction entre les individus, produit par-là même leurs propres rôles. Il produit du «genre» car il reflète la signification sexuée du partage du travail familial pour les acteurs. On rejoint par là une conception interactionniste qui a été prise en compte dans le schéma théorique au niveau de l'interaction conjugale (niveau meso).
} 
Les deux sections de ce chapitre traitent respectivement de la France et de l'Allemagne. Dans chaque cas, nous avons privilégié une étude chronologique du partage du travail parental, depuis les années 1970 à aujourd'hui. Pour chacune des périodes, nous étudions le partage des tâches parentales en relation avec le partage du travail domestique et professionnel. 


\section{SECTION III.A LE PARTAGE DU TRAVAIL FAMILIAL EN FRANCE}

\section{SOUS-SECTION III.A.1 LA PARENTALITE AU SEIN DU DOMESTIQUE DANS LES ANNEES 1970 ET 1980}

Dans les années 1970, la spécification des genres détermine un partage strict des tâches : le travail domestique et parental pour la femme, le professionnel pour l'homme. Si les femmes actives passent moins de temps au travail domestique que les femmes au foyer, leur comportement en matière d'activité ménagère n'est pas différent. Les femmes effectuent l'ensemble des tâches et il y a peu de tâches qu'elles n'effectuent pas. Le principal déterminant de leur participation au travail domestique est plus lié à la taille du ménage qu'à leur activité professionnelle (Rousse ; Roy 1981).

La participation des hommes n'augmente pas en fonction du volume de travail ménager à effectuer, contrairement à leur engagement professionnel : «le nombre d'heures travaillées par le chef de ménage est à peu près croissant avec la taille du ménage (...) le mari travaille en moyenne 42 heures et demi quand il y a un enfant, $44 \mathrm{~h}$ quand il y en a au moins trois» (Rousse ; Roy $1981: 65)^{160}$. Les hommes ne prennent jamais, soit très rarement, une ou l'ensemble des tâches ménagères à leur compte (Enquête «Emploi du temps» 1974-1975, Rousse ; Roy 1981). Leur participation est très réduite, de l'ordre de 10 heures par semaine (Chastand 1982 : 69), ils n'effectuent pas certaines tâches ménagères et en délèguent une grande partie. Mais un homme participe plus si sa femme est active que si elle est au foyer (Rousse ; Roy 1981). Le partage conjugal du domestique est lié au partage du professionnel.

Encadré $\mathrm{n}^{\circ} 7 \quad$ Note méthodologique sur la mesure du travail parental et domestique, en France

Jusqu'à la fin des années 1990, le travail parental n'est pas construit comme une catégorie de la connaissance. Par contre, le travail domestique est largement discuté en relation au travail professionnel (il contient alors les activités parentales). La première mesure marchande du travail domestique a eu lieu en France au début des années 1980 (Chadeau, Fouquet 1981). Elle a permis de repérer son importance volumique mais aussi sa valeur marchande (qui, en fonction des options théoriques choisies montre une grande variabilité de mesures).

Pour les personnes seules, la délégation du travail domestique vers l'extérieur est importante, surtout pour les hommes «isolés» (jeunes adultes ou veufs). Si les hommes seuls prennent en charge certaines tâches, ce sont essentiellement celles de l'entretien de la maison (dont la vaisselle), contrairement au soin du linge qui est fortement délégué à l'extérieur, à un service ou à une personne de la famille. « $40 \%$ des hommes jeunes «isolés» confient le repassage à l'extérieur alors que seulement $8 \%$ de jeunes femmes célibataires font de même,

\footnotetext{
${ }^{160}$ Aujourd'hui encore, le surplus de travail domestique qui incombe à la femme, lorsqu'il y a des enfants dans le ménage, est supérieur au surplus de travail professionnel pour l'homme (Barrère-Maurisson ; Marchand ; Rivier 2000).
} 
elles sont plus de $80 \%$ à assumer cette tâche alors que $20 \%$ seulement de leurs homologues masculins font eux-mêmes le travail et que $20 \%$ encore s'en dispensent totalement» (Rousse ; Roy 1981 : 62). Dans le cas des femmes seules, il n'y pas de travail ménager qu'elles ne fassent pas (au contraire des hommes), elles font peu appel à l'externalisation (Rousse ; Roy 1981).

Le travail domestique est composé de différentes tâches ménagères dont chacune appartient à un registre sociologique différent. Chaque tâche domestique est un enjeu dans la négociation car elle touche directement la définition des rôles dans la famille.

L'entretien du linge et le ménage demandent un savoir-faire ${ }^{161}$ particulier, lié aux règles d'hygiène. Des différentes tâches qui composent le soin du linge, le repassage permet la meilleure observation des pratiques. En effet, il clôture les gestes des différentes étapes et en permet donc un contrôle à posteriori. Il reflète tout à la fois les normes du propre, du rangé et de l'ordre (J. C. Kaufmann 1992).

La cuisine appartient à un autre registre sociologique, mais elle nécessite également un savoir-faire spécifique. Dans le milieu ouvrier par exemple, les femmes d'origine rurale maintiennent des savoir-faire d'origine paysanne qui se distinguent de ceux d'origine citadine (Chastand 1982). Par ailleurs, la cuisine est la seule tâche domestique qui soit directement liée à un moment de convivialité. En effet, «faire la cuisine» est une activité qui peut s'effectuer à plusieurs, en même temps que mettre la table par exemple, et elle anticipe le repas, moment partagé au centre de la vie familiale.

La vaisselle semble être du même registre que celui des courses : elles ne demandent pas de savoir-faire particulier, surtout lorsqu'elles sont découplées de la préparation des menus. Elles s'appuient sur les habitudes de consommations du ménage et ne nécessitent pas de véritables négociations, elles sont donc relativement faciles à exécuter. Elles sont de nature flexible car elles s'effectuent à l'extérieur de la maison et peuvent s'associer à d'autres activités, en particulier pendant les trajets liés au professionnel.

Notre objet est de vérifier si la répartition à l'intérieur de la catégorie des activités féminines ${ }^{162}$ évolue vers plus de mixité : or, la vaisselle est pour nous un indicateur pertinent pour montrer comment et quand évoluent les pratiques. La part masculine atteint $22,4 \%$ en 1999 : elle est en limite des catégories des activités «mixtes» pour lesquelles la part de l'homme est supérieure à 35\% (Brousse 1999: 140). En ce sens, les changements de pratiques liées à la vaisselle, nous renseignent sur la direction du processus : vers plus de mixité ou vers une plus grande spécialisation des rôles.

Le partage de la vaisselle est plus égalitaire que pour les autres tâches pour trois raisons. Premièrement, les résistances des femmes sont moindre, car le partage ne met pas en

\footnotetext{
${ }^{161}$ La distinction des tâches en fonction du critère de savoir-faire est importante car elle nous renseigne sur le mode de partage des tâches. Ainsi, pour les tâches relatives nécessitant un apprentissage particulier, le savoirfaire a été transmis et reproduit de génération en génération, faisant partie intégrale du processus de socialisation. Il inscrit une logique sexuée des rôles. En revanche, une tâche domestique qui ne fait pas appel à un savoir-faire particulier fera plus facilement l'objet d'un partage égalitaire.

${ }^{162}$ C. Brousse (1999) a classé les tâches ménagères en trois catégories: les activités à dominante féminine «préparer les repas», «faire la vaisselle», «faire le ménage et nettoyer», «effectuer le repassage», «s'occuper des enfants et des autres membres du ménage», les activités à dominante masculine «bricoler», «jardiner»; et les activités mixtes «faire les courses», «prendre en charge les démarches administratives», «prendre en charge les comptes du ménage», «prendre en charge l'éducation des enfants».
} 
danger leur identité sociale, basée sur une culture du domestique (savoir-faire), puisque cette tâche ne nécessite pas de compétence particulières. Par ailleurs, un faible niveau requis de savoir-faire, signifie une faible injonction à agir et un faible niveau d'exigence ${ }^{163}$; ceci prédispose à un plus grand partage de cette activité. Deuxièmement, le peu de savoir-faire à acquérir facilite l'apprentissage de la tâche et signifie une moindre résistance des hommes. Troisièmement, les règles conjugales qui définissent le protocole d'exécution sont facilement négociables car la tâche est de moindre complexité.

Inversement, le soin au linge est lié à un savoir-faire traditionnel dont les origines remontent à «l'Hygiénisme» du XIX siècle. A cette époque, les médecins acquièrent un droit de regard sur l'organisation sociale publique et privée.

Dans les couches populaires, l'enjeu était d'ordre public : il fallait agir contre le paupérisme alors assimilé à la criminalité et à la démoralisation. Les épidémies de choléra (en particulier celle de 1832), renforcent l'idée dominante d'une nécessaire ingérence dans les pratiques familiales des couches populaires (Cicchelli-Pugeault ; Cicchelli 1998:8).

L'ingérence dans les familles bourgeoises «contribue plus largement à rendre possible une réorganisation des rapports familiaux au profit de la figure féminine (...). Promue auxiliaire du médecin, l'épouse-mère l'aide à lutter contre la médecine populaire. Dans le même temps, elle conquiert un nouveau statut social, une reconnaissance de ses fonctions maternelles et éducatives» (Cicchelli-Pugeault ; Cicchelli 1998 : 8). La femme se fait alors le relais du médecin, elle est chargée d'appliquer les règles d'hygiène nécessaires à la survie des individus et du groupe familial. On retrouve des traces de cette tradition dans les pratiques des années 1950 : le linge doit être bouilli, puis blanchi (la blancheur était la garantie visible du respect des règles d'hygiène), la femme doit porter des vêtements propres pour s'occuper du linge (par peur de la contamination). Ces quelques exemples montrent comment les pratiques du linge se rattachent à une longue tradition dont la femme a été le vecteur privilégié. Elle détient le savoir-faire traditionnel et la signification des pratiques. Par voie de conséquence, l'entretien du linge est devenu traditionnellement le domaine des femmes. Il constitue le «pôle féminin» des activités domestiques (Zarca 1990).

Dans ce contexte, l'entretien du linge prend une signification particulière : par la plus longue tradition des savoir-faire, le nombre de tâches à accomplir, la complexité d'exécution et l'enjeu symbolique, le soin au linge, en particulier le repassage, est l'indicateur type d'un partage traditionnel des rôles basés sur une différence sexuée très forte.

Dans les années 1970, environ une femme sur deux s'occupe du linge contre moins de 5\% des hommes (Roy 1989 : 9). Ces pratiques se prolongent dans les années $1990: 88 \%$ des couples répondent que la lessive est effectuée par la femme, 9\% par les deux et 2\% par l'homme (cf. sondage La Croix).

On retrouve cette spécialisation à toutes les étapes: le tri du linge, le repassage, l'étendage... ${ }^{164}$. Si l'homme intervient dans ces tâches, c'est essentiellement sous forme de «coup de main». Cette aide ponctuelle n'engage qu'un minimum de compétence et elle se subordonne à la responsabilité de la femme. Cette stratégie a pour fonction de respecter une

\footnotetext{
${ }^{163}$ Nous faisons référence au schéma de production des pratiques domestiques de J. C. Kaufmann (1992) : les manières d'agir trouvent leurs forces structurantes dans l'intériorisation des idées et des représentations sociales ainsi que dans l'apprentissage des techniques domestiques. Ce capital de manières produit un schéma producteur de pratiques: «les injonctions à agir». La confrontation, dans le couple, des différents capitaux de manières et des niveaux d'exigence déterminent de quelle manière vont s'exprimer les injonctions à agir des deux partenaires et donc quel mode de partage va être choisi.

${ }^{164}$ Au début des années 1980, si les pères participent un peu plus dans les familles nombreuses, il n'en reste pas moins qu'ils ont en commun de ne s'occuper en rien du linge (Roy 1982).
} 
distribution spécialisée des compétences et des tâches entre les conjoints. Elle s'oppose donc aux méthodes basées sur l'interchangeabilité des rôles (J. C. Kaufmann 1992 : 87). Cette stratégie de participation en «coup de main» s'est maintenue malgré l'introduction de la mécanisation des tâches dans les années 1960, ce qui n'a pas remis en question le partage dans le couple ${ }^{165}$.

En résumé, le partage n'est pas un phénomène homogène, il dépend du sexe de l'individu, de la forme de famille, mais aussi de la nature de la tâche domestique. Certaines tâches font l'objet d'un partage égalitaire, d'autres au contraire obéissent à une règle de partage traditionnel. La nature de la tâche illustre à la fois les mécanismes identitaires au sein de la famille et les résistances familiales qui leur sont associées (cf. Figure III.2). Elle révèlent la spécification et la mixité des rôles dans la famille et leur négociation. Aussi, le repassage et la vaisselle sont deux indicateurs révélateurs des pratiques de partage des tâches domestiques.

Figure 17 Répartition des tâches domestiques selon les résistances dans le couple

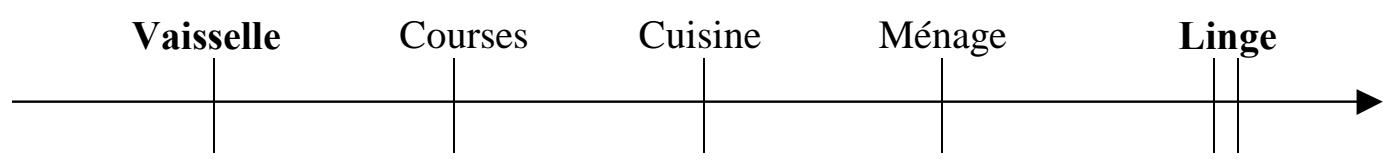

faibles résistances familiales pour un partage égalitaire du travail domestique fortes résistances familiales pour un partage égalitaire du travail domestique

Toutes les tâches ne sont pas partagées de la même manière par tout le monde. «Les femmes et les hommes ont toujours des rôles domestiques distincts, les activités liées au linge sont exclusivement assurées par les femmes, mais le partage des tâches est un peu moins inégalitaire : si le volume global du temps domestique est resté stable, les activités plutôt

\footnotetext{
${ }^{165}$ L'histoire de l'entretien du linge connaît trois grandes périodes :

La première, marquée par des techniques traditionnelles de lavage, se prolonge jusqu'à la seconde guerre mondiale, elle se distingue par une professionnalisation des différentes tâches (les laveuses, les blanchisseuses...), par un travail collectif et salarié. Chaque étape du lavage représente un travail distinct, corporel et contraignant. La lessive est l'objet d'une journée complète de travail pendant laquelle l'exécution de toutes autres tâches ménagères est suspendue. Elle avait lieu à un rythme mensuel.

La seconde période entre la seconde moitié des années 1940 et le milieu des années 1960 correspond à un bouleversement des techniques et des habitudes par l'introduction de la mécanisation des tâches de lavage. La première machine automatique à laver le linge «Constructa» a été présentée en Allemagne en 1951, il faudra attendre le milieu des années 1960 pour que cet objet ménager ne soit plus un objet de luxe (Silberzahn-Jandt 1991), mais entre à grande échelle dans les ménages allemands.

L'introduction de la machine à laver le linge dans les années 1950, a modifié les techniques de lavage, les normes d'hygiène et les représentations du propre (Silberzahn-Jandt 1991: 71-86). Les bouleversements techniques des années 1950 ont brisé les habitudes traditionnelles d'entretien du linge, cette phase de rupture avec la tradition n'a pourtant pas été propice à l'émergence de nouvelles pratiques. Il n'y a pas eu de réaffectation des tâches dans le couple, le modèle de partage sexué des tâches s'est maintenu malgré les bouleversements techniques (Bussemer 1986 : 315). La troisième période, depuis la fin des années 1960 à nos jours, se caractérise par une stagnation des innovations techniques. Parallèlement, le partage n'a pas évolué, il est resté très inégalitaire.
} 
masculines (bricolage, rangement) ont augmenté tandis que les féminines (cuisine, vaisselle, linge) ont diminué» entre 1975 et 1985 (Grimler, Roy, 1987). Certaines tâches domestiques comme le linge et la vaisselle sont deux extrêmes d'un continuum de modalités de partage. Elles révèlent que le partage et la spécification des rôles ne sont pas immuables mais se négocie. Ce processus semble aller vers plus d'égalité, l'idéal-type en étant les pratiques liées à la vaisselle. En revanche, les résistances à ce mouvement se situent dans la spécialisation sexuée des pratiques. La forme de famille exacerbe ce processus : le concubinage est une forme de famille qui pratique un partage plus égalitaire que les formes de famille plus institutionnelles.

Mais les plus grandes variations de pratiques concernent le travail parental entre les années 1980 et 1990. En 10 ans les pratiques ont évolué : «en particulier les jeunes pères s'occupent d'avantage qu'auparavant de leur premier enfant même si le temps moyen qu'il lui consacre reste relativement faible, trois quarts d'heure en 1986, contre une demi-heure en 1975» (Roy 1989). Au milieu des années 1980, les mères consacrent près de trois fois plus de temps aux activités parentales que les pères «l'écart est plus grand quand l'enfant est en bas âge. Dans un foyer où les deux conjoints travaillent, la mère consacre deux heures trente par jour à son enfant s'il a moins de deux ans, le père trois quarts d'heure» (Roy 1989 : 9). Mais «des changements d'attitude se sont produits dans la manière d'être père. Les comportements pendant la grossesse, la participation des pères à l'accouchement (plus de 80\%), le rapprochement entre le père et l'environnement quotidien du bébé, la paternité relationnelle, de proximité, avec une conception de l'autorité plus souple, plus consensuelle, participent des nouvelles manières d'être père » (Castelain-Meunier, 1998). 


\section{SOUS-SECTION III.A.2 LE TRAVAIL PARENTAL, LE TRAVAIL DOMESTIQUE ET LE TRAVAIL PROFESSIONNEL DANS LES ANNEES 1990-2000}

\section{III.A.2.1 Un partage hétérogène du travail parental}

Encadré $n^{\circ} 8 \quad$ Note méthodologique sur les mesures du travail domestique et parental pour les années 1990 et 2000 , en France

Les données sur le travail domestique, que nous traitons ici, sont tirées d'une exploitation secondaire de l'enquête Emploi du temps de l'INSEE. Le travail domestique y est calculé à partir du temps consacré au ménage, au repassage, à la cuisine (ordinaire et de réception), aux courses, à la vaisselle, au soin aux enfants et aux adultes, au bricolage, au jardinage, au soin aux animaux. On voit ici que, pour l'INSEE, le soin aux enfants est une notion interne au travail domestique.

Nous avons également exécuté une exploitation statistique des fichiers de base de l'enquête emploi du temps de l'INSEE (collecte de 1998-1999) pour étudier les pratiques de délégation $\mathrm{du}$ parental et du domestique. Nous avons alors retenu une définition restrictive du domestique qui exclut le soin aux enfants, la cuisine de réception, le bricolage et le jardinage (qui à notre avis relèvent du loisir ${ }^{166}$ ). Le travail domestique est alors composé de différentes tâches ménagères : le linge, le ménage, la cuisine, la vaisselle, les courses.

Quant aux catégories d'activités parentales et de temps parental, elles ne sont apparues comme catégories constituées qu'en 2000, à travers le travail du groupe Division Familiale du Travail du MATISSE (Barrère-Maurisson, Marchand, Rivier 2000). Il n'avait jamais été identifié et quantifié auparavant.

Le questionnaire de l'enquête avait été délibérément constitué de façon à pouvoir cerner l'ensemble des activités et des temps consacrés aux enfants. Ainsi, on a enfin isolé le temps spécifiquement parental qui regroupe «toutes les activités effectuées par les parents avec et pour les enfants» (cf. Encadré 6). Nous avons repris et continué ici l'exploitation statistique des données du groupe DFT-MATISSE pour nous renseigner sur le temps parental et son partage entre les parents.

Pour leur part, les enquêtes emploi du temps de l'INSEE identifient 4 temps de vie : le temps professionnel, le temps domestique, le temps physiologique et le temps de loisir (Dumontier, Pan ké-Shon 1999). Aussi, à la différence de l'enquête DFT-MATISSE-1999, la notion de temps parental dans l'enquête Emploi du Temps de l'INSEE, est dilué à l'intérieur du temps domestique et du temps libre. C'est pourquoi, aujourd'hui, on tente de le reconstituer à travers des exploitations secondaires (cf. Algava, 2002).

\footnotetext{
${ }^{166}$ En effet, nos critères de définition qualifient ces trois dernières activités comme des loisirs : premièrement, faire la cuisine pour une réception, faire du bricolage et faire du jardinage ont un caractère exceptionnel ; deuxièmement, la satisfaction retirée de ces activités est très importante, beaucoup plus que pour les autres activités ménagères : $72 \%$ des individus qui ont effectué ces activités, considèrent que le bricolage est une activité agréable, $77 \%$ pour l'activité «faire la cuisine pour une réception» et $81 \%$ pour le jardinage, les autres activités courantes ont une proportion de réponse à l'item «agréable» inférieur à $40 \%$ (calculs de l'auteur), ce critère de satisfaction isole très clairement ces trois activités dont la nature est très proche d'un loisir.
} 
Les résultats de C. Brousse montrent que la part des hommes dans les soins aux enfants a augmenté de plus de 5 points entre 1986 et 1999. Ce phénomène n'a pas d'équivalence dans aucune autre tâche des catégories «féminines» et «mixtes» (Brousse :1999 : 140). Mais il reste inégalement réparti entre les pères et les mères (cf. Tableau 20). En couple, les mères effectuent deux fois plus de temps parental que les pères, respectivement $25 \mathrm{~h} 40$ minutes contre $12 \mathrm{~h} 40$ pour les hommes en moyenne par semaine ${ }^{167}$.

Tableau 20 Temps parental hebdomadaire selon le type de famille (en heures et minutes par semaine)

\begin{tabular}{|r|c|}
\hline \multicolumn{1}{|c|}{ TYPE DE FAMILLE } & TEMPS PARENTAL MOYEN ${ }^{\mathbf{1 6 8}}$ PAR INDIVIDU \\
\hline Parents monoparentaux & $20 \mathrm{~h} 57 \mathrm{mn}$ \\
\hline Parents en couple avec enfants & $19 \mathrm{~h} 27 \mathrm{mn}$ \\
\hline Dont pères & $12 \mathrm{~h} 41 \mathrm{mn}$ \\
\hline Dont mères & $25 \mathrm{~h} 37 \mathrm{mn}$ \\
\hline Ensemble & $19 \mathrm{~h} 37 \mathrm{mn}$ \\
\hline
\end{tabular}

Source : enquête MATISSE -DFT - 1999 (Barrère-Maurisson ; Marchand ; Rivier 2000)

Le travail parental se décompose en quatre types de tâches (cf. Tableau 21): premièrement, les tâches de sociabilité parentale qui recouvrent les activités de présence auprès des enfants ou de temps consacré aux adolescents (jeux et activités à l'extérieur...) ; deuxièmement, le travail parental domestique qui concerne essentiellement les petits enfants puisqu'il s'agit de leur faire prendre les repas, de les habiller et de faire leur toilette; troisièmement, le travail parental „taxi“ qui est calculé à partir des tâches qui consistent à accompagner les enfants à leurs activités régulières, école ou autre; et enfin, le travail parental scolaire qui est consacré à l'aide aux devoirs (Barrère-Maurisson ; Marchand; Rivier, 2002).

$\underline{\text { Tableau } 21 \quad \text { Les quatre temps parentaux en heure et minutes }}{ }^{169}$

\begin{tabular}{|l|c|}
\hline Temps de sociabilité parentale & $19 \mathrm{~h} 56 \mathrm{mn}$ \\
\hline Temps parental domestique & $10 \mathrm{~h} 12 \mathrm{mn}$ \\
\hline Temps parental «taxi» & $5 \mathrm{~h} 20 \mathrm{mn}$ \\
\hline Temps parental scolaire & $3 \mathrm{~h} 49 \mathrm{mn}$ \\
\hline
\end{tabular}

Source : enquête MATISSE -DFT 1999 (Barrère-Maurisson ; Marchand ; Rivier 2000)

\footnotetext{
${ }^{167}$ L'exploitation des données a montré que le nombre de familles monoparentales hommes est encore faible. Nous avons pu cependant estimer que les comportements des familles monoparentales hommes sont proches de ceux des familles monoparentales femmes, le type de famille ayant ici un impact plus important sur les comportements que les différences de genre.

${ }^{168}$ Il s'agit du temps moyen hebdomadaire consacré par les parents à l'ensemble des tâches parentales.

${ }^{169}$ Cette mesure correspond au temps moyen consacré à cette tâche (calculée sur l'ensemble des individus qui l'effectuent).
} 
Chaque trajet lié au professionnel est associé à des activités particulières, elles sont réparties de façon différente pour les hommes et les femmes (Fermanian ; Lagarde 1999). Les femmes sont beaucoup plus nombreuses à relier le trajet professionnel à une autre activité, et ce, plus le matin que le soir. Le trajet du matin est souvent lié à la conduite des enfants à l'école («temps parental taxi») et les activités du soir sont surtout liées aux courses. La participation des hommes, qui augmente en présence des enfants, concerne plus les trajets du matin (Fermanian; Lagarde 1999 : 107) et donc du «temps parental taxi».

Chaque type d'activité parentale se partage de façon différente dans le couple. Pour avoir une mesure de ce partage, on a calculé la part respective des pères et des mères par rapport au temps parental global moyen du couple $\mathrm{e}^{170}$.

Les tâches parentales se répartissent de façon hétérogène: le temps de sociabilité parentale est le moins inégalement réparti, deux cinquièmes pour les pères et trois cinquièmes pour les mères, contrairement au temps de travail parental domestique qui fait encore l'objet d'une répartition très inégalitaire ${ }^{171}$ (environ un tiers pour le père, deux tiers pour les mères) (cf. Tableau 22).

Tableau 22 Partage du temps parental dans les couples, en $\%$

\begin{tabular}{|l|c|c|c|}
\hline & $\begin{array}{c}\text { Part effectuée } \\
\text { par les pères }\end{array}$ & $\begin{array}{c}\text { Part effectuée } \\
\text { par les mères }\end{array}$ & Total \\
\hline Temps de sociabilité parentale & 40 & 60 & $100 \%$ \\
\hline Temps parental scolaire & 37 & 63 & $100 \%$ \\
\hline Temps parental taxi & 35 & 65 & $100 \%$ \\
\hline Temps parental domestique & 31 & 69 & $100 \%$ \\
\hline Temps parental total & 33 & 66 & $100 \%$ \\
\hline
\end{tabular}

Source : enquête MATISSE -DFT - 1999

Lorsque l'on compare le temps parental moyen par individu au temps parental «normé» ${ }^{172}$, il apparaît une différence d'environ vingt heures par semaine (cf. Tableau 23). Cela signifie que les personnes interrogées soit ne sont pas concernées, soit n'effectuent pas seules les tâches (qui sont alors partagées, déléguées, externalisées ou non assumées).

\footnotetext{
${ }^{170}$ Les chiffres représentent le temps moyen des pères et des mères (en couple) rapporté au temps parental du couple. Ce dernier est construit à partir de la somme des temps moyens des mères en couple et des pères en couple (il s'agit d'une construction, à posteriori, car les partenaires respectifs n'ont pas été interrogés dans l'enquête).

${ }^{171}$ Les activités consacrées aux enfants ne font pas l'objet d'une journée particulière, elles ne modifient pas la structure de l'emploi du temps, comme les tâches domestiques (E. Maurin 1989). Cependant, il nous semble important de différencier le week-end du reste de la semaine : effectivement, le week-end a un rythme particulier qui détermine une participation plus importante des hommes aux tâches parentales.

${ }^{172}$ Le temps parental normé est calculé à partir de la somme des temps moyens des parents consacrés à chaque tâche parentale. Cette valeur est une construction (d'où le terme de «normé») car elle représente le temps théorique qu'effectuerait un parent s'il se consacrait réellement à toutes les tâches parentales (suivant la valeur moyenne de l'ensemble des parents qui se consacrent effectivement à ces tâches).
} 


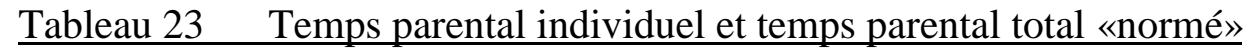

\begin{tabular}{|c|c|c|}
\hline Type de famille & $\begin{array}{l}\text { Temps parental } \\
\text { moyen par } \\
\text { individu }\end{array}$ & $\begin{array}{l}\text { Temps individuel en \% du } \\
\text { temps «normé» } \\
\text { (39 } \mathrm{h} 18 \mathrm{mn})\end{array}$ \\
\hline PARENTS & 19 h $37 \mathrm{mn}$ & 49,9 \\
\hline Dont pères & $12 \mathrm{~h} 59 \mathrm{mn}$ & 33,0 \\
\hline Dont mères & $24 \mathrm{~h} 41 \mathrm{mn}$ & 62,8 \\
\hline PARENTS EN COUPLE & $19 \mathrm{~h} 27 \mathrm{mn}$ & 49,5 \\
\hline Dont pères & $12 \mathrm{~h} 41 \mathrm{mn}$ & 32,3 \\
\hline Dont mères & $25 \mathrm{~h} 37 \mathrm{mn}$ & 65,2 \\
\hline PARENTS MONOPARENTAUX & $20 \mathrm{~h} 57 \mathrm{mn}$ & 53,3 \\
\hline
\end{tabular}

Source : enquête MATISSE -DFT - 1999 (Barrère-Maurisson ; Marchand ; Rivier 2000)

Les chefs de famille monoparentale consacrent, chaque semaine, une heure et demie de plus à leurs enfants que les parents en couple. Ils doivent effectuer «seuls» les tâches parentales alors que, dans un couple, le temps parental effectué par le père s'ajoute à celui dispensé par la mère (du fait du partage des tâches). À eux deux, ils fournissent l'équivalent de $97 \%$ du temps parental total «normé», contre $53 \%$ pour les familles monoparentales. Pour ces dernières, le surplus de temps parental individuel (par rapport à une personne en couple) est plutôt redistribué en faveur des activités de sociabilité parentale qu'en faveur du temps scolaire.

À l'intérieur des couples, les différences sont très importantes et les mères sont deux fois plus présentes auprès des enfants que les pères. Dans les familles monoparentales, les pratiques ne diffèrent pas fondamentalement qu'il s'agisse d'un homme ou d'une femme. Il y a donc un effet de «genre» qui joue sur la durée des tâches parentales dans les couples mais qui n'existe plus dans le cas des familles monoparentales.

Autrement dit, il y a un effet de «couple» sur les pratiques des femmes : être dans une logique de partage fait ressortir les pratiques sexuées à la fois de façon quantitative et qualitative. C'est dans la répartition des temps parentaux domestiques et de sociabilité que la spécification des rôles est la plus visible : proportionnellement, les pères s'impliquent plus dans les activités de sociabilité que dans toute autre tâche parentale, les activités parentales domestiques, elles, restant typiquement dévolues aux mères. 
Figure 18 Répartition des tâches parentales selon les résistances dans le couple

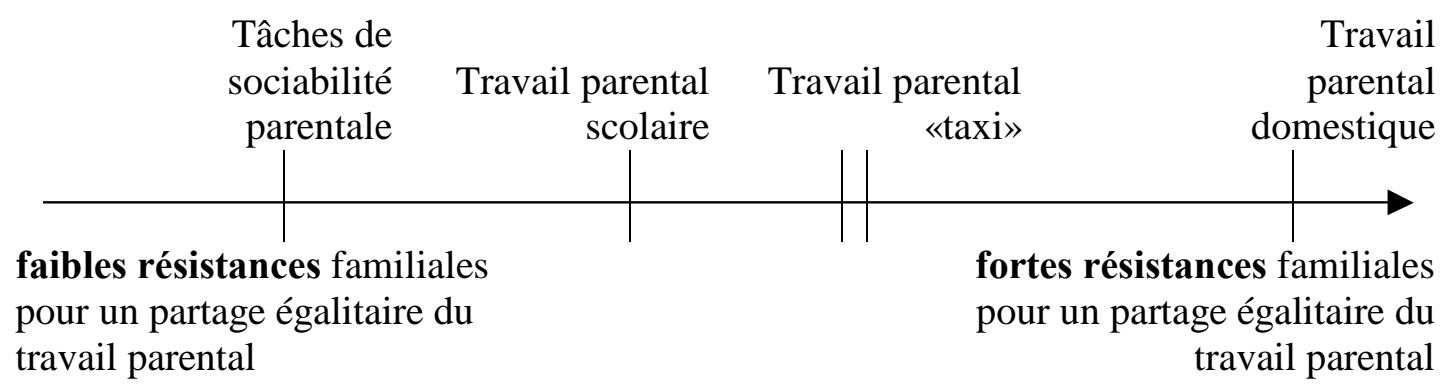

Le partage du travail parental, encore inégalitaire, l'est de moins de moins sous l'influence des pratiques masculines, qui restent cependant hétérogènes. On peut dégager deux logiques opposées de partage du travail parental : la première, pour laquelle il y a une moindre résistance à un partage égalitaire, concerne les tâches d'ordre relationnel, et la seconde, pour laquelle les résistances familiales sont plus importantes, est liée aux tâches organisationnelles et domestiques $^{173}$ (cf. Figure 18 ci-dessus).

\section{III.A.2.2 Le temps parental et le temps professionnel}

La montée du travail féminin dans les années 1970 et 1980, change le partage des tâches à l'intérieur du couple. Les années 1980 confirment l'inscription des femmes sur le marché du travail, d'abord à le temps partiel. M.-A. Barrère-Maurisson a mis en évidence l'évolution du partage du travail professionnel, depuis un modèle conjugal à un apporteur, vers un modèle à deux apporteurs, d'abord inégaux puis, depuis peu, équivalents (1992: 207). Cependant, le travail professionnel n'est pas partagé à égalité dans le couple, même si la norme de la double activité professionnelle à temps complet est maintenant majoritaire.

La répartition des tâches en deux catégories, le travail professionnel d'un côté (rémunéré) et le travail domestique et parental de l'autre (non-rémunéré), met en évidence le déséquilibre global dans le partage du «travail familial» ${ }^{174}$, du à la répartition inégalitaire du professionnel : ainsi, sur une journée théorique de 24 heures, les femmes font en moyenne $11 \mathrm{~h}$ de travail dont : 4h20 de temps professionnel, $4 \mathrm{~h} 30$ de temps domestique et $2 \mathrm{~h} 10$ de temps parental ; il leur reste alors 13 heures pour le hors travail (moins de 4 heures pour le temps personnel et plus de 9 heures pour le temps physiologique). Les hommes, eux, effectuent

\footnotetext{
${ }^{173}$ Cette constatation polarise l'impact des politiques publiques sur le partage du travail parental : premièrement, tous services, institutionnalisés ou non, qui agissent sur les transports, les facilités d'accès, la proximité des lieux, ainsi que sur les réseaux de communication, de même que les services liés à l'externalisation et la délégation du domestique, influencent la répartition du travail parental. Mais, leur action modifie le volume du travail, peu sa répartition entre les genres. Deuxièmement, une action qui agirait sur les activités de socialisation familiale (par une disponibilité en temps pour des activités conjointes), toucherait directement la logique de partage du travail parental, encourageant les pratiques masculines d'encadrement, d'éducation et de socialisation (et donc un partage plus égalitaire).

${ }^{174}$ Nous rappellerons notre définition du «travail familial» qui décrit l'ensemble du travail négocié, travail parental, domestique et professionnel, au sein de la famille.
} 
moins de $10 \mathrm{~h}$ de travail dont : $6 \mathrm{~h} 30$ de professionnel, $2 \mathrm{~h} 10$ de domestique et $1 \mathrm{~h}$ de parental, contre 14 heures de hors-travail (4 heures 30 de temps personnel et plus de 9 h30 de temps physiologique) (Barrère-Maurisson ; Minni ; Rivier ; 2001).

Le temps consacré à l'activité professionnelle est directement lié au parental. L'influence de la présence de jeunes enfants se fait réellement sentir à partir des naissances de rang deux, «lorsque les membres d'un couple exercent tous les deux une activité salariée, il faut au moins deux jeunes enfants (de moins de 6 ans) pour faire baisser la durée moyenne hebdomadaire du travail de la femme : cette diminution est alors d'une heure en moyenne ; elle est compensée par un allongement équivalent du travail du conjoint.» (Maurin : 96). On voit de grande différence entre les catégories socioprofessionnelles ${ }^{175}$. Cet effet de réduction de temps de travail est très visible pour les femmes cadres : elles avaient peu recours au temps partiel avant la naissance, contrairement aux autres catégories socioprofessionnelles qui travaillaient à temps partiel même sans enfant (les ouvrières ou les employées) (Fermanian ; Lagarde 1999).

Le parental influence également les mécanismes de partage du professionnel dans le couple. L'effet d'entraînement de la durée du travail professionnel de l'homme sur celle de la femme est maximal lorsque les enfants sont plus âgés (plus de 6 ans). Inversement, il est beaucoup plus faible lorsque les enfants sont plus jeunes. Il varie suivant les catégories socioprofessionnelles : si l'homme appartient à la même catégorie professionnelle que sa conjointe, sa durée hebdomadaire de travail enregistre une baisse (en présence d'au moins un enfant). Si la situation professionnelle est supérieure à celle de sa femme, son temps de travail s'allonge en présence d'enfant(s) (Fermanian ; Lagarde 1999) ${ }^{176}$. Par ailleurs, l'inactivité de la femme influence l'activité professionnelle de l'homme: la durée hebdomadaire de travail pour les cadres et les professions intermédiaires l'allonge, et ce, plus encore, en présence de jeunes enfants.

Par contre, les horaires de travail professionnel (et non de la durée) sont indépendants (Fermanian ; Lagarde 1999).

Le tableau 24 (ci-dessous) représente les parts respectives du temps parental et du temps professionnel en fonction du sexe et de la présence d'un partenaire. Le temps parental moyen (calculé sur l'ensemble des parents) représente un tiers du temps global (temps parental ajouté au temps professionnel). Cette proportion est légèrement plus importante pour les familles monoparentales, elle est la plus élevée pour les mères en couple, et elle est d'environ un cinquième pour les pères.

\footnotetext{
${ }^{175}$ La répartition du travail domestique varie en fonction des catégories socioprofessionnelles. Les indépendants, les agriculteurs, et à moindre degré les ouvriers, participent le moins aux tâches ménagères. Par ailleurs, ce sont dans les milieux où les femmes se consacrent le plus aux tâches ménagères que les hommes y participent le moins. Par contre, un homme cadre participe aussi souvent qu'un employé mais se contente d'avantage d'une participation modeste (E. Maurin 1989 : 39). On retrouve une stratégie du «coup de main».

${ }^{176}$ Ces variations de temps ne peuvent survenir que dans les professions qui offrent une certaine flexibilité d'horaire, comme les cadres et les professions intermédiaires (Fermanian ; Lagarde 1999).
} 
Tableau 24 Répartition du temps professionnel et du temps parental, en \%

\begin{tabular}{|r|c|c|l|}
\hline & Temps parental & Temps professionnel & Total \\
\hline PARENTS & $\mathbf{3 3 , 3}$ & $\mathbf{6 6 , 7}$ & $\mathbf{1 0 0}$ \\
\hline Dont pères & 23,7 & 76,3 & 100 \\
\hline Dont mères & 40,3 & 59,7 & 100 \\
\hline PARENTS EN COUPLE AVEC ENFANTS & 33,9 & 66,1 & 100 \\
\hline Dont pères & 23,9 & 76,1 & 100 \\
\hline Dont mères & 40,7 & 59,3 & 100 \\
\hline PARENTS MONOPARENTAUX & 34,6 & 65,4 & 100 \\
\hline
\end{tabular}

Source : enquête MATISSE - DFT 1999

Le temps consacré au travail parental varie en fonction de l'activité professionnelle. Le tableau 25 montre que le parent a une activité professionnelle plus importante lorsque le temps parental se réduit. Mais il ne s'agit pas d'une simple disponibilité en temps qui se transfère d'une activité sur l'autre, car il varie de façon différente suivant le genre : les hommes actifs, à temps complet, effectuent $12 \mathrm{~h} 05$ de temps parental en moyenne contre $20 \mathrm{~h} 15$ pour les femmes dans la même situation.

Tableau 25 Temps parental selon l'activité professionnelle (en heures et minutes par semaine $)^{(*)}$

\begin{tabular}{|l|l|}
\hline Homme actif à temps complet & $12 \mathrm{~h} 05$ \\
\hline Femme active à temps complet & $20 \mathrm{~h} 15$ \\
\hline Femme active à temps partiel & $23 \mathrm{~h} 20$ \\
\hline Femme inactive & $29 \mathrm{~h} 15$ \\
\hline
\end{tabular}

${ }^{(*)}$ Individus en couples

Source : enquête MATISSE-DFT-1999 (Barrère-Maurisson ; Marchand ; Rivier 2000)

Les femmes passent une demi-heure par jour ( 7 minutes pour leurs partenaires) pour réaliser le travail parental domestique (cf. Tableau 26 et 27). La charge éducative et affective des enfants, ce qui équivaut au temps parental scolaire et au temps parental de sociabilité, représente en moyenne 8 minutes par jour pour les mères et 6 minutes pour les pères (Brousse 1999 : 139). 
Tableau 26 Temps parental moyen (soins et activités de sociabilité) en minutes par jour pour les personnes vivant en couple

\begin{tabular}{|l|c|c|c|c|}
\hline \multirow{2}{*}{} & \multicolumn{2}{|c|}{ Hommes } & \multicolumn{2}{c|}{ Femmes } \\
\cline { 2 - 5 } & 1986 & 1999 & 1986 & 1999 \\
\hline Soins aux enfants et aux adultes & 6 & 7 & 34 & 28 \\
\hline Jeux, éducation des enfants & 5 & 6 & 9 & 10 \\
\hline
\end{tabular}

Source : Brousse (1999: 139)

Tableau 27 Soins aux enfants et aux adultes en heures et en minutes par jour en 1986 et 1999

\begin{tabular}{|l|c|c|c|c|}
\hline & $\begin{array}{c}\text { Femmes } \\
\text { actives }\end{array}$ & $\begin{array}{c}\text { Femmes au } \\
\text { foyer }\end{array}$ & $\begin{array}{c}\text { Hommes } \\
\text { inactifs }\end{array}$ & $\begin{array}{c}\text { Hommes } \\
\text { actifs }\end{array}$ \\
\hline «Emploi du temps» 1985-1986 & 24 & 32 & 5 & 9 \\
\hline «Emploi du temps» 1997- 1998 & 27 & 26 & 6 & 11 \\
\hline
\end{tabular}

Sources : Dumontier 1999

Dans les couples à deux actifs, le nombre d'enfants est un facteur déterminant dans la répartition des tâches parentales et domestiques : la part de temps attribuée aux tâches domestiques dans les couples croît avec le nombre d'enfants. L'âge, et plus particulièrement la limite des 6 ans, est un autre facteur clef. Plus les enfants sont jeunes, plus la part de temps parental est importante. Or, plus la part de travail parental domestique est importante, plus le partage est inégalitaire: en effet, plus il y a d'enfants (et d'enfants jeunes), plus la participation masculine est faible.

\section{III.A.2.3 Le travail domestique des pères $(1980)$ et des mères $(1990)$}

Au cours des années 1980, les écarts importants de pratiques entre les hommes et les femmes, se sont réduites pour certaines tâches : une proportion élevée d'hommes s'occupe de la cuisine, de la vaisselle (Grimler, Roy 1987 ; Roy 1989) et des courses (dites tâches «négociables» (Zarca 1990)). En 1998, la participation des hommes au domestique est la plus sensible dans le cas de la vaisselle : les femmes déclarent faire l'activité seules à $45 \%$, en partenariat à $43 \%$ et $11 \%$ la délèguent au partenaire (les hommes déclarent participer à cette tâche à 68\% $)^{177}$. Selon les données de l'enquête «INSEE Emploi du temps 98-99», 55,1\% des hommes déclarent avoir fait la vaisselle dans les quatre dernières semaines contre $89,3 \%$ pour les femmes.

\footnotetext{
177 L'institut CSA a réalisé pour le journal «La Croix» un sondage exclusif en 1998 auprès d'un échantillon national représentatif de 623 personnes mariées ou vivant en couple sur le thème du partage des tâches domestiques.
} 
En 1998, les femmes effectuent l'ensemble des tâches domestiques dans une proportion supérieure à $88 \%$, quelle que soit leur situation familiale. Quant aux hommes, leur participation commence à être lisible sur certains travaux domestiques. En effet, la majorité d'entre eux participent dans le cas de la vaisselle et des courses (respectivement 55,1\% et 77\%). Par contre, 50,7\% d'entre eux n'effectuent pas la cuisine, 55,3\% le ménage et $84,7 \%$ le repassage (cf. Tableau 28). Les pratiques spécifiques se maintiennent en fonction du sexe, mais aussi en fonction de la tâche.

Tableau 28 La participation aux activités ménagères par sexe en 1998, en \%

\begin{tabular}{|l|c|c|c|c|c|}
\hline & Les courses & La vaisselle & La cuisine & Le ménage & Le repassage \\
\hline HoMMES \\
\hline Oui & $\mathbf{7 7 , 0}$ & $\mathbf{5 5 , 1}$ & 49,3 & 44,7 & 15,3 \\
\hline Non & 23,0 & 44,9 & $\mathbf{5 0 , 7}$ & $\mathbf{5 5 , 3}$ & $\mathbf{8 4 , 7}$ \\
\hline Total & $100 \%$ & $100 \%$ & $100 \%$ & $100 \%$ & $100 \%$ \\
\hline FEMMES & 94,7 & 89,3 & 97,2 & 95,3 & 88,2 \\
\hline Oui & 5,3 & 10,7 & 2,8 & 4,7 & 11,8 \\
\hline Non & $100 \%$ & $100 \%$ & $100 \%$ & $100 \%$ & $100 \%$ \\
\hline Total &
\end{tabular}

Question : Au cours des quatre dernières semaines, avez-vous fait les activités suivantes pour votre ménage?

Source : INSEE Enquête Emploi du temps 98-99, MATISSE

La forme de famille est un autre facteur qui détermine les mécanismes de partage du travail domestique : le fait d'être seul ou en couple a plus d'effet sur les pratiques des hommes que sur celle des femmes.

En 1975, dès qu'il y avait présence féminine au foyer, c'est la femme qui effectuait l'ensemble des travaux ménagers (Rousse ; Roy 1981). Et si l'homme marié participait un peu plus que les hommes seuls aux tâches de l'entretien de la maison, il s'agissait plutôt d'un «coup de main» (Rousse ; Roy 1981).

A l'heure actuelle, toutes choses égales par ailleurs, les hommes en couple sans enfant consacrent 22 minutes en moins aux travaux domestiques que les hommes seuls. Quant aux femmes en couple sans enfant, elles passent 53 minutes de plus aux tâches domestiques que les femmes seules. L'écart est grand. Il l'est d'autant plus que, chez les personnes seules, les femmes ont déjà des pratiques supérieures à celles des hommes (Brousse 1999). En 1998, les hommes seuls consacrent aux tâches ménagères les deux tiers du temps que les femmes seules leur consacrent (Dumontier, Pan ké-Shon 1999).

Au moment de la mise en couple, la participation des hommes change de nature : en effet, ils effectuent plus d'activités à dominante féminine que lorsqu'ils sont seuls, soit $47 \%$ de leur temps domestique, contre $28 \%$ lorsqu'ils sont en couple. Inversement, la part d'activités à tendance masculine est plus grande lorsqu'ils sont en couple, avec $41 \%$ de leur temps, contre 19\% lorsqu'ils sont seuls (Brousse 1999). La mise en couple a pour effet d'allonger le temps de travail domestique de façon importante pour les femmes et creuse les écarts de sexe déjà existants. Elle renforce le clivage sexué des activités. 
«Être en couple» signifie une baisse de l'engagement domestique par rapport aux personnes seules : c'est le phénomène de partage. Le report sur le partenaire est beaucoup plus important pour les hommes, mais il existe aussi pour les femmes. Les différences entre les femmes seules et celles en couple sont faibles : 2,6 points entre les femmes seules et les femmes en couples concubins ; 5,1 points entre les femmes seules et les femmes mariées. Les écarts sont beaucoup plus importants dans le cas des hommes, respectivement 27,1 et 45,1 points (cf. Tableau 29).

De la même manière, la forme du couple joue un rôle important ${ }^{178}$. Dans le cas de la vaisselle, les personnes seules participent plus que les personnes en couple; les concubins plus que les couples mariés. Cette observation se vérifie sur l'ensemble des individus, quel que soit le sexe (cf. Tableau 29). La forme de famille est d'autant plus importante pour les hommes : les concubins participent plus que les hommes mariés. On observe la même relation pour les femmes, mais dans une moindre mesure. L'institutionnalisation du couple semble donc renforcer la spécialisation des rôles, en particulier sous l'influence déterminante des pratiques masculines : la spécialisation dans le couple est surtout due à la désaffectation des hommes.

Tableau 29 Participation à la vaisselle en fonction de la situation conjugale et du sexe de la personne, en \%

\begin{tabular}{|c|c|c|c|c|}
\hline & Couple marié & Couple concubin & Seul & Ensemble \\
\hline \multicolumn{5}{|c|}{ HOMMES } \\
\hline Oui & 44,1 & 62,1 & 89,2 & 55,1 \\
\hline Non & 55,9 & 37,9 & 10,8 & 44,9 \\
\hline Total & $100 \%$ & $100 \%$ & $100 \%$ & $100 \%$ \\
\hline \multicolumn{5}{|c|}{ FEMMES } \\
\hline Oui & 87,5 & 90,0 & 92,6 & 89,3 \\
\hline Non & 12,5 & 10,0 & 7,4 & 10,7 \\
\hline Total & $100 \%$ & $100 \%$ & $100 \%$ & $100 \%$ \\
\hline \multicolumn{5}{|c|}{ ENSEMBLE } \\
\hline Oui & 65,8 & 76,1 & 91,3 & 73,3 \\
\hline Non & 34,2 & 23,9 & 8,7 & 26,7 \\
\hline Total & $100 \%$ & $100 \%$ & $100 \%$ & $100 \%$ \\
\hline
\end{tabular}

Question : Au cours des quatre dernières semaines, avez-vous fait la vaisselle pour votre ménage?

Source : INSEE Enquête Emploi du temps 98-99, MATISSE

Les pratiques sexuées du repassage se distinguent de celles concernant la vaisselle. Les hommes réduisent leur participation de façon beaucoup plus importante que dans le cas de la vaisselle.

\footnotetext{
${ }^{178}$ Plusieurs variables influencent les pratiques de partage. Le sexe, la situation professionnelle, l'état marital, l'âge et le nombre d'enfants ont un rôle principal sur les temps consacrés aux activités d'une journée. Le niveau socioculturel des personnes joue un rôle secondaire (Rousse ; Roy 1981) par rapport aux déterminants premiers. Pour J. C. Kaufmann (1992) qui privilégie une approche par l'acteur, l'âge (jeunes couples, couples de plus de 50 ans), et les classes sociales (entre classes populaires et les classes plus dotées culturellement) sont déterminants.
} 
Les femmes ont une forte participation au repassage lorsqu'elles sont mariées, moindre lorsqu'elles sont en concubinage, et relativement plus faible lorsqu'elles sont seules. Les chiffres montrent une tendance inverse dans le cas de la vaisselle : en couple, il y a un report sur le partenaire. Les écarts de pratiques des femmes mariées, en concubinage et seules sont de 2,5 points dans le cas de la vaisselle, alors qu'ils sont plus importants dans le cas du repassage (la différence entre les femmes mariées et les femmes concubines est de 6,2 points). Pour les femmes, la forme du couple a plus d'influence dans le cas du repassage que dans celui de la vaisselle, en d'autres termes, les formes de famille institutionnalisées renforcent d'autant plus la logique sexuée que les pratiques sont traditionnelles, l'effet étant moindre sur les tâches «négociables».

Tableau 30 Participation au repassage en fonction de la situation conjugale et du sexe de la personne, en \%

\begin{tabular}{|l|c|c|c|c|}
\hline & Couple marié & Couple concubin & Seul & Ensemble \\
\hline \multicolumn{5}{|l|}{ HOMMES } \\
\hline Oui & 6,5 & 17,1 & 45,3 & 15,3 \\
\hline Non & 93,5 & 82,9 & 54,7 & 84,7 \\
\hline Total & $100 \%$ & $100 \%$ & $100 \%$ & $100 \%$ \\
\hline FEMMES & 91,8 & 85,6 & 81,8 & 88,2 \\
\hline Oui & 8,2 & 14,4 & 18,2 & 11,8 \\
\hline Non & $100 \%$ & $100 \%$ & $100 \%$ & $100 \%$ \\
\hline Total & 49,1 & 51,5 & 68,3 & 54,1 \\
\hline ENSEMBLE & 50,9 & 48,5 & 31,7 & 45,9 \\
\hline Oui & $100 \%$ & $100 \%$ & $100 \%$ & $100 \%$ \\
\hline Non
\end{tabular}

Question : Au cours des quatre dernières semaines, avez-vous fait le repassage pour votre ménage?

Source : INSEE Enquête Emploi du temps 98-99, MATISSE

Le couple n'est pas un simple facteur de rigidification des pratiques sexuées. En effet, il a une influence variée selon le sexe de l'individu et la nature de la tâche. Pour les femmes, avoir un partenaire, et surtout le fait d'être mariée, renforce leur participation, dans le cas du repassage, mais peu dans celui de la vaisselle. Cette dernière se partage plus dans les couples, que se soit pour les hommes ou pour les femmes (les femmes avec un conjoint font moins la vaisselle que les femmes seules).

Par ailleurs, la mise en couple est un moment privilégié pendant lequel le partage du travail familial se négocie très activement, plus que dans toutes autres phases de la vie familiale. En effet, l'individu et le couple élaborent alors des règles de partage qui peuvent se distancier fortement des règles sociales (et permettre une mixité). «La participation, toujours limitée mais grandissante, des hommes aux tâches ménagères, se traduit notamment par les expérimentations fréquentes (surtout dans les débuts du couple) des méthodes égalitaires de répartition tâche par tâche» (J. C. Kaufmann 1992 : 88). De plus, les pratiques des concubins se distinguent des couples mariés, car elles s'élaborent fréquemment par résistance par rapport 
au phénomène d'intégration ménagère ${ }^{179}$. Par-là, les concubins développent des comportements plus égalitaires que les couples mariés.

L'homme participe à 22\% lorsqu'il est le seul à exercer une activité professionnelle dans le couple, à 35\% quand les deux travaillent, à 39\% quand les deux ne travaillent pas ${ }^{180}$, et 53\% lorsque seule la femme à un emploi (Brousse 1999 : 142). «L'économie domestique n'obéit (...) pas aux mêmes règles selon que la mère exerce ou non une activité professionnelle. Dans les familles où la mère est active occupée, le nombre d'enfants conditionne largement le temps consacré à l'unité domestique.» Ce qui n'est pas le cas dans les familles où la femme n'exerce pas d'activité professionnelle (Brousse 1999 : 143). Lorsque la femme est au foyer, elle réalise les $4 / 5$ du travail domestique, alors que, dans la même situation, l'homme n'en effectue que la moitié.

Le volume horaire du travail domestique a augmenté entre 1975 et 1985, quels que soient le sexe et le statut professionnel. Cependant, la progression est plus importante pour les hommes : ils participent une heure et demi de plus lorsqu'ils sont inactifs, et 40 minutes de plus lorsqu'ils sont actifs (respectivement 20 et 15 minutes supplémentaires pour les femmes). Lors de la décennie suivante, entre 1985 et 1997, les chiffres confirment la participation grandissante des hommes, environ 10 minutes supplémentaires, quel que soit le statut professionnel $^{181}$ (cf. Tableau 31).

En 1985, une femme au foyer consacre 6 h20 au travail ménager ${ }^{182}$ (ici, le travail domestique comprend les activités parentales ainsi que les activités de semi-loisir), une femme active $4 \mathrm{~h} 15$ et un homme actif $2 \mathrm{~h} 10$.

\footnotetext{
${ }^{179}$ En référence à J.C.Kaufmann (1992 : 56), l'intégration ménagère signifie la structuration des habitudes et l'enfermement dans les rôles spécialisés, mécanismes dont les jeunes couples cherchent à se protéger en réduisant la densification ménagère au minimum au moment de la mise en couple.

${ }^{180}$ Les couples de retraités partagent mieux le travail que les couples à deux actifs.

${ }^{181}$ Les chiffres du Tableau 31 expriment des volumes horaires journaliers calculés sur la semaine. Or les weekends ont un rythme particulier: la participation des hommes est plus importante pendant le week-end que pendant le reste de la semaine, et en particulier pour les hommes actifs (Grimler 1989: 46). Pour les femmes au foyer, le samedi ressemble à une journée de la semaine (Grimler 1989 : 46). Pour elles, le travail domestique diminue uniquement le dimanche. Il s'agit de la journée la plus égalitaire (Brousse 1999 : 146).

$18220 \%$ des femmes actives se consacrent à tous instants de la matinée et de l'après-midi aux tâches ménagères,

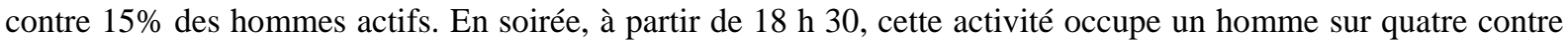
une femme sur deux (Grimler $1989:$ 42).
} 
Tableau 31 Volume horaire consacré au travail domestique en fonction du sexe et de la situation professionnelle

\begin{tabular}{|c|c|c|c|c|}
\hline & $\begin{array}{c}\text { Femmes } \\
\text { actives }\end{array}$ & $\begin{array}{c}\text { Femmes au } \\
\text { foyer }\end{array}$ & $\begin{array}{c}\text { Hommes } \\
\text { inactifs }\end{array}$ & $\begin{array}{c}\text { Hommes } \\
\text { actifs }\end{array}$ \\
\hline «Emploi du temps» $1974-1975^{(1)}$ & $4 \mathrm{~h}$ & $6 \mathrm{~h}$ & $2 \mathrm{~h} 30$ & $1 \mathrm{~h} 30$ \\
\hline «Emploi du temps» $1985-1986^{(2)}$ & $4 \mathrm{~h} 15$ & $6 \mathrm{~h} 20$ & $4 \mathrm{~h}$ & 2 h 10 \\
\hline «Emploi du temps» $1985-1986^{(3)}$ & $3 \mathrm{~h} 49$ & 5 h 16 & $2 \mathrm{~h} 45$ & $1 \mathrm{~h} 51$ \\
\hline «Emploi du temps»1997- $1998^{(3)}$ & $3 \mathrm{~h} 48$ & $4 \mathrm{~h} 47$ & 2 h 55 & $1 \mathrm{~h} 59$ \\
\hline
\end{tabular}

Sources : ${ }^{(1)}$ (Rousse ; Roy 1981); ${ }^{(2)}$ (Roy 1989) ; ${ }^{(3)}$ (Dumontier 1999)

Le plus grand bouleversement dans les pratiques des hommes se situe entre les années 1970 et 1980. Pour les femmes, les années 1990 sont les plus significative : leur participation a diminué avec une demi-heure de travail domestique en moins pour les femmes au foyer.

Entre les années 1970 et 1980, l'écart de pratique entre les hommes et les femmes s'est réduit car la participation des hommes a augmenté plus vite que celle des femmes.

Dans les années 1990, les différences se sont encore réduites sous l'action conjuguée d'une participation grandissante (même si moindre) des hommes et une stabilisation, voire une diminution de l'investissement ménager des femmes (surtout visible chez les femmes au foyer). Or il devient nécessaire de dissocier ce qui relève strictement du domestique et ce qui relève du parental, car il semble que leur plus grand engagement domestique soit due à leur participation au travail parental. Quant aux femmes, elles maintiendraient le parental mais réduiraient le domestique, comme l'indique les chiffres des femmes au foyer. 


\section{SOUS-SECTION III.A.3 LA DELEGATION DU TRAVAIL FAMILIAL}

A l'heure actuelle, une égalité entre les hommes et les femmes ne peut se concevoir, en France, que par rapport à la norme d'activité professionnelle à temps plein. La proportion de couple à deux actifs équivalents est très importante et majoritaire sur l'ensemble des familles. Aussi, le partage du travail familial au sein du couple se définit en fonction de la norme du double temps professionnel à temps complet, pour l'homme comme pour la femme. Cette logique implique une délégation et une externalisation des tâches familiales, en particulier pendant la journée.

Le recours aux réseaux d'entraide semble ne pas avoir évolué ces cinq dernières années, comme si la capacité d'aide informelle et gratuite était saturée (Aliaga, Flipo 2000). Par contre, on voit se développer, en particulier sous l'impulsion des politiques publiques, un accès aux services payants pour toutes les couches sociales, dont les ménages plus modestes (Aliaga, Flipo 2000).

\section{III.A.3.1 Les réseaux de solidarité}

Moins de $10 \%$ des ménages reçoivent une aide informelle pour les tâches familiales : $8,3 \%$ pour les courses et les transports (l'aide la plus importante), 7,8\% pour le travail parental et $6,2 \%$ pour les tâches quotidiennes ${ }^{183}$ (cf. Tableau 32).

Tableau 32 Proportion de ménages qui ont reçu une aide informelle dans les quatre dernières semaines, en \%

\begin{tabular}{|l|c|c|c|}
\hline & Oui & Non & Total \\
\hline Aide extérieure pour les courses, transport & 8,3 & 91,7 & 100 \\
\hline Aide extérieure pour s'occuper des enfants & 7,8 & 92,2 & 100 \\
\hline Aide extérieure pour les tâches quotidiennes & 6,2 & 93,8 & 100 \\
\hline Aide extérieure pour le jardin ou les animaux & 5,7 & 94,3 & 100 \\
\hline Aide extérieure pour bricoler & 5,6 & 94,4 & 100 \\
\hline Aide extérieure pour les soins aux adultes & 0,7 & 99,3 & 100 \\
\hline
\end{tabular}

Question : Au cours des quatre dernières semaines, avez-vous fait appel à certaines aides de personnes extérieures (voisins, parents, amis), ni professionnelles, ni institutionnelles ?

Source : INSEE Enquête Emploi du temps 98-99, MATISSE

Ces contributions informelles sont essentiellement des aides d'appoint (mensuelles ou hebdomadaires) (cf. Tableau 33). Le soutien pour le travail parental est le plus fréquent : $32,8 \%$ des ménages reçoivent cette aide avec une fréquence supérieure à 5 fois par mois. Elle est journalière, voire biquotidienne dans 3,7\% des cas (cf. Annexe 16). La contribution du

\footnotetext{
${ }^{183}$ Les soins aux adultes ne font pratiquement jamais l'objet d'une aide extérieure : moins d'1\% des ménages reçoivent un soutien dans ce sens.
} 
réseau d'entraide aux tâches quotidiennes est sensiblement équivalente à celle du parental, bien que la proportion des ménages qui la reçoivent biquotidiennement soit supérieure avec $7,5 \%$.

Tableau 33 Fréquence du recours à l'aide informelle, en \%

\begin{tabular}{|l|c|c|c|}
\hline \multirow{2}{*}{ NATURE DE L'AIDE } & \multicolumn{3}{|l|}{$\begin{array}{l}\text { nombre de fois où le ménage à fait appel à une aide } \\
\text { extérieure dans l'intervalle des quatre dernières } \\
\text { semaines }\end{array}$} \\
\cline { 2 - 4 } & $\begin{array}{l}\text { AIDE FREQUENTE } \\
\text { (1) }\end{array}$ & AIDE D'APPOINT $^{(\mathbf{2})}$ & Total \\
\hline Aide pour s'occuper des enfants & $\mathbf{3 2 , 8}$ & 67,2 & $100 \%$ \\
\hline Aide pour les tâches quotidiennes & 29 & 71 & $100 \%$ \\
\hline Aide pour les courses, transport & 18,6 & 81,4 & $100 \%$ \\
\hline
\end{tabular}

${ }^{(1)}$ Aide fréquente : plus de 4 fois par mois

${ }^{(2)}$ Aide d'appoint : de une à 4 fois par mois

Source : INSEE Enquête Emploi du temps 98-99, MATISSE

Certaines formes de ménage concentrent l'aide extérieure : 16,2\% des personnes seules ont fait appel à une assistance informelle pour le travail familial quotidien et $21 \%$ pour les courses et le transport. Aucune autre forme de famille ne reçoit dans la même proportion, un soutien extérieur pour les tâches domestiques (cf. Tableau 34).

Tableau 34 Recours à l'aide informelle extérieure en fonction de la forme de famille, en \%

\begin{tabular}{|l|c|c|c|c|c|c|c|c|c|}
\hline \multirow{2}{*}{ ForMES DE FAMILLES } & \multicolumn{3}{|c|}{ TACHES } & \multicolumn{3}{c|}{$\begin{array}{c}\text { COURSES, } \\
\text { QUOTIDIENNES }\end{array}$} & \multicolumn{3}{c|}{$\begin{array}{c}\text { S'OCCUPER } \\
\text { DES ENFANTS }\end{array}$} \\
\cline { 2 - 10 } & Oui & Non & Total & Oui & Non & Total & Oui & Non & Total \\
\hline Personne seule & $\mathbf{1 6 , 2}$ & 83,8 & $100 \%$ & $\mathbf{2 1 , 0}$ & 79,0 & $100 \%$ & 0,1 & 99,9 & $100 \%$ \\
\hline $\begin{array}{l}\text { Familles } \\
\text { monoparentales }\end{array}$ & $\mathbf{7 , 9}$ & 92,1 & $100 \%$ & $\mathbf{1 2 , 4}$ & 87,6 & $100 \%$ & $\mathbf{1 4 , 2}$ & 85,8 & $100 \%$ \\
\hline Couple sans enfant & 4,5 & 95,5 & $100 \%$ & $\mathbf{6 , 4}$ & 93,6 & $100 \%$ & - & - & - \\
\hline Couple avec enfant(s) & 2,9 & 97,1 & $100 \%$ & 3,6 & 96,4 & $100 \%$ & $\mathbf{1 7 , 2}$ & 82,8 & $100 \%$ \\
\hline Total & $\mathbf{6 , 2}$ & 93,8 & $100 \%$ & 8,3 & 91,7 & $100 \%$ & 7,8 & 92,2 & $100 \%$ \\
\hline
\end{tabular}

Source : INSEE Enquête Emploi du temps 98-99, MATISSE

La seconde forme de ménage qui reçoit de l'aide extérieure dans une proportion relativement importante, est celle des familles monoparentales. Dans le cas des tâches quotidiennes, elles sont un peu plus soutenues par un réseau informel que la moyenne, 7,9\% contre $6,2 \%$. Elles reçoivent une assistance extérieure pour les courses et le transport dans une proportion de $12,4 \%$, soit plus du double que les couples avec enfants.

Par contre, ce sont les couples qui font le plus appel à un soutien extérieur pour le travail parental, respectivement $17,2 \%$ pour les couples et $14,2 \%$ pour les familles monoparentales. Les couples avec enfants est la forme de famille qui fait le moins appel à une contribution 
informelle pour les travaux quotidiens, les courses et les transports, ils concentrent ce type d'aide pour le travail parental.

Le réseau d'entraide des ménages est d'abord composé par la famille (très fréquemment), puis par les amis, par les voisins, et enfin par les collègues (très rarement). Lorsque le ménage fait appel à une aide extérieure pour effectuer les tâches quotidiennes, c'est tout d'abord la famille qui est sollicitée dans $82,3 \%$ des cas, puis les amis avec 13,8\%, en enfin, peu souvent, les voisins avec 3,2\%. Le travail parental est délégué en premier lieu à la famille avec $79,8 \%$, aux amis avec $11,8 \%$ et aux voisins avec $8 \%$ (cf. Tableau 35). Mais les voisins sont relativement plus sollicités pour le travail parental que pour toute autre tâche. Les tâches quotidiennes sont proportionnellement plus souvent le lot de la famille

Tableau 35 Composition du réseau qui apporte l'aide au foyer, en \%

\begin{tabular}{|l|c|c|c|c|c|}
\hline \multicolumn{1}{|c|}{ NATURE DE L'AIDE } & FAMILLE & AMIS & VOISINS & COLLEGUES & TOTAL \\
\hline Aide pour les tâches quotidiennes & $\mathbf{8 2 , 3}$ & $\mathbf{1 3 , 8}$ & 3,2 & 0,7 & 100,0 \\
\hline Aide à s'occuper des enfants & $\mathbf{7 9 , 8}$ & $\mathbf{1 1 , 8}$ & $\mathbf{8 , 0}$ & 0,4 & 100,0 \\
\hline Aide pour les courses, transport & 71,6 & 19,7 & 8,0 & 0,7 & 100,0 \\
\hline
\end{tabular}

Source : INSEE Enquête Emploi du temps 98-99, MATISSE

Si globalement la famille reste le premier fournisseur de solidarité, c'est l'affinité plus que la proximité qui détermine le service rendu. Cependant, dans le cas du travail parental, les voisins ont une participation relativement plus importante, indiquant que la proximité est un facteur déterminant dans la délégation du travail parental.

\section{III.A.3.2 La délégation du domestique}

Près de $9 \%$ des ménages font appel à une aide professionnelle rémunérée pour les tâches ménagères (INSEE Enquête Emploi du temps 1998, MATISSE). 70\% de ces ménages le font dans un volume horaire inférieur ou égal à 5 heures par semaine (cf. Figure 19 ). 
Figure 19 Nombre d'heures d'aide rémunérée par semaine

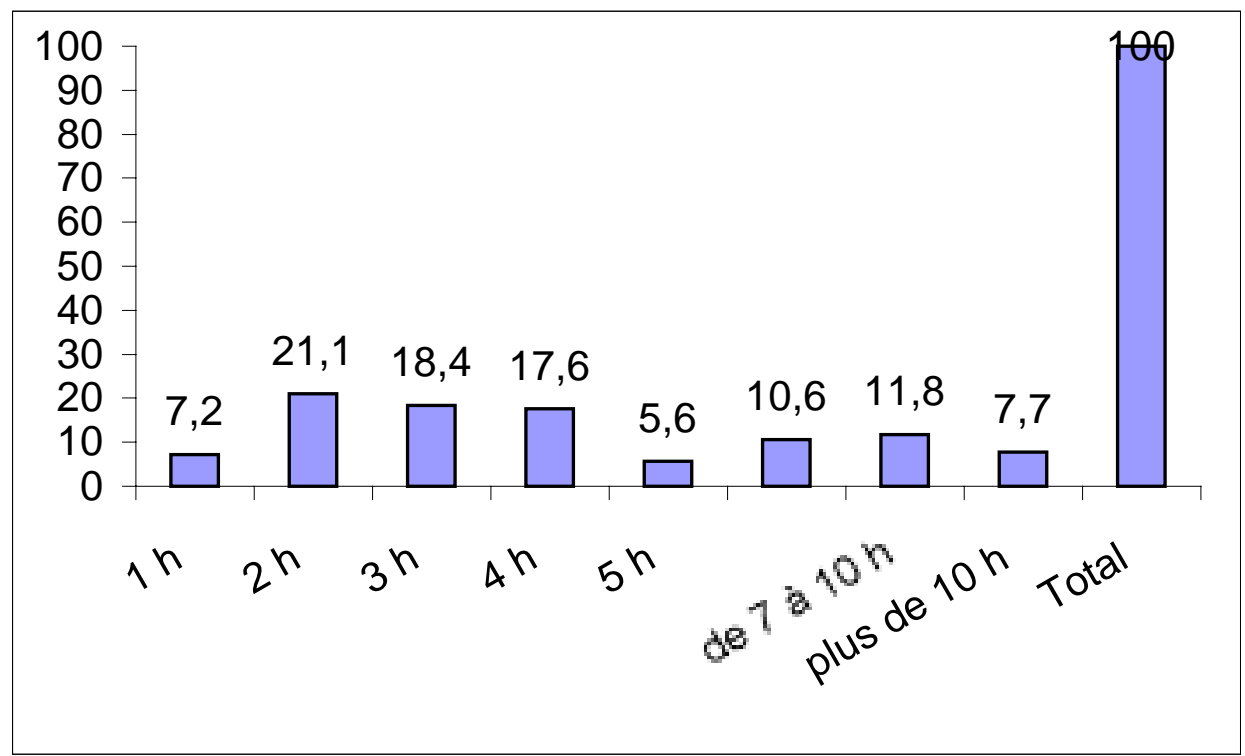

Source : INSEE Enquête Emploi du temps 98-99, MATISSE

Sur l'ensemble des ménages concernés, l'aide rémunérée s'élève à 5 heures environ en moyenne, et pour 2,8\% d'entre eux, l'aide dépasse 20 heures par mois. En 1995, un peu plus de $5 \%$ des ménages emploient une femme de ménage, et pour la majorité d'entre eux, avec un volume horaire de 2 à 4 heures par semaine. Ces chiffres sont relativement stables depuis les années 1980 (Flipo, Hourriez 1995). Il semble donc que la délégation du travail domestique par le biais de l'aide rémunérée se soit maintenue, voire légèrement développée au cours des années 1990. Les chèques emplois services, introduits en 1994, ont certainement contribué à donner à ce type d'aide une place non négligeable dans les stratégies de conciliation des ménages. Parmi les ménages qui payent un service à domicile, la part de ceux qui utilisent le chèque emploi service s'est élevée en trois ans, pour atteindre plus de 10\% en 1999 (Aliaga, Flipo 2000).

La proportion de ménages qui déclarent faire appel aux services marchands est beaucoup plus variable et importante (jusqu'à 60\%) que la part de ménages qui font appel à l'aide informelle $8 \%$ ou à l'aide institutionnelle (aide rémunérée) $9 \%$. La part de ménage qui recourent aux services marchands, dépasse, quelle que soit la tâche, la proportion de ménages qui utilisent les deux autres modes de délégation. Il ne s'agit pas d'un service d'appoint, mais d'une structure nécessaire à la délégation du travail familial. En ce sens, il est important de concevoir les modes de délégation du travail familial, qu'ils soient marchands, familiaux ou publics, de façon complémentaire et simultanée.

$57,5 \%$ des ménages ont recours au pressing, 10,4\% à la laverie automatique, $9,8 \%$ à la livraison des courses et $8,8 \%$ à la livraison des plats cuisinés (cf. Tableau 36). Le soin du 
linge ${ }^{184}$ est donc délégué dans une proportion qui n'a pas d'égal dans l'ensemble des tâches ménagères ${ }^{185}$. Déjà dans les années 1990 , «le recours aux services extérieurs - femmes de ménages, pressing,... - comme substitut complet au travail domestique ${ }^{186}$ est presque inexistant à deux exceptions près : le repassage est délégué dans $8 \%$ des cas, le lavage du linge dans 6\%» (Grimler, Roy 1990).

Tableau $36 \quad$ Recours aux services marchands par les ménages, en \%

\begin{tabular}{|l|c|c|c|}
\hline \multicolumn{1}{|c|}{ SERVICES MARCHANDS } & OUI & NON & TOTAL \\
\hline Recours au pressing & $\mathbf{5 7 , 5}$ & 42,5 & 100,0 \\
\hline Recours à la laverie automatique & $\mathbf{1 0 , 4}$ & 89,6 & 100,0 \\
\hline Recours à la livraison de vos courses & $\mathbf{9 , 8}$ & 90,2 & 100,0 \\
\hline Recours à la livraison de plats cuisinés & $\mathbf{8 , 8}$ & 91,2 & 100,0 \\
\hline
\end{tabular}

Source : INSEE Enquête Emploi du temps 98-99, MATISSE

Le linge est, dans l'absolu, l'une des tâches la plus fréquemment déléguée et externalisée, vers les services ou la famille. Environ $30 \%$ des ménages qui ont recours à ce type de service le font fréquemment. Cependant, en 1998, la fréquence du recours montre qu'il est surtout un service annuel. Le repassage qui est l'une des tâches la moins égalitaire au sein du couple est en fait la tâche domestique qui est la plus déléguée à l'extérieur du ménage. En effet, on a vu que le partage égalitaire du repassage se heurtait à de grandes résistances familiales. De fait, c'est la délégation vers l'extérieur qui réduit les inégalités, en jouant principalement sur le volume de travail à partager.

\section{III.A.3.3 La délégation du parental}

La majorité des ménages qui ont des enfants, ont d'abord recours à des modes de garde informels, et ce d'autant plus que les enfants sont scolarisés : 43,2\% des ménages mobilisent un membre de la famille pour la garde des enfants de moins de 6 ans, la proportion est de $61,3 \%$ pour les enfants scolarisés (cf. Tableau 37). Puis, dans le cas de l'aide institutionnalisée, les modes de garde individualisés (une personne rémunérée ou une nourrice) sont préférés aux modes de gardes collectifs, probablement pour leur plus grande flexibilité. Pour les enfants scolarisés, si le réseau informel joue un grand rôle, il n'en reste pas moins que la garde rémunérée ou les centres collectifs concernent environ $20 \%$ des ménages. Aussi, on voit que les structures d'accueil pour les enfants scolarisés jouent un rôle important dans la stratégie de gestion du parental de nombreux ménages.

\footnotetext{
${ }^{184}$ Le recours au pressing est hebdomadaire pour 3,9\% des ménages, mensuel pour $28,6 \%$ et annuel pour $67,5 \%$. Quant à celui de la laverie, 11,4\% des ménages l'utilisent de façon hebdomadaire, 15\% mensuellement et 73,6\% annuellement.

${ }^{185}$ Par contre, la livraison des courses, lorsque ce service est utilisé, est plus fréquente : $62 \%$ des ménages y ont recours une fois par mois, 9,8\% des ménages l'utilisent au moins une fois par semaine. Et lorsque les ménages se font livrer des plats cuisinés, ils le font avec une fréquence importante : 25,9\% y ont recours une fois par mois, $12,2 \%$ deux fois par mois et $9,2 \%$ une ou plusieurs fois par semaine.

${ }^{186}$ Souligné par nous
} 
$\underline{\text { Tableau } 37 \text { Modes de gardes informels et institutionnalisés suivant l'âge de l'enfant, en \% }}$

\begin{tabular}{|l|c|c|c|c|c|c|}
\hline \multirow{2}{*}{} & \multicolumn{2}{|c|}{$\begin{array}{c}\text { ENFANTS AGES DE } \\
\text { MOINS DE 6 ANS }\end{array}$} & \multicolumn{3}{|c|}{$\begin{array}{c}\text { ENFANTS } \\
\text { SCOLARISES }\end{array}$} \\
\cline { 2 - 6 } & Oui & Non & Total & Oui & Non & Total \\
\hline
\end{tabular}

MODES DE GARDE INFORMELS

\begin{tabular}{|l|l|l|l|l|l|l|}
\hline Garde par une personne du ménage & $\mathbf{4 3 , 2}$ & 56,8 & 100,0 & $\mathbf{6 1 , 3}$ & 38,7 & 100,0 \\
\hline Garde non rémunérée par un(e) ami(e) & 17,9 & 82,1 & 100,0 & 33,2 & 66,8 & 100,0 \\
\hline
\end{tabular}

MODES DE GARDE INSTITUTIONNALISES

\begin{tabular}{|l|c|c|c|c|c|c|}
\hline Garde par une nourrice & 17,1 & 82,9 & 100,0 & - & - & - \\
\hline Garde au domicile par personne rémunérée & 8,6 & 91,4 & 100,0 & - & - & - \\
\hline Garde dans une crèche & 7,9 & 92,1 & 100,0 & - & - & - \\
\hline Garde dans une crèche parentale & 1,2 & 98,8 & 100,0 & - & - & - \\
\hline Garde par une personne rémunérée & - & - & - & $\mathbf{2 1 , 3}$ & 78,7 & 100,0 \\
\hline Garde en centre de loisirs, étude, halte & - & - & - & $\mathbf{2 0 , 0}$ & 80,0 & 100,0 \\
\hline
\end{tabular}

Question : Si vous avez des jeunes enfants (non scolarisés et scolarisés), quel est le mode de garde que vous avez choisi ? Plusieurs réponses sont possibles.

Source : INSEE Enquête Emploi du temps 98-99, MATISSE

Le nombre d'heures de garde rémunérée varie en fonction de l'activité professionnelle des parents. Les ménages, dont le répondant exerce une activité professionnelle à temps complet, sont légèrement plus sur-représentés, avec $12,4 \%$ contre $10,8 \%$ en moyenne, dans la catégorie des 35 heures et plus (cf. Tableau 38). Les salariés à temps partiel sont, pour leur part, plus représentés dans la catégorie des 5 à 10 heures. Quant aux inactifs, ils ont plus recours à une garde rémunérée pour un volume horaire compris entre 1 et 5 heures, avec 39,3\% contre $26,1 \%$ en moyenne. 
Tableau 38 Nombre d'heures de garde rémunérée par semaine en fonction de l'activité professionnelle, en \%

\begin{tabular}{|l|c|c|c|c|c|c|}
\hline \multirow{2}{*}{$\begin{array}{c}\text { ACTIVITE } \\
\text { PROFESSIONNELLE }\end{array}$} & \multicolumn{6}{|c|}{ NOMBRE D'HEURES DE GARDE REMUNEREE } \\
\cline { 2 - 8 } & $\begin{array}{c}\text { de 1 à 5 } \\
\text { heures }\end{array}$ & $\begin{array}{c}\text { de 5 à 10 } \\
\text { heures }\end{array}$ & $\begin{array}{c}\text { de 10 à 19 } \\
\text { heures }\end{array}$ & $\begin{array}{c}\text { entre 19 et } \\
35 \text { heures }\end{array}$ & $\begin{array}{c}\text { plus de 35 } \\
\text { heures }\end{array}$ & Total \\
\hline $\begin{array}{l}\text { Exerce une profession à } \\
\text { temps plein }\end{array}$ & 24,8 & 24,7 & 16,3 & 21,8 & 12,4 & $100 \%$ \\
\hline Salariés à temps partiel & 29,5 & 30,8 & 12,4 & 19,1 & 8,2 & $100 \%$ \\
\hline Chômeur & 23,6 & 5,6 & 11,1 & 55,8 & 3,8 & $100 \%$ \\
\hline Inactif & 39,3 & 27,4 & 15,0 & 18,3 & - & $100 \%$ \\
\hline Ensemble & 26,1 & 25,2 & 15,3 & 22,6 & 10,8 & $100 \%$ \\
\hline
\end{tabular}

Source : INSEE Enquête Emploi du temps 98-99, MATISSE

${ }^{(*)}$ Limite d'âge fixée à 65 ans

Le recours à un mode de garde rémunérée ne doit pas être uniquement interprété comme un moyen de conciliation entre temps professionnel et familial, il n'est pas une simple réaffectation des temps d'un domaine sur l'autre. En effet, 55,8\% des chômeurs, et c'est le résultat le plus surprenant, font appel, entre 19 et 35 heures par semaine, à une aide rémunérée pour la garde des enfants (contre 22,6\% sur l'ensemble des individus). Le chômage montre que le facteur explicatif n'est pas lié à une logique de disponibilité, ou de réaffectation, des temps.

Les couples se distinguent des familles monoparentales par le nombre d'heures de garde rémunérée par semaine. La plus grande différence concerne la tranche horaire comprise entre 5 et 10 heures : les couples sont sous-représentés dans cette catégorie avec une proportion de $24,4 \%$, contre 38,9\% pour les familles monoparentales (cf. Tableau 39). A l'inverse, les couples font appel à ce service dans un volume horaire supérieur à 35 heures, dans 11,3\% des cas, contre $2,7 \%$ pour les familles monoparentales. 
Tableau 39 Nombres d'heures de garde rémunérée par semaine en fonction de la forme de famille, en \%

\begin{tabular}{|l|c|c|c|c|c|c|}
\hline & \multicolumn{7}{|c|}{ NOMBRE D'HEURES DE GARDE REMUNEREE } \\
\cline { 2 - 7 } FORME DE FAMILLES & $\begin{array}{c}\text { de } 1 \text { à } 5 \\
\text { heures }\end{array}$ & $\begin{array}{c}\text { de } 5 \text { à } 10 \\
\text { heures }\end{array}$ & $\begin{array}{c}\text { de } 10 \text { à } 19 \\
\text { heures }\end{array}$ & $\begin{array}{c}\text { de } 19 \text { à } 35 \\
\text { heures }\end{array}$ & $\begin{array}{c}\text { plus de } 35 \\
\text { heures }\end{array}$ & Total \\
\hline $\begin{array}{l}\text { Couple avec } \\
\text { enfant(s) }\end{array}$ & 26,4 & $\mathbf{2 4 , 4}$ & 15,1 & 22,8 & $\mathbf{1 1 , 3}$ & $100 \%$ \\
\hline $\begin{array}{l}\text { Familles } \\
\text { monoparentales }\end{array}$ & 23,8 & $\mathbf{3 8 , 9}$ & 14,0 & 20,5 & $\mathbf{2 , 7}$ & $100 \%$ \\
\hline Ensemble & 26,1 & 25,2 & 15,3 & 22,6 & 10,8 & $100 \%$ \\
\hline
\end{tabular}

Source : INSEE Enquête Emploi du temps 98-99, MATISSE

Pour les couples, la délégation du travail parental signifie une garde rémunérée beaucoup plus longue. Ce qui est lié à la double norme du temps complet. Quant aux familles monoparentales, il s'agit d'une solution d'appoint, dont l'accès limité peut être dû à un coût trop élevé de l'aide (ou d'un emploi à temps partiel). Il faut également rappeler que les familles monoparentales ont un accès limité à l'aide informelle venant accentuer ces différences (Déchaux 1996 ; Crenner 1999).

Les données du tableau 40 confirment que les familles monoparentales accèdent moins souvent aux modes informels de garde que les couples avec enfants. De plus, elles ont moins souvent recours à une nourrice et à une personne rémunérée pour les enfants scolarisés, tandis que le rôle des structures collectives est proportionnellement plus important.

Par contre, pour les jeunes enfants, les familles monoparentales font plus souvent appel à une aide rémunérée à domicile, $13 \%$ contre $10,2 \%$ pour les couples. Ces chiffres décrivent la tendance actuelle suivant laquelle les ménages plus modestes utilisent un peu plus souvent les services individuels qu'en 1996 (Aliaga, Flipo 2000). 
Tableau 40 Modes de garde des enfants en fonction de la forme de famille, en $\%$

\begin{tabular}{|c|c|c|c|c|c|c|}
\hline \multirow[t]{2}{*}{ MODES DE GARDE } & \multicolumn{3}{|c|}{$\begin{array}{c}\text { FAMILLES } \\
\text { MONOPARENTALES }\end{array}$} & \multicolumn{3}{|c|}{$\begin{array}{l}\text { COUPLES AVEC } \\
\text { ENFANTS }\end{array}$} \\
\hline & Oui & Non & Total & Oui & Non & Total \\
\hline \multicolumn{7}{|l|}{ ENFANTS DE MOINS DE 6 ANS } \\
\hline Garde par une personne du ménage & 21,2 & 78,8 & $100 \%$ & 51,3 & 48,7 & $100 \%$ \\
\hline Garde non rémunérée par un(e) ami(e) & 10,3 & 89,7 & $100 \%$ & 22,1 & 77,9 & $100 \%$ \\
\hline Garde par une nourrice & 8,4 & 91,6 & $100 \%$ & 21,3 & 78,7 & $100 \%$ \\
\hline Garde au domicile par personne rémunérée & 13,0 & 85,8 & $100 \%$ & 10,2 & 89,8 & $100 \%$ \\
\hline Garde dans une crèche & 9,7 & 90,3 & $100 \%$ & 9,1 & 90,9 & $100 \%$ \\
\hline Garde dans une crèche parentale & 1,4 & 98,6 & $100 \%$ & 1,5 & 98,5 & $100 \%$ \\
\hline \multicolumn{7}{|l|}{ ENFANTS SCOLARISES } \\
\hline Garde par une personne du ménage & 42,4 & 57,6 & $100 \%$ & 62,3 & 37,7 & $100 \%$ \\
\hline Garde non rémunérée par un(e) ami(e) & 38,2 & 61,8 & $100 \%$ & 33,1 & 66,9 & $100 \%$ \\
\hline Garde par une personne rémunérée & 20,4 & 79,6 & $100 \%$ & 21,9 & 78,1 & $100 \%$ \\
\hline Garde en centre de loisirs, étude, halte & 26,4 & 73,6 & $100 \%$ & 20,1 & 79,9 & $100 \%$ \\
\hline
\end{tabular}

Source : INSEE Enquête Emploi du temps 98-99, MATISSE

En France, deux types de mesures développent les services domestiques et les emplois familiaux. Une première série de mesures tente de réduire le coût du travail pour les ménages par l'abaissement de la TVA et l'exonération des charges patronales. Une autre série de mesures contribue à la «solvabilisation» de la demande, par l'exonération fiscale des ménages employeurs; on nommera, entre autre mesure, les chèques emploi service (CES) et le développement des titres emploi service (TES) (Le Feuvre 2000). L'enjeu du chèque emploi service $^{187}$ était de simplifier les contraintes administratives de l'embauche, il a connu depuis son introduction un vif succès et notamment auprès de ménages bi-actifs de haut niveau et de personnes âgées valides et relativement aisées (Le Feuvre 2000).

Sans jouer sur les mécanismes de répartition à l'intérieur du ménage (par une augmentation de la participation masculine), l'externalisation d'une charge de travail effectuée par les femmes permet cependant de corriger le fort déséquilibre.

Actuellement, les stratégies de conciliation des couples avec enfants montrent un usage important de la délégation du travail parental, domaine pour lequel ils reçoivent le plus d'aide informelle. Ce besoin est d'autant plus important que la norme dominante pour le travail professionnel dans les couples est un double temps complet. Ces familles favorisent des modes de garde personnalisée, plutôt que des structures d'accueil collectives, pour la garde des enfants de moins de 6 ans, tandis que l'accueil collectif a un rôle relativement important pour les enfants scolarisés. Par ailleurs, nous avons montré que la logique de proximité est déterminante, pour la délégation du travail parental, donnant une importance particulière aux crèches et garderies d'entreprises ainsi qu'aux complexes scolaires pour les enfants plus âgés.

\footnotetext{
${ }^{187}$ Le développement de ces services correspond aux politiques sociales cherchant à développer des nouveaux secteurs d'activité et à favoriser l'emploi (Barrère-Maurisson, Buffier-Morel, Rivier 2001).
} 
Les familles monoparentales ont à la fois un problème d'accés au réseau informel pour la délégation du travail parental et un moindre recours aux modes de garde institutionnalisée. Une garde individuelle rémunérée est un peu plus souvent un moyen de conciliation que pour les couples, mais pour un volume horaire bien inférieur. 


\title{
SECTION III.B LE PARTAGE DU TRAVAIL FAMILIAL EN
}

\section{ALLEMAGNE}

\section{SOUS-SECTION III.B.1 LE TEMPS PARENTAL ET LE TEMPS DOMESTIQUE DANS LES ANNEES 1960 ET 1970}

\author{
Encadré $\mathrm{n}^{\circ} 9 \quad$ Note méthodologique sur la mesure du travail domestique et parental dans \\ les années 1960 et 1970, en Allemagne
}

Entre 1960 et 1970, dans l'ancienne Allemagne de l'Ouest, le relevé des données sur le domestique n'est pas standardisé. Les différentes enquêtes utilisent le plus souvent une catégorisation des tâches domestiques fondée sur les «fonctions» familiales et isolent quatre types d'activités :

Ce premier domaine d'activité concerne la production de biens et de marchandises ainsi que les soins aux membres du ménage (liés aux besoins physiques des individus) («hauswirtschaftlich»).

Le deuxième domaine regroupe les activités domestiques d'organisation, de prévoyance, d'information, de coordination et d'administration du ménage («haushälterisch»).

Le troisième concerne les activités relationnelles et socialisantes, qu'elles soient orientées vers les membres de la famille (en particulier les enfants) ou vers la parenté ou le voisinage... («beziehungsorientiert»). Il s'agit également de la «fonction de refuge», orientée principalement vers les besoins de l'homme actif («psychischer Spannungsausgleich»). En effet, dans la littérature féministe des années 70 , on parle du travail domestique en différenciant les activités d'ordre matériel, technique et physique, de celles d'ordre psychique et relationnel (Kettschau $1980: 41$ ), tout en les revendiquant comme travail.

Enfin, le quatrième domaine regroupe les activités d'ordre, de coordination et de ritualisation de la vie familiale («Gestaltungsarbeit»). Il regroupe l'ensemble des activités qui, selon l'expression de (Kettschau 1980 : 42-43), transforment le «ménage» en «foyer» («aus einem Haus ein Heim machen»).

Les domaines d'activité comme «les courses», «le ménage», «la cuisine» et «les soins du linge» sont identifiés de la même façon dans les enquêtes citées dans la section ci-dessous (IZS, Deist et Böhner, Zander, Pross et Schöps). En revanche, les tâches domestiques, les loisirs, et la catégorie «Autre», sont construites différement selon les auteurs (cf. Annexe 14).

Les activités d'administration, de prévision, de planification et d'information (faire les menus, les courses, tenir les comptes du ménage, mais aussi démarcher et s'informer auprès des institutions) sont consignées dans la catégorie de «haushälterische Tätigkeit» qui n'est évaluée que dans les enquêtes de Zander et de Deist/Böhner.

Il faut attendre la fin des années 1970 pour voir apparaître le travail domestique comme une catégorie de la connaissance (Ostner 1978). Le terme de travail était jusqu'alors compris en référence au monde productif (le travail comme travail rémunéré). I. Ostner, a mis en relation les deux catégories de la connaissance, profession et travail domestique : le travail domestique ne pouvant faire l'objet d'une professionnalisation des tâches domestiques à 
l'intérieur de la famille (l'externalisation des fonctions étant cependant possibles) il devenait vain de chercher une équivalence des deux domaines (1978).

Les activités relatives à la socialisation et aux soins des enfants ne constituent pas une catégorie en soi. Les tâches parentales sont éclatées sur l'ensemble des activités. Globalement, le temps parental est étudié à partir du temps passé aux soins («Pflege»), à l'encadrement («Betreuung»), et à l'éducation des enfants («Erziehung»). Peu d'études prennent en compte le relationnel ou le temps de sociabilité parentale («Beziehungsarbeit») (Kettschau 1980: 84).

La catégorie du parental montre de nombreuses variations, moins liées aux activités qu'aux méthodes de recueil. En ce qui concerne l'évaluation du temps parental, il est difficile de comparer les femmes actives aux femmes inactives sans tenir compte de la taille du ménage. En effet, les ménages où les femmes sont actives ont un moins grand nombre d'enfants lesquels sont d'ailleurs plus âgés. Le volume de travail lié aux activités domestiques est donc moins important. Cependant, et nous reprendrons les arguments de Kettschau (1980) sur ce point, la nature des tâches change lorsque les enfants vieillissent, et si les tâches relèvent moins du domestique, le volume de travail parental reste important.

Les enquêtes de Zander et de Deist/Böhner ${ }^{188}$ saisissent l'ensemble des activités de soins, d'encadrement et d'éducation effectuées auprès de l'enfant (activités de supervision pendant les activités de jeux et d'apprentissage, soins corporels). Pross et Schoeps s'interrogent également sur le temps passé auprès des enfants dans les activités d'encadrement (jeux et devoirs scolaires). Seule l'enquête IZS tient compte du temps passé à parler, lire et expliquer, à jouer (à l'intérieur comme à l'extérieur), et à se promener. Le temps passé auprès des enfants malades est également précisé dans cette enquête.

Dans les années 1960 et 1970, deux domaines d'activités ne font pas partie de la mesure des activités parentales : le premier domaine concerne le relationnel, le second, les activités qui ne sont pas faites directement avec l'enfant, mais pour l'enfant, et relèvent de la catégorie «gestaltende Tätigkeiten» (que l'on pourrait traduire par l'ensemble des tâches immatérielles d'organisation) (Kettschau 1980 : 86). Cette dernière catégorie comprend les discussions parentales sur le thème de l'éducation, les rencontres avec les professeurs, la fonction de médiation dans certains conflits, toutes les activités de relationnel par rapport à l'extérieur et aux autres institutions de socialisation, mais également toutes les activités de relationnel à l'intérieur de la famille qui selon Kettschau dépasse les activités de sociabilité parentales pour prendre en compte les pures activités de relationnel (comme consoler par exemple) ${ }^{189}$.

Dans les années 1970, la mesure des activités parentales est limitée aux besoins physiques et d'éducation de l'enfant. La prise en compte des tâches de sociabilité (telles que nous les avons présentées dans l'enquête DFT en France), n'apparaît qu'à la fin des années

\footnotetext{
${ }^{188}$ L'enquête de Zander a un échantillon réduit et non représentatif : il est essentiellement composé de ménages citadins de quatre personnes. On remarque par ailleurs une sous-estimation globale des temps, non seulement au niveau du travail domestique, mais également au niveau du travail parental. On interprétera donc les chiffres de cette enquête avec beaucoup de précaution. En revanche, les enquêtes (IZS ; Pross/Schoeps) se basent sur un échantillon représentatif et leur mesure du temps servira de référence.

${ }^{189}$ Kettschau (1980 : 84) estime que si le domaine relationnel a été négligé dans les années 1970, la faute en revenait à la construction théorique et méthodologique des recherches qui n'avaient pas poursuivi de réflexion systématique sur le lien entre d'un côté les activités et de l'autre les fonctions du ménage.
} 
$1970^{190}$, avec l'émergence d'un discours sur la nature des tâches immatérielles et relationnelles (comme celle d'un travail).

Le tableau 41 rassemble les temps consacrés aux différentes activités parentales dans les années 1960 et 1970. Ces mesures sont à prendre avec beaucoup de précaution, car elles montrent de fortes variations. En 1972, le temps parental des mères au foyer est supérieur d'une heure à celui des mères actives. Les hommes ont un temps parental d'une heure quand leur conjointe est inactive et de $2 \mathrm{~h} 10$, soit près du double, quand elle est active.

Tableau 41 Temps parental hebdomadaire des femmes actives, inactives et des hommes, en heures et minutes

\begin{tabular}{|c|c|c|c|c|c|}
\hline Temps parental & $\begin{array}{c}\text { IZS } \\
1965-1966\end{array}$ & $\begin{array}{c}\text { Zander } \\
1972\end{array}$ & $\begin{array}{l}\text { Pross } \\
1973\end{array}$ & $\begin{array}{c}\text { Schoeps } \\
1974\end{array}$ & $\begin{array}{l}\text { Deist } \\
1976\end{array}$ \\
\hline Temps parental des femmes au foyer & 10h 55 & $6 h 30$ & $21 \mathrm{~h}$ & - & $12 \mathrm{~h}$ \\
\hline Temps parental des femmes actives & $8 \mathrm{~h} 20$ & $5 h 40$ & - & $17 \mathrm{~h}$ & - \\
\hline Temps parental des hommes & $1 \mathrm{~h} 50$ & \begin{tabular}{l|l}
$1 \mathbf{h}^{(1)}$ & $\mathbf{2 h} \mathbf{1 0}$ \\
$(2)$
\end{tabular} & & & \\
\hline
\end{tabular}

${ }^{(1)}$ Hommes dont la femme est inactive

(2) Hommes dont la femme est active

Source : d'après Kettschau (1980 : 113-115)

La participation des hommes, lorsque la femme est au foyer, s'est maintenue, voire a légèrement diminué entre 1965 et 1972 (cf. Tableau 42).

Par contre, la participation des hommes a pratiquement doublé lorsque la femme est active : de $21,7 \%$ en 1965 à $38,6 \%$ en 1972 . Le statut professionnel de la femme fait varier la participation des hommes aux tâches parentales qui, en 1972, approche $40 \%$ du travail parental féminin.

Tableau 42 Temps parental des hommes en pourcentage du temps parental des femmes

\begin{tabular}{|l|c|c|}
\hline \multirow{2}{*}{} & \multicolumn{2}{|c|}{ PARTICIPATION DES HOMMES } \\
\cline { 2 - 3 } & Femme au foyer & Femme active \\
\hline $\begin{array}{l}\text { Activités parentales } \\
\text { Enquête IZS 1965-66 }\end{array}$ & $\mathbf{1 6 , 6 \%}$ & $\mathbf{2 1 , 7 \%}$ \\
\hline $\begin{array}{l}\text { Activités parentales } \\
\text { Enquête Zander 1972 }\end{array}$ & $\mathbf{1 5 , 4 \%}$ & $\mathbf{3 8 , 6 \%}$ \\
\hline
\end{tabular}

Source : d'après Kettschau (1980 : 118-120)

La proportion entre les activités parentales et les activités domestiques reste relativement constante pour les pères et les mères (cf. Tableau 43). En 1965, la part de

\footnotetext{
${ }^{190}$ Mais déjà dans les années 1970, la recherche internationale «enquête budget temps» a construit la catégorie de sociabilité à partir du temps consacré aux enfants (lecture discussion, activités de jeu à l'intérieur comme à l'extérieur, promenade).
} 
l'activité parentale par rapport à l'ensemble des tâches ménagères équivaut à $16,5 \%$ pour les hommes et à 17,4\% pour les femmes, qu'elles soient actives ou inactives.

En 1972, dans le cas où la femme est au foyer, les hommes participent plus aux activités domestiques qu'aux activités parentales. Par contre, les activités parentales prennent une plus grande importance pour les pères lorsque la femme est active : ils consacrent un quart de leur temps ménager aux activités parentales.

Tableau 43 Proportions relatives des activités domestiques et parentales, en \%, en fonction de la situation professionnelle

\begin{tabular}{|c|c|c|c|c|c|c|c|c|c|}
\hline \multirow[b]{2}{*}{ ACTIVITES } & \multicolumn{3}{|c|}{ IZS 1965-66 } & \multicolumn{4}{|c|}{ Zander 1972} & \multicolumn{2}{|c|}{\begin{tabular}{|l|} 
Pross/Schoeps \\
$1973-74$ \\
\end{tabular}} \\
\hline & $\begin{array}{l}\text { Femme } \\
\text { au foyer }\end{array}$ & $\begin{array}{l}\text { Femme } \\
\text { active }\end{array}$ & Homme & $\begin{array}{l}\text { Femme } \\
\text { au foyer }\end{array}$ & $\begin{array}{c}\text { Femme } \\
\text { active }\end{array}$ & $\begin{array}{l}\text { Hommes } \\
\text { /femme } \\
\text { au foyer }\end{array}$ & $\begin{array}{c}\text { Hommes } \\
\text { /femme } \\
\text { active }\end{array}$ & $\begin{array}{c}\text { Femme } \\
\text { au } \\
\text { foyer }\end{array}$ & $\begin{array}{c}\text { Femme } \\
\text { active }\end{array}$ \\
\hline $\begin{array}{l}\text { Activités } \\
\text { parentales }\end{array}$ & 17,4 & 17,4 & 16,5 & 13,5 & 14,4 & 13,7 & 25 & 29,4 & 30,1 \\
\hline $\begin{array}{l}\text { Activités } \\
\text { domestiques }\end{array}$ & 82,6 & 82,6 & 83,5 & 86,5 & 85,6 & 86,3 & 75 & 70,6 & 69,9 \\
\hline Total & $100 \%$ & $100 \%$ & $100 \%$ & $100 \%$ & $100 \%$ & $100 \%$ & $100 \%$ & $100 \%$ & $100 \%$ \\
\hline
\end{tabular}

Source : d'après Kettschau (1980 : 118-120)

L'activité féminine provoque une réorganisation et une réaffectation des temps domestiques sur certains postes. Mais c'est le rapport entre le domestique et le parental qui change profondément, ce dernier prend une part plus importante pour l'homme quand la femme est active.

Le temps domestique des femmes au foyer est d'environ 51 heures, et celui des femmes actives de 39 heures 30 (cf. Tableau 44). Ces chiffres montrent qu'il n'y a pas eu de grandes évolutions entre 1965 et 1974 et que le temps domestique est resté constant. La répartition des tâches à l'intérieur du travail domestique prend la forme suivante : le temps maximum est consacré au ménage, puis à la cuisine et aux activités annexes, au linge et aux activités de bricolage, jardinage..., enfin aux courses. Cette répartition des tâches se maintient quels que soient les relevés des différentes enquêtes et quelle que soit la situation professionnelle de la femme.

En 1965, les femmes au foyer consacrent 18 heures 20 minutes au ménage contre 14 heures pour les femmes actives (cf. Tableau 44). La cuisine ${ }^{191}$ représente un poste qui s'élève à 15 heures 20 minutes pour les femmes au foyer et à 11 heures $10 \mathrm{mn}$ pour les femmes actives. Quant au linge ${ }^{192}$, les femmes au foyer lui consacrent 7 heures 20, les femmes actives

\footnotetext{
${ }^{191}$ Sur l'ensemble des tâches domestiques, la cuisine est considérée comme l'activité la plus agréable par l'ensemble des femmes (Kettschau 1980 : 135). Cette tâche est associée à une moindre fatigue corporelle; elle est créative et en lien direct avec un produit concret. Selon Kettschau, elle concentre les responsabilités de la femme (et de la mère) pour le bien être physique et psychique des membres de la famille; elle reçoit une reconnaissance directe par rapport au travail fourni et visible.

192 «Le repassage est une tâche ressentie comme une corvée, dans l'ensemble des enquêtes, il est l'activité la moins appréciée. Et s'il n'apparaît pas en première place, il se situe après faire la vaisselle et le nettoyage» (Kettschau 1980 : 137).
} 
6 heures 30. Si on compare ces chiffres avec les années 1973-1974, on s'aperçoit que le changement le plus important concerne le linge puisque les femmes au foyer ne lui consacrent plus que 4 heures 30 et les femmes actives 4 heures.

Tableau 44 Temps des femmes inactives et actives consacré aux taches domestiques en heures et minutes

\begin{tabular}{|c|c|c|c|c|c|c|}
\hline \multirow[b]{2}{*}{ ACTIVITES } & \multicolumn{2}{|c|}{ IZS 1965-66 } & \multicolumn{2}{|c|}{ Zander 1972} & \multicolumn{2}{|c|}{$\begin{array}{l}\text { Pross/Schoeps } \\
1973-74\end{array}$} \\
\hline & $\begin{array}{l}\text { Femmes } \\
\text { au foyer }\end{array}$ & $\begin{array}{c}\text { Femmes } \\
\text { actives }\end{array}$ & $\begin{array}{l}\text { Femmes } \\
\text { au foyer }\end{array}$ & $\begin{array}{c}\text { Femmes } \\
\text { actives }\end{array}$ & $\begin{array}{l}\text { Femmes } \\
\text { au foyer }\end{array}$ & $\begin{array}{c}\text { Femmes } \\
\text { actives }\end{array}$ \\
\hline Ménage & 18h 20 & $14 \mathrm{~h}$ & $15 \mathrm{~h}$ & $12 \mathrm{~h} 50$ & $28 h^{(193)}$ & $19 \mathrm{~h}$ \\
\hline Cuisine et activités annexes & 15h 20 & 11h 10 & $11 \mathrm{~h}$ & $9 h 55$ & $15 \mathrm{~h}$ & $16 \mathrm{~h} 30$ \\
\hline Linge & 7 h 20 & $6 h 30$ & $5 \mathrm{~h} 10$ & $4 \mathrm{~h}$ & $4 h 30$ & $4 h$ \\
\hline Autres & $6 \mathrm{~h} 00$ & $3 \mathrm{~h} 40$ & $5 \mathrm{~h} 10$ & $2 \mathrm{~h} 50$ & - & - \\
\hline Courses & 4h 10 & $3 h 45$ & $3 h 50$ & $3 \mathrm{~h} 30$ & $3 \mathrm{~h}$ & \\
\hline Activités de planification & - & - & 1h 20 & Oh 50 & - & - \\
\hline Total $^{(*)}$ du temps domestique & $51 \mathrm{~h} 10$ & $39 \mathrm{~h} 20$ & $41 \mathrm{~h} 30$ & $33 \mathrm{~h} 50$ & $50 \mathrm{~h} 30$ & $39 \mathrm{~h} 30$ \\
\hline
\end{tabular}

- non évalué

${ }^{(*)}$ Les totaux ont été effectués avec les valeurs non arrondies des différentes activités. Pour cette raison, le total peut ne pas être strictement égale à la somme des temps pour chaque activité.

Source : Kettschau (1980 : 122)

Le tableau 45 indique la part du temps consacré à chaque poste d'activité par rapport au temps domestique total. Un des résultats principaux du tableau est de montrer la réduction constante du temps consacré au linge, au cours des années 1960 et 1970. Les activités de cuisine et de ménage restent relativement égales et les courses ont légèrement augmenté entre 1965 et 1972.

Par ailleurs, on a une inversion des postes de travail entre celui du ménage et celui de la cuisine entre les femmes de l'ancienne Allemagne de l'Est et celle de l'Ouest. Par contre, le temps consacré au linge est proportionnellement le même : on retrouve le chiffre de $16,5 \%$ en 1965 (ce qui correspond à la part consacré par les femmes actives de l'Allemagne de l'Ouest).

\footnotetext{
${ }^{193}$ Dans cette enquête, les activités de bricolage, de jardinage etc... se retrouvent dans la catégorie «ménage» et «entretien de la maison» et non pas dans une catégorie «autre», ce qui explique le fort décalage des chiffres.
} 
Tableau 45 Part du temps consacré à chaque tâche domestique par les femmes inactives et actives, en \%.

\begin{tabular}{|l|c|c|c|c|c|c|}
\hline \multirow{2}{*}{\multicolumn{2}{c|}{ ACTIVITES }} & \multicolumn{2}{l|}{ IZS 1965-1966 } & \multicolumn{2}{l|}{ Zander 1972 } & \multicolumn{2}{l|}{$\begin{array}{l}\text { Pross/Schoeps } \\
\text { 1973-1974 }\end{array}$} \\
\cline { 2 - 7 } & $\begin{array}{c}\text { Femmes } \\
\text { au foyer }\end{array}$ & $\begin{array}{c}\text { Femmes } \\
\text { actives }\end{array}$ & $\begin{array}{c}\text { Femmes } \\
\text { au foyer }\end{array}$ & $\begin{array}{c}\text { Femmes } \\
\text { actives }\end{array}$ & $\begin{array}{c}\text { Femmes } \\
\text { au foyer }\end{array}$ & $\begin{array}{c}\text { Femmes } \\
\text { actives }\end{array}$ \\
\hline Ménage & 35,7 & 36,1 & 36,1 & 37,9 & 55,4 & 48,1 \\
\hline $\begin{array}{l}\text { Cuisine } \\
\text { et activités annexes }\end{array}$ & 29,9 & 28,5 & 26,5 & 29,3 & 29,7 & 41,8 \\
\hline Linge & $\mathbf{1 4 , 3}$ & $\mathbf{1 6 , 5}$ & $\mathbf{1 2 , 5}$ & $\mathbf{1 1 , 8}$ & $\mathbf{8 , 9}$ & $\mathbf{1 0 , 1}$ \\
\hline Autres & 11,9 & 9,4 & 12,5 & 8,3 & - & - \\
\hline Courses & 8,2 & 9,4 & 9,2 & 10,4 & 5,9 & - \\
\hline Activités de planification & - & - & 3,1 & 2,4 & - & - \\
\hline Total du temps domestique & $100 \%$ & $100 \%$ & $100 \%$ & $100 \%$ & $100 \%$ & $100 \%$ \\
\hline
\end{tabular}

Source : d'après Kettschau $1980: 122$

En Allemagne de l'Ouest, la situation de la femme au foyer est discutée par rapport au travail professionnel. Le temps qu'elles passent à effectuer les tâches domestiques et parentales, est comparativement plus important que celui des femmes actives. Or, le temps des femmes actives pour remplir ces tâches est utilisé comme une référence maximum du temps nécessaire pour les effectuer. Il s'agit donc là d'une interprétation sur l'efficience et la productivité du travail domestique des femmes au foyer. Cette discussion a d'autant plus de sens qu'une fraction du mouvement féministe en Allemagne de l'Ouest revendique une rémunération de ce travail. De fait, sa quantification s'effectue à partir des critères du travail professionnel et salarié.

A la même période, en Allemagne de l'Est (cf. Encadré n ${ }^{\circ} 10$ ), les chiffres sont interprétés différemment. On part du principe que les femmes actives cumulent un double temps de travail professionnel et domestique. Le référent implicite est l'activité professionnelle de la femme, et de sa double charge de travail. Dans ce cas, il manque un temps nécessaire aux femmes actives et les activités domestiques sont effectuées plus rapidement (ou non effectuées).

Ainsi, la femme au foyer est au centre de la norme dans l'ancienne allemangne de l'Ouest et la femme active au centre de l'analyse dans l'ancienne Allemagne de l'Est.

Le tableau 46 présente les temps absolus (en heures et minutes) et relatifs (en \%), consacrés par les hommes aux différentes activités domestiques, en fonction de la situation professionnelle de la femme. Le temps destiné au bricolage, au jardinage, mais aussi aux activités de correspondance avec les administrations, de gestion et de tenue des comptes, représente 6 heures 50 soit 74,7\% de leur temps domestique global. Les activités liées aux courses et au ménage ( 7 et $11 \%$ ) occupent la seconde place dans l'emploi du temps des 
hommes, soit un volume horaire d'une 1 heure. La cuisine est une activité «résiduelle» (moins d'une demi-heure). Quant au linge, cette activité est pratiquement inexistante dans l'emploi du temps des hommes.

La participation des hommes aux différentes activités domestiques varie en fonction de la situation professionnelle des femmes. Sa participation, aux catégories «autres» et «ménage», reste constant en temps absolu mais baisse légèrement proportionnellement aux autres activités lorsque la femme est au foyer. Par contre, sa participation devient plus importante au niveau de la cuisine, 7,7\% lorsque la femme est au foyer et 18,2\% lorsqu'elle est active. Sa participation aux courses est également plus importante avec 9,5\%, et 15,2\% lorsqu'elle est active. Enfin, sa participation aux activités de planification, de correspondance et de gestion diminue lorsque la femme est active. L'activité professionnelle de la femme change le profil de la participation des hommes, qui, tout en restant typiquement masculine, l'est moins dans le cas où la femme est active : moins d'activités d'administration et plus d'activités domestiques.

Tableau 46 Temps absolus (en heures et minutes) et relatifs (en \%) consacrés par les hommes aux différentes activités domestiques en fonction de la situation professionnelle de la femme

\begin{tabular}{|c|c|c|c|c|c|c|}
\hline \multirow{4}{*}{$\begin{array}{c}\text { ACTIVITES } \\
\text { Autres }\end{array}$} & \multirow{3}{*}{\multicolumn{2}{|c|}{$\begin{array}{l}\text { IZS 1965-66 } \\
\text { Participation des } \\
\text { hommes }\end{array}$}} & \multirow{2}{*}{\multicolumn{4}{|c|}{$\begin{array}{l}\text { Zander } 1972 \\
\text { Participation des hommes selon la situation } \\
\text { professionnelle de leur femme }\end{array}$}} \\
\hline & & & & & & \\
\hline & & & \multicolumn{2}{|c|}{ femme au foyer } & \multicolumn{2}{|c|}{ femme active } \\
\hline & $6 \mathrm{~h} 50^{(194)}$ & 74,7 & $2 \mathrm{~h} 50$ & 44,4 & $2 \mathrm{~h} 50$ & 42,4 \\
\hline Courses & $1 \mathrm{~h} 00$ & 11,0 & Oh 35 & 9,5 & $1 \mathrm{~h} 00$ & 15,2 \\
\hline Ménage & Oh 40 & 7,7 & $1 \mathrm{~h} 00$ & 15,9 & Oh 55 & 13,6 \\
\hline Cuisines & Oh 25 & 4,4 & Oh 30 & 7,9 & 1h 10 & 18,2 \\
\hline Linge & Oh 10 & 2,2 & Oh 00 & 0,0 & Oh 00 & 0,0 \\
\hline $\begin{array}{c}\text { Activités } \\
\text { d'administration }\end{array}$ & - & - & 1h 25 & 22,2 & Oh 40 & 10,6 \\
\hline Total & $9 \mathrm{~h} 05$ & $100 \%$ & $6 h 20$ & $100 \%$ & $6 h 35$ & $100 \%$ \\
\hline
\end{tabular}

- non évalué

Source : Kettschau 1980:118-120

\footnotetext{
${ }^{194}$ Cette valeur associe les catégories «autres» et «activités de planification» de l'enquête Zander.
} 
Encadré $\mathrm{n}^{\circ} 10 \quad$ Le budget temps en DDR dans les années 1970

Dans l'enquête budget temps effectuée en Allemagne de l'Est en 1970, le travail parental relevait à la fois du domestique et du temps de libre (pour les activités liées à la socialisation et à l'éducation) (Lippold $171: 42-43$ ).

En 1965 , le temps total consacré aux activités domestiques équivaut à $4 \mathrm{~h}$ par jour (calculé sur l'ensemble des individus) et à 5,5 h pour les femmes. Elles consacrent $32,7 \%$ de leur temps total à la préparation des repas et $25,5 \%$ au ménage, tandis que l'inverse est vrai sur l'ensemble des individus : le ménage occupe le premier poste d'activité avec 32,5\%, suivit de la préparation des repas avec $27,5 \%$. A cette différence près, la structure de la répartition des tâches ne varie pas de façon significative. En revanche, le temps attribué à chaque activité est plus important pour les femmes (cf. Annexe 15).

Pour évaluer la participation des hommes, on se référera aux résultats de l'enquête internationale «emploi du temps» de 1965-1966. Dans le cas de l'ancienne Allemagne de l'Est, les hommes, qu'ils soient mariés ou non, effectuaient $20 \%$ du temps global consacré à l'ensemble des tâches domestiques (Lippold 1971 : 115).

A la fin des années 1960, les femmes de l'Allemagne de l'Est revendiquent, dans un discours égalitaire et d'émancipation, la nécessité de réduire le travail domestique. La promotion de l'égalité entre les sexes, composante forte de l'idéologie socialiste, passait par un ensemble de conditions qui devaient permettre aux femmes de participer à la vie sociale et professionnelle. En ce sens, la société devait leur faciliter l'accès au marché du travail. La conciliation entre famille et activité professionnelle relevait certes du domaine privé mais était également un enjeu social. Dans ce contexte, la société socialiste s'intéressait donc à la répartition des tâches au sein du ménage ${ }^{195}$ et envisageait certaines alternatives, comme la collectivisation des tâches ménagères. En discutant le partage des activités au sein des familles, l'idéologie socialiste souhaite corriger ce qu'elle appelait «l'héritage du passé»: en d'autres termes, soustraire le partage des tâches au processus de reproduction sociale, héritée du modèle bourgeois de l'ancienne Allemagne de l'Ouest (et de sa répartition sexuée des tâches).

\footnotetext{
${ }^{195}$ D'où la préoccupation, dans les régimes socialistes, d'une connaissance approfondie des emplois du temps des individus. De fait, un relevé des emplois du temps, lors d'enquêtes représentatives, a eu lieu, pendant la période socialiste, à intervalle régulier.
} 


\section{SOUS-SECTION III.B.2 LA PARENTALITE ENTRE DOMESTIQUE ET PROFESSIONNEL (1990 ET 2000)}

\section{III.B.2.1 Le partage des tâches parentales}

L'ensemble du travail parental étudié ici comprend 17 activités, dont le mode de répartition est consigné dans la Figure 20. Le nombre de tâches effectuées principalement par la mère est supérieur à celles effectuées exclusivement par le père. Il ne s'occupe jamais seul des activités de soins et de «travail parental domestique», tandis que la mère les prend en charge dans leur ensemble. Statistiquement, moins d'un père sur deux effectue seul une activité parentale (les autres ne prennent aucune activité à leur charge) ${ }^{196}$. Globalement, la mère exécute seule 9,1 activités en moyenne. C'est exactement la même proportion d'activités qui est exécutée conjointement par les deux parents.

L'ensemble des activités de jeux et de sociabilité est effectué en majeure partie par les deux parents. Ces activités, plus ludiques, sont celles pour lesquelles la participation des hommes est la plus importante. Pour les activités parentales domestiques et de soins, le père a essentiellement une fonction de soutien, plus que de prise en charge. Enfin, l'engagement du père est très faible pour les activités sans contact direct avec l'enfant.

\footnotetext{
${ }^{196}$ Les hommes surévaluent légèrement l'engagement masculin et partenarial (et sous-évaluent la participation féminine). Ils confirment que les tâches parentales pour les enfants en dessous de trois ans sont exécutées essentiellement par la mère (LBS $2000: 8$ ).
} 
Figure 20 Qui s'occupe du parental ?

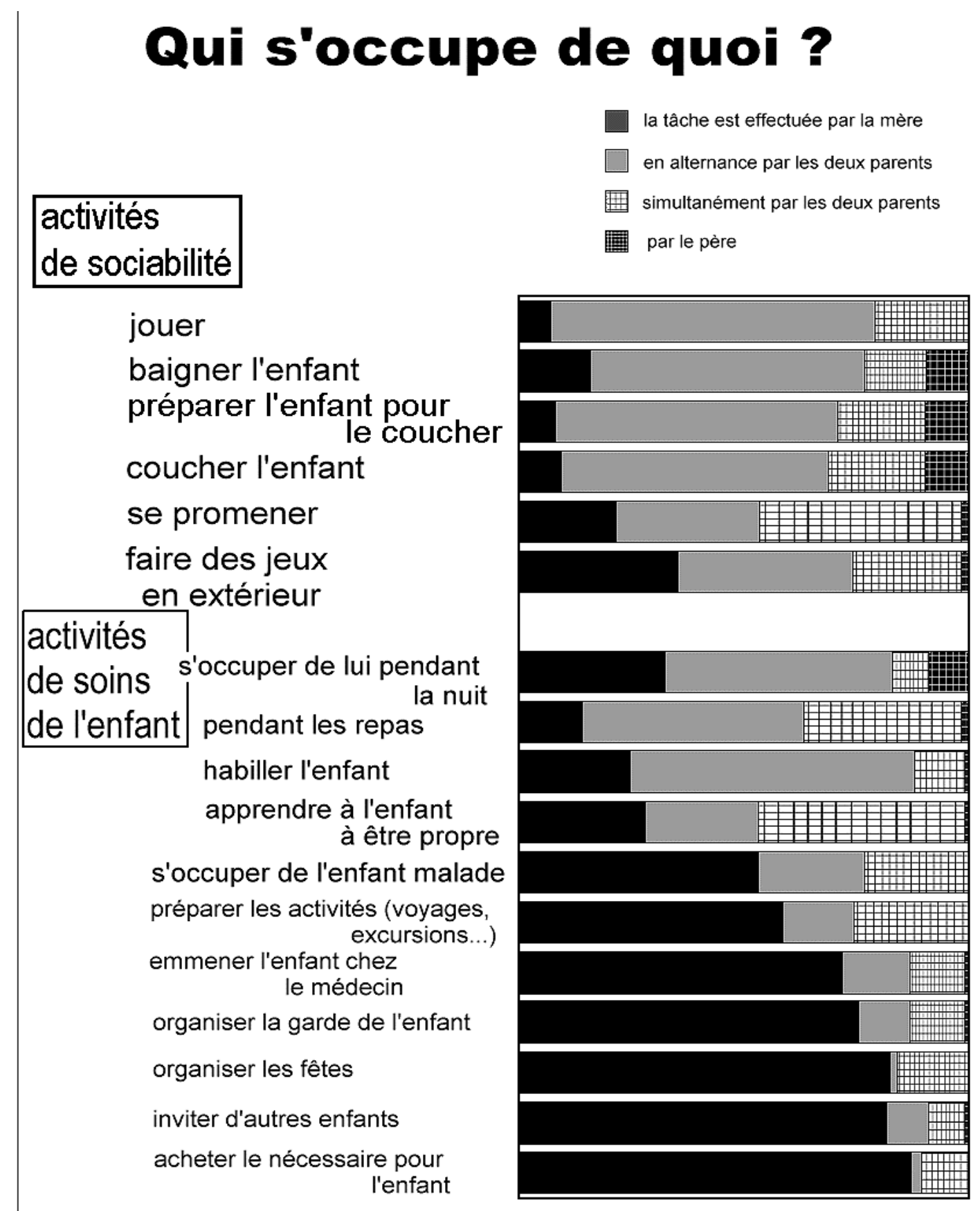

Source : LBS «Familien Studie Übergang zur Elternschaft» $(2000: 10)$ 
La participation du père change en fonction de l'âge et du développement de l'enfant (cf. Tableau 47).

Lorsque l'enfant est plus âgé, le nombre d'activités partagées entre le père et la mère augmente, et ce, pour presque toutes les tâches. De nouveaux domaines de participation apparaissent, en particulier liés à l'alimentation (et à la fin de l'allaitement), mais aussi au bain ou aux soins corporels (LBS 1998b). La tâche pour qui le «mode de partage» à le plus augmenté entre la naissance et les 18 mois de l'enfant, concerne l'aide pendant les repas de l'enfant. Seules les tâches «s'informer sur l'éducation et le développement de l'enfant» et «acheter le nécessaire pour l'enfant», deviennent plus inégalitaires en défaveur des femmes (la proportion du «mode partagé» diminue).

Tableau 47 Evolution du partage des tâches parentales $\left(^{*}\right)$

\begin{tabular}{|l|c|c|c|c|c|c|c|c|}
\hline \multirow{2}{*}{ LES TACHES PARENTALES } & \multicolumn{3}{|l|}{4 MOIS APRES LA NAISSANCE } & \multicolumn{3}{l|}{18 MOIS APRES LA NAISSANCE } \\
\cline { 2 - 11 } & la mère & les deux & le père & total & la mère & les deux & le père & total \\
\hline Jouer avec l'enfant & 4 & 95 & 1 & 100 & 5 & 94 & 1 & 100 \\
\hline Le coucher & 27 & 72 & 1 & 100 & 25 & 71 & 4 & 100 \\
\hline Le baigner & 26 & $\mathbf{6 5}$ & 9 & 100 & 17 & $\mathbf{8 1}$ & 2 & 100 \\
\hline Le changer & 39 & $\mathbf{6 0}$ & 1 & 100 & 24 & $\mathbf{7 6}$ & 0 & 100 \\
\hline L'habiller & 51 & 48 & 1 & 100 & 48 & 51 & 0 & 100 \\
\hline $\begin{array}{l}\text { Organiser la garde de } \\
\text { l'enfant }\end{array}$ & 54 & $\mathbf{4 6}$ & 0 & 100 & 70 & $\mathbf{3 0}$ & 0 & 100 \\
\hline $\begin{array}{l}\text { S'informer sur l'éducation } \\
\text { et le développement de } \\
\text { l'enfant }\end{array}$ & 56 & 44 & 0 & 100 & $\mathbf{6 3}$ & 37 & 0 & 100 \\
\hline $\begin{array}{l}\text { S'occuper de l'enfant } \\
\text { pendant ses repas }\end{array}$ & 64 & $\mathbf{3 5}$ & 1 & 100 & 21 & $\mathbf{8 0}$ & 0 & 100 \\
\hline $\begin{array}{l}\text { Accompagner l'enfant } \\
\text { chez le médecin }\end{array}$ & 66 & 34 & 0 & 100 & 71 & 29 & 0 & 100 \\
\hline $\begin{array}{l}\text { Acheter le nécessaire pour } \\
\text { l'enfant }\end{array}$ & 71 & 29 & 0 & 100 & $\mathbf{8 1}$ & 19 & 0 & 100 \\
\hline $\begin{array}{l}\text { S'occuper de l'enfant } \\
\text { pendant la nuit }\end{array}$ & 79 & $\mathbf{2 0}$ & 1 & 100 & 54 & $\mathbf{4 3}$ & 2 & 100 \\
\hline
\end{tabular}

(*) proportion de couples pour lesquels la femme a la responsabilité principale de la tâche, les deux partenaires à part égale, et les couples où l'homme a l'entière responsabilité de la tâche (en \%)

Source : d'après les résultats de l'enquête LBS-Familien-Studie „Übergang zur Elternschaft“, 1998b. 
Encadré $\mathrm{n}^{\circ} 11 \quad$ Note méthodologique sur la mesure du travail domestique et parental dans les années 1990 et 2000, en Allemagne

Dans l'ancienne Allemagne de l'Ouest, le travail parental n'est pas constituée comme une catégorie de la connaissance. Mais une recomposition de l'étude de Blättel-Mink, Kramer et Mischau (2000), a permis de faire émerger les catégories de «sociabilité parentale», de tâches parentales «taxi» et «domestiques» permettant une comparaison quasiment terme à terme avec les données françaises du groupe DFT-MATISSE.

Par ailleurs, il existe une difficulté de traduction de la notion de «temps parental» vers l'allemand. En effet, une traduction littérale par «Elternzeit» n'est pas possible car elle désigne la nouvelle mesure de congé parental depuis 2001. Aussi, nous proposons d'utiliser le terme de «Kinderzeit» («le temps de l'enfant»).

Nous proposons également d'utiliser la notion d' «elterliche Arbeit» pour rendre compte de la notion de «travail parental». Cette expression permet de souligner l'idée de travail, centrale dans le contexte du «travail familial».

Les données du projet «Soziale Lage und Lebensführung von Frauen - eine vergleichende regionale Analyse in zwei Landkreisen Baden-Württembergs» (Blättel-Mink ; Kramer ; Mischau 2000 : 25) permet une comparaison quasiment terme à terme avec l'étude française sur le temps parental du groupe Division Familiale du Travail (Barrère-Maurisson ; Marchand ; Rivier $2000^{\circ}$ ). L'ensemble des activités parentales se regroupe en trois catégories: la première regroupe les travaux effectués pour l'enfant (équivalent au «travail parental domestique» de l'enquête française); la deuxième concerne les trajets effectués avec l'enfant (équivalence au «travail parental taxi») et la troisième catégorie concerne toutes les occupations faites en présence de l'enfant, par exemple, se promener, jouer, consoler (activités de «sociabilité parentale»).

$83,1 \%$ des femmes déclarent prendre à leur charge les tâches parentales domestiques, $14 \%$ déclarent les partager à part égale avec leur partenaire et $2 \%$ indiquent que l'homme les fait seul (cf. Figure 21). Le temps parental «taxi» est également réparti de façon très inégalitaire : $76,7 \%$ des femmes prennent en charge cette activité ; 20,3\% partagent à part égale avec leur partenaire et $2 \%$ des femmes déclarent que c'est l'homme qui l'effectue.

Un autre profil se dessine dans le cas du travail parental de sociabilité : les femmes prennent en charge ces activités dans la moitié des cas (47, 9\%). Pour l'autre moitié (49,5\%), les deux partenaires se partagent la responsabilité à part égale; et l'homme effectue seul ces tâches dans 1,6\% des cas. C'est donc pour les activités de jeux, de sociabilité et de relationnel, que l'on voit apparaître une pratique majoritaire de partage égalitaire entre les parents, comme en France; et la participation masculine se concentre essentiellement sur les activités de sociabilité. 
Figure $21 \quad$ Les modes de partage du travail parental, en Allemagne

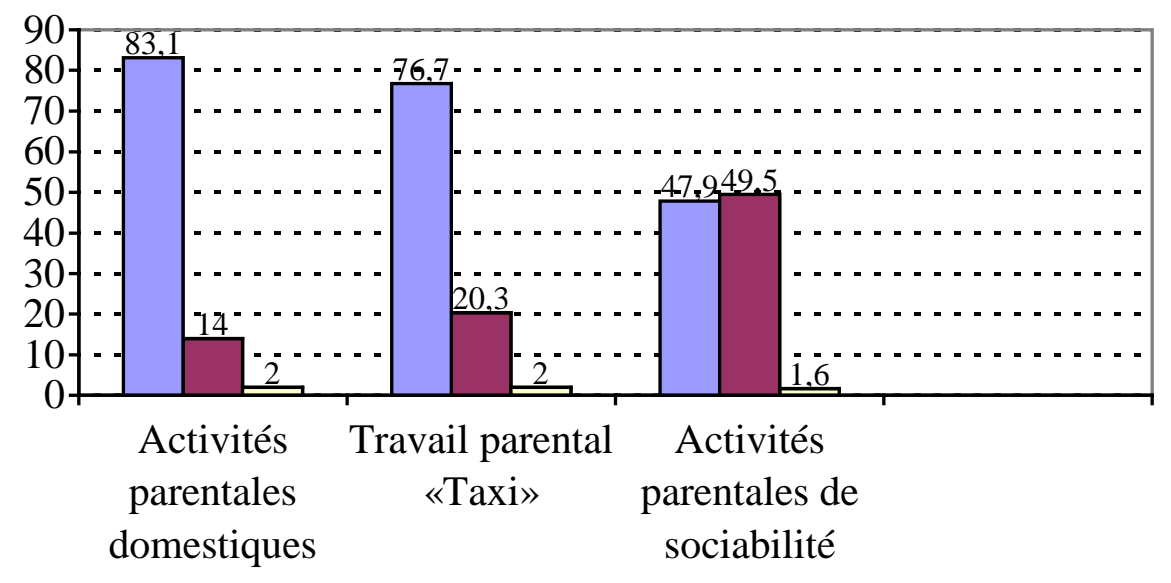

$\square$ principalement la femme $\square$ partage égalitaire $\square$ principalement l'homme

Source : Blättel-Mink ; Kramer ; Mischau (2000 : 25)

Le graphique suivant indique les types de partage du travail parental que les mères souhaiteraient dans leur couple (cf. Figure 22 ).

$55,7 \%$ des femmes souhaitent un partage égalitaire du «travail parental domestique», tandis que 39,9\% souhaitent s'en occuper exclusivement. Dans le cas du «travail parental taxi» : 60,5\% des femmes s'expriment pour un partage de la tâche et plus d'un tiers $(35,4 \%)$ pour leur responsabilité exclusive. Enfin $81,3 \%$ des femmes veulent un partage égalitaire des tâches de «sociabilité parentale» et $14,6 \%$ souhaitent s'en occuper seules. Il y a un fort consensus au niveau des représentations féminines sur un partage égalitaire du «travail parental de sociabilité».

Figure 22 Les souhaits des femmes relatifs aux modes de partage du travail parental

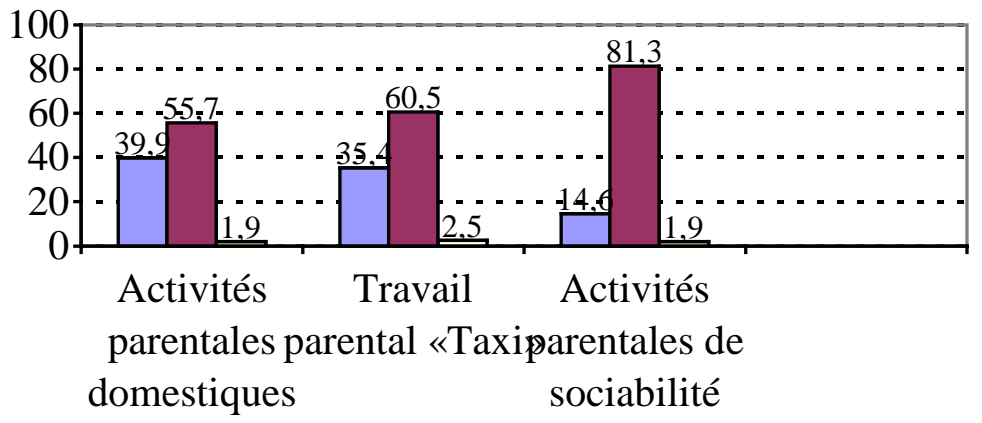

$\square$ principalement la femme $\square$ partage égalitaire $\square$ principalement l'homme

Source : Blättel-Mink ; Kramer ; Mischau 2000 : 25

Quant à la participation exclusive des hommes sur les tâches parentales, elles n'excédent pas $3 \%$ des cas. En pratique, suivant les déclarations des femmes, $1,5 \%$ et $2 \%$ 
des pères s'occupent seuls de certaines activités parentales(cf. Figure $21, \S$ précédent). C'est dans la même proportion que les femmes souhaitent effectivement donner la pleine responsabilité de ces tâches à l'homme ( 2 et 2,5\%) (cf. Figure 22 ci-dessus). La délégation exclusive au partenaire n'est ni pratiquée ni souhaitée, les écarts sont inférieurs à 0,5 points. Il existe donc une adéquation entre les pratiques déclarées par les femmes et leurs représentations.

Globalement, la majorité des femmes revendique un partage plus égalitaire du travail parental, quelle que soit la tâche. Mais ce n'est pas pour les activités qu'elles ont majoritairement à leur charge, qu'elles revendiquent le plus un partage égalitaire. Au contraire, elles souhaitent renforcer le partage dans le cas de la «sociabilité parentale», alors qu'il est déjà quasiment majoritaire dans les couples. A l'inverse, elles le revendiquent peu pour les «tâches parentales domestiques» (quelles effectuent à 83\%). C'est aussi pour ces tâches que la part de celles qui souhaitent exclusivement s'en occuper seules est la plus forte $^{197}$.

En d'autres termes, plus l'activité est inégalement partagée, plus la proportion des femmes qui s'occupent seules de ces tâches est importante, et plus le pourcentage de femmes qui revendiquent ces tâches pour elles-mêmes est important. Inversement, plus le partage des tâches entre les deux partenaires est égalitaire, plus la proportion de femmes qui revendiquent ce partage augmente.

La norme égalitaire, comme mode de partage des tâches parentales, est consensuelle, mais les résistances sont encore fortes envers les tâches parentales domestiques et «taxi». Le domestique continue donc à être un référent important pour l'identité féminine et explique ces résistances importantes face au partage. En revanche, les domaines du relationnel et de la sociabilité ont tendance à être plus partagés.

Deux logiques différentes de partage des tâches parentales se côtoient : la première fait référence à un partage traditionnel des tâches, à la polarisation des rôles. Elle s'observe dans l'orientation du travail des femmes pour le domestique. La seconde logique des rapports parentaux, plus égalitaire, s'exprime dans la mixité d'exécution des tâches relationnelles et de sociabilité. On observe donc une polarisation des modes de partage en fonction de la nature des tâches et des logiques de genre (cf. Figure 23).

\footnotetext{
${ }^{197}$ Cette tendance est encore plus visible à travers les réponses des femmes qui effectuent exclusivement les tâches «parentales donmestiques» (et non plus sur l'ensemble des femmes). 49,6\% d'entre elles souhaitent maintenir l'exclusivité féminine, $48 \%$ préfèrent un partage égalitaire. Moins de la moitié des femmes souhaitent un partage partenarial. Dans le cas du «temps parental taxi», ces dernières deviennent majoritaires avec 51,1\% contre $47,6 \%$ qui souhaitent exercer seules ces tâches. La norme égalitaire est largement dominante pour les «tâches de sociabilité parentale»: 67,6\% souhaitent un partage égalitaire, contre 29,8\% qui préfèrent maintenir le rôle prépondérant de la mère. $2,8 \%$ des femmes préfèrent déléguer ce travail à l'extérieur plutôt qu'à l'intérieur de la famille en particulier au partenaire (cette part ne représente que 0,7\%) (Blättel-Mink ; Kramer ; Mischau 2000).
} 
Figure $23 \quad$ Les logiques de partage du travail parental

\begin{tabular}{|c|}
\hline MODE \\
D'EXECUTION : \\
\hline
\end{tabular}

La mère
a l'exclusivité
d'exécution

Les deux parents

se partagent

les tâches

$$
\begin{gathered}
\text { partage traditionnel } \\
\text { des tâches en } \\
\text { fonction du sexe : la } \\
\text { «logique du } \\
\text { domestique» }
\end{gathered}
$$

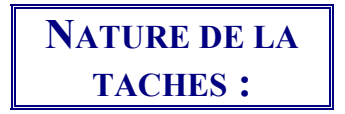

Tâches parentales à orientation domestique

Tâches parentales de sociabilité et de nature relationnelle

\section{RAPPORTS \\ PARENTAUX :}

Rapports

parentaux

inégalitaires
Partage

égalitaire
Parité

parentale

\author{
Le père \\ a l'exclusivité \\ d'exécution
}

L'activité professionnelle de la femme modifie le partage du parental dans le couple. Lorsque la mère travaille, les tâches parentale domestiques sont prises en charge par la femme à 46,7\%, elles sont partagées à part égale entre les deux partenaires dans 36,7\% des cas, l'homme effectue principalement cette activité dans $10 \%$ des cas, et les tâches sont déléguées dans $3,3 \%$ des cas.

Lorsque la femme est active à temps partiel ou au foyer, la participation des hommes diminue. La part du partage égalitaire, par rapport aux autres modes de partage est plus faible : 17,2\% lorsque la femme travaille à temps partiel, contre 8,9\% lorsqu'elle est au foyer. Ces dernières ont l'exclusivité des tâches dans 88,7\% des cas (Blättel-Mink ; Kramer ; Mischau $2000: 28$ ).

Plus la femme est présente sur le marché du travail, plus les tâches parentales domestiques sont déléguées au père (qui les effectue seul dans $13,8 \%$ des cas) ${ }^{198}$.

L'impact de l'âge varie de façon linéaire : plus la femme est âgée, plus la tendance à un mode de partage égalitaire augmente. Ceci s'explique par un changement de structure des besoins de l'enfant, où le temps parental domestique diminue au profit du «temps parental taxi» et de «sociabilité».

\footnotetext{
${ }^{198}$ Le niveau de formation, comme l'âge, n'est pas déterminant sur le mode de partage choisi. Par contre, plus le nombre d'enfants est important et plus le travail sera partagé. Plus le revenu est faible, plus il est probable que la femme s'occupe seule de ces tâches (Blättel-Mink ; Kramer ; Mischau 2000 : 25-26).
} 
Lorsque la femme est active à temps complet, les tâches de sociabilité parentale se partagent à $62,1 \%$, respectivement 57,3\% lorsqu'elle est à temps partiel. Lorsqu'elle est au foyer, elle s'occupe seule de ces tâches dans la majorité des couples (un partage égalitaire devenant alors minoritaire) (Blättel-Mink ; Kramer ; Mischau 2000 : 25-26).

Encadré $\mathrm{n}^{\circ} 12$ Les modifications de la répartition des tâches dans l'ancienne Allemagne de l'Est

À la fin des années 1980, dans l'ancienne Allemagne l'Est, la répartition du travail familial était relativement égalitaire entre les sexes, la durée étant relativement équivalente, malgré une spécification des tâches.

Sous l'idéologie socialiste, l'accès à une activité professionnelle pour la femme, au même titre que l'homme, permettait une solidarité entre les sexes et un partage du travail domestique et parental plus égalitaire, l'exercice d'une activité professionnelle se faisant sur la base d'un double temps complet pour les couples.

Le contrat de genre qui existait au sein de l'ancienne Allemagne de l'Est s'est modifié dans les années 1990. Suite à la réunification, de nombreuses familles, dans l'ancienne Allemagne de l'Est, ont été confronté au chômage. La perte de l'emploi (principalement pour la femme) a signifié, dans de nombreuses situations, un retour à une pratique inégalitaire de partage, avec la reconduction des femmes au foyer (Scherf, Ingrid ; Winkler, Gunnar, 1994).

\section{III.B.2.2 Une paternité individuelle et conjugale, une maternité consensuelle et sociale}

Les représentations individuelles de la parentalité sont étudiées ici à partir de quatre dimensions (LBS 2000) : la première est liée à l'intérêt porté à l'enfant : s'intéresser, consacrer du temps, câliner, jouer, lire, entreprendre quelque chose seul avec lui, s'amuser. La seconde est relative à l'éducation : être conséquent, féliciter l'enfant, être patient, lui apprendre les bonnes manières, réfléchir sur les modes d'éducation, montrer de la sérénité. La troisième concerne le climat familial : être prêt à aider le partenaire, exprimer son attachement affectif, soutenir le mode éducatif du partenaire, la stabilité du couple, éviter les disputes. La quatrième dimension traite des rôles traditionnels de genre : renoncement à la carrière professionnelle, renoncement à ses propres intérêts, superviser l'enfant, offrir un bien être matériel, garantir le revenu familial.

L'exercice de la parentalité fait l'objet d'un large consensus culturel chez les individus. En effet, les hommes et les femmes, dans leur grande majorité, ont répondu de la même manière : pour l'ensemble des personnes interviewées, le père a la responsabilité de garantir le revenu familial et le bien être de la famille ; sa fonction principale est celle de nourricier (LBS 2000 : 4). Parallèlement, il y a un consensus qui définit le renoncement de la carrière pour les femmes comme un élément constitutif de la maternité.

Les représentations individuelles du concept de paternité et de maternité sont indépendantes du niveau de formation des personnes interrogées ${ }^{199}$. Une compréhension

\footnotetext{
${ }^{199}$ Le niveau de formation a un rôle important: mais s'il détermine les représentations, il agit peu sur les pratiques de partage des tâches entre les individus (Blättel - Mink, Kramer, Mischau 2000). Ces résultats remettent en cause les interprétations centrées sur l'importance de la formation et de la qualification comme déterminants de l'action sociale.
} 
traditionnelle des rapports de genre ne corrèle pas avec le niveau de formation (pour les pères et pour les mères) : un niveau faible de formation n'implique pas une adhésion plus forte à une répartition des rôles suivant le modèle traditionnel. On remarquera cependant une petite variation entre le niveau de formation et les réponses des hommes : plus l'homme a un niveau de formation élevé plus il adhère à une réflexion sur le style d'éducation à donner à l'enfant (LBS 2000).

«L'homme nouveau»n'est pas seulement une figure rhétorique mais une réalité qui peut être chiffrée. $19 \%$ des hommes adhèrent à cette nouvelle image où l'homme aide aux tâches domestiques et ne se définit plus comme le seul apporteur de la famille ou comme l'unique soutien de famille (Zulehner ; Volz 1998) ; il redéfinit en partie les rapports de genre où les idées de partenariat dominent ; et le relationnel devient très important dans les rapports parentaux.

Selon P. M. Zulehner, la norme de «l'homme nouveau» ne s'imposera pas comme norme dominante car les hommes de plus de 50 ans et les jeunes de 20 ans partagent plutôt une image traditionnelle de l'homme ; cette norme ne pourra pas s'établir véritablement, de part le manque général de repères dans la définition des rôles, et de part la concurrence entre les hommes et les femmes provoqué par le chômage. Cependant, il nous semble difficile d'argumenter sur ces deux catégories, lesquelles n'étant pas (plus ou pas encore) directement confrontées à la paternité. Par ailleurs, les plus de 50 ans sont les représentants des valeurs traditionnelles de la génération précédente. Quant aux plus jeunes, c'est la confrontation entre les représentations des jeunes hommes et des jeunes femmes qui crée la nouvelle norme des rapports de genre. Or, il semble que les jeunes femmes montrent au contraire des valeurs très émancipées et volontaires (BRIGITTE 1999) qui pourront venir heurter les représentations plutôt traditionnelles des jeunes hommes.

Le «Münchener Stadttinstitut für Frühpädagogik», «l'institut pédagogique de la petite enfance de Munich» a réalisé une étude, commanditée par le ministère fédéral de la famille, sur les pères : «Quelle compréhension ont-ils de leur rôle ? ». Les résultats montrent que les $2 / 3$ des pères se considèrent comme éducateurs. Ils favorisent les fonctions sociales et instrumentales («faire avec l'enfant» et «faire pour l'enfant»), plus que celles liées au monde professionnel (carrière et revenus). Cette représentation est généralisée sur l'ensemble des pères et des mères et ces aspects montrent un consensus important sur les compétences sociales des pères. En revanche, 1/3 se définissent avant tout par leur rôle de soutien de famille (Fthenakis ; Minsel 2001).

\section{Encadré $n^{\circ} 13 \quad$ Le «père éducateur» et le «père nourricier»}

Les deux auteurs ont constitué un concept sociologique de la paternité à partir de quatre dimensions (Fthenakis ; Minsel 2001) : premièrement la fonction sociale (prendre du temps avec l'enfant, faire une activité avec lui) ; deuxièmement, la fonction d'apporteur ; troisièmement, la fonction instrumentale (faire quelque chose pour l'enfant) ; quatrièmement, le renoncement à une carrière professionnelle.

A partir de ces quatre fonctions, deux types de paternité ont été définis : le «père éducateur» et le «père nourricier». Une personne adhère au modèle du «père éducateur» lorsque la moyenne des points obtenus aux fonctions sociales et instrumentales est supérieure à celle obtenue aux deux autres. Inversement, lorsque les scores sont plus importants pour les notions 
d'apporteur et de renoncement à la carrière, la personne adhère au type du «père nourricier». $71 \%$ des hommes préfèrent la représentation du père éducateur (respectivement $75 \%$ pour les femmes). Par contre, $29 \%$ des pères se reconnaissent dans l'image du père nourricier (respectivement $25 \%$ pour les femmes).

Cinq facteurs individuels déterminent l'engagement des pères et leur participation parentale au sein du couple.

Premièrement, l'orientation plus ou moins traditionnelle des rôles influence la participation des pères, la position de la mère étant déterminante: plus elle a une représentation traditionnelle, moins le père prend en charge une activité parentale (LBS 2000). Inversement, la participation parentale des pères est supérieure lorsque la mère a une représentation égalitaire des rôles, c'est à dire qu'elle adhère à une représentation de la paternité pour laquelle les fonctions sociales et le renoncement à la carrière (pour le père) dominent (Fthenakis; Minsel 2001). Les nouvelles pratiques des pères («les nouveaux pères») dépendent donc de la mère et de ses dispositions en faveur d'un partage partenarial des tâches parentales. Un partage égalitaire du travail parental sous-entend donc une conception conjugale commune, fondée sur la norme partenariale (Fthenakis ; Minsel 2001).

Deuxièmement, la structure conjugale a également une influence sur la participation des pères : plus la personne définit de la même manière ses attentes par rapport aux rôles paternel et maternel (pour lui-même et son conjoint), plus les activités sont partagées simultanément par les deux parents (LBS 2000).

Troisièmement, le sens que le père donne à la maternité et à la paternité a un rôle sur ses propres pratiques (ce qui n'est pas vrai pour la mère) (LBS 2000). Lorsque le père définit la parentalité comme un ensemble d'activités et de devoirs semblables pour les deux parents, il participe plus aux activités parentales. Par ailleurs, sa façon d'être père influence la satisfaction qu'éprouve sa partenaire de sa participation. Inversement, cela n'influence ni la façon dont le père perçoit l'exercice du rôle maternel par sa conjointe, ni la satisfaction qu'il a de la manière dont elle l'exerce.

Le phénomène est différent dans le cas de la maternité : la définition de la parentalité par les femmes n'a pas d'influence sur leur propre façon d'exercer leur rôle maternel. En d'autres termes, la forme de la maternité est moins dépendante de la représentation individuelle et des convictions de la mère (LBS 2000); et c'est le jugement de la mère qui a une influence sur la participation du père et l'exercice de son rôle, non l'inverse.

Quatrièmement, la participation du père est d'autant plus importante que la grossesse correspond à un moment favorable dans la biographie de l'homme (par rapport à son âge, à ses ambitions personnelles et professionnelles et à celles de sa partenaire) (LBS 1998b). Cette dépendance entre l'engagement ultérieur du père et sa position vis-à-vis de la grossesse est particulière à la paternité. En effet, pour la mère, sa façon d'exercer la maternité est indépendante de ses positions individuelles et de ses orientations. Ceci montre de quelle manière la maternité est fortement structurée culturellement et comment elle est indépendante des orientations et des attentes personnelles de la mère, contrairement à la paternité.

Cinquièmement, la participation du père dépend également des caractéristiques et du développement de l'enfant. L'impression subjective du père «si l'enfant est difficile ou agréable» détermine son engagement parental, contrairement à la mère pour laquelle on attend 
un engagement important, quelles que soient les difficultés rencontrées avec l'enfant (LBS 1998b).

L'engagement parental des pères est également lié à la relation conjugale, à sa qualité et aux dispositions des mères. Plus l'homme rapporte que sa relation conjugale est de bonne qualité, plus le nombre d'activités effectuées en commun est important. Par ailleurs, plus la mère lui reconnaît un ensemble de qualités lui permettant de répondre aux besoins de l'enfant, plus le nombre d'activités, que l'homme effectue seul, augmente. La mère détermine l'accès du partenaire en l'autorisant ou non à s'occuper activement de l'enfant (LBS 1998b).

La participation du père est aussi fonction du temps qu'il consacre à l'activité professionnelle dans une relation simple de disponibilité en temps (LBS 2000), mais également en fonction du stress dont s'accompagne sa charge professionnelle (LBS 1998b).

En résumé, à l'heure actuelle, la fonction d'apporteur et de nourricier (par la garantie du revenu de la famille) fonde toujours le caractère principal de la fonction paternelle pour lequel il existe encore un consensus très fort, hérité d'une conception plus ancienne des rapports et des rôles. Le processus de traditionnalisation des rôles et des rapports de genre au moment de la naissance du premier enfant en est l'expression ${ }^{200}$ (cf. paragraphes suivants).

A côté de cette dimension fortement normalisée, deux autres dimensions sont apparues au cours de ces trente dernières années (LBS 1998b) : la participation du père aux soins à l'enfant et la qualité de la relation parentale. Mais elles ne font pas l'objet d'un consensus social et la participation du père à ces différentes activités est en pleine définition. Beaucoup d'hommes qui deviennent pères choisissent, à l'heure actuelle, de façon très consciente, une forme de parentalité qui les distingue de celle exercée par leur propre père, sans savoir pour autant de quelle manière remplir ce nouveau rôle. Ces pratiques sont essentiellement influencées par des facteurs individuels et conjugaux, beaucoup plus que par des facteurs macro-sociologiques. Il n'y a pas encore de domaine et de tâches particulières que l'homme effectue seul, il y a cependant un grand nombre de tâches effectuées par les deux conjoints. On voit, au niveau des pratiques, que les pères participent autant que les mères sur l'ensemble des tâches de sociabilité parentales.

Quant à la maternité, elle reste très normée et peu individualisée. Les facteurs sociologiques ont un poids plus important que les facteurs personnels et individuels (contrairement à la paternité). Les devoirs et les obligations liés à la responsabilité parentale des mères dépendent beaucoup plus des représentations sociales que des attentes et des convictions du partenaire ${ }^{201}$.

\footnotetext{
${ }^{200}$ La venue des enfants oblige à redéfinir le partage des tâches, on parle alors d'une orientation traditionnelle du partage (J. C. Kaufmann 1992 ; Glaude, de Singly 1986).

${ }^{201}$ Il n'a pas été possible de mettre en évidence un lien d'interdépendance simple et fort entre le comportement éducatif un fois adulte et celui vécu pendant sa propre enfance. Apparement, ce serait le développement de sa propre personnalité et les résultats d'une réflexion critique par rapport au nouveau système normatif auquel est confronté l'individu à l'âge adulte, qui aurait le plus de poids (LBS 2000).
} 


\section{III.B.2.3 La «traditionnalisation» des rôles parentaux à la naissance de l'enfant}

Le passage à la parentalité s'accompagne d'une polarisation traditionnelle des rôles, la mère est alors la première exécutante des tâches parentales à la naissance de l'enfant. Elle s'occupe seule de plus de la moitié des tâches, alors qu'elle avait évalué, de façon idéale, ce type de participation (être seule à avoir la responsabilité de l'activité) à seulement 20\% (Fthenakis ; Minsel 2001). Pour le père, le processus de «traditionnalisation» signifie un désengagement domestique et un investissement professionnel (LBS 1998b). Cependant, les partenaires qui avaient une représentation plus égalitaire des rôles avant la naissance, partagent en plus grande proportion les soins aux enfants, venant nuancer ce processus général de «traditionnalisation».

Les deux chercheurs ont mis en évidence un modèle idéal selon lequel s'oriente l'ensemble des représentations des individus : 70\% des tâches liées à l'enfant doivent être partagées de façon égale entre les deux parents, $20 \%$ effectuées par la mère et $10 \%$ par le père $^{202}$ (Fthenakis ; Minsel 2001). Ce modèle a des caractéristiques de mixité très marqué et le pourcentage souhaité de tâches à effectuer en commun, a la particularité de rester constant $(70 \%)$. En revanche, ce sont les pourcentages des tâches qui relèvent de la mère et du père qui varient selon les cas : lorsque l'enfant est plus jeune, la part idéale de la mère augmente légèrement faisant diminuer celle du père (Fthenakis; Minsel 2001). Cette repésentation idéale de partage est plus présente dans les couples qui favorisent les fonctions sociales et instrumentales et un moindre investissement professionnel du père.

Pour les familles ayant des enfants en âge d'être scolarisés, ce modèle idéal de partage de la parentalité change légèrement: $72 \%$ des tâches parentales sont attribuées aux deux partenaires, $21 \%$ à la mère et $8 \%$ au père (Fthenakis ; Minsel 2001).

A côté de ce modèle idéal, $45 \%$ des tâches sont effectivement partagées à deux, $44 \%$ reviennent à la femme et $11 \%$ à l'homme. Quelques mois après que l'enfant ait intégré l'école, la répartition des tâches devient encore plus traditionnelle : 43\% des tâches sont effectuées à deux, 48\% par la mère et $9 \%$ par le père (Fthenakis ; Minsel 2001).

\section{III.B.2.4 L'exercice de la parentalité et la «traditionnalisation» des rôles domestiques}

Plus de la moitié des activités domestiques est prise en charge par les femmes, en particulier la cuisine, la lessive et le ménage, même si la femme travaille à temps complet. La majorité des femmes disent s'attendre à effectuer plus de travail domestique que l'homme. Inversement, l'homme attend que la femme prenne en charge plus de travail domestique que lui-même (Rohmann 2000).

\footnotetext{
${ }^{202}$ Ces proportions se retrouvent sur l'ensemble des couples, ceux qui n'ont pas encore d'enfant, de jeunes enfants ou d'enfant en âge d'être scolarisés.
} 
La Figure 24 fait apparaître trois groupes d'activités domestiques, classées en fonction de la participation décroissante des femmes :

Le premier groupe qui comprend le linge, la cuisine, le ménage et la vaisselle, est celui où la femme prend majoritairement la tâche à sa charge. La délégation de cette tâche au conjoint, que ce soit dans un mode égalitaire ou exclusif pour l'homme, est très faible, surtout pour le repassage. Les deux pourcentages sont inférieurs à la part de la délégation à une autre personne du foyer (6\%). Pour cette activité, plus de $85 \%$ des femmes répondent prendre cette tâche à leur compte. Le partage est le plus traditionnel pour les activités liées au linge : le linge (lessive etc...) est très peu délégué, que ce soit à l'extérieur ou au partenaire.

La cuisine est également peu déléguée par les femmes (elles l'effectuent à 80\%) et le partenariat comme mode de partage, représente seulement 13,5\% des cas. Les femmes s'occupent exclusivement du ménage (70 à $80 \%$ ), qu'il s'agisse du nettoyage ou des activités de rangement. Le partenariat représente $11,4 \%$, et l'externalisation de la tâche 10,1\% des cas.

Le rangement est effectué sur un mode partenarial dans $21,6 \%$ des cas, et la délégation extérieure est relativement moins importante que pour le ménage, avec $6,6 \%$. La vaisselle est prise majoritairement en charge par les femmes avec 56,1\%. En revanche, dans un tiers des cas, elle est effectuée en partenariat. C'est pour cette tâche domestique que la participation exclusive des hommes est la plus forte avec $6,7 \%$.

Le second groupe d'activités est particulier puisqu'il ne contient que les courses : les femmes ne sont pas majoritaires dans la prise en charge de cette activité. Le partage égalitaire y est très important et la participation des hommes est supérieure à celle qu'ils ont dans toute autre activité domestique. Les femmes ne prennent majoritairement cette tâche à leur compte que dans $46,4 \%$ des cas. Elle est effectuée en partenariat dans $40,1 \%$ des cas et l'homme l'effectue seul dans $11,6 \%$ des cas.

Enfin, le troisième groupe d'activités concerne le soin de la voiture et les réparations de l'appartement ou de la maison. Dans cette catégorie d'activités dites «à dominante masculine», l'entretien de la voiture et les réparations sont prises en charge principalement par l'homme dans 60 et $70 \%$ des cas. La délégation à une personne extérieure est importante, $16,9 \%$ pour la voiture et $10 \%$ pour la maison. Ce qui n'équivaut à aucune autre délégation professionnelle des tâches domestiques sauf pour le ménage qui est délégué à 10,1\%. 
Figure $24 \quad$ Les modes de partage du travail domestique, en Allemagne

Les modes de partage du travail domestique, en Allemagne

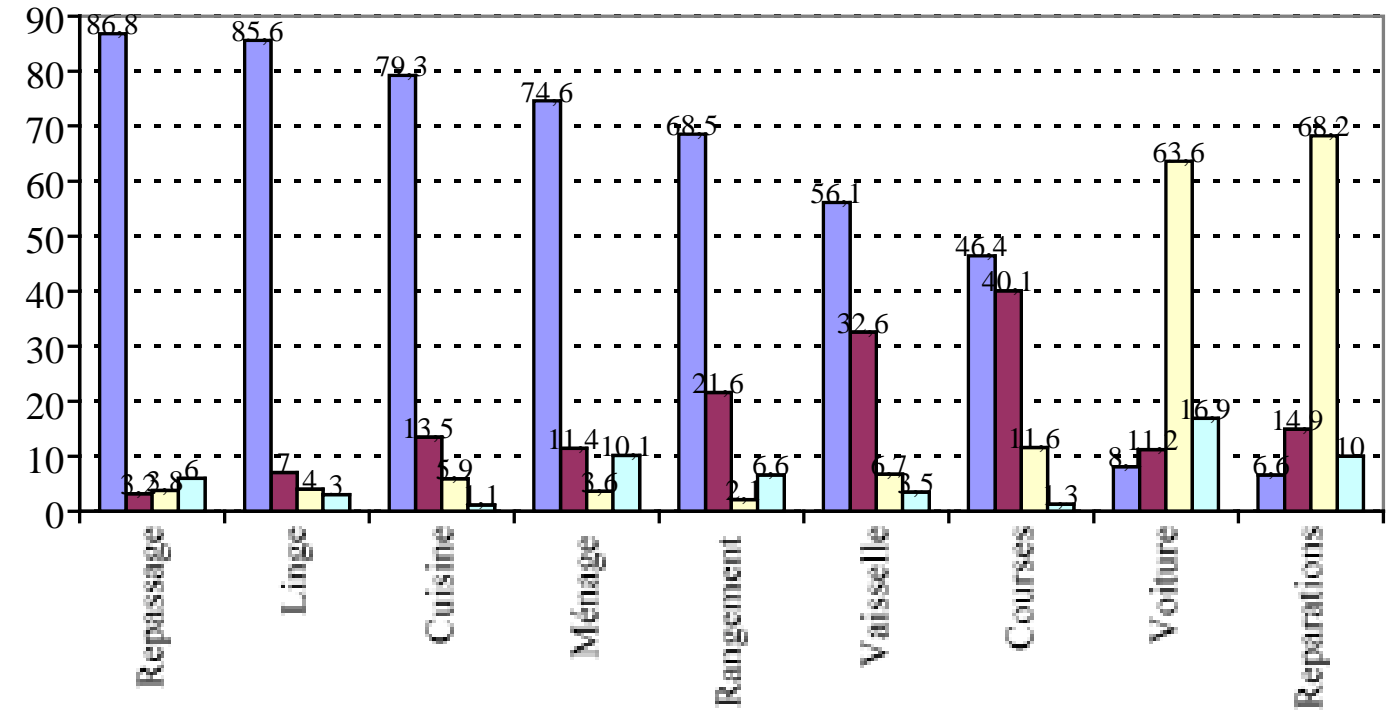

$\square$ principalement la femme $\square$ partage égalitaire $\square$ principalement l'homme $\square$ Autre personne

Source : d'après Blättel-Mink ; Kramer ; Mischau 2000 : 28

Par ailleurs, la baisse de la prise en charge des tâches par la femme s'accompagne d'une augmentation de la part du mode partenarial.

La Figure 25 représente les modes de partage souhaités par les femmes pour chaque tâche domestique (seules les réponses des femmes qui effectuent cette tâche sont prises en compte). Elle illustre les revendications des femmes, et en particulier les souhaits de maintien du monopole féminin, mais aussi les résistances et les avancées de la norme égalitaire au sein des couples.

Ainsi, les femmes revendiquent majoritairement le monopôle des tâches liées au linge (que ce soit le repassage et la lessive) et $52,5 \%$ des femmes qui effectuent seules la cuisine, souhaitent maintenir ce mode exclusif de répartition. Dans le cas du ménage, 41,3\% déclarent vouloir s'occuper principalement de cette tâche. Par contre 20,4\% d'entre elles souhaitent déléguer cette tâche à l'extérieur. La somme de ces deux modes de partage est supérieure à une délégation souhaitée au conjoint. On observe le même phénomène dans le cas du rangement. Pour l'ensemble de ces tâches, le monopôle de la femme se maintient comme valeur sociale $\mathrm{s}^{203}$.

\footnotetext{
${ }^{203}$ Les réponses des femmes qui prennent exclusivement la tâche à leur charge montrent encore plus nettement cette tendance. Elles souhaitent très majoritairement maintenir un mode de partage inégalitaire (où la femme a la responsabilité principale de la tâche) : 74,9\% d'entre elles pour la lessive et le linge, $67 \%$ pour la cuisine, 66,9\% pour le repassage, $58,1 \%$ pour les courses et $55,7 \%$ pour le ménage. Pour le rangement et la vaisselle, les moyennes sont inférieures à la moyenne mais largement supérieures à celles données sur l'ensemble des femmes : 48,8\% dans le cas du rangement et $42,3 \%$ dans le cas de la vaisselle. Sur l'ensemble des activités, le souhait d'un partage égalitaire va de $18,8 \%$ pour la lessive à $37,7 \%$ pour le rangement. La vaisselle fait exception avec $43,1 \%$ où ce souhait est majoritaire par rapport à celles qui souhaitaient maintenir le monopôle de la femme (42,3\%) (Blättel-Mink ; Kramer ; Mischau 2000).
} 
Par contre, la répartition de la vaisselle et des courses appartient à une autre logique. Pour ces deux activités, les femmes souhaitent majoritairement une prise en charge partenariale (respectivement $57,3 \%$ et $56,4 \%$ ). C'est également pour ces tâches que la délégation au partenaire est souhaitée à $8,2 \%$ et $13 \%$ (contre $4,5 \%$ dans le cas des autres activités domestiques).

Figure $25 \quad$ Les souhaits des femmes allemandes relatifs aux modes de partage du travail domestique

Les souhaits des femmes allemandes relatifs aux modes de partage du travail domestique

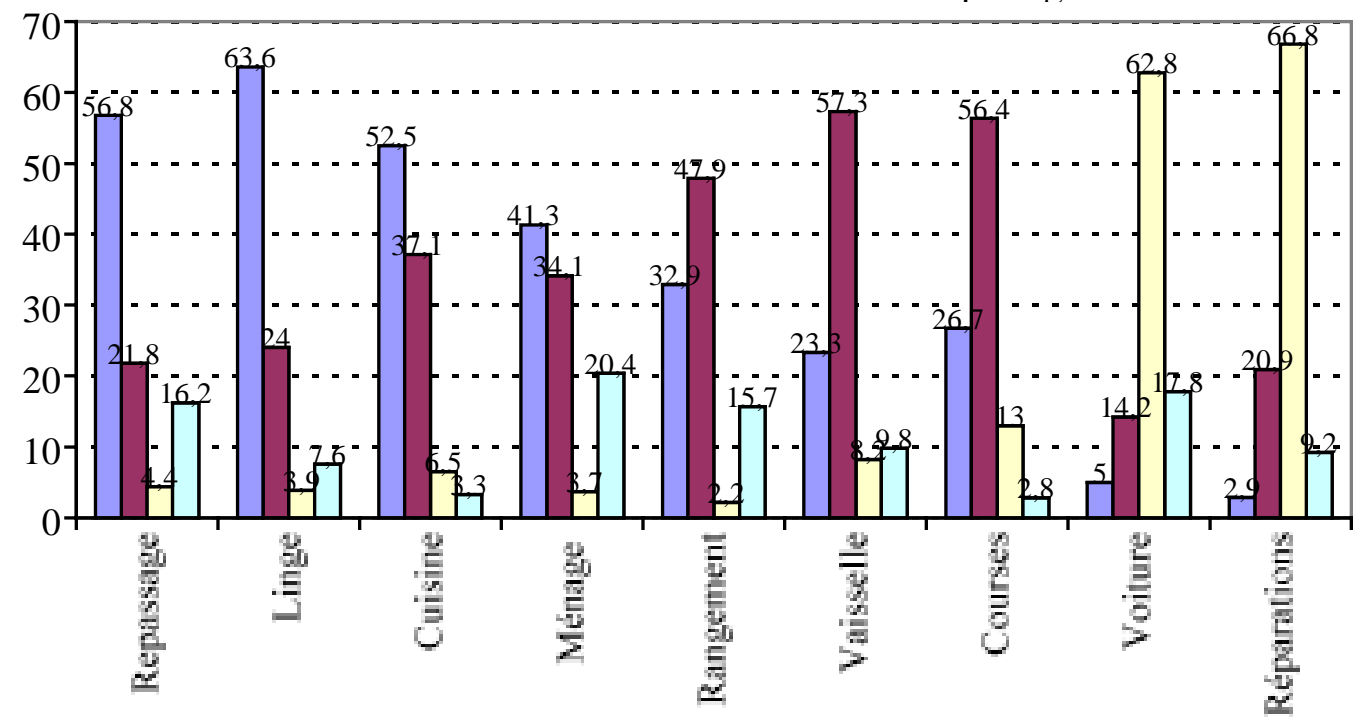

$\square$ principalement la femme $\square$ partage égalitaire $\square$ principalement l'homme $\square$ Autre personne

Source : d'après Blättel-Mink ; Kramer ; Mischau (2000 : 28)

Les différences entre les souhaits (cf. Figure 25) et les pratiques (cf. Figure 24) indiquent que des modes de partage des activités à dominante masculine fonctionnent avec une logique particulière. En effet, les écarts entre les souhaits et les pratiques ne dépassent pas 6 points, toutes catégories confondues. La différence entre la participation exclusive des hommes, réelle et souhaitée est inférieure à 1,5 points. Enfin, la différence entre la délégation extérieure réelle et souhaitée est inférieure ou égale à 1 point $^{204}$. En résumé, les modes de partage des activités à dominante masculine montrent une relative adéquation entre les souhaits des femmes et les pratiques réelles des couples ${ }^{205}$.

Les écarts entre les souhaits et les pratiques réelles de partage des tâches à dominante féminine (travail domestique) atteignent 21,3 points. Si on compare la part des femmes qui répondent pratiquer un partage égalitaire avec la part de celles qui le souhaite, les écarts moyens sont de 28,6 points. Si une décharge est souhaitée par les femmes, c'est premièrement, sous la forme d'un partage de la responsabilité, puis vers une délégation à

\footnotetext{
${ }^{204}$ Il semble que l'impact du revenu de la personne soit important. Il favorise, dans le cas des femmes, une exterrnalisation des tâches masculines (Blättel-Mink ; Kramer ; Mischau 2000).

${ }^{205}$ L'intégration plus ou moins grande de la femme sur le marché du travail, ainsi que son propre revenu, ont une influence sur le mode réel du partage des tâches à l'intérieur du couple mais peu sur les représentations. En effet, seuls l'âge et le niveau de formation déterminent les représentations et les modes souhaités de répartition du travail ; l'impact de l'activité professionnelle n'étant pas significatif (Blättel-Mink ; Kramer ; Mischau 2000).
} 
l'extérieur au couple, et enfin, très rarement, vers le partenaire (en lui donnant la responsabilité principale de la tâche). Concernant la catégorie «Autre personne extérieure au ménage» les pratiques et les souhaits atteignent 5,7 points de différence en moyenne sur l'ensemble ces sept activités à dominante féminine (l'écart le plus grand concerne le ménage avec 10,3 points).

Dans l'ensemble des activités domestiques à dominante féminine, une catégorie constituée du ménage, du rangement et de la vaisselle, se distingue des autres par la plus forte demande de délégation à l'extérieur, par les plus forts écarts entre les pratiques et les souhaits (lorsque la femme effectue seule la tâche), et par l'inadéquation la plus importante entre pratique et souhait pour le partage partenarial. Or, nous rappellerons que ces trois activités forment le pôle le plus traditionnel du travail domestique.

L'inadéquation entre les souhaits et les pratiques de partage est beaucoup plus importante dans le cas du parental que dans le cas du domestique, avec respectivement 30 à 40 points d'écart contre 15 et 35 points pour le domestique (cf. Figure 21 et Figure 22).

Le tableau 48 montre la répartition du travail domestique avant et après la naissance du premier l'enfant (LBS 1997). En fait, il n'y a pas de nouvelle redistribution des tâches dans le couple au moment des naissances de deuxième ou de troisième rang, la spécialisation des rôles qui apparaît à la première naissance se maintenant quasiment définitivement. Au moment de la naissance, l'homme se désengage de certaines tâches en particulier des «Courses» «Ranger, nettoyer», alors qu'elles étaient majoritairement partagées précédemment. Pour les tâches, «courses quotidiennes», «cuisiner», «faire la liste des courses», «laver le linge», «repasser», la faible participation, déjà moindre, des hommes, s'accentue. C'est au moment de la première naissance que s'effectuent les clivages décisifs concernant l'attribution des rôles de l'homme et de la femme. 
Tableau $48 \quad$ Influence de la première naissance sur le partage des tâches domestiques, en \%

\begin{tabular}{|c|c|c|c|c|c|c|c|c|}
\hline \multirow{3}{*}{$\begin{array}{c}\text { TACHES } \\
\text { DOMESTIQUES }\end{array}$} & \multicolumn{8}{|c|}{$\begin{array}{c}\text { PROPORTION DE COUPLE } \\
\text { REPARTI EN FONCTION DU MODE DE PARTAGE }^{(1)}\end{array}$} \\
\hline & \multicolumn{4}{|c|}{ Pendant la grossesse } & \multicolumn{4}{|c|}{4 mois après la naissance } \\
\hline & les deux & la mère & le père & total & les deux & la mère & le père & total \\
\hline Contacts amicaux & 89 & 11 & 0 & 100 & 87 & 13 & 0 & 100 \\
\hline Planifier les vacances & 89 & 9 & 2 & 100 & 85 & 12 & 3 & 100 \\
\hline Courses & 82 & 15 & 3 & 100 & 66 & 26 & 8 & 100 \\
\hline S'occuper des invités & 80 & 18 & 2 & 100 & 74 & 26 & 0 & 100 \\
\hline Ranger, nettoyer & 76 & 24 & 0 & 100 & 34 & 64 & 2 & 100 \\
\hline Faire la vaisselle & 74 & 25 & 1 & 100 & 53 & 47 & 0 & 100 \\
\hline & & & & & & & & \\
\hline $\begin{array}{l}\text { Administrations et } \\
\text { correspondance }\end{array}$ & 66 & 15 & 19 & 100 & 62 & 19 & 19 & 100 \\
\hline $\begin{array}{l}\text { Coups de téléphones } \\
\text { «désagréables» }\end{array}$ & 60 & 15 & 25 & 100 & 60 & 17 & 23 & 100 \\
\hline Penser aux anniversaires & 55 & 44 & 1 & 100 & 49 & 49 & 2 & 100 \\
\hline S'occuper des poubelles & 52 & 11 & 37 & 100 & 57 & 17 & 26 & 100 \\
\hline Gestion des finances & 52 & 9 & 39 & 100 & 45 & 16 & 39 & 100 \\
\hline Courses quotidiennes & 40 & 59 & 1 & 100 & 21 & 78 & 1 & 100 \\
\hline S'occuper des cadeaux & 40 & 51 & 0 & 100 & 38 & 62 & 0 & 100 \\
\hline Cuisiner & 39 & 58 & 3 & 100 & 25 & 75 & 0 & 100 \\
\hline Faire la liste des courses & 37 & 62 & 1 & 100 & 21 & 78 & 1 & 100 \\
\hline Laver le linge, repasser & 32 & 67 & 1 & 100 & 18 & 82 & 0 & 100 \\
\hline $\begin{array}{l}\text { Réparer la maison ou } \\
\text { l'appartement }\end{array}$ & 25 & 1 & 74 & 100 & 27 & 1 & 71 & 100 \\
\hline Entretenir la voiture & 21 & 4 & 75 & 100 & 20 & 4 & 76 & 100 \\
\hline
\end{tabular}

${ }^{(1)}$ Il y a trois modes de partage : la femme a la responsabilité principale de la tâche, les deux partenaires partagent à part égale, l'homme a la responsabilité exclusive de la tâche.

Source : d'après les résultats de l'enquête LBS-Familien-Studie „Übergang zur Elternschaft“ 1997

\section{III.B.2.5 L'exercice de la parentalité et la situation professionnelle des parents}

Sur l'ensemble des couples étudiés (LBS 1998a), les hommes et les femmes avaient, avant la naissance de l'enfant, la même situation professionnelle : $81 \%$ étaient actifs et $17 \%$ en formation, même si les femmes travaillaient un peu moins avec 29,9 heures par semaine contre 33,4 heures pour les hommes. Or, à la naissance du premier enfant, le temps travaillé par les femmes passe à 7,4 heures par semaine, celui des hommes augmente pour atteindre 
39,9 heures. A la naissance du deuxième enfant, leur temps professionnel se réduit encore pour atteindre 5,2 heures par semaine; celui des hommes se rallonge (43,5 heures). La naissance d'un enfant a un impact très marqué sur le volume horaire travaillé par les femmes qui diminue, et sur l'engagement des hommes dans le professionnel qui augmente.

Quatre facteurs déterminent la reprise professionnelle par la femme après la naissance du premier enfant (LBS 1998a). Les deux premiers facteurs sont d'ordre individuel et subjectif $^{206}$.

Le premier facteur est la confrontation de l'attractivité des rôles professionnel et familial : plus l'activité professionnelle était satisfaisante, plus la probabilité d'un retour sur le marché du travail est forte. De même, un moindre intérêt pour un rôle maternel et de femme au foyer augmente les chances d'une reprise de la profession. A l'inverse, une naissance pourra être l'occasion de ne plus exercer une profession peu satisfaisante. Enfin, de nombreuses femmes ne souhaitent pas reprendre une activité professionnelle et préfèrent se consacrer à la sphère familiale.

Le second facteur concerne l'impact de la représentation individuelle de la «meilleure» éducation pour l'enfant.

Le niveau de revenu est le troisième facteur qui détermine le retour à l'activité professionnelle : plus le niveau de revenu a été important plus il y aura un retour rapide à l'activité professionnelle.

Enfin, le quatrième facteur est de nature structurelle: les offres de prise en charge des enfants en bas âge et les modes de garde possibles.

Les déterminants individuels et structurels ont plus de poids dans la décision des mères à reprendre une activité professionnelle que dans la participation de l'homme aux tâches familiales. Inversement, l'activité professionnelle de la femme influence l'engagement du père à la prise en charge des activités parentales de soins. En effet, la participation des pères aux activités de routine (activités parentales domestiques comme habiller, changer, nourrir etc...) augmentent en fonction du temps professionnel de la mère (LBS 1998a : 6).

Les pères ont plus souvent des activités seuls ${ }^{207}$ avec leurs enfants quand la mère est active et qu'elle adhère au concept du «père éducateur». De plus, un père actif participe d'autant plus aux activités liées à l'enfant que sa motivation professionnelle est importante. Enfin, plus les pères partagent de tâches avec leur conjointe plus ils sont accessibles pendant leur temps de travail aux problèmes liés à la famille (Fthenakis; Minsel 2001). Lorsque les deux parents sont actifs $^{208}$, le parental est plus partagé et les sphères familiale et professionnelle ne sont plus strictement opposées.

Inversement, lorsque la femme est au foyer, les sphères familiale et professionnelle se comprennent comme deux sphères exclusives. Une motivation plus importante du père pour le travail professionnel, a pour conséquence de séparer davantage la sphère professionnelle de la sphère familiale : ils sont beaucoup moins accessibles pour des problèmes liés à la famille pendant les temps de travail (Fthenakis ; Minsel 2001). L'activité professionnelle des couples

\footnotetext{
${ }^{206}$ Les souvenirs de la mère du mode d'éducation qu'elle a reçu et de l'attitude de sa propre mère par rapport au marché du travail sont déterminants.

${ }^{207}$ L'ensemble des activités auxquelles le père participe se fait dans la majorité des cas en présence de la mère (Fthenakis ; Minsel 2001 : 13).

${ }^{208}$ Sur l'ensemble des couples dont l'enfant est en âge d'être scolarisé, 93\% des pères et $17 \%$ des mères travaillent à temps plein, la moitié des mères étant au foyer (l'échantillon est représentatif) (Fthenakis ; Minsel 2001).
} 
favorise alors un plus grand partage des tâches parentales et une conception de la sphère familiale et la sphère professionnelle dans la complémentarité ${ }^{209}$.

Deux enquêtes (au moment de la grossesse, puis, un an et demi après la naissance de l'enfant) permettent de voir l'impact de la venue de l'enfant sur la carrière professionnelle de la femme et donc d'étudier l'ampleur du phénomène de «traditionnalisation» au moment de la naissance de l'enfant (LBS 1998a). Les femmes sont regroupées selon quatre trajectoires professionnelles.

Le premier groupe (33\%) est constitué des mères qui étaient actives pendant la grossesse et qui ont maintenu leur activité professionnelle après la naissance de l'enfant (ce qui est surtout le cas des mères au moment de la première naissance). La participation du partenaire aux tâches ménagères, diminue au moment de la reprise professionnelle de la femme. Le phénomène de «traditionnalisation» se maintient au-delà de la reprise d'une activité professionnelle par la femme.

Le second groupe (29\%) est composé des mères qui n'étaient actives ni au moment de la naissance ni un an et demi après (ce qui est surtout le cas des femmes au moment de la deuxième et troisième naissance). Dans ce cas, la répartition des rôles est traditionnelle. A l'arrivée d'un autre enfant, la charge des tâches domestiques et parentales augmente pour les femmes, les hommes ne participent pas plus au moment des naissances successives mais augmentent au contraire leur engagement professionnel.

Le troisième groupe $(28 \%)$ comprend les mères qui sont actives au moment de la naissance et inactives un an et demi après. La sortie des femmes de l'activité professionnelle signifie une nouvelle répartition des tâches domestiques en leur défaveur.

Enfin, le quatrième groupe $(10 \%)$ représente les mères qui n'étaient pas actives au moment de la naissance mais qui ont repris une activité professionnelle un an et demi après (essentiellement des femmes avec plusieurs enfants). Pour elles, la charge domestique n'augmente pas, leurs conjoints participant légèrement plus.

\footnotetext{
${ }^{209}$ Les stratégies de délégation du travail domestique et parental sont plus limitées d'en France. Par exemple, les mesures «Haushaltsscheck» et «Dienstleistungsgutschein» (les équivalents des chèques emploi service en France), ont peu de succès auprès des ménages (Birkhölzer, Lorenz 2000).
} 


\section{CONCLUSION}

Dans ce chapitre, nous avons étudié les mécanismes individuels et conjugaux qui déterminent l'exercice de la parentalité pour les pères et les mères. Nous avons vu que la paternité et la maternité ne peuvent se concevoir l'un sans l'autre. La construction des rôles à l'intérieur de la famille s'ajoute donc à une définition extérieure (bien qu'intériorisée) en s'imposant à l'individu ${ }^{210}$.

L'exercice de la parentalité dépend aussi de la participation des pères et des mères au marché du travail, et de leur participation au travail domestique (au sens strict). Enfin, les possibilités de délégation des tâches familiales (au sens large, c'est-à-dire du domestique et du parental) par le biais de l'Etat-providence et du réseau informel, influencent les modes de partage du travail familial et donc l'exercice de la parentalité.

\section{L'EXERCICE DE LA PARENTALITE EN FRANCE}

En France, dans les années 1970, les tâches parentales sont définies comme faisant partie du travail domestique. Le partage du travail parental n'est pas discuté en soi. Le domestique (au sens large) est alors analysé en rapport avec l'activité professionnelle et en fonction des différences de genre. On observe alors que les femmes actives ont les mêmes pratiques que les femmes au foyer. L'influence de l'activité professionnelle est moins déterminante que la forme du ménage. Les hommes participent peu au domestique, et en présence d'enfant, leur participation professionnelle augmente. Par un phénomène de partage au niveau conjugal, ils participent légèrement plus si la femme travaille. A l'intérieur du travail domestique, (au sens strict), les hommes participent uniquement à certaines tâches, essentiellement la vaisselle et les courses. Le phénomène de partage du domestique est très polarisé, il est pratiquement exclusivement effectué par les femmes; le ménage et le linge sont les tâches les plus sexuellement marquées.

Entre les années 1980 et 1990, les pratiques de partage ont évolué au niveau du parental (toujours défini au sein du domestique). Ces changements sont dus essentiellement à la participation plus importante des pères.

Le partage du domestique (au sens large) se comprend toujours en opposition avec le professionnel qui domine comme référent (surtout pour l'homme). La polarisation de l'activité domestique demeure. Mais si la nature de la contribution des hommes change peu, leur participation a légérement augmenté au cours des années 1980. Elle est déterminée par la forme de famille, il participe plus au sein d'un couple de concubins, que d'un couple marié. Il participe plus quand la femme est active. Par ailleurs, la femme a réduit sa participation au cours des années 1990, ce qui ne s'était jamais produit auparavant. Le domestique est en mouvement, mais bien moins que les autres sphères parentale et professionnelle ${ }^{211}$.

\footnotetext{
${ }^{210}$ J.C. Kaufmann (1992) explique le décalage entre les idées égalitaires et les pratiques encore très inégalitaires, par le fait qu'il s'agit de deux niveaux différents de l'action sociale, les deux ayant une réalité mais le poids des gestes étant le plus déterminant, il détermine la lenteur des évolutions des pratiques.

${ }^{211}$ Après le domestique, le dernier bastion des inégalités est sans doute celui des loisirs et du temps personnel. En effet, les évolutions du temps de loisir entre 1986 et 1999 montrent une augmentation globale de 30 minutes
} 
Dans les années 1990, une véritable rupture théorique et sociologique a lieu. En théorie, les activités parentales se distinguent des tâches domestiques, au point de devenir une catégorie en soi. Nous avons alors identifié le «travail parental» comme l'ensemble des activités effectuées «pour» et «en présence» de l'enfant («elterliche Arbeit») dont nous distinguons quatre catégories en fonction de la nature des tâches : la «sociabilité parentale», le «travail parental 'taxi'», le «travail parental domestique» et le «travail parental scolaire».

En pratique, depuis les années 1980, les comportements au sein des familles changent et les mécanismes de partage se modifient. Mais les résistances sont nombreuses, et les évolutions allient très clairement des éléments traditionnels et modernes (voir innovants).

Le travail parental se partage encore de façon inégalitaire au sein du couple, un tiers est effectué par les pères, deux tiers par les mères (en 1999). La répartition est relativement plus égalitaire dans le cas de la «sociabilité parentale» (40\% pour le père, $60 \%$ pour la mère) et dans le cas du temps «parental taxi» (idem). Les inégalités sont plus prononcées dans le cas $\mathrm{du} \ll$ travail parental domestique».

Le partage est un processus hétérogène qui dépend de la nature de la tâche : les évolutions les plus marquantes concernent la participation grandissante des pères aux «tâches parentales domestiques « (domaine qui était exclusivement réservé aux femmes auparavant) : en revanche, leur plus grande participation aux tâches de «sociabilité parentale» a toujours été caractéristique de leur investissement parental.

Au cours des deux dernières décennies, le professionnel et le parental n'est plus le domaine réservé d'un «genre» particulier. Dans la famille, le partage du professionnel, même de façon inégalitaire, est un fait acquis, il n'est plus l'exclusivité des hommes. Dans les années 1990, la majorité des mères sont actives à temps complet : 72,3\% pour les mères de deux enfants et $51 \%$ pour celles de trois enfants et plus (ces dernières n'étaient que $16 \%$ en 1982 (Données Sociales, 1999)). De fait, les couples parentaux sont surtout des couples à deux actifs équivalents. L'investissement professionnel des pères dépend de la situation professionnelle de la mère (active ou inactive) et de leurs catégories socioprofessionnelles respectives. Par ailleurs, la présence d'enfant (surtout à partir du second) signifie un allongement de la participation professionnelle des pères (mais faible) et une réduction de celle de la mère.

Enfin, les stratégies de délégation du parental, de part l'activité professionnelle des parents, sont importantes pour les familles. Elles sont complexes, regroupant à la fois les solutions institutionnelles (collectives et individuelles) et informelles.

\section{L'EXERCICE DE LA PARENTALITE EN ALLEMAGNE}

En Allemagne, jusque dans les années 1970, la mesure des activités parentales est surtout effectuée à partir que ce que nous avons appelé le «travail parental domestique». Les autres tâches comme le relationnel et la sociabilité ne sont pas prises en compte. La participation des pères équivaut au milieu des années 1960 à environ 16\% du temps parental des mères. Dans les années 1970, leur participation semble avoir augmenté, mais uniquement dans le cas où la mère travaille.

du temps de loisir (Dumontier, Pan Ké Shon, 1999), néanmoins la différence entre les hommes et les femmes est très importante et s'est maintenue au cours de la dernière décennie. 
L'engagement des hommes dans le travail domestique varie en fonction des tâches, constituant une forte polarisation des rôles. Cependant, l'activité professionnelle de la femme s'accompagne d'une plus grande implication de leur part, même dans des tâches féminines comme la cuisine et les courses.

Depuis les années 1980, l'exercice de la parentalité est au centre d'un processus dont les règles socio-normatives sont en pleine redéfinition.

La participation du père aux tâches parentales est directement liée aux attitudes et aux pratiques de la mère. Celle-ci permet une participation plus importante des pères lorsque ellemême adhère à une conception du père comme «éducateur» et non comme «nourricier». Le processus inverse n'est pas vérifié : la propre conception de la paternité par l'homme a peu d'influence sur son rôle et sur celui de sa partenaire.

Une seule fonction paternelle est définie par les conventions sociales : son rôle de «nourricier» (d' «apporteur»), qui fait, encore aujourd'hui, l'objet d'un large consensus social. Quant aux autres fonctions, elles deviennent de plus en plus valorisées sur le plan social : la fonction éducative et celle liée aux soins de l'enfant («tâches domestiques parentales»). Cependant, elles ne font pas l'objet d'une codification sociale définie, laissant une place plus importante aux facteurs individuels et conjugaux. Les déterminants microsociologiques ont un poids important dans la construction de la paternité, montrant l'instabilité culturelle à son égard (F.X. Kaufmann 1996). En revanche, l'ensemble des fonctions de la maternité est nettement codifié et normalisé : les facteurs macrosociologiques sont relativement plus déterminants que les facteurs individuels.

Aujourd'hui, le modèle des «nouveaux pères» (un tiers des pères) rencontre de nombreuses résistances, dont les représentations des mères et la forme de l'activité professionnelle des mères. Dans les années 1990, les familles partagent le travail professionnel sur le mode d'un apporteur et demi et l'inactivité des mères mariées est encore importante. Or, il semble que seul l'exercice d'un temps complet de la mère s'accompagne d'un engagement paternel plus important.

Au-delà des différences de mesures, on voit que les tâches de «sociabilité parentale» sont largement partagées. Le partage du «travail parental domestique» reste inégalitaire ${ }^{212}$ en défaveur des mères (comme en France). L'activité professionnelle des mères modifie le partage du parental mais uniquement dans le cas d'un temps complet : les tâches «parentales domestiques» sont alors plus souvent déléguées au père et les activités de «sociabilité parentale» se partagent plus entre les deux parents. Dans le cas d'un temps partiel ou d'une inactivité, la participation des pères diminue.

La naissance de l'enfant provoque une «traditionnalisation» des rôles conjugaux : bien que les représentations des pères et des mères s'accordent sur un partage paritaire du parental, des pratiques inégalitaires s'institutionnalisent dès la naissance. La venue de l'enfant polarise également les pratiques du domestique, la mère effectue seule certaines tâches qui étaient partagées auparavant. Une naissance change aussi les comportements relatifs au professionnel : les mères diminuent très fortement leur participation, les pères l'augmentent (ces tendances sont plus fortes qu'en France).

\footnotetext{
${ }^{212}$ Cependant, une comparaison des modes de partage réels et souhaités montre que ce sont pour les tâches qui sont les mieux réparties que les mères souhaitent davantage de partage contrairement aux tâches inégalement réparties pour lesquelles elles sont plus nombreuses à souhaiter maintenir le rapport inégalitaire.
} 
Jusque dans les années 1970, le modèle familialiste, qui délègue les fonctions éducatives à la femme, domine. Il faudra attendre les années 1980 pour voir apparaître un autre modèle plus égalitaire dans la prise en charge des tâches éducatives et de soins auprès de l'enfant. Certaines activités parentales, comme la «sociabilité parentale», sont dorénavant largement partagées et l'exclusivité stricte des tâches «parentales domestiques» a disparu.

Ce nouveau partage s'inscrit définitivement dans les pratiques des années 2000, il fait suite aux représentations de parité parentale, présentes depuis les années 1980 dans les discours. Les années 1990 prolongent donc les changements de l'exercice de la paternité, amorcés précédemment, et modifient l'exercice du parental et du domestique pour les mères.

\section{LA REDEFINITION DU TRAVAIL FAMILIAL}

Jusque dans les années 1970, on entendait par «travail familial» l'ensemble indifférencié des tâches ménagères effectuées par la femme au sein du foyer. Cette définition trouve son origine dans la spécialisation traditionnelle des rôles et dans le modèle dominant de la femme au foyer.

Dans les années 1980, le «travail familial» est discuté en terme d'articulation entre le travail domestique et le travail professionnel. Cette avancée théorique était concomitante à l'entrée massive des femmes sur le marché du travail. Les travaux de M.-A. BarrèreMaurisson ont participé à repenser définitivement la famille dans une relation dynamique entre les sphères de «production» et de «reproduction» (1992).

Depuis les années 1980 et le début des années 1990, le couple conjugal est au centre de l'analyse. Cette position théorique permet de mettre en avant le phénomène de partage du «travail familial» dans le couple, il n'est plus le seul lot de la femme active. L'activité professionnelle de l'homme est repensée en dynamique avec celui de la femme et inversement (Fermanian, Lagarde 1999).

Puis, la compréhension du «travail familial» s'est modifiée récemment avec l'introduction de la notion de «travail parental». Les travaux du groupe Division Familiale du Travail ont montré qu'il était nécessaire de distinguer le travail domestique du travail parental, ce dernier ayant été jusqu'à présent considéré et inclus dans le domestique (BarrèreMaurisson ; Marchand ; Rivier 2000). Cette prise en compte du temps parental coïncide avec l'idée dominante de la place centrale de l'enfant dans nos sociétés contemporaines.

L'ensemble des analyses traditionnelles de la famille se fonde sur une opposition forte entre la «sphère familiale» et la «sphère professionnelle»: avec d'un côté la famille (comprenant le domestique, et dont on a récemment isolé le parental) et de l'autre le professionnel (cf. Figure 26). Dans ce contexte, on parle de «conciliation famille-travail», de «sphère rémunérée/non-rémunérée» ou de «travail/hors travail» ${ }^{213} \ldots$

\footnotetext{
${ }^{213}$ Ces catégories sont peu satisfaisantes et ouvrent une discussion sur la signification de la notion de travail, de rémunération et d'activité. Les notions de travail/hors travail étaient une tentative, grâce à la notion de travail parental, de recomposer ces catégories (Barrère-Maurisson, Minni, Rivier 2001).
} 
Figure $26 \quad$ L'articulation «Famille»-«Travail»

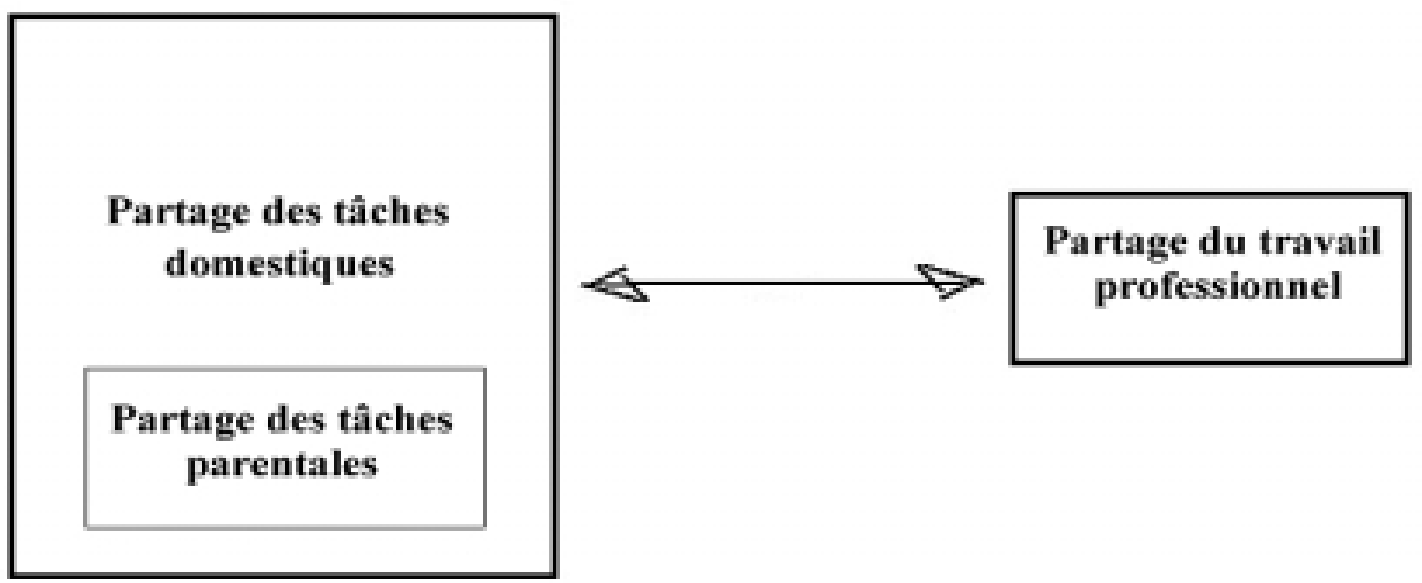

Aujourd'hui, nous proposons de redéfinir le «travail familial» à partir de la notion de travail $^{214}$. Il concerne alors l'ensemble des activités qui sont effectuées «dans» et «pour» la famille. L'ensemble du «travail familial» est un enjeu de négociation dans le couple et la famille. Sa masse globale dépend de la structure de la famille (en couple, avec ou sans enfant, personne seule) mais également des exigences individuelles et des normes sociales. L'affectation des tâches et des temps, entre le professionnel, le domestique et le parental, pour chaque individu et entre les membres du ménage, se discute au sein de la sphère familiale en fonction de mécanismes complexes. Il est composé de l'ensemble des tâches, c'est à dire de la masse de travail négocié à l'intérieur de la famille, il contient donc l'ensemble du travail professionnel, du travail domestique et du travail parental, partagés à un niveau individuel et conjugal (cf. Figure 27).

Figure 27 Le partage du «travail familial» en fonction de la notion de travail

\section{Partage du travail familial}

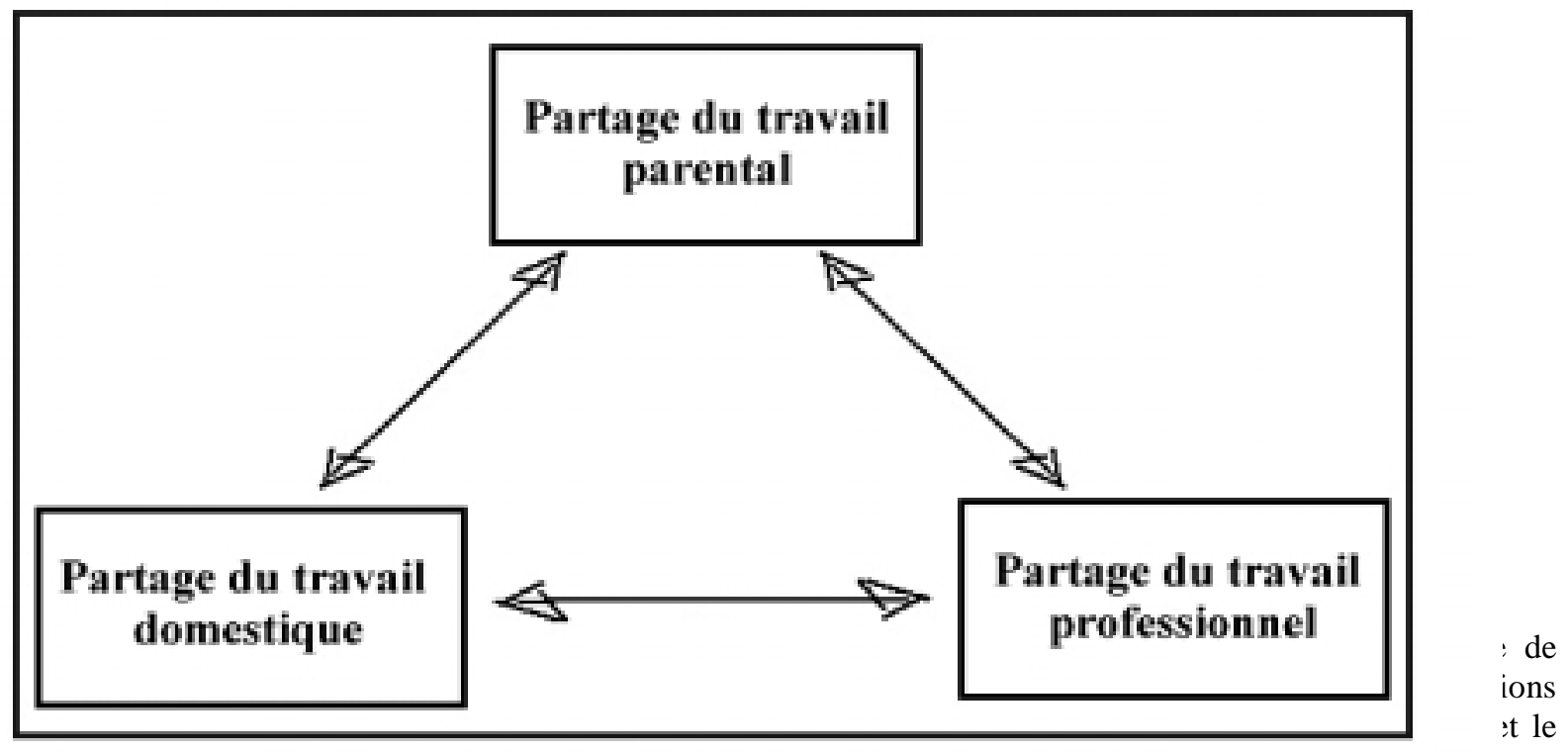


La notion de «travail familial» illustre le parti pris selon lequel le travail est défini depuis la sphère familiale, lieu de négociation, et non plus seulement depuis le marché du travail, lieu de définition du travail professionnel. Le travail professionnel n'est plus une notion exogène à la famille, mais bien une masse de travail qui est négociée et partagée en son sein. En ce sens, notre concept intègre la relation qui existe entre la famille et le marché du travail, identifiée par la théorie de la «Division Familiale du Travail» (Barrère-Maurisson 1992), tout en la prolongeant pour prendre en compte la relation entre l'exercice de la parentalité et le partage du travail domestique. La parentalité et ses modes de partage sont alors analysés comme un élément du travail familial. En d'autres termes, on parlera de «travail familial» pour exprimer la négociation du partage des tâches au sein de la famille, tout en gardant la référence au marché du travail.

Définir le «travail familial» comme un volume de tâches négociées au sein de la famille (le domestique, le parental et le professionnel), permet de dépasser l'opposition entre famille et marché du travail ${ }^{215}$. De cette manière, on place le couple et la négociation conjugale au centre du mécanisme de répartition des tâches et des temps. L'avantage en distinguant ces trois grandes catégories d'activités familiales, est d'éviter de les hiérarchiser et de comprendre, par exemple, le temps domestique comme un temps résiduel du temps professionnel.

Nous proposons donc par-là une compréhension des modèles familiaux qui dépasse les oppositions strictes «famille/profession», «rôles masculins/rôles féminins».

Le partage du «travail familial» ainsi redéfini, montre une différenciation de plus en plus grande des tâches les unes par rapports aux autres. Dans ce contexte, nous rappelons le phénomène d'autonomisation de la logique du parental par rapport aux autres logiques familiales, que nous avons identifié dans le chapitre précédent. Ainsi, le parental prend une valeur en soit au sein du «travail familial»; il est l'objet d'un partage entre les pères et les mères, non plus suivant une logique de différenciation des rôles, mais suivant un partage des tâches, des fonctions et des rôles, dans une relative neutralité de genre (même si les inégalités et la spécialisation entres les sexes sont encore importantes). Au niveau des pratiques, on est encore loin d'un exercice paritaire de la parentalité, mais elles ont évolué depuis un modèle fondé sur un partage sexué de ,travail familial», vers un modèle de partage où la place relative des parents est renégociée en fonction du principe d'égalitée ${ }^{16}$. Le processus de construction d'une nouvelle paternité modifie les repères très institutionnalisés des pratiques des mères, lesquelles, inversement, sont déterminantes pour la paternité.

\section{LA REGULATION DU PARTAGE DU «TRAVAIL FAMILIAL»}

En France, le partage du travail parental est indéniablement lié au partage du travail professionnel sur la norme du temps complet, pour les mères et les pères. Le partage du «travail familial» est marqué par la coupure entre d'un côté, la sphère professionnelle où règne la plus grande égalité de répartition entre conjoint, et de l'autre, le travail parental et le travail domestique qui se distinguent par une répartition plus inégalitaire. La participation quasiment égalitaire dans la sphère professionnelle, indifféremment du sexe, attribue une

\footnotetext{
${ }^{215}$ En concevant le travail professionnel comme une composante du travail familial, il est possible de discuter du partage du travail rémunéré et non-rémunéré comme un phénomène endogène à la famille.

${ }^{216}$ Les pratiques montrent de nombreuses inégalités, mais la référence aux valeurs égalitaires est forte et très présente, quels que soient la forme familiale et l'âge des individus (J. C. Kaufmann 92).
} 
fonction d'apporteur aux hommes et aux femmes. Dans ce contexte, on leur reconnaît les mêmes fonctions familiales et l'égalité entre les parents est alors la conséquence indirecte de l'égalité professionnelle (d'abord affirmée dans les normes, puis, de plus en plus dans les pratiques). L'accumulation, pour les deux parents, de l'ensemble des fonctions familiales («parentalité conciliée» pour les deux parents) pousse à un partage égalitaire.

Le partage du «travail familial» à l'intérieur du couple s'effectue d'abord en fonction du travail professionnel, lui-même défini à partir de la logique de la double activité professionnelle à temps complet pour les hommes et les femmes. Ce dernier est la norme de l'organisation des tâches et des temps du «travail familial» dans les ménages en France (Anxo et al. 1998). Le partage s'effectue ensuite en fonction du travail parental, et enfin, en fonction du travail domestique. Cette hiérarchie coïncide avec les avancées égalitaires au sein des familles : c'est d'abord le travail professionnel qui est réparti le plus égalitairement, suivi du travail parental (en particulier sous l'impulsion des pères qui participent de plus en plus), et enfin du travail domestique. Les résistances intrafamiliales pour un partage égalitaire du travail domestique sont encore très importantes, même si la mixité s'annonce sur certaines tâches, en particulier lorsque les savoir-faire domestiques qui les accompagnent sont faibles.

La délégation et l'externalisation du «travail familial»s'effectuent en fonction de la nature de la tâche.

La première logique concerne les tâches partagées sur un mode traditionnel : le linge est délégué dans une proportion importante pour compenser un partage qui n'est pas effectué au sein du ménage. Dans ce cas, une délégation à l'extérieur diminue le volume global de la tâche sans pour autant jouer sur les mécanismes de répartition.

La seconde logique concerne la délégation des tâches qui sont largement partagées comme les courses : dans ce cas, l'externalisation vient appuyer une répartition déjà égalitaire des tâches en allégeant la charge de travail pour le couple à la manière d'un troisième intervenant. Par conséquent, l'externalisation et la délégation, si elles permettent une meilleure conciliation des tâches dans le «travail familial», entre le professionnel, le parental et le domestique, en diminuant le volume de certaines tâches, elles ne modifient pas les mécanismes de partage intraconjugal et peuvent maintenir des rapports inégalitaires.

En Allemagne, le modèle de partage du travail professionnel dans le couple suit celui de l'actif et demi. Le partage du travail parental n'est pas discuté en termes égalitaires mais en termes de différenciation des rôles au sein de la famille. L'abandon quasiment définitif du modèle à un apporteur prioritaire montre la tension sociale actuelle entre, d'un côté une participation constante des femmes au marché du travail bouleversant les rapports de genre et, de l'autre, le maintien de la différenciation sexuée des fonctions à l'intérieur de couple parental. La répartition du «travail familial» montre une inversion de l'investissement professionnel et parental, entre les hommes et femmes, qui se fonde sur l'idée d'une équivalence du travail (et non pas d'une égalité de fonction comme en France). Le partage du parental résulte de la répartition entre les sexes du travail, par délégation sur l'un des parents (et non pas sur un mode partagé). Cela pousse à une complémentarité entre les sexes qui justifie alors une distinction sexuée des fonctions laquelle renforce le partage spécifique du parental. C'est donc essentiellement le lien entre le parental et le professionnel qui explique la répartition des tâches familiales et peu celui du travail domestique.

Ainsi, la notion de «travail familial» permet de montrer que la régulation actuelle s'effectue entre le parental et le marché du travail et non pas entre le parental et le domestique 
ou entre le domestique et le professionnel (cf. Figure 28). Aujourd'hui, l'expression de «conciliation entre la famille et le travail» doit donc être avant tout comprise comme la relation entre le parental et le professionnel.

Figure $28 \quad$ La régulation du partage du «travail familial»

\section{Partage du travail familial}

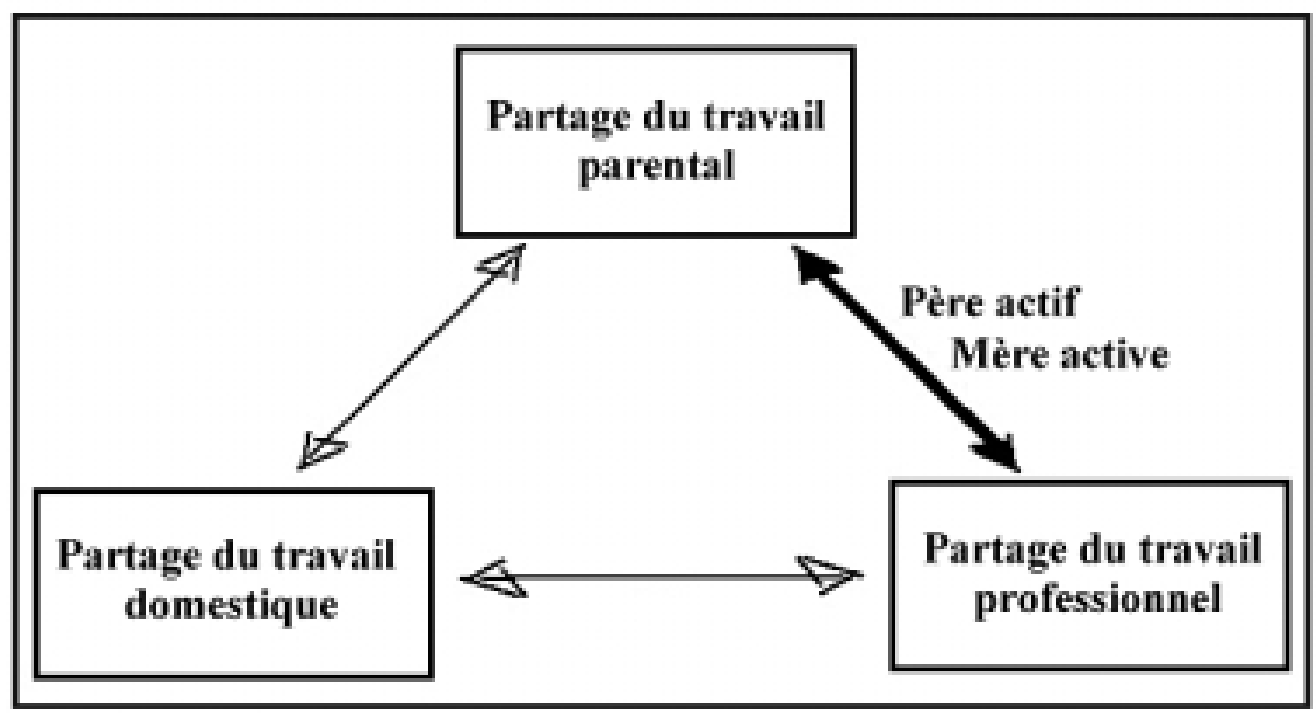

Historiquement, le premier mode de régulation du partage du «travail familial» (d'abord compris comme l'ensemble (indifférencié) des tâches domestiques) était fondé sur le statut de «mère au foyer» et de «l'homme actif» (jusque dans les années 1970).

Le second mode de régulation du «travail familial», toujours fondé sur une opposition entre le «domestique» et le «professionnel», était centré sur les statuts de la «travailleuse» [«femme active» pour l'Allemagne et de la «mère active» pour la France] et de «l'homme actif».

Enfin, depuis les années 1990, la régulation du partage familial (compris à partir du partage du travail parental, professionnel, et domestique) est centrée sur le statut de la «mère active» et du «père actif», en France comme en Allemagne.

C'est l'exercice de la parentalité conciliée qui est au centre de la régulation des rapports familiaux. Le statut de «parent actif», tant pour les pères que pour les mères, devient central. 


\section{CONCLUSION : LE PARENTALISME COMME MODE DE REGULATION?}

En ce début de XXIe siècle, les questions concernant la famille et sa place dans nos sociétés sont toujours d'actualité. Nombreuses sont les interrogations sur ses fonctions et son devenir dans une société confrontée à la «crise du marché du travail» et à la «crise de l'Etatprovidence» : la «flexibilisation» des horaires de travail des salarié(e)s et de leurs difficultés à «concilier la vie professionnelle et la vie familiale»; le problème de la dénatalité et de ses conséquences sur le financement des retraites ou le vieillissement de la population. Plus encore que le souci d'une «crise de la famille» qui annoncerait «la fin de cette institution», c'est l'existence et la fragilité du lien entre l'enfant et ses parents, première expression du «contrat de génération», qui est, plus que jamais, au centre des préoccupations (F.X Kaufmann 1997a). Dans ce contexte, l'objet de notre travail est de contribuer à expliquer les changements familiaux, les évolutions du lien entre la famille et le marché du travail, entre la famille et l'Etat-providence, en France et en Allemagne.

Introduction : Comment la parentalité metelle en lumière les changements

du lien familial?

Chapitre I : Le cadre théorique et les formes de parentalité : la parentalité exclusive et la parentalité conciliée

Chapitre II : Les normes de parentalité : la coparentalité française et la biparentalité allemande

Chapitre III : Les pratiques de parentalité dans les familles et le partage du travail familial

Conclusion : Le parentalisme comme mode de régulation?
Quel est le mode de régulation des rapports familiaux, des relations famille/marché du travail, et des liens famille/Etat-providence, dans les deux contextes sociétaux français et allemand?

Face aux récentes transformations familiales, qui mettent à mal les concepts classiques de la sociologie de la famille, nous proposons une nouvelle approche théorique qui contribue à expliquer les changements du lien familial : pour la première fois, ce n'est plus le couple conjugal, ni l'enfant, mais la relation entre l'enfant et le couple parental qui est au centre 
de l'analyse, comme indicateur des changements familiaux ${ }^{217}$. A cet effet, nous définissons le concept de parentalité ${ }^{218}$, comme l'ensemble des rapports sociaux entre l'enfant et ses parents et nous proposons d'étudier la façon dont la parentalité se construit, dans ses formes, ses norme et ses pratiques. Notre étude tient compte également des relations entre la famille et le marché du travail, entre la famille et l'Etat-providence, car elles sont des éléments constitutifs de la parentalité.

Nous avons alors établi une typologie des rapports entre l'enfant et ses parents suivant quatre catégories : une «parentalité bilatérale», une «parentalité unilatérale», une «parentalité première» et une «parentalité composée» (cf. Chapitre I). Les deux premières catégories sont fonction de l'existence ou non d'un couple parental pour l'enfant, les deux dernières, de l'origine biologique ou sociale de la relation. Elles permettent de restituer les situations de fait dans les familles et se distancient des dénominations classiques fondées sur la forme du lien conjugal (en terme de légitimité et d'illégitimité) et sur le statut juridique des individus (marié, célibataire, divorcé ou veuf).

Par ailleurs, nous avons vu que les modes de participation des parents au marché du travail et les mesures des politiques familiales construisent activement deux formes de parentalité : la «parentalité conciliée» et la «parentalité exclusive». La «parentalité conciliée» signifie que le parent exerce à la fois un rôle parental et professionnel et la «parentalité exclusive» que le parent remplit des fonctions parentales sans exercer de profession.

Le modèle de la «parentalité exclusive» est plus présent en l'Allemagne de l'Ouest, et la «parentalité conciliée» s'y effectue en référence au travail professionnel à temps partiel pour les mères. Dans l'ancienne Allemagne de l'Est et en France, la «parentalité conciliée» est comprise en référence au travail professionnel à temps complet, pour les pères et les mères. L'exercice de la «parentalité exclusive» est limité aux mères de jeunes enfants.

Dans les deux pays, la généralisation de la «parentalité conciliée» montre qu'il n'y a pas «une conciliation des rôles parentaux et professionnels», mais une accumulation des deux rôles pour les mères et pour les pères. De plus, les dernières mesures en politique familiale en France (comme le congé de paternité) et les nouveaux objectifs politiques en Allemagne (le développement des centres d'accueil) montrent que c'est la prise en compte du statut de parent pour les pères et les mères qui est aujourd'hui au centre de la régulation politique des rapports familiaux.

Les rapports sociaux entre l'enfant et ses parents sont construits socialement par le droit de la famille (cf. chapitre II) et par les politiques familiales (cf. Chapitre I et Chapitre II). Grâce à la comparaison sociétale, nous avons isolé deux variations différentes de la norme de parentalité que nous appelons la «coparentalité» française et la «biparentalité» allemande (cf. Chapitre II). La longue tradition allemande de non-ingérence par rapport à l'unité conjugale caractèrise la façon dont la parentalité est construite socialement: pendant longtemps, les arrangements parentaux (au sein de la famille unie ou désunie) relevaient strictement de la sphère privée. De fait, la notion de «biparentalité» exprime la prise en

\footnotetext{
${ }^{217}$ Le pouvoir explicatif de ce concept permet de mieux comprendre la relation de la famille au marché du travail et à l'Etat-providence, contribuant ainsi à une meilleure connaissance des changements sociaux dans leur ensemble.

${ }^{218}$ En Allemagne, la notion de parentalité («Elternschaft») est désormais passée dans le langage courant. Elle a été reprise dans le cadre des politiques familiales pour le développement des structures d'accueil des jeunes enfants mais également dans la campagne présidentielle (2002) du chancelier G. Schröder (cf. Bundeskanzleramt 2002), signifiant que la parentalité devient un objet de l'action publique.
} 
compte de la relation entre l'enfant et chacun de ces deux parents, et la non-intervention publique au niveau de l'arrangement parental. En revanche, en France, outre la régulation du lien entre l'enfant et ses deux parents, le couple parental est également l'objet de l'action publique suivant une norme de coopération parentale, nous parlerons alors de «coparentalité».

En France et en Allemagne, les parents exercent majoritairement une profession, ce qui contribue à une prise en compte de plus en plus forte de l'exercice de la parentalité dans le monde professionnel. Inversement, du point de vue de la famille, le professionnel est un travail qui se négocie, au même titre que les autres tâches.

La notion originale de «travail familial» que nous avons alors construite, exprime ce nouveau point de vue (cf. Chapitre III). Le «travail familial» regroupe l'ensemble des tâches qui sont effectuées «dans» et "pour» le ménage et qui relèvent du parental, du domestique et du professionnel. Aussi, la famille est le lieu de négociation du partage du «travail parental» (que nous avons isolé et mesuré pour la première fois en France en 1999 (Barrère-Maurisson, Rivier 2000)), du travail domestique, et du travail professionnel. Grâce à cette notion, il est de plus en plus évident que l'exercice de la parentalité dépend du partage du travail domestique, mais également de l'exercice d'une activité professionnelle. Le «travail familial» montre que l'opposition des sphères entre la famille et le professionnel est un construit théorique que nous avons remis ici en question.

Cette notion permet de comprendre en quoi le partage ne s'effectue pas uniquement en fonction des différences de genre, mais également en fonction de l'exercice de la parentalité : en d'autres termes, un partage entre les hommes et les femmes, mais surtout entre les pères et les mères, et ce, non plus comme un processus individuel et sexué, mais comme une négociation conjugale et parentale.

Suite aux nouveaux comportements familiaux et à l'apparition du divorce comme risque familial, la référence au conjugal s'est estompée et l'enfant est devenu l'unité de référence redéfinissant l'orientation du droit de la famille et des politiques familiales. Il convenait, dès lors, de préserver un droit à l'enfant à voir ses deux parents, qu'ils habitent ensemble ou non. Ainsi, les nouvelles orientations normatives sont centrées sur la «relation entre l'enfant et ses deux parents». C'est pourquoi, nous proposons d'appeler ce nouveau mode de régulation des rapports familiaux, le «parentalisme» (Barrère-Maurisson, Rivier 2002) ou «Parentalismus» en allemand. En accédant à ce degré d'abstraction, on met en évidence un modèle de compréhension qui n'est plus du registre de l'observation (Commaille, de Singly, 1997 ; Maurice 2000), mais une modélisation de la réalité sociale dépassant les différences sociétales.

Cependant, premièrement, la norme de parentalité prend des significations sociétales différentes en France en Allemagne : la «coparentalité» et la «biparentalité». Deuxièmement, en France, le parentalisme fait suite à une période de familialisme qui s'étend jusqu'à la fin des années 1960, puis au féminisme entre les années 1970 et 1980, pour émerger au début des années 1990. En Allemagne, le parentalisme prolonge une forme radicale de «conjugalisme» depuis la période d'après-guerre jusqu'au début des années 1980, puis une forme modérée de «conjugalisme» jusque dans les années 1980, pour apparaître au milieu des années 1990 (cf. Figure 29 en fin de conclusion). 


\section{LES FORMES, LES NORMES ET LES PRATIQUES DE PARENTALITE}

\section{Comment les évolutions socio-démographiques de la famille, les modes de participation au marché du travail et le cadre socio-politique déterminent-ils les formes de parentalité ?}

Nous avons défini la parentalité comme les rapports sociaux entre l'enfant et ses deux parents. Cette notion est au croisement des positions théoriques qui rendent compte des changements de la famille à partir du lien conjugal ou à partir du point de vue de l'enfant. Or, chacune d'entre elles se heurtent à une dynamique familiale de plus en plus complexe: il n'est plus possible de décrire la multiplication des formes de famille du seul point de vue du couple conjugal ; et les situations deviennent trop compliquées à saisir du seul point de vue l'enfant. Désormais, la complexité des relations familiales, par dissociation des processus de conjugalité et de filiation mais aussi par recomposition, conduit à un nécessaire renversement de perspective.

On doit ainsi passer d'une catégorisation de la famille fondée sur le couple (qui tentait ensuite de mettre en relation les parents et les enfants) et d'une catégorisation où l'enfant est au centre (confronté à la multiplication des situations), à une catégorisation où la parentalité est la nouvelle unité de référence. En cela, on dépasse deux contradictions théoriques : premièrement, le fait que la conjugalité se dissocie de la filiation, et deuxièmement, l'affirmation que l'enfant appartienne à une seule et unique famille tandis que ses liens avec les deux parents restent indissociables.

Or, la parentalité rend compte de ces situations complexes car elle tient compte du fait que l'enfant pourra, à un moment donné, appartenir à différentes unités familiales. Elle intègre donc l'exercice concomitant de différentes formes de parentalité et permet ainsi de saisir le déroulement des séquences de l'histoire familiale d'une manière dynamique.

Les rapports entre l'enfant et ses parents se regroupent en quatre catégories qui se fondent sur deux caractéristiques des rapports sociaux au sein des familles actuelles : la première est fonction de l'existence ou non d'un couple parental, la seconde décrit l'origine biologique ou sociale de la relation à l'enfant. Nous avons alors identifié quatre formes d'exercice de la parentalité : une «parentalité bilatérale» lorsque l'enfant entretient des relations avec les deux parents (que le couple soit uni ou désuni), une «parentalité unilatérale» lorsque la séparation conjugale se sanctionne par la perte de contact avec le deuxième parent, une "parentalité première» lorsque l'enfant vit toujours avec le couple parental d'origine et une «parentalité composée» où le biologique et le social sont mêlés pour l'enfant (cf. Chapitre I).

Nous avons alors montré que ces catégories permettent de définir une typologie des formes de familles qui est proche des pratiques sociologiques des individus ${ }^{219}$. On voit alors, par exemple, que les couples développent de nouvelles stratégies d'institutionnalisation de l'exercice de la «parentalité bilatérale» (par les reconnaissances paternelles et les reconnaissances conjointes prénatales) qui viennent s'ajouter aux comportements de légitimation de la filiation par le mariage.

\footnotetext{
${ }^{219}$ Elles se distancient des dénominations classiques fondées sur la forme du lien conjugal (en terme de légitimité et d'illégitimité) et du statut juridique des individus (marié, célibataire, divorcé ou veuf).
} 
Les modes de participation des parents au marché du travail, construisent, depuis le point de vue de la famille, deux modes d'exercice de la parentalité : la «parentalité conciliée», qui signifie que le parent exerce à la fois un rôle parental et professionnel, et la «parentalité exclusive» qui indique que le parent exerçe des fonctions parentales mais pas d'activité professionnelle.

Le modèle de la «parentalité exclusive» est plus représenté en l'Allemagne de l'Ouest, il correspond à «mode temporaire» ${ }^{220}$ dans la biographie professionnelle, mais également à un «mode durable». La «parentalité conciliée» pour les mères s'y effectue en référence au travail professionnel à temps partiel. Dans l'ancienne Allemagne de l'Est, la «parentalité conciliée» est comprise en référence au travail professionnel à temps complet pour les pères et les mères. L'exercice de la «parentalité exclusive» est limité aux mères de jeunes enfants de moins de trois ans. On retrouve une situation similaire en France.

Nous prolongeons les modèles d'un couple à un apporteur principal, à 2 apporteurs équivalents et à deux apporteurs inéquivalents (Ostner 1995) qui ont montré que la répartition du travail professionnel à l'intérieur des familles est une stratégie de couple fondée sur «la division familiale du travail» (Barrère-Maurisson 1992). Ainsi, l'évolution de la participation des parents au marché du travail montre l'apparition de différentes constellations : un modèle à un parent comme apporteur principal, un modèle à deux parents apporteurs suivant la sousdistinction entre apporteurs équivalents ou inéquivalents.

Nous avons alors montré que l'exercice du travail parental pouvait être exclusif pour les femmes, et toujours une forme conciliée pour les hommes. La conciliation du travail professionnel et parental n'est plus un problème féminin et privé (Théry 1998), mais concerne les pères, les mères, le couple parental et la collectivité. Dans les deux pays, la généralisation de la "parentalité conciliée» montre ainsi qu'il n'y a pas une «conciliation» des rôles parentaux et professionnels, mais une accumulation des deux rôles pour les mères et pour les pères.

De plus, l'Etat-providence, par les différentes mesures de politique familiale, construit les rapports entre l'enfant et ses parents, à trois niveaux différents :

Le premier niveau concerne les mesures directes pour l'aide à la prise en charge de la présence et de l'entretien de l'enfant : les congés de maternité et de paternité en France, ou une large reconnaissance des relations de parentalité d'un parent avec ses enfants dans le calcul des allocations familiales comme en Allemagne.

Le second niveau des mesures favorise une «parentalité exclusive». Cette orientation est plus marquée en Allemagne où le congé parental est plus généreux. Cependant, la «parentalité exclusive» en tant que mode stable d'exercice de la parentalité n'y est plus majoritaire.

Enfin, le troisième niveau d'intervention concerne l'ensemble des mesures de conciliation entre la famille et le marché du travail en favorisant la délégation du travail parental. Cette dernière est très largement instituée en France pour les jeunes enfants (ou les très jeunes enfants). Elle est restreinte voire inexistante en Allemagne (à l'exception de l'Allemagne de l'Est).

Les catégories «de parentalité conciliée» et de «parentalité exclusive» ont permis de montrer qu'il $y$ avait au sein de la société française une prise en compte de la

\footnotetext{
${ }^{220}$ par l'intermédiaire du congé parental d'éducation (limité le plus souvent à trois ans)
} 
«parentalité conciliée» des pères. En Allemagne, à l'heure actuelle, on prend simultanément en compte la "parentalité conciliée» des mères et des pères. Le modèle d'une famille à deux «parentalités conciliées» s'affirme en France, il émerge en Allemagne. Aujourd'hui, c'est la prise en compte du statut de parent, pour les pères et les mères qui est au centre des dernières mesures en politique familiale : le congé de paternité en France, le développement des centres d'accueil et les campagnes récentes d'opinion pour la reconnaissance de la «parentalité conciliée» des pères en Allemagne.

Comment se définissent les modes d'exercice de la parentalité en France et en Allemagne pour les familles unies et désunies : quel est le traitement social du risque familial ?

La construction du rapport entre l'enfant et ses deux parents est au centre des politiques familiales et du droit de la famille. La forme de la protection sociale du risque familial montre l'émergence d'une norme de «parentalité bilatérale» centrée sur le couple parental (l'institutionnalisation de la parenté dépasse des distinctions de genre, et favorise le consensus social sur l'égalité des droits et des devoirs des pères et des mères). La régulation de ce risque incite les parents de familles désunies et recomposées à maintenir le contact avec l'enfant. C'est l'existence ou non du lien entre le parent et l'enfant qui est décisif, peu si les familles sont unies ou séparées, légitimes ou naturelles, cohabitantes ou non. L'exercice d'une «parentalité unilatérale» devient alors une exception, qui, en prenant une place marginale renforce d'autant plus la force normative de la «parentalité bilatérale». Sur ce principe commun de la reconnaissance de la parentalité comme le référent social de l'organisation des rapports familiaux, deux variations sociétales se dégagent : la «coparentalité» française qui insiste sur les rapports de coopération éventuelle entre les parents et un partage égalitaire des tâches parentales, et la «biparentalité» allemande qui se caractérisée par un objectif de noningérence quant à l'organisation des rôles parentaux, à l'image de la réticence du législateur allemand à réguler les rapports de sexe dans le couple conjugal. On observe une complémentarité des rôles, autour de la prédominance encore forte de la femme (la priorité féminine dans l'exercice de la parentalité pouvant justifier une pratique unilatérale) (cf. Chapitre II). Mais les différences sociétales entre la France et l'Allemagne portent sur les termes de l'arrangement parental, pas sur son existence.

L'étude de la parentalité montre la capacité d'innovation des familles contemporaines dans la création du lien parental. Inversement, les familles suivent un ensemble de prescriptions sociales qui régulent les comportements familiaux, seule la forme de cette régulation a changé. En d'autres termes, le recentrage de l'analyse, non plus sur le lien conjugal, mais sur la forme des rapports entre les parents et les enfants, a fait émerger un nombre important de continuités. Les phénomènes du «démariage» modifient directement les rapports entre les parents et les enfants (on rappelle que les formes actuelles de parentalité en sont le produit («unilatérale/bilatérale», «première/composée»)) mais ne les font pas pour autant disparaître. Deux formes sociétales de régulation du changement familial apparaissent en France et en Allemagne: le modèle français s'articule autour de la norme de «coparentalité»; le modèle allemand est centré sur la «biparentalité». 
Quels déterminants influencent les modes d'exercice de la parentalité dans les familles ? Pourquoi la parentalité est-elle indissociable de la manière dont la famille partage le travail domestique et professionnel ?

Les pratiques des familles déterminent la manière dont la parentalité devient une réalité sociale ; à ce titre, nous avons choisi d'étudier le partage des tâches parentales dans les couples. Pour cela, nous définissons le «travail parental» comme l'ensemble des activités effectuées «pour» et «en présence» de l'enfant, que nous distinguons en quatre catégories, en fonction de la nature des tâches : la «sociabilité parentale», le «travail parental 'taxi'», le «travail parental domestique» et le «travail parental scolaire» (Barrère-Maurisson, Marchand, Rivier 2000) (cf. Chapitre III).

Après une longue période de «répartition traditionnelle des rôles», les comportements au sein de la famille changent au cours des années 1970, modifiant les mécanismes de partage dans les années 1980. On observe alors, en France et en Allemagne, une tendance générale vers une répartition plus égalitaire du parental (malgré des inégalités encore persistantes).

Ces changements de pratiques sont dus essentiellement à la participation plus importante des pères, entre les années 1980 et 1990. La différence d'investissement des pères et des mères dans le parental montrent encore de grands écarts, mais la «sociabilité parentale» est presque répartie de façon égalitaire et les tâches «parentales domestiques» commencent aussi à se partager (alors qu'elles étaient très exclusives auparavant).

Dans la famille, le professionnel n'est plus l'exclusivité des hommes, même s'il reste inégalitaire. La participation grandissante des pères au parental et la participation de plus en plus généralisée des mères au professionnel définissent les nouvelles pratiques de partage. Le professionnel et le parental ne sont plus le domaine réservé d'un «genre» particulier.

Le partage du domestique est lui-aussi en mouvement, mais bien moins que les autres sphères parentale et professionnelle. La participation des hommes au domestique a augmenté au cours des années 1980 (sous l'influence de la cohabitation et de l'activité professionnelle des femmes). En France, la femme a réduit sa participation au cours des années 1990 (ce qui ne s'était jamais produit).

Ainsi, nous avons montré que le parental prend une valeur en soit, qu'il est l'objet d'un partage entre les pères et les mères, non plus en fonction d'une logique de différenciation des rôles, mais en fonction d'un partage des tâches (même si les inégalités et la spécialisation entres les sexes sont encore importantes). Au niveau des pratiques, on est encore loin d'un exercice paritaire de la parentalité, mais les attitudes évoluent depuis un modèle institué sur un partage sexué des tâches, vers un modèle de partage où la place relative des parents est renégociée en fonction du principe d'égalité.

Il apparaît alors que la famille est le lieu de négociation du partage du «travail parental», du travail domestique et du travail professionnel, nous parlons alors de «travail familial» (cf. Chapitre III). La notion de «travail familial» illustre le parti pris selon lequel le travail est défini à partir de la sphère familiale, lieu de négociation, et non plus seulement à partir du marché du travail, lieu de définition du travail professionnel. En d'autres termes, on parlera de «travail familial» pour exprimer la négociation du partage de l'ensemble des tâches au sein de la famille, tout en tenant compte du lien au marché du travail : le travail professionnel n'est plus une notion exogène à la famille, mais bien une masse de travail qui y est négociée pour être partagée. En distinguant ces trois grandes catégories d'activités familiales, nous évitons de les hiérarchiser et de comprendre le 
partage uniquement en référence au professionnel. Cette position permet aussi de dépasser les oppositions en termes de genre (qui différencie des rôles masculins et les rôles féminins) et en termes de «sphères» (qui oppose la «famille»au «marché du travail»).

Historiquement, le premier mode de régulation du partage du travail familial était fondé sur le statut de «mère au foyer» et de «l'homme actif» (jusque dans les années 1970). Le second mode de régulation du «travail familial», toujours fondé sur une opposition entre le «domestique» et le «professionnel», il était centré sur les statuts de la «travailleuse» («femme active» pour l'Allemagne et de la «mère active» pour la France) et de «l'homme actif» (comme le montre les études contrastées des régimes de protection sociale (Martin 1999; Ostner 2001)). Aujourd'hui, c'est le lien entre le professionnel et le parental qui est au centre de la régulation du partage du «travail familial», il est relatif aux statuts de «parent actif» pour les deux parents.

Au cours des trois chapitres précédents, on a vu que les rapports entre l'enfant et ses parents sont au centre du lien familial. Les formes, les normes et les pratiques de parentalité qui le constituent, sont construites par la famille, le marché du travail, l'Etat-providence et leurs relations respectives. La régulation de ce processus de construction sociale de la parentalité (par les différents acteurs sociaux) prend aujourd'hui la forme particulière du «parentalisme». 


\section{LE FAMILIALISME, LE FEMINISME ET LE PARENTALISME COMME MODES DE REGULATION EN FRANCE}

Les années 1950 représentent une période idéale typique du partage sexué des rôles au sein des familles. La répartition du «travail familial» reconnaissait une séparation stricte entre les sphères de la production et de la reproduction, lesquelles étaient le domaine réservé d'un membre du couple. Les années 1950, «l'âge d'or du familialisme» (Lenoir 1985a, 1985b), connaissaient un mode de régulation des rapports familiaux, basé sur le lien conjugal légitime, la parentalité s'y inscrivant de façon quasiment exclusive.

Entre la période d'après-guerre et les années 1970, la famille est comprise comme une institution fondée sur l'autorité du chef de famille ; l'homme est le seul actif prioritaire. Les rapports de générations sont fondés sur des rapports d'allégeance.

Le «familialisme», morale de la vie sociale, a ses bases dans l'influence de l'Eglise, dans l'entreprise familiale comme lieu de production, et dans l'exclusion des femmes de la scolarisation et du travail professionnel. Ce courant prône la famille nombreuse, l'ordre moral fondé sur le respect des hiérarchies, la tradition chrétienne, la morale catholique, la propriété, la femme comme épouse et mère, les valeurs du foyer (Lenoir 1985a). Les mesures développées au cours de la première période des Trente Glorieuses ont été justifiées par la promotion des familles d'au moins trois enfants et le combat des tendances démographiques de dénatalité. Puis, le «familialisme» (Lenoir 1991:176) s'est centré sur le maintien et la préservation de la famille à deux enfants, par des mesures diverses (fiscales, aide au logement, etc...), suite à la baisse régulière du taux de fécondité. Le risque familial se définissait alors autour de deux dimensions : préserver la natalité et consolider la famille nucléaire.

Avec les changements qui s'amorcent à la fin des années 1960, les «bases sociales du familialisme» se trouvent définitivement ébranlées (Lenoir 1985b). Les changements dans les rapports de sexe, la montée des valeurs égalitaires, la participation des femmes au marché du travail, ont contribué à changer le mode de régulation des rapports sociaux. En France, la politique familiale, tout d'abord axée sur un objectif nataliste mettant au premier plan une position «familialiste», s'oriente désormais vers la protection de la femme, sous l'influence de ces évolutions qui ont transformé la famille et le marché du travail.

Ainsi, l'Etat cessait de conformer les familles à un modèle unique dont l'évolution sociale s'éloignait, bousculé par le développement du travail féminin et l'acceptation encore timide des familles monoparentales : la politique des transferts sociaux prenait le pas sur celle de péréquation des charges familiales (Prost 1984 : 21) ; l'unité conjugale est devenue une valeur en soi, plaçant les rapports de genre au centre de la société.

Au début des années 1970, les femmes sont de plus en plus présentes sur le marché du travail, le partage du travail professionnel devient majoritairement un modèle à un actif et demi (données du recensement de 1975 et de 1982). Les rapports d'autorité conjugale, basés sur la subordination légale et sociale de la femme (au foyer), sont remis en question. La montée des considérations égalitaires, oriente l'action politique vers une moindre discrimination de la femme qui devient l'objet des politiques publiques (Commaille 1992). Avec le développement des divorces, la politique familiale s'est centrée sur la défense des droits de la femme : femme seule («isolée»), célibataire et surtout «femme chef de famille» de ce que l'on a alors appelé «les familles monoparentales». On parlera alors d'une période de 
«féminisme» pour qualifier la politique familiale des années 1970 et 1980 (Commaille 1996 : 126). L'attention est portée au couple et à sa non-existence éventuelle (l'Etat jouant là un rôle de subsidiarité par rapport à la solidarité du couple), plutôt qu'à la famille comme lieu de reproduction. Le «féminisme», mode de régulation des rapports sociaux dans les années 19701980, place les différences de genre, unité constitutive de la famille conjugale, au cœur des relations sociales.

L'éclatement des formes traditionnelles de famille a progressivement amené la société à se préoccuper non plus de la situation de la femme comme objet de risque, mais de celle de l'enfant. Suite aux nouveaux comportements familiaux et à l'apparition du divorce, la référence au conjugal s'est estompée dans les années 1990, et l'enfant est devenu l'unité de référence, redéfinissant l'orientation du droit et des politiques familiales. Il convenait dès lors de préserver à l'enfant un droit à avoir ses deux parents, qu'ils cohabitent ou non. Ainsi, les nouvelles orientations de la politique familiale sont centrées sur la relation entre l'enfant et ses parents dans un mouvement de «retour du politique à propos de la famille» (Martin 1998). Nous proposons d'appeler ce nouveau mode de régulation des rapports familiaux le «parentalisme». Aussi, nous sommes passé du «féminisme» au «parentalisme» lorsque l'enfant a été reconnu comme l'unité de référence de notre société, plaçant la parentalité au cœur des rapports familiaux. L'entrée dans le parentalisme dans les années 1990 est conséquente à une réflexion sur les rapports de filiation, sur les conséquences des désunions familiales (Théry 1998, Dekeuwer-Defossez 1999) et de la manière dont l'intérêt de l'enfant domine l'organisation de la famille dissociée (Théry 1993 : 143).

On s'est alors interrogé sur la place relative de la mère, du père et de l'enfant au sein des familles (Castelain-Meunier 1998, Neyrand 2001). La participation des familles au marché du travail, a poussé à reconnaître l'égalité des sexes qui devient un fait acquis au niveau normatif et de plus en plus, au niveau des pratiques. Le modèle de partage du travail familial est celui du couple à deux actifs équivalents. "Le rôle professionnel accru des femmes, même s'il n'a pas modifié sensiblement leur place dans les rapports domestiques a, au moins dans les esprits, affecté celle des hommes. Ces derniers ne sont plus désormais les seuls à travailler à l'extérieur, ce qui en théorie ne les exonèrent plus aussi légitimement des affaires domestiques. » (Blöss 2001 : 56). La question de l'égalité des rôles et des fonctions entre les hommes et les femmes, d'abord comprise à partir du marché professionnel, a aujourd'hui pour signification une égalité des rôles et des fonctions domestiques et parentales.

Le caractère indissoluble du couple mère-enfant, crée à partir de la deuxième moitié du XXe siècle pour promouvoir les options natalistes de l'état et protéger l'enfant (par la garantie de la pérennité de ce lien, en particulier face au risque du divorce) (Blöss 2001 : 63) est largement critiquée. Cette remise en cause du lien indissoluble mère-enfant ou de «l'idéologie du maternage» (Norvez (1990) (citée par Blöss 2001 : 63)) est une des caractéristiques de la nouvelle régulation. La notion de travail parental et sa mesure, l'égalitarisme parental, l'investissement des «nouveaux pères», montrent que l'analyse ne se fait plus en terme de genre, comme sous le «féminisme», mais en terme de parental (père/mère).

Il est important de souligner que cette tendance vers l'égalité des rapports parentaux, s'accompagne de nombreux mécanismes de reproduction de l'inégalité parentale, dont l'action publique, elle-même, à travers l'institutionnalisation active de la dominance du lien maternel, depuis la moitié du XXe siècle (Blöss 2001). Mais les nouvelles orientations 
politiques concrétisent la logique égalitaire du parental par la valorisation de la paternité, en particulier la réforme récente du congé de paternité.

Avec l'entrée dans le «parentalisme» dans les années 1990, ce n'est plus la famille légitime qui fonde les rapports familiaux et sociaux, ni les rapports de genre et avec eux les différenciations de sexe qui forment l'unité centrale de la famille, mais les rapports de l'enfant à ses deux parents ${ }^{221}$. La famille se comprend alors, non plus en fonction de la conjugalité ou de la filiation, mais à partir de leur relation respective dans la parentalité.

En résumé, après une période où les préoccupations liées à la famille déterminaient le FAMILIALISME comme un mode de régulation des changements sociaux, après le FEMINISME fondé sur les intérêts de la femme et sa place dans la société, on a vu, depuis les années 1980, se déplacer de nouveau le sens des interactions institutionnelles vers l'enfant : désormais, c'est le rapport entre l'enfant et ses parents qui définit les nouvelles orientations du mode de régulation institutionnelle, ce que nous avons appelé le PARENTALISME (cf. Figure 29). Il régule les interactions sociales, à un niveau microsociologique (l'enfant), mesosociologique (entre les parents) et macrosociologique (entre la famille, le marché du travail et l'Etat).

\footnotetext{
${ }^{221}$ Au temps du «familialisme», la famille était institutionnalisée autour de l'autorité paternelle. Puis à l'époque du «féminisme», elle s'est fondée sur l'autorité conjugale. Aujourd'hui sous le «parentalisme», elle est organisée à travers l'autorité parentale (et particulièrement du principe de l'autorité parentale conjointe à partir de 1993). De la même manière, le risque familial, qui détermine les orientations de l'action publique, a changé de signification. Le risque familial se définissait à partir des objectifs natalistes et de la consolidation de la famille nucléaire sous le «familialisme», puis à partir de la protection de la femme face au divorce sous le «féminisme». Désormais, il concerne la protection de l'enfant face aux conséquences des séparations parentales et en particulier au maintien des relations de l'enfant avec ses deux parents.
} 


\section{LE CONJUGALISME ET LE PARENTALISME COMME MODES DE REGULATION EN ALLEMAGNE}

En Allemagne, le régime national-socialiste a fait subir une inflexion à l'organisation des rapports sociaux et familiaux, faisant de la cellule familiale un lieu de reproduction dépossédée des fonctions de socialisation, d'éducation et d'élevage de l'enfant qui relevaient alors de l'Etat. Cette période a profondément marqué l'orientation des politiques publiques en Allemagne. En réaction au régime totalitaire, la non-ingérence de l'Etat par rapport à la sphère familiale devient un consensus social (Bast, Ostner 1992).

Par la suite, il est renforcé par une forte distanciation politique par rapport au régime socialiste de l'Allemagne de l'Est fondé également sur l'instrumentalisation de la cellule familiale. Le couple conjugal (dans sa version légitime) est alors érigé en valeur sociale, l'action publique optant pour une non-ingérence dans les arrangements familiaux et conjugaux et a fortiori parentaux. Ne cherchant donc pas, comme en France, à établir des rapports égalitaires de genre, l'action publique obéit au principe implicite de non-ingérence.

Aussi, depuis la sortie de la guerre jusqu'aux début des années 1980, le mode de régulation des rapports sociaux a pris la forme du «conjugalisme», au sens de Schultheis : la forme politique «conjugaliste» de la famille place son point fort sur le couple marié, la France a une politique familialiste au sens de «pro-famille» (Schultheis : 150).

La première période du «conjugalisme», plus radicale, s'étend de l'après-guerre jusqu'aux années 1980, la seconde, plus modérée, des années 1980 au milieu des années 1990. La valeur sociale du couple conjugal légitime, subordonnée à une distinction et une complémentarité des sexes, se prolonge jusque dans les années 1980.

Puis, les séparations et les remises en couple se font de plus en plus nombreuses au cours des années 1980. Le risque familial du divorce se généralise. Les mères participent au marché du travail suivant la norme du temps partiel et le modèle à un actif et demi devient plus commun. A la même période, la place des pères dans les familles est rediscutée. En 1989, la réunification a contribué à éroder davantage la valeur sociale du couple légitime. En revanche, elle a peu changé les comportements démographiques de l'ancienne Allemagne de l'Ouest. Pour, l'Allemagne de l'Est, les comportements de divorce, de séparation et de natalité se sont modifiés. Seules les familles naturelles se sont maintenues avec leur comportement de natalité spécifique. Depuis le milieu des années 1990, nous sommes alors entrés dans un nouveau mode de régulation, celui du «parentalisme». On est passé définitvement d'une référence au couple légitime sous le «conjugalisme», à une centralité de l'enfant de plus en plus importante dans les rapports sociaux.

Aujourd'hui, depuis les années 1990 en France et du milieu des années 1990 en Allemagne, le «parentalisme» place la parentalité au centre de la régulation des rapports familiaux. La construction des rapports entre l'enfant et ses parents, quelle que soit la forme familiale, est au centre de la régulation politique et juridique. Mais cette dernière prend deux formes sociétales différentes, la «coparentalité» et la «biparentalité» (cf. Figure 29). 
Figure $29 \quad$ Les modes de régulation des rapports sociaux en France et en Allemagne

France

L’unité conjugale

FAMILIALISME

[1945-70]

L'institution familiale -

Le couple légitime

Les rapports

égalitaires de genre

Le travail

professionnel des

femmes

FEMINISME

[1970-80]

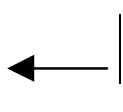

La femme
Allemagne*

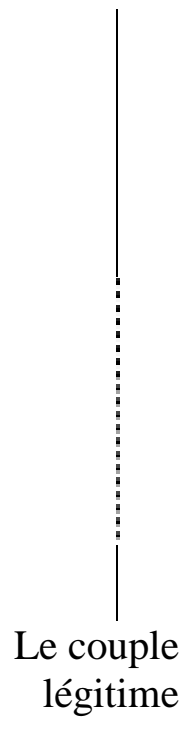

LA PERIODE NATIONALE-

SOCIALISTE [1933-45]

Régime autoritaire

d'organisation des rapports

sociaux

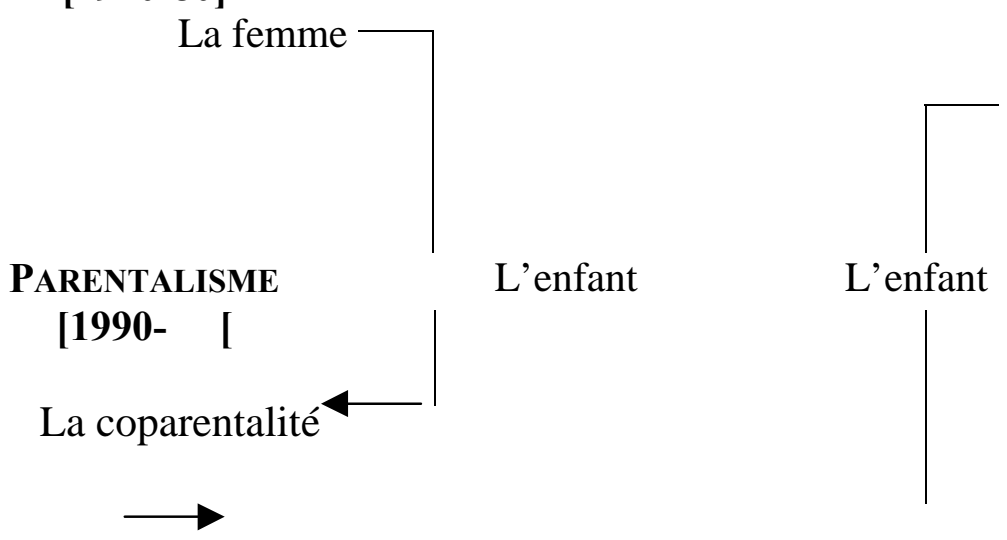

[1980-1989-1995]

\section{Parentalisme \\ [1995- [}

La biparentalité

* La figure 29 représente les évolutions des modes de régulation des rapports sociaux dans l'ancienne Allemagne de l'Ouest, puis depuis la réunification, celles de l'Allemagne réunifiée. 
La «coparentalité» française se définit à partir de la généralisation, pour l'ensemble des individus, d'un exercice de «parentalité conciliée». Le partage du travail parental se discute en terme de répartition égalitaire, relayée par un discours contemporain sur l'égalité professionnelle. La norme de coparentalité sous-entend le principe de coopération entre les deux parents, qu'ils soient séparés ou non, il devient un objet de l'action publique en soi. L'ingérence publique avait placé les discriminations de sexe et la femme comme objet politique sous le «féminisme». De la même manière, le père est devenu l'objet des politiques familiales (comme dans le cas du congé de paternité) sous le «parentalisme».

En Allemange, la norme de «biparentalité» sous-entend que c'est alors la relation de l'enfant à chacun de ses deux parents qui est l'objet de l'action publique et non le couple parental. (même implicitement comme en France). Effectivement, d'un côté l'enfant est une valeur sociale en soi et la parentalité est l'objet des politiques familiales et du droit. De l'autre, il y a une non-ingérence par rapport au couple conjugal. Le système de politique familiale favorise d'abord un exercice de la parentalité exclusive. De fait, l'intervention de l'état se fait encore en faveur de la mère, néanmoins, de façon moins marquée, remettant en question la différenciation stricte des fonctions au sein du couple parental.

Au tournant du XXe siècle, le «parentalisme»s'inscrit en France et en Allemagne comme le mode de régulation des rapports familiaux. Or, les relations entre la famille et le marché du travail mais également entre la famille et l'Etat-providence, institutionnalisent un mode précis d'exercice de parentalité. On voit donc dès à présent que le «parentalisme» régule un ensemble de relations sociales qui ne relèvent plus uniquement de la stricte sphère familiale.

«Si le «parentalisme» est en mesure de s'ériger en mode de régulation sociale, c'est aussi parce qu'il rend compte des évolutions, non seulement familiales, mais dans les places et les rôles respectifs des hommes et des femmes. La plus grande égalisation des rôles et des charges entre les deux sexes en est un signe. Et les politiques ne peuvent qu'accompagner la parité parentale et domestique, comme elles ont accompagné dernièrement la parité professionnelle ; par le développement de l'accueil à la petite enfance (de manière publique, associative ou entreprenariale), de l'aide aux services domestiques (par les chèques emploisservices, les chèques services et les services domestiques sur le lieu de travail). Ainsi tendent à se mettre en place de plus en plus des convergences entre les différentes politiques (familiales, sociales, d'emploi, d'entreprise, d'égalité, des temps) autour d'un nouveau consensus: la garantie de la parentalité, expression de l'égalité entre les hommes et les femmes dans un souci de pérennité et d'équilibre social »(Barrère-Maurisson, Rivier 2002). En Allemagne, la thématisation des politiques des temps des villes (Mückenberger 2001), l'annonce d'une politique familiale axée sur le développement des modes de garde $^{222}$ (Bundeskanzleramt 2002) semblent alors s'interprèter comme les premiers signes d'une prise en compte du statut de «parent» dans l'ensemble des sphères sociales, quelque soit le sexe des individus.

\footnotetext{
222 A l'occasion de la campagne présidentielle, le candidat G. Schröder (parti socialiste), actuellement en fonction, a été le premier chancelier allemand à discuter explicitement d'une politique familiale.
} 


\section{BIBLIOGRAPHIE}

\section{OUVRAGES GENERAUX ET SPECIALISES}

BARRERE-MAURISSON, Marie-Agnès, 1992: La division familiale du travail - La vie en double, Paris : Presses Universitaires de France, Collection "Economie en liberté"

BLÄTtEl-MinK, Birgit ; KRAMER, Caroline ; MisCHAU, Anina, 2000 : Innerfamiliäre Arbeitsteilung - Wunsch und Wirklichkeit - Die Sicht der Frauen, Wechselwirkungen, Jahrbuch aus Lehre und Forschung der Universität Stuttgart

BLASIUS, Dirk, 1987 : Ehescheidung in Deutschland 1794-1945 : Scheidung und Scheidungsrecht in historischer Perspektive, Göttingen : Vandenhoeck u. Ruprecht

BORRILlO, Daniel ; FASSIN, Eric, (sous la direction de) 1999 : Au delà du PACS - L'expertise familiale à l'épreuve de l'homosexualité, Paris : Presses Universitaires de France

Burguiere, André ; Klapisch-Zuber, Christiane ; Segalen, Martine ; Zonabend, Françoise, 1986 : Histoire de la famille - 3. Le choc des modernités, Paris : Armand Colin

Castelain-Meunier, Christine, 1998 : Pères, mères, enfants, Collection Dominos, Flammarion

CASTELAIN-MEUNIER, Christine, 1992 : Cramponnez-vous les pères - les hommes face à leur femme et à leurs enfants, Paris : Albin Michel

COEnEn-Huther, Josette ; Kellerhals, Jean ; Allmen, Malik (von), 1994 : Les réseaux de solidarité dans la famille, Lausanne : Edition réalités sociales

Commaille, Jacques ; MARTIN, Claude, 1998: Les enjeux politiques de la famille, Coll. "Société", Paris : Bayard Éditions

CommaILle, Jacques, 1996 : Misère de la famille, question d'Etat, Paris : Presse de Sciences Po

Commaille, Jacques, 1994 : L'esprit sociologique des lois, Thèse de doctorat Paris I, Paris : Presses Universitaires de France

DANIEL, Christine ; PALIER, Bruno, 2002 : La protection sociale en Europe, le temps des réformes, Paris : La documentation

DEKEUWER-DEFOSSEZ, Françoise, 1999 : Rénover le droit de la famille - Propositions pour un droit adapté aux réalités et aux aspirations de notre temps, Rapport au Garde des Sceaux, Ministre de la Justice, Collection des rapports officiels, Paris : La Documentation Française, Éditions O. Jacob

ESPING-ANDERSEN, Gøsta, 1990 : The Three Worlds of Welfare Capitalism, Cambridge : Polity Press

FAGNANI, Jeanne, 2001 : Un travail et des enfants : Petit arbitrage et grand dilemme, Paris : Bayard Edition

FEnet, Francine ; LEPRINCE, Frédérique; PERIER, Liliane, 2001 : Les modes d'accueil des jeunes enfants - Concilier vie familiale, vie professionnelle et vie sociale, Actualités sociales hebdomadaires, ${ }^{\circ}$ 187-189, Supplément septembre 2001

HACK-UNTERKIRCHER, Elke, 1976: Der organisierbare Haushalt. Grundlagen der Einzelwirtschaftslehre des privaten Haushalts, Essen

HERPIN, Nicolas ; OLIER, Lucile, 1997 : Les familles monoparentales: Aidées, mais fragilisées - France, Portrait Social 1997-1998, Paris : INSEE 
INSEE, 2000 : Enquête emploi, Paris

INSEE, 1999 : Données sociales, Paris

INSEE, 1995 : Les femmes. Portrait social, collection contours et caractères, avec le service des droits des femmes, Paris

INSEE, 1994: Les familles monoparentales, Portrait Social, Contours et caractères, INSEE,Paris : Hachette

INSEE, 1992 : Enquête Emploi, Paris

INSEE, 1989 : Enquête Budget Famille, Paris

JENSON, Jane, 2000 : L'Enfant citoyen ? Les politiques sociales et les familles, Lien social et politiques, $\mathrm{n}^{\circ} 44$

KaufMann, Franz-Xaver, 1997a : Herausforderungen des Sozialstaates, Frankfurt/Main : Suhrkamp

KaufMann, Franz-Xaver, 1996 : Modernisierungsschübe, Familie und Sozialstaat, München : R. Oldenbourg Verlag

KAUFMANN, Franz-Xaver, 1995 : Zukunft der Familie im vereinten Deutschland : gesellschaftliche und politische Bedingungen, München : Beck

KAUFMANN, Jean-Claude, 1992: La trame conjugale - Analyse du couple par son linge, Essais et Recherches, Paris : Nathan

KÖNIG, René, 1946 : Materialien zur Soziologie der Familie, Bern

Kontos, Silvia ; WALSER, Karin, 1979 : ...weil nur zählt, was Geld einbringt. Probleme der Hausfrauenarbeit, Gelnhausen, Berlin, Stein

KOPPETSCH, Cornelia ; BURKART, Günter, 1999 : Die Illusion der Emanzipation : zur Wirksamkeit latenter Geschlechtsnormen im Milieuvergleich, Koppetsch, C. (éd.), Konstanz : UVK

LAMPERT, Heinz, 1996 : Priorität für die Familie - Plädoyer für ein rationale Familienpolitik, Soziale Orientierung, Band 10, Berlin : Duncker \& Humblot

LE PLAY, Pierre, 1875 : L'Organisation de la famille selon le vrai modèle signalé par l'histoire de toutes les races et de tous les temps, Paris - Tours

LEGALl, Didier ; MARTIN, Claude, 1987 : Les familles monoparentales - Evolution et traitement social, Paris : Les éditions ESF

LimBACH, Jutta, 1988 : Familie ohne Ehe, Schrift des Deutschen Juristinnenbundes, Band 3, Limbach, J. ; Schwenzer, I. (éds.), Frankfurt/Main; München : Schweitzer

LIPPOLD, Gerhard [et al.], 1971 : Das Zeitbudget der Bevölkerung, Schriftenreihe Planung und Leitung der Volkswirtschaft, Heft 42, Berlin (Ost) : Verlag Die Wirtschaft

MARTIN, Claude, 1996 : Qui doit nourrir l'enfant quand les parents sont séparés ? Une comparaison France - Angleterre, Paris : CNAF

MITTERAUER, Michael ; SIERDER, Reinhard, 1991 : Vom Patriarchat zur Partnerschaft - Zum Strukturwandel der Familie (4.Aufl.), München : Beck

NEUBAUER, Erika, 1989 : Alleinerziehende Mütter und Väter - eine Analyse der Gesamtsituation, Bundesministerium für Jugend, Familie, Frauen und Gesundheit (éd.), Schriftenreihe Band 219, Stuttgart : Kohlhammer

NEYRAND, Gérard, 2001 : L'enfant, la mère et la question des pères : Un bilan critique de l'évolution des savoirs sur la petite enfance, Paris : Presses Universitaires de France

OsTNER, Ilona, 1978 : Beruf und Hausarbeit - Die Arbeit der Frau in unserer Gesellschaft, Frankfurt/Main : Campus

OUVRAGE COLLECTIF, 2000 : Homoparentalités, États des lieux, ESF

PARSONS, Talcott, 1955 : Eléments pour une sociologie de l'action, Paris : Plon (1949)

PITROU, Agnès, 1972 : La famille dans la vie de tous les jours

RIEHL, Wilhelm H., 1861 : Die Familie, Stuttgart : J.G. Gotta'scher Verlag 
ROHMANN, Elke, 2000 : Gerechtigkeitserleben und Erwartungserfüllung in Partnerschaften, Rohmann, E. (ed.), Frankfurt /Main : Lang

Ronsin, Francis, 1992 : Les divorciaires - Affrontements politiques et conceptions du mariage dans la France du XIXème siècle, Paris : Aubier

RosenBAuM, Heidi, 1973 : Familie als Gegenstruktur zur Gesellschaft: Kritik grundlegender theoretischer Ansätze der westdeutschen Familiensoziologie, Stuttgart : Enke

RousSEL, Louis, 1989 : La famille incertaine, Evreux : Editions O. Jacob

SCHNEIDER, Norbert F. ; RosenkranZ, Doris ; LimMER, Ruth, 1998 : Nichtkonventionelle Lebensformen. Entstehung, Entwicklung, Konsequenzen, Opladen : Leske + Budrich

SEgAlEn, Martine, 1993 : Sociologie de la famille, 3ème édition revue et augmentée, Paris : Armand Colin

SILBERZAHN-JANDT, Gudrun, 1991: Wasch-Maschine : zum Wandel von Frauenarbeit im Haushalt, Marburg : Jonas Verlag

SINGLY, François de, 1993 : Sociologie de la famille contemporaine, Paris : Nathan

Singly, François de ; SCHUlthEIS, Franz, 1991 : Affaires de famille, Affaires d'Etat, Nancy : Edition de l'Est

StATISTISCHES BUNDESAMT, 2002a : Statistisches Jahrbuch 2001, Wiesbaden

StATISTISCHES BundeSAMT, 2001 : Leben und Arbeiten in Deutschland - Ergebnisse des Mikrozensus 2000, Wiesbaden

SULLEROT, Evelyne, 1992 : Quels pères ? Quels fils ?, Mesnil-sur-l'Estrée : Fayard

THERY, Irène, 1998 : Couple, filiation et parenté aujourd'hui, le droit face aux mutations de la famille et de la vie privée, Rapport à la Ministre de l'Emploi de la Solidarité et au Garde des Sceaux, Ministre de la Justice, Paris : La Documentation Française, Éditions O. Jacob

THERY, Irène, 1993 : Le démariage, Paris : Éditions O. Jacob

VASKOVICS, Laszlo A. ; Lipinski, H, 1996-1998 : Familiale Lebenswelten und Bildungsarbeit. Interdisziplinäre Bestandsaufnahme 1 u. 2 (= Ehe und Familie im sozialen Wandel, Bde. 1 u. 2), Didaktische Erfahrungen und Materialien (= Ehe und Familie im sozialen Wandel, Bd. 3), in : Ehe und Familie im sozialen Wandel, Opladen : Leske + Budrich

VASKOVICS, Laszlo A., 1997 : Familienleitbilder und Familienrealitäten, Opladen : Leske + Budrich

VASKOVICS, Laszlo ; RosT, Harald, 1999: Väter und Erziehungsurlaub, Bundesministerium für Familie, Senioren, Frauen und Jugend (éd.), Schriftenreihe Bd. 179, Stuttgart : Kohlhammer

WeBER, Marianne, 1907 : Ehefrau und Mutter in der Rechtsentwicklung, Tübingen : J. C. B. Mohr (Paul Siebeck)

WERnER, Heinz ; PIOTROWSKI-ROCHEFORT, Anne ; KÖNIG, Ingeborg, 1991 : Glossar zur Arbeitsmarkt- und Berufsforschung Französisch-Deutsch / Deutsch-Französisch Begriffe zu Arbeitsmarkt, Bildung und Sozialem, Nürnberg : Institut für Arbeitsmarktund Berufsforschung der Bundesanstalt für Arbeit

Zulehner, Paul ; Volz, Rainer, 1998 : Die Männerstudie "Männer im Aufbruch - Wie Männer sich selbst und wie Frauen sie sehen" - Ein Forschungsbericht, Ostfildern : Schwabenverlag 


\section{ARTICLES ET CONTRIBUTIONS A DES OUVRAGES COLLECTIFS}

Aliaga, Christel ; FLIPO, Anne, 2000 : «Les services de proximité se développent au profit des enfants», INSEE Première, $\mathrm{n}^{\circ} 704$

AlgaVA, Élisabeth, 2002 : «Quel temps pour les activités parentales ?», Études et résultats DRESS, Ministère de l'Emploi et de la Solidarité, $\mathrm{n}^{\circ} 162$ (mars)

AnXo, Dominique ; Boulin, Jean-Yves ; Lallement, Michel ; LefeVre, Gilbert ; Silvera, Rachel, 1998 : «Recomposition du temps de travail, rythmes sociaux et modes de vie Une comparaison France-Suède», Travail et emploi, $\mathrm{n}^{\circ} 74$

ARNAUD, André-Jean, 1994: «Le droit comme produit. Présentation du dossier sur la production de la norme juridique», Droit et société, $\mathrm{n}^{\circ}$ 27, p. 231 - 345

BARRERE-MAURISSON, Marie-Agnès ; RIVIER, Sabine 2002 : «Temps parental, parentalité et «parentalisme», à propos des nouvelles pratiques, institutions et régulations en matière de famille», Cahier de la MSE, Université de Paris I - CNRS - MATISSE - Groupe Division Familiale du Travail, ${ }^{\circ} 2002.42$

Barrere-Maurisson, Marie-Agnès ; Buffier-Morel, Martine ; Rivier, Sabine, 2001 : «Partage des temps et des tâches dans les ménages», sous la direction de Marie-Agnès Barrère-Maurisson, série Cahier travail et emploi, Paris : La Documentation Française

Barrere-MAurisson, Marie-Agnès ; Minni, Claude ; RIVIER, Sabine, 2001 : «Le partage des temps pour les hommes et les femmes - ou comment conjuguer travail rémunéré, non-rémunéré et non-travail», Premières synthèses, Ministère de l'Emploi et de la Solidarité - DARES, mars 2001 - $\mathrm{n}^{\circ} 11.1$ [mentionné dans Le Monde du 8 mars 2001]

BARRERE-MAURISSON, Marie-Agnès ; MARCHAND, Olivier ; RIVIER, Sabine, 2000 : «Temps de travail, temps parental - La charge parentale : un travail à mi-temps», Premières synthèses, Ministère de l'Emploi et de la Solidarité - DARES, $\mathrm{n}^{\circ} 20.1$ [mentionné en première page dans Le Monde du 27 mai 2000]

BARRERE-MAURISSON, Marie-Agnès, 1987: «Structures économiques et structures familiales : Émergence et construction d'une relation», L'année sociologique

BAST, Kerstin ; OSTNER, Ilona, 1992: «Ehe und Familie in der Sozialpolitik der DDR und BRD - ein Vergleich», in : Schmähl, W., Sozialpolitik im Prozess der deutschen Vereinigung, Frankfurt / New York : Campus

Beaumel, Catherine ; KerJosse, Roselyne ; Toulemon, Laurent, 1999 : «Des mariages, des couples et des enfants», INSEE Première, $\mathrm{n}^{\circ} 624$ (janvier)

BECK-GERNSHEIM, Elisabeth, 1992 : «Arbeitsteilung, Selbstbild und Lebensentwurf. Neue Konfliktlagen in der Familie», Kölner Zeitschrift für Soziologie und Sozialpsychologie, 2. Jg., Heft 44

BeckmanN, Petra, 2001 : «Neue Väter braucht das Land ! - Wie stehen die Chancen für eine stärkere Beteiligung der Männer am Erziehungsurlaub ?», Bundesanstalt für Arbeit (éd.), IAB Werkstattbericht, 6

BEGEOT, François; FERNANDEZ-CORDON, Juan Antonio, 1997: «La convergence démographique au delà des différences nationales», in: La question familiale en Europe, Paris : L'Harmattan 
BLÖSS, Thierry, 2001 : «L'égalité parentale au cœur des contradictions de la vie privée et des politiques publiques», in : La dialectique des rapports hommes-femmes, Sociologie d'aujourd'hui, Paris : Presses Universitaires de France

BoDE, Lutz, 1999 : «Die Fähigkeit zur Kooperation - und bist du nicht willig...», FamRZ, Heft 21

BouRdiEU, Pierre, 1996 : «Des familles sans nom», Actes de la Recherche en sciences sociales, $\mathrm{n}^{\circ} 113$ (juin)

BOURDIEU, Pierre, 1993 : «A propos de la famille comme catégorie réalisée», Actes de la recherche en sciences sociales, $\mathrm{n}^{\circ} 100$

BREITHAUPT, Marianne, 1993 : «Der aufhaltsame Aufstieg des gemeinsamen Sorgerechts für geschiedene Eltern», Kritische Justiz, Heft 4

BRousSE, Cécile, 1999: «La répartition du travail domestique entre conjoints reste très largement spécialisée et inégale», INSEE France Portrait social, INSEE

BUSSEMER, Ulrike ; MEYER, Sibylle, 1986 : «Zur Sozialgeschichte der Haushaltstechnik im 20 Jahrhundert», in : Dalhoff, J. (éd.), Frauenmacht in der Geschichte : Beiträge des Historikerinnentreffens 1985 zur Frauengeschichtsforschung, Geschichtsdidaktik : Studien, Materialien 41, Düsseldorf : Schwann

CAbantous, Alain, 1990 : «La fin des patriarches», in : Delumeau, J. ; Roche, D. (éds.), Histoire des pères et de la paternité, Paris : Larousse

CADORET, Anne, 2000 : «La parenté aujourd'hui : Agencement de la filiation et de l'alliance», Sociétés contemporaines, $\mathrm{n}^{\circ} 38$

Chadeau, Ann; FouQuet, Annie, 1981: «Peut-on mesurer le travail domestique ?», Economie et statistique, $\mathrm{n}^{\circ} 136$, INSEE

Chastand, Antoine, 1982: «Activités professionnelle et domestique des femmes de milieu ouvrier», INSEE, Economie et statistiques, $\mathrm{n}^{\circ} 141$

Commaille, Jacques, 1993 : «La fonction heuristique du droit pour une connaissance en sciences sociales sur la famille», Solidarité santé, $\mathrm{n}^{\circ} 4$

Commaille, Jacques, 1992 : «La régulation politique de la famille», in : Singly, F. de, La famille, l'état des savoirs, Paris : La Découverte

COUET, Christine, 1996 : «Les naissances hors mariage», INSEE, Données Sociales

CRENNER, Emmanuelle, 1999 : «Famille, je vous aide», INSEE Première, $\mathrm{n}^{\circ} 631$

DAGUET, Fabienne, 1996 : «Mariage, divorce et union libre», INSEE Première, $\mathrm{n}^{\circ} 482$

DECHAUX, Jean-Hugues, 1996 : «Les services de la parenté : fonctions, régulation, effets», in : sous la direction de Jean-Claude Kaufmann, Faire ou faire-faire ? Familles et services, Rennes : Presses Universitaires de Rennes

DECHAUX, Jean-Hugues, 1995 : «Orientations théoriques en sociologie de la famille : Autour de cinq ouvrages récents», Revue française de la sociologie, $\mathrm{n}^{\circ} \mathrm{XXXVI-3}$

DIENEL, Christiane, 1996 : «Politique familiale en Allemagne : les récents changements», Recherches et prévision, $\mathrm{n}^{\circ} 44$

D'IRIBARNE, Philippe, 1991 : «Culture et effet sociétal», Revue française de la sociologie, ${ }^{\circ}$ XXXII

DumONTIER, Françoise ; PAN Ke SHON, Jean-Louis, 2000 : «Enquête Emploi du Temps 19981999, Description des activités quotidiennes», INSEE Résultats, ${ }^{\circ}$ 693-694

Dumontier, Françoise ; PAN Ke ShON, Jean-Louis, 1999: «En 13 ans, moins de temps contraints et plus de loisirs», INSEE Première, $\mathrm{n}^{\circ} 675$

DURKHEIM, Emile, 1975 : «La famille conjugale», in : Karady, V. (éd.), Textes III - Fonctions sociales et institutions, Paris : Les Editions de Minuit

EGGEN, Bernd, 1995 : «Familienpolitische Leistungen der Länder Deutschlands für Familien mit Kindern unter drei Jahren», Sozialer Fortschritt n ${ }^{\circ} 10$ 
ENGELBRECH, Gerhard ; JungKunst, Maria, 2001 : «Alleinerziehende Frauen haben besondere Beschäftigungsprobleme», IAB Kurzbericht, 2 / 16.2.2001

FAGNANI, Jeanne, 1996 : «Les politiques familiales dans la dynamique sociale européenne», Solidarité santé, $\mathrm{n}^{\circ} 1$

FAGNANI, Jeanne, 1996 : «Travail et fécondité en France et en Allemagne de l'Ouest», Revue Française des Affaires Sociales, $\mathrm{n}^{\circ} 2$

FERMANIAN, Jean-David ; LAGARDE, Sylvie, 1999 : «Les horaires de travail dans le couple», Economie et statistique, $\mathrm{n}^{\circ}$ 321-322

Festy, Patrick ; VALETAS, Marie-France, 1993 : «Les pensions alimentaires à l'épreuve de la recomposition familiale», in : Meulders-Klein, M.-Th. ; Théry, I., Les Recompositions Familiales Aujourd'hui, Essais et recherches, Paris : Nathan

FLIPO, Anne ; HourRIEZ, Jean-Michel, 1995 : «Recourir à une femme de ménage», INSEE Première, $\mathrm{n}^{\circ} 411$

GALTIER, Bénédicte, 1999 : «Les temps partiels : Entre emplois choisis et emplois "faute de mieux"», Économie et statistique, $\mathrm{n}^{\circ} 321-322$

Glaude, Michel ; Singly, Francois de, 1986: «L'organisation domestique, "pouvoir et négociation"», Économie et statistique, $\mathrm{n}^{\circ} 187$

GRIMLER, Ghislaine, 1989: «Des journées les plus contraignantes aux journées les plus paisibles», Économie et statistiques, $\mathrm{n}^{\circ} 227$

GRIMLER, Ghislaine ; ROY, Caroline, 1990 : «Activités domestiques : faire, acheter, faire faire ou ne pas faire ?», INSEE Première, $\mathrm{n}^{\circ} 109$

GRIMLER, Ghislaine ; ROY, Caroline, 1987 : «Les emplois du temps en France en 1985-1986», Premiers résultats, $\mathrm{n}^{\circ} 100$

GROSSFELD, B., 1991 : «Die Einkommensteuer, geschichtliche Grundlage und rechtsvergleichender Ansatz», Recht und Staat, 504-505, Tübingen : Mohr

HANTRAIS, Linda, 1994 : «Comparing Family Policy in Britain, France and Germany», Revue Int. Soc. Pol., $\mathrm{n}^{\circ} 2$

HeILIGER, Anita, 1992 : «Zur Problematik einer Konzeption nachehelicher gemeinsamer elterlicher Sorge als Regelfall im Kontext einer geplanten Reform des Kindschaftsrechts», FamRZ, Heft 9 / 1992

HERDEGEN, Mathias, 1993 : «Die Aufnahme besonderer Rechte des Kindes in die Verfassung», FamRZ, Heft 4 / 1993

KAUFMANN, Franz-Xaver, 1997b : «Les Etats providence européens dans leur rapport avec la famille», in : sous la direction de Commaille, J. ; Singly, F. de, La question familiale en europe, Paris : L'Harmattan

KaUfMANN, Franz-Xaver, 1995 : «Zur Einführung: Sozialpolitik im französisch-deutschen Vergleich», Zeitschrift für Sozialreform, Heft 11/12

KAUfMANN, Franz-Xaver, 1993 : «Familienpolitik in Europa», in : Bundesministerium für Familien und Senioren (éd.), 40 Jahre Familienpolitik in der Bundesrepublik Deutschland, Rückblick / Ausblick, Neuwied : Luchterhand

KAUfMANn, Franz-Xaver, 1988 : Vorwort in : Schultheis, F., Sozialgeschichte der französischen Familienpolitik, Frankfurt/Main : Campus

Kaufmann, Otto ; Reinhard, Hans Joachim ; Pardell Vea, Agnès ; Dyspersyn, Michel; BALlANDI, Gian Guido ; CALAFA, Laura ; HANTRAIS, Linda, 1994 : «Comparing Family Policy in Britain, France and Germany», Revue Int. Soc. Pol, 2

KÜNZLER, Jan ; WALTER, Wolfgang, 2001 : «Arbeitsteilung in Partnerschaften - Theoretische Ansätze und empirische Befunde», in : Huinink, J. ; Strohmeier, K. P. ; Wagner, M., Solidarität in Partnerschaft und Familie, Würzburg : Ergon 
KÜNZLER, Jan, 1999 : «Arbeitsteilung in Ehen und nichtehelichen Lebensgemeinschaften», in : Klein, Th. ; Lauterbach, W. [éds.], Nichteheliche Lebensgemeinschaft, Opladen : Leske + Budrich

KüNZLER, Jan, 1995a : «Geschlechtsspezifische Arbeitsteilung - Die Beteiligung von Männern im Haushalt im internationalen Vergleich», Zeitschrift für Frauenforschung 13

KÜNZLER, Jan, 1995b : «Familiale Arbeitsteilung in der Bundesrepublik Deutschland 1988», in : Gerhardt, U. ; Hardil, St. ; Lucke, D. ; Nauck, B. [éds.], Familie der Zukunft, Lebensbedingungen und Lebensformen, Reihe Sozialstrukturanalyse, Band 6, Opladen : Leske + Budrich

LEFAUCHEUR, Nadine, 1997 : «Qui doit nourrir l'enfant de parents non mariés ou démariés ?», CNAF, Recherches et prévisions, $\mathrm{n}^{\circ} 47$

LEFAUCHEUR, Nadine, 1991 : «La famille monoparentale et l'état : Petite généalogie du traitement social des risques familiaux» in : sous la direction de Singly, F. de ; Schultheis, F., Affaires de famille, Affaires d'Etat, Nancy : Edition de l'Est

LENOIR, René, 1991a : «Family Policy in France since 1938», in : Amblee, J. S., The French Welfare State: Surviving Social and Ideological Change, New York : New York University Press

LENOIR, René, 1991b : «Politique familiale et construction sociale de la famille», Revue française de science politique, $\mathrm{n}^{\circ} 6$

LENOIR, René, 1985a: «L'effondrement des bases sociales du familialisme», Actes de la recherches en sciences sociales, $\mathrm{n}^{\circ} 57-58$

LENOIR, René, 1985b : «Transformations du familialisme et reconversions morales», Actes de la recherches en sciences sociales, $\mathrm{n}^{\circ} 59$

LERIDON, Henri, 1993 : «Recomposer les familles dans les sources statistiques», in : Les Recompositions Familiales Aujourd'hui, Essais et Recherches, Paris : Nathan

LESSENICH, Stephan ; OSTNER, Ilona, 1995 : «Die institutionelle Dynamik 'dritter Wege' Zur Entwicklung der Familienpolitik in 'katholischen' Wohlfahrtsstaaten am Beispiel Deutschlands und Frankreichs», Zeitschrift für Sozialreform, Heft 11/12

LEWIS, Jane, 1993 : «Introduction : Women, work, family and social policies», in : Lewis, J., Europe, Women and Social Policies in Europe. Work, Family and the State, Aldershot: Edward Elgar (Gower House)

LiMBACH, Jutta, 1988 : «Die Suche nach dem Kindeswohl - Ein Lehrstück der soziologischen Jurisprudenz», Zeitschrift für Rechtssoziologie, Bd.9, Heft 2

LIMBACH, Jutta, 1981 : «Das Eheleitbild in der Jurisprudenz», Lebenswelt und soziale Probleme, Frankfurt/Main : Campus

LOHKAMP-HIMMIGHOFEN, Marlene, 1993 : «Ansätze zur Förderung der Vereinbarkeit von Familie und Beruf», in : Bundesministerium für Familie und Senioren (éd.), Zwölf Wege der Familienpolitik in der Europäischen Gemeinschaft, Schriftenreihe Band 22.1, Teil 8, Stuttgart : Kohlhammer

LUTHIN, Horst., 1996 : «Richterliche Vorurteile gegen gemeinsames Sorgerecht?», FamRZ, Heft 19 / 1996

MACCOBY, Eleanor ; MNOOKIN, Robert, 1995 : «Die Schwierigkeiten der Sorgerechtsregelung», FamRZ, Heft 1 / 1995

MARTIN, Claude, 1999 : «Comparer les politiques familiales en Europe», Recherches et prévisions, $\mathrm{n}^{\circ} 55$, Paris : CNAF

MARTIN, Claude, 1997a : «L'après-divorce. Lien familial et vulnérabilité », Rennes : Presses Universitaires de Rennes 
MARTIN, Claude, 1997b : «L'action publique en direction des ménages monoparentaux. Une comparaison France / Royaume-Uni», CNAF, Recherches et prévisions, ${ }^{\circ} 47$

Maurice, Marc ; Sellier, François ; Silvestre, Jean-Jacques, 1992 : «Analyse sociétale et cultures nationales : Réponse à Philippe d'Iribarne», Revue française de la sociologie, $\mathrm{n}^{\circ}$ XXXIII

MAURICE, Marc, 1989 : «Méthode comparative et analyse sociétale : Les implications théoriques des comparaisons internationales», Sociologie du travail, $\mathrm{n}^{\circ} 2$

MAURIN, Eric, 1989: «Types de pratiques quotidiennes, type de journée et déterminantes sociaux de la vie quotidienne», Économie et statistiques, $\mathrm{n}^{\circ} 223$

MENDRAS, Henri, 1997 : «Les familles monoparentales, familles recomposées et réseau familial», Observations and Diagnostics Economics, Revue de l'OCDE, ${ }^{\circ} 62$

MEULDERS-KLEIN, Marie-Thérèse; THERY, Irène, 1993: «La parenté aux frontières de l'amitié : Statut et rôle du beau-parent dans les familles recomposées», in : Les recompositions familiales aujourd'hui, Essais et recherches, Paris : Nathan

MEULDERS-KLEIN, Marie-Thérèse, 1993 : «Le temps des recompositions familiales», in : Les recompositions familiales aujourd'hui, Essais et recherches, Paris : Nathan

MitTERAUER, Michael, 1979 : «Faktoren des Wandels historischer Familienformen», in : Pross, H. (éd.), Familie - wohin?, Reinbek : Rowohlt

Motzer, Stefan, 1999 : «Die gerichtliche Praxis der Sorgerechtsentscheidung seit der Neufassung von $\S 1671$ BGB», FamRZ, Heft 17

MulliEZ, Jacques, 1990 : «La volonté d'un homme», in : Delumeau, J. ; Roche, D., Histoire des pères et de la paternité, Paris : Larousse

MunOZ-PereZ, Francisco ; PrIOUX, France, 1999a : «Les enfants nés hors mariage et leurs parents. Reconnaissances et légitimations depuis 1965», Population, $n^{\circ} 54$ (3)

MunOZ-PereZ, Francisco ; PriOuX, France, 1999b : «Reconnaissances et légitimations des enfants nés hors mariage depuis 1965. Des comportements différents selon l'âge des parents et leur milieu social», Population, $\mathrm{n}^{\circ} 54$ (6)

NeIDHARDT, Friedhelm, 1974 : «Strukturbedingungen und Probleme familiarer Sozialisation», in : Claessens, D. ; Milhoffer, P. (éds.), Familiensoziologie, Frankfurt/Main : Athenäum

NeubAuer, Erika, 1994 : «Alleinerziehende in den zwölf Ländern der EG», Aus Politik und Zeitgeschichte, B 7-8 / 94

NEUBAUER, Erika, 1993 : «Alleinerziehende als Zielgruppe der Familienpolitik», in: Bundesministerium für Familie und Senioren (éd.), Zwölf Wege der Familienpolitik in der Europäischen Gemeinschaft, Schriftenreihe Band 22.1, Stuttgart: Kohlhammer

OELKERS, Harald ; KASTEN, Hartwig, 1994 : «Das gemeinsame Sorgerecht nach Scheidung in der Praxis des Amtsgerichts Hamburg - Familiengericht», FamRZ, Heft 17

OELSCHLÄGER, Angelika; SCHUNTER-KLEEMANN Susanne, 1992 : «Frauen als Erwerbsarbeiterinnen - Frauen als Familienarbeiterinnen - Frauen, Eltern, Familien als Empfänger sozialer Leistungen», in : Schunter-Kleemann, S. (éd.), Herrenhaus Europa - Geschlechterverhältnisse im Wohlfahrtsstaat, Berlin : Sigma

OSTNER, Ilona, 2002 : «Régimes de protection sociale, taux d'activité des femmes et famille», in : Daniel, C. ; Palier, B. (éds.), La protection sociale en Europe, le temps des réformes, Paris : La documentation

OSTNER, Ilona, 1995: «Arm ohne Ehemann? Sozialpolitische Regulierung von Lebenschancen für Frauen im internationalen Vergleich», in : Bundeszentrale für politische Bildung (éd.), Aus Politik und Zeitgeschichte, B 36-37

OSTNER, Ilona, 1993 : «Slow motion : Women work and the family in Germany», in : Lewis, J. (éd.), Women and Social Policies in Europe : Work, Family and the State, Aldershot :

E. Elgar (Gower House) 
Prost, Antoine, 1984: «L'évolution de la politique familiale en France de 1938 à 1981», Mouvement social, $\mathrm{n}^{\circ} 129$

Rousse, Hélène ; Roy, Caroline, 1981 : «Activités ménagères et cycle de vie», Économie et statistiques, $\mathrm{n}^{\circ} 131$

Roy, Caroline, 1989 : «La gestion du temps des hommes et des femmes, des actifs et des inactifs», Économie et statistiques, $\mathrm{n}^{\circ} 223$

Roy, Caroline, 1983 : «La journée des citadins», Économie et statistiques, n $^{\circ} 158$

RoY, Caroline, 1982 : «L'emploi du temps des mères et pères de familles nombreuses», Économie et statistiques, $\mathrm{n}^{\circ} 141$

RuBELLIN-DEVICHI, Jacqueline, 1996 : «Le principe de l'intérêt de l'enfant dans la loi et la jurisprudence française», $J D J, \mathrm{n}^{\circ} 159$

SALGO, Ludwig, 1996 : «Zur gemeinsamen elterlichen Sorge nach Scheidung als Regelfall ein Zwischenruf», FamRZ, Heft 8

SCHUlTHEIS, Franz, 1995 : «Die Familie: Eine Kategorie des Sozialrechts? Ein deutschfranzösischer Vergleich», Zeitschrift für Sozialreform, Heft 11/12

SCHULTHEIS, Franz, 1992: «Inerties structurelles et ambivalences idéologiques : La protection sociale allemande face au nouveaux risques familiaux», Recherche sociale

SCHULTHEIS, Franz, 1987 : «Fatale Strategien und ungeplante Konsequenzen beim Aushandeln 'familiärer Risiken' zwischen Mutter, Kind und 'Vater Staat'», Soziale Welt, Göttingen : Otto Schwarz

SCHÜTZE, Yvonne, 1988 : «Zur Veränderung im Eltern-Kind-Verhältnis seit der Nachkriegszeit», in : Nave-Herz, R. (éd.), Wandel und Kontinuität der Familie in der $B R D$, Stuttgart : Enke

SILVERA, Rachel, 1998 : «Les femmes et la diversification du temps de travail : Nouveaux enjeux, nouveau risque », Revue française des affaires sociales, $\mathrm{n}^{\circ} 3$

SINGLY, François de, 1976: «La lutte conjugale pour le pouvoir domestique», Revue française de la sociologie, $\mathrm{n}^{\circ} \mathrm{XVII}$

SøRENSEN, Aage B., 1990 : «Zur geschlechtsspezifischen Struktur von Armut», in : Leibfried, S., Voges, W. (éds.), Armut im modernen Wohlfahrtsstaat, Sonderheft 32/1992 der Kölner Zeitschrift für Soziologie und Sozialpsychologie, Opladen : Westdeutscher Verlag

SOZIALISTISCHER FRAUENBUND, 1978: «Wir wollen keinen Lohn für Hausarbeit», in : Sozialistischer Frauenbund (éd.), Frauen als bezahlte und unbezahlte Arbeitskräfte, Beitrage zur 2. Berliner Sommeruniversität für Frauen 1977, Berlin

STÖCKER, Hans A., 1992 : «Die UNO-Kinderkonvention und das deutsche Familienrecht», FamRZ, Heft 3

STÖCKER, Hans A., 1992 : «Schlusswort», FamRZ, Heft 8

THERY, Irène, 1998 : «Un état des lieux a interpréter, démariage et filiation», in : Faut-il banaliser le divorce ?, Dialogue recherches cliniques et sociologiques sur le couple et la famille, $\mathrm{n}^{\circ} 141$

Ullmann, Christian, 1992 : «Die UNO-Kinderkonvention und das deutsche Familienrecht», FamRZ, Heft 2

VILLAC, Michel, 1993 : «La politique familiale - Débats autour d'une définition», Solidarité santé : Études statistiques, $\mathrm{n}^{\circ} 4$

VILLAC, Michel ; RENAUDAT, Evelyne, 1991 : «L'Allocation de soutien familial.

L'intervention de l'Etat dans la gestion privée de l'après-divorce» in : sous la direction de Singly , F de ; Schultheis,, F., Affaires de famille, Affaires d'Etat, Nancy : Edition de l'Est 
VILLENEUVE-GoKalP, Catherine, 1999: «La double famille des enfants de parents séparés», Population, $\mathrm{n}^{\circ} 1$

VILLENEUVE-GOKALP, Catherine, 1993 : «De la famille d'origine à la famille recomposée», in : Meulders-Klein, M.-Th. ; Théry, I., Les recompositions familiales aujourd'hui, Essais et recherches, Paris : Nathan

VOEGELI, Wolfgang, 1991: «Familles monoparentales et 'Etat - Père'. Tendances à la socialisation des risques familiaux», in : sous la direction de Singly, F. de ; Schultheis, F., Affaires de famille, Affaires d'Etat, Nancy : Edition de l'Est

VoGES, Wolfgang ; OSTNER, Ilona, 1995 : «Wie arm sind alleinerziehende Frauen?», in : Bieback, K-J. ; Milz, H. (éds.), Neue Armut, Frankfurt/Main : Campus

WAGENITZ, Thomas ; BARTH, Thomas, 1996 : «Die Änderung der Familie als Aufgabe für den Gesetzgeber», FamRZ, Heft 10

WALTER, Wolfgang, 1995 : «Familienberichterstattung und familienpolitischer Diskurs», in : Gerhardt, U. ; Hradil, St. ; Lucke, D. ; Nauck, B. (éds.), Familie der Zukunft, Lebensbedingungen und Lebensformen, Reihe Sozialstrukturanalyse, Band 6, Opladen : Leske + Budrich

WHITEFORD, Peter, BRADSHAW, Jonathan, 1994 : «Prestations et incitations offertes aux parents seuls : analyse comparative», Revue internationale de sécurité sociale, $\mathrm{n}^{\circ}$ 3-4

ZARCA, Bernard, 1990 : «La division du travail domestique - Poids du passé et tensions au sein du couple», Économie et statistique, $\mathrm{n}^{\circ} 228$

\section{NOTES, DOCUMENTS, COMMUNICATIONS, RAPPORTS ET THESES}

BAUER, R., (éd.), 1992 : «Bundessozialhilfegesetz», München : R. Oldenburg

BIELENSKI, Harald ; KAUPPINEN, Timo, 2000 : Concilier vie familiale et vie professionnelle: Répartition du temps de travail entre homme et femme, Fondation européenne pour l'amélioration des conditions de vie et de travail

BIRKHÖLZER, Karl ; LORENZ, Günther, 2000 : Beschäftigung, Familie und GemeinwesenAktivitäten : Ein Vergleich der Chancen für Frauen und Männer, European Foundation for the improvement of Living and Working Conditions

BRIGITTE, 1999 : Frauen Gestern, Heute, Morgen - Interpretationen aus 25 Jahren BrigitteForschung

BundeSAnSTALT FÜR ARBEIT, 2002 : Merkblatt Kindergeld, Bundesamt für Finanzen (éd.)

BundeSMinisterium FÜR FAMILIE, SENIOREN, FrAuEN Und JugEnd, 2001 : Erziehungsgeld, Elternzeit - Das neue Bundeserziehungsgeldgesetz für Eltern mit Kindern ab Geburtsjahrgang 2001, Bundesministerium für Familie, Senioren, Frauen und Jugend (éd.), Berlin

Bundesministerium FÜR FAMILIE, SENIOREN, FRAUEN UND JUGEND, 2000 : Mutterschutzgesetz - Leitfaden zum Mutterschutz, Bundesministerium für Familie, Senioren, Frauen und Jugend (éd.), Berlin

BUNDESMINISTERIUM FÜR FAMILIE UND SENIOREN, 1994 : Familien und Familienpolitik im geeinten Deutschland -Zukunft des Humanvermögens - Fünfter Familienbericht, Bundesministerium für Familie und Senioren (éd.), Bonn

BUNDESVERFASSUNGSGERICHT 1998 : «Erfolglose Verfassungsbeschwerde betreffend "Sonderurlaub für Niederkunft der nichtehelichen Lebensgefährtin"», Bundesverfassungsgericht (éd.), Pressemitteilung, Nr. 39 / 98 vom 17. April 1998 
COLlOQUE «Coparentalité», 1997 : Organisé par la commission Typhaon avec le concours de l'ordre des avocats de Paris, le syndicat de la magistrature et le syndicat des avocats de France, Maison du Barreau de Paris, 12 juin 1997, une partie des actes ont été publiés dans la revue DiALOGUE, 1998 : Faut-il banaliser le divorce?

DAS PARLAMENT N $15-16$ 9/ 16 avril 1993

DAS PARLAMENT N ${ }^{\circ} 43 / 28$ octobre 1978

DEKEUWER-DEFOSSEZ, Françoise, 1999 : Rénover le droit de la famille - Propositions pour un droit adapté aux réalités et aux aspirations de notre temps, Rapport au Garde des Sceaux, Ministre de la Justice, Collection des rapports officiels, Paris : La Documentation Française, Editions O. Jacob

ENGSTLER, Heribert, 1999 : Die Familie im Spiegel der amtlichen Statistik, Mutterschutzgesetz - Leitfaden zum Mutterschutz - Lebensformen, Familienstrukturen, wirtschaftliche Situation der Familien und familiendemographische Entwicklung in Deutschland, sur la demande du 'Bundesministerium für Familie, Senioren, Frauen und Jugend', et la coopération du 'Statistisches Bundesamt', Bonn

ENTRETIEN avec Gérard Neyrand, propos recueillis par Pascale Kremer, Le Monde, 25 novembre 2000

ENTRETIEN avec Gérard Poussin, propos recueillis par Sébastien Amine, Ouest France, mai 2000

ENTRETIEN avec Madame le Juge aux Affaires Familiales, Vice-Présidente du Tribunal de Grande Instance de Paris, propos recueillis par Sabine Rivier dans le cadre d'entretien d'expert, Paris, 1997

ENTRETIEN avec Michel Tort, propos recueillis par Blandine Grosjean, à l'occasion de la proposition de loi sur la réforme sur la transmission du nom de famille, Libération, 8 février 2001

ENTRETIEN avec Monsieur le directeur du service de recouvrement des pensions alimentaires, propos recueillis par Sabine Rivier dans le cadre d'entretien d'expert, Göttingen, 1997

ENTRETIEN avec Monsieur le Juge aux Affaires Familiales, propos recueillis par Sabine Rivier dans le cadre d'entretien d'expert, tribunal Göttingen, 1997

ENTRETIEN de C. Brinck avec M. von Renesse, TAZ, Heft 846 du 17 mai 1996

FAMRZ, 1999a : Décision du tribunal OLG de Karlsruhe du 23. 09. 1998, Heft 3

FAMRZ, 1999b : Décision du tribunal OLG de Braunschweig du 14. 10. 1998, Heft 3, p. 184

FAMRZ 1998 : Décision du tribunal OLG Groß-Gerau du 22.10.1997, Heft 8

FAMRZ 1995a : Décision du tribunal OLG Hamm du 31.10.1994, Heft 5, p. 314

FAMRZ, 1995b: Décision du tribunal OLG (Oberlandesgericht) Bamberg du 14.3.1995, Heft 23, p. 1509

FthenAKIS, Wassilios E. ; Minsel, Beate, 2001: «Die Rolle des Vaters in der Familie», Bundesministerium für Familie, Senioren, Frauen und Jugend (éd.), Staatsinstitut für Frühpädagogik, München : IFP

FULCHIRON, Hugues, 1997 : La mise en œuvre du droit de l'enfant a être élevé par ces deux parents et la généralisation de l'autorité de l'exercice en commun de l'autorité parentale, Rapport pour le Ministère de la Justice

HANTRAIS Linda; LETABLIER Marie-Thérèse, 1995: La relation famille-emploi. Une comparaison des modes d'ajustement en Europe, Centre d'études de l'emploi : Dossier $\mathrm{n}^{\circ} 6$, Nouvelle série

HUININK, Johannes, 2001: Hausarbeit in Partnerschaften - Determinanten und Bewältigungsstrategien geschlechtstypischer Arbeitsteilung in nichtehelichen und ehelichen Lebensgemeinschaften, Arbeitsbericht DFG, Rostock : Universität Rostock 
HuLLEN, Gert, 1995 : «Frauenbiographie - Ergebnisse der retrospektiven Befragung zur Familien- und Erwerbsbiographie 35 - bis 60 jähriger Frauen», BiB, Materialen zur Bevölkerungswissenschaft, Heft 83

INSTITUT DER DEUTSCHEN WIRTSCHAFT KÖLN, 2001 : Zahlen zur wirtschaftlichen Entwicklung der Bundesrepublik Deutschland, Institut der deutschen Wirtschaft Köln (éd.), Köln : deutscher Instituts-Verlag

JO : Assemblée nationale, 7 mai 1987, p.954

JoCHHEIM, Barbara, 1995: Trennung, Scheidung, Unterhalt von A-Z, WRS-Ratgeber, Freiburg : WRS

Kettschau, Irmhild, 1980 : Wie viel Arbeit macht ein Familienhaushalt? - Zur Analyse von Inhalt, Umfang und Verteilung der Hausarbeit heute, Dissertation (Thèse de doctorat), Dortmund

KURZ-SCHERF, Ingrid ; WINKLER, Gunnar, 1994 : Sozialreport 1994 - Daten und Fakten zur sozialen Lage in den neuen Bundesländern, Kurz-Scherf, I. ; Winkler, G. (éds.), Sozialwissenschaftliches Forschungszentrum Berlin-Brandenburg e.V, GSFP Gesellschaft für sozialwissenschaftliche Forschung und Publizistik, Berlin

LANDTAGSDRUCKSACHE, $1993: 11 / 2157$

LBS, 1997: «Aufgaben- und Rollenverteilung zwischen Frau und Mann», LBS (Landesbausparkasse)-Familien-Studie "Übergang zur Elternschaft", LBS-Initiative „Junge Familie“, sous la direction de Fthenakis, W. E ; Engfer, A., Report 2 / 97

LBS, 1998a : «Die Vereinbarkeit von Familie und Beruf», LBS (Landesbausparkasse) Familien-Studie "Übergang zur Elternschaft", LBS-Initiative „Junge Familie“ sous la direction de Fthenakis, W. E ; Engfer, A., Report $1 / 98$

LBS, 1998b : «Die Chancen der Vaterschaft», LBS (Landesbausparkasse)-Familien-Studie "Übergang zur Elternschaft", LBS-Initiative „Junge Familie“, sous la direction de Fthenakis, W. E ; Engfer, A., Report 2 / 98

LBS, 2000: «Vorstellung von Vaterschaft und Mutterschaft - zwei Begriffe für eine gemeinsame Verantwortung», LBS (Landesbausparkasse)-Familien-Studie "Übergang zur Elternschaft", LBS-Initiative „Junge Familie“ sous la direction de Fthenakis, W. E ; Engfer, A., Report 1 / 2000

LE FEUVRE, N., 2000 : Emploi, famille et citoyenneté - un nouvel équilibre pour les hommes et les femmes, European Foundation for the Improvement of Living and Working Conditions

MARTIN-PAPINEAU, Nadine, 1994 : Les familles monoparentales dans le champs politique français -Emergence, construction, captations d'un problème (1968-88), thèse de doctorat à l'Institut de Science Politique de Paris

MiNISTERE DE L'EMPLOI ET DE LA SOLIDARITE, 2000 : «La filiation des enfants nés hors mariage - trente ans d'évolution (1965-1994)», in: 29e rapport sur la situation démographique en France, INED

MÜCKENBERGER, Ulrich 2001 : «Des politiques du temps de travail aux politiques du temps urbain», communication, colloque Sphères privée et professionnelle : vers une recomposition des rôles et des actions, 8-9 novembre 2001, Gembloux (Belgique)

PRESSE- UND INFORMATIONSAMT DER BUNDESREGIERUNG, 1995 : Information für Familien Familienpolitik in 55 Stichworten (2. unveränderte Auflage), Presse- und Informationsamt der Bundesregierung (éd.), Reihe Ratschläge und Hinweise, Bonn PROCES VERBAL de la séance du 7 avril 1981, n 280 Sénat

Roll, Jo, 1992 : Lone parent families in the European Community, European Familiy and Social Policy Unit, London 
Rosenbaum, Heidi, 1978 : «Seminar Familie und Gesellschaftsstruktur», in : Materialien zu den sozio-ökonomischen Bedingungen von Familienformen, Rosenbaum, H. (éd.), Frankfurt/Main : Suhrkamp

SAYN, Isabelle, 1996 : Enfant à charge et parent isolé ou les difficultés de mise en ouvre des critères de fait, Note de synthèse, Lyon : CNAF

SOZIALPOLITISCHE UMSCHAU, 1998 : Presse- und Informationsamt der Bundesregierung (éd.), Bonn, $\mathrm{n}^{\circ} 239 / 98$

SOZIALPOLITISCHE UMSCHAU, 1998b: Presse- und Informationsamt der Bundesregierung (éd.), Bonn, n $241 / 98$

SOZIALPOLITISCHE UMSCHAU, 1998c: Presse- und Informationsamt der Bundesregierung (éd.), Bonn, $n^{\circ} 243 / 98$

SOZIALPOLITISCHE UMSCHAU, 1998d: Presse- und Informationsamt der Bundesregierung (éd.), Bonn, $n^{\circ} 244$ / 98

SOZIALPOLITISCHE UMSCHAU, 1997a : Presse- und Informationsamt der Bundesregierung (éd.), Bonn, nº 68 / 97

SOZIALPOLITISCHE UMSCHAU, 1997b: Presse- und Informationsamt der Bundesregierung (éd.), Bonn, $\mathrm{n}^{\circ} 70$ / 97

SOZIALPOLITISCHE UMSCHAU, 1997c : Presse- und Informationsamt der Bundesregierung (éd.), Bonn, nº 75 / 97

SOZIALPOLITISCHE UMSCHAU, 1995 : Presse- und Informationsamt der Bundesregierung (éd.), Bonn, $\mathrm{n}^{\circ} 233$ / 95

STATISTISCHES BUNDESAMT, 2002b : «Erwerbstätigenquote von Frauen entwickelt sich uneinheitlich», Pressemitteilung, 7 mars 2002

STATISTISCHES BUNDESAMT, 2001: «Statement von Präsident Johann Hahlen zur Pressekonferenz "Leben und Arbeiten in Deutschland - Ergebnisse des Mikrozensus 2000"», Mitteilung für die Presse, 19 avril 2001.

STATISTISCHES BunDESAMT, 2001a : «Erwerbstätigkeit von Müttern», Mitteilung für die Presse, 10 mai 2001

StATISTISCHES BundeSAMT, 2001b : «Neuer Hochstand bei den Ehescheidungen im Jahr 2000», Mitteilung für die Presse, 6 septembre 2001

STATISTISCHES BUNDESAMT, 2001c : «Zahl der Einzelkinder seit den 50er Jahren nahezu unverändert», Mitteilung für die Presse, 19 septembre 2001

StATISTISCHES BundeSAMT, 2000 : "Ergebnisse des Mikrozensus 1999", Mitteilung für die Presse, avril 2000

STATISTISCHES BUNDESAMT, 2000a : «1999 - 7\% weniger Ehepaare mit Kinder als 1991», Mitteilung für die Presse, 14 avril 2000

STATISTISCHES BUNDESAMT, 2000b : «Immer weniger Kinder suchen Ostereier», Mitteilung für die Presse, 19 avril 2000

StATISTISCHES BundeSAMT, 2000c : «Mütter im erwerbsfähigen Alter», Mitteilung für die Presse, 11 mai 2000

STATISTISCHES BUNDESAMT, 2000d : «Immer mehr nichteheliche Lebensgemeinschaften», Mitteilung für die Presse, 26 mai 2000

StATISTISCHES BundESAMT, 2000e : «1999 weniger Geburten, aber mehr Eheschließungen als im Vorjahr», Mitteilung für die Presse, 6 juillet 2000

STATISTISCHES BUNDESAMT, 1999: «2,5\% mehr Ehescheidungen in Deutschland im Jahr 1998», Mitteilung für die Presse, 3 août 1999

United NATiOns, 2000 : World's Women 2000 - Trends and Statistics, Department of Economic and Social Affairs Statistics Division, New York 
ADMINISTRATION FRANÇAISE, 2002 : «Quelles sont les voies de recours d'un fonctionnaire auquel l'administration n'a pas versé le supplément familial de traitement?», <http://vosquestions.service-public.fr/fiche/2535.htm>, site de l'administration française service-public, le portail de l'administration française, CIRA, date d'actualisation de la page : février 02, date de consultation de la page : 21 février 02

ADMINISTRATION FRANÇAISE, 2001 : «Famille», <http://vosdroits.service-public.fr/ARBO/10$\underline{\mathrm{NX} 10 . h \mathrm{html}}>$, site de l'administration française service-public, le portail de l'administration française, La Documentation Française 2001, date de consultation 15 février 2002

ADMINISTRATION FRANÇAISE, 2000 : «Fonction publique: rémunération», <http://vosdroits.service-public.fr/ARBO/05030702-FXTRA413.html >, site de l'administration française service-public, le portail de l'administration française, La Documentation Française 2000, date de création de la page : 2000, date de consultation de la page : 21 février 02

AIDH, 2002a: «La Déclaration des droits de l'enfant», $<$ http://www.droitshumains.org/Biblio/Trait_internat/Declar_DE.htm>, Site de l'association internet pour la défense et la promotion des droits de l'homme (Aidh), dernière mise à jour du site : mardi 26 février 2002

AIDH, 2002b: «La Convention internationale des droits de l'enfant », $<$ http://www.droitshumains.org/DE/Convention.htm>, Site de l'association internet pour la défense et la promotion des droits de l'homme (Aidh), dernière mise à jour du site : mardi 26 février 2002

AOK, 2001, «Sonderurlaub», <http://www.sbb.aok.de/ct/364000.html $>$, site AOK-online Business, dernière modification de la page : 13 novembre 2001

BUNDESKANZLERAMT, 2002: «Familie: Bessere Kinderbetreuung wird Schwerpunkt der Familienpolitik», 〈http://www.bundeskanzler.de/Kanzler-Aktuell-.7718.htm>, Kanzler News du 18 avril 2002, site du Bundeskanzleramt 〈http://www.bundeskanzler.de〉

Bundesministerium für FAmilie, Senioren, Frauen Und Jugend, 2002 : «Balance von Beruf und Familie ist Top-Thema», Pressemitteilung der Kampagne "Mehr Spielraum für Väter",

<http://www.bmfsfj.de/top/dokumente/Pressemitteilung/ix 71085.htm?template=single \&id=71085\&script=1\&ixepf $=$ 71085 $>$, Site du Bundesministerium für Familie, Senioren, Frauen und Jugend <http://www.bmfsfj.de $>$, date de consultation 5 mars 2002

BUNDESMINISTERIUM FÜR FAMILIE, SENIOREN, FRAUEN UND JUGEND, 2002 :

$<$ http://www.bmfsfj.de/dokumente/Pressemitteilung/ix_67769_4911.htm>, site du Bundesministerium für Familie, Senioren, Frauen und Jugend, date de création de la page : 23 janvier 2002, date de consultation 18 février 2002

BUNDESMINISTERIUM FÜR FAMILIE, SENIOREN, FRAUEN UND JUGEND, 2002 : 50 Jahre

Mutterschutzgesetz - eine Erfolgsgeschichte, 367/02,

<http://www.bmfsfj.de/dokumente/Pressemitteilung/ix_67769.htm>, site du

Bundesministerium für Familie, Senioren, Frauen und Jugend

BUNDESMINISTERIUM FÜR FAMILIE, SENIOREN, FRAUEN UND JUGEND, 2001 : «351/01: 14

Wochen Mutterschutz auch bei vorzeitiger Geburt-Bundesregierung beschließt

Gesetzesänderung»,

<http://www.bmfsfj.de/dokumente/Pressemitteilung/ix_64484_4893.htm>, site du

Bundesministerium für Familie, Senioren, Frauen und Jugend 
BUNDESMINISTERIUM FÜR FAMILIE, SENIOREN, FRAUEN UND JUGEND, 2000 : «Mutterschutzgesetz - MuSchG», <http://www.bmfsfj.de/dokumente/Artikel/ix_27550.htm>, site du Bundesministerium für Familie, Senioren, Frauen und Jugend, date de création de la page : 28 décembre 2000

BUNDESVERFASSUNGSGERICHT 1998b : «1BvR 1872/94 vom 8.1.1998, Absatz-Nr. (1 - 10)», <http://www.bverfg.de>, Bundesministerium für Familie, Senioren, Frauen und Jugend, date de création de la page : 5 décembre 2001, date de consultation : 18 février 2002

CAF, 2001 : «Toutes les prestations», site Le site des Caisses d'Allocations Familiales : prestations familiales et aides au logement, formulaires à télécharger, adresses, rôle et services des CAF, <http://www.caf.fr/catalogue>, date de mise à jour : 8 octobre 2001, date de consultation : 14 février 2002

CNAMTS, 2002 : «Congé paternité», <http://www.cnamts.fr/ass/pater/sompater.htm>, site de la Caisse nationale d'assurance maladie des travailleurs salariés, date de mise à jour du site : 16 février 2002, date de consultation : 17 février 2002

COMMUNAUTES EUROPEENNES, 2002 : «La protection sociale dans les Etats membres de l'UE et de l'Espace économique européen - Situation au 1er janvier 2001 et évolution», $<$ http://europa.eu.int/comm/employment_social/missoc2001/index_fr.htm>, MISSOC : Système d'information mutuelle sur la protection sociale des Etats membre de l'EU et de l'EEE, date de consultation : 18 février 2002

COMMUNAUTES EUROPEENNES, 2002 : «Soziale Sicherheit \& soziale Integration», <http://europa.eu.int/comm/employment_social/missoc2001/missoc_87_de.htm>, site Europa, L'union européenne en ligne, date de consultation : 18 février 2002

MINISTERE DE L'EMPLOI ET DE SOLIDARITE, 2001 : «Conférence de la famille », $<$ http://www.social.gouv.fr/famille-enfance/doss_pr/34_010611.htm>, site du Ministère de l'emploi et de solidarité, date d'actualisation de la page : juin 2001, date de consultation : 1 novembre 2001

MINISTERE DE L'EMPLOI ET DE SOLIDARITE, 1998 : «Annexe / Le quotient familial», <http://www.social.gouv.fr/htm/actu/secu/980922cca.htm>, site du Ministère de l'emploi et de solidarité, date de création : 20 février 1998, date de consultation : 15 février 2002

MinISTERE DE L'ÉCONOMIE, DES FINANCES ET DE L'INDUSTRIE, 1998 : «Aide18», <http://www.finances.gouv.fr/calcul_impot/98/aide18.htm>, site du Ministère de l'Économie, des Finances et de l'Industrie, date de modification du site : 31 janvier 2002, date de consultation : 15 février 2002

NIEDERSÄCHSISCHES LANDESAMT FÜR BEZÜGE UND VERSORGUNG, 2001 : « Anpassung der Besoldungs- und Versorgungsbezüge ab 01.01.2002», $\langle$ http://www.nlbv.de/inhalt/besoldung/tabellen/tabellenwerke.html $>$, date d'actualisation de la page : 11 décembre 01 , date de consultation de la page : 21 février 02

PATRIMOINE.COM, 2002 : «Patrimoine.com - impôts - comment calculez votre impôt sur le revenu-accueil», 〈http://www.patrimoine.com/infos/gui57.html>, date de mise à jour : février 2002, date de consultation : 15 février 2002

STATISTISCHES BunDESAMT, 2001d : «Bevölkerung - Eheschließungen, Geborene, Gestorbene - Ehescheidungen», <http://www.destatis.de/basis/d/bevoe/bevoetab1.htm>, site du Statistisches Bundesamt Deutschland, date de mise à jour : 10 octobre 2001 


\section{INDEX DES SIGLES UTILISES}

ACEPP Association des Collectifs Enfant-Parents-Professionnelle

AFEAMA Aide à la Famille pour l'Emploi d'une Assistante Maternelle Agrée

AFPA Association nationale pour la Formation Professionnelle pour Adulte

AGED Allocation de Garde d'Enfant à Domicile

ALR Allgemeines Landrecht für die preussischen Staaten

ANPE Agence Nationale Pour l'Emploi

$\mathrm{AO}$

Allocation Orphelin

APE

Allocation Parentale d'Education

API

Allocation pour Parent Isolé

APJE

Allocation Parentale pour Jeune Enfant

Art.

Article

ASF

Allocation de Soutien Familial

$\mathrm{BA}$

Bundesanstalt für Arbeit

BFK

Bundesfamilienkasse

BGB

Bürgerliches Gesetzbuch

$\mathrm{BiB}$

Bundesinstitut für Bevölkerungsforschung

BMA

Bundesministerium für Arbeit und Sozialordnung

BMFSFJ Bundesministerium für Familie, Senioren, Frauen und Jugend

BO

Bulletin Officiel

BSHG Bundessozialhilfegesetz

BverfG Bundesverfassungsgericht

C. civ. Code Civil

CAF Caisses d'Allocations Familiales

CDU Christlich Demokratische Union

CNAF Caisse Nationale d'Allocations Familiales

Cnamts Caisse nationale d'assurance maladie des travailleurs salariés

CNRS Centre National de la Recherche Scientifique

CREDES Centre de Recherche d'Etudes et de Documentation en Economie de la Santé

CSU Christlich Soziale Union

DARES Direction de l'Animation de la Recherche des Etudes et des Statistiques

DFT Division Familiale du Travail

DGB Deutscher Gewerkschaftsbund

DREES Direction de la Recherche, des Etudes, de l'Evaluation et des Statistiques

FDP

FMCP

GESIS

HLU

IAB

ifo

Freie Demokratische Partei

Fédération des Mouvements de la Conditions Paternelle

Gesellschaft Sozialwissenschaftlicher Infrastruktureinrichtungen

Hilfe zum Lebensunterhalt

Institut für Arbeitsmarkt- und Berufsforschung

ILO

Institut für Wirtschaftsforschung, München

INED Insitut National d'Etudes Démographiques

INSEE Institut National de la Statistique et des Etudes Economiques

IRES Institut de Recherches Economiques et Sociales

IRESCO Institut de recherche sur les sociétés contemporaines 
IW Köln Institut der deutschen Wirtschaft, Köln

IZ Informationszentrum Sozialwissenschaften

JAF Juge aux Affaires Familiales

JO Journal Officiel

KindRG Kindschaftsrechtsgesetz

LASMAS Laboratoire d'Analyse Secondaire et de Méthodes Appliquées à la Sociologie

LBS Familienstudie Übergang zur Elternschaft

LIS Luxembourg Income Study

LPartG Lebenspartnerschaftsgesetz

MATISSE Modélisation Appliquée, Transformations Institutionnelles et Stratégies SocioEconomiques

MittAB Mitteilungen aus der Arbeitsmarkt- und Berufsforschung

OECD Organisation for economic cooperation and developpement

OLG Oberlandesgericht

OSCE Office statistique des communautés européennes

PACS Pacte Civil de Solidarité

PS Parti Socialiste

RMI Revenu Minimum d'Insertion

SPD Sozialdemokratische Partei Deutschlands

StBA Statistisches Bundesamt

TMO Signe d'une société d'enquête et de sondage (Paris)

UCANSS Union des Caisses Nationales de Sécurité Sociale

UNAF Union Nationale des Associations Familiales

URSSAF Union de Recouvrement des côtisations de Sécurité Sociale et d'Allocations Familiales

UVG Unterhaltvorschußgesetz

VAMV Verband Alleinerziehender Mütter und Väter

WZB Wissenschaftszentrum Berlin für Sozialforschung 


\section{ANNEXES}

\section{ANNEXE 1 Annexe méthodologique}

ANNEXE 2 Types de famille répartis en fonction de la situation conjugale et de la présence d'enfants, en Allemagne

ANNEXE 3 Familles naturelles, en Allemagne

ANNEXE 4 La dénatalité en Allemagne et en France

ANNEXE 5 Taux d'activité des femmes en fonction de leur situation matrimoniale et de l'âge des enfants, en Allemagne

ANNEXE 6 Femmes actives, en fonction de la situation familiale, de l'âge et du nombre d'enfants, en Allemagne

ANNEXE 7 Activité professionnelle à temps complet et à temps partiel, en Allemagne

ANNEXE 8 Activité professionnelle en fonction du volume horaire travaillé par semaine

ANNEXE 9

Activité professionnelle marginale en fonction du sexe et du statut matrimonial, en Allemagne

ANNEXE 10

Répartition des femmes et des hommes en fonction de leur participation au marché du travail, en Allemagne

ANNEXE 11 Répartition des femmes et des hommes actifs en fonction du statut familial et du sexe, en Allemagne

ANNEXE 12 Règles d'imposition en France en fonction de la situation familiale et du nombre de personne à charge

ANNEXE 13 Chronologie juridique de la mise en place de la parentalité en France et en Allemagne

ANNEXE 14 Construction de la catégorie «Autre» dans les enquêtes IZS-Zander

ANNEXE 15

Les temps des hommes et des femmes consacrés aux activités ménagères en DDR 1965

ANNEXE 16 Fréquence de l'aide du réseau informel 


\section{ANNEXE 1 Annexe méthodologique}

La codirection scientifique du travail de recherche par le Prof. Dr. I. Ostner, Institut für Sozialpolitik de l'Université Georg-August de Göttingen, et par Dr. M.-A. BarrèreMaurisson, CNRS-MATISSE Université de Paris I-Sorbonne, spécialistes des comparaisons internationales et de la place la famille dans les régulations, ainsi que des séjours prolongés dans les deux pays (cf. Tableau ci-dessous) ont permis d'acquérir une compréhension approfondie de la logique sociétale de chaque pays et d'éviter l'écueil d'éthnocentricité. La cotutelle de thèse a été institutionnalisée par la signature d'un contrat de cotutelle entre les deux directrices de thèse et les présidents d'université.

Séjours à Université de Paris I-Sorbonne et à l'Université Georg-August de Göttingen dans le cadre de la cotutelle de thèse

\begin{tabular}{|c|c|c|c|}
\hline \multicolumn{4}{|c|}{ Séjours à MATISSE (Paris) } \\
\hline $\begin{array}{l}1997 \\
\text { 14nov97-22nov97 } \\
\text { 30nov97-15déc97 }\end{array}$ & $\begin{array}{l}\text { 1998-1999 } \\
\text { 21dec-27jan 98 } \\
\text { 9fev98-1mars98 } \\
\text { 21mars98-16nov98 } \\
\text { 6mars99-23avr99 } \\
\text { 5juin99-10juin99 } \\
\text { 13 sept 99-1oct99 } \\
\end{array}$ & $\begin{array}{l}\mathbf{2 0 0 0} \\
24 \text { fevr00-14avr00 } \\
12 \text { juin00-1juil01 } \\
1 \text { oct00-29oct00 } \\
19 \text { nov00-3dec00 }\end{array}$ & $\begin{array}{l}\mathbf{2 0 0 1} \\
\text { 11mars-31mars01 } \\
\text { 21juin01-3juillet01 } \\
\text { 24août01-13sept01 } \\
\text { 1nov01-19nov01 }\end{array}$ \\
\hline \multicolumn{4}{|c|}{ Séjours à l'institut de politiques sociales (Göttingen) } \\
\hline $\begin{array}{l}1997 \\
28 \text { oct-14nov97 } \\
\text { 22nov-30nov } 97 \\
\text { 15déc-21dec97 }\end{array}$ & \begin{tabular}{|l} 
1998-1999 \\
27jan98-9fev98 \\
16nov98-6mars99 \\
24avr99-5juin99 \\
10juin99-13sept99
\end{tabular} & $\begin{array}{l}\mathbf{2 0 0 0} \\
\text { 1oct99-24fev00 } \\
\text { 14avr00-12juin00 } \\
\text { 1juil00-1oct00 } \\
\text { 29oct00-19nov00 }\end{array}$ & $\begin{array}{l}\mathbf{2 0 0 1} \\
\text { 3dec00-11mars01 } \\
\text { 1avr01-21juin01 } \\
\text { 3juil01-23août01 } \\
\text { 14sept01-2nov01 } \\
\text { 20nov01-juin02 } \\
\end{array}$ \\
\hline
\end{tabular}

Dans le contexte allemand de la cotutelle, nous avons été associée à l'école doctorale de Göttingen «l'avenir du modèle social européen» dont l'objectif est d'identifier les modèles sociaux et leur viabilité face aux changements actuels. Dans ce contexte, nos travaux ont contribué à penser la place de la famille dans les arrangements sociaux contemporains. Notre coopération avec l'école doctorale nous a permis de travailler plus particulièrement sur l'articulation entre Famille et Etat, par une analyse des politiques sociales.

Dans le cadre de la cotutelle française nous sommes membre du groupe de travail «la division familiale du travail» de l'institut français MATISSE de l'université Paris I. En son sein, il nous a été permis d'approfondir le lien institutionnel entre la famille et le marché du travail, de réaliser et d'exploiter en exclusivité les données nationales sur le partage du travail parental, domestique et professionnel au sein des familles françaises. Ce travail d'exploitation a donné lieu à plusieurs publications (Barrère-Maurisson, Buffier-Morel, Rivier 2001 ; Barrère-Maurisson, Marchand, Rivier 2000 ; Barrère-Maurisson, Minni, Rivier 2001 ; Barrère-Maurisson, Rivier 2002) et différentes communications à des colloques scientifiques. 
Les données empiriques pour la comparaison sociétale sont constitués à partir de l'analyse des textes de loi relatifs à l'autorité parentale et des décisions jurisprudentielles, de l'analyse des politiques familiales et de l'analyse secondaire des enquêtes budget- temps.

Les données primaires dans le cas de la France, sont extraites de l'exploitation statistique, effectuée par nous même, de deux enquêtes :

1. la première exploitation concerne l'enquête nationale du Groupe Division Familiale du Travail intitulée «La division du travail dans les familles entre activités rémunérées et non rémunérées, le rôle des politiques», volet national du réseau «European Network on Policies and the Division of Unpaid and Paid Work» de l'université de Tilburg (auquel la DARES et le Service des Droits des Femmes apportent leur soutien depuis 1995).

L'échantillon de plus de 1000 chefs de ménage (ou conjoints) âgés de 20 à 49 ans, est représentatif de la population, stratifiée selon les cinq critères suivants : la région INSEE (en huit catégories), la taille de l'agglomération, le sexe (croisé avec le fait d'être actif ou inactif), l'âge (en trois tranches) et la catégorie socioprofessionnelle. Le redressement s'est appuyé sur les données du «recensement de la population» de 1990, et les résultats ont été confrontés, pour validation, avec des informations plus récentes issues de «l'enquête emploi» de l'INSEE. Cette confrontation s'est révélée satisfaisante.

Toutes les catégories de ménage sont prises en considération, y compris les ménages ne comportant pas de couple (personnes seules, chefs de famille monoparentale, notamment). Il était en effet intéressant de connaître l'organisation des personnes sans conjoint pour les tâches de la vie quotidienne, et ce, d'autant plus que cette catégorie représente une part non négligeable de la population étudiée (plus d'un tiers).

L'enquête a été réalisée par l'agence TMO. Elle s'est déroulée sur le territoire national au cours de deux semaines. Il s'agit d'une enquête réalisée en face-à-face, en deux temps : une partie en présence de l'enquêteur et une partie en auto-administré. Les enquêteurs ont réalisé la première partie de l'enquête au domicile des interviewés, leur ont ensuite expliqué comment remplir les parties qui demandaient un effort de réflexion plus important, et ont relevé les questionnaires deux à trois jours plus tard.

Le questionnaire est une adaptation au cas français du modèle standard européen élaboré par le réseau en langue anglaise. Il a totalement été retravaillé par le Groupe Division Familiale du Travail, en fonction de plusieurs objectifs : coller au mieux à la réalité française, maintenir cependant l'esprit du document original pour rendre possible la comparaison internationale, permettre le rapprochement avec l'enquête INSEE «Emploi du temps 1998-1999» dont on a retenu un certain nombre de questions et d'items. Le questionnaire final comprend plusieurs cahiers :

A - informations personnelles, composition du ménage

$\mathrm{B}$ - activité professionnelle

$\mathrm{C}$ - gestion du temps et répartition des rôles domestiques

$\mathrm{D}$ - conciliation entre vie de famille et vie professionnelle

$\mathrm{S}$ - revenus

E - habitat, réseaux de solidarité et vie associative

F - opinions (à mesurer notamment dans le cadre de diverses échelles d'attitude).

Ce sont les cahiers A, B, D, S, E qui ont été remplis en présence de l'enquêteur.

Préalablement, une enquête-test a été réalisée sur un petit échantillon, ce qui, avec les remarques et suggestions de TMO, a permis l'adaptation finale du questionnaire. 
L'exploitation des données nationales a été effectuée à l'aide de tris croisés (en ce qui concerne les variables individuelles et des ménages) et de l'évaluation des carnets d'activités et du relevé des temps passés à chaque activité.

Les calculs des temps ont permis de conclure sur le partage du travail familial en France (cf. Chapitre III).

2. La seconde analyse statistique traite de l'«Enquête Emploi du temps 1998-1999» de l'INSEE ont complété les données précédentes.

Les données empiriques pour l'Allemagne sont extraites d'une exploitation secondaire des enquêtes bugdet-temps et de nombreux projets sur les familles et le partage des tâches. 
ANNEXE 2 Nombre absolu (en milliers) des types de famille répartis en fonction de la situation conjugale et de la présence d'enfants, en 1991 et 2000, en Allemagne

\begin{tabular}{|c|c|c|c|c|c|c|c|}
\hline & \begin{tabular}{|l} 
Familles \\
naturelles \\
sans enfant
\end{tabular} & $\begin{array}{l}\text { Couples } \\
\text { mariés sans } \\
\text { enfant }\end{array}$ & $\begin{array}{l}\text { Personnes } \\
\text { vivant } \\
\text { seules }\end{array}$ & $\begin{array}{l}\text { Familles } \\
\text { naturelles } \\
\text { avec } \\
\text { enfant(s) }\end{array}$ & $\begin{array}{l}\text { Couples } \\
\text { mariés } \\
\text { avec } \\
\text { enfant(s) }\end{array}$ & $\begin{array}{l}\text { Familles } \\
\text { monoparen } \\
\text { tales }\end{array}$ & Ensemble \\
\hline \multicolumn{8}{|c|}{ Ancienne Allemagne de l'Est } \\
\hline 1991 & 147 & 1687 & 1839 & 180 & 2288 & 502 & 6643 \\
\hline 2000 & 268 & 1822 & 2412 & 252 & 1715 & 542 & 7011 \\
\hline \multicolumn{8}{|c|}{ Ancienne Allemagne de l'Ouest } \\
\hline 1991 & 868 & 6706 & 10019 & 198 & 8811 & 1660 & 28262 \\
\hline 2000 & 1.222 & 7778 & 11337 & 371 & 8140 & 1803 & 30651 \\
\hline \multicolumn{8}{|c|}{ Allemagne réunifiée } \\
\hline 1991 & 1.015 & 8.393 & 11.858 & 378 & 11.099 & 2.162 & 34905 \\
\hline 2000 & 1.490 & 9.600 & 13.749 & 623 & 9.855 & 2.345 & 37662 \\
\hline
\end{tabular}

Source: nos propres calculs à partir des données de l'enquête Mikrozensus 2000 (Statistisches Bundesamt 2001 : 61-68-71)

ANNEXE 3 Nombre absolu (en milliers) des familles naturelles avec enfant et sans enfant, en Allemagne

Dans l'ancienne Allemagne de l'Ouest

\begin{tabular}{|l|l|l|l|l|l|}
\hline & 1982 & 1985 & 1988 & 1989 & 1990 \\
\hline Sans enfant & 445 & 616 & 723 & 745 & 856 \\
\hline Avec enfant & 71 & 70 & 97 & 97 & 107 \\
\hline Ensemble & 516 & 686 & 820 & 842 & 963 \\
\hline
\end{tabular}

En Allemagne

\begin{tabular}{|l|l|l|l|l|l|l|l|l|l|}
\hline & 1991 & 1992 & 1993 & 1994 & 1996 & 1997 & 1998 & 1999 & 2000 \\
\hline Sans enfant & 1015 & 1076 & 1146 & 1196 & 1316 & 1375 & 1425 & 1460 & 1489 \\
\hline Avec enfant & 378 & 409 & 436 & 462 & 508 & 530 & 557 & 594 & 624 \\
\hline Ensemble & 1393 & 1485 & 1582 & 1658 & 1824 & 1905 & 1982 & 2054 & 2113 \\
\hline
\end{tabular}

(Statistisches Bundesamt 2002a : 64) 


\section{ANNEXE 4 La dénatalité en Allemagne et en France}

L'Allemagne a été marquée en 1996 par une reprise des naissances. C'est la première augmentation depuis les années 1990 ; elle est plus marquée dans les nouveaux Bundesländer $(11,5 \%)$ que pour l'ancienne Allemagne de l'Ouest où le nombre de naissances avait constamment mais faiblement diminué depuis 1991 ; 1996 est donc la première augmentation avec 2,9\% de naissances en plus (Sozialpolitische Umschau 1997b). La tendance se maintient en 1997 : sur l'ensemble de l'Allemagne il y a 2,7 \% de naissance en plus, respectivement $2,1 \%$ pour l'Allemagne de l'Ouest et 7,5\% pour l'ancienne Allemagne de l'Est où le chiffre de 1997 représente environ $55 \%$ du nombre de naissances en 1990 (Sozialpolitische Umschau 1998b). L'augmentation s'est maintenue en 1998.

Depuis 1999 et 2000, le nombre de naissances a de nouveau diminué sur l'ensemble de l'Allemagne (-2,7\% entre 1998 et 1999) (Statistisches Bundesamt 2001d), essentiellement en Allemagne de l'Ouest depuis 1998 (Statistisches Bundesamt 2002a : 67-69), tandis que l'ancienne Allemagne de l'Est a vu une reprise constante des naissances depuis 1995 sans pour autant avoir retrouvé le niveau de 1990 (Statistisches Bundesamt 2002a : 68 ; Statistisches Bundesamt 2000e).

La majorité des enfants vivent en présence d'un frère ou d'une sœur. Les données de l'enquête Mikrozensus de 1995 montrent que $31 \%$ des enfants sur l'ensemble de l'Allemagne, sont des enfants uniques ${ }^{223}, 44,6 \%$ vivent avec un frère ou une sœur, $17 \%$ avec deux frères et sœurs, et 7,4 \% avec 3 ou plus de frères et sœurs (Sozialpolitische Umschau 1998c).

La proportion d'enfant unique sur l'ensemble de l'Allemagne réunifiée se maintient en 2000, avec une proportion de $30 \%$ d'enfant unique sur l'ensemble des enfants vivant dans les familles. On observe quelques différences entre l'ancienne Allemagne de l'Est et l'ancienne Allemagne de l'Ouest ${ }^{224}: 31 \%$ des enfants sont des enfants uniques dans l'ancienne Allemagne de l'Ouest, respectivement $33 \%$ pour l'ancienne Allemagne de l'Est ; $44 \%$ vivent avec un frère ou une sœur, respectivement $50 \%$ pour l'ancienne Allemagne de l'Est : $18 \%$ des enfants à l'Ouest vivent avec deux frères et sœurs, contre $13 \%$ en ancienne Allemagne de l'Est. Enfin $8 \%$ des enfants vivent dans une famille nombreuse avec plus de trois frères et sœurs, contre 4\% en Allemagne de l'Est (Sozialpolitische Umschau 1995). Les fratries sont donc moins importantes dans l'ancienne Allemagne de l'Est où la proportion d'enfant unique est légèrement plus importante mais la fratrie de deux enfants reste majoritaire. Le nombre d'enfant varie avec la forme du ménage : $54 \%$ des couples mariés ont deux enfants ou plus, 68\% des familles monoparentales ou cohabitantes ont un seul enfant (Engstler 1999: 44).

\footnotetext{
${ }^{223}$ Sur l'ensemble des enfants uniques, dans l'ancienne Allemagne de l'Ouest, $71 \%$ vivent au sein d'un couple marié, $23 \%$ au sein d'une famille monoparentale dont le chef de famille est une femme, (ces chiffres comprennent la proportion de familles naturelles et recomposées), $6 \%$ vivent dans une famille monoparentale dont le chef de famille est un homme (il peut y avoir des comptes doubles avec les familles cohabitantes) (Statistisches Bundesamt 2001c). Pour l'ancienne Allemagne de l'Est, la proportion d'enfants uniques vivant au sein d'un couple marié est moins importante (62\%), $32 \%$ au sein d'une famille monoparentale, cohabitante ou recomposée dont les chefs de famille est une femme, et $6 \%$ au sein d'une famille monoparentale dont le chef de famille est un homme (Statistisches Bundesamt 2001c).

${ }^{224}$ Dans l'ancienne Allemagne de l'Est, les enfants ont moins de frères et sœur, et sont plus souvent enfant unique, ce qui s'explique par la forte baisse des naissances (conséquente aux nouvelles conditions économiques et sociales) (Engstler $1999: 32$ ).
} 
L'indice conjoncturel de fécondité en France et en Allemagne suit la tendance décroissante qui s'observe sur l'ensemble de l'Union européenne ${ }^{225}$ : la fécondité a diminué de 47\% entre 1965 et 1994 (Bégeot, Fernandez-Cordon 1997 : 37). Mais si ses évolutions sont semblables, les différences sont très fortes entre les deux pays : la France a un des indices conjoncturels de fécondité les plus forts d'Europe et l'Allemagne un des plus faibles (Hantrais 1994 : 139).

Le taux de fécondité à la fin des années 1990 atteint 1,7 pour la France contre 1,44 en moyenne sur l'Europe. «La proportion d'enfants de moins de trois ans y est également parmi les plus importantes : 3,7 \% de la population française pour 3,2\% en moyenne dans les quinze pays de l'union européenne.» (Fenet, Leprince, Périer 2001 : 12). En France, le taux de natalité a constamment diminué depuis la fin du baby-boom dans le milieu des années 1960. Le taux de natalité avait continué à diminuer dans les années 1990, et même à chuter en 1992 et au début de l'année 1993. Il atteint son niveau le plus bas en 1994. Un redressement s'est confirmé au cours du second semestre 1995 par une hausse de 2,5\% par rapport à 1994 (INSEE Première, 1996) ${ }^{226}$.

En résumé, depuis les années 1970, on a une baisse constante du nombre d'enfants. Malgré ce phénomène de dénatalité, la parentalité concerne toujours la majorité des ménages. On observe, depuis années 1990, et en particulier depuis 1995, des phénomènes de reprise de la natalité. Lorsqu'il y a parentalité au sein d'un ménage, dans la majeure partie des cas cela concerne plusieurs enfants.

\footnotetext{
${ }^{225}$ La première transition démographique a été vécue par tous les pays de l'Europe : forte baisse de la mortalité, la fécondité n'ayant fait que s'adapter. La seconde, depuis les années 1960, concerne les conditions mêmes de la reproduction : la baisse de la fécondité. Néanmoins, les diversités nationales composent avec ce processus général (Bégeot, Fernandez-Cordon 1997 : 33).

${ }^{226}$ L'âge moyen des mères à la naissance de leur premier enfant diminue jusqu'en 1975 en France. Il augmente par la suite pour atteindre 28,8 ans pour les mères françaises en 1994 (INSEE Première 1996). L'âge de la maternité est passé à un peu plus de 29 ans en 2001 (respectivement 28,3 ans en 1990) (Fenet, Leprince, Périer $2001: 13)$.
} 
ANNEXE 5 Le taux d'activité des femmes en fonction de leur situation matrimoniale et de l'âge des enfants en Allemagne, en pourcentage

\begin{tabular}{|c|c|c|c|c|c|c|c|}
\hline \multirow[t]{2}{*}{ Enfant dans la famille } & \multirow[t]{2}{*}{ Ensemble } & \multirow[t]{2}{*}{ Mariée } & \multirow{2}{*}{$\begin{array}{l}\text { Personne seule } \\
\text { et famille } \\
\text { monoparentale }\end{array}$} & \multicolumn{4}{|l|}{ dont } \\
\hline & & & & Célibataire & Mariée $^{3}$ & Veuve & Divorcée \\
\hline \multicolumn{8}{|l|}{ Avril 1991} \\
\hline Ensemble (toutes femmes actives) & 57,1 & 54,4 & 61,9 & 63,4 & 66,0 & 36,4 & 72,8 \\
\hline Femmes actives sans enfant ${ }^{1}$ & 56,5 & 51,1 & 60,5 & 62,9 & 67,7 & 30,3 & 69,4 \\
\hline Avec un enfant de moins de 6 ans & 50,1 & 48,4 & 62,0 & 65,1 & 52,8 & 54,7 & 60,6 \\
\hline Avec un enfant de moins de 18 ans & 59,1 & 57,3 & 70,7 & 70,4 & 63,2 & 61,1 & 74,7 \\
\hline \multicolumn{8}{|l|}{ Mai 2000} \\
\hline Ensemble (toutes femmes actives) & 57,8 & 56,7 & 59,4 & 59,8 & 64,9 & 36,8 & 64,9 \\
\hline Femmes actives sans enfant ${ }^{1}$ & 54,0 & 49,1 & 57,6 & 59,5 & 64,3 & 30,8 & 63,2 \\
\hline Avec un enfant de moins de 6 ans & 51,8 & 51,7 & 52,4 & 52,9 & 47,8 & 53,3 & 54,0 \\
\hline Avec un enfant de moins de 18 ans & 63,3 & 62,5 & 67,2 & 63,1 & 64,5 & 63,6 & 72,4 \\
\hline
\end{tabular}

Source : résultats de l'enquête Mikrozensus 2000 (Statistisches Bundesamt 2002b) (Femmes âgées entre 15 et 65 ans)

${ }^{1}$ sans enfant célibataire vivant au foyer

${ }^{2}$ Personnes seules sans enfant, familles monoparentales (et comprend également les familles naturelles)

${ }^{3}$ Mariée (mais séparée) 
ANNEXE 6 Femmes actives, en mai 2000, en fonction de la situation familiale, de l'âge et du nombre d'enfants ${ }^{4}$

\begin{tabular}{|c|c|c|c|c|c|c|c|}
\hline \multirow[t]{2}{*}{ Taux d'activité des femmes } & \multirow[t]{2}{*}{ Ensemble } & \multirow[t]{2}{*}{ Mariée } & \multirow{2}{*}{$\begin{array}{l}\begin{array}{l}\text { Personne seule et } \\
\text { famille } \\
\text { monoparentale }\end{array} \\
\end{array}$} & \multicolumn{4}{|l|}{ dont } \\
\hline & & & & Célibataire & Mariée $^{3}$ & Veuve & Divorcée \\
\hline Femmes actives avec enfant $(\mathrm{s})^{4}$ & 62,8 & 62,0 & 66,6 & 63,9 & 65,4 & 52,4 & 73,3 \\
\hline Femmes actives sans enfant $^{1}$ & 54,0 & 49,1 & 57,6 & 59,5 & 64,3 & 30,8 & 63,2 \\
\hline Ensemble (toutes femmes actives) & 57,8 & 56,7 & 59,4 & 59,8 & 64,9 & 36,8 & 67,9 \\
\hline \multicolumn{8}{|l|}{ En fonction du nombre d'enfant } \\
\hline Avec un enfant & 65,5 & 64,2 & 69,1 & 68,3 & 68,2 & 50,0 & 76,7 \\
\hline Avec deux enfants & 64,2 & 64,0 & 66,1 & 53,4 & 67,6 & 60,8 & 72,3 \\
\hline Avec trois enfants & 52,8 & 53,1 & 50,7 & 30,4 & 46,2 & 50,0 & 59,1 \\
\hline \multicolumn{8}{|l|}{ En fonction de l'âge des enfants } \\
\hline moins de 18 ans & 63,3 & 62,5 & 67,2 & 63,1 & 64,5 & 63,6 & 72,4 \\
\hline entre 15 et 18 ans & 70,2 & 69,6 & 73,0 & 72,8 & 70,2 & 63,7 & 75,9 \\
\hline moins de quinze ans & 61,5 & 60,6 & 65,3 & 61,8 & 62,8 & 61,8 & 70,1 \\
\hline moins de six ans & 51,8 & 51,7 & 52,4 & 52,9 & 47,8 & 53,3 & 54,0 \\
\hline moins de trois ans & 48,3 & 48,6 & 46,5 & 47,5 & 41,0 & - & 46,0 \\
\hline
\end{tabular}

Source : Statistisches Bundesamt 2002a : 109 (Femmes âgées entre 15 et 65 ans)

${ }^{1}$ sans enfant célibataire vivant au foyer

${ }^{2}$ Personnes seules sans enfant, familles monoparentales (et comprend également les familles naturelles)

${ }^{3}$ Mariée (mais séparée)

${ }^{4}$ sans limite d'âge pour l'enfant 
ANNEXE 7 Activité professionnelle à temps complet et à temps partiel des salariés, en 1991 et 2000 , en Allemagne, en \%

\begin{tabular}{|c|c|c|c|c|c|c|c|c|c|}
\hline & \multicolumn{3}{|c|}{ Allemagne réunifiée } & \multicolumn{3}{|c|}{$\begin{array}{lll}\text { Ancienne } & \text { Allemagne } & \text { de } \\
\text { l'Ouest } & & \end{array}$} & \multicolumn{3}{|c|}{ Ancienne Allemagne de l'Est } \\
\hline & Ensemble & Hommes & Femmes & Ensemble & Hommes & Femmes & Ensemble & Hommes & Femmes \\
\hline \multicolumn{10}{|l|}{1991} \\
\hline $\begin{array}{l}\text { Temps } \\
\text { complet }\end{array}$ & 86,0 & 97,9 & 60,8 & 84,6 & 97,7 & 65,7 & 91,1 & 98,8 & 82,5 \\
\hline $\begin{array}{l}\text { Temps } \\
\text { partiel }\end{array}$ & 14,0 & 2,1 & 30,2 & 15,4 & 2,3 & 34,3 & 8,9 & 1,2 & 17,5 \\
\hline Ensemble & 100 & 100 & 100 & 100 & 100 & 100 & 100 & 100 & 100 \\
\hline \multicolumn{10}{|l|}{2000} \\
\hline $\begin{array}{l}\text { Temps } \\
\text { complet }\end{array}$ & 80,2 & 95,2 & 61,7 & 78,5 & 95,0 & 58,1 & 87,4 & 96,1 & 77,2 \\
\hline $\begin{array}{l}\text { Temps } \\
\text { partiel }\end{array}$ & 19,8 & 4,8 & 38,3 & 21,5 & 5,0 & 41,9 & 12,6 & 3,9 & 22,8 \\
\hline Ensemble & 100 & 100 & 100 & 100 & 100 & 100 & 100 & 100 & 100 \\
\hline
\end{tabular}

Source : résultats de l'enquête Mikrozensus 2000 (Statistisches Bundesamt 2001 : 29)

ANNEXE 8 Activité professionnelle en fonction du volume horaire travaillé par semaine, pour les hommes et les femmes, en \%

\begin{tabular}{|l|l|l|}
\hline Volume horaire travaillé par semaine & Hommes & Femmes \\
\hline Moins de 21 heures & 4,2 & 26,3 \\
\hline Entre 21 heures et 39 heures & 39,9 & 44,0 \\
\hline Entre 40 et 44 heures & 37,1 & 23,2 \\
\hline Plus de 45 heures & 18,8 & 6,5 \\
\hline Ensemble & $100 \%$ & $100 \%$ \\
\hline
\end{tabular}

Source : Statistisches Bundesamt (2002a : 103) (données pour mai 2000)

ANNEXE 9 Activité professionnelle marginale en fonction du sexe et du statut matrimonial, en Allemagne, en \%

\begin{tabular}{|l|l|l|}
\hline & Femme & Homme \\
\hline Marié(e) & 75,0 & 45,4 \\
\hline Célibataire & 15,4 & 47,2 \\
\hline Divorcé(e) & 5 & 5,1 \\
\hline Veuf(ve) & 4,7 & 2,2 \\
\hline Ensemble & $100 \%$ & $100 \%$ \\
\hline
\end{tabular}

Source : Statistisches Bundesamt (2001:31) 
ANNEXE 10 La répartition des femmes et des hommes en fonction de leur participation au marché du travail, en Allemagne en 1991 et 2000*

\begin{tabular}{|c|c|c|c|c|}
\hline & $\begin{array}{l}\text { Actifs } \\
\text { occupés }\end{array}$ & $\begin{array}{l}\text { Personne à la recherche } \\
\text { d'un emploi }\end{array}$ & Inactif & Ensemble \\
\hline \multicolumn{5}{|c|}{ Femmes } \\
\hline 1991 & 57,0 & 5,1 & 37,9 & $100 \%$ \\
\hline 2000 & 57,7 & 6,3 & 36,0 & $100 \%$ \\
\hline \multicolumn{5}{|c|}{ Hommes } \\
\hline 1991 & 78,4 & 4,5 & 17,1 & $100 \%$ \\
\hline 2000 & 72,8 & 7,1 & 20,1 & $100 \%$ \\
\hline
\end{tabular}

Source : Statistisches Bundesamt (2001: 37)

* Population âgée entre 15 et 65 ans

ANNEXE 11 La répartition des femmes et des hommes actifs en fonction du statut familial et du sexe, en Allemagne, en \%

\begin{tabular}{|l|l|l|l|}
\hline Statut familial & Hommes & Femmes & Ensemble \\
\hline Célibataire & 33,4 & 28,3 & 31,1 \\
\hline Marié & 60,2 & 59,8 & 60,0 \\
\hline Veuf & 0,7 & 2,8 & 1,7 \\
\hline Divorcé & 5,7 & 9,1 & 7,2 \\
\hline Ensemble & $100 \%$ & $100 \%$ & $100 \%$ \\
\hline
\end{tabular}

Source : Statistisches Bundesamt (2002a : 106) (données pour mai 2000)

ANNEXE 12 Règles d'imposition en France en fonction de la situation familiale et du nombre de personne à charge

\begin{tabular}{|l|l|l|l|l|l|l|}
\hline & \multicolumn{5}{l|}{ nombre de parts } \\
\hline \multicolumn{4}{|l|l|l|}{ Le nombre de personnes à charge } \\
\hline Situation familiale & 0 & 1 & 2 & 3 & 4 \\
\hline $\begin{array}{l}\text { Couple marié soumis à imposition } \\
\text { commune }\end{array}$ & 2 parts & 2,5 parts & 3 parts & 4 parts & 5 parts \\
\hline Veuf ou veuve & 1 part & 2,5 parts & 3 parts & 4 parts & 5 parts \\
\hline $\begin{array}{l}\text { Célibataire, divorcé et assimilé (3) ne } \\
\text { vivant pas en concubinage }\end{array}$ & 1 part & 2 parts & 2,5 parts & 3,5 parts & 4,5 parts \\
\hline $\begin{array}{l}\text { Célibataire, divorcé et assimilé (3) vivant } \\
\text { en concubinage }\end{array}$ & 1 part & 1,5 part & 2 parts & 3 parts & 4 parts \\
\hline
\end{tabular}

Source : (Patrimoine.com 2002)

Sans personne à charge, un couple marié soumis à l'imposition commune prétend à 2 parts ; une personne veuve à 1 part ; une personne célibataire, divorcé ou assimilé ne vivant pas en concubinage, à 1 part ; et une personne célibataire, divorcé et assimilé vivant en concubinage également à 1 part. 
ANNEXE 13 Chronologie juridique de la mise en place de la parentalité en France et en Allemagne

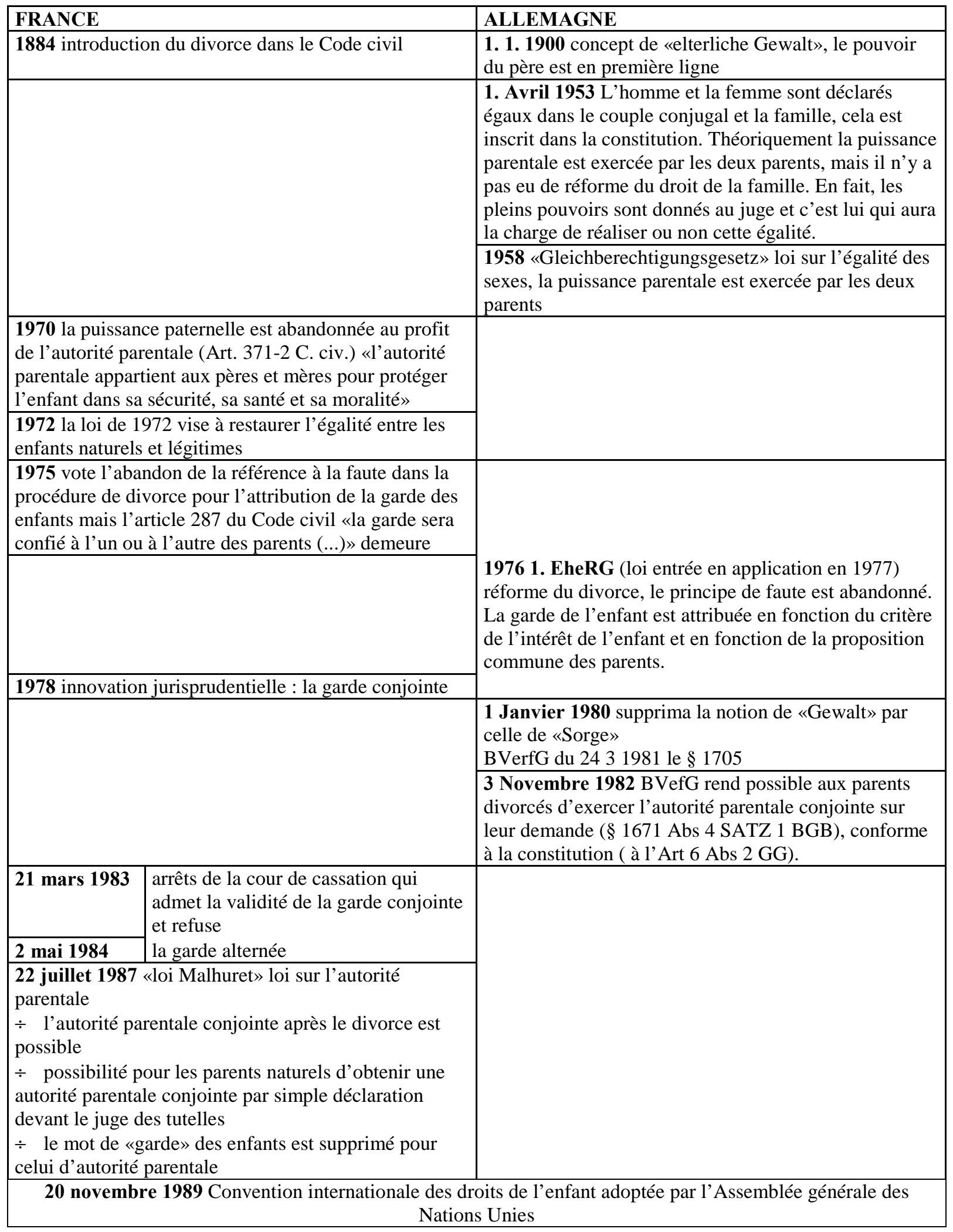


7 août 1990 Ratification de la convention internationale des droits de l'enfant loi du 26. 6. 1990 (entrée en application le 1.1.1991)

Kinder und Jugendhilfegesetz (KJHG) contribue à placer l'enfant et le jeune adulte comme sujet de droit (par exemple, un chapitre de la loi est consacré à la protection des données personnelles concernant la personne de l'enfant)

2 octobre 1990 Ratification de la convention internationale des droits de l'enfant par la DDR

7 mai 1991 BVerfG la cours décide que le $\S 1738$ I BGB (à la légitimation de l'enfant par le père, la mère perd obligatoirement son autorité parentale, qui est donnée au père), contredit la constitution Art. 6II et V de la GG (droit des parents)

6 mars 1992 Ratification de la convention internationale des droits de l'enfant par l'Allemagne réunifiée

\section{Janvier 1993}

$\div$ l'autorité parentale sera par principe exercée en commun après le divorce

$\div$ pour les familles naturelles, c'est aussi le principe qui sera appliqué si les deux parents ont reconnu

l'enfant, il leur faut cependant faire la preuve de leur vie commune
7 Avril 1995 BVerfg le père naturel est aussi porteur des droits parentaux.

1 Janvier 1996 un droit à une place dans une institution de garderie

1998 KindRG Kindschaftsrechtsgesetz Réforme des droits de l'enfant abandon des notions de filiation légitime et naturelle L'autorité parentale conjointe comme principe

1 Août 2001 LPartG Lebenspartnerschaftsgesetz 
ANNEXE 14 Construction de la catégorie «Autre» dans les enquêtes IZS et Zander

\begin{tabular}{|l|l|}
\hline \multicolumn{1}{|c|}{ IZS } & \multicolumn{1}{c|}{ Zander } \\
\hline activités de jardinage & activités de jardinage \\
soins des animaux & soins des animaux \\
tenue des comptes & dans une autre catégorie \\
lettres administratives & dans une autre catégorie \\
chauffage & - \\
chercher de l'eau & - \\
soins des personnes âgées et malades & - \\
travaux de réparation & - \\
& Couture et autres travaux manuels \\
& Tapisser et autres travaux de bricolage et de \\
& construction \\
\hline
\end{tabular}

Le relevé exhaustif des activités ménagères et leur regroupement en catégories plus agrégées ne permet pas une transparence sur la construction des catégories dans l'enquête IZS.

De fait, la construction de la catégorie «Autre» est différente selon les auteurs : Zander y intégre la couture et jardinage et les définis comme loisir, Deist/Böhner par contre les considère comme des activités domestiques ; pour Pross et Schöps, l'administration des stocks, les travaux de réparation, d'entretien, de jardinage, de réparation du linge sont considérées comme des loisirs.

ANNEXE 15 Les temps des hommes et des femmes consacrés aux activités ménagères en DDR, en 1965

\begin{tabular}{|c|c|c|c|c|}
\hline \multirow[b]{2}{*}{ Activités } & \multicolumn{2}{|l|}{ Par individu } & \multicolumn{2}{|c|}{ Pour les femmes } \\
\hline & $\begin{array}{l}\text { En heures } \\
\text { et minutes } \\
\text { par jour }\end{array}$ & $\begin{array}{l}\text { Part de } \\
\text { chaque } \\
\text { activité }\end{array}$ & $\begin{array}{l}\text { En heures } \\
\text { et minutes } \\
\text { par jour }\end{array}$ & $\begin{array}{l}\text { Part de } \\
\text { chaque } \\
\text { activité }\end{array}$ \\
\hline Ménage $^{1}$ & 1h 20 & 32,5 & $1 \mathrm{~h} 25$ & 25,5 \\
\hline Préparation des repas ${ }^{2}$ & 1h 05 & 27,5 & $1 \mathrm{~h} 50$ & 32,7 \\
\hline Lessive, soin et réparation du linge & Oh 40 & 17,5 & Oh 55 & 16,4 \\
\hline $\begin{array}{l}\text { Courses, consulter les services } \\
\text { administrations }{ }^{3}\end{array}$ & Oh 30 & 12,5 & Oh 40 & 12,7 \\
\hline $\begin{array}{l}\text { Chauffage, réparation et } \\
\text { autres activités dans le ménage }\end{array}$ & Oh 10 & 5,0 & Oh 40 & 12,7 \\
\hline Jardinage et s'occuper des animaux & Oh 10 & 5,0 & & \\
\hline Ensemble & 4h 00 & $100 \%$ & $5 \mathrm{~h} 30$ & $100 \%$ \\
\hline
\end{tabular}

Source : d'après Lippold 1971 (113 et 116)

${ }^{1}$ dont les tâches liées à la vaisselle, mais sans celles qui relèvent de la lessive

2 «faire la vaisselle» est compris dans la première catégorie

${ }^{3}$ sans les temps d'attente

${ }^{4}$ dont s'occuper de la voiture 
ANNEXE 16 Fréquence de l'aide du réseau informel, en \%

\begin{tabular}{|c|c|c|c|c|c|c|c|c|}
\hline \multirow[b]{3}{*}{ Nature de l'aide } & \multicolumn{8}{|c|}{$\begin{array}{l}\text { nombre de fois où le ménage a fait appel à une aide extérieure } \\
\text { dans l'intervalle des quatre dernières semaines }\end{array}$} \\
\hline & \multicolumn{4}{|c|}{ «Aide d'appoint» } & \multicolumn{3}{|c|}{ «Aide fréquente» } & \multirow[b]{2}{*}{ Total } \\
\hline & 1 fois & 2 fois & 3 fois & 4 fois & $\begin{array}{l}\text { de } 5 \\
14 \text { fois }\end{array}$ & $\begin{array}{l}\text { de } 15 \\
29 \text { fois }\end{array}$ & $\begin{array}{l}\text { de } 30 \\
60 \text { fois }\end{array}$ & \\
\hline $\begin{array}{l}\text { Aide pour s'occuper des } \\
\text { enfants }\end{array}$ & 21,5 & 19,3 & 6,4 & 19,8 & 18,2 & 10,9 & 3,7 & 100,0 \\
\hline $\begin{array}{l}\text { Aide pour les tâches } \\
\text { quotidiennes }\end{array}$ & 27,8 & 17,5 & 5,3 & 20,5 & 14,4 & 7,1 & 7,5 & 100,0 \\
\hline $\begin{array}{l}\text { Aide pour les courses, } \\
\text { transport }\end{array}$ & 26,4 & 20,7 & 6,7 & 27,6 & 13,0 & 3,5 & 2,1 & 100,0 \\
\hline
\end{tabular}

Source : INSEE Enquête Emploi du temps 98-99, MATISSE 


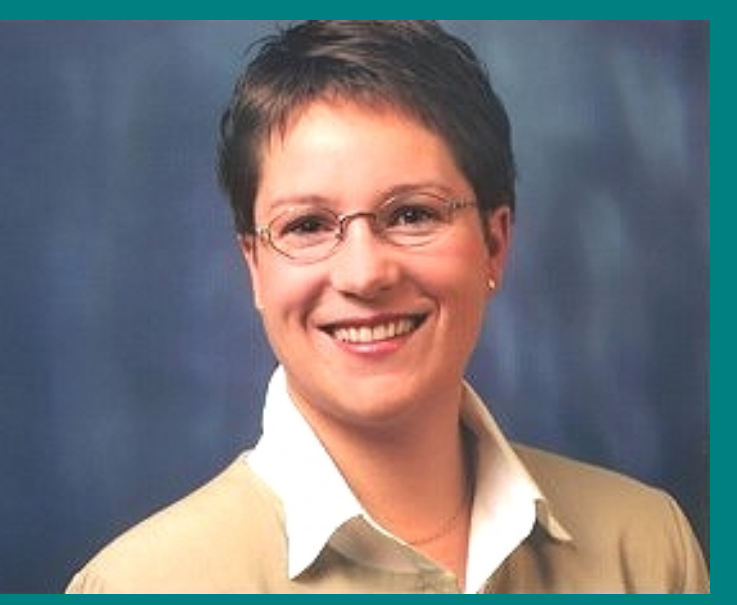

Sabine Rivier se consacre, dans le cadre de ses activités scientifiques, à l'approche sociologique comparative. Sa thèse de doctorat lui a donné l'occasion d'approfondir les termes de la comparaison sociétale entre la France et l'Allemagne, contribuant ainsi à l'analyse des liens entre le marché du travail, la famille et l'Etat-providence, et plus généralement à l'étude du changement social. Son intérêt pour ces deux pays s'était déjà affirmé lors de ses activités scientifiques au sein du réseau européen «Gender and European Welfare Regimes» (Université de Brême). Par la suite, elle est restée fidèle à la méthode comparative, et l'a intégrée dans ses travaux de recherche : à partir de 1997, elle commençait son

doctorat de sciences sociales en cotutelle franco-allemande à l'université de Paris 1 - Sorbonne (auprès de sa directrice de thèse française Dr. Marie-Agnès Barrère-Maurisson) et à l'université Georg-August de Göttingen (auprès de sa directrice de thèse allemande Prof. Dr. Ilona Ostner), où elle a été associée à l'école doctorale «l'avenir du modèle social européen» (Die Zukunft des europäischen Sozialmodells). À côté de ces activités de recherche, Sabine Rivier a été, à différentes reprises, chargée d'enseignement à la faculté sociologique de l'université de Göttingen. Au cours de son doctorat, Sabine Rivier a été allocataire de recherche du Ministère de l'éducation nationale, de l'enseignement supérieur et de la recherche, et boursière de l'Office allemand d'échanges universitaires (DAAD). Elle a toujours favorisé la recherche au cœur de réseaux internationaux, convaincue de la valeur scientifique de telles contributions : dans le cadre du programme «European Network on Policies and the Division of Unpaid and Paid Work», elle a été responsable de l'évaluation statistique de la partie nationale française. Depuis lors, elle est membre du groupe de recherche «Division Familiale du Travail» (MATISSE - Université Paris I-Sorbonne) au sein duquel elle contribue activement au travail empirique et théorique.

Sabine Rivier, Jahrgang 1972, widmet sich im Rahmen ihrer wissenschaftlichen Arbeit besonders der komparativen sozialwissenschaftlichen Forschung. Die Promotionsarbeit gab ihr die Gelegenheit, sich intensiv mit dem Vergleich Frankreichs und Deutschlands zu beschäftigen, um zum Verständnis der Wechselbeziehungen zwischen Arbeitsmarkt, Familie und Wohlfahrtsstaat und darüber hinaus des Sozialwandels beizutragen. Die Beschäftigung mit diesen beiden Ländern hat zu einem frühen Zeitpunkt in der wissenschaftlichen Karriere der Autorin begonnen. In Bremen arbeitete sie als wissenschaftliche Assistentin am europäischen Projekt „Gender and European Welfare Regimes“. Sie blieb der vergleichenden Methode auch in der Folge treu, und hat schon sie auch praktisch in ihre wissenschaftliche Arbeit integriert: 1997 begann sie ihre Doktorarbeit im Rahmen eines deutschfranzösischen Promotionsverfahrens zugleich an der Universität Paris I- Sorbonne (bei Dr. Marie-Agnès Barrère-Maurisson) und an der Georg-August Universität Göttingen (bei Prof. Dr. Ilona Ostner), wo sie am Graduiertenkolleg „Die Zukunft des europäischen Sozialmodells“ assoziiert war. Neben der Forschungstätigkeit war Sabine Rivier mehrfach Lehrbeauftragte der sozialwissenschaftlichen Fakultät der Universität Göttingen. Während der Promotion war Sabine Rivier Stipendiatin des Ministeriums für Ausbildung und Forschung Frankreichs und erhielt darüber hinaus ein Stipendium des DAAD. Sie hat immer die Arbeit in internationalen Netzwerken favorisiert, überzeugt von der Relevanz der Ergebnisse solcher wissenschaftlicher Kooperationen: im Rahmen des Programms „European Network on Policies and the Division of Unpaid and Paid Work" war sie für die Ausarbeitung und Auswertung des französischen Teils zuständig. Seitdem war sie Mitglied der Forschungsgruppe „Division Familiale du Travail“" (MATISSE - Universität Paris I-Sorbonne) in der sie einen wichtigen Teil der empirischen und theoretischen Arbeit leistete. 